Spectra S4: The information of single-peptide-based protein identifications in the large-scale experiment (part 2). 
MS/MS Fragmentation of LGAGYGLLSEAELEVVPIIAK

Found in sp|Q15642|CIP4_HUMAN, Cdc42-interacting protein 4 OS=Homo sapiens GN=TRIP10 PE=1 SV=3

Match to Query 77577: 2429.4from(810.8073,3+)

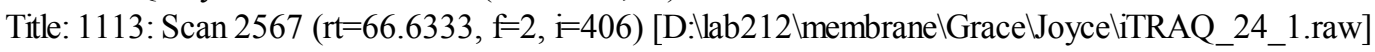

Data File:Submitted from 20120508-1(merge) by Mascot Daemon on JOYCE-VAIO

Monoisotopic mass of neutral peptide $\mathrm{Mr}$ (calc): 2429.4

Variable modifications:

K21 iTRAQ4plex (K)

Ions Score: 51.41 Expect: 0.002

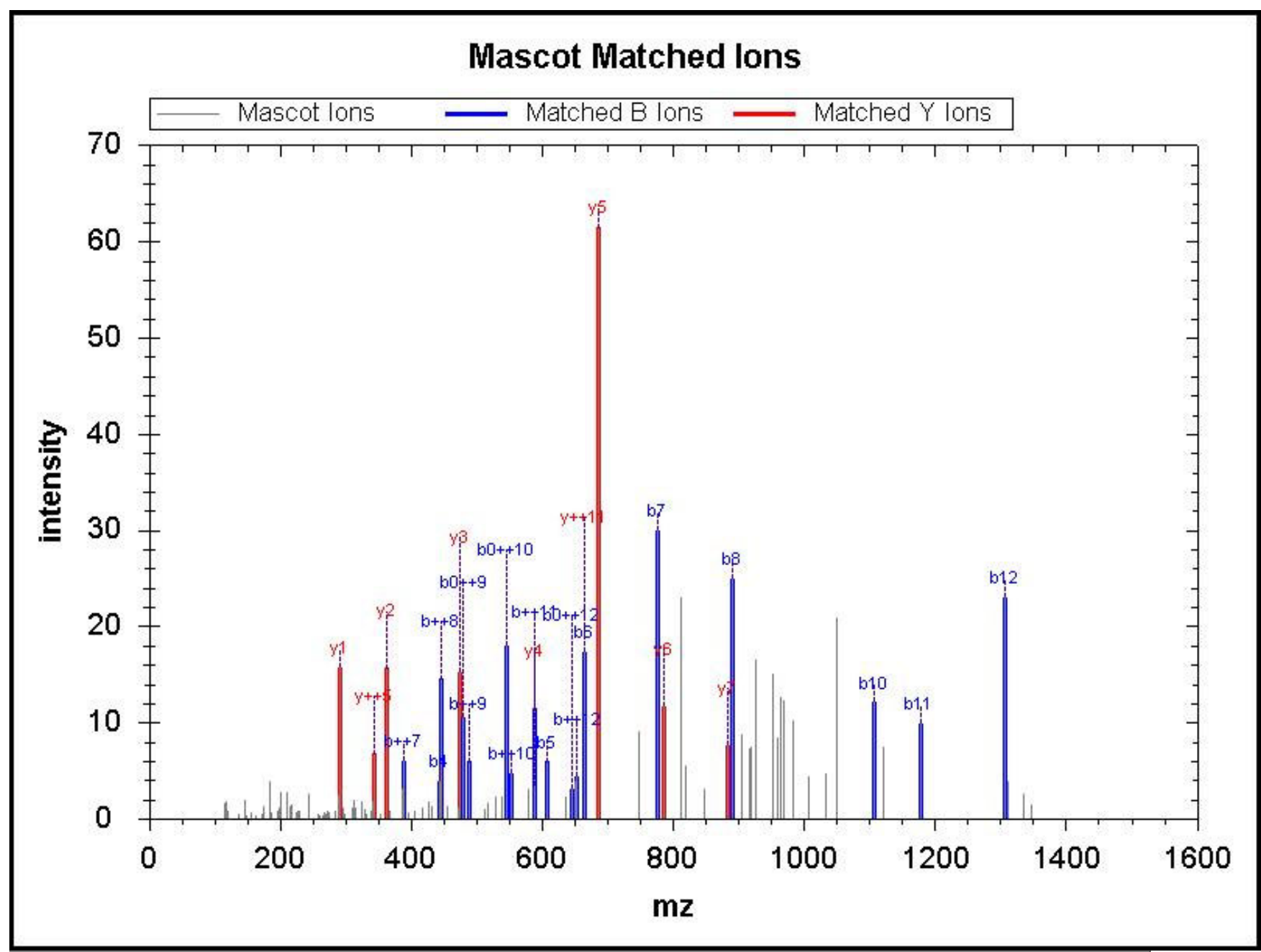

\begin{tabular}{|r|r|r|r|r|r|r|r|r|r|r|r|r|}
\hline \hline No & $\mathrm{b}$ & $\mathrm{b}++$ & $\mathrm{b} 0$ & $\mathrm{~b} 0++$ & $\mathrm{Seq}$ & $\mathrm{y}$ & $\mathrm{y}++$ & $\mathrm{y}^{*}$ & $\mathrm{y}^{*++}$ & $\mathrm{y} 0$ & $\mathrm{y} 0++$ & $\mathrm{RevNo}$ \\
\hline 1 & 258.19 & 129.60 & & & $\mathrm{~L}$ & & & & & & & 21 \\
\hline 2 & 315.21 & 158.11 & & & $\mathrm{G}$ & $2,173.22$ & $1,087.12$ & $2,156.20$ & $1,078.60$ & $2,155.21$ & $1,078.11$ & 20 \\
\hline 3 & 386.25 & 193.63 & & & $\mathrm{~A}$ & $2,116.20$ & $1,058.60$ & $2,099.18$ & $1,050.09$ & $2,098.19$ & $1,049.60$ & 19 \\
\hline 4 & 443.27 & 222.14 & & & $\mathrm{G}$ & $2,045.17$ & $1,023.09$ & $2,028.14$ & $1,014.57$ & $2,027.15$ & $1,014.08$ & 18 \\
\hline 5 & 606.34 & 303.67 & & & $\mathrm{Y}$ & $1,988.14$ & 994.58 & $1,971.12$ & 986.06 & $1,970.13$ & 985.57 & 17 \\
\hline 6 & 663.36 & 332.18 & & & $\mathrm{G}$ & $1,825.08$ & 913.04 & $1,808.05$ & 904.53 & $1,807.07$ & 904.04 & 16 \\
\hline 7 & 776.44 & 388.72 & & & $\mathrm{~L}$ & $1,768.06$ & 884.53 & $1,751.03$ & 876.02 & $1,750.05$ & 875.53 & 15 \\
\hline 8 & 889.53 & 445.27 & & & $\mathrm{~L}$ & $1,654.97$ & 827.99 & $1,637.95$ & 819.48 & $1,636.96$ & 818.99 & 14 \\
\hline 9 & 976.56 & 488.78 & 958.55 & 479.78 & $\mathrm{~S}$ & $1,541.89$ & 771.45 & $1,524.86$ & 762.94 & $1,523.88$ & 762.44 & 13 \\
\hline 10 & $1,105.60$ & 553.30 & $1,087.59$ & 544.30 & $\mathrm{E}$ & $1,454.86$ & 727.93 & $1,437.83$ & 719.42 & $1,436.85$ & 718.93 & 12 \\
\hline 11 & $1,176.64$ & 588.82 & $1,158.63$ & 579.82 & A & $1,325.82$ & 663.41 & $1,308.79$ & 654.90 & $1,307.81$ & 654.41 & 11 \\
\hline 12 & $1,305.68$ & 653.34 & $1,287.67$ & 644.34 & $\mathrm{E}$ & $1,254.78$ & 627.89 & $1,237.75$ & 619.38 & $1,236.77$ & 618.89 & 10 \\
\hline 13 & $1,418.76$ & 709.89 & $1,400.75$ & 700.88 & L & $1,125.74$ & 563.37 & $1,108.71$ & 554.86 & $1,107.73$ & 554.37 & 9 \\
\hline 14 & $1,547.81$ & 774.41 & $1,529.80$ & 765.40 & E & $1,012.65$ & 506.83 & 995.63 & 498.32 & 994.64 & 497.82 & 8 \\
\hline & & & & & & & & & & & &
\end{tabular}




\begin{tabular}{|c|c|c|c|c|c|c|c|c|c|c|}
\hline 15 & 1,6 & 823.94 & $1,628.87$ & 814.94 & V & 883.61 & 442.31 & 866.58 & 433.80 & 7 \\
\hline 16 & $1,745.94$ & 873.48 & $1,727.93$ & 864.47 & V & 784.54 & 392.77 & 767.51 & 384.26 & 6 \\
\hline 17 & $1,843.00$ & 922.00 & $1,824.99$ & 913.00 & $\mathrm{P}$ & 685.47 & 343.24 & 668.45 & 334.73 & 5 \\
\hline 18 & $1,956.08$ & 978.54 & $1,938.07$ & 969.54 & I & 588.42 & 294.71 & 571.39 & 286.20 & 4 \\
\hline 19 & $2,069.17$ & $1,035.09$ & $2,051.15$ & $1,026.08$ & I & 475.34 & 238.17 & 458.31 & 229.66 & 3 \\
\hline 20 & $2,140.20$ & $1,070.60$ & $2,122.19$ & $1,061.60$ & A & 362.25 & 181.63 & 345.23 & 173.12 & 2 \\
\hline 21 & & & & & K & 291.21 & 146.11 & 274.19 & 137.60 & 1 \\
\hline
\end{tabular}

Query 69518 Hit 1

MS/MS Fragmentation of KVSQEILELLNTTTAK

Found in sp|Q86U44|MTA70_HUMAN, N6-adenosine-methyltransferase $70 \mathrm{kDa}$ subunit OS=Homo sapiens GN=METTL3 PE=1 $\mathrm{SV}=2$

Match to Query 69518: 2219.313from(740.7783,3+)

Title: 915: Scan 1987 ( $\mathrm{r}=54.3811, \mathrm{f}=3, \mathrm{i}=309)$ [D:lab212 $\backslash$ membranelGracelJoyceliTRAQ_45_2.raw]

Data File:Submitted from 20120508-1(merge) by Mascot Daemon on JOYCE-VAIO

Monoisotopic mass of neutral peptide $\operatorname{Mr}($ calc): 2219.313

Variable modifications:

$\mathrm{K} 1$ iTRAQ4plex (K)

K16 :TRAQ4plex (K)

Ions Score: 51.35 Expect: 0.002

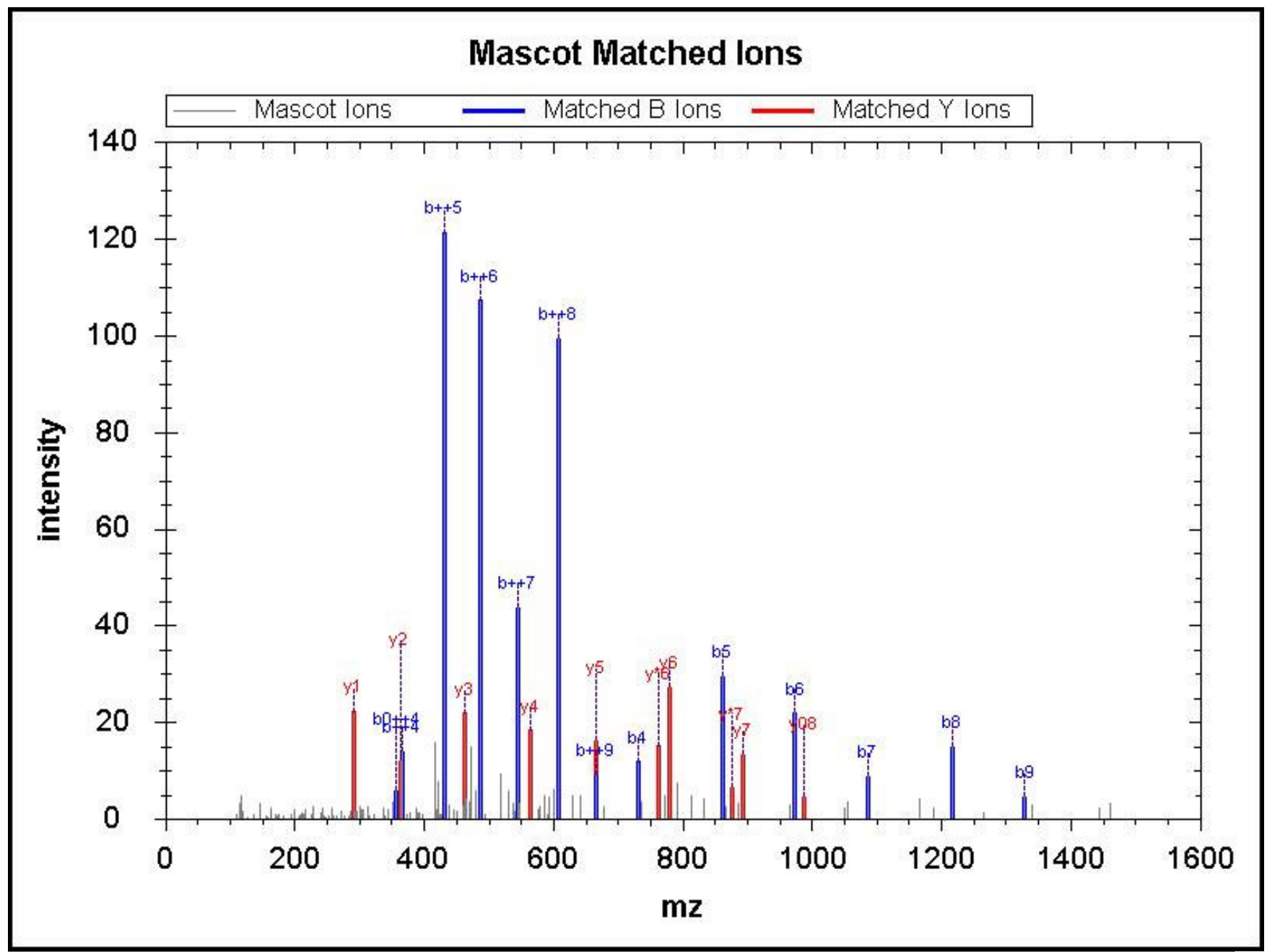

\begin{tabular}{|r|c|c|c|c|c|c|c|c|c|c|c|r|r|r|}
\hline \hline No & $\mathrm{b}$ & $\mathrm{b}++$ & $\mathrm{b}^{*}$ & $\mathrm{~b}^{*++}$ & $\mathrm{b} 0$ & $\mathrm{~b} 0++$ & Seq & $\mathrm{y}$ & $\mathrm{y}++$ & $\mathrm{y}^{*}$ & $\mathrm{y}^{*++}$ & $\mathrm{y} 0$ & $\mathrm{y} 0++$ & $\mathrm{RevNo}$ \\
\hline 1 & 417.31 & 209.16 & 400.28 & 200.64 & & & $\mathrm{~K}$ & & & & & & & 16 \\
\hline 2 & 516.37 & 258.69 & 499.35 & 250.18 & & & $\mathrm{~V}$ & $1,804.02$ & 902.51 & $1,786.99$ & 894.00 & $1,786.01$ & 893.51 & 15 \\
\hline 3 & 603.41 & 302.21 & 586.38 & 293.69 & 585.40 & 293.20 & $\mathrm{~S}$ & $1,704.95$ & 852.98 & $1,687.92$ & 844.47 & $1,686.94$ & 843.97 & 14 \\
\hline 4 & 731.47 & 366.24 & 714.44 & 357.72 & 713.45 & 357.23 & $\mathrm{Q}$ & $1,617.92$ & 809.46 & $1,600.89$ & 800.95 & $1,599.91$ & 800.46 & 13 \\
\hline 5 & 860.51 & 430.76 & 843.48 & 422.24 & 842.50 & 421.75 & $\mathrm{E}$ & $1,489.86$ & 745.43 & $1,472.83$ & 736.92 & $1,471.85$ & 736.43 & 12 \\
\hline
\end{tabular}




\begin{tabular}{|r|r|r|r|r|r|r|r|r|r|r|r|r|r|r|r|r|}
\hline 6 & 973.59 & 487.30 & 956.57 & 478.79 & 955.58 & 478.29 & $\mathrm{I}$ & $1,360.82$ & 680.91 & $1,343.79$ & 672.40 & $1,342.81$ & 671.91 & 11 \\
\hline 7 & $1,086.68$ & 543.84 & $1,069.65$ & 535.33 & $1,068.67$ & 534.84 & $\mathrm{~L}$ & $1,247.73$ & 624.37 & $1,230.71$ & 615.86 & $1,229.72$ & 615.36 & 10 \\
\hline 8 & $1,215.72$ & 608.36 & $1,198.69$ & 599.85 & $1,197.71$ & 599.36 & $\mathrm{E}$ & $1,134.65$ & 567.83 & $1,117.62$ & 559.31 & $1,116.64$ & 558.82 & 9 \\
\hline 9 & $1,328.80$ & 664.91 & $1,311.78$ & 656.39 & $1,310.79$ & 655.90 & $\mathrm{~L}$ & $1,005.61$ & 503.31 & 988.58 & 494.79 & 987.60 & 494.30 & 8 \\
\hline 10 & $1,441.89$ & 721.45 & $1,424.86$ & 712.93 & $1,423.88$ & 712.44 & $\mathrm{~L}$ & 892.52 & 446.76 & 875.50 & 438.25 & 874.51 & 437.76 & 7 \\
\hline 11 & $1,555.93$ & 778.47 & $1,538.90$ & 769.96 & $1,537.92$ & 769.46 & $\mathrm{~N}$ & 779.44 & 390.22 & 762.41 & 381.71 & 761.43 & 381.22 & 6 \\
\hline 12 & $1,656.98$ & 828.99 & $1,639.95$ & 820.48 & $1,638.97$ & 819.99 & $\mathrm{~T}$ & 665.39 & 333.20 & 648.37 & 324.69 & 647.38 & 324.20 & 5 \\
\hline 13 & $1,758.03$ & 879.52 & $1,741.00$ & 871.00 & $1,740.01$ & 870.51 & $\mathrm{~T}$ & 564.35 & 282.68 & 547.32 & 274.16 & 546.34 & 273.67 & 4 \\
\hline 14 & $1,859.07$ & 930.04 & $1,842.05$ & 921.53 & $1,841.06$ & 921.03 & $\mathrm{~T}$ & 463.30 & 232.15 & 446.27 & 223.64 & 445.29 & 223.15 & 3 \\
\hline 15 & $1,930.11$ & 965.56 & $1,913.08$ & 957.05 & $1,912.10$ & 956.55 & $\mathrm{~A}$ & 362.25 & 181.63 & 345.23 & 173.12 & & & 2 \\
\hline 16 & & & & & & & $\mathrm{~K}$ & 291.21 & 146.11 & 274.19 & 137.60 & & & 1 \\
\hline
\end{tabular}

Query 65714 Hit 1

MS/MS Fragmentation of AFVEFLTDEIKEEK

Found in sp $|\mathrm{O} 35658| \mathrm{C} 1 \mathrm{QBP}$ _MOUSE, Complement component 1 Q subcomponent-binding protein

Match to Query 65714: 2129.167from(710.7294,3+)

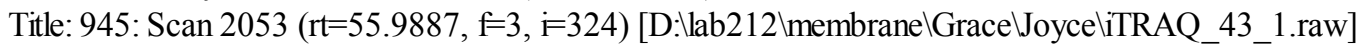

Data File:Submitted from 20120508-1(merge) by Mascot Daemon on JOYCE-VAIO

Monoisotopic mass of neutral peptide Mr(calc): 2129.167

Variable modifications:

K11 :iTRAQ4plex (K)

K14 :iTRAQ4plex (K)

Ions Score: 51.12 Expect: 0.006

\section{Mascot Matched lons}

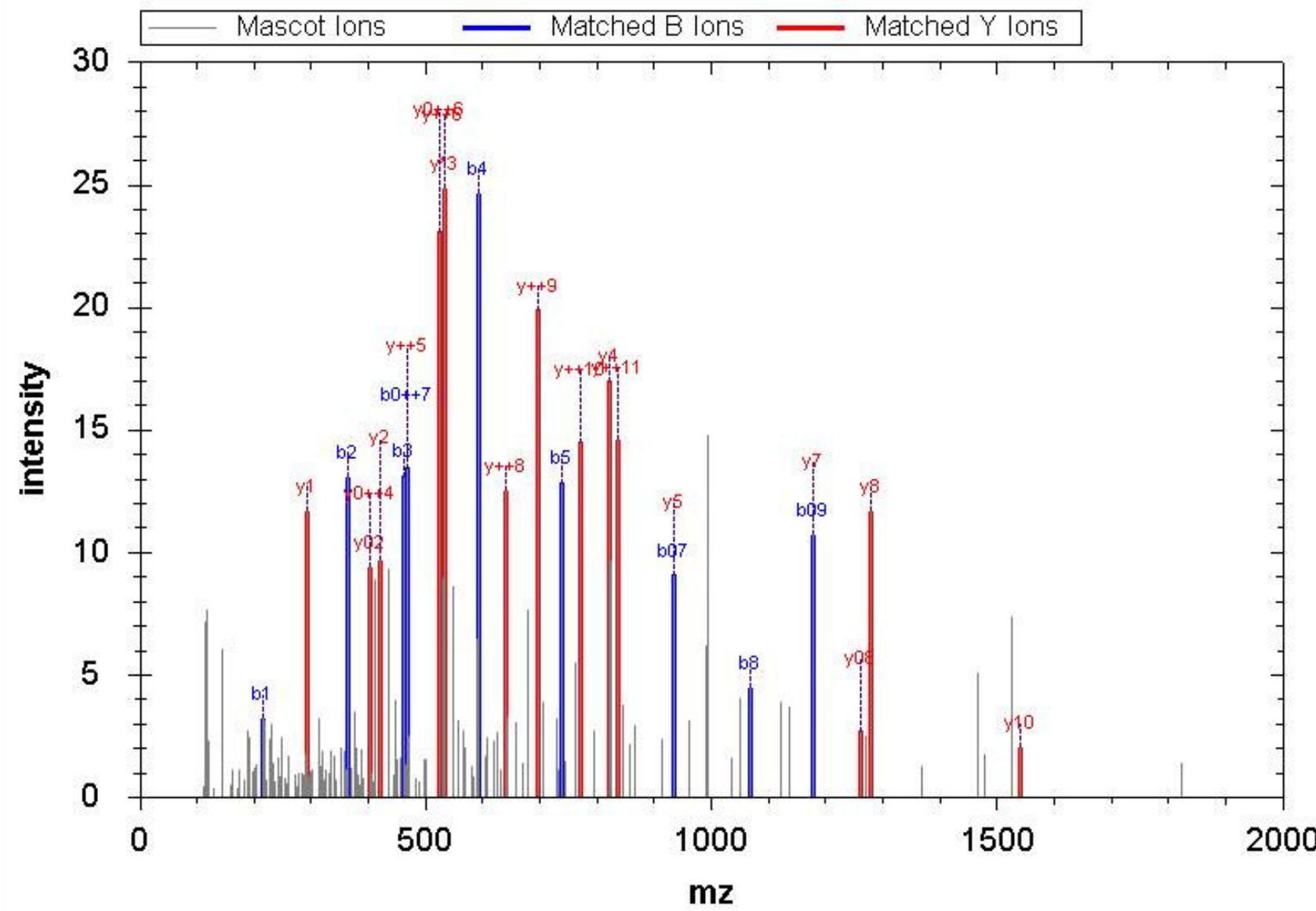

\begin{tabular}{|r|c|c|c|c|c|c|c|c|c|c|c|r|r|r|}
\hline \hline No & $\mathrm{b}$ & $\mathrm{b}++$ & $\mathrm{b}^{*}$ & $\mathrm{~b}^{*++}$ & $\mathrm{b} 0$ & $\mathrm{~b} 0++$ & Seq & $\mathrm{y}$ & $\mathrm{y}++$ & $\mathrm{y}^{*}$ & $\mathrm{y}^{*++}$ & $\mathrm{y} 0$ & $\mathrm{y} 0++$ & $\mathrm{RevNo}$ \\
\hline 1 & 216.15 & 108.58 & & & & & A & & & & & & & 14 \\
\hline 2 & 363.21 & 182.11 & & & & & F & $1,915.03$ & 958.02 & $1,898.00$ & 949.51 & $1,897.02$ & 949.01 & 13 \\
\hline
\end{tabular}




\begin{tabular}{|r|r|r|r|r|r|r|r|r|r|r|r|r|r|r|}
3 & 462.28 & 231.65 & & & & & $\mathrm{~V}$ & $1,767.96$ & 884.48 & $1,750.94$ & 875.97 & $1,749.95$ & 875.48 & 12 \\
\hline 4 & 591.33 & 296.17 & & & 573.32 & 287.16 & $\mathrm{E}$ & $1,668.89$ & 834.95 & $1,651.87$ & 826.44 & $1,650.88$ & 825.95 & 11 \\
\hline 5 & 738.39 & 369.70 & & & 720.38 & 360.70 & $\mathrm{~F}$ & $1,539.85$ & 770.43 & $1,522.82$ & 761.92 & $1,521.84$ & 761.42 & 10 \\
\hline 6 & 851.48 & 426.24 & & & 833.47 & 417.24 & $\mathrm{~L}$ & $1,392.78$ & 696.89 & $1,375.76$ & 688.38 & $1,374.77$ & 687.89 & 9 \\
\hline 7 & 952.53 & 476.77 & & & 934.52 & 467.76 & $\mathrm{~T}$ & $1,279.70$ & 640.35 & $1,262.67$ & 631.84 & $1,261.69$ & 631.35 & 8 \\
\hline 8 & $1,067.55$ & 534.28 & & & $1,049.54$ & 525.27 & $\mathrm{D}$ & $1,178.65$ & 589.83 & $1,161.62$ & 581.32 & $1,160.64$ & 580.82 & 7 \\
\hline 9 & $1,196.60$ & 598.80 & & & $1,178.58$ & 589.80 & $\mathrm{E}$ & $1,063.62$ & 532.32 & $1,046.60$ & 523.80 & $1,045.61$ & 523.31 & 6 \\
\hline 10 & $1,309.68$ & 655.34 & & & $1,291.67$ & 646.34 & $\mathrm{I}$ & 934.58 & 467.79 & 917.55 & 459.28 & 916.57 & 458.79 & 5 \\
\hline 11 & $1,581.88$ & 791.44 & $1,564.85$ & 782.93 & $1,563.87$ & 782.44 & $\mathrm{~K}$ & 821.50 & 411.25 & 804.47 & 402.74 & 803.49 & 402.25 & 4 \\
\hline 12 & $1,710.92$ & 855.96 & $1,693.89$ & 847.45 & $1,692.91$ & 846.96 & $\mathrm{E}$ & 549.30 & 275.15 & 532.27 & 266.64 & 531.29 & 266.15 & 3 \\
\hline 13 & $1,839.96$ & 920.48 & $1,822.94$ & 911.97 & $1,821.95$ & 911.48 & $\mathrm{E}$ & 420.26 & 210.63 & 403.23 & 202.12 & 402.25 & 201.63 & 2 \\
\hline 14 & & & & & & & $\mathrm{~K}$ & 291.21 & 146.11 & 274.19 & 137.60 & & & 1 \\
\hline
\end{tabular}

Query 50714 Hit 1

\section{MS/MS Fragmentation of EEGHAGPDDNEEVMR}

Found in sp|P29083|T2EA_HUM AN, General transcription factor IIE subunit 1 OS=Homo sapiens GN=GTF2E1 PE=1 SV=2 Match to Query 50714: 1827.782 from(610.2681,3+)

Title: 100: Sum of 2 scans in range $337(\mathrm{rt}=16.923, \mathrm{f}=4, \mathrm{i}=55)$ to $338(\mathrm{rt}=16.9484, \mathrm{f}=4, \mathrm{i}=56)$

[D:lab212 \membranelGrace JoyceliTRAQ_38_2.raw]

Data File:Submitted from 20120508-1(merge) by Mascot Daemon on JOYCE-VAIO

Monoisotopic mass of neutral peptide $\mathrm{Mr}(\mathrm{calc}): 1827.782$

Variable modifications:

Ions Score: 51.11 Expect: 0.003

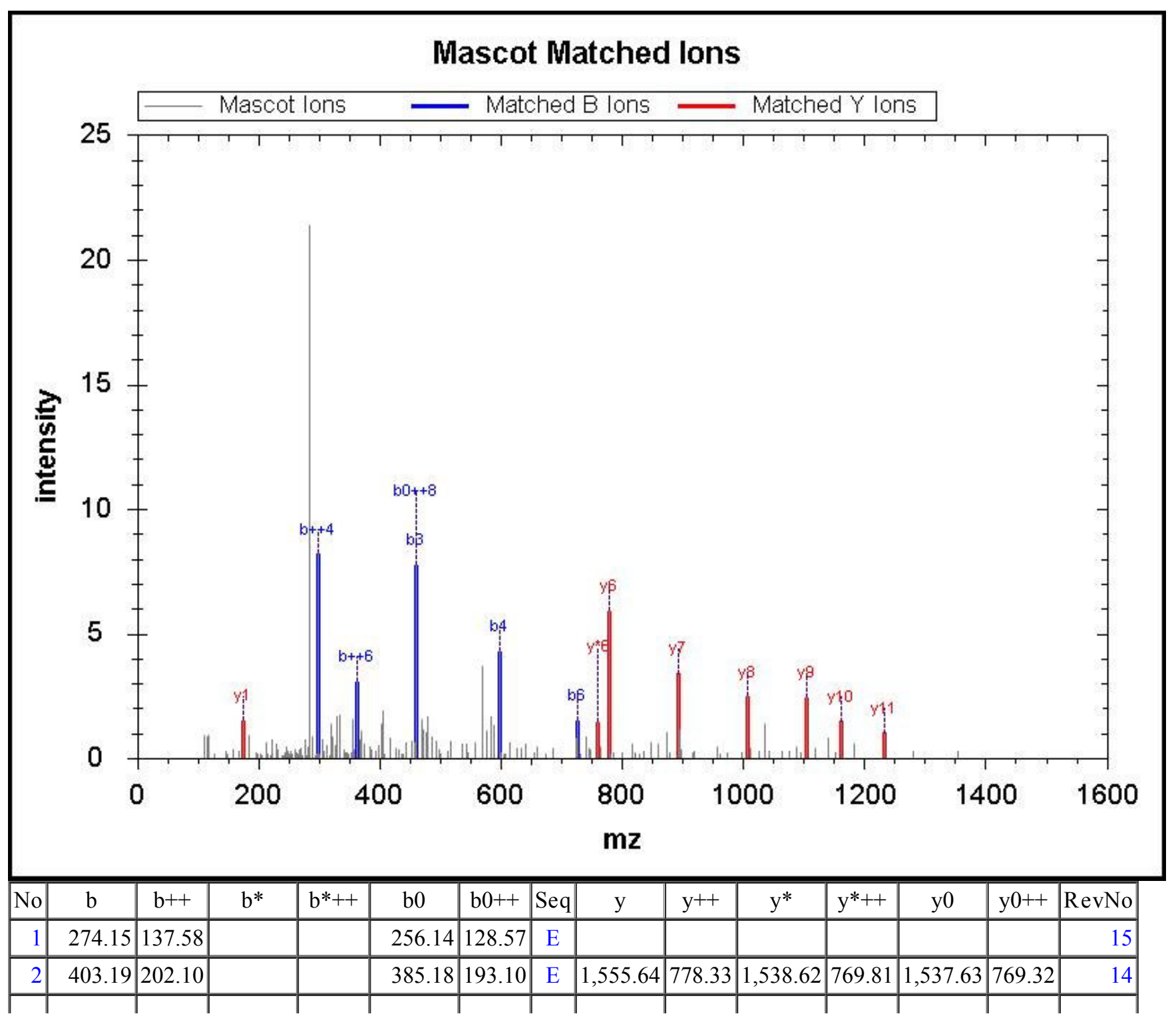




\begin{tabular}{|r|r|r|r|r|r|r|r|r|r|r|r|r|r|r|r|r|r|}
\hline 3 & 460.22 & 230.61 & & & 442.21 & 221.61 & $\mathrm{G}$ & $1,426.60$ & 713.80 & $1,409.58$ & 705.29 & $1,408.59$ & 704.80 & 13 \\
\hline 4 & 597.27 & 299.14 & & & 579.26 & 290.14 & $\mathrm{H}$ & $1,369.58$ & 685.29 & $1,352.55$ & 676.78 & $1,351.57$ & 676.29 & 12 \\
\hline 5 & 668.31 & 334.66 & & & 650.30 & 325.65 & $\mathrm{~A}$ & $1,232.52$ & 616.76 & $1,215.49$ & 608.25 & $1,214.51$ & 607.76 & 11 \\
\hline 6 & 725.33 & 363.17 & & & 707.32 & 354.17 & $\mathrm{G}$ & $1,161.48$ & 581.25 & $1,144.46$ & 572.73 & $1,143.47$ & 572.24 & 10 \\
\hline 7 & 822.39 & 411.70 & & & 804.38 & 402.69 & $\mathrm{P}$ & $1,104.46$ & 552.73 & $1,087.44$ & 544.22 & $1,086.45$ & 543.73 & 9 \\
\hline 8 & 937.41 & 469.21 & & & 919.40 & 460.20 & $\mathrm{D}$ & $1,007.41$ & 504.21 & 990.38 & 495.70 & 989.40 & 495.20 & 8 \\
\hline 9 & $1,052.44$ & 526.72 & & & $1,034.43$ & 517.72 & $\mathrm{D}$ & 892.38 & 446.70 & 875.36 & 438.18 & 874.37 & 437.69 & 7 \\
\hline 10 & $1,166.48$ & 583.75 & $1,149.46$ & 575.23 & $1,148.47$ & 574.74 & $\mathrm{~N}$ & 777.36 & 389.18 & 760.33 & 380.67 & 759.35 & 380.18 & 6 \\
\hline 11 & $1,295.53$ & 648.27 & $1,278.50$ & 639.75 & $1,277.52$ & 639.26 & $\mathrm{E}$ & 663.31 & 332.16 & 646.29 & 323.65 & 645.30 & 323.15 & 5 \\
\hline 12 & $1,424.57$ & 712.79 & $1,407.54$ & 704.27 & $1,406.56$ & 703.78 & $\mathrm{E}$ & 534.27 & 267.64 & 517.24 & 259.13 & 516.26 & 258.63 & 4 \\
\hline 13 & $1,523.64$ & 762.32 & $1,506.61$ & 753.81 & $1,505.63$ & 753.32 & $\mathrm{~V}$ & 405.23 & 203.12 & 388.20 & 194.60 & & & 3 \\
\hline 14 & $1,654.68$ & 827.84 & $1,637.65$ & 819.33 & $1,636.67$ & 818.84 & $\mathrm{M}$ & 306.16 & 153.58 & 289.13 & 145.07 & & & 2 \\
\hline 15 & & & & & & & $\mathrm{R}$ & 175.12 & 88.06 & 158.09 & 79.55 & & & 1 \\
\hline
\end{tabular}

Query 33852 Hit 1

MS/MS Fragmentation of ALSAETESHIYR

Found in sp $\mid$ O14569|C56D2_HUM AN, Cytochrome b561 domain-containing protein 2 OS=Homo sapiens GN=CYB561D2 PE=1 SV=1 Match to Query 33852: 1519.774 from(507.5987,3+)

Title: 304: Sum of 2 scans in range $729(\mathrm{rt}=26.0218, \mathrm{f}=4, \mathrm{~F}=195)$ to $730(\mathrm{rt}=26.0472, \mathrm{f}=4, \mathrm{~F}=196)$

[D:lab212 \membranelGrace JoyceliTRAQ_39_2.raw]

Data File:Submitted from 20120508-1(merge) by Mascot Daemon on JOYCE-VAIO

Monoisotopic mass of neutral peptide $\operatorname{Mr}(\mathrm{calc}): 1519.774$

Variable modifications:

Ions Score: 51.07 Expect: 0.007

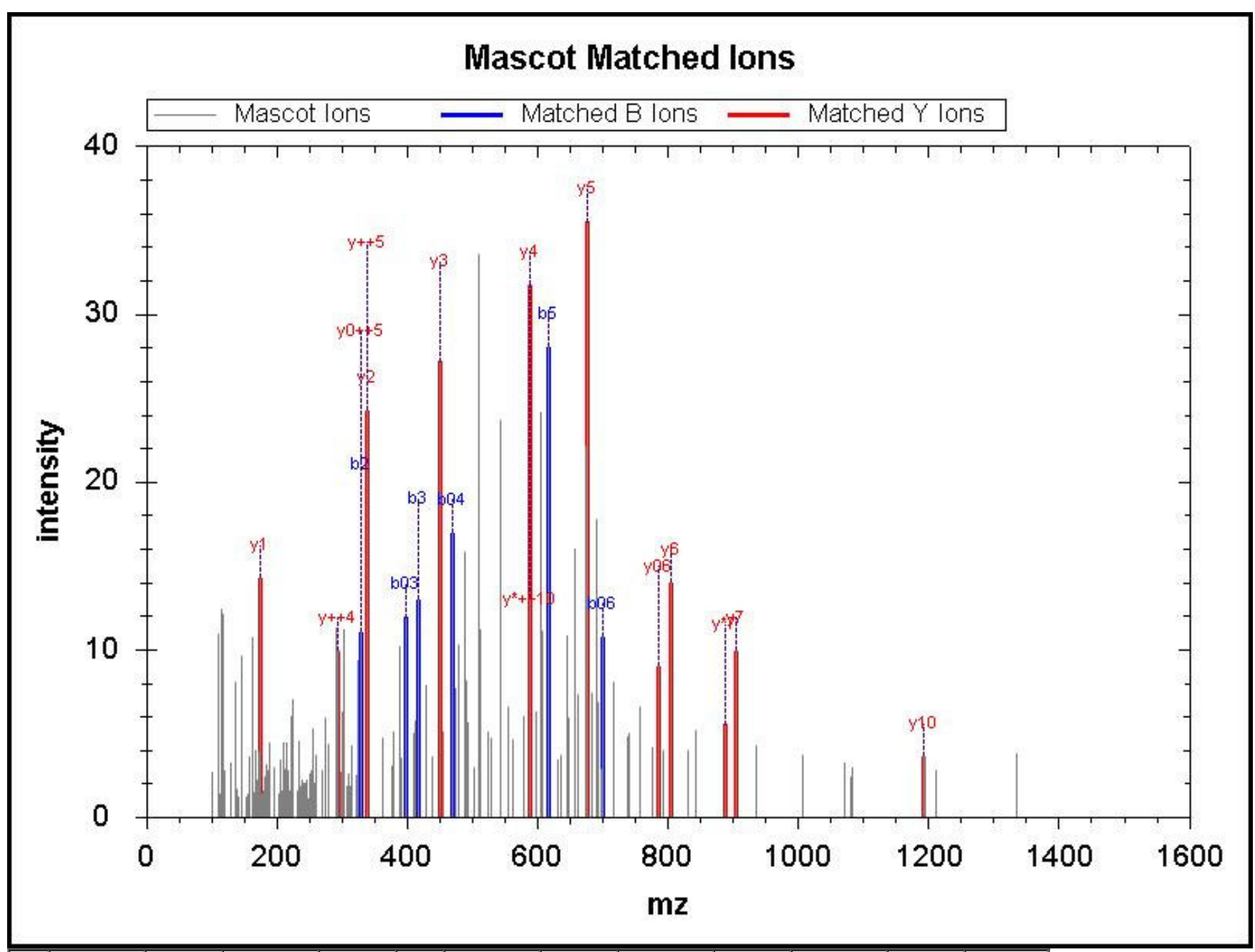

\begin{tabular}{|c|c|c|c|c|c|c|c|c|c|c|c|c|}
\hline \hline No & $\mathrm{b}$ & $\mathrm{b}++$ & $\mathrm{b} 0$ & $\mathrm{~b} 0++$ & Seq & $\mathrm{y}$ & $\mathrm{y}++$ & $\mathrm{y}^{*}$ & $\mathrm{y}^{*++}$ & $\mathrm{y} 0$ & $\mathrm{y} 0++$ & $\mathrm{RevNo}$ \\
\hline & & & & & & & & & & & &
\end{tabular}




\begin{tabular}{|r|r|r|r|r|r|r|r|r|r|r|r|r|}
\hline 1 & 216.15 & 108.58 & & & A & & & & & & & 12 \\
\hline 2 & 329.23 & 165.12 & & & L & $1,305.64$ & 653.33 & $1,288.62$ & 644.81 & $1,287.63$ & 644.32 & 11 \\
\hline 3 & 416.26 & 208.63 & 398.25 & 199.63 & S & $1,192.56$ & 596.78 & $1,175.53$ & 588.27 & $1,174.55$ & 587.78 & 10 \\
\hline 4 & 487.30 & 244.15 & 469.29 & 235.15 & A & $1,105.53$ & 553.27 & $1,088.50$ & 544.75 & $1,087.52$ & 544.26 & 9 \\
\hline 5 & 616.34 & 308.67 & 598.33 & 299.67 & E & $1,034.49$ & 517.75 & $1,017.46$ & 509.24 & $1,016.48$ & 508.74 & 8 \\
\hline 6 & 717.39 & 359.20 & 699.38 & 350.19 & T & 905.45 & 453.23 & 888.42 & 444.71 & 887.44 & 444.22 & 7 \\
\hline 7 & 846.43 & 423.72 & 828.42 & 414.71 & E & 804.40 & 402.70 & 787.37 & 394.19 & 786.39 & 393.70 & 6 \\
\hline 8 & 933.46 & 467.24 & 915.45 & 458.23 & S & 675.36 & 338.18 & 658.33 & 329.67 & 657.35 & 329.18 & 5 \\
\hline 9 & $1,070.52$ & 535.77 & $1,052.51$ & 526.76 & H & 588.33 & 294.67 & 571.30 & 286.15 & & & 4 \\
\hline 10 & $1,183.61$ & 592.31 & $1,165.60$ & 583.30 & I & 451.27 & 226.14 & 434.24 & 217.62 & & & 3 \\
\hline 11 & $1,346.67$ & 673.84 & $1,328.66$ & 664.83 & Y & 338.18 & 169.59 & 321.16 & 161.08 & & & 2 \\
\hline 12 & & & & & R & 175.12 & 88.06 & 158.09 & 79.55 & & & 1 \\
\hline
\end{tabular}

Query 48712 Hit 1

MS/MS Fragmentation of HLVLLDTAQAAAAGHR

Found in sp|O00754|MA2B1_HUMAN, Lysosomal alpha-mannosidase OS=Homo sapiens GN=MAN2B1 PE=1 SV=3 Match to Query 48712: 1786.996from(596.6726,3+)

Title: 466: Scan 1153 ( $\mathrm{rt}=35.1716, \mathrm{f}=3, \mathrm{i}=163)$ [D:lab212 \membranelGrace JoyceliTRAQ_48_2.raw]

Data File:Submitted from 20120508-1(merge) by Mascot Daemon on JOYCE-VAIO

Monoisotopic mass of neutral peptide $\mathrm{Mr}$ (calc): 1786.996

Variable modifications:

Ions Score: 50.86 Expect: 0.006

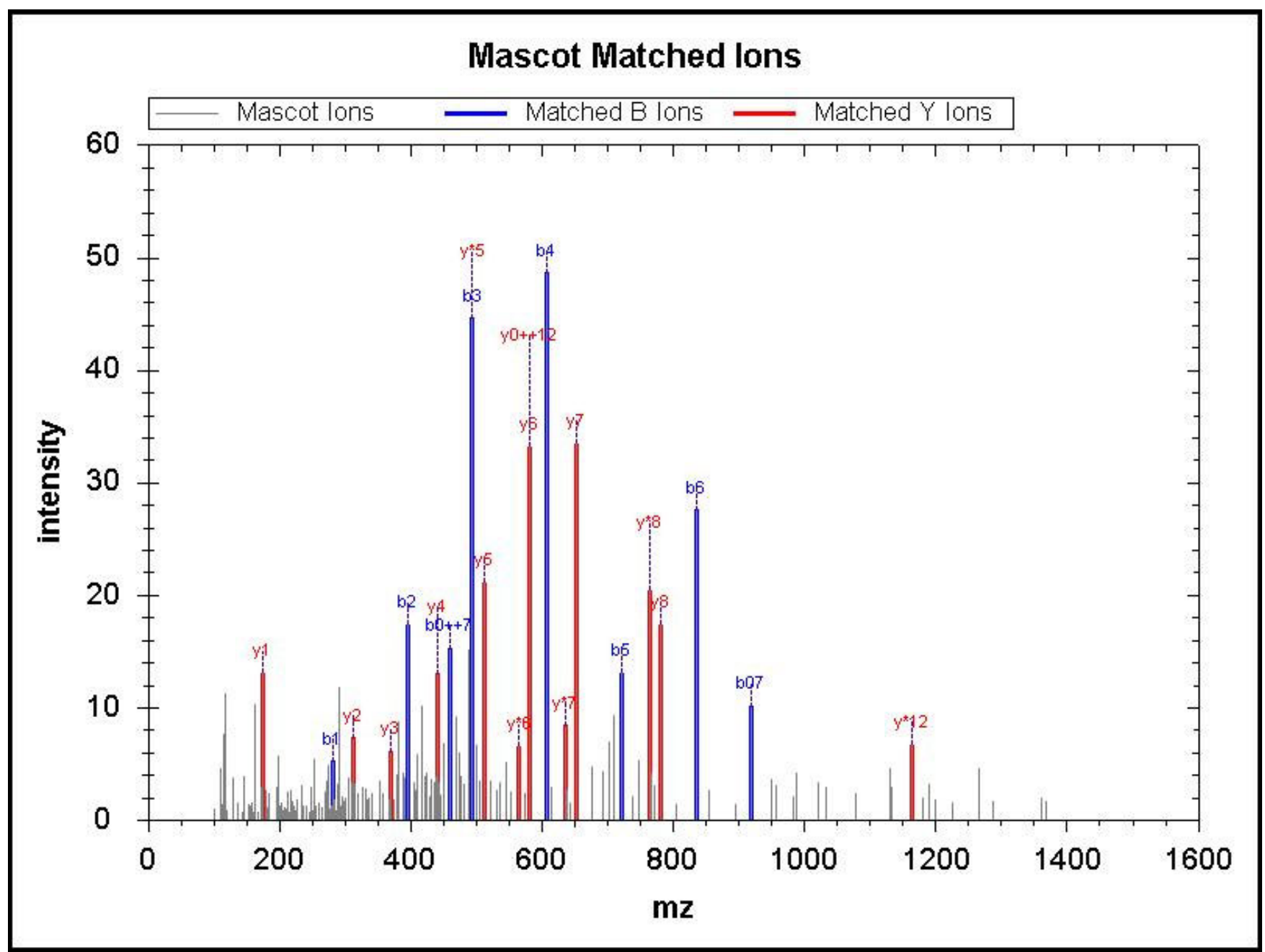

\begin{tabular}{|r|c|c|c|c|c|r|c|c|c|c|c|r|r|r|}
\hline \hline No & $\mathrm{b}$ & $\mathrm{b}++$ & $\mathrm{b}^{*}$ & $\mathrm{~b}++$ & $\mathrm{b} 0$ & $\mathrm{~b} 0++$ & Seq & $\mathrm{y}$ & $\mathrm{y}++$ & $\mathrm{y}^{*}$ & $\mathrm{y}^{*++}$ & $\mathrm{y} 0$ & $\mathrm{y} 0++$ & $\mathrm{RevNo}$ \\
\hline 1 & 282.17 & 141.59 & & & & & $\mathrm{H}$ & & & & & & & 16 \\
\hline 2 & 395.25 & 198.13 & & & & & L & $1,506.84$ & 753.92 & $1,489.81$ & 745.41 & $1,488.83$ & 744.92 & 15 \\
\hline & & & & & & & & & & & & & &
\end{tabular}




\begin{tabular}{|r|r|r|r|r|r|r|r|r|r|r|r|r|r|r|}
\hline 3 & 494.32 & 247.66 & & & & & $\mathrm{~V}$ & $1,393.75$ & 697.38 & $1,376.73$ & 688.87 & $1,375.74$ & 688.38 & 14 \\
\hline 4 & 607.40 & 304.21 & & & & & $\mathrm{~L}$ & $1,294.69$ & 647.85 & $1,277.66$ & 639.33 & $1,276.68$ & 638.84 & 13 \\
\hline 5 & 720.49 & 360.75 & & & & & $\mathrm{~L}$ & $1,181.60$ & 591.30 & $1,164.58$ & 582.79 & $1,163.59$ & 582.30 & 12 \\
\hline 6 & 835.52 & 418.26 & & & 817.51 & 409.26 & $\mathrm{D}$ & $1,068.52$ & 534.76 & $1,051.49$ & 526.25 & $1,050.51$ & 525.76 & 11 \\
\hline 7 & 936.56 & 468.79 & & & 918.55 & 459.78 & $\mathrm{~T}$ & 953.49 & 477.25 & 936.46 & 468.74 & 935.48 & 468.24 & 10 \\
\hline 8 & $1,007.60$ & 504.30 & & & 989.59 & 495.30 & $\mathrm{~A}$ & 852.44 & 426.73 & 835.42 & 418.21 & & & 9 \\
\hline 9 & $1,135.66$ & 568.33 & $1,118.63$ & 559.82 & $1,117.65$ & 559.33 & $\mathrm{Q}$ & 781.41 & 391.21 & 764.38 & 382.69 & & & 8 \\
\hline 10 & $1,206.70$ & 603.85 & $1,189.67$ & 595.34 & $1,188.69$ & 594.85 & $\mathrm{~A}$ & 653.35 & 327.18 & 636.32 & 318.66 & & & 7 \\
\hline 11 & $1,277.73$ & 639.37 & $1,260.71$ & 630.86 & $1,259.72$ & 630.37 & $\mathrm{~A}$ & 582.31 & 291.66 & 565.28 & 283.15 & & & 6 \\
\hline 12 & $1,348.77$ & 674.89 & $1,331.74$ & 666.38 & $1,330.76$ & 665.88 & $\mathrm{~A}$ & 511.27 & 256.14 & 494.25 & 247.63 & & & 5 \\
\hline 13 & $1,419.81$ & 710.41 & $1,402.78$ & 701.89 & $1,401.80$ & 701.40 & $\mathrm{~A}$ & 440.24 & 220.62 & 423.21 & 212.11 & & & 4 \\
\hline 14 & $1,476.83$ & 738.92 & $1,459.80$ & 730.40 & $1,458.82$ & 729.91 & $\mathrm{G}$ & 369.20 & 185.10 & 352.17 & 176.59 & & & 3 \\
\hline 15 & $1,613.89$ & 807.45 & $1,596.86$ & 798.93 & $1,595.88$ & 798.44 & $\mathrm{H}$ & 312.18 & 156.59 & 295.15 & 148.08 & & & 2 \\
\hline 16 & & & & & & & $\mathrm{R}$ & 175.12 & 88.06 & 158.09 & 79.55 & & & 1 \\
\hline
\end{tabular}

Query 94284 Hit 1

MS/MS Fragmentation of LQLFIQEVNHAVEETSHQALQNMPK

Found in sp|P83436|COG7_HUMAN, Conserved oligomeric Golgi complex subunit 7 OS=Homo sapiens GN=COG7 PE=1 SV=1 Match to Query 94284:3191.669from(1064.897,3+)

Title: 927: Scan 2724 (rt=68.3447, $\mathrm{f}=2, \mathrm{i}=437)$ [D:lab212 $\backslash$ membranelGracelJoyceliTRAQ_43_2.raw]

Data File:Submitted from 20120508-1(merge) by Mascot Daemon on JOYCE-VAIO

Monoisotopic mass of neutral peptide $\operatorname{Mr}($ calc): 3191.669

Variable modifications:

K25 :TRAQ4plex (K)

Ions Score: 50.78 Expect: 0.006

\section{Mascot Matched lons}

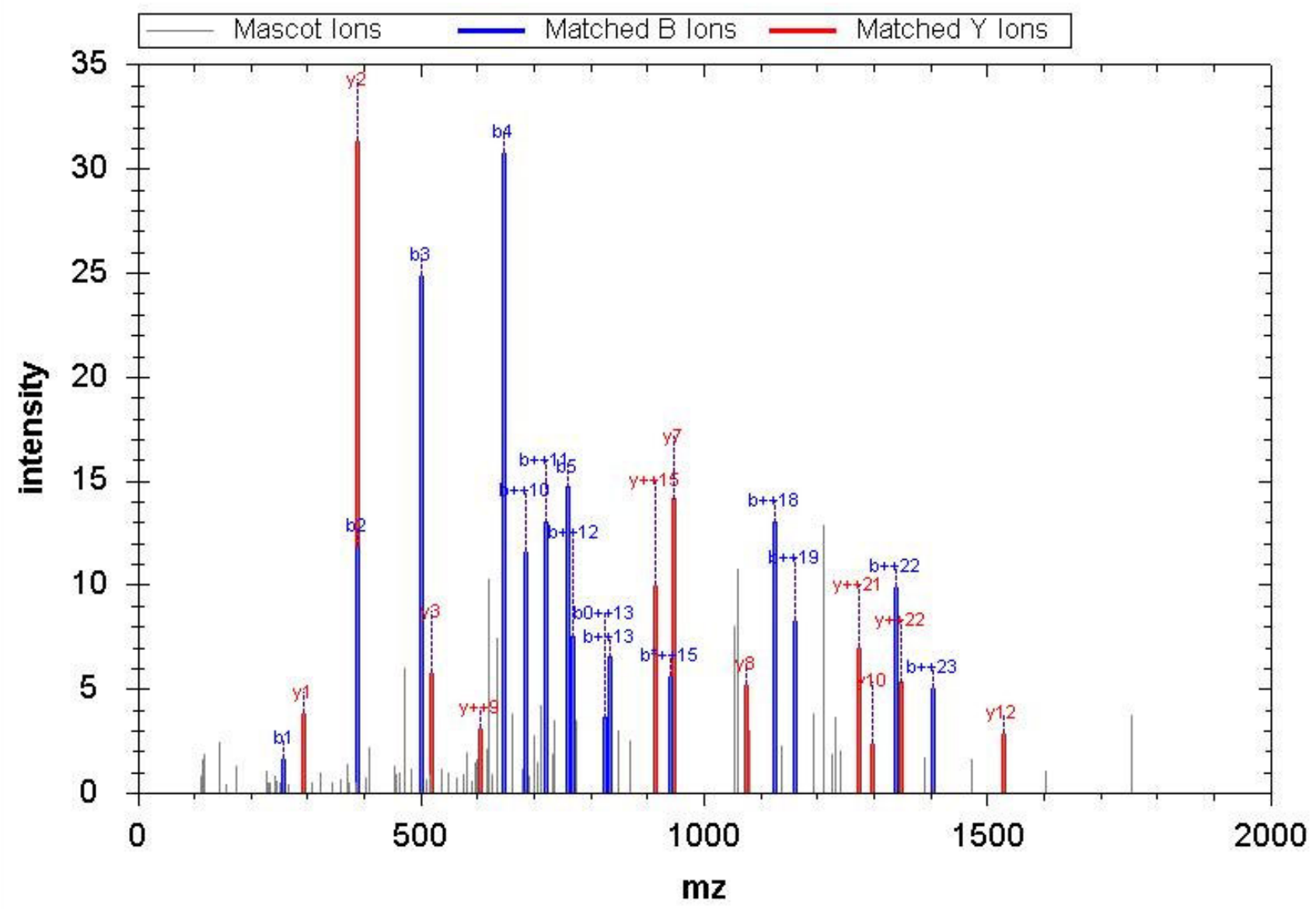




\begin{tabular}{|c|c|c|c|c|c|c|c|c|c|c|c|c|c|c|}
\hline No & $\mathrm{b}$ & $\mathrm{b}++$ & $b^{*}$ & $b^{*++}$ & b0 & $\mathrm{b} 0++$ & Seq & $\mathrm{y}$ & $\mathrm{y}^{++}$ & $\mathrm{y}^{*}$ & $\mathrm{y}^{*++}$ & y0 & $\mathrm{y} 0++$ & RevNo \\
\hline 1 & 258.19 & 129.60 & & & & & $\mathrm{~L}$ & & & & & & & 25 \\
\hline 2 & 386.25 & 193.63 & 369.23 & 185.12 & & & Q & $2,935.48$ & $1,468.25$ & $2,918.46$ & $1,459.73$ & $2,917.47$ & $1,459.24$ & 24 \\
\hline 3 & 499.34 & 250.17 & 482.31 & 241.66 & & & $\mathrm{~L}$ & $2,807.43$ & $1,404.22$ & $2,790.40$ & $1,395.70$ & $2,789.42$ & $1,395.21$ & 23 \\
\hline 4 & 646.40 & 323.71 & 629.38 & 315.19 & & & $\mathrm{~F}$ & $2,694.34$ & $1,347.67$ & $2,677.32$ & $1,339.16$ & $2,676.33$ & $1,338.67$ & 22 \\
\hline 5 & 759.49 & 380.25 & 742.46 & 371.73 & & & I & $2,547.27$ & $1,274.14$ & $2,530.25$ & $1,265.63$ & $2,529.26$ & $1,265.14$ & 21 \\
\hline 6 & 887.55 & 444.28 & 870.52 & 435.76 & & & Q & $2,434.19$ & $1,217.60$ & $2,417.16$ & $1,209.09$ & $2,416.18$ & $1,208.59$ & 20 \\
\hline 7 & $1,016.59$ & 508.80 & 999.56 & 500.29 & 998.58 & 499.79 & $\mathrm{E}$ & $2,306.13$ & $1,153.57$ & $2,289.10$ & $1,145.06$ & $2,288.12$ & $1,144.56$ & 19 \\
\hline 8 & $1,115.66$ & 558.33 & $1,098.63$ & 549.82 & $1,097.65$ & 549.33 & V & $2,177.09$ & $1,089.05$ & $2,160.06$ & $1,080.53$ & $2,159.08$ & $1,080.04$ & 18 \\
\hline 9 & $1,229.70$ & 615.35 & $1,212.67$ & 606.84 & $1,211.69$ & 606.35 & $\mathrm{~N}$ & $2,078.02$ & $1,039.51$ & $2,060.99$ & $1,031.00$ & $2,060.01$ & $1,030.51$ & 17 \\
\hline 10 & $1,366.76$ & 683.88 & $1,349.73$ & 675.37 & $1,348.75$ & 674.88 & $\mathrm{H}$ & $1,963.98$ & 982.49 & $1,946.95$ & 973.98 & $1,945.97$ & 973.49 & 16 \\
\hline 11 & $1,437.80$ & 719.40 & $1,420.77$ & 710.89 & $1,419.79$ & 710.40 & A & $1,826.92$ & 913.96 & $1,809.89$ & 905.45 & $1,808.91$ & 904.96 & 15 \\
\hline 12 & $1,536.87$ & 768.94 & $1,519.84$ & 760.42 & $1,518.85$ & 759.93 & V & $1,755.88$ & 878.44 & $1,738.86$ & 869.93 & $1,737.87$ & 869.44 & 14 \\
\hline 13 & $1,665.91$ & 833.46 & $1,648.88$ & 824.94 & $1,647.90$ & 824.45 & $\mathrm{E}$ & $1,656.81$ & 828.91 & $1,639.79$ & 820.40 & $1,638.80$ & 819.90 & 13 \\
\hline 14 & $1,794.95$ & 897.98 & $1,777.92$ & 889.47 & $1,776.94$ & 888.97 & $\mathrm{E}$ & $1,527.77$ & 764.39 & $1,510.74$ & 755.88 & $1,509.76$ & 755.38 & 12 \\
\hline 15 & $1,896.00$ & 948.50 & $1,878.97$ & 939.99 & $1,877.99$ & 939.50 & $\mathrm{~T}$ & $1,398.73$ & 699.87 & $1,381.70$ & 691.35 & $1,380.72$ & 690.86 & 11 \\
\hline 16 & $1,983.03$ & 992.02 & $1,966.00$ & 983.51 & $1,965.02$ & 983.01 & S & $1,297.68$ & 649.34 & $1,280.65$ & 640.83 & $1,279.67$ & 640.34 & 10 \\
\hline 17 & $2,120.09$ & $1,060.55$ & $2,103.06$ & $1,052.04$ & $2,102.08$ & $1,051.54$ & $\mathrm{H}$ & $1,210.65$ & 605.83 & $1,193.62$ & 597.31 & & & 9 \\
\hline 18 & $2,248.15$ & $1,124.58$ & $2,231.12$ & $1,116.06$ & $2,230.14$ & $1,115.57$ & Q & $1,073.59$ & 537.30 & $1,056.56$ & 528.79 & & & 8 \\
\hline 19 & $2,319.19$ & $1,160.10$ & $2,302.16$ & $1,151.58$ & $2,301.17$ & $1,151.09$ & A & 945.53 & 473.27 & 928.50 & 464.76 & & & 7 \\
\hline 20 & $2,432.27$ & $1,216.64$ & $2,415.24$ & $1,208.12$ & $2,414.26$ & $1,207.63$ & L & 874.49 & 437.75 & 857.47 & 429.24 & & & 6 \\
\hline 21 & $2,560.33$ & $1,280.67$ & $2,543.30$ & $1,272.15$ & $2,542.32$ & $1,271.66$ & Q & 761.41 & 381.21 & 744.38 & 372.70 & & & 5 \\
\hline 22 & $2,674.37$ & $1,337.69$ & $2,657.34$ & $1,329.18$ & $2,656.36$ & $1,328.68$ & $\mathrm{~N}$ & 633.35 & 317.18 & 616.32 & 308.67 & & & 4 \\
\hline 23 & $2,805.41$ & $1,403.21$ & $2,788.38$ & $1,394.70$ & $2,787.40$ & $1,394.20$ & $\mathrm{M}$ & 519.31 & 260.16 & 502.28 & 251.64 & & & 3 \\
\hline 24 & $2,902.46$ & $1,451.74$ & $2,885.44$ & $1,443.22$ & $2,884.45$ & $1,442.73$ & $\mathrm{P}$ & 388.27 & 194.64 & 371.24 & 186.12 & & & 2 \\
\hline 25 & & & & & & & K & 291.21 & 146.11 & 274.19 & 137.60 & & & 1 \\
\hline
\end{tabular}

Query 93980 Hit 1

MS/MS Fragmentation of ALYFYEQAITTPAMAVSHIMLESYK

Found in sp|Q9UNS2|CSN3_HUM AN, COP9 signalosome complex subunit 3 OS=Homo sapiens GN=COPS3 PE=1 SV=3

Match to Query 93980:3164.616from(792.1612,4+)

Title: 1211: Sum of 2 scans in range $2805(\mathrm{rt}=72.0248, \mathrm{f}=2, \mathrm{i}=472)$ to $2806(\mathrm{rt}=72.0503, \mathrm{f}=2, \mathrm{i}=473)$

[D:llab212 \membranelGracelJoyceliTRAQ_32_2.raw]

Data File:Submitted from 20120508-1(merge) by Mascot Daemon on JOYCE-VAIO

Monoisotopic mass of neutral peptide Mr(calc): 3164.616

Variable modifications:

K25 :iTRAQ4plex (K)

Ions Score: 50.67 Expect: 0.007 


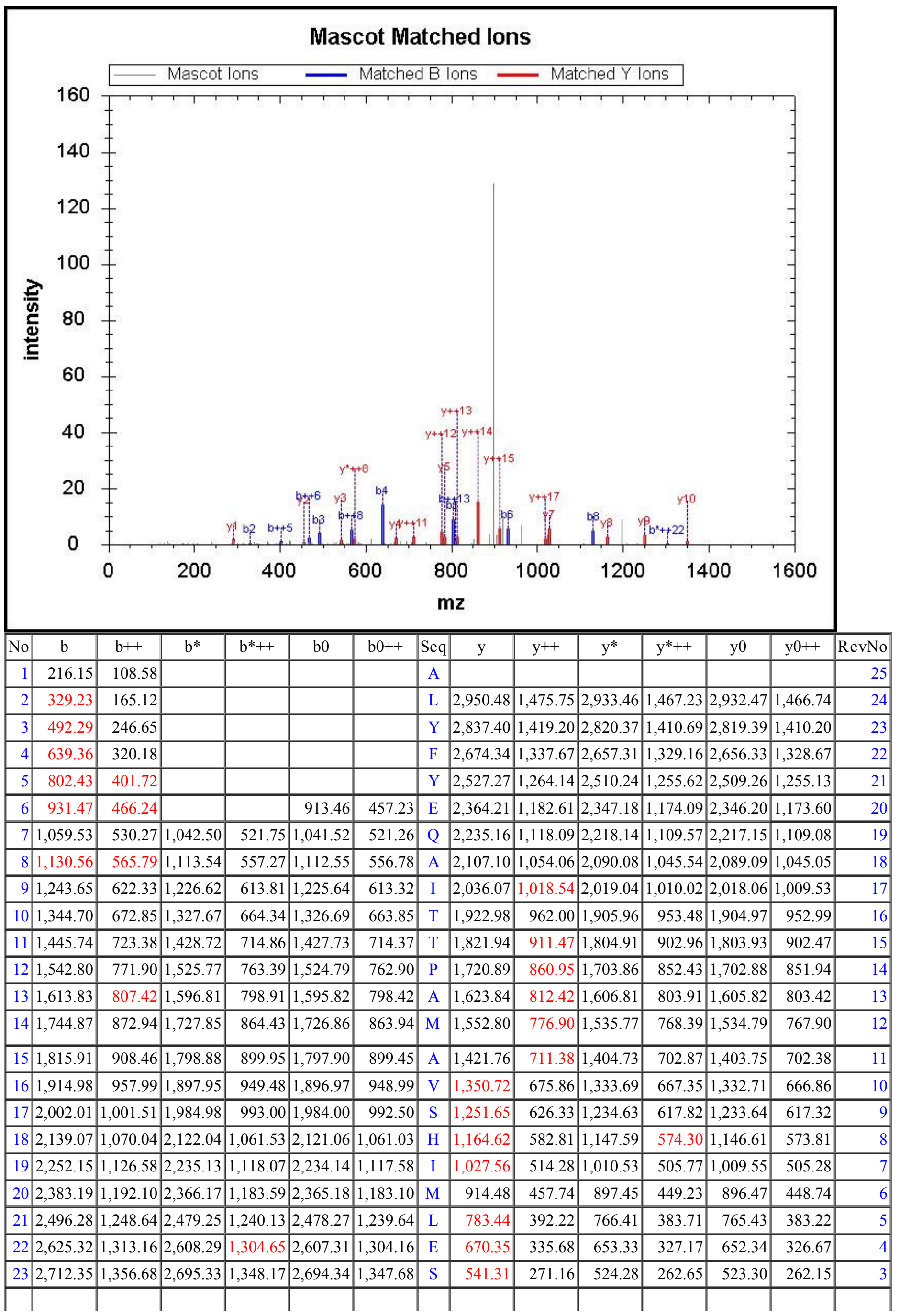




\begin{tabular}{|r|r|r|r|r|r|r|r|r|r|r|r|r|r|r|r|}
\hline 24 & $2,875.42$ & $1,438.21$ & $2,858.39$ & $1,429.70$ & $2,857.41$ & $1,429.21$ & $\mathrm{Y}$ & 454.28 & 227.64 & 437.25 & 219.13 & & & 2 \\
\hline 25 & & & & & & & $\mathrm{~K}$ & 291.21 & 146.11 & 274.19 & 137.60 & & & 1 \\
\hline
\end{tabular}

Query 15054 Hit 1

MS/MS Fragmentation of LVLLFSGK

Found in sp|Q15126|PM VK_HUM AN, Phosphomevalonate kinase OS=Homo sapiens GN=PMVK PE=1 SV=3

Match to Query 15054: 1163.75 from(582.8824,2+)

Title: 769: Sum of 2 scans in range 1800 ( $\mathrm{rt}=49.4978, \mathrm{f}=4, \mathrm{i}=509)$ to 1801 ( $\mathrm{rt}=49.5232, \mathrm{f}=4, \mathrm{i}=510$ )

[D:llab212\membranelGrace \JoyceliTRAQ_29_1.raw]

Data File:Submitted from 20120508-1(merge) by Mascot Daemon on JOYCE-VAIO

Monoisotopic mass of neutral peptide $\mathrm{Mr}$ (calc): 1163.75

Variable modifications:

K8 :iTRAQ4plex (K)

Ions Score: 50.51 Expect: 0.003

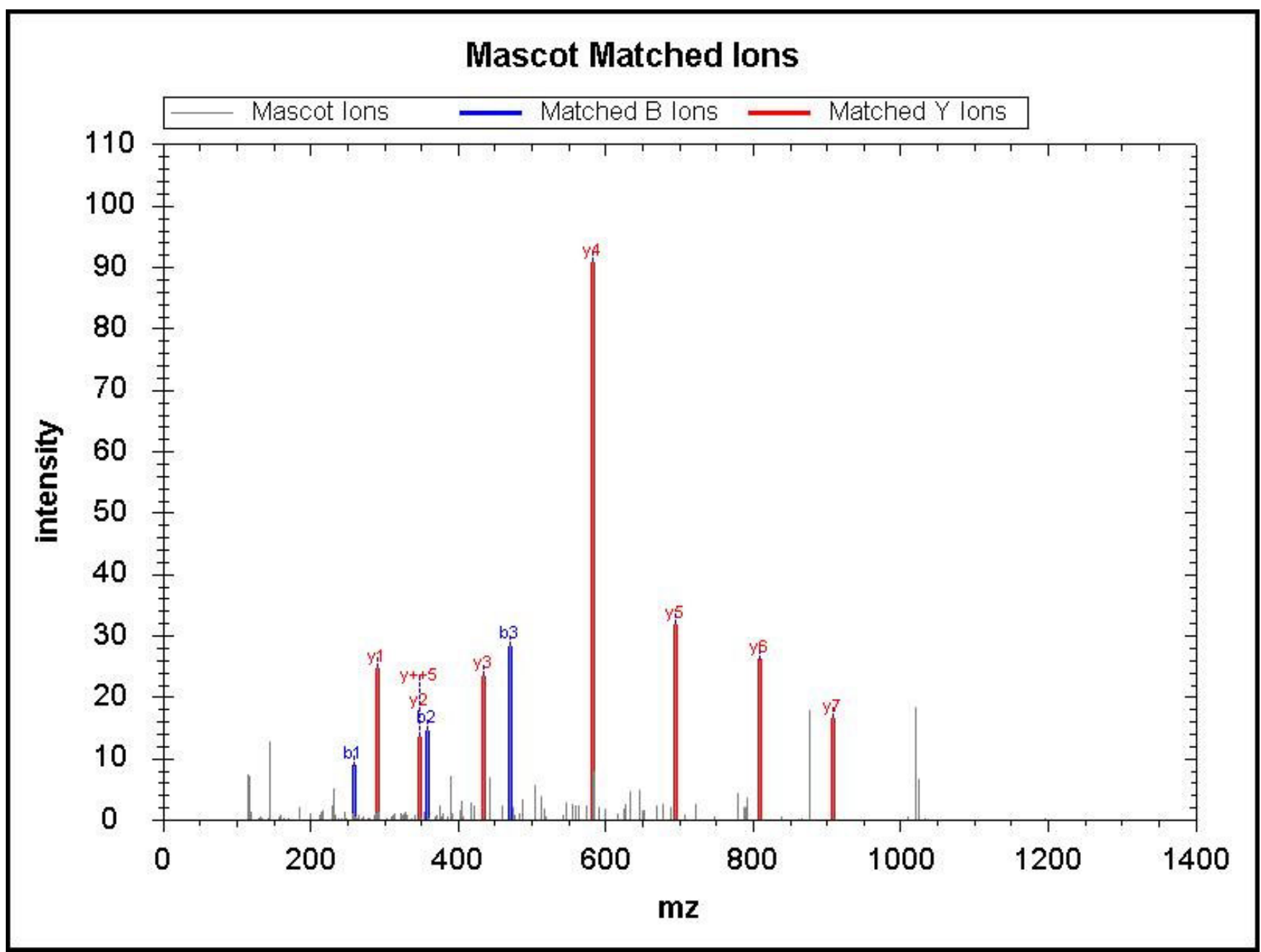

\begin{tabular}{|r|c|c|c|c|c|c|c|c|c|c|r|r|}
\hline \hline No & $\mathrm{b}$ & $\mathrm{b}++$ & $\mathrm{b} 0$ & $\mathrm{~b} 0++$ & Seq & $\mathrm{y}$ & $\mathrm{y}++$ & $\mathrm{y}^{*}$ & $\mathrm{y}^{*++}$ & $\mathrm{y} 0$ & $\mathrm{y} 0++$ & RevNo \\
\hline 1 & 258.19 & 129.60 & & & $\mathrm{~L}$ & & & & & & & 8 \\
\hline 2 & 357.26 & 179.13 & & & $\mathrm{~V}$ & 907.57 & 454.29 & 890.55 & 445.78 & 889.56 & 445.28 & 7 \\
\hline 3 & 470.35 & 235.68 & & & $\mathrm{~L}$ & 808.50 & 404.76 & 791.48 & 396.24 & 790.49 & 395.75 & 6 \\
\hline 4 & 583.43 & 292.22 & & & $\mathrm{~L}$ & 695.42 & 348.21 & 678.39 & 339.70 & 677.41 & 339.21 & 5 \\
\hline 5 & 730.50 & 365.75 & & & $\mathrm{~F}$ & 582.34 & 291.67 & 565.31 & 283.16 & 564.33 & 282.67 & 4 \\
\hline 6 & 817.53 & 409.27 & 799.52 & 400.26 & $\mathrm{~S}$ & 435.27 & 218.14 & 418.24 & 209.62 & 417.26 & 209.13 & 3 \\
\hline 7 & 874.55 & 437.78 & 856.54 & 428.77 & $\mathrm{G}$ & 348.24 & 174.62 & 331.21 & 166.11 & & & 2 \\
\hline 8 & & & & & $\mathrm{~K}$ & 291.21 & 146.11 & 274.19 & 137.60 & & & 1 \\
\hline
\end{tabular}

Query 70660 Hit 1 
Found in sp|Q9NVX0|HAUS2_HUMAN, HAUS augmin-like complex subunit 2 OS=Homo sapiens GN=HAUS2 PE=1 SV=1 Match to Query 70660: 2248.21 from(750.4106,3+)

Title: 1408: Sum of 2 scans in range $3125(\mathrm{rt}=79.5561, \mathrm{f}=2, \mathrm{i}=517)$ to $3126(\mathrm{rt}=79.5815, \mathrm{f}=2, \mathrm{i}=518)$

[D:llab212 \membranelGrace JoyceliTRAQ_26_2.raw]

Data File:Submitted from 20120508-1(merge) by Mascot Daemon on JOYCE-VAIO

Monoisotopic mass of neutral peptide $\mathrm{Mr}$ (calc): 2248.21

Variable modifications:

K17 :iTRAQ4plex (K)

Ions Score: 50.48 Expect: 0.007

\section{Mascot Matched lons}

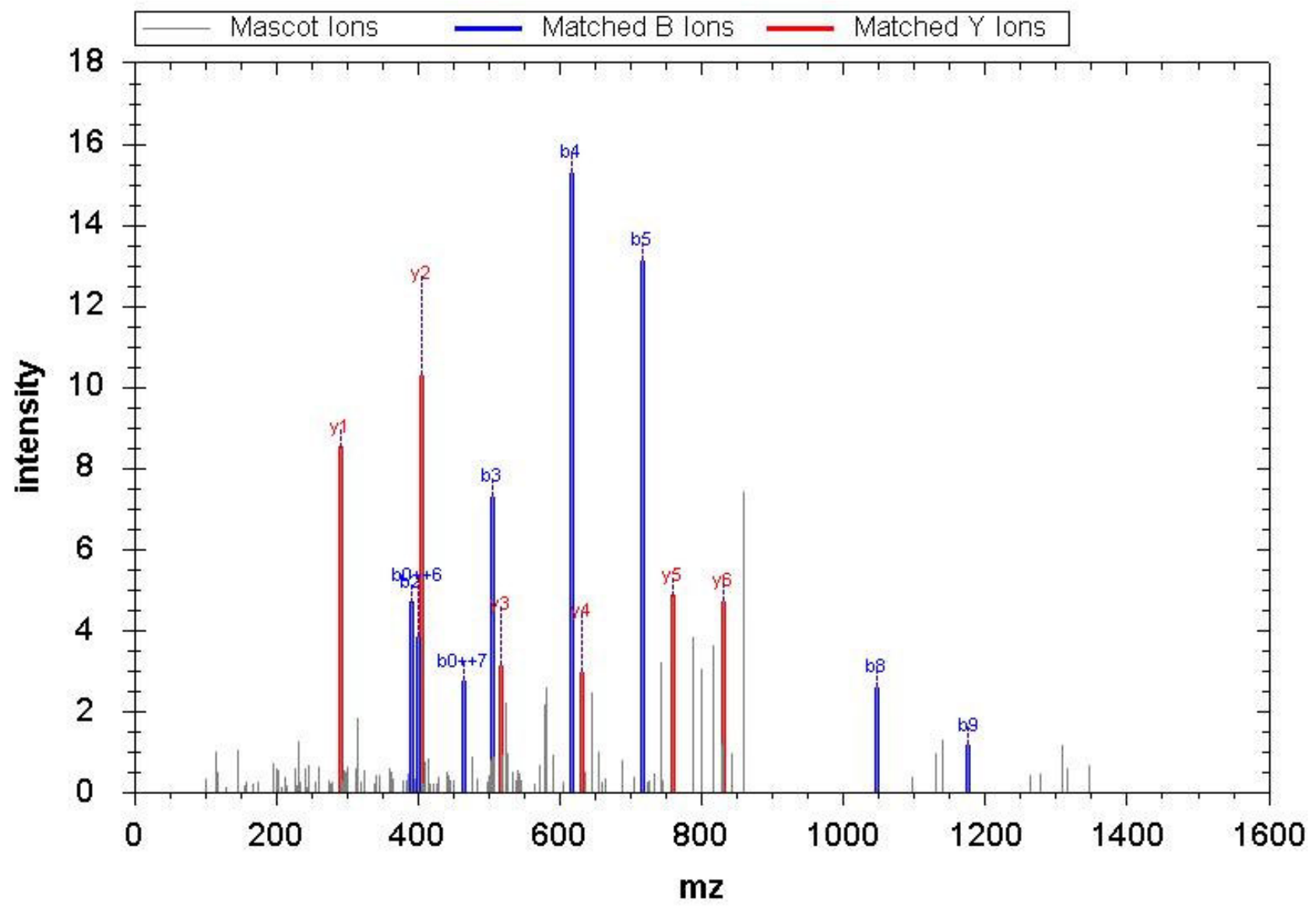

\begin{tabular}{|r|r|r|r|r|r|r|r|r|r|r|r|r|r|r|}
\hline $\mathrm{No}$ & $\mathrm{b}$ & $\mathrm{b}++$ & $\mathrm{b}^{*}$ & $\mathrm{~b}++$ & $\mathrm{b} 0$ & $\mathrm{~b} 0++$ & $\mathrm{Seq}$ & $\mathrm{y}$ & $\mathrm{y}++$ & $\mathrm{y}^{*}$ & $\mathrm{y}^{*++}$ & $\mathrm{y} 0$ & $\mathrm{y} 0++$ & $\mathrm{RevNo}$ \\
\hline 1 & 276.15 & 138.58 & & & & & $\mathrm{M}$ & & & & & & & 17 \\
\hline 2 & 391.18 & 196.09 & & & 373.17 & 187.09 & $\mathrm{D}$ & $1,974.08$ & 987.54 & $1,957.05$ & 979.03 & $1,956.07$ & 978.54 & 16 \\
\hline 3 & 504.26 & 252.63 & & & 486.25 & 243.63 & $\mathrm{I}$ & $1,859.05$ & 930.03 & $1,842.02$ & 921.52 & $1,841.04$ & 921.02 & 15 \\
\hline 4 & 617.34 & 309.18 & & & 599.33 & 300.17 & $\mathrm{~L}$ & $1,745.97$ & 873.49 & $1,728.94$ & 864.97 & $1,727.95$ & 864.48 & 14 \\
\hline 5 & 716.41 & 358.71 & & & 698.40 & 349.70 & $\mathrm{~V}$ & $1,632.88$ & 816.94 & $1,615.85$ & 808.43 & $1,614.87$ & 807.94 & 13 \\
\hline 6 & 817.46 & 409.23 & & & 799.45 & 400.23 & $\mathrm{~T}$ & $1,533.81$ & 767.41 & $1,516.79$ & 758.90 & $1,515.80$ & 758.40 & 12 \\
\hline 7 & 946.50 & 473.76 & & & 928.49 & 464.75 & E & $1,432.77$ & 716.89 & $1,415.74$ & 708.37 & $1,414.75$ & 707.88 & 11 \\
\hline 8 & $1,047.55$ & 524.28 & & & $1,029.54$ & 515.27 & T & $1,303.72$ & 652.36 & $1,286.70$ & 643.85 & $1,285.71$ & 643.36 & 10 \\
\hline 9 & $1,176.59$ & 588.80 & & & $1,158.58$ & 579.80 & E & $1,202.67$ & 601.84 & $1,185.65$ & 593.33 & $1,184.66$ & 592.84 & 9 \\
\hline 10 & $1,305.64$ & 653.32 & & & $1,287.63$ & 644.32 & E & $1,073.63$ & 537.32 & $1,056.61$ & 528.81 & $1,055.62$ & 528.31 & 8 \\
\hline 11 & $1,418.72$ & 709.86 & & & $1,400.71$ & 700.86 & L & 944.59 & 472.80 & 927.56 & 464.29 & 926.58 & 463.79 & 7 \\
\hline 12 & $1,489.76$ & 745.38 & & & $1,471.75$ & 736.38 & A & 831.51 & 416.26 & 814.48 & 407.74 & 813.49 & 407.25 & 6 \\
\hline 13 & $1,618.80$ & 809.90 & & & $1,600.79$ & 800.90 & E & 760.47 & 380.74 & 743.44 & 372.22 & 742.46 & 371.73 & 5 \\
\hline 14 & $1,732.84$ & 866.93 & $1,715.82$ & 858.41 & $1,714.83$ & 857.92 & N & 631.43 & 316.22 & 614.40 & 307.70 & & & 4 \\
\hline 15 & $1,845.93$ & 923.47 & $1,828.90$ & 914.95 & $1,827.92$ & 914.46 & I & 517.38 & 259.20 & 500.36 & 250.68 & & & 3 \\
\hline 16 & $1,959.01$ & 980.01 & $1,941.98$ & 971.50 & $1,941.00$ & 971.00 & L & 404.30 & 202.65 & 387.27 & 194.14 & & & 2 \\
\hline
\end{tabular}


Query 72926 Hit 1

MS/MS Fragmentation of FIAHVPVPSQQEIEEALVR

Found in sp|Q9ULR0|ISY1_HUMAN, Pre-mRNA-splicing factor ISY1 homolog OS=Homo sapiens GN=ISY1 PE=1 SV=3

Match to Query 72926: 2305.247from(769.423,3+)

Title: 848: Sum of 2 scans in range $1903(\mathrm{rt}=52.2309, \mathrm{f}=4, \mathrm{i}=569)$ to $1904(\mathrm{rt}=52.2563, \mathrm{f}=4, \mathrm{i}=570)$

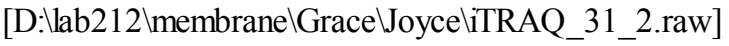

Data File:Submitted from 20120508-1(merge) by Mascot Daemon on JOYCE-VAIO

Monoisotopic mass of neutral peptide Mr(calc): 2305.247

Variable modifications:

Ions Score: 50.43 Expect: 0.007

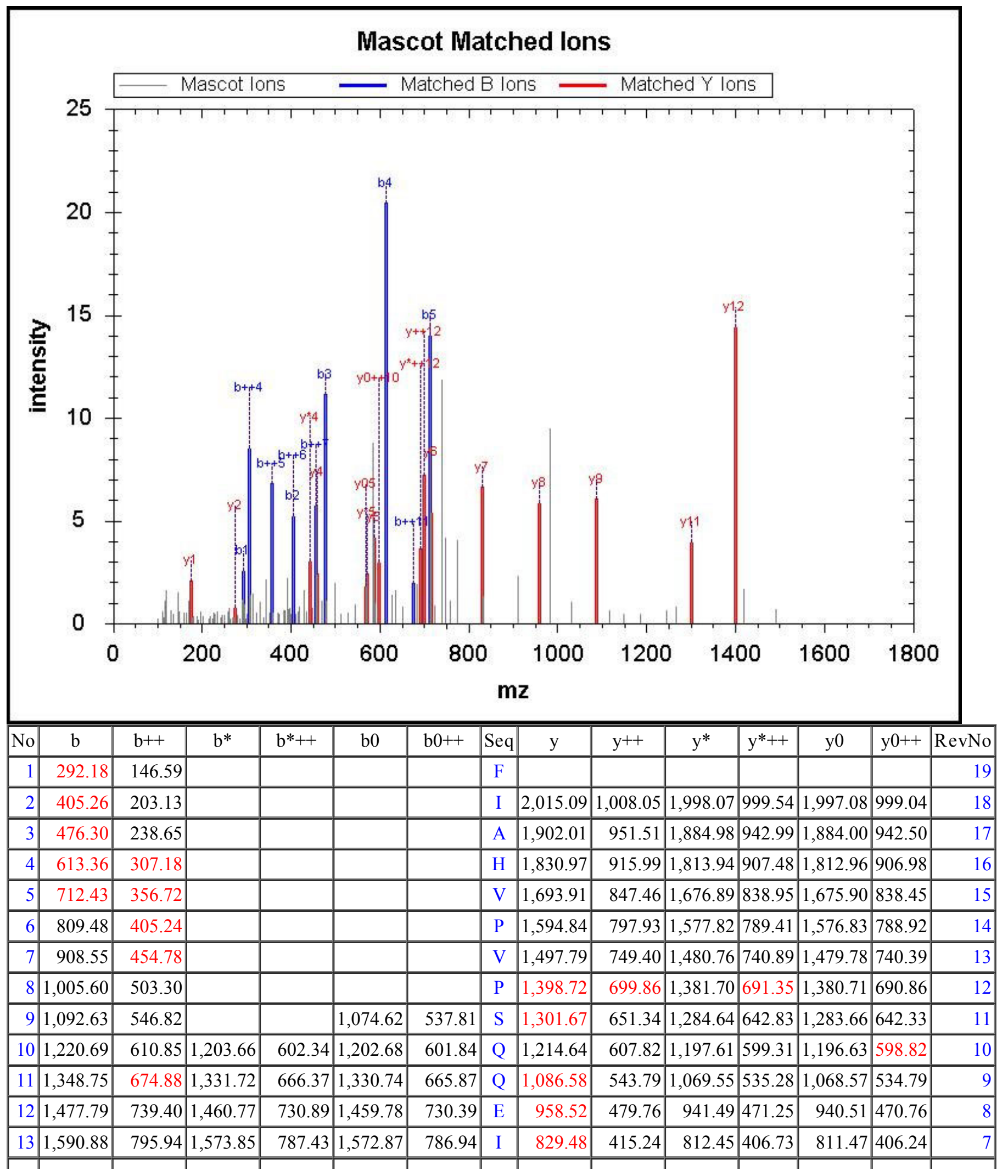




\begin{tabular}{|r|r|r|r|r|r|r|r|r|r|r|r|r|r|r|}
14 & $1,719.92$ & 860.46 & $1,702.89$ & 851.95 & $1,701.91$ & 851.46 & $\mathrm{E}$ & 716.39 & 358.70 & 699.37 & 350.19 & 698.38 & 349.70 & 6 \\
\hline 15 & $1,848.96$ & 924.98 & $1,831.93$ & 916.47 & $1,830.95$ & 915.98 & $\mathrm{E}$ & 587.35 & 294.18 & 570.32 & 285.67 & 569.34 & 285.17 & 5 \\
\hline 16 & $1,920.00$ & 960.50 & $1,902.97$ & 951.99 & $1,901.99$ & 951.50 & $\mathrm{~A}$ & 458.31 & 229.66 & 441.28 & 221.14 & & & 4 \\
\hline 17 & $2,033.08$ & $1,017.04$ & $2,016.06$ & $1,008.53$ & $2,015.07$ & $1,008.04$ & $\mathrm{~L}$ & 387.27 & 194.14 & 370.24 & 185.63 & & & 3 \\
\hline 18 & $2,132.15$ & $1,066.58$ & $2,115.12$ & $1,058.07$ & $2,114.14$ & $1,057.57$ & $\mathrm{~V}$ & 274.19 & 137.60 & 257.16 & 129.08 & & & 2 \\
\hline 19 & & & & & & & $\mathrm{R}$ & 175.12 & 88.06 & 158.09 & 79.55 & & & 1 \\
\hline
\end{tabular}

Query 45252 Hit 1

MS/MS Fragmentation of VLVDGEEHVGFLK

Found in sp|Q9NPA0|CO024_HUMAN, UPF0480 protein C15orf24 OS=Homo sapiens GN=C15orf24 PE=1 SV=1

Match to Query 45252: 1728.912from(577.3113,3+)

Title: 580: Scan 1286 ( $\mathrm{rt}=38.7193, \mathrm{f}=3, \mathrm{i}=200)$ [D:lab212 ImembranelGrace JoyceliTRAQ40.raw]

Data File:Submitted from 20120508-1(merge) by Mascot Daemon on JOYCE-VAIO

Monoisotopic mass of neutral peptide $\mathrm{Mr}$ (calc): 1728.912

Variable modifications:

K13 :TRAQ4plex (K)

Ions Score: 50.39 Expect: 0.008

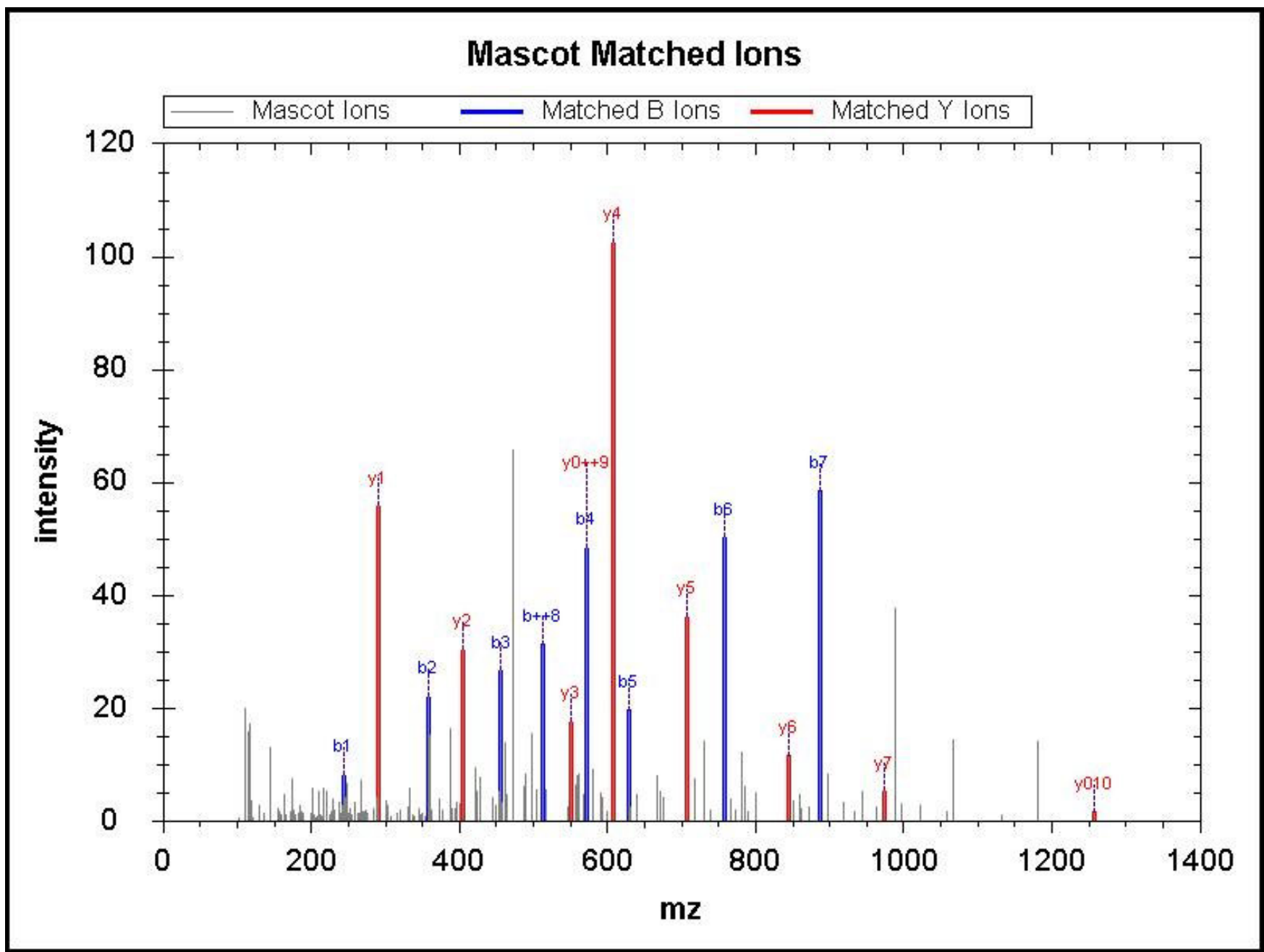

\begin{tabular}{|r|c|c|c|c|c|c|c|c|c|c|r|r|}
\hline \hline No & $\mathrm{b}$ & $\mathrm{b}++$ & $\mathrm{b} 0$ & $\mathrm{~b} 0++$ & $\mathrm{Seq}$ & $\mathrm{y}$ & $\mathrm{y}++$ & $\mathrm{y}^{*}$ & $\mathrm{y} *++$ & $\mathrm{y} 0$ & $\mathrm{y} 0++$ & RevNo \\
\hline 1 & 244.18 & 122.59 & & & $\mathrm{~V}$ & & & & & & & 13 \\
\hline 2 & 357.26 & 179.13 & & & $\mathrm{~L}$ & $1,486.80$ & 743.90 & $1,469.78$ & 735.39 & $1,468.79$ & 734.90 & 12 \\
\hline 3 & 456.33 & 228.67 & & & $\mathrm{~V}$ & $1,373.72$ & 687.36 & $1,356.69$ & 678.85 & $1,355.71$ & 678.36 & 11 \\
\hline 4 & 571.36 & 286.18 & 553.35 & 277.18 & $\mathrm{D}$ & $1,274.65$ & 637.83 & $1,257.62$ & 629.32 & $1,256.64$ & 628.82 & 10 \\
\hline 5 & 628.38 & 314.69 & 610.37 & 305.69 & $\mathrm{G}$ & $1,159.62$ & 580.32 & $1,142.60$ & 571.80 & $1,141.61$ & 571.31 & 9 \\
\hline 6 & 757.42 & 379.21 & 739.41 & 370.21 & $\mathrm{E}$ & $1,102.60$ & 551.80 & $1,085.57$ & 543.29 & $1,084.59$ & 542.80 & 8 \\
\hline 7 & 886.46 & 443.74 & 868.45 & 434.73 & $\mathrm{E}$ & 973.56 & 487.28 & 956.53 & 478.77 & 955.55 & 478.28 & 7 \\
\hline 8 & $1,023.52$ & 512.27 & $1,005.51$ & 503.26 & $\mathrm{H}$ & 844.52 & 422.76 & 827.49 & 414.25 & & & 6 \\
\hline & & & & & & & & & & & &
\end{tabular}




\begin{tabular}{|r|r|r|r|r|r|r|r|r|r|r|r|r|}
9 & $1,122.59$ & 561.80 & $1,104.58$ & 552.79 & $\mathrm{~V}$ & 707.46 & 354.23 & 690.43 & 345.72 & & & 5 \\
\hline 10 & $1,179.61$ & 590.31 & $1,161.60$ & 581.30 & $\mathrm{G}$ & 608.39 & 304.70 & 591.36 & 296.18 & & & 4 \\
\hline 11 & $1,326.68$ & 663.84 & $1,308.67$ & 654.84 & $\mathrm{~F}$ & 551.37 & 276.19 & 534.34 & 267.67 & & & 3 \\
\hline 12 & $1,439.77$ & 720.39 & $1,421.75$ & 711.38 & $\mathrm{~L}$ & 404.30 & 202.65 & 387.27 & 194.14 & & & 2 \\
\hline 13 & & & & & $\mathrm{~K}$ & 291.21 & 146.11 & 274.19 & 137.60 & & & 1 \\
\hline
\end{tabular}

Query 73054 Hit 1

MS/MS Fragmentation of GQTVEDLLEVLSDIDEMSR

Found in sp|Q8N201|INT1_HUM AN, Integrator complex subunit 1 OS=Homo sapiens GN=INTS1 PE=1 SV=2

Match to Query 73054:2308.123from(770.3816,3+)

Title: 898: Sum of 2 scans in range $2847(\mathrm{rt}=71.4021, \mathrm{f}=4, \mathrm{i}=487)$ to $2848(\mathrm{rt}=71.4275, \mathrm{f}=4, \mathrm{i}=488)$

[D:llab212 \membranelGracelJoyceliTRAQ19.raw]

Data File:Submitted from 20120508-1(merge) by Mascot Daemon on JOYCE-VAIO

Monoisotopic mass of neutral peptide $\mathrm{Mr}$ (calc): 2308.123

Variable modifications:

M17 :Oxidation (M), 63.99828

Ions Score: 50.33 Expect: 0.008

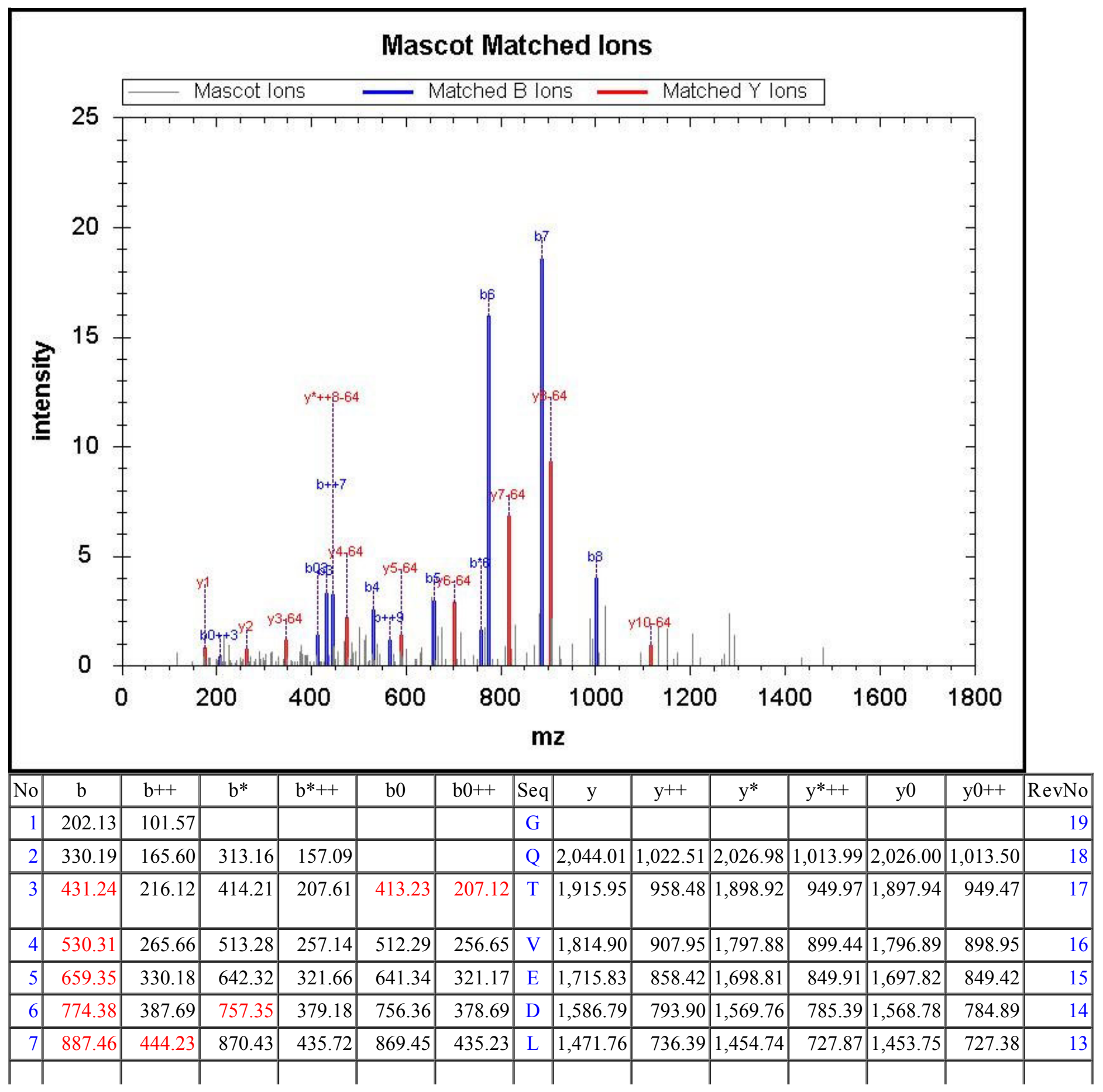




\begin{tabular}{|r|r|r|r|r|r|r|r|r|r|r|r|r|r|r|r|r|}
\hline 8 & $1,000.54$ & 500.78 & 983.52 & 492.26 & 982.53 & 491.77 & $\mathrm{~L}$ & $1,358.68$ & 679.84 & $1,341.65$ & 671.33 & $1,340.67$ & 670.84 & 12 \\
\hline 9 & $1,129.59$ & 565.30 & $1,112.56$ & 556.78 & $1,111.58$ & 556.29 & $\mathrm{E}$ & $1,245.60$ & 623.30 & $1,228.57$ & 614.79 & $1,227.58$ & 614.30 & 11 \\
\hline 10 & $1,228.65$ & 614.83 & $1,211.63$ & 606.32 & $1,210.64$ & 605.83 & $\mathrm{~V}$ & $1,116.55$ & 558.78 & $1,099.53$ & 550.27 & $1,098.54$ & 549.77 & 10 \\
\hline 11 & $1,341.74$ & 671.37 & $1,324.71$ & 662.86 & $1,323.73$ & 662.37 & $\mathrm{~L}$ & $1,017.48$ & 509.25 & $1,000.46$ & 500.73 & 999.47 & 500.24 & 9 \\
\hline 12 & $1,428.77$ & 714.89 & $1,411.74$ & 706.38 & $1,410.76$ & 705.88 & $\mathrm{~S}$ & 904.40 & 452.70 & 887.37 & 444.19 & 886.39 & 443.70 & 8 \\
\hline 13 & $1,543.80$ & 772.40 & $1,526.77$ & 763.89 & $1,525.79$ & 763.40 & $\mathrm{D}$ & 817.37 & 409.19 & 800.34 & 400.67 & 799.36 & 400.18 & 7 \\
\hline 14 & $1,656.88$ & 828.94 & $1,639.85$ & 820.43 & $1,638.87$ & 819.94 & $\mathrm{I}$ & 702.34 & 351.67 & 685.32 & 343.16 & 684.33 & 342.67 & 6 \\
\hline 15 & $1,771.91$ & 886.46 & $1,754.88$ & 877.94 & $1,753.90$ & 877.45 & $\mathrm{D}$ & 589.26 & 295.13 & 572.23 & 286.62 & 571.25 & 286.13 & 5 \\
\hline 16 & $1,900.95$ & 950.98 & $1,883.92$ & 942.47 & $1,882.94$ & 941.97 & $\mathrm{E}$ & 474.23 & 237.62 & 457.20 & 229.11 & 456.22 & 228.61 & 4 \\
\hline 17 & $1,983.99$ & 992.50 & $1,966.96$ & 983.98 & $1,965.98$ & 983.49 & $\mathrm{M}$ & 345.19 & 173.10 & 328.16 & 164.58 & 327.18 & 164.09 & 3 \\
\hline 18 & $2,071.02$ & $1,036.01$ & $2,053.99$ & $1,027.50$ & $2,053.01$ & $1,027.01$ & $\mathrm{~S}$ & 262.15 & 131.58 & 245.12 & 123.07 & 244.14 & 122.57 & 2 \\
\hline 19 & & & & & & & $\mathrm{R}$ & 175.12 & 88.06 & 158.09 & 79.55 & & & 1 \\
\hline
\end{tabular}

Query 53264 Hit 1

MS/MS Fragmentation of VNPDMNFEVFIHK

Found in sp|Q9HB90|RRAGC_HUMAN, Ras-related GTP-binding protein C OS=Homo sapiens GN=RRAGC PE=1 SV=1 Match to Query 53264: 1876.942 from(626.6545,3+)

Title: 764: Sum of 2 scans in range $1665(\mathrm{rt}=47.2628, \mathrm{f}=4, \mathrm{i}=517)$ to $1666(\mathrm{rt}=47.2882, \mathrm{f}=4, \mathrm{i}=518)$

[D:lab212 \membranelGrace JoyceliTRAQ40.raw]

Data File:Submitted from 20120508-1 (merge) by Mascot Daemon on JOYCE-VAIO

Monoisotopic mass of neutral peptide $\operatorname{Mr}($ calc): 1876.942

Variable modifications:

K13 :iTRAQ4plex (K)

Ions Score: 50.32 Expect: 0.009

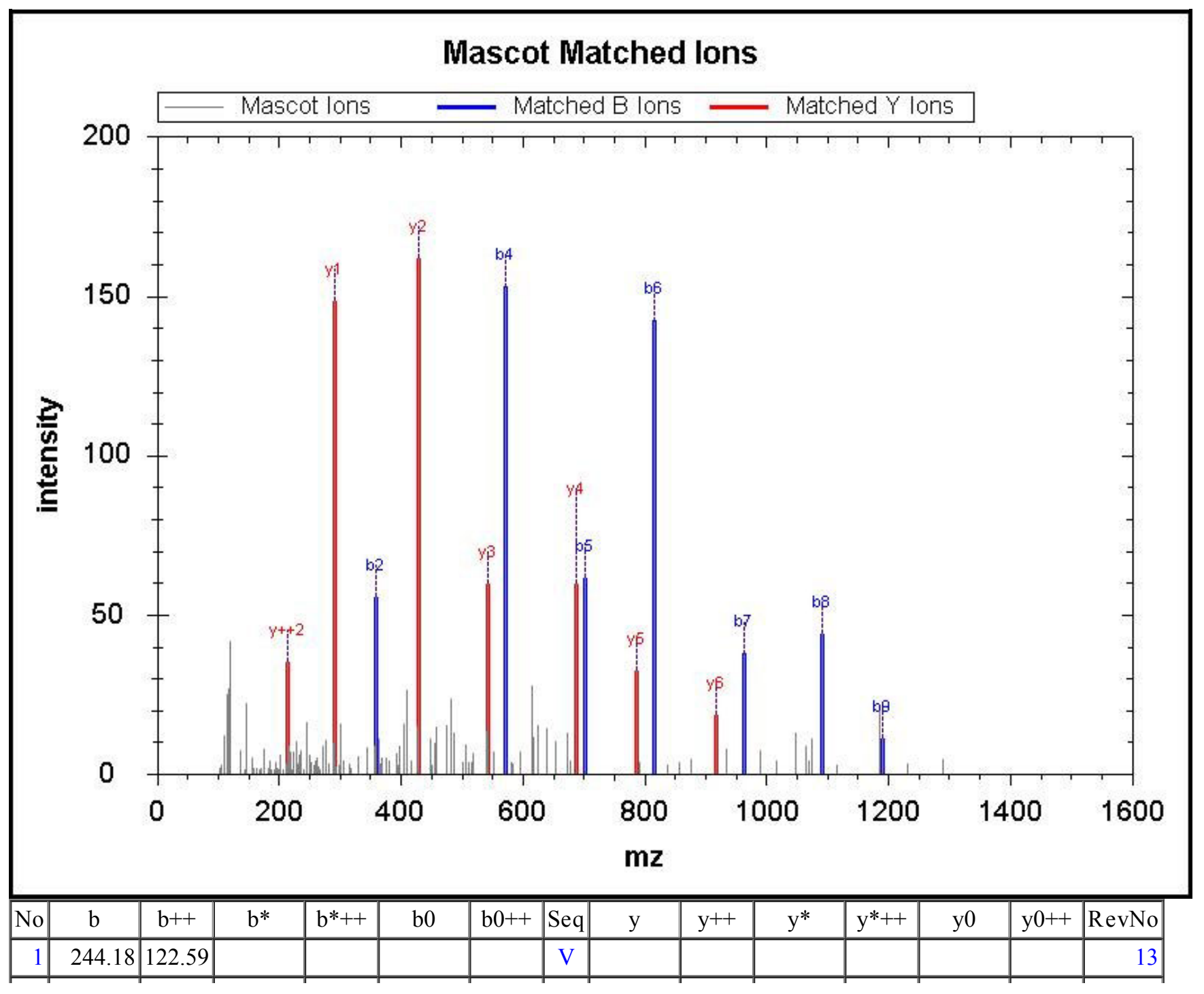




\begin{tabular}{|r|r|r|r|r|r|r|r|r|r|r|r|r|r|r|}
2 & 358.22 & 179.61 & 341.19 & 171.10 & & & $\mathrm{~N}$ & $1,634.81$ & 817.91 & $1,617.79$ & 809.40 & $1,616.80$ & 808.90 & 12 \\
\hline \hline 3 & 455.27 & 228.14 & 438.25 & 219.63 & & & $\mathrm{P}$ & $1,520.77$ & 760.89 & $1,503.74$ & 752.37 & $1,502.76$ & 751.88 & 11 \\
\hline 4 & 570.30 & 285.65 & 553.27 & 277.14 & 552.29 & 276.65 & $\mathrm{D}$ & $1,423.72$ & 712.36 & $1,406.69$ & 703.85 & $1,405.71$ & 703.36 & 10 \\
\hline 5 & 701.34 & 351.17 & 684.31 & 342.66 & 683.33 & 342.17 & $\mathrm{M}$ & $1,308.69$ & 654.85 & $1,291.66$ & 646.34 & $1,290.68$ & 645.84 & 9 \\
\hline 6 & 815.38 & 408.20 & 798.36 & 399.68 & 797.37 & 399.19 & $\mathrm{~N}$ & $1,177.65$ & 589.33 & $1,160.62$ & 580.81 & $1,159.64$ & 580.32 & 8 \\
\hline 7 & 962.45 & 481.73 & 945.43 & 473.22 & 944.44 & 472.72 & $\mathrm{~F}$ & $1,063.61$ & 532.31 & $1,046.58$ & 523.79 & $1,045.60$ & 523.30 & 7 \\
\hline 8 & $1,091.49$ & 546.25 & $1,074.47$ & 537.74 & $1,073.48$ & 537.25 & $\mathrm{E}$ & 916.54 & 458.77 & 899.51 & 450.26 & 898.53 & 449.77 & 6 \\
\hline 9 & $1,190.56$ & 595.79 & $1,173.54$ & 587.27 & $1,172.55$ & 586.78 & $\mathrm{~V}$ & 787.49 & 394.25 & 770.47 & 385.74 & & & 5 \\
\hline 10 & $1,337.63$ & 669.32 & $1,320.61$ & 660.81 & $1,319.62$ & 660.31 & $\mathrm{~F}$ & 688.43 & 344.72 & 671.40 & 336.20 & & & 4 \\
\hline 11 & $1,450.72$ & 725.86 & $1,433.69$ & 717.35 & $1,432.71$ & 716.86 & $\mathrm{I}$ & 541.36 & 271.18 & 524.33 & 262.67 & & & 3 \\
\hline 12 & $1,587.77$ & 794.39 & $1,570.75$ & 785.88 & $1,569.76$ & 785.39 & $\mathrm{H}$ & 428.27 & 214.64 & 411.25 & 206.13 & & & 2 \\
\hline 13 & & & & & & & $\mathrm{~K}$ & 291.21 & 146.11 & 274.19 & 137.60 & & & 1 \\
\hline
\end{tabular}

Query 37666 Hit 1

\section{MS/MS Fragmentation of HLEEIVHVEQGR}

Found in sp|Q01433|AMPD2_HUM AN, AMP deaminase 2 OS=Homo sapiens GN=AMPD2 PE=1 SV=2

Match to Query 37666: 1588.849 from(530.6238,3+)

Title: 335: Scan 764 ( $\mathrm{rt}=26.8968, \mathrm{f}=3, \mathrm{i}=114)$ [D:lab212 $\backslash$ membranelGracelJoyceliTRAQ_50_2.raw]

Data File:Submitted from 20120508-1(merge) by Mascot Daemon on JOYCE-VAIO

Monoisotopic mass of neutral peptide $\operatorname{Mr}($ calc): 1588.849

Variable modifications:

Ions Score: 50.28 Expect: 0.008

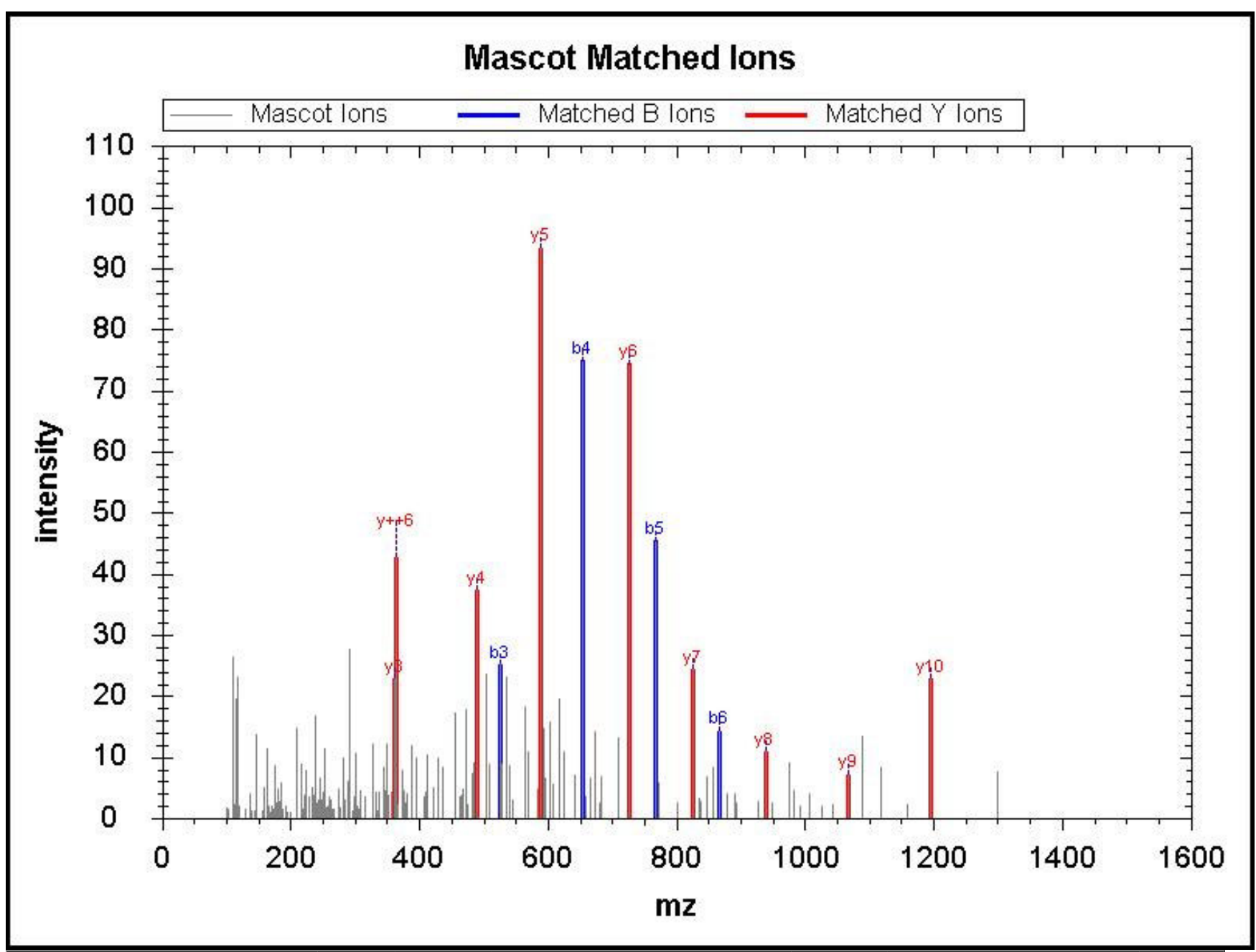

\begin{tabular}{|r|c|c|c|c|c|c|c|c|c|c|c|r|r|r|}
\hline \hline No & $\mathrm{b}$ & $\mathrm{b}++$ & $\mathrm{b}^{*}$ & $\mathrm{~b}^{*++}$ & $\mathrm{b} 0$ & $\mathrm{~b} 0++$ & Seq & $\mathrm{y}$ & $\mathrm{y}++$ & $\mathrm{y}^{*}$ & $\mathrm{y}^{*++}$ & $\mathrm{y} 0$ & $\mathrm{y} 0++$ & $\mathrm{RevNo}$ \\
\hline 1 & 282.17 & 141.59 & & & & & $\mathrm{H}$ & & & & & & & 12 \\
\hline 2 & 395.25 & 198.13 & & & & & L & $1,308.69$ & 654.85 & $1,291.66$ & 646.34 & $1,290.68$ & 645.84 & 11 \\
\hline 3 & 524.29 & 262.65 & & & 506.28 & 253.65 & E & $1,195.61$ & 598.31 & $1,178.58$ & 589.79 & $1,177.60$ & 589.30 & 10 \\
\hline
\end{tabular}




\begin{tabular}{|r|r|r|r|r|r|r|r|r|r|r|r|r|r|r|r|}
4 & 653.34 & 327.17 & & & 635.33 & 318.17 & $\mathrm{E}$ & $1,066.56$ & 533.79 & $1,049.54$ & 525.27 & $1,048.55$ & 524.78 & 9 \\
\hline 5 & 766.42 & 383.71 & & & 748.41 & 374.71 & $\mathrm{I}$ & 937.52 & 469.26 & 920.49 & 460.75 & 919.51 & 460.26 & 8 \\
\hline 6 & 865.49 & 433.25 & & & 847.48 & 424.24 & $\mathrm{~V}$ & 824.44 & 412.72 & 807.41 & 404.21 & 806.43 & 403.72 & 7 \\
\hline 7 & $1,002.55$ & 501.78 & & & 984.54 & 492.77 & $\mathrm{H}$ & 725.37 & 363.19 & 708.34 & 354.67 & 707.36 & 354.18 & 6 \\
\hline 8 & $1,101.62$ & 551.31 & & & $1,083.61$ & 542.31 & $\mathrm{~V}$ & 588.31 & 294.66 & 571.28 & 286.15 & 570.30 & 285.65 & 5 \\
\hline 9 & $1,230.66$ & 615.83 & & & $1,212.65$ & 606.83 & $\mathrm{E}$ & 489.24 & 245.12 & 472.22 & 236.61 & 471.23 & 236.12 & 4 \\
\hline 10 & $1,358.72$ & 679.86 & $1,341.69$ & 671.35 & $1,340.71$ & 670.86 & $\mathrm{Q}$ & 360.20 & 180.60 & 343.17 & 172.09 & & & 3 \\
\hline 11 & $1,415.74$ & 708.37 & $1,398.71$ & 699.86 & $1,397.73$ & 699.37 & $\mathrm{G}$ & 232.14 & 116.57 & 215.11 & 108.06 & & & 2 \\
\hline 12 & & & & & & & $\mathrm{R}$ & 175.12 & 88.06 & 158.09 & 79.55 & & & 1 \\
\hline
\end{tabular}

Query 52691 Hit 1

MS/MS Fragmentation of IQHAVQLATEPLEK

Found in sp|P49589|SYCC_HUM AN, Cysteine--tRNA ligase

Match to Query 52691: 1864.068 from(622.3633,3+)

Title: 548: Scan 1226 ( $\mathrm{rt}=37.2987, \mathrm{f}=3, \mathrm{i}=191$ ) [D:llab212 membranelGracelJoyceliTRAQ_40_1.raw]

Data File:Submitted from 20120508-1(merge) by Mascot Daemon on JOYCE-VAIO

Monoisotopic mass of neutral peptide $\mathrm{Mr}$ (calc): 1864.068

Variable modifications:

K14 :TRAQ4plex (K)

Ions Score: 50.27 Expect: 0.005

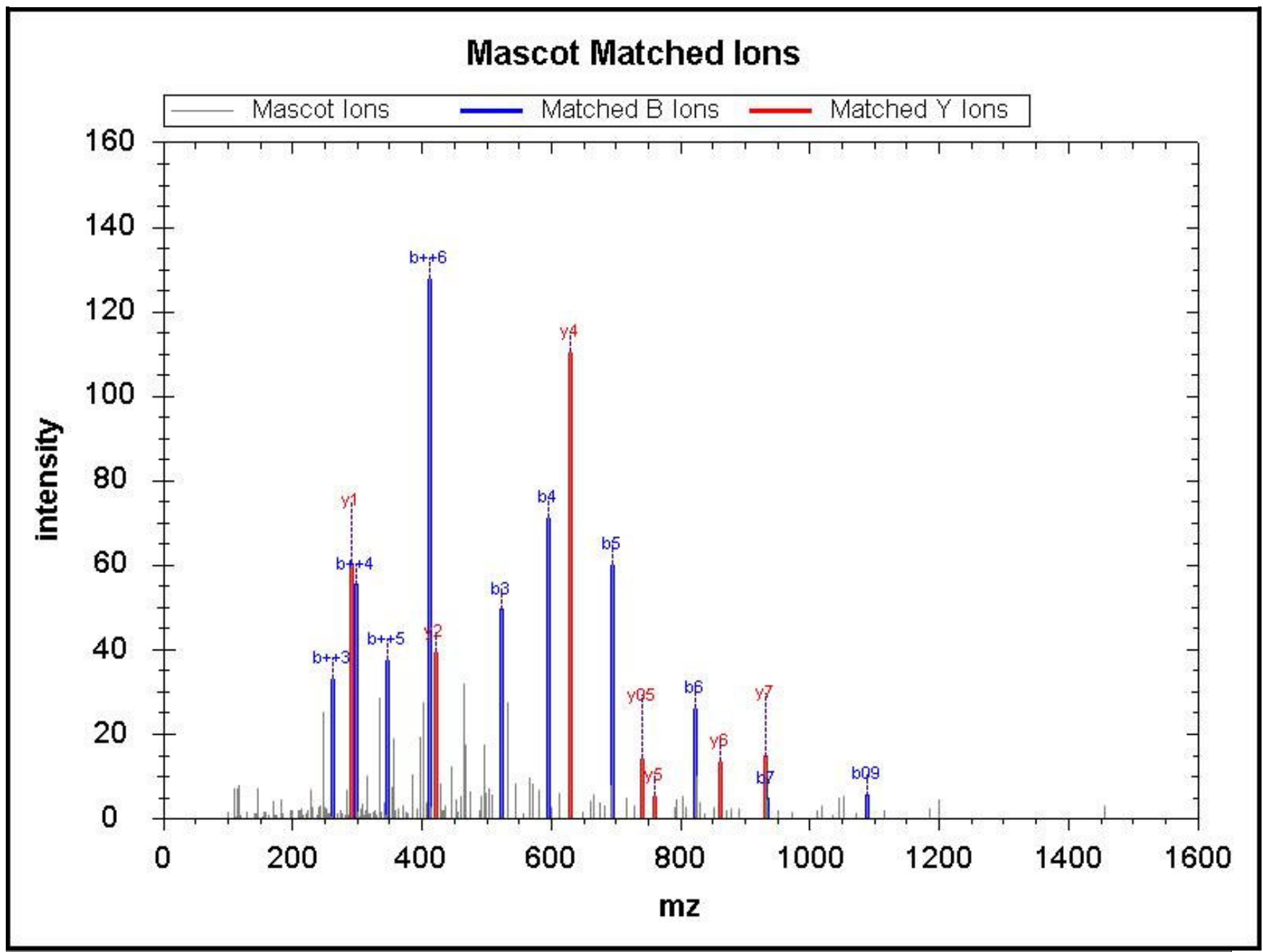

\begin{tabular}{|r|c|c|c|c|c|c|c|c|c|c|c|c|c|r|}
\hline \hline $\mathrm{No}$ & $\mathrm{b}$ & $\mathrm{b}++$ & $\mathrm{b}^{*}$ & $\mathrm{~b}^{*++}$ & $\mathrm{b} 0$ & $\mathrm{~b} 0++$ & Seq & $\mathrm{y}$ & $\mathrm{y}++$ & $\mathrm{y}^{*}$ & $\mathrm{y}^{*++}$ & $\mathrm{y} 0$ & $\mathrm{y} 0++$ & $\mathrm{RevNo}$ \\
\hline 1 & 258.19 & 129.60 & & & & & $\mathrm{I}$ & & & & & & & 14 \\
\hline 2 & 386.25 & 193.63 & 369.23 & 185.12 & & & $\mathrm{Q}$ & $1,607.89$ & 804.45 & $1,590.86$ & 795.93 & $1,589.88$ & 795.44 & 13 \\
\hline 3 & 523.31 & 262.16 & 506.28 & 253.65 & & & $\mathrm{H}$ & $1,479.83$ & 740.42 & $1,462.80$ & 731.90 & $1,461.82$ & 731.41 & 12 \\
\hline 4 & 594.35 & 297.68 & 577.32 & 289.16 & & & $\mathrm{~A}$ & $1,342.77$ & 671.89 & $1,325.74$ & 663.38 & $1,324.76$ & 662.88 & 11 \\
\hline 5 & 693.42 & 347.21 & 676.39 & 338.70 & & & $\mathrm{~V}$ & $1,271.73$ & 636.37 & $1,254.71$ & 627.86 & $1,253.72$ & 627.36 & 10 \\
\hline
\end{tabular}




\begin{tabular}{|r|r|r|r|r|r|r|r|r|r|r|r|r|r|r|r|}
6 & 821.47 & 411.24 & 804.45 & 402.73 & & & $\mathrm{Q}$ & $1,172.66$ & 586.84 & $1,155.64$ & 578.32 & $1,154.65$ & 577.83 & 9 \\
\hline \hline 7 & 934.56 & 467.78 & 917.53 & 459.27 & & & $\mathrm{~L}$ & $1,044.61$ & 522.81 & $1,027.58$ & 514.29 & $1,026.60$ & 513.80 & 8 \\
\hline 8 & $1,005.60$ & 503.30 & 988.57 & 494.79 & & & $\mathrm{~A}$ & 931.52 & 466.26 & 914.50 & 457.75 & 913.51 & 457.26 & 7 \\
\hline 9 & $1,106.64$ & 553.83 & $1,089.62$ & 545.31 & $1,088.63$ & 544.82 & $\mathrm{~T}$ & 860.48 & 430.75 & 843.46 & 422.23 & 842.47 & 421.74 & 6 \\
\hline 10 & $1,235.69$ & 618.35 & $1,218.66$ & 609.83 & $1,217.68$ & 609.34 & $\mathrm{E}$ & 759.44 & 380.22 & 742.41 & 371.71 & 741.43 & 371.22 & 5 \\
\hline 11 & $1,332.74$ & 666.87 & $1,315.71$ & 658.36 & $1,314.73$ & 657.87 & $\mathrm{P}$ & 630.39 & 315.70 & 613.37 & 307.19 & 612.38 & 306.70 & 4 \\
\hline 12 & $1,445.82$ & 723.42 & $1,428.80$ & 714.90 & $1,427.81$ & 714.41 & $\mathrm{~L}$ & 533.34 & 267.17 & 516.31 & 258.66 & 515.33 & 258.17 & 3 \\
\hline 13 & $1,574.87$ & 787.94 & $1,557.84$ & 779.42 & $1,556.86$ & 778.93 & $\mathrm{E}$ & 420.26 & 210.63 & 403.23 & 202.12 & 402.25 & 201.63 & 2 \\
\hline 14 & & & & & & & $\mathrm{~K}$ & 291.21 & 146.11 & 274.19 & 137.60 & & & 1 \\
\hline
\end{tabular}

Query 25904 Hit 1

MS/MS Fragmentation of LLLEQILNK

Found in sp|O43913|ORC5_HUM AN, Origin recognition complex subunit 5 OS=Homo sapiens GN=ORC5 PE=1 SV=1

Match to Query 25904: 1370.78from(686.3972,2+)

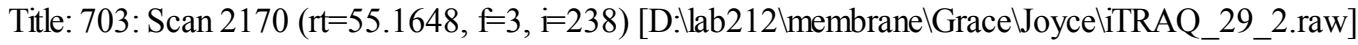

Data File:Submitted from 20120508-1(merge) by Mascot Daemon on JOYCE-VAIO

Monoisotopic mass of neutral peptide $\operatorname{Mr}$ (calc): 1370.78

Variable modifications:

K9 :TTRAQ4plex (K)

Ions Score: 50.24 Expect: 0.006

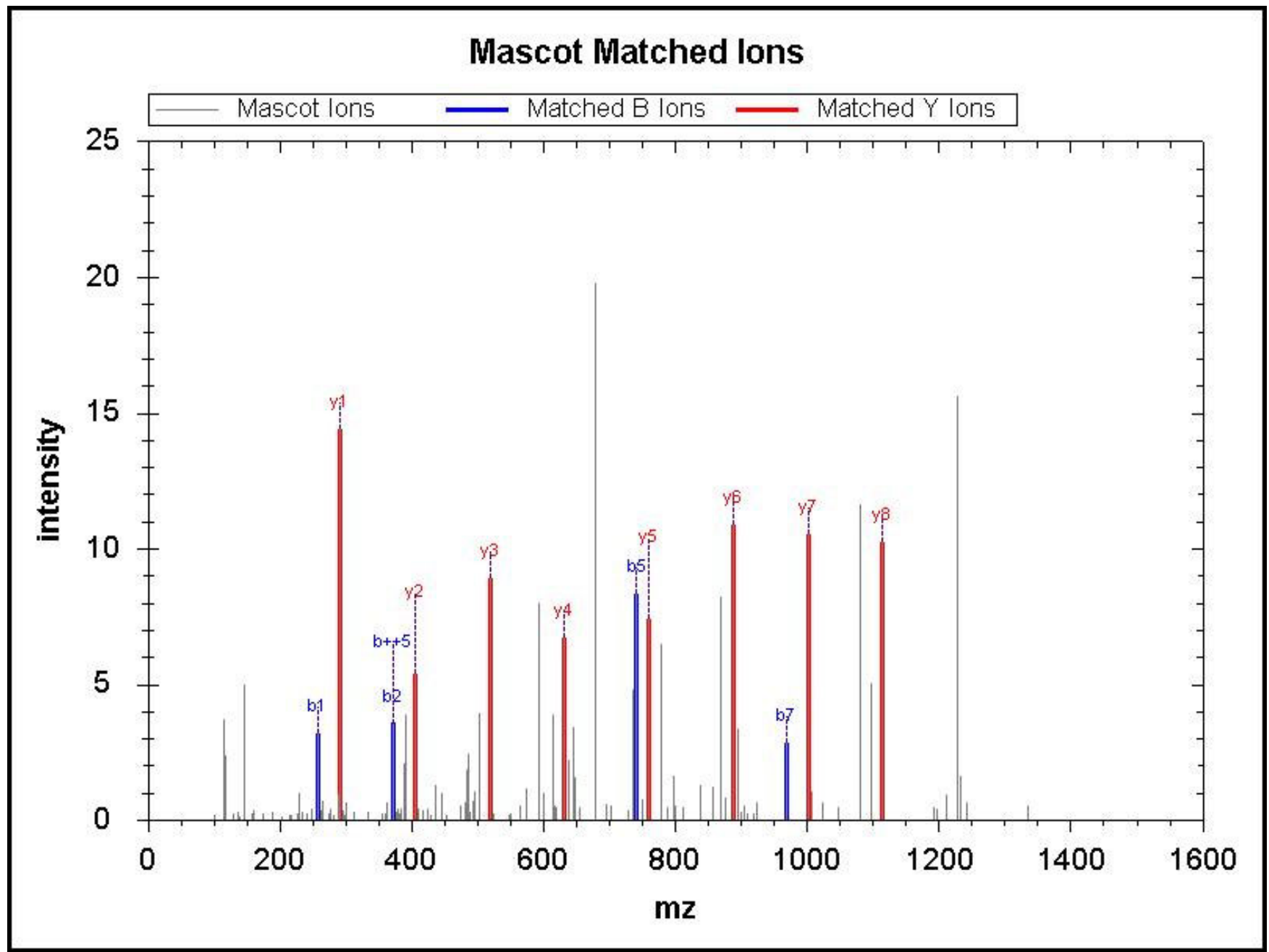

\begin{tabular}{|r|c|c|c|c|c|r|r|r|r|r|r|r|r|r|}
\hline \hline No & $\mathrm{b}$ & $\mathrm{b}++$ & $\mathrm{b}^{*}$ & $\mathrm{~b} *++$ & $\mathrm{b} 0$ & $\mathrm{~b} 0++$ & Seq & $\mathrm{y}$ & $\mathrm{y}++$ & $\mathrm{y}^{*}$ & $\mathrm{y}^{*++}$ & $\mathrm{y} 0$ & $\mathrm{y} 0++$ & $\mathrm{RevNo}$ \\
\hline 1 & 258.19 & 129.60 & & & & & L & & & & & & & \\
\hline 2 & 371.28 & 186.14 & & & & & L & $1,114.70$ & 557.85 & $1,097.67$ & 549.34 & $1,096.68$ & 548.85 & 8 \\
\hline 3 & 484.36 & 242.68 & & & & & L & $1,001.61$ & 501.31 & 984.58 & 492.80 & 983.60 & 492.30 & 7 \\
\hline 4 & 613.40 & 307.21 & & & 595.39 & 298.20 & E & 888.53 & 444.77 & 871.50 & 436.25 & 870.52 & 435.76 & 6 \\
\hline 5 & 741.46 & 371.23 & 724.44 & 362.72 & 723.45 & 362.23 & Q & 759.48 & 380.25 & 742.46 & 371.73 & & & \\
\hline
\end{tabular}




\begin{tabular}{|r|r|r|r|r|r|r|r|r|r|r|r|r|r|r|}
6 & 854.55 & 427.78 & 837.52 & 419.26 & 836.54 & 418.77 & $\mathrm{I}$ & 631.43 & 316.22 & 614.40 & 307.70 & & & 4 \\
\hline \hline 7 & 967.63 & 484.32 & 950.60 & 475.81 & 949.62 & 475.31 & $\mathrm{~L}$ & 518.34 & 259.67 & 501.32 & 251.16 & & & 3 \\
\hline 8 & $1,081.67$ & 541.34 & $1,064.65$ & 532.83 & $1,063.66$ & 532.34 & $\mathrm{~N}$ & 405.26 & 203.13 & 388.23 & 194.62 & & & 2 \\
\hline 9 & & & & & & & $\mathrm{~K}$ & 291.21 & 146.11 & 274.19 & 137.60 & & & 1 \\
\hline
\end{tabular}

Query 40807 Hit 1

MS/MS Fragmentation of VLGQPHHELDSK

Found in sp|Q96DH6|MSI2H_HUMAN, RNA-binding protein Musashi homolog 2 OS=Homo sapiens GN=MSI2 PE=1 SV=1 Match to Query 40807: 1646.9from(549.9739,3+)

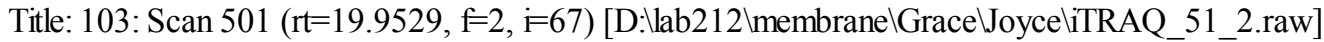

Data File:Submitted from 20120508-1(merge) by Mascot Daemon on JOYCE-VAIO

Monoisotopic mass of neutral peptide $\mathrm{Mr}$ (calc): 1646.9

Variable modifications:

K12 :iTRAQ4plex (K)

Ions Score: 50.21 Expect: 0.008

\section{Mascot Matched lons}

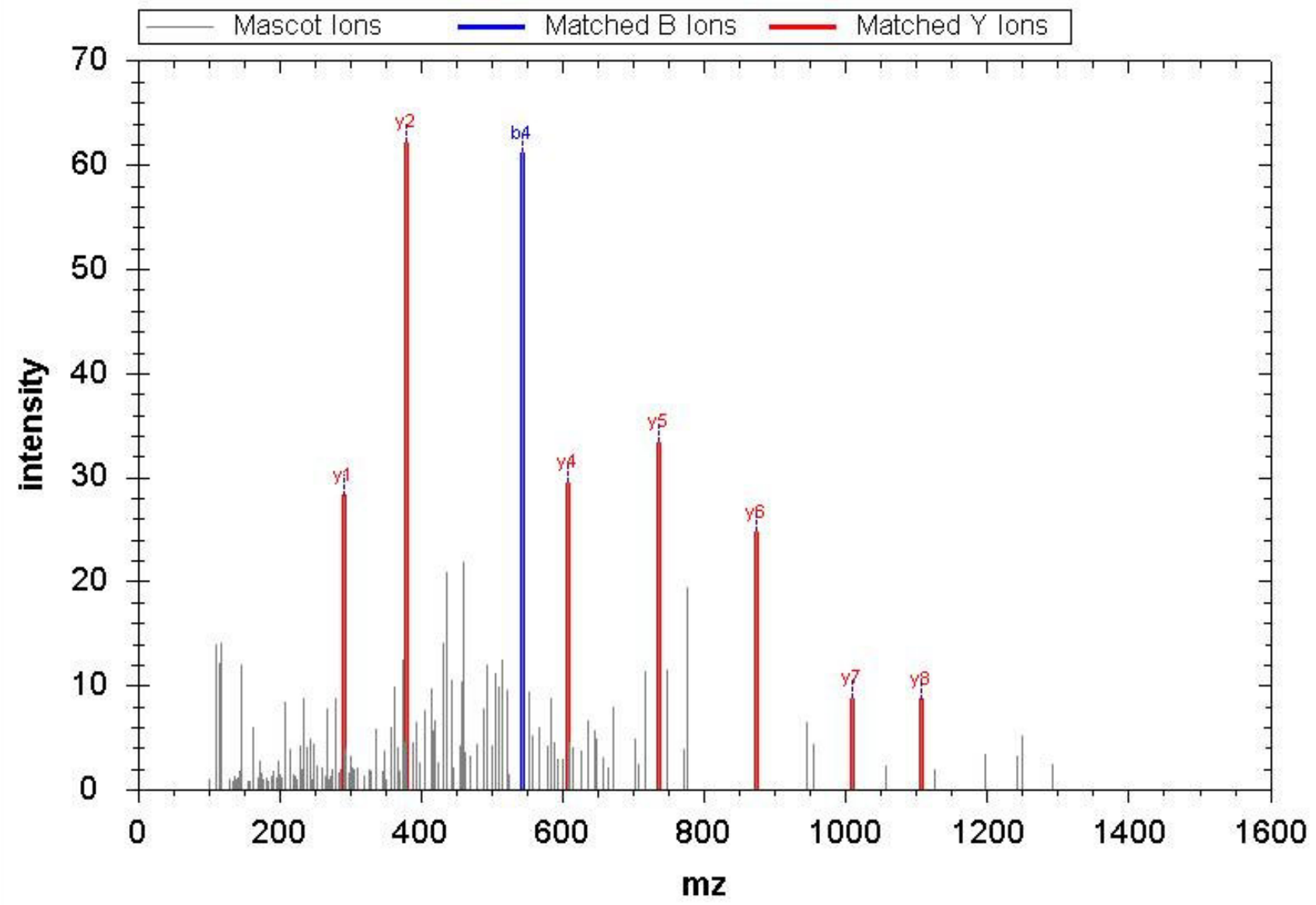

\begin{tabular}{|r|c|c|c|c|c|r|c|c|c|c|c|r|r|r|}
\hline \hline No & $\mathrm{b}$ & $\mathrm{b}++$ & $\mathrm{b}^{*}$ & $\mathrm{~b}^{*++}$ & $\mathrm{b} 0$ & $\mathrm{~b} 0++$ & Seq & $\mathrm{y}$ & $\mathrm{y}++$ & $\mathrm{y}^{*}$ & $\mathrm{y}^{*++}$ & $\mathrm{y} 0$ & $\mathrm{y} 0++$ & $\mathrm{RevNo}$ \\
\hline 1 & 244.18 & 122.59 & & & & & $\mathrm{~V}$ & & & & & & & 12 \\
\hline 2 & 357.26 & 179.13 & & & & & $\mathrm{~L}$ & $1,404.74$ & 702.87 & $1,387.71$ & 694.36 & $1,386.72$ & 693.87 & 11 \\
\hline 3 & 414.28 & 207.65 & & & & & $\mathrm{G}$ & $1,291.65$ & 646.33 & $1,274.62$ & 637.82 & $1,273.64$ & 637.32 & 10 \\
\hline 4 & 542.34 & 271.67 & 525.32 & 263.16 & & & Q & $1,234.63$ & 617.82 & $1,217.60$ & 609.31 & $1,216.62$ & 608.81 & 9 \\
\hline 5 & 639.39 & 320.20 & 622.37 & 311.69 & & & P & $1,106.57$ & 553.79 & $1,089.54$ & 545.28 & $1,088.56$ & 544.78 & 8 \\
\hline 6 & 776.45 & 388.73 & 759.43 & 380.22 & & & H & $1,009.52$ & 505.26 & 992.49 & 496.75 & 991.51 & 496.26 & 7 \\
\hline 7 & 913.51 & 457.26 & 896.49 & 448.75 & & & H & 872.46 & 436.73 & 855.43 & 428.22 & 854.45 & 427.73 & 6 \\
\hline 8 & $1,042.56$ & 521.78 & $1,025.53$ & 513.27 & $1,024.54$ & 512.78 & E & 735.40 & 368.20 & 718.37 & 359.69 & 717.39 & 359.20 & 5 \\
\hline 9 & $1,155.64$ & 578.32 & $1,138.61$ & 569.81 & $1,137.63$ & 569.32 & L & 606.36 & 303.68 & 589.33 & 295.17 & 588.35 & 294.68 & 4 \\
\hline 10 & $1,270.67$ & 635.84 & $1,253.64$ & 627.32 & $1,252.66$ & 626.83 & D & 493.27 & 247.14 & 476.25 & 238.63 & 475.26 & 238.14 & 3 \\
\hline
\end{tabular}




\begin{tabular}{|l|l|l|l|l|l|l|l|l|l|l|l|l|l|r|}
11 & $1,357.70$ & 679.35 & $1,340.67$ & 670.84 & $1,339.69$ & 670.35 & $\mathrm{~S}$ & 378.25 & 189.63 & 361.22 & 181.11 & 360.24 & 180.62 & 2 \\
\hline 12 & & & & & & & $\mathrm{~K}$ & 291.21 & 146.11 & 274.19 & 137.60 & & & 1 \\
\hline
\end{tabular}

Query 20499 Hit 1

MS/MS Fragmentation of SEVAAENHLR

Found in sp|Q14554|PDIA5_HUMAN, Protein disulfide-isomerase A5 OS=Homo sapiens GN=PDIA5 PE=1 SV=1

Match to Query 20499: 1268.658from(423.8932,3+)

Title: 101: Sum of 2 scans in range $324(\mathrm{rt}=16.8319, \mathrm{f}=4, \mathrm{i}=63)$ to $325(\mathrm{rt}=16.8574, \mathrm{f}=4, \mathrm{i}=64)$

[D:lab212 \membranelGrace JoyceliTRAQ_41_2.raw]

Data File:Submitted from 20120508-1(merge) by Mascot Daemon on JOYCE-VAIO

Monoisotopic mass of neutral peptide $\mathrm{Mr}$ (calc): 1268.658

Variable modifications:

Ions Score: 50.15 Expect: 0.007

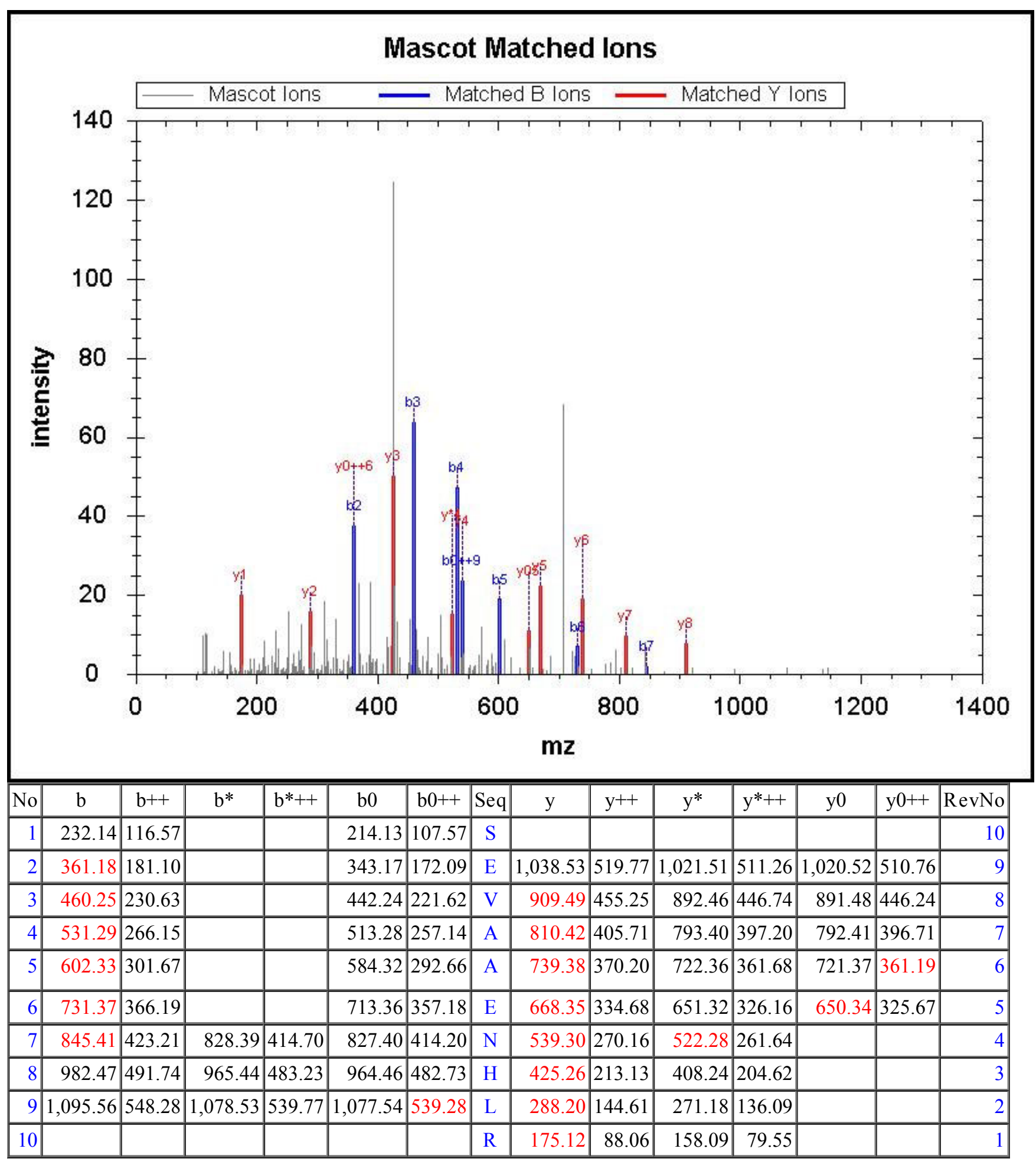


MS/MS Fragmentation of SQLQDTLIHLIK

Found in sp|Q9NPI6|DCP1A_HUMAN, mRNA-decapping enzyme 1A OS=Homo sapiens GN=DCP1A PE=1 SV=2

Match to Query 43405: 1696.01 from(566.3438,3+)

Title: 850: Scan 1852 ( $\mathrm{r}=51.4046, \mathrm{f}=3$, i=292) [D:lab212 membranelGracelJoyceliTRAQ_40_1.raw]

Data File:Submitted from 20120508-1(merge) by Mascot Daemon on JOYCE-VAIO

Monoisotopic mass of neutral peptide $\mathrm{Mr}$ (calc): 1696.01

Variable modifications:

K12 :TRAQ4plex (K)

Ions Score: 50.14 Expect: 0.004

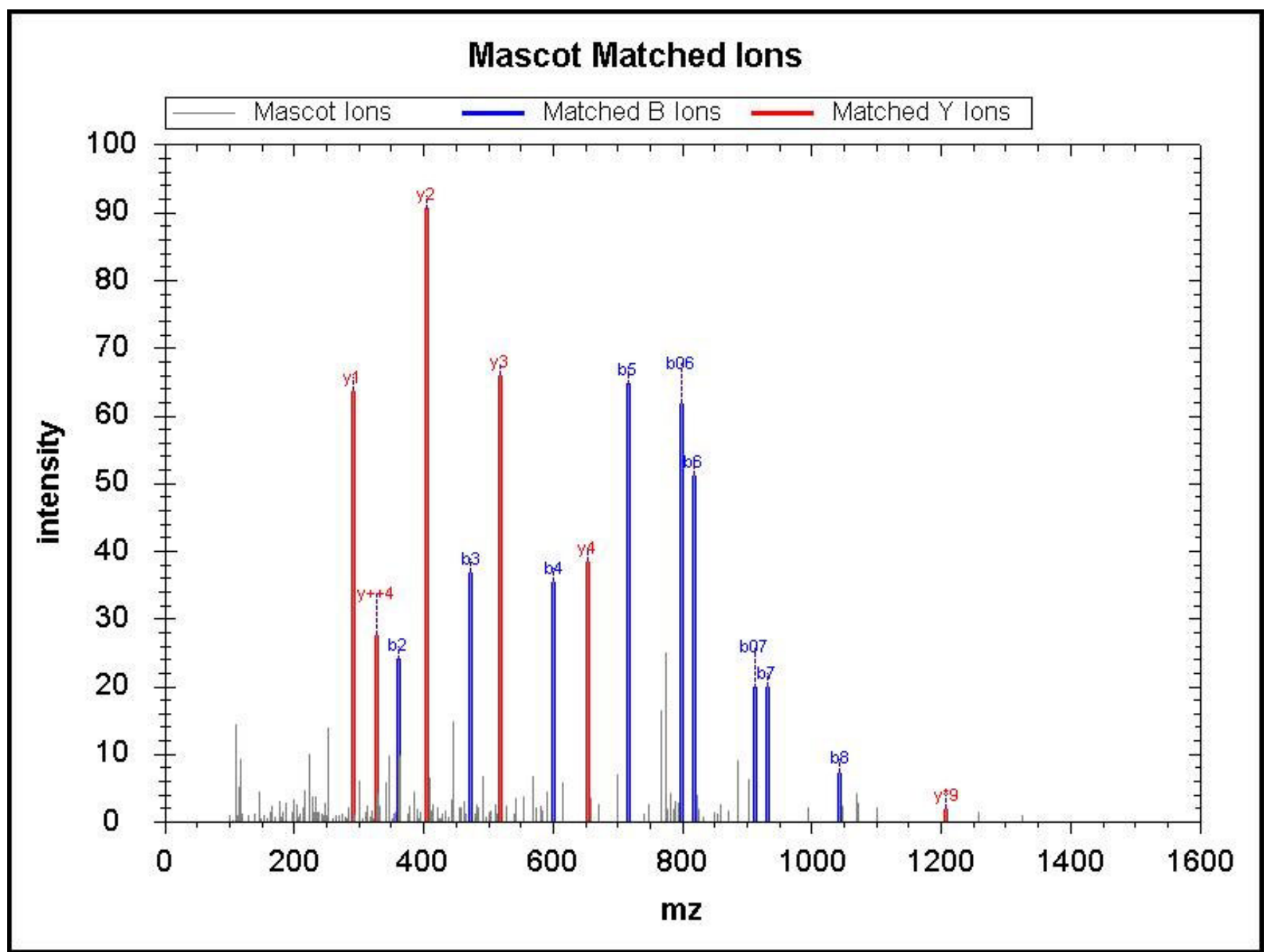

\begin{tabular}{|c|c|c|c|c|c|c|c|c|c|c|c|c|c|c|}
\hline No & $\mathrm{b}$ & $\mathrm{b}++$ & $b^{*}$ & $b^{*++}$ & b0 & $\mathrm{b} 0++$ & Seq & $\mathrm{y}$ & $\mathrm{y}++$ & $y^{*}$ & $\mathrm{y}^{*++}$ & y0 & y0++ & RevNo \\
\hline 1 & 232.14 & 116.57 & & & 214.13 & 107.57 & $\mathrm{~S}$ & & & & & & & 12 \\
\hline 2 & 360.20 & 180.60 & 343.17 & 172.09 & 342.19 & 171.60 & Q & $1,465.89$ & 733.45 & $1,448.86$ & 724.93 & $1,447.88$ & 724.44 & 11 \\
\hline 3 & 473.28 & 237.15 & 456.26 & 228.63 & 455.27 & 228.14 & $\mathrm{~L}$ & $1,337.83$ & 669.42 & $1,320.80$ & 660.90 & $1,319.82$ & 660.41 & 10 \\
\hline 4 & 601.34 & 301.17 & 584.32 & 292.66 & 583.33 & 292.17 & Q & $1,224.74$ & 612.88 & $1,207.72$ & 604.36 & $1,206.73$ & 603.87 & 9 \\
\hline 5 & 716.37 & 358.69 & 699.34 & 350.18 & 698.36 & 349.68 & D & $1,096.68$ & 548.85 & $1,079.66$ & 540.33 & $1,078.67$ & 539.84 & 8 \\
\hline 6 & 817.42 & 409.21 & 800.39 & 400.70 & 799.41 & 400.21 & $\mathrm{~T}$ & 981.66 & 491.33 & 964.63 & 482.82 & 963.65 & 482.33 & 7 \\
\hline 7 & 930.50 & 465.75 & 913.47 & 457.24 & 912.49 & 456.75 & $\mathrm{~L}$ & 880.61 & 440.81 & 863.58 & 432.30 & & & 6 \\
\hline 8 & $1,043.59$ & 522.30 & $1,026.56$ & 513.78 & $1,025.57$ & 513.29 & I & 767.53 & 384.27 & 750.50 & 375.75 & & & 5 \\
\hline 9 & $1,180.64$ & 590.83 & $1,163.62$ & 582.31 & $1,162.63$ & 581.82 & $\mathrm{H}$ & 654.44 & 327.72 & 637.42 & 319.21 & & & 4 \\
\hline 10 & $1,293.73$ & 647.37 & $1,276.70$ & 638.85 & $1,275.72$ & 638.36 & L & 517.38 & 259.20 & 500.36 & 250.68 & & & 3 \\
\hline 11 & $1,406.81$ & 703.91 & $1,389.79$ & 695.40 & $1,388.80$ & 694.90 & I & 404.30 & 202.65 & 387.27 & 194.14 & & & 2 \\
\hline 12 & & & & & & & K & 291.21 & 146.11 & 274.19 & 137.60 & & & 1 \\
\hline
\end{tabular}

Query 69579 Hit 1 


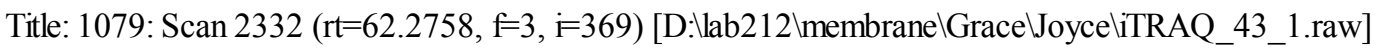

Data File:Submitted from 20120508-1(merge) by Mascot Daemon on JOYCE-VAIO

Monoisotopic mass of neutral peptide $\mathrm{Mr}$ (calc): 2220.247

Variable modifications:

K4 :iTRAQ4plex (K)

K14 :iTRAQ4plex (K)

Ions Score: 50.12 Expect: 0.005

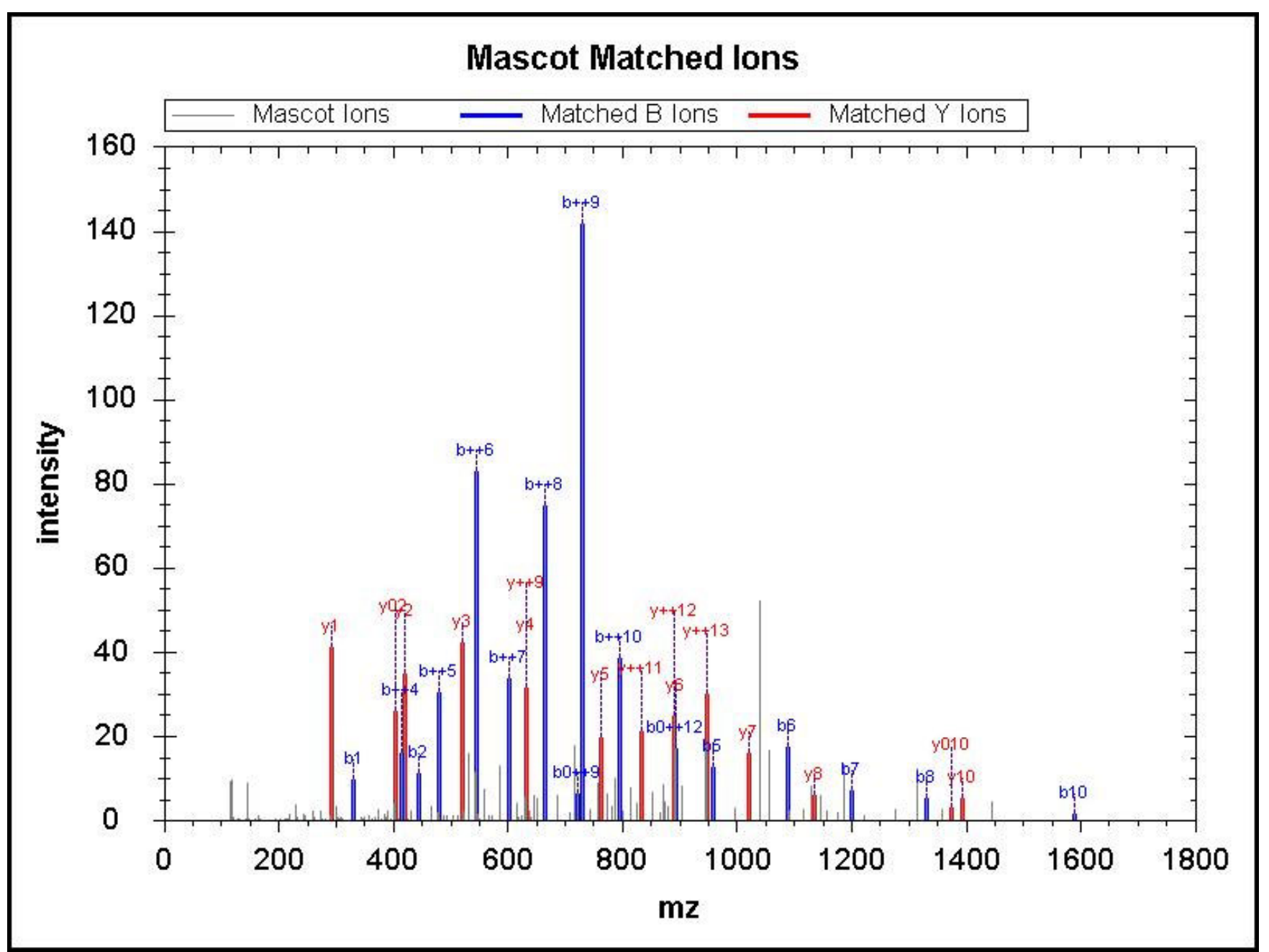

\begin{tabular}{|c|c|c|c|c|c|c|c|c|c|c|c|c|c|c|}
\hline No & $\mathrm{b}$ & $\mathrm{b}++$ & $b^{*}$ & $\mathrm{~b}^{*++}$ & b0 & b0++ & Seq & $\mathrm{y}$ & $\mathrm{y}++$ & $y^{*}$ & $\mathrm{y}^{*++}$ & y0 & $\mathrm{y} 0++$ & RevNo \\
\hline 1 & 331.19 & 166.10 & & & & & W & & & & & & & 14 \\
\hline 2 & 444.27 & 222.64 & & & & & $\mathrm{~L}$ & $1,891.07$ & 946.04 & $1,874.04$ & 937.53 & $1,873.06$ & 937.03 & 13 \\
\hline 3 & 557.36 & 279.18 & & & & & I & $1,777.99$ & 889.50 & $1,760.96$ & 880.98 & $1,759.98$ & 880.49 & 12 \\
\hline 4 & 829.55 & 415.28 & 812.53 & 406.77 & & & K & $1,664.90$ & 832.95 & $1,647.88$ & 824.44 & $1,646.89$ & 823.95 & 11 \\
\hline 5 & 958.60 & 479.80 & 941.57 & 471.29 & 940.59 & 470.80 & $\mathrm{E}$ & $1,392.70$ & 696.86 & $1,375.68$ & 688.34 & $1,374.69$ & 687.85 & 10 \\
\hline 6 & $1,087.64$ & 544.32 & $1,070.61$ & 535.81 & $1,069.63$ & 535.32 & $\mathrm{E}$ & $1,263.66$ & 632.33 & $1,246.64$ & 623.82 & $1,245.65$ & 623.33 & 9 \\
\hline 7 & $1,200.72$ & 600.87 & $1,183.70$ & 592.35 & $1,182.71$ & 591.86 & $\mathrm{~L}$ & $1,134.62$ & 567.81 & $1,117.59$ & 559.30 & $1,116.61$ & 558.81 & 8 \\
\hline 8 & $1,329.77$ & 665.39 & $1,312.74$ & 656.87 & $1,311.76$ & 656.38 & $\mathrm{E}$ & $1,021.54$ & 511.27 & $1,004.51$ & 502.76 & $1,003.53$ & 502.27 & 7 \\
\hline 9 & $1,458.81$ & 729.91 & $1,441.78$ & 721.39 & $1,440.80$ & 720.90 & E & 892.49 & 446.75 & 875.47 & 438.24 & 874.48 & 437.74 & 6 \\
\hline 10 & $1,589.85$ & 795.43 & $1,572.82$ & 786.91 & $1,571.84$ & 786.42 & M & 763.45 & 382.23 & 746.42 & 373.72 & 745.44 & 373.22 & 5 \\
\hline 11 & $1,702.93$ & 851.97 & $1,685.91$ & 843.46 & $1,684.92$ & 842.96 & $\mathrm{~L}$ & 632.41 & 316.71 & 615.38 & 308.20 & 614.40 & 307.70 & 4 \\
\hline 12 & $1,802.00$ & 901.50 & $1,784.97$ & 892.99 & $1,783.99$ & 892.50 & $\mathrm{~V}$ & 519.33 & 260.17 & 502.30 & 251.65 & 501.32 & 251.16 & 3 \\
\hline 13 & $1,931.04$ & 966.03 & $1,914.02$ & 957.51 & $1,913.03$ & 957.02 & E & 420.26 & 210.63 & 403.23 & 202.12 & 402.25 & 201.63 & 2 \\
\hline 14 & & & & & & & K & 291.21 & 146.11 & 274.19 & 137.60 & & & 1 \\
\hline
\end{tabular}

Query 66941 Hit 1 
Match to Query 66941:2158.294from(720.4386,3+)

Title: 731: Scan 1644 ( $\mathrm{rt}=46.6217, \mathrm{f}=2, \mathrm{i}=266)$ [D:llab212 $\backslash$ membranelGrace JoyceliTRAQ_44_1.raw]

Data File:Submitted from 20120508-1(merge) by Mascot Daemon on JOYCE-VAIO

Monoisotopic mass of neutral peptide Mr(calc): 2158.294

Variable modifications:

K2 :iTRAQ4plex (K)

$\mathrm{K} 15$ :iTRAQ4plex (K)

Ions Score: 50.05 Expect: 0.002

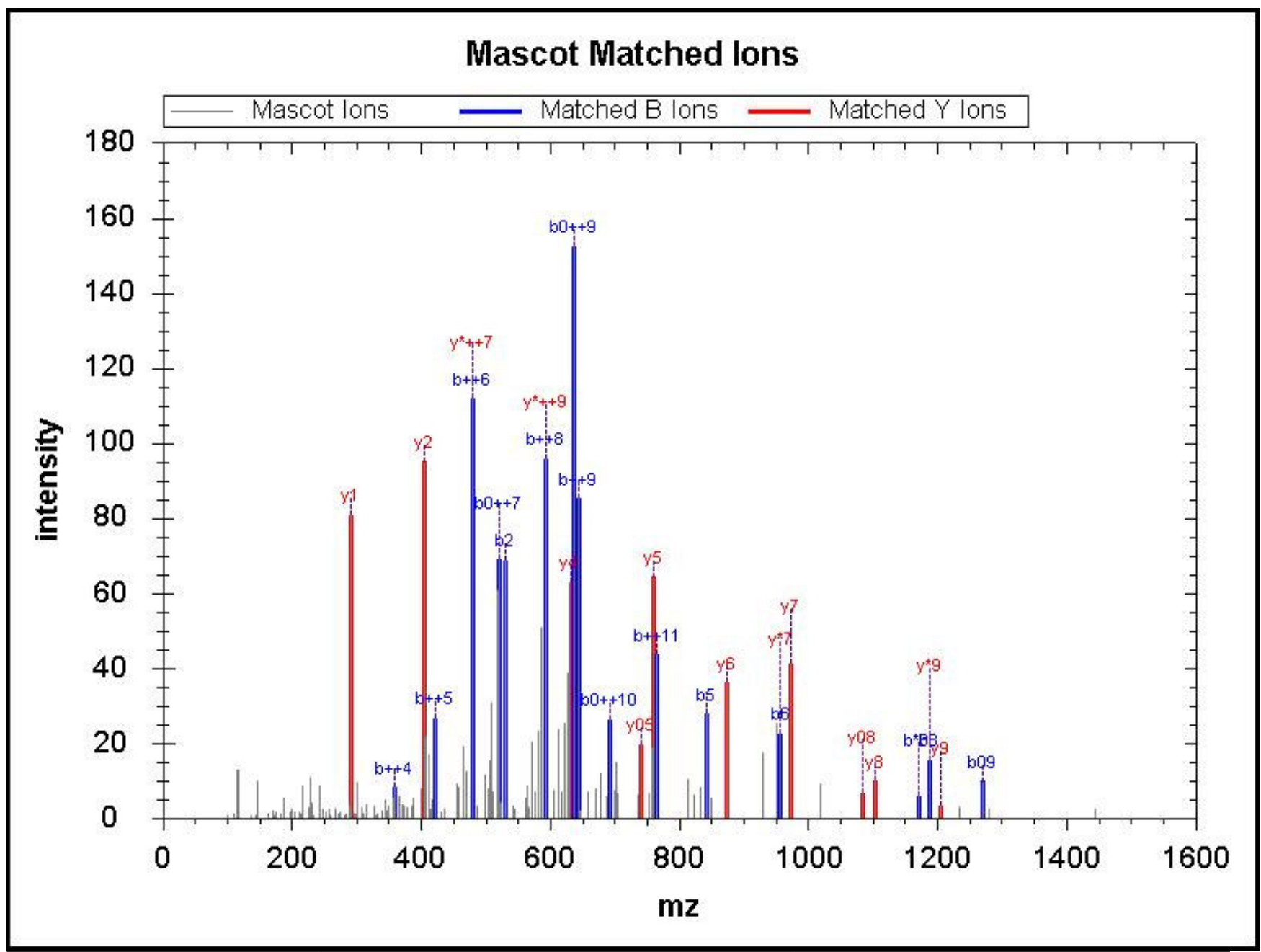

\begin{tabular}{|r|c|c|c|c|c|c|c|c|c|r|r|r|r|r|}
\hline $\mathrm{No}$ & $\mathrm{b}$ & $\mathrm{b}++$ & $\mathrm{b} *$ & $\mathrm{~b}++$ & $\mathrm{b} 0$ & $\mathrm{~b} 0++$ & $\mathrm{Seq}$ & $\mathrm{y}$ & $\mathrm{y}++$ & $\mathrm{y}^{*}$ & $\mathrm{y}^{*++}$ & $\mathrm{y} 0$ & $\mathrm{y} 0++$ & $\mathrm{RevNo}$ \\
\hline 1 & 258.19 & 129.60 & & & & & $\mathrm{I}$ & & & & & & & 15 \\
\hline 2 & 530.39 & 265.70 & 513.36 & 257.19 & & & $\mathrm{~K}$ & $1,902.11$ & 951.56 & $1,885.09$ & 943.05 & $1,884.10$ & 942.56 & 14 \\
\hline 3 & 627.44 & 314.23 & 610.42 & 305.71 & & & $\mathrm{P}$ & $1,629.92$ & 815.46 & $1,612.89$ & 806.95 & $1,611.91$ & 806.46 & 13 \\
\hline 4 & 714.48 & 357.74 & 697.45 & 349.23 & 696.46 & 348.74 & $\mathrm{~S}$ & $1,532.87$ & 766.94 & $1,515.84$ & 758.42 & $1,514.85$ & 757.93 & 12 \\
\hline 5 & 842.53 & 421.77 & 825.51 & 413.26 & 824.52 & 412.77 & $\mathrm{Q}$ & $1,445.83$ & 723.42 & $1,428.81$ & 714.91 & $1,427.82$ & 714.41 & 11 \\
\hline 6 & 956.58 & 478.79 & 939.55 & 470.28 & 938.57 & 469.79 & $\mathrm{~N}$ & $1,317.77$ & 659.39 & $1,300.75$ & 650.88 & $1,299.76$ & 650.39 & 10 \\
\hline 7 & $1,057.62$ & 529.32 & $1,040.60$ & 520.80 & $1,039.61$ & 520.31 & $\mathrm{~T}$ & $1,203.73$ & 602.37 & $1,186.71$ & 593.86 & $1,185.72$ & 593.36 & 9 \\
\hline 8 & $1,186.67$ & 593.84 & $1,169.64$ & 585.32 & $1,168.66$ & 584.83 & $\mathrm{E}$ & $1,102.68$ & 551.85 & $1,085.66$ & 543.33 & $1,084.67$ & 542.84 & 8 \\
\hline 9 & $1,287.71$ & 644.36 & $1,270.69$ & 635.85 & $1,269.70$ & 635.36 & $\mathrm{~T}$ & 973.64 & 487.32 & 956.61 & 478.81 & 955.63 & 478.32 & 7 \\
\hline 10 & $1,400.80$ & 700.90 & $1,383.77$ & 692.39 & $1,382.79$ & 691.90 & L & 872.59 & 436.80 & 855.57 & 428.29 & 854.58 & 427.80 & 6 \\
\hline 11 & $1,529.84$ & 765.42 & $1,512.82$ & 756.91 & $1,511.83$ & 756.42 & E & 759.51 & 380.26 & 742.48 & 371.75 & 741.50 & 371.25 & 5 \\
\hline 12 & $1,642.93$ & 821.97 & $1,625.90$ & 813.45 & $1,624.92$ & 812.96 & L & 630.47 & 315.74 & 613.44 & 307.22 & & & 4 \\
\hline 13 & $1,756.01$ & 878.51 & $1,738.98$ & 870.00 & $1,738.00$ & 869.50 & L & 517.38 & 259.20 & 500.36 & 250.68 & & & 3 \\
\hline 14 & $1,869.09$ & 935.05 & $1,852.07$ & 926.54 & $1,851.08$ & 926.05 & L & 404.30 & 202.65 & 387.27 & 194.14 & & & 2 \\
\hline 15 & & & & & & & K & 291.21 & 146.11 & 274.19 & 137.60 & & & 1 \\
\hline
\end{tabular}


MS/MS Fragmentation of LNISEVDEQVR

Found in sp|Q01432|AMPD3 HUMAN, AMP deaminase 3 OS=Homo sapiens GN=AMPD3 PE=1 SV=1

Match to Query 29962: 1445.71 from(723.8621,2+)

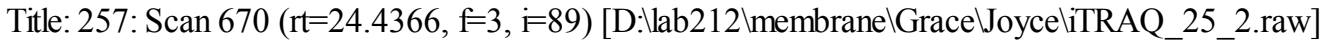

Data File:Submitted from 20120508-1(merge) by Mascot Daemon on JOYCE-VAIO

Monoisotopic mass of neutral peptide $\mathrm{Mr}$ (calc): 1445.71

Variable modifications:

Q9 :Deamidated (NQ)

Ions Score: 49.93 Expect: 0.008

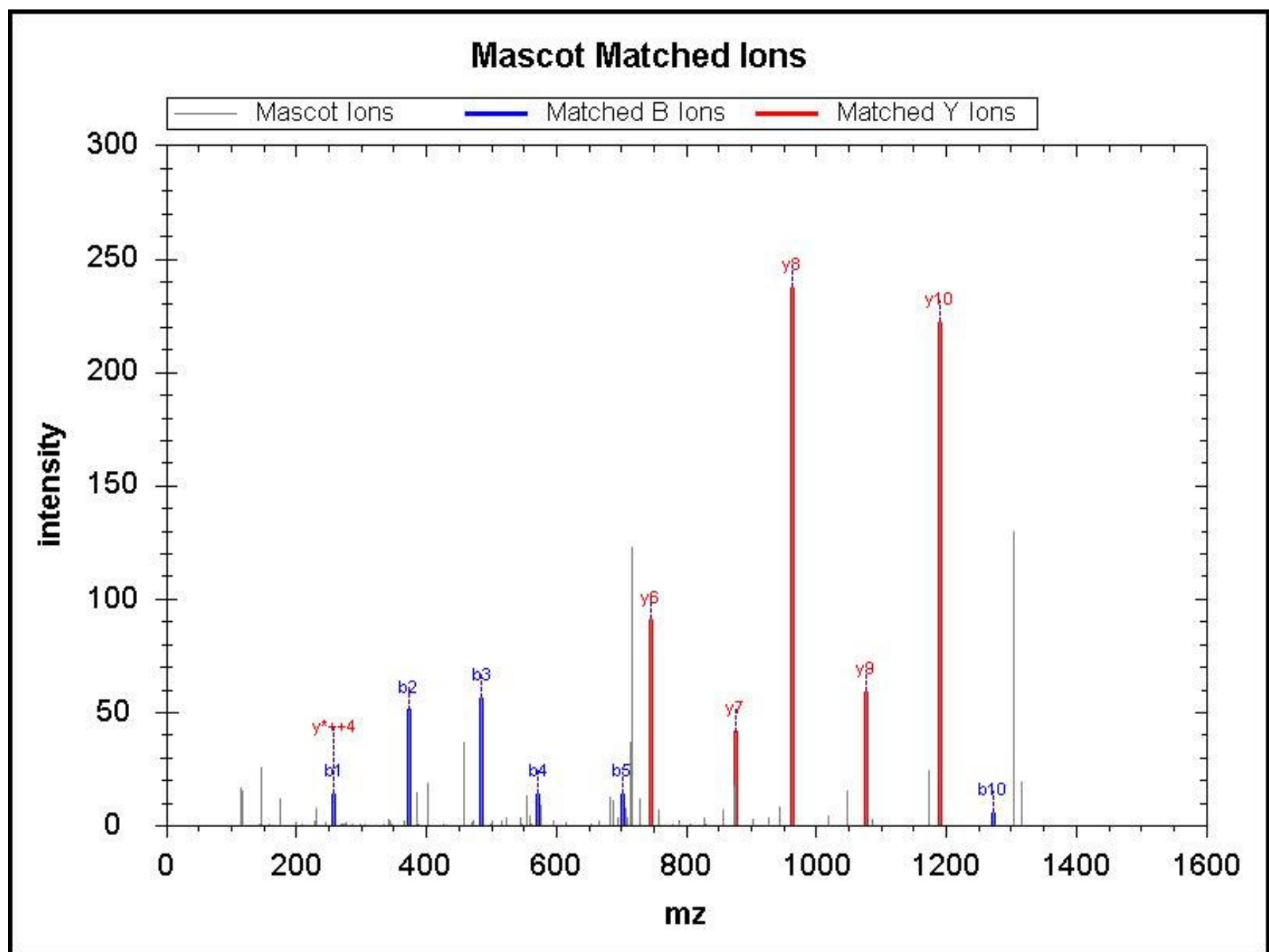

\begin{tabular}{|c|c|c|c|c|c|c|c|c|c|c|c|c|c|c|}
\hline No & $\mathrm{b}$ & $\mathrm{b}++$ & $b^{*}$ & $b^{*++}$ & b0 & b0++ & Seq & $\mathrm{y}$ & $\mathrm{y}++$ & $\mathrm{y}^{*}$ & $\mathrm{y}^{*++}$ & y0 & y0++ & RevNo \\
\hline 1 & 258.19 & 129.60 & & & & & $\mathrm{~L}$ & & & & & & & 11 \\
\hline 2 & 372.24 & 186.62 & 355.21 & 178.11 & & & $\mathrm{~N}$ & $1,189.57$ & 595.29 & $1,172.54$ & 586.78 & $1,171.56$ & 586.28 & 10 \\
\hline 3 & 485.32 & 243.16 & 468.29 & 234.65 & & & I & $1,075.53$ & 538.27 & $1,058.50$ & 529.75 & $1,057.52$ & 529.26 & 9 \\
\hline 4 & 572.35 & 286.68 & 555.33 & 278.17 & 554.34 & 277.67 & $\mathrm{~S}$ & 962.44 & 481.72 & 945.42 & 473.21 & 944.43 & 472.72 & 8 \\
\hline 5 & 701.40 & 351.20 & 684.37 & 342.69 & 683.38 & 342.20 & E & 875.41 & 438.21 & 858.38 & 429.70 & 857.40 & 429.20 & 7 \\
\hline 6 & 800.46 & 400.74 & 783.44 & 392.22 & 782.45 & 391.73 & $\mathrm{~V}$ & 746.37 & 373.69 & 729.34 & 365.17 & 728.36 & 364.68 & 6 \\
\hline 7 & 915.49 & 458.25 & 898.46 & 449.74 & 897.48 & 449.24 & D & 647.30 & 324.15 & 630.27 & 315.64 & 629.29 & 315.15 & 5 \\
\hline 8 & $1,044.53$ & 522.77 & $1,027.51$ & 514.26 & $1,026.52$ & 513.76 & $\mathrm{E}$ & 532.27 & 266.64 & 515.25 & 258.13 & 514.26 & 257.63 & 4 \\
\hline 9 & $1,173.58$ & 587.29 & $1,156.55$ & 578.78 & $1,155.56$ & 578.29 & Q & 403.23 & 202.12 & 386.20 & 193.61 & & & 3 \\
\hline 10 & $1,272.64$ & 636.83 & $1,255.62$ & 628.31 & $1,254.63$ & 627.82 & V & 274.19 & 137.60 & 257.16 & 129.08 & & & 2 \\
\hline 11 & & & & & & & $\mathrm{R}$ & 175.12 & 88.06 & 158.09 & 79.55 & & & 1 \\
\hline
\end{tabular}

Query 48188 Hit 1

MS/MS Fragmentation of KLEEALM ADILSR

Found in sp|Q9UBB5|MBD2_HUMAN, Methyl-CpG-binding domain protein 2 OS=Homo sapiens GN=MBD2 PE=1 SV=1 Match to Query 48188: 1776.007from(593.0096,3+)

Title: 934: Sum of 2 scans in range 2029 ( $\mathrm{rt}=55.4513, \mathrm{f}=4, \mathrm{~F}=630)$ to $2030(\mathrm{rt}=55.4767, \mathrm{f}=4, \mathrm{i}=631$ ) 
[D:llab212 \membranelGrace JoyceliTRAQ_43_1.raw]

Data File:Submitted from 20120508-1(merge) by Mascot Daemon on JOYCE-VAIO

Monoisotopic mass of neutral peptide $\operatorname{Mr}(\mathrm{calc}): 1776.007$

Variable modifications:

K1 :TRAQ4plex (K)

Ions Score: 49.86 Expect: 0.007

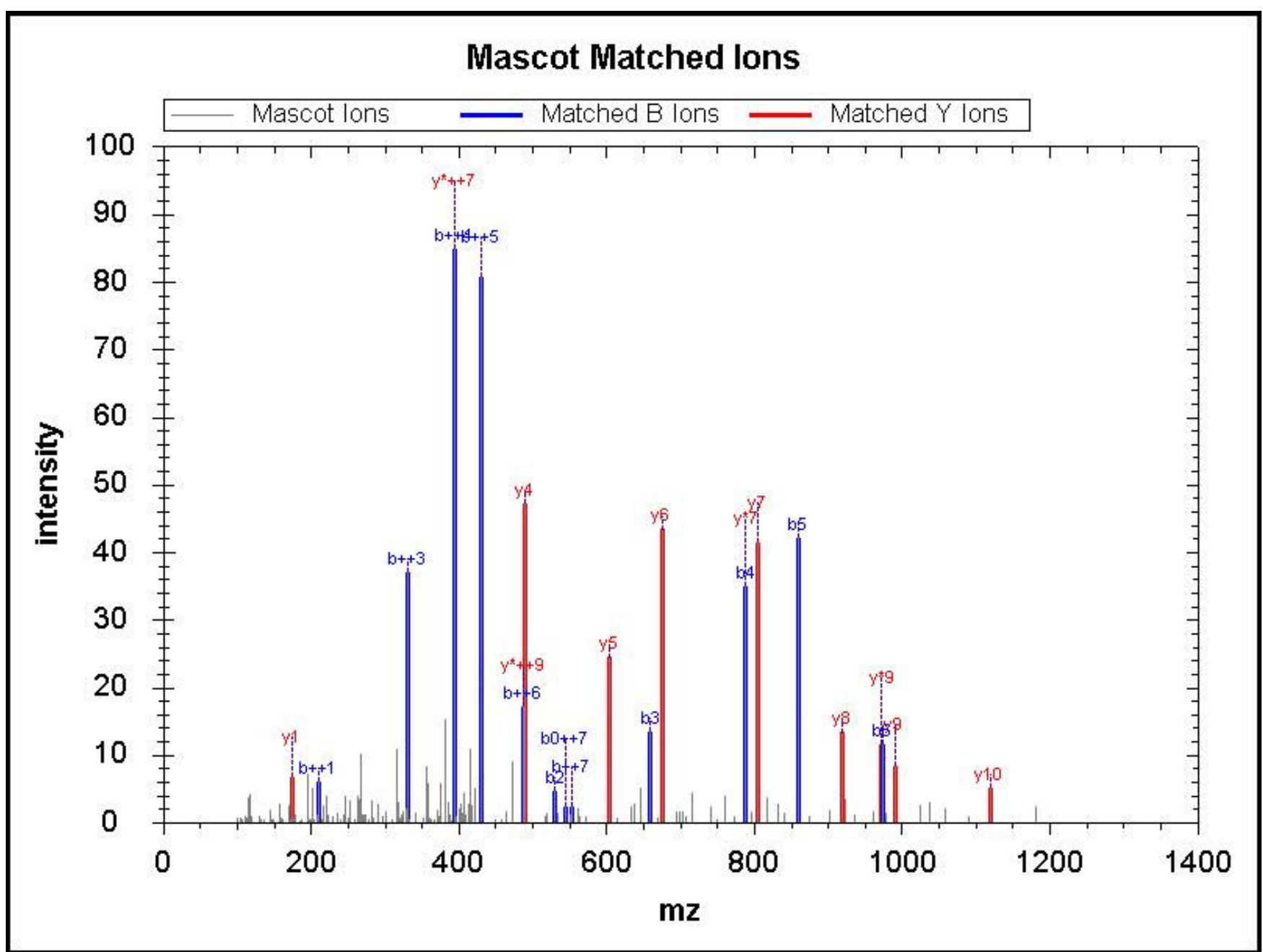

\begin{tabular}{|r|c|c|c|c|c|c|c|c|c|c|c|r|r|r|}
\hline \hline $\mathrm{No}$ & $\mathrm{b}$ & $\mathrm{b}++$ & $\mathrm{b}^{*}$ & $\mathrm{~b}++$ & $\mathrm{b} 0$ & $\mathrm{~b} 0++$ & $\mathrm{Seq}$ & $\mathrm{y}$ & $\mathrm{y}++$ & $\mathrm{y}^{*}$ & $\mathrm{y}^{*++}$ & $\mathrm{y} 0$ & $\mathrm{y} 0++$ & $\mathrm{RevNo}$ \\
\hline 1 & 417.31 & 209.16 & 400.28 & 200.64 & & & $\mathrm{~K}$ & & & & & & & 13 \\
\hline 2 & 530.39 & 265.70 & 513.36 & 257.19 & & & $\mathrm{~L}$ & $1,360.71$ & 680.86 & $1,343.69$ & 672.35 & $1,342.70$ & 671.86 & 12 \\
\hline 3 & 659.43 & 330.22 & 642.41 & 321.71 & 641.42 & 321.21 & $\mathrm{E}$ & $1,247.63$ & 624.32 & $1,230.60$ & 615.81 & $1,229.62$ & 615.31 & 11 \\
\hline 4 & 788.48 & 394.74 & 771.45 & 386.23 & 770.47 & 385.74 & $\mathrm{E}$ & $1,118.59$ & 559.80 & $1,101.56$ & 551.28 & $1,100.58$ & 550.79 & 10 \\
\hline 5 & 859.51 & 430.26 & 842.49 & 421.75 & 841.50 & 421.25 & $\mathrm{~A}$ & 989.54 & 495.28 & 972.52 & 486.76 & 971.53 & 486.27 & 9 \\
\hline 6 & 972.60 & 486.80 & 955.57 & 478.29 & 954.59 & 477.80 & $\mathrm{~L}$ & 918.51 & 459.76 & 901.48 & 451.24 & 900.50 & 450.75 & 8 \\
\hline 7 & $1,103.64$ & 552.32 & $1,086.61$ & 543.81 & $1,085.63$ & 543.32 & $\mathrm{M}$ & 805.42 & 403.22 & 788.40 & 394.70 & 787.41 & 394.21 & 7 \\
\hline 8 & $1,174.67$ & 587.84 & $1,157.65$ & 579.33 & $1,156.66$ & 578.84 & A & 674.38 & 337.70 & 657.36 & 329.18 & 656.37 & 328.69 & 6 \\
\hline 9 & $1,289.70$ & 645.35 & $1,272.67$ & 636.84 & $1,271.69$ & 636.35 & $\mathrm{D}$ & 603.35 & 302.18 & 586.32 & 293.66 & 585.34 & 293.17 & 5 \\
\hline 10 & $1,402.79$ & 701.90 & $1,385.76$ & 693.38 & $1,384.77$ & 692.89 & $\mathrm{I}$ & 488.32 & 244.66 & 471.29 & 236.15 & 470.31 & 235.66 & 4 \\
\hline 11 & $1,515.87$ & 758.44 & $1,498.84$ & 749.93 & $1,497.86$ & 749.43 & L & 375.24 & 188.12 & 358.21 & 179.61 & 357.22 & 179.12 & 3 \\
\hline 12 & $1,602.90$ & 801.95 & $1,585.88$ & 793.44 & $1,584.89$ & 792.95 & S & 262.15 & 131.58 & 245.12 & 123.07 & 244.14 & 122.57 & 2 \\
\hline 13 & & & & & & & R & 175.12 & 88.06 & 158.09 & 79.55 & & & 1 \\
\hline
\end{tabular}

Query 56216 Hit 1

MS/MS Fragmentation of FLVLATDGLWETMHR

Found in sp|Q9P0J 1|PDP1_HUMAN, [Pyruvate dehydrogenase [acetyl-transferring]]-phosphatase 1

Match to Query 56216: 1932.005from(645.0089,3+)

Title: 1038: Sum of 2 scans in range $2298(\mathrm{rt}=61.1505, \mathrm{f}=4, \mathrm{i}=697)$ to $2299(\mathrm{rt}=61.1759, \mathrm{f}=4, \mathrm{i}=698)$ 
[D:llab212 \membranelGrace JoyceliTRAQ_33_1.raw]

Data File:Submitted from 20120508-1(merge) by Mascot Daemon on JOYCE-VAIO

Monoisotopic mass of neutral peptide $\operatorname{Mr}($ calc): 1932.005

Variable modifications:

Ions Score: 49.7 Expect: 0.010

\section{Mascot Matched lons}

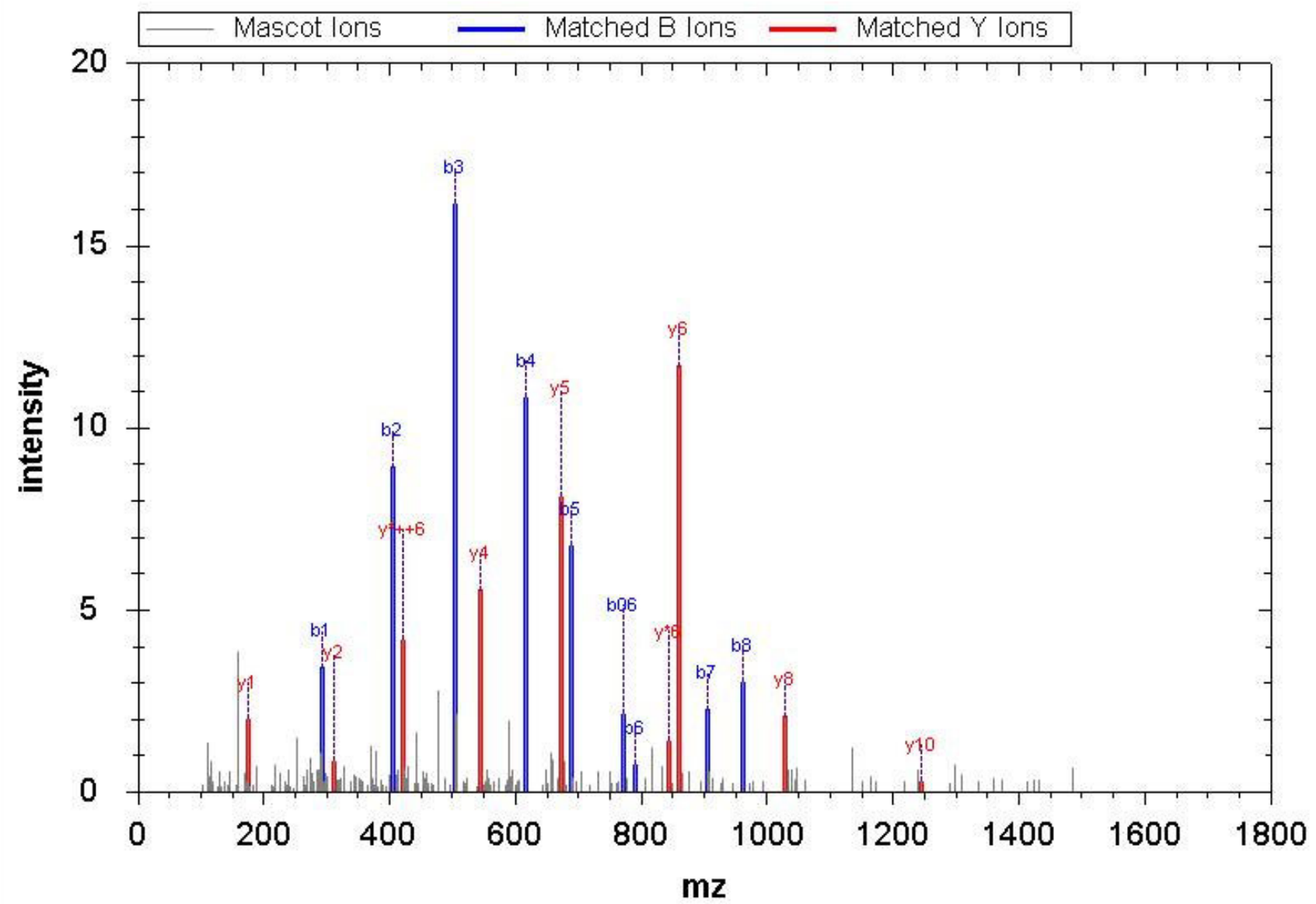

\begin{tabular}{|r|c|c|c|c|c|c|c|c|c|c|r|r|}
\hline $\mathrm{No}$ & $\mathrm{b}$ & $\mathrm{b}++$ & $\mathrm{b} 0$ & $\mathrm{~b} 0++$ & $\mathrm{Seq}$ & $\mathrm{y}$ & $\mathrm{y}++$ & $\mathrm{y}^{*}$ & $\mathrm{y}^{*++}$ & $\mathrm{y} 0$ & $\mathrm{y} 0++$ & RevNo \\
\hline 1 & 292.18 & 146.59 & & & $\mathrm{~F}$ & & & & & & & 15 \\
\hline 2 & 405.26 & 203.13 & & & $\mathrm{~L}$ & $1,641.84$ & 821.42 & $1,624.82$ & 812.91 & $1,623.83$ & 812.42 & 14 \\
\hline 3 & 504.33 & 252.67 & & & $\mathrm{~V}$ & $1,528.76$ & 764.88 & $1,511.73$ & 756.37 & $1,510.75$ & 755.88 & 13 \\
\hline 4 & 617.41 & 309.21 & & & $\mathrm{~L}$ & $1,429.69$ & 715.35 & $1,412.66$ & 706.84 & $1,411.68$ & 706.34 & 12 \\
\hline 5 & 688.45 & 344.73 & & & $\mathrm{~A}$ & $1,316.61$ & 658.81 & $1,299.58$ & 650.29 & $1,298.59$ & 649.80 & 11 \\
\hline 6 & 789.50 & 395.25 & 771.49 & 386.25 & $\mathrm{~T}$ & $1,245.57$ & 623.29 & $1,228.54$ & 614.77 & $1,227.56$ & 614.28 & 10 \\
\hline 7 & 904.53 & 452.77 & 886.52 & 443.76 & $\mathrm{D}$ & $1,144.52$ & 572.76 & $1,127.49$ & 564.25 & $1,126.51$ & 563.76 & 9 \\
\hline 8 & 961.55 & 481.28 & 943.54 & 472.27 & $\mathrm{G}$ & $1,029.49$ & 515.25 & $1,012.47$ & 506.74 & $1,011.48$ & 506.25 & 8 \\
\hline 9 & $1,074.63$ & 537.82 & $1,056.62$ & 528.81 & $\mathrm{~L}$ & 972.47 & 486.74 & 955.45 & 478.23 & 954.46 & 477.73 & 7 \\
\hline 10 & $1,260.71$ & 630.86 & $1,242.70$ & 621.85 & $\mathrm{~W}$ & 859.39 & 430.20 & 842.36 & 421.68 & 841.38 & 421.19 & 6 \\
\hline 11 & $1,389.75$ & 695.38 & $1,371.74$ & 686.38 & $\mathrm{E}$ & 673.31 & 337.16 & 656.28 & 328.64 & 655.30 & 328.15 & 5 \\
\hline 12 & $1,490.80$ & 745.90 & $1,472.79$ & 736.90 & $\mathrm{~T}$ & 544.27 & 272.64 & 527.24 & 264.12 & 526.26 & 263.63 & 4 \\
\hline 13 & $1,621.84$ & 811.42 & $1,603.83$ & 802.42 & $\mathrm{M}$ & 443.22 & 222.11 & 426.19 & 213.60 & & & 3 \\
\hline 14 & $1,758.90$ & 879.95 & $1,740.89$ & 870.95 & $\mathrm{H}$ & 312.18 & 156.59 & 295.15 & 148.08 & & & 2 \\
\hline 15 & & & & & $\mathrm{R}$ & 175.12 & 88.06 & 158.09 & 79.55 & & & 1 \\
\hline
\end{tabular}

Query 65417 Hit 1 
Match to Query 65417: 2123.15from(708.7241,3+)

Title: 902: Sum of 2 scans in range $2015(\mathrm{rt}=54.7534, \mathrm{f}=4, \mathrm{i}=605)$ to $2016(\mathrm{rt}=54.7788, \mathrm{f}=4, \mathrm{i}=606)$

[D:llab212\membranelGrace JoyceliTRAQ_31_2.raw]

Data File:Submitted from 20120508-1(merge) by Mascot Daemon on JOYCE-VAIO

Monoisotopic mass of neutral peptide $\mathrm{Mr}$ (calc): 2123.15

Variable modifications:

Ions Score: 49.7 Expect: 0.009

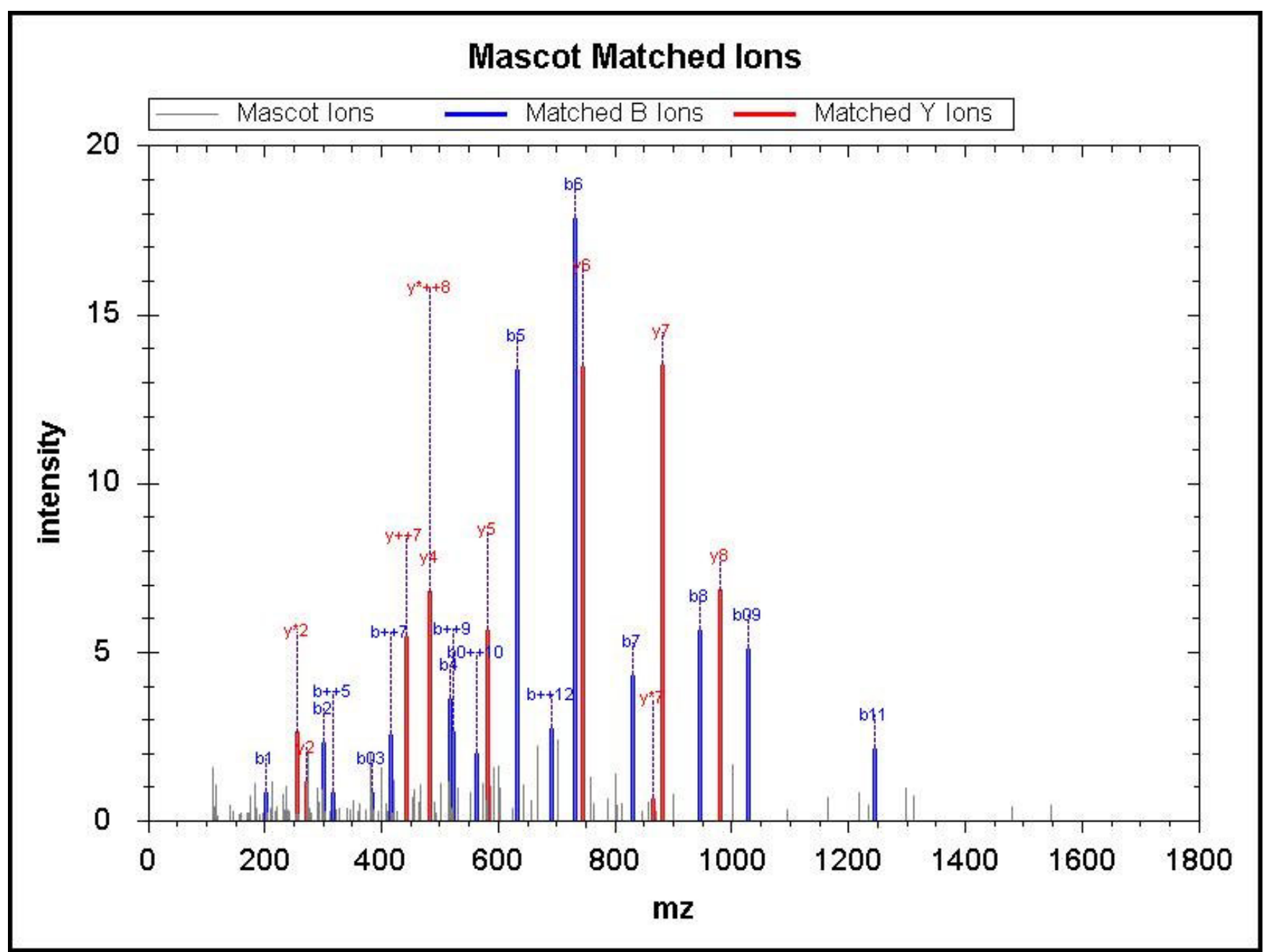

\begin{tabular}{|c|c|c|c|c|c|c|c|c|c|c|c|c|c|c|}
\hline No & $\mathrm{b}$ & $\mathrm{b}++$ & $b^{*}$ & $\mathrm{~b}^{*++}$ & b0 & b0++ & Seq & $\mathrm{y}$ & $y++$ & $y^{*}$ & $\mathrm{y}^{*++}$ & y0 & y0++ & RevNo \\
\hline 1 & 202.13 & 101.57 & & & & & G & & & & & & & 18 \\
\hline 2 & 301.20 & 151.10 & & & & & V & $1,923.03$ & 962.02 & $1,906.01$ & 953.51 & $1,905.02$ & 953.02 & 17 \\
\hline 3 & 402.25 & 201.63 & & & 384.24 & 192.62 & $\mathrm{~T}$ & $1,823.96$ & 912.49 & $1,806.94$ & 903.97 & $1,805.95$ & 903.48 & 16 \\
\hline 4 & 517.27 & 259.14 & & & 499.26 & 250.14 & D & $1,722.92$ & 861.96 & $1,705.89$ & 853.45 & $1,704.91$ & 852.96 & 15 \\
\hline 5 & 631.32 & 316.16 & 614.29 & 307.65 & 613.31 & 307.16 & $\mathrm{~N}$ & $1,607.89$ & 804.45 & $1,590.86$ & 795.94 & $1,589.88$ & 795.44 & 14 \\
\hline 6 & 730.39 & 365.70 & 713.36 & 357.18 & 712.37 & 356.69 & V & $1,493.85$ & 747.43 & $1,476.82$ & 738.91 & $1,475.84$ & 738.42 & 13 \\
\hline 7 & 829.45 & 415.23 & 812.43 & 406.72 & 811.44 & 406.23 & V & $1,394.78$ & 697.89 & $1,377.75$ & 689.38 & $1,376.77$ & 688.89 & 12 \\
\hline 8 & 944.48 & 472.74 & 927.45 & 464.23 & 926.47 & 463.74 & $\mathrm{D}$ & $1,295.71$ & 648.36 & $1,278.68$ & 639.85 & $1,277.70$ & 639.35 & 11 \\
\hline 9 & $1,045.53$ & 523.27 & $1,028.50$ & 514.75 & $1,027.52$ & 514.26 & $\mathrm{~T}$ & $1,180.68$ & 590.85 & $1,163.66$ & 582.33 & $1,162.67$ & 581.84 & 10 \\
\hline 10 & $1,144.60$ & 572.80 & $1,127.57$ & 564.29 & $1,126.59$ & 563.80 & $\mathrm{~V}$ & $1,079.64$ & 540.32 & $1,062.61$ & 531.81 & & & 9 \\
\hline 11 & $1,243.66$ & 622.34 & $1,226.64$ & 613.82 & $1,225.65$ & 613.33 & V & 980.57 & 490.79 & 963.54 & 482.27 & & & 8 \\
\hline 12 & $1,380.72$ & 690.87 & $1,363.70$ & 682.35 & $1,362.71$ & 681.86 & $\mathrm{H}$ & 881.50 & 441.25 & 864.47 & 432.74 & & & 7 \\
\hline 13 & $1,543.79$ & 772.40 & $1,526.76$ & 763.88 & $1,525.78$ & 763.39 & $\mathrm{Y}$ & 744.44 & 372.72 & 727.41 & 364.21 & & & 6 \\
\hline 14 & $1,642.86$ & 821.93 & $1,625.83$ & 813.42 & $1,624.84$ & 812.93 & V & 581.38 & 291.19 & 564.35 & 282.68 & & & 5 \\
\hline 15 & $1,739.91$ & 870.46 & $1,722.88$ & 861.94 & $1,721.90$ & 861.45 & $\mathrm{P}$ & 482.31 & 241.66 & 465.28 & 233.14 & & & 4 \\
\hline 16 & $1,852.99$ & 927.00 & $1,835.97$ & 918.49 & $1,834.98$ & 917.99 & $\mathrm{~L}$ & 385.26 & 193.13 & 368.23 & 184.62 & & & 3 \\
\hline 17 & $1,950.05$ & 975.53 & $1,933.02$ & 967.01 & $1,932.03$ & 966.52 & $\mathrm{P}$ & 272.17 & 136.59 & 255.15 & 128.08 & & & 2 \\
\hline & & & & & & & & & & & & & & \\
\hline
\end{tabular}


Query 21846 Hit 1

MS/MS Fragmentation of AFSQR SSLVR

Found in sp|Q86TJ 5|ZN554_HUMAN, Zinc finger protein 554 OS=Homo sapiens GN=ZNF554 PE=2 SV=1

Match to Query 21846: 1293.677from(647.8459,2+)

Title: 254: Scan 663 ( $\mathrm{rt}=24.2812, \mathrm{f}=3, \mathrm{i}=88)$ [D:llab212 'membranelGrace JoyceliTRAQ_25_2.raw]

Data File:Submitted from 20120508-1(merge) by Mascot Daemon on JOYCE-VAIO

Monoisotopic mass of neutral peptide $\mathrm{Mr}$ (calc): 1293.677

Variable modifications:

Ions Score: 49.67 Expect: 0.009

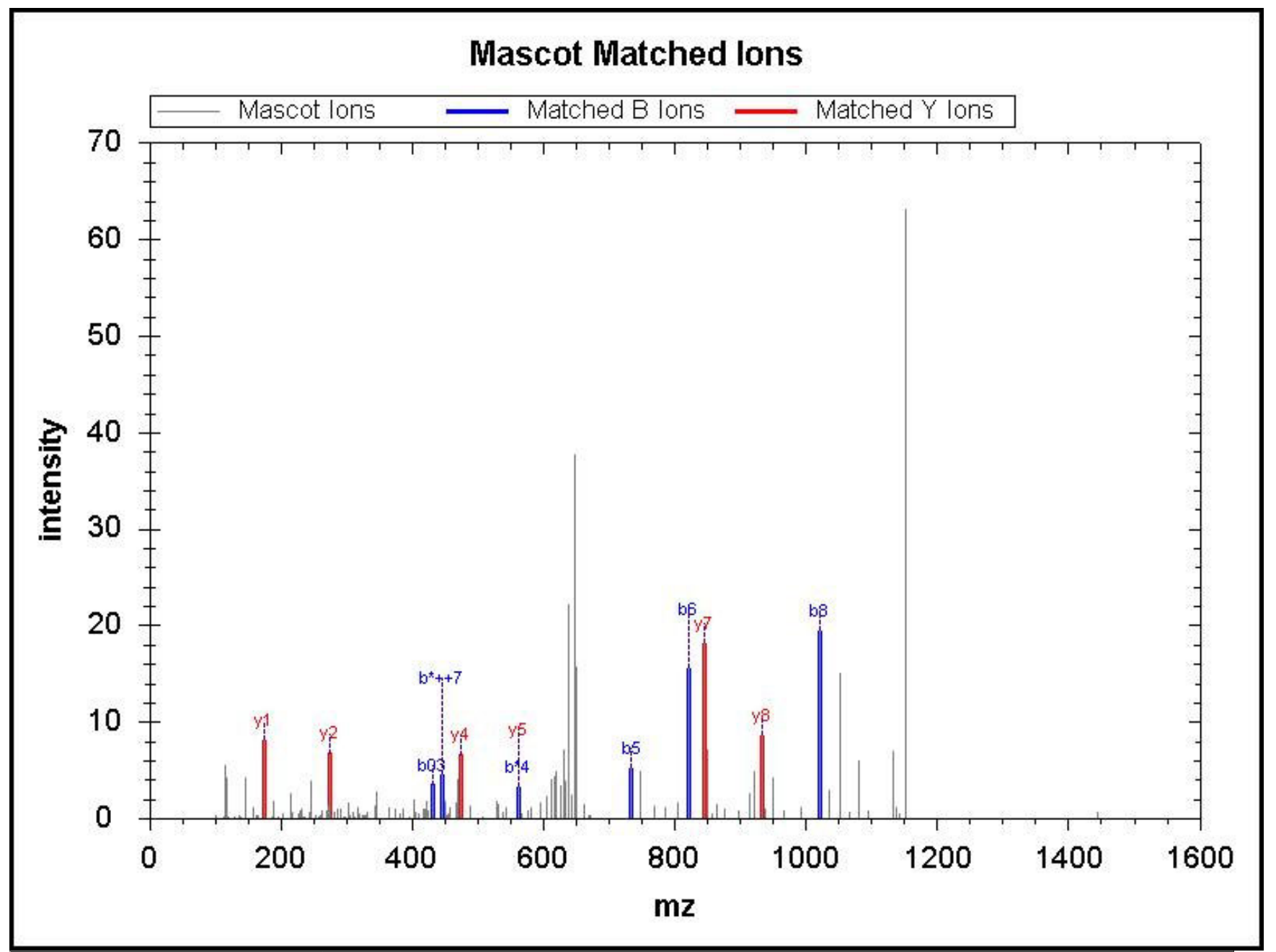

\begin{tabular}{|r|c|c|c|c|r|r|r|r|r|r|r|r|r|r|}
\hline \hline No & $\mathrm{b}$ & $\mathrm{b}++$ & $\mathrm{b}^{*}$ & $\mathrm{~b}++$ & $\mathrm{b} 0$ & $\mathrm{~b} 0++$ & Seq & $\mathrm{y}$ & $\mathrm{y}++$ & $\mathrm{y}^{*}$ & $\mathrm{y}^{*++}$ & $\mathrm{y} 0$ & $\mathrm{y} 0++$ & $\mathrm{RevNo}$ \\
\hline 1 & 216.15 & 108.58 & & & & & $\mathrm{~A}$ & & & & & & & 10 \\
\hline 2 & 363.21 & 182.11 & & & & & $\mathrm{~F}$ & $1,079.60$ & 540.30 & $1,062.57$ & 531.79 & $1,061.59$ & 531.30 & 9 \\
\hline 3 & 450.25 & 225.63 & & & 432.24 & 216.62 & $\mathrm{~S}$ & 932.53 & 466.77 & 915.50 & 458.25 & 914.52 & 457.76 & 8 \\
\hline 4 & 578.31 & 289.66 & 561.28 & 281.14 & 560.29 & 280.65 & $\mathrm{Q}$ & 845.50 & 423.25 & 828.47 & 414.74 & 827.48 & 414.25 & 7 \\
\hline 5 & 734.41 & 367.71 & 717.38 & 359.19 & 716.40 & 358.70 & $\mathrm{R}$ & 717.44 & 359.22 & 700.41 & 350.71 & 699.43 & 350.22 & 6 \\
\hline 6 & 821.44 & 411.22 & 804.41 & 402.71 & 803.43 & 402.22 & $\mathrm{~S}$ & 561.34 & 281.17 & 544.31 & 272.66 & 543.32 & 272.17 & 5 \\
\hline 7 & 908.47 & 454.74 & 891.44 & 446.23 & 890.46 & 445.73 & $\mathrm{~S}$ & 474.30 & 237.66 & 457.28 & 229.14 & 456.29 & 228.65 & 4 \\
\hline 8 & $1,021.55$ & 511.28 & $1,004.53$ & 502.77 & $1,003.54$ & 502.28 & $\mathrm{~L}$ & 387.27 & 194.14 & 370.24 & 185.63 & & & 3 \\
\hline 9 & $1,120.62$ & 560.82 & $1,103.60$ & 552.30 & $1,102.61$ & 551.81 & $\mathrm{~V}$ & 274.19 & 137.60 & 257.16 & 129.08 & & & 2 \\
\hline 10 & & & & & & & $\mathrm{R}$ & 175.12 & 88.06 & 158.09 & 79.55 & & & 1 \\
\hline
\end{tabular}

Query 56495 Hit 1 
Match to Query 56495: 1936.16from(646.3941,3+)

Title: 1198: Sum of 2 scans in range $2752(\mathrm{rt}=70.8572, \mathrm{f}=3, \mathrm{i}=422)$ to $2753(\mathrm{rt}=70.8826, \mathrm{f}=3, \mathrm{i}=423)$

[D:lab212 \membranelGrace JoyceliTRAQ_37_1.raw]

Data File:Submitted from 20120508-1(merge) by Mascot Daemon on JOYCE-VAIO

Monoisotopic mass of neutral peptide $\mathrm{Mr}$ (calc): 1936.16

Variable modifications:

K16 :iTRAQ4plex (K)

Ions Score: 49.66 Expect: 0.003

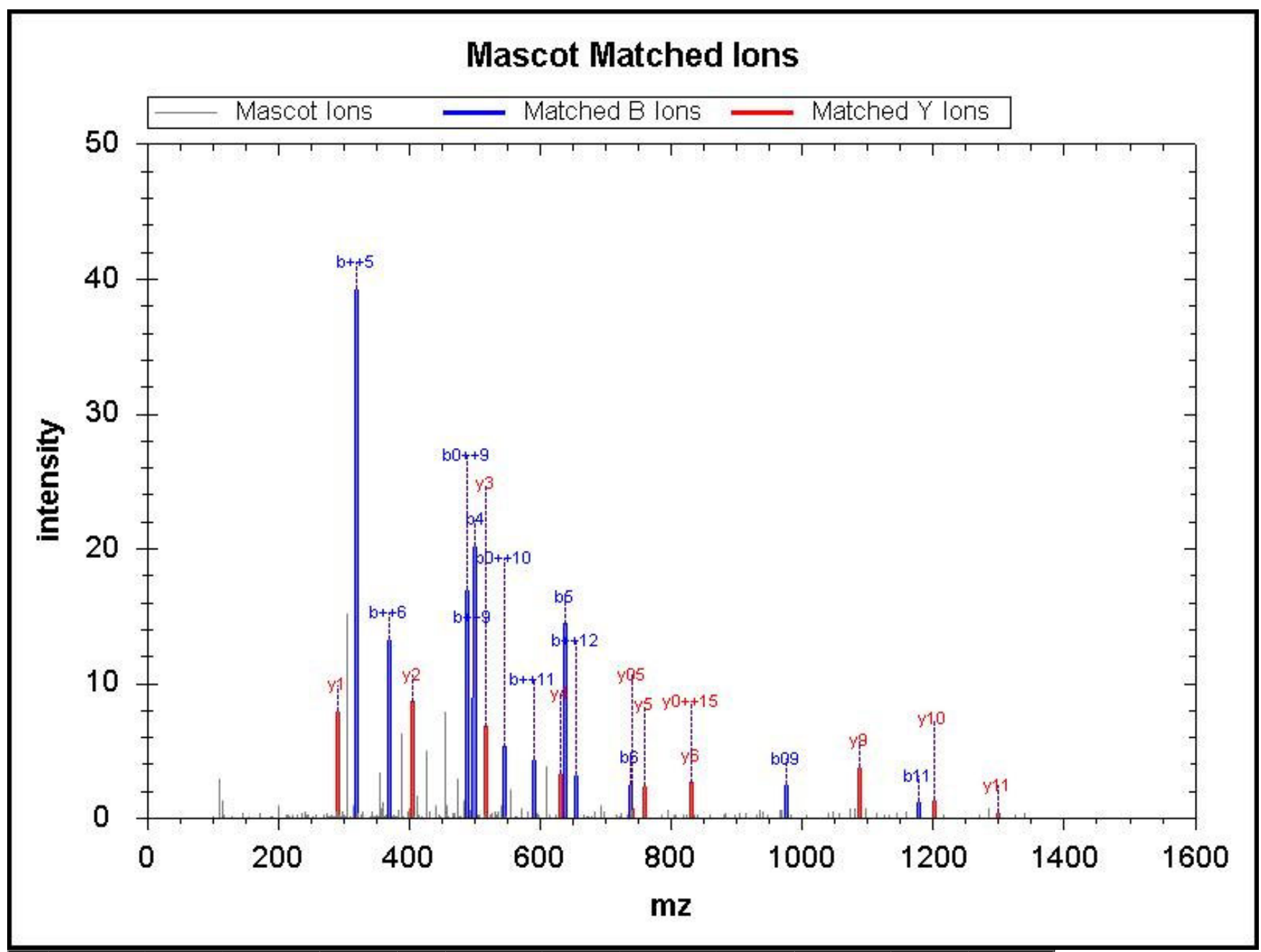

\begin{tabular}{|r|c|c|c|c|c|c|c|c|c|c|r|r|}
\hline \hline $\mathrm{No}$ & $\mathrm{b}$ & $\mathrm{b}++$ & $\mathrm{b} 0$ & $\mathrm{~b} 0++$ & $\mathrm{Seq}$ & $\mathrm{y}$ & $\mathrm{y}++$ & $\mathrm{y} *$ & $\mathrm{y}++$ & $\mathrm{y} 0$ & $\mathrm{y} 0++$ & RevNo \\
\hline 1 & 260.14 & 130.57 & 242.13 & 121.57 & $\mathrm{D}$ & & & & & & & 16 \\
\hline 2 & 317.16 & 159.08 & 299.15 & 150.08 & $\mathrm{G}$ & $1,678.04$ & 839.52 & $1,661.01$ & 831.01 & $1,660.03$ & 830.52 & 15 \\
\hline 3 & 388.19 & 194.60 & 370.18 & 185.60 & $\mathrm{~A}$ & $1,621.02$ & 811.01 & $1,603.99$ & 802.50 & $1,603.01$ & 802.01 & 14 \\
\hline 4 & 501.28 & 251.14 & 483.27 & 242.14 & $\mathrm{~L}$ & $1,549.98$ & 775.49 & $1,532.95$ & 766.98 & $1,531.97$ & 766.49 & 13 \\
\hline 5 & 638.34 & 319.67 & 620.33 & 310.67 & $\mathrm{H}$ & $1,436.90$ & 718.95 & $1,419.87$ & 710.44 & $1,418.89$ & 709.95 & 12 \\
\hline 6 & 737.41 & 369.21 & 719.40 & 360.20 & $\mathrm{~V}$ & $1,299.84$ & 650.42 & $1,282.81$ & 641.91 & $1,281.83$ & 641.42 & 11 \\
\hline 7 & 850.49 & 425.75 & 832.48 & 416.74 & $\mathrm{I}$ & $1,200.77$ & 600.89 & $1,183.74$ & 592.37 & $1,182.76$ & 591.88 & 10 \\
\hline 8 & 907.51 & 454.26 & 889.50 & 445.25 & $\mathrm{G}$ & $1,087.68$ & 544.35 & $1,070.66$ & 535.83 & $1,069.67$ & 535.34 & 9 \\
\hline 9 & 994.54 & 497.78 & 976.53 & 488.77 & $\mathrm{~S}$ & $1,030.66$ & 515.84 & $1,013.64$ & 507.32 & $1,012.65$ & 506.83 & 8 \\
\hline 10 & $1,107.63$ & 554.32 & $1,089.62$ & 545.31 & $\mathrm{~L}$ & 943.63 & 472.32 & 926.60 & 463.81 & 925.62 & 463.31 & 7 \\
\hline 11 & $1,178.67$ & 589.84 & $1,160.65$ & 580.83 & $\mathrm{~A}$ & 830.55 & 415.78 & 813.52 & 407.26 & 812.54 & 406.77 & 6 \\
\hline 12 & $1,307.71$ & 654.36 & $1,289.70$ & 645.35 & $\mathrm{E}$ & 759.51 & 380.26 & 742.48 & 371.75 & 741.50 & 371.25 & 5 \\
\hline 13 & $1,420.79$ & 710.90 & $1,402.78$ & 701.89 & $\mathrm{I}$ & 630.47 & 315.74 & 613.44 & 307.22 & & & 4 \\
\hline 14 & $1,533.88$ & 767.44 & $1,515.87$ & 758.44 & $\mathrm{~L}$ & 517.38 & 259.20 & 500.36 & 250.68 & & & 3 \\
\hline 15 & $1,646.96$ & 823.98 & $1,628.95$ & 814.98 & $\mathrm{~L}$ & 404.30 & 202.65 & 387.27 & 194.14 & & & 2 \\
\hline 16 & & & & & $\mathrm{~K}$ & 291.21 & 146.11 & 274.19 & 137.60 & & & 1 \\
\hline
\end{tabular}


MS/MS Fragmentation of ILYEGTHLDPER

Found in sp|Q5RI15|FA36A_HUMAN, Protein FAM36A OS=Homo sapiens GN=FAM36A PE=1 SV=2

Match to Query 37514: 1585.82from(529.6139,3+)

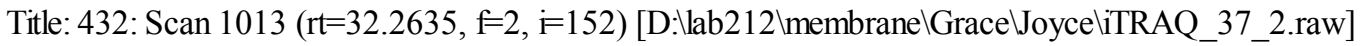

Data File:Submitted from 20120508-1(merge) by Mascot Daemon on JOYCE-VAIO

Monoisotopic mass of neutral peptide $\mathrm{Mr}$ (calc): 1585.82

Variable modifications:

Ions Score: 49.57 Expect: 0.009

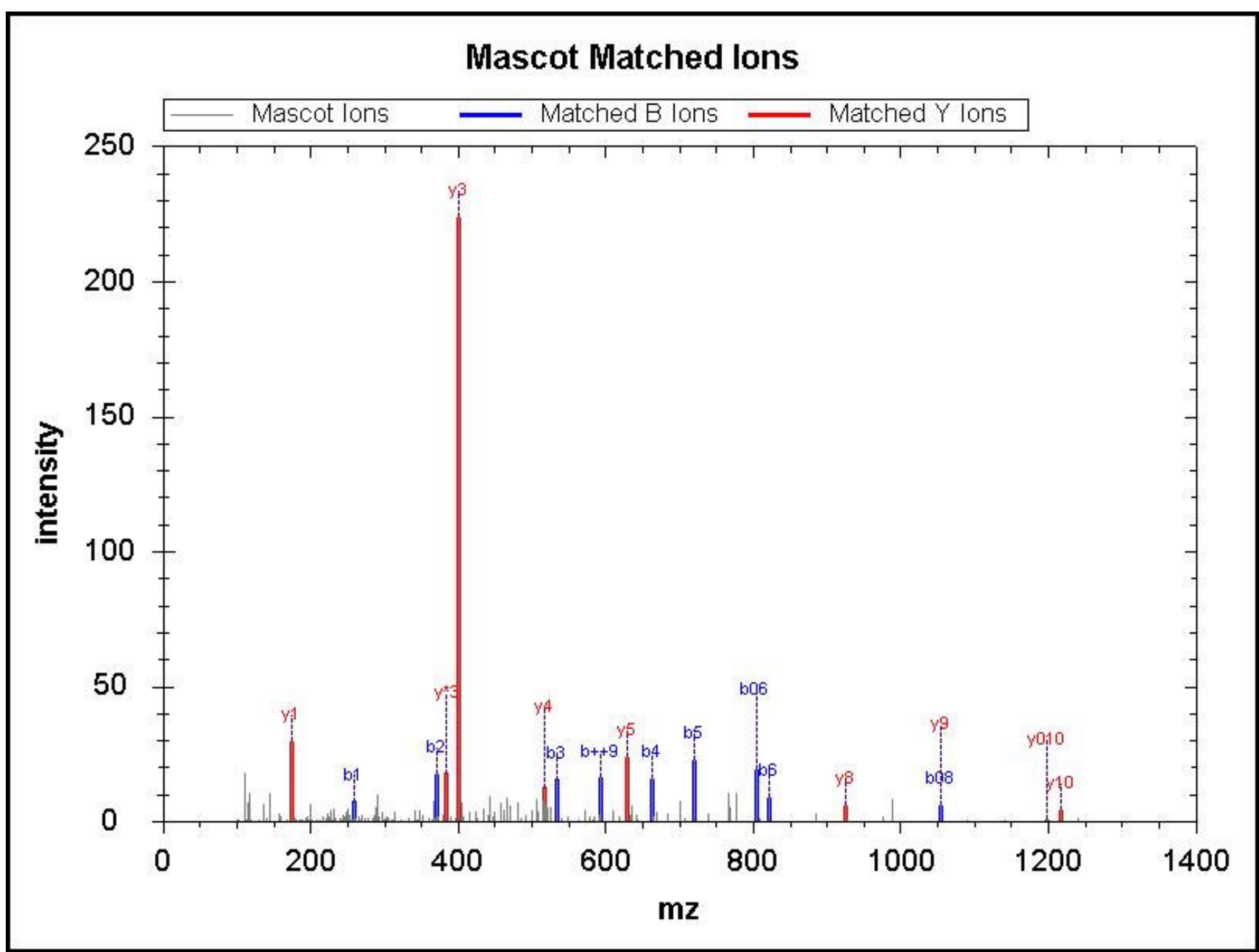

\begin{tabular}{|r|c|c|c|c|c|c|c|c|c|c|r|r|}
\hline No & $\mathrm{b}$ & $\mathrm{b}++$ & $\mathrm{b} 0$ & $\mathrm{~b} 0++$ & $\mathrm{Seq}$ & $\mathrm{y}$ & $\mathrm{y}++$ & $\mathrm{y}^{*}$ & $\mathrm{y}^{*++}$ & $\mathrm{y} 0$ & $\mathrm{y} 0++$ & RevNo \\
\hline 1 & 258.19 & 129.60 & & & $\mathrm{I}$ & & & & & & & 12 \\
\hline 2 & 371.28 & 186.14 & & & $\mathrm{~L}$ & $1,329.64$ & 665.33 & $1,312.62$ & 656.81 & $1,311.63$ & 656.32 & 11 \\
\hline 3 & 534.34 & 267.67 & & & $\mathrm{Y}$ & $1,216.56$ & 608.78 & $1,199.53$ & 600.27 & $1,198.55$ & 599.78 & 10 \\
\hline 4 & 663.38 & 332.20 & 645.37 & 323.19 & $\mathrm{E}$ & $1,053.50$ & 527.25 & $1,036.47$ & 518.74 & $1,035.49$ & 518.25 & 9 \\
\hline 5 & 720.40 & 360.71 & 702.39 & 351.70 & $\mathrm{G}$ & 924.45 & 462.73 & 907.43 & 454.22 & 906.44 & 453.73 & 8 \\
\hline 6 & 821.45 & 411.23 & 803.44 & 402.22 & $\mathrm{~T}$ & 867.43 & 434.22 & 850.41 & 425.71 & 849.42 & 425.21 & 7 \\
\hline 7 & 958.51 & 479.76 & 940.50 & 470.75 & $\mathrm{H}$ & 766.38 & 383.70 & 749.36 & 375.18 & 748.37 & 374.69 & 6 \\
\hline 8 & $1,071.60$ & 536.30 & $1,053.58$ & 527.30 & $\mathrm{~L}$ & 629.33 & 315.17 & 612.30 & 306.65 & 611.31 & 306.16 & 5 \\
\hline 9 & $1,186.62$ & 593.81 & $1,168.61$ & 584.81 & $\mathrm{D}$ & 516.24 & 258.62 & 499.21 & 250.11 & 498.23 & 249.62 & 4 \\
\hline 10 & $1,283.68$ & 642.34 & $1,265.66$ & 633.34 & $\mathrm{P}$ & 401.21 & 201.11 & 384.19 & 192.60 & 383.20 & 192.11 & 3 \\
\hline 11 & $1,412.72$ & 706.86 & $1,394.71$ & 697.86 & $\mathrm{E}$ & 304.16 & 152.58 & 287.13 & 144.07 & 286.15 & 143.58 & 2 \\
\hline 12 & & & & & $\mathrm{R}$ & 175.12 & 88.06 & 158.09 & 79.55 & & & 1 \\
\hline
\end{tabular}

Query 36622 Hit 1 
Match to Query 36622: 1568.812 from(523.9446,3+)

Title: 391: Scan 927 ( $\mathrm{rt}=30.3291, \mathrm{f}=3, \mathrm{i}=139$ ) [D:llab212 $\backslash$ membranelGracelJoyceliTRAQ_37_2.raw]

Data File:Submitted from 20120508-1(merge) by Mascot Daemon on JOYCE-VAIO

Monoisotopic mass of neutral peptide $\mathrm{Mr}$ (calc): 1568.812

Variable modifications:

Ions Score: 49.52 Expect: 0.009

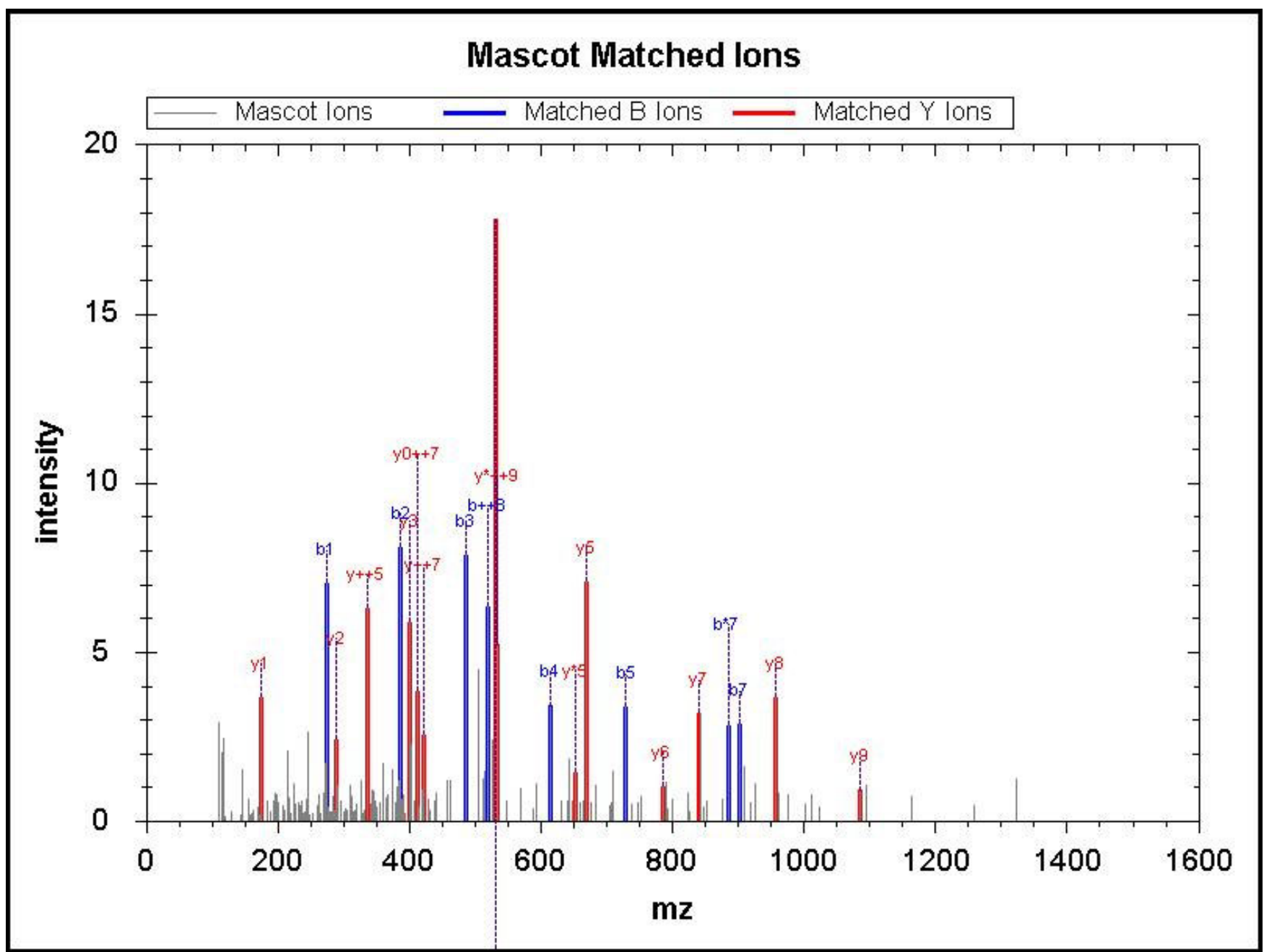

\begin{tabular}{|r|c|c|c|c|c|c|c|c|c|c|c|c|r|r|}
\hline \hline $\mathrm{No}$ & $\mathrm{b}$ & $\mathrm{b}++$ & $\mathrm{b} *$ & $\mathrm{~b}^{*++}$ & $\mathrm{b} 0$ & $\mathrm{~b} 0++$ & $\mathrm{Seq}$ & $\mathrm{y}$ & $\mathrm{y}++$ & $\mathrm{y}^{*}$ & $\mathrm{y}^{*++}$ & $\mathrm{y} 0$ & $\mathrm{y} 0++$ & $\mathrm{RevNo}$ \\
\hline 1 & 274.15 & 137.58 & & & 256.14 & 128.57 & $\mathrm{E}$ & & & & & & & 12 \\
\hline 2 & 387.24 & 194.12 & & & 369.23 & 185.12 & $\mathrm{I}$ & $1,296.67$ & 648.84 & $1,279.65$ & 640.33 & $1,278.66$ & 639.83 & 11 \\
\hline 3 & 486.30 & 243.66 & & & 468.29 & 234.65 & $\mathrm{~V}$ & $1,183.59$ & 592.30 & $1,166.56$ & 583.78 & $1,165.58$ & 583.29 & 10 \\
\hline 4 & 614.36 & 307.69 & 597.34 & 299.17 & 596.35 & 298.68 & $\mathrm{Q}$ & $1,084.52$ & 542.76 & $1,067.49$ & 534.25 & $1,066.51$ & 533.76 & 9 \\
\hline 5 & 729.39 & 365.20 & 712.36 & 356.69 & 711.38 & 356.19 & $\mathrm{D}$ & 956.46 & 478.73 & 939.44 & 470.22 & 938.45 & 469.73 & 8 \\
\hline 6 & 786.41 & 393.71 & 769.38 & 385.20 & 768.40 & 384.70 & $\mathrm{G}$ & 841.43 & 421.22 & 824.41 & 412.71 & 823.42 & 412.22 & 7 \\
\hline 7 & 901.44 & 451.22 & 884.41 & 442.71 & 883.43 & 442.22 & $\mathrm{D}$ & 784.41 & 392.71 & 767.39 & 384.20 & 766.40 & 383.71 & 6 \\
\hline 8 & $1,038.50$ & 519.75 & $1,021.47$ & 511.24 & $1,020.49$ & 510.75 & $\mathrm{H}$ & 669.39 & 335.20 & 652.36 & 326.68 & & & 5 \\
\hline 9 & $1,169.54$ & 585.27 & $1,152.51$ & 576.76 & $1,151.53$ & 576.27 & $\mathrm{M}$ & 532.33 & 266.67 & 515.30 & 258.15 & & & 4 \\
\hline 10 & $1,282.62$ & 641.81 & $1,265.60$ & 633.30 & $1,264.61$ & 632.81 & $\mathrm{I}$ & 401.29 & 201.15 & 384.26 & 192.63 & & & 3 \\
\hline 11 & $1,395.71$ & 698.36 & $1,378.68$ & 689.84 & $1,377.70$ & 689.35 & $\mathrm{I}$ & 288.20 & 144.61 & 271.18 & 136.09 & & & 2 \\
\hline 12 & & & & & & & $\mathrm{R}$ & 175.12 & 88.06 & 158.09 & 79.55 & & & 1 \\
\hline
\end{tabular}

Query 79295 Hit 1

MS/MS Fragmentation of FKPGVISEELQDALGVTDK

Found in sp|Q6NZY4|ZCHC8_HUMAN, Zinc finger CCHC domain-containing protein 8 OS=Homo sapiens GN=ZCCHC8 PE=1 SV=2 Match to Query 79295: 2477.38from(826.8008,3+)

Title: 930: Sum of 2 scans in range 2014 ( $\mathrm{rt}=54.9947, \mathrm{f}=4, \mathrm{i}=616)$ to 2015 ( $\mathrm{rt}=55.0201, \mathrm{f}=4, \mathrm{i}=617$ )

[D:lab212 \membranelGrace JoyceliTRAQ_41_1.raw]

Data File:Submitted from 20120508-1(merge) by Mascot Daemon on JOYCE-VAIO 


\section{Mascot Matched lons}

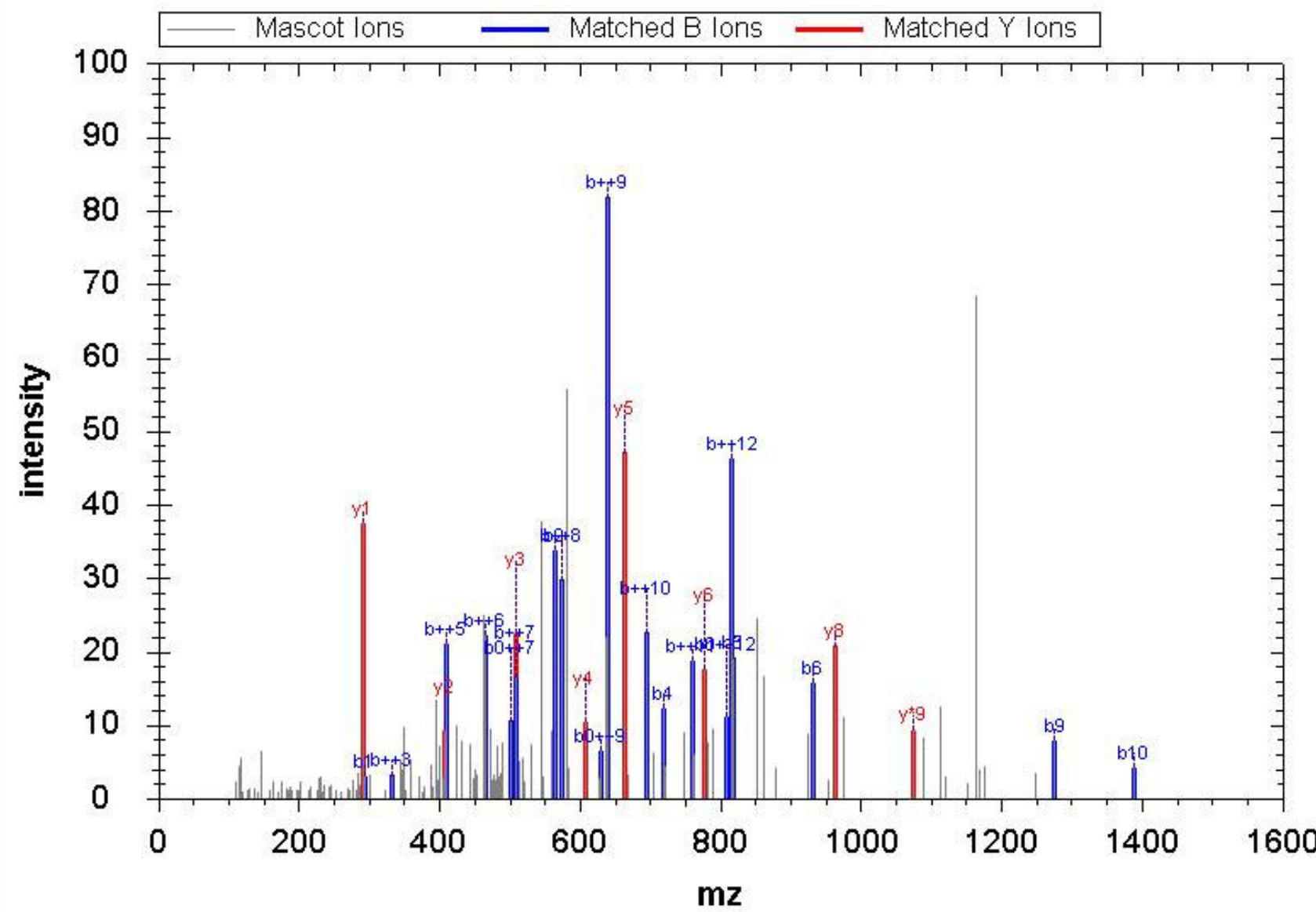

\begin{tabular}{|c|c|c|c|c|c|c|c|c|c|c|c|c|c|c|}
\hline $\mathrm{o}$ & $b$ & ++ & $\mathrm{b}^{*}$ & k++ & b0 & $\mathrm{b} 0++$ & Seq & $\mathrm{y}$ & $y++$ & $\mathrm{y}^{*}$ & $\mathrm{y}^{*++}$ & y0 & $\mathrm{y} 0++$ & evNo \\
\hline 1 & 292.18 & 146.59 & & & & & F & & & & & & & 19 \\
\hline 2 & 564.37 & 282.69 & 7.35 & 274.18 & & & $\mathrm{~K}$ & 87.21 & $1,094.11$ & $2,170.18$ & $1,085.60$ & $2,169.20$ & $1,085.10$ & 18 \\
\hline 3 & 661.43 & 331.22 & 644.40 & 322.70 & & & $\mathrm{P}$ & $1,915.01$ & 958.01 & $1,897.99$ & 949.50 & $1,897.00$ & 949.01 & 17 \\
\hline 4 & 718.45 & 359.73 & 701.42 & 351.21 & & & G & 1,81 & 909.48 & $1,800.93$ & 900.97 & $1,799.95$ & 900.48 & 1 \\
\hline 5 & 817.52 & 409.26 & 800.49 & 400.75 & & & $\mathrm{~V}$ & $1,760.94$ & 880.97 & $1,743.91$ & 872.46 & $1,742.93$ & 871.97 & 15 \\
\hline 6 & 930.60 & 465.80 & 913.57 & 457.29 & & & I & $1,661.87$ & 831.44 & $1,644.84$ & 822.93 & $1,643.86$ & 822.43 & 14 \\
\hline 7 & $1,017.63$ & 509.32 & 00.61 & 500.81 & | & 500.22 & $\mathrm{~S}$ & $1,548.79$ & 774.90 & $1,531.76$ & 66.38 & $1,530.78$ & 765.8 & 15 \\
\hline 8 & $1,146.68$ & 3.84 & 29.65 & 565.33 & $\mid$ & 564.84 & $\mathrm{E}$ & $1,401 . / 0$ & 731.38 & $1,444.73$ & 2.87 & $1,443.74$ & 722. & 12 \\
\hline 9 & $1,275.72$ & 638.36 & 9 & 629.85 & & 6 & $E$ & 71 & 666.86 & 1,3 & 658.35 & .70 & 657.8 & \\
\hline 10 & $1,388.80$ & 694.91 & $1,371.78$ & 686.39 & 1,370 & 685.90 & $\mathrm{~L}$ & $1,203.67$ & 602.34 & $1,186.64$ & 593.83 & $1,185.66$ & 593.3 & 10 \\
\hline 11 & $1,516.86$ & 758.93 & 99.83 & 750.42 & $1,498.85$ & 749.93 & Q & $1,090.59$ & 545.80 & $1,073.56$ & 537.28 & $1,072.58$ & 536.79 & \\
\hline 12 & $1,631.89$ & 816.45 & 514.86 & 807.93 & $1,613.88$ & 807.44 & $\mathrm{D}$ & 962.53 & 481.77 & 945.50 & 473.25 & 944.52 & 472.76 & \\
\hline 13 & $1,702.93$ & 851.97 & $1,685.90$ & 843.45 & $1,684.91$ & 842.96 & A & 847.50 & 424.25 & 830.47 & 415.74 & 829.49 & 415.25 & \\
\hline 14 & $1,816.01$ & 908.51 & $1,798.98$ & 900.00 & $1,798.00$ & 899.50 & $\mathrm{~L}$ & 776.46 & 388.74 & 759.44 & 380.22 & 758.45 & 379.73 & \\
\hline 15 & $1,873.03$ & 937.02 & $1,856.00$ & 928.51 & $1,855.02$ & 928.01 & G & 663.38 & 332.19 & 646.35 & 323.68 & 645.37 & 323.19 & \\
\hline 16 & $1,972.10$ & 986.55 & $1,955.07$ & 978.04 & $1,954.09$ & 977.55 & $\mathrm{~V}$ & 606.36 & 303.68 & 589.33 & 295.17 & 588.35 & 294.68 & \\
\hline 17 & $2,073.15$ & $1,037.08$ & $2,056.12$ & $1,028.56$ & $2,055.14$ & $1,028.07$ & $\mathrm{~T}$ & 507.29 & 254.15 & 490.26 & 245.64 & 489.28 & 245.14 & \\
\hline 18 & $2,188.17$ & $1,094.59$ & $2,171.15$ & $1,086.08$ & $2,170.16$ & $1,085.59$ & D & 406.24 & 203.62 & 389.22 & 195.11 & 388.23 & 194.62 & \\
\hline 19 & & & & & & & $\mathrm{~K}$ & 291.21 & 146.11 & 274.19 & 137.60 & & & \\
\hline
\end{tabular}


MS/MS Fragmentation of ELQHAALGGTATR

Found in sp $|\mathrm{O} 14828| \mathrm{SCAM} 3$ HUM AN, Secretory carrier-associated membrane protein $3 \mathrm{OS}=$ Homo sapiens GN=SCAMP3 PE=1 $\mathrm{SV}=3$

Match to Query 31228: 1467.788from(490.27,3+)

Title: 160: Scan 464 ( $\mathrm{rt}=19.7768, \mathrm{f}=2, \mathrm{i}=67)$ [D:llab212 $\backslash$ membranelGrace JoyceliTRAQ_38_1.raw]

Data File:Submitted from 20120508-1(merge) by Mascot Daemon on JOYCE-VAIO

Monoisotopic mass of neutral peptide $\mathrm{Mr}$ (calc): 1467.788

Variable modifications:

Ions Score: 49.48 Expect: 0.009

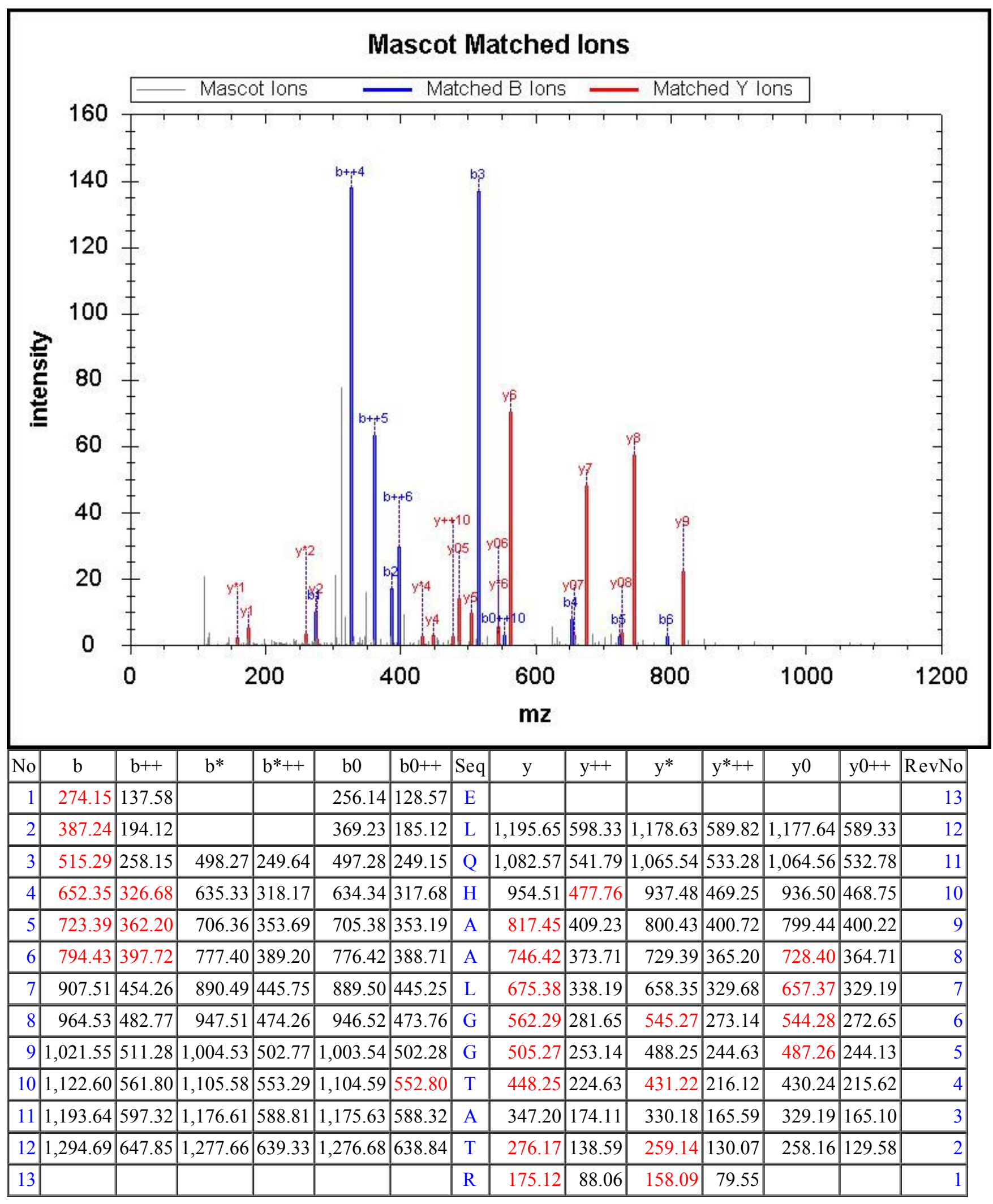

Query 31118 Hit 1 
MS/MS Fragmentation of TTLLAGDIEHPR

Found in sp|Q07954|LRP1_HUM AN, Prolow-density lipoprotein receptor-related protein 1 OS=Homo sapiens GN=LRP1 PE=1 SV=2 Match to Query 31118: 1465.801 from(489.6076,3+)

Title: 385: Scan 916 ( $\mathrm{rt}=30.0637, \mathrm{f}=2, \mathrm{i}=137)$ [D:llab212 membranelGraceJoyceliTRAQ_37_1.raw]

Data File:Submitted from 20120508-1(merge) by Mascot Daemon on JOYCE-VAIO

Monoisotopic mass of neutral peptide $\operatorname{Mr}($ calc): 1465.801

Variable modifications:

Ions Score: 49.47 Expect: 0.009

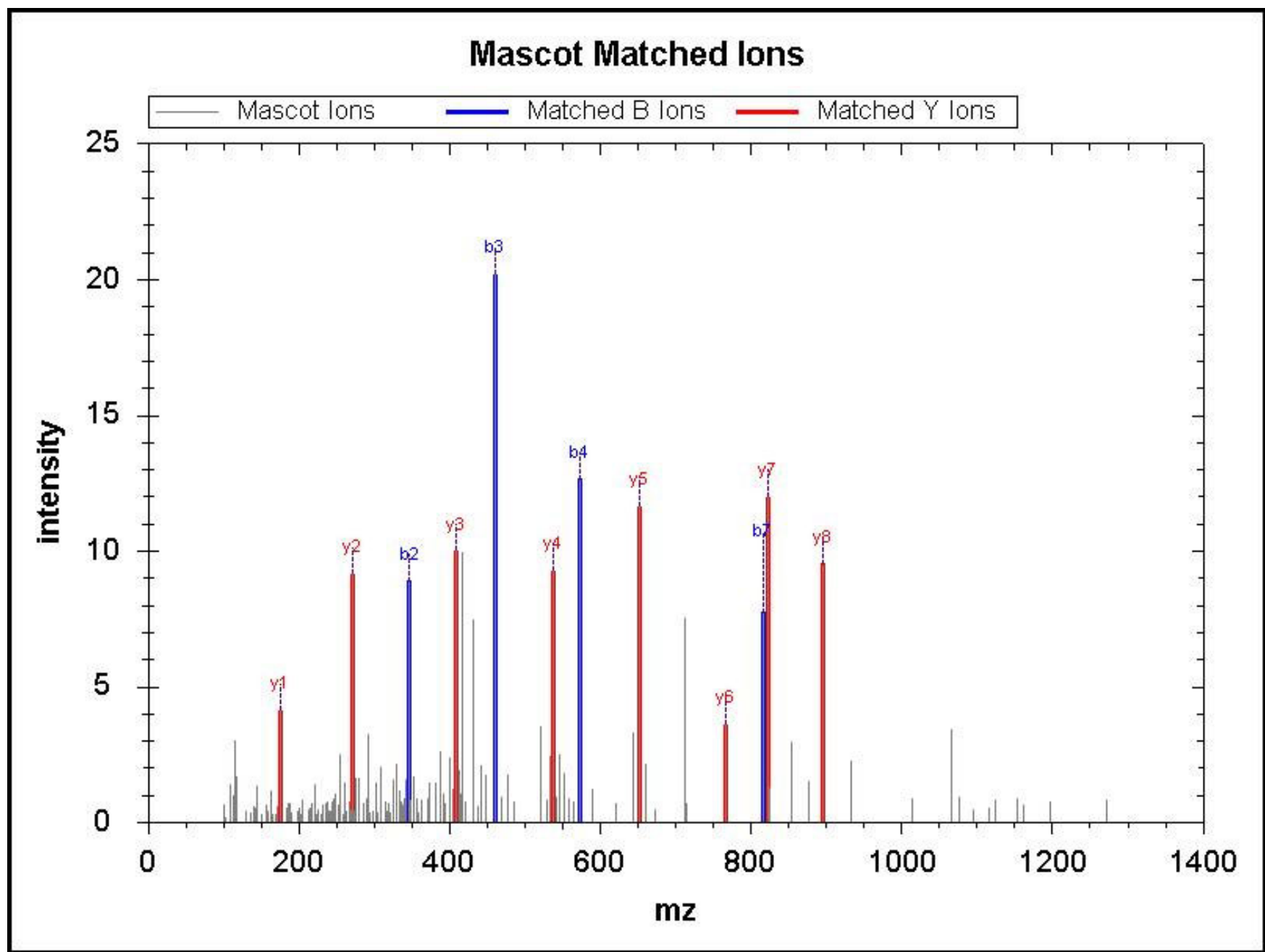

\begin{tabular}{|r|c|c|c|c|c|c|c|c|c|c|r|r|}
\hline \hline No & $\mathrm{b}$ & $\mathrm{b}++$ & $\mathrm{b} 0$ & $\mathrm{~b} 0++$ & $\mathrm{Seq}$ & $\mathrm{y}$ & $\mathrm{y}++$ & $\mathrm{y}^{*}$ & $\mathrm{y} *++$ & $\mathrm{y} 0$ & $\mathrm{y} 0++$ & RevNo \\
\hline 1 & 246.16 & 123.58 & 228.15 & 114.58 & $\mathrm{~T}$ & & & & & & & 12 \\
\hline 2 & 347.20 & 174.11 & 329.19 & 165.10 & $\mathrm{~T}$ & $1,221.66$ & 611.33 & $1,204.63$ & 602.82 & $1,203.65$ & 602.33 & 11 \\
\hline 3 & 460.29 & 230.65 & 442.28 & 221.64 & $\mathrm{~L}$ & $1,120.61$ & 560.81 & $1,103.58$ & 552.30 & $1,102.60$ & 551.80 & 10 \\
\hline 4 & 573.37 & 287.19 & 555.36 & 278.18 & $\mathrm{~L}$ & $1,007.53$ & 504.27 & 990.50 & 495.75 & 989.52 & 495.26 & 9 \\
\hline 5 & 644.41 & 322.71 & 626.40 & 313.70 & $\mathrm{~A}$ & 894.44 & 447.73 & 877.42 & 439.21 & 876.43 & 438.72 & 8 \\
\hline 6 & 701.43 & 351.22 & 683.42 & 342.21 & $\mathrm{G}$ & 823.41 & 412.21 & 806.38 & 403.69 & 805.40 & 403.20 & 7 \\
\hline 7 & 816.46 & 408.73 & 798.45 & 399.73 & $\mathrm{D}$ & 766.38 & 383.70 & 749.36 & 375.18 & 748.37 & 374.69 & 6 \\
\hline 8 & 929.54 & 465.27 & 911.53 & 456.27 & $\mathrm{I}$ & 651.36 & 326.18 & 634.33 & 317.67 & 633.35 & 317.18 & 5 \\
\hline 9 & $1,058.58$ & 529.80 & $1,040.57$ & 520.79 & $\mathrm{E}$ & 538.27 & 269.64 & 521.25 & 261.13 & 520.26 & 260.63 & 4 \\
\hline 10 & $1,195.64$ & 598.33 & $1,177.63$ & 589.32 & $\mathrm{H}$ & 409.23 & 205.12 & 392.20 & 196.61 & & & 3 \\
\hline 11 & $1,292.70$ & 646.85 & $1,274.69$ & 637.85 & $\mathrm{P}$ & 272.17 & 136.59 & 255.15 & 128.08 & & & 2 \\
\hline 12 & & & & & $\mathrm{R}$ & 175.12 & 88.06 & 158.09 & 79.55 & & & 1 \\
\hline
\end{tabular}

Query 56871 Hit 1

MS/MS Fragmentation of HMDFLSDFEMMLQR

Found in sp|Q96ST2|IWS1_HUMAN, Protein IWS1 homolog OS=Homo sapiens GN=IWS1 PE=1 SV=2

Match to Query 56871: 1942.889from(648.6369,3+) 
Title: 1116: Sum of 2 scans in range $2511(\mathrm{rt}=65.6578, \mathrm{f}=2, \mathrm{i}=413)$ to $2512(\mathrm{rt}=65.6832, \mathrm{f}=2, \mathrm{i}=414)$

[D:lab212 \membranelGrace $\backslash J$ oyceliTRAQ 38 2.raw]

Data File:Submitted from 20120508-1(merge) by Mascot Daemon on JOYCE-VAIO

Monoisotopic mass of neutral peptide Mr(calc): 1942.889

Variable modifications:

Ions Score: 49.39 Expect: 0.007

\section{Mascot Matched lons}

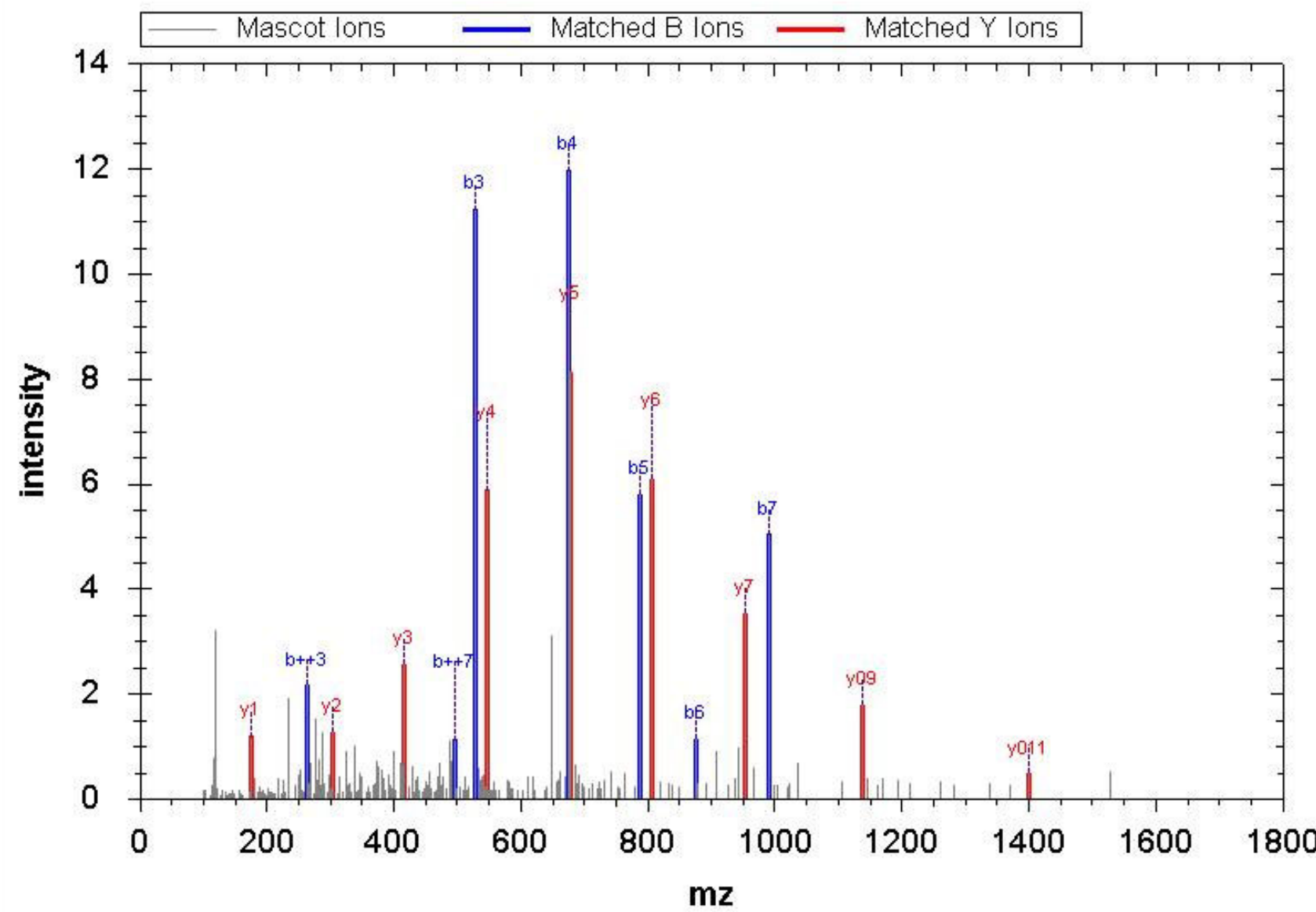

\begin{tabular}{|c|c|c|c|c|c|c|c|c|c|c|c|c|c|c|}
\hline No & $\mathrm{b}$ & $\mathrm{b}++$ & $\mathrm{b}^{*}$ & $b^{*++}$ & b0 & $\mathrm{b} 0++$ & Seq & y & $\mathrm{y}++$ & $\mathrm{y}^{*}$ & $\mathrm{y}^{*++}$ & y0 & $\mathrm{y} 0++$ & RevNo \\
\hline 1 & 282.17 & 141.59 & & & & & $\mathrm{H}$ & & & & & & & 14 \\
\hline 2 & 413.21 & 207.11 & & & & & $\mathrm{M}$ & $1,662.73$ & 831.87 & $1,645.71$ & 823.36 & $1,644.72$ & 822.86 & 13 \\
\hline 3 & 528.24 & 264.62 & & & 510.23 & 255.62 & $\mathrm{D}$ & $1,531.69$ & 766.35 & $1,514.67$ & 757.84 & $1,513.68$ & 757.34 & 12 \\
\hline 4 & 675.30 & 338.16 & & & 657.29 & 329.15 & $\mathrm{~F}$ & $1,416.67$ & 708.84 & $1,399.64$ & 700.32 & $1,398.65$ & 699.83 & 11 \\
\hline 5 & 788.39 & 394.70 & & & 770.38 & 385.69 & $\mathrm{~L}$ & $1,269.60$ & 635.30 & $1,252.57$ & 626.79 & $1,251.59$ & 626.30 & 10 \\
\hline 6 & 875.42 & 438.21 & & & 857.41 & 429.21 & S & $1,156.51$ & 578.76 & $1,139.49$ & 570.25 & $1,138.50$ & 569.75 & 9 \\
\hline 7 & 990.45 & 495.73 & & & 972.44 & 486.72 & $\mathrm{D}$ & $1,069.48$ & 535.24 & $1,052.45$ & 526.73 & $1,051.47$ & 526.24 & 8 \\
\hline 8 & $1,137.52$ & 569.26 & & & $1,119.51$ & 560.26 & $\mathrm{~F}$ & 954.45 & 477.73 & 937.43 & 469.22 & 936.44 & 468.73 & 7 \\
\hline 9 & $1,266.56$ & 633.78 & & & $1,248.55$ & 624.78 & $\mathrm{E}$ & 807.39 & 404.20 & 790.36 & 395.68 & 789.37 & 395.19 & 6 \\
\hline 10 & $1,397.60$ & 699.30 & & & $1,379.59$ & 690.30 & $\mathrm{M}$ & 678.34 & 339.67 & 661.32 & 331.16 & & & 5 \\
\hline 11 & $1,528.64$ & 764.82 & & & $1,510.63$ & 755.82 & $\mathrm{M}$ & 547.30 & 274.15 & 530.28 & 265.64 & & & 4 \\
\hline 12 & $1,641.72$ & 821.37 & & & $1,623.71$ & 812.36 & $\mathrm{~L}$ & 416.26 & 208.63 & 399.24 & 200.12 & & & 3 \\
\hline 13 & $1,769.78$ & 885.39 & $1,752.76$ & 876.88 & $1,751.77$ & 876.39 & Q & 303.18 & 152.09 & 286.15 & 143.58 & & & 2 \\
\hline 14 & & & & & & & $\mathrm{R}$ & 175.12 & 88.06 & 158.09 & 79.55 & & & 1 \\
\hline
\end{tabular}

Query 13496 Hit 1 
Title: 64: Sum of 2 scans in range $254(\mathrm{rt}=15.0732, \mathrm{f}=4, \mathrm{i}=37)$ to $255(\mathrm{rt}=15.0986, \mathrm{f}=4, \mathrm{i}=38)$

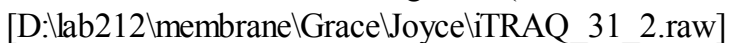

Data File:Submitted from 20120508-1(merge) by Mascot Daemon on JOYCE-VAIO

Monoisotopic mass of neutral peptide Mr(calc): 1133.619

Variable modifications:

K8 :iTRAQ4plex (K)

Ions Score: 49.24 Expect: 0.011

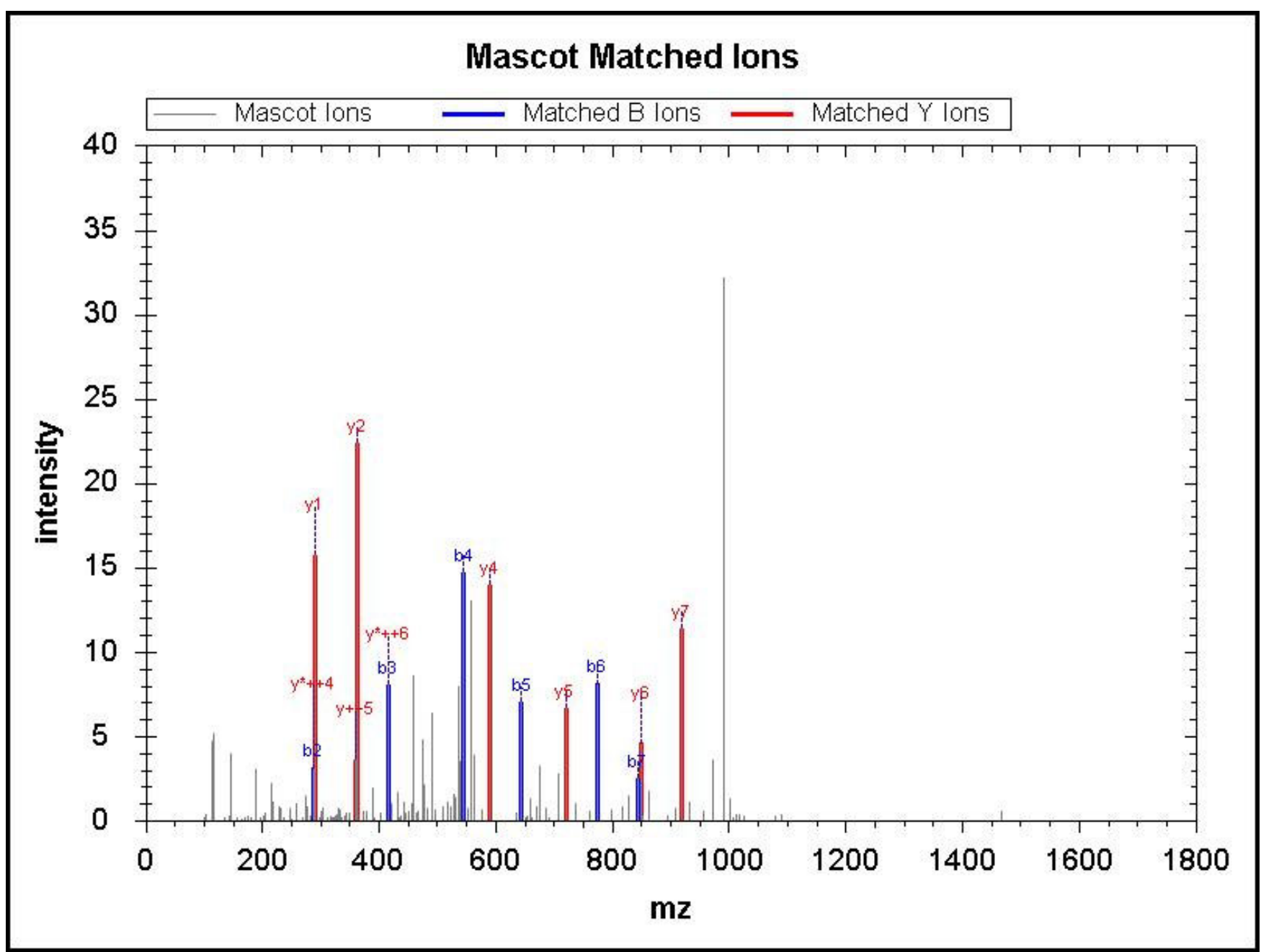

\begin{tabular}{|r|c|c|c|c|c|c|c|c|c|c|r|r|}
\hline No & $\mathrm{b}$ & $\mathrm{b}++$ & $\mathrm{b} 0$ & $\mathrm{~b} 0++$ & Seq & $\mathrm{y}$ & $\mathrm{y}++$ & $\mathrm{y}^{*}$ & $\mathrm{y}^{*++}$ & $\mathrm{y} 0$ & $\mathrm{y} 0++$ & RevNo \\
\hline 1 & 216.15 & 108.58 & & & A & & & & & & & 8 \\
\hline 2 & 287.18 & 144.10 & & & A & 919.49 & 460.25 & 902.46 & 451.73 & 901.47 & 451.24 & 7 \\
\hline 3 & 416.23 & 208.62 & 398.22 & 199.61 & $\mathrm{E}$ & 848.45 & 424.73 & 831.42 & 416.21 & 830.44 & 415.72 & 6 \\
\hline 4 & 545.27 & 273.14 & 527.26 & 264.13 & $\mathrm{E}$ & 719.41 & 360.21 & 702.38 & 351.69 & 701.40 & 351.20 & 5 \\
\hline 5 & 644.34 & 322.67 & 626.33 & 313.67 & $\mathrm{~V}$ & 590.36 & 295.69 & 573.34 & 287.17 & 572.35 & 286.68 & 4 \\
\hline 6 & 773.38 & 387.19 & 755.37 & 378.19 & $\mathrm{E}$ & 491.29 & 246.15 & 474.27 & 237.64 & 473.28 & 237.15 & 3 \\
\hline 7 & 844.42 & 422.71 & 826.41 & 413.71 & A & 362.25 & 181.63 & 345.23 & 173.12 & & & 2 \\
\hline 8 & & & & & K & 291.21 & 146.11 & 274.19 & 137.60 & & & 1 \\
\hline
\end{tabular}

Query 61002 Hit 1

MS/MS Fragmentation of LHLLDQVFFQELLK

Found in sp|Q7Z6J4|FGD2_HUMAN, FYVE

Match to Query 61002: 2030.178from(677.7333,3+)

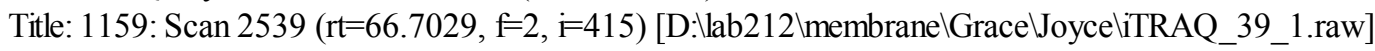

Data File:Submitted from 20120508-1(merge) by Mascot Daemon on JOYCE-VAIO

Monoisotopic mass of neutral peptide Mr(calc): 2030.178

Variable modifications:

K14 :iTRAQ4plex (K)

Ions Score: 49.1 Expect: 0.005 
Mascot Matched lons

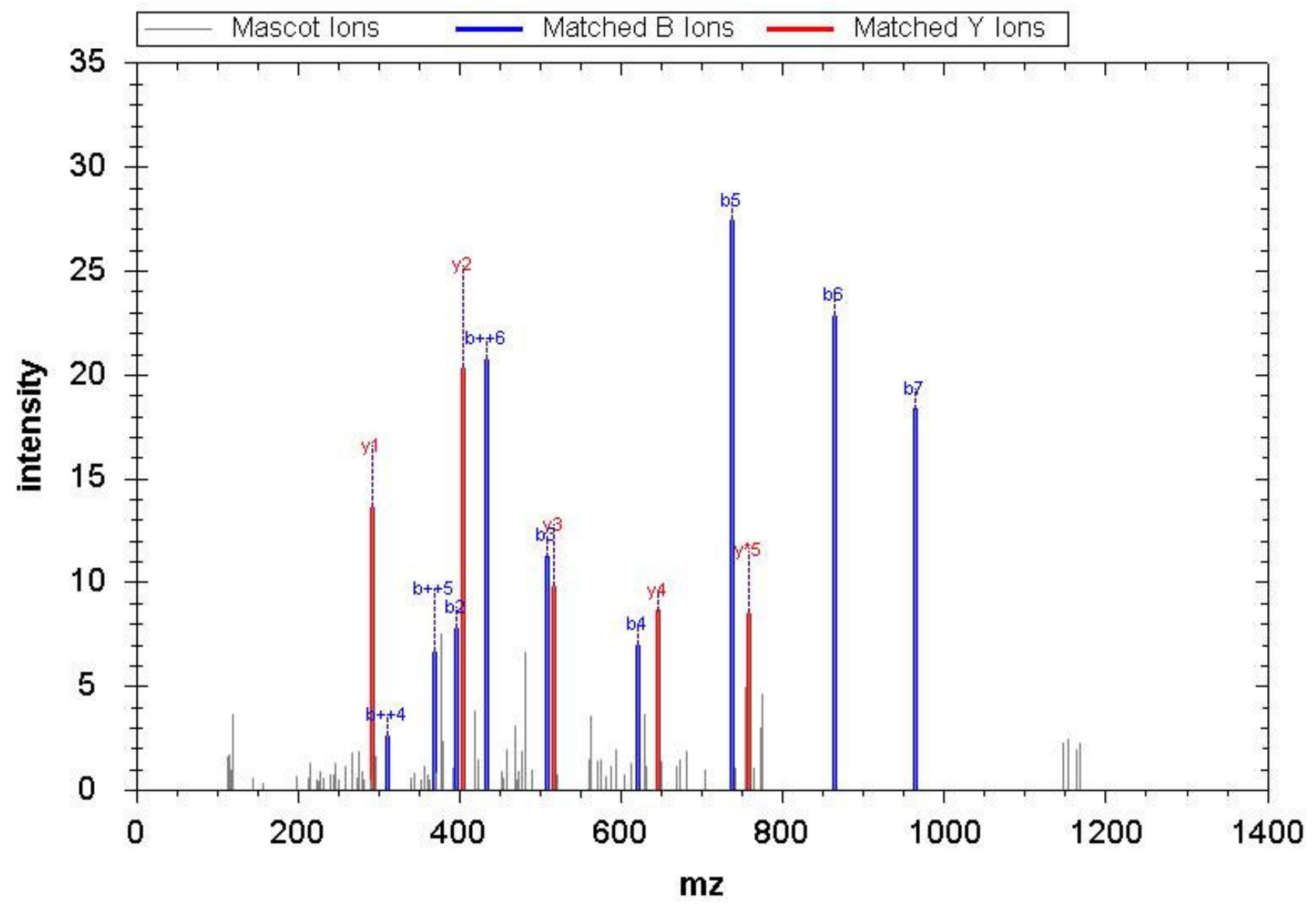

\begin{tabular}{|c|c|c|c|c|c|c|c|c|c|c|c|c|c|c|}
\hline No & $\mathrm{b}$ & $\mathrm{b}++$ & $b^{*}$ & $\mathrm{~b}^{*++}$ & b0 & b0++ & Seq & $\mathrm{y}$ & $\mathrm{y}++$ & $y^{*}$ & $\mathrm{y}^{*++}$ & y0 & y0++ & RevNo \\
\hline 1 & 258.19 & 129.60 & & & & & $\mathrm{~L}$ & & & & & & & 14 \\
\hline 2 & 395.25 & 198.13 & & & & & $\mathrm{H}$ & $1,774.00$ & 887.50 & $1,756.98$ & 878.99 & $1,755.99$ & 878.50 & 13 \\
\hline 3 & 508.34 & 254.67 & & & & & $\mathrm{~L}$ & $1,636.94$ & 818.98 & $1,619.92$ & 810.46 & $1,618.93$ & 809.97 & 12 \\
\hline 4 & 621.42 & 311.21 & & & & & $\mathrm{~L}$ & $1,523.86$ & 762.43 & $1,506.83$ & 753.92 & $1,505.85$ & 753.43 & 11 \\
\hline 5 & 736.45 & 368.73 & & & 718.44 & 359.72 & D & $1,410.77$ & 705.89 & $1,393.75$ & 697.38 & $1,392.76$ & 696.89 & 10 \\
\hline 6 & 864.51 & 432.76 & 847.48 & 424.24 & 846.50 & 423.75 & Q & $1,295.75$ & 648.38 & $1,278.72$ & 639.86 & $1,277.74$ & 639.37 & 9 \\
\hline 7 & 963.57 & 482.29 & 946.55 & 473.78 & 945.56 & 473.29 & $\mathrm{~V}$ & $1,167.69$ & 584.35 & $1,150.66$ & 575.84 & $1,149.68$ & 575.34 & 8 \\
\hline 8 & $1,110.64$ & 555.83 & $1,093.62$ & 547.31 & $1,092.63$ & 546.82 & $\mathrm{~F}$ & $1,068.62$ & 534.81 & $1,051.59$ & 526.30 & $1,050.61$ & 525.81 & 7 \\
\hline 9 & $1,257.71$ & 629.36 & $1,240.68$ & 620.85 & $1,239.70$ & 620.35 & $\mathrm{~F}$ & 921.55 & 461.28 & 904.53 & 452.77 & 903.54 & 452.27 & 6 \\
\hline 10 & $1,385.77$ & 693.39 & $1,368.74$ & 684.88 & $1,367.76$ & 684.38 & Q & 774.48 & 387.75 & 757.46 & 379.23 & 756.47 & 378.74 & 5 \\
\hline 11 & $1,514.81$ & 757.91 & $1,497.79$ & 749.40 & $1,496.80$ & 748.90 & $\mathrm{E}$ & 646.43 & 323.72 & 629.40 & 315.20 & 628.41 & 314.71 & 4 \\
\hline 12 & $1,627.90$ & 814.45 & $1,610.87$ & 805.94 & $1,609.89$ & 805.45 & $\mathrm{~L}$ & 517.38 & 259.20 & 500.36 & 250.68 & & & 3 \\
\hline 13 & $1,740.98$ & 870.99 & $1,723.95$ & 862.48 & $1,722.97$ & 861.99 & $\mathrm{~L}$ & 404.30 & 202.65 & 387.27 & 194.14 & & & 2 \\
\hline 14 & & & & & & & K & 291.21 & 146.11 & 274.19 & 137.60 & & & 1 \\
\hline
\end{tabular}

Query 24609 Hit 1

\section{MS/MS Fragmentation of SFEDIHHYR}

Found in sp|P01116|RASK_HUMAN, GTPase KRas OS=Homo sapiens GN=KRAS PE=1 SV=1

Match to Query 24609: 1346.656 from(449.8928,3+)

Title: 212: Scan $526(\mathrm{rt}=21.4652, \mathrm{f}=3, \mathrm{i}=73)$ [D:llab212 \membranelGrace $\backslash$ JoyceliTRAQ_53_2.raw]

Data File:Submitted from 20120508-1(merge) by Mascot Daemon on JOYCE-VAIO

Monoisotopic mass of neutral peptide Mr(calc): 1346.656

Variable modifications:

Ions Score: 48.96 Expect: 0.009 


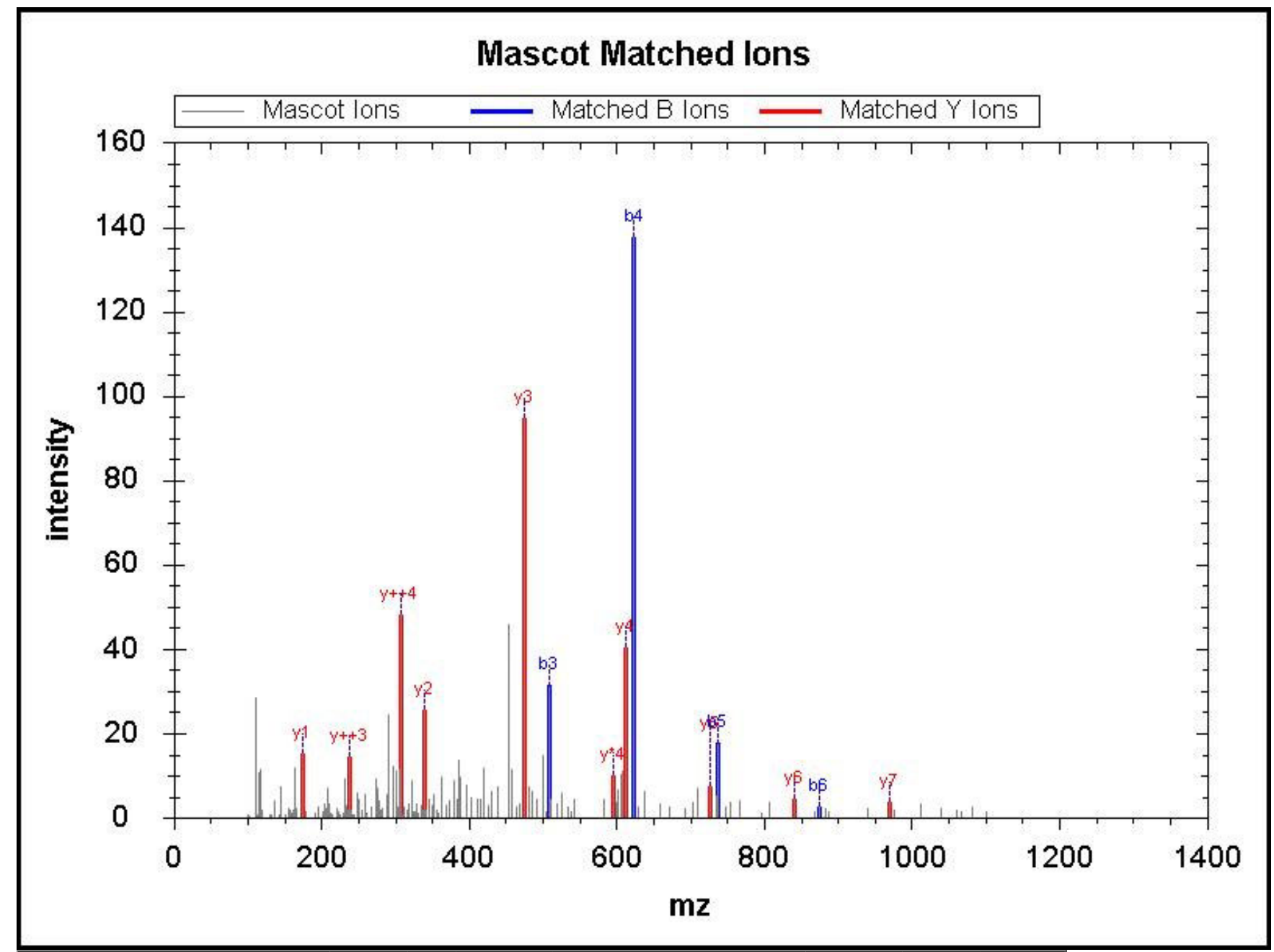

\begin{tabular}{|r|c|c|c|c|c|c|c|c|c|c|r|r|}
\hline \hline No & $\mathrm{b}$ & $\mathrm{b}++$ & $\mathrm{b} 0$ & $\mathrm{~b} 0++$ & $\mathrm{Seq}$ & $\mathrm{y}$ & $\mathrm{y}++$ & $\mathrm{y}^{*}$ & $\mathrm{y} *++$ & $\mathrm{y} 0$ & $\mathrm{y} 0++$ & $\mathrm{RevNo}$ \\
\hline 1 & 232.14 & 116.57 & 214.13 & 107.57 & $\mathrm{~S}$ & & & & & & & 9 \\
\hline 2 & 379.21 & 190.11 & 361.20 & 181.10 & $\mathrm{~F}$ & $1,116.52$ & 558.76 & $1,099.50$ & 550.25 & $1,098.51$ & 549.76 & 8 \\
\hline 3 & 508.25 & 254.63 & 490.24 & 245.62 & $\mathrm{E}$ & 969.45 & 485.23 & 952.43 & 476.72 & 951.44 & 476.23 & 7 \\
\hline 4 & 623.28 & 312.14 & 605.27 & 303.14 & $\mathrm{D}$ & 840.41 & 420.71 & 823.38 & 412.20 & 822.40 & 411.70 & 6 \\
\hline 5 & 736.36 & 368.69 & 718.35 & 359.68 & $\mathrm{I}$ & 725.38 & 363.20 & 708.36 & 354.68 & & & 5 \\
\hline 6 & 873.42 & 437.21 & 855.41 & 428.21 & $\mathrm{H}$ & 612.30 & 306.65 & 595.27 & 298.14 & & & 4 \\
\hline 7 & $1,010.48$ & 505.74 & 992.47 & 496.74 & $\mathrm{H}$ & 475.24 & 238.12 & 458.21 & 229.61 & & & 3 \\
\hline 8 & $1,173.54$ & 587.28 & $1,155.53$ & 578.27 & $\mathrm{Y}$ & 338.18 & 169.59 & 321.16 & 161.08 & & & 2 \\
\hline 9 & & & & & $\mathrm{R}$ & 175.12 & 88.06 & 158.09 & 79.55 & & & 1 \\
\hline
\end{tabular}

Query 22205 Hit 1

MS/MS Fragmentation of TIQAHEGFVR

Found in sp|Q9NV06|DCA13_HUMAN, DDB1- and CUL4-associated factor 13 OS=Homo sapiens GN=DCAF13 PE=1 SV=2 Match to Query 22205: 1300.62 from $(434.5472,3+)$

Title: 247: Sum of 2 scans in range $574(\mathrm{rt}=22.679, \mathrm{f}=4, \mathrm{i}=165)$ to $575(\mathrm{rt}=22.7044, \mathrm{f}=4, \mathrm{i}=166)$

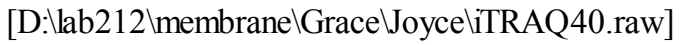

Data File:Submitted from 20120508-1(merge) by Mascot Daemon on JOYCE-VAIO

Monoisotopic mass of neutral peptide Mr(calc): 1300.62

Variable modifications:

Ions Score: 48.85 Expect: 0.007 


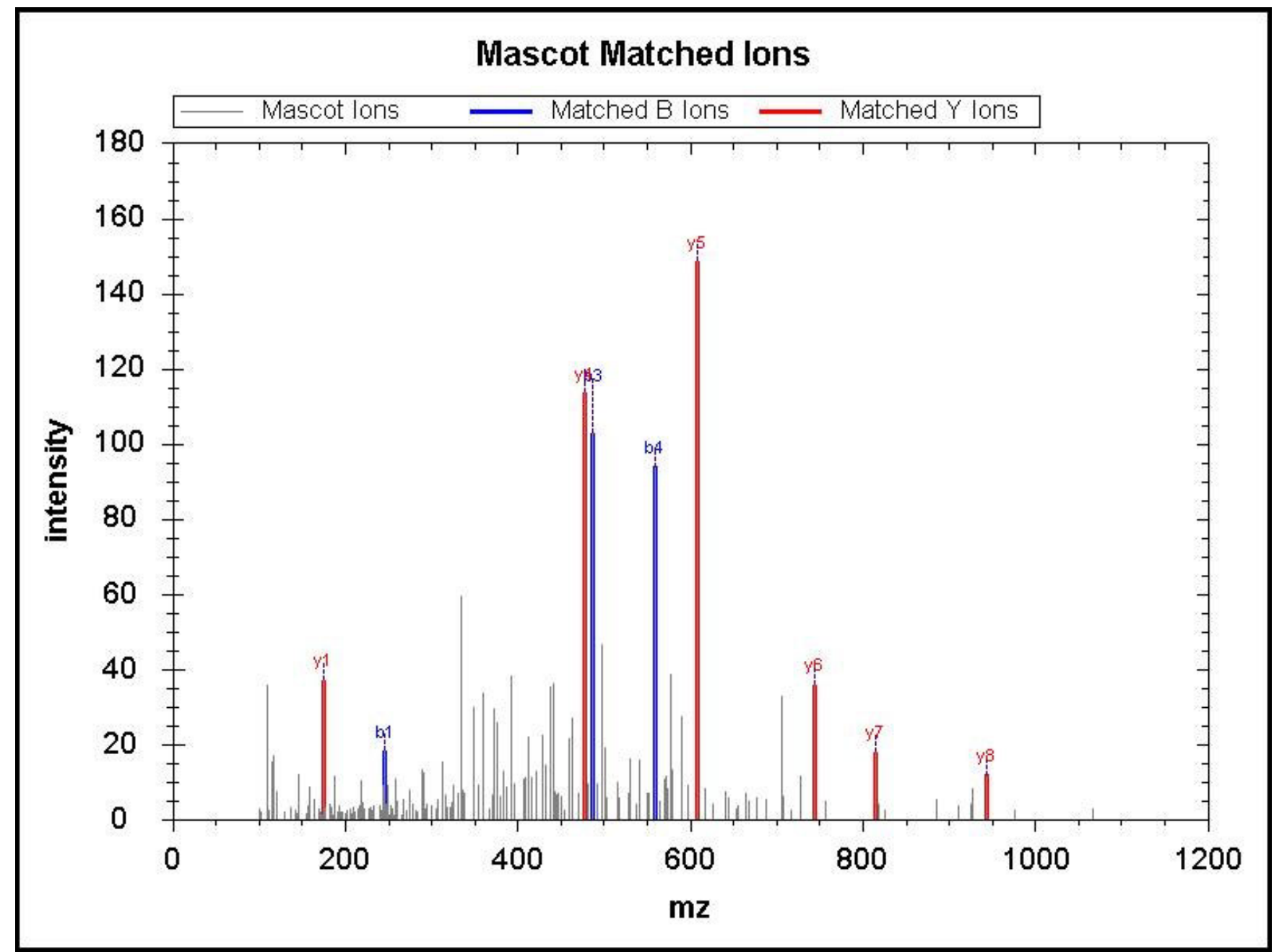

\begin{tabular}{|c|c|c|c|c|c|c|c|c|c|c|c|c|c|c|}
\hline No & $\mathrm{b}$ & $\mathrm{b}++$ & $b^{*}$ & $b^{*++}$ & b0 & $\mathrm{b} 0++$ & Seq & $\mathrm{y}$ & $\mathrm{y}++$ & $y^{*}$ & $\mathrm{y}^{*++}$ & y0 & $\mathrm{y} 0++$ & RevNo \\
\hline 1 & 246.16 & 123.58 & & & 228.15 & 114.58 & $\mathrm{~T}$ & & & & & & & 10 \\
\hline 2 & 359.24 & 180.12 & & & 341.23 & 171.12 & I & $1,056.56$ & 528.78 & $1,039.53$ & 520.27 & $1,038.55$ & 519.78 & 9 \\
\hline 3 & 487.30 & 244.15 & 470.27 & 235.64 & 469.29 & 235.15 & Q & 943.47 & 472.24 & 926.45 & 463.73 & 925.46 & 463.24 & 8 \\
\hline 4 & 558.34 & 279.67 & 541.31 & 271.16 & 540.33 & 270.67 & A & 815.42 & 408.21 & 798.39 & 399.70 & 797.41 & 399.21 & 7 \\
\hline 5 & 695.40 & 348.20 & 678.37 & 339.69 & 677.39 & 339.20 & $\mathrm{H}$ & 744.38 & 372.69 & 727.35 & 364.18 & 726.37 & 363.69 & 6 \\
\hline 6 & 824.44 & 412.72 & 807.41 & 404.21 & 806.43 & 403.72 & $\mathrm{E}$ & 607.32 & 304.16 & 590.29 & 295.65 & 589.31 & 295.16 & 5 \\
\hline 7 & 881.46 & 441.23 & 864.43 & 432.72 & 863.45 & 432.23 & G & 478.28 & 239.64 & 461.25 & 231.13 & & & 4 \\
\hline 8 & $1,028.53$ & 514.77 & $1,011.50$ & 506.25 & $1,010.52$ & 505.76 & $\mathrm{~F}$ & 421.26 & 211.13 & 404.23 & 202.62 & & & 3 \\
\hline 9 & $1,127.60$ & 564.30 & $1,110.57$ & 555.79 & $1,109.59$ & 555.30 & V & 274.19 & 137.60 & 257.16 & 129.08 & & & 2 \\
\hline 10 & & & & & & & $\mathrm{R}$ & 175.12 & 88.06 & 158.09 & 79.55 & & & 1 \\
\hline
\end{tabular}

Query 64839 Hit 1

\section{MS/MS Fragmentation of EKDGEFSVLQLVGMLR}

Found in sp|P29317|EPHA2_HUMAN, Ephrin type-A receptor 2 OS=Homo sapiens GN=EPHA2 PE=1 SV=2

Match to Query 64839: 2108.155from(703.7255,3+)

Title: 1133: Sum of 2 scans in range $2436(\mathrm{rt}=64.5664, \mathrm{f}=4, \mathrm{i}=757)$ to $2437(\mathrm{rt}=64.5918, \mathrm{f}=4, \mathrm{i}=758)$

[D:lab212 \membranelGrace JoyceliTRAQ_40_1.raw]

Data File:Submitted from 20120508-1(merge) by Mascot Daemon on JOYCE-VAIO

Monoisotopic mass of neutral peptide $\mathrm{Mr}$ (calc): 2108.155

Variable modifications:

K2 :iTRAQ4plex (K)

Ions Score: 48.79 Expect: 0.010 


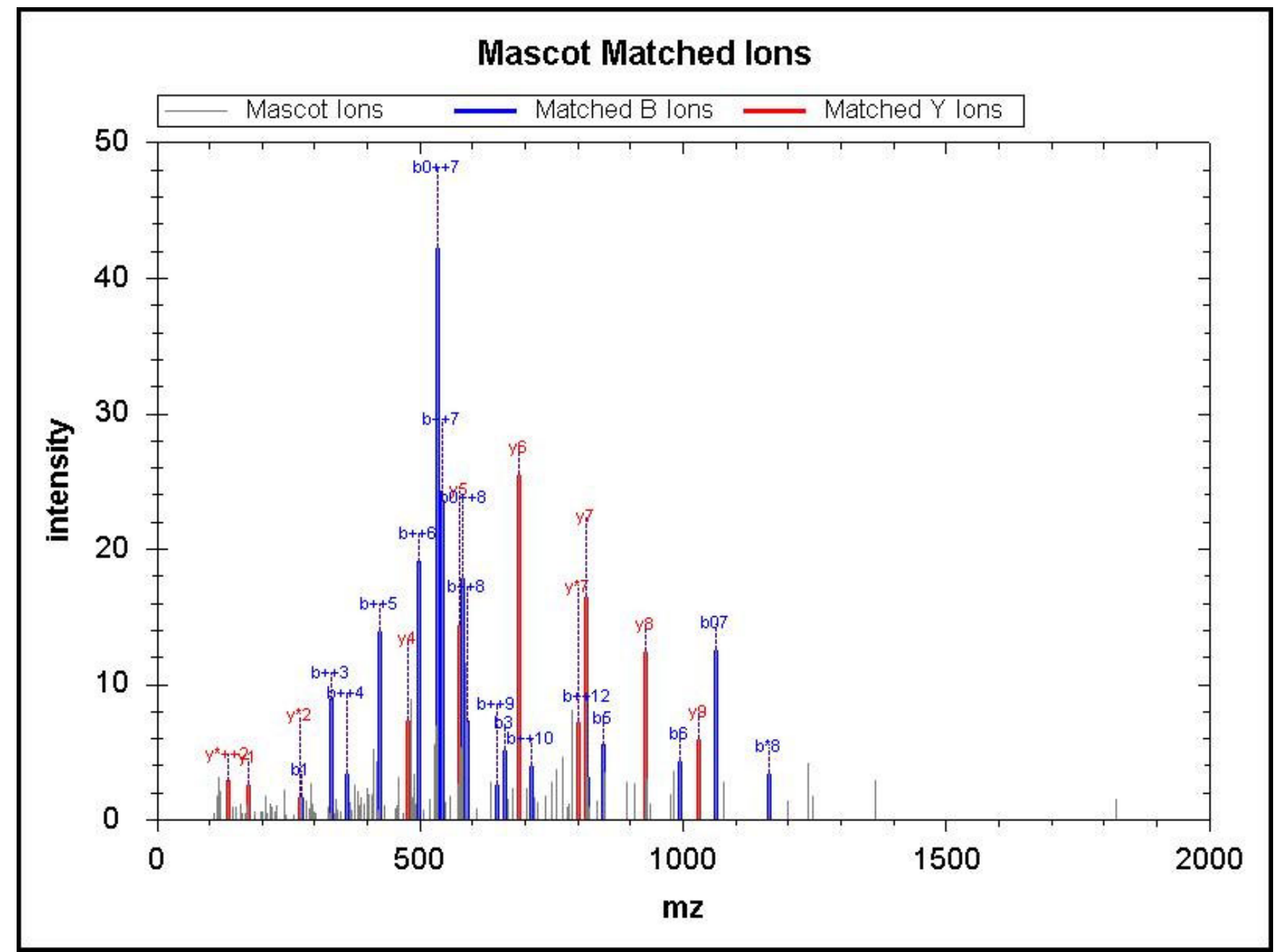

\begin{tabular}{|c|c|c|c|c|c|c|c|c|c|c|c|c|c|c|}
\hline No & $\mathrm{b}$ & $\mathrm{b}++$ & $b^{*}$ & $b^{*++}$ & b0 & $\mathrm{b} 0++$ & Seq & $\mathrm{y}$ & $\mathrm{y}++$ & $y^{*}$ & $y^{*++}$ & y0 & $\mathrm{y} 0++$ & RevNo \\
\hline 1 & 274.15 & 137.58 & & & 256.14 & 128.57 & $\mathrm{E}$ & & & & & & & 16 \\
\hline 2 & 546.35 & 273.68 & 529.32 & 265.16 & 528.34 & 264.67 & $\mathrm{~K}$ & $1,836.02$ & 918.51 & $1,818.99$ & 910.00 & $1,818.01$ & 909.51 & 15 \\
\hline 3 & 661.38 & 331.19 & 644.35 & 322.68 & 643.37 & 322.19 & $\mathrm{D}$ & $1,563.82$ & 782.41 & $1,546.79$ & 773.90 & $1,545.81$ & 773.41 & 14 \\
\hline 4 & 718.40 & 359.70 & 701.37 & 351.19 & 700.39 & 350.70 & G & $1,448.79$ & 724.90 & $1,431.77$ & 716.39 & $1,430.78$ & 715.89 & 13 \\
\hline 5 & 847.44 & 424.22 & 830.41 & 415.71 & 829.43 & 415.22 & $\mathrm{E}$ & $1,391.77$ & 696.39 & $1,374.74$ & 687.88 & $1,373.76$ & 687.38 & 12 \\
\hline 6 & 994.51 & 497.76 & 977.48 & 489.24 & 976.50 & 488.75 & $\mathrm{~F}$ & $1,262.73$ & 631.87 & $1,245.70$ & 623.35 & $1,244.72$ & 622.86 & 11 \\
\hline 7 & $1,081.54$ & 541.27 & $1,064.51$ & 532.76 & $1,063.53$ & 532.27 & $\mathrm{~S}$ & $1,115.66$ & 558.33 & $1,098.63$ & 549.82 & $1,097.65$ & 549.33 & 10 \\
\hline 8 & $1,180.61$ & 590.81 & $1,163.58$ & 582.29 & $1,162.60$ & 581.80 & V & $1,028.63$ & 514.82 & $1,011.60$ & 506.30 & & & 9 \\
\hline 9 & $1,293.69$ & 647.35 & $1,276.67$ & 638.84 & $1,275.68$ & 638.34 & $\mathrm{~L}$ & 929.56 & 465.28 & 912.53 & 456.77 & & & 8 \\
\hline 10 & $1,421.75$ & 711.38 & $1,404.72$ & 702.87 & $1,403.74$ & 702.37 & Q & 816.48 & 408.74 & 799.45 & 400.23 & & & 7 \\
\hline 11 & $1,534.84$ & 767.92 & $1,517.81$ & 759.41 & $1,516.82$ & 758.92 & $\mathrm{~L}$ & 688.42 & 344.71 & 671.39 & 336.20 & & & 6 \\
\hline 12 & $1,633.90$ & 817.46 & $1,616.88$ & 808.94 & $1,615.89$ & 808.45 & V & 575.33 & 288.17 & 558.31 & 279.66 & & & 5 \\
\hline 13 & $1,690.93$ & 845.97 & $1,673.90$ & 837.45 & $1,672.91$ & 836.96 & G & 476.26 & 238.64 & 459.24 & 230.12 & & & 4 \\
\hline 14 & $1,821.97$ & 911.49 & $1,804.94$ & 902.97 & $1,803.96$ & 902.48 & $\mathrm{M}$ & 419.24 & 210.13 & 402.22 & 201.61 & & & 3 \\
\hline 15 & $1,935.05$ & 968.03 & $1,918.02$ & 959.52 & $1,917.04$ & 959.02 & $\mathrm{~L}$ & 288.20 & 144.61 & 271.18 & 136.09 & & & 2 \\
\hline 16 & & & & & & & $\mathrm{R}$ & 175.12 & 88.06 & 158.09 & 79.55 & & & 1 \\
\hline
\end{tabular}

Query 24487 Hit 1

MS/MS Fragmentation of YGLLVGGAASHR

Found in sp|Q15274|NADC HUM AN, Nicotinate-nucleotide pyrophosphorylase [carboxylating] OS=Homo sapiens GN=QPRT PE=1 $\mathrm{SV}=3$

Match to Query 24487: 1343.744from(448.9219,3+)

Title: 383: Sum of 2 scans in range $909(\mathrm{rt}=29.9275, \mathrm{f}=4, \mathrm{i}=251)$ to $910(\mathrm{rt}=29.9529, \mathrm{f}=4, \mathrm{i}=252)$

[D:lab212ไmembranelGrace JoyceliTRAQ_37_2.raw]

Data File:Submitted from 20120508-1(merge) by Mascot Daemon on JOYCE-VAIO 


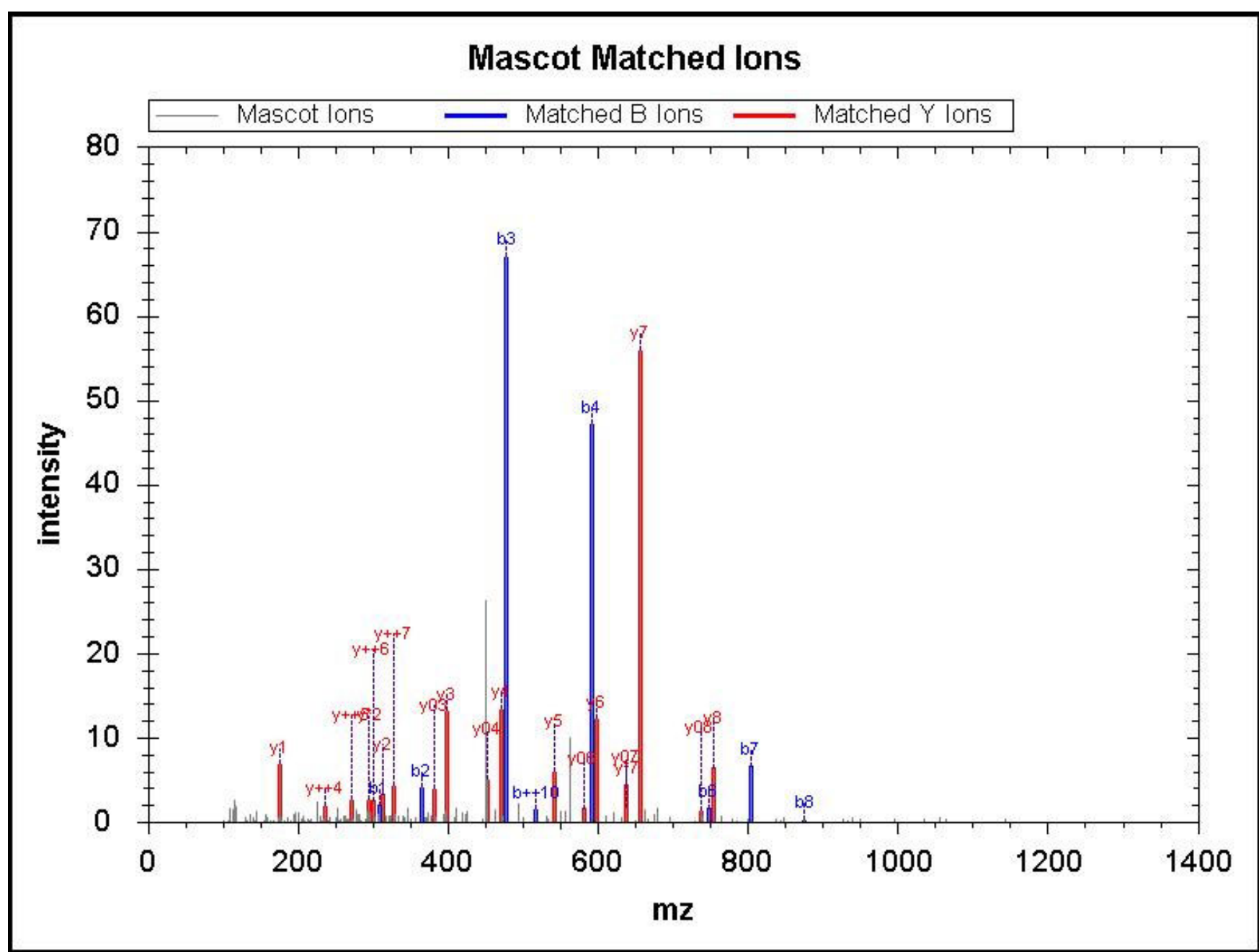

\begin{tabular}{|r|r|r|r|r|r|r|r|r|r|r|r|r|}
\hline No & $\mathrm{b}$ & $\mathrm{b}++$ & $\mathrm{b} 0$ & $\mathrm{~b} 0++$ & $\mathrm{Seq}$ & $\mathrm{y}$ & $\mathrm{y}++$ & $\mathrm{y} *$ & $\mathrm{y}^{*++}$ & $\mathrm{y} 0$ & $\mathrm{y} 0++$ & RevNo \\
\hline 1 & 308.17 & 154.59 & & & $\mathrm{Y}$ & & & & & & & 12 \\
\hline 2 & 365.19 & 183.10 & & & $\mathrm{G}$ & $1,037.59$ & 519.30 & $1,020.56$ & 510.78 & $1,019.57$ & 510.29 & 11 \\
\hline 3 & 478.28 & 239.64 & & & $\mathrm{~L}$ & 980.56 & 490.79 & 963.54 & 482.27 & 962.55 & 481.78 & 10 \\
\hline 4 & 591.36 & 296.18 & & & $\mathrm{~L}$ & 867.48 & 434.24 & 850.45 & 425.73 & 849.47 & 425.24 & 9 \\
\hline 5 & 690.43 & 345.72 & & & $\mathrm{~V}$ & 754.40 & 377.70 & 737.37 & 369.19 & 736.38 & 368.70 & 8 \\
\hline 6 & 747.45 & 374.23 & & & $\mathrm{G}$ & 655.33 & 328.17 & 638.30 & 319.65 & 637.32 & 319.16 & 7 \\
\hline 7 & 804.47 & 402.74 & & & $\mathrm{G}$ & 598.31 & 299.66 & 581.28 & 291.14 & 580.30 & 290.65 & 6 \\
\hline 8 & 875.51 & 438.26 & & & $\mathrm{~A}$ & 541.28 & 271.15 & 524.26 & 262.63 & 523.27 & 262.14 & 5 \\
\hline 9 & 946.55 & 473.78 & & & A & 470.25 & 235.63 & 453.22 & 227.11 & 452.24 & 226.62 & 4 \\
\hline 10 & $1,033.58$ & 517.29 & $1,015.57$ & 508.29 & S & 399.21 & 200.11 & 382.18 & 191.60 & 381.20 & 191.10 & 3 \\
\hline 11 & $1,170.64$ & 585.82 & $1,152.63$ & 576.82 & H & 312.18 & 156.59 & 295.15 & 148.08 & & & 2 \\
\hline 12 & & & & & R & 175.12 & 88.06 & 158.09 & 79.55 & & & 1 \\
\hline
\end{tabular}

Query 8386 Hit 1

MS/MS Fragmentation of LFVIGGK

Found in sp $|\mathrm{Q} 8 \mathrm{NAB} 2| \mathrm{KBTB} 3$ HUMAN, Kelch repeat and BTB domain-containing protein 3 OS=Homo sapiens GN=KBTBD3 PE=2 $\mathrm{SV}=2$

Match to Query 8386: 1020.653 from(511.3337,2+)

Title: 548: Sum of 2 scans in range 1226 ( $\mathrm{rt}=37.2906, \mathrm{f}=4, \mathrm{i}=369)$ to $1227(\mathrm{rt}=37.316, \mathrm{f}=4, \mathrm{i}=370)$

[D:llab212 \membranelGrace JoyceliTRAQ_33_2.raw]

Data File:Submitted from 20120508-1(merge) by Mascot Daemon on JOYCE-VAIO

Monoisotopic mass of neutral peptide $\mathrm{Mr}$ (calc): 1020.653

Variable modifications: 


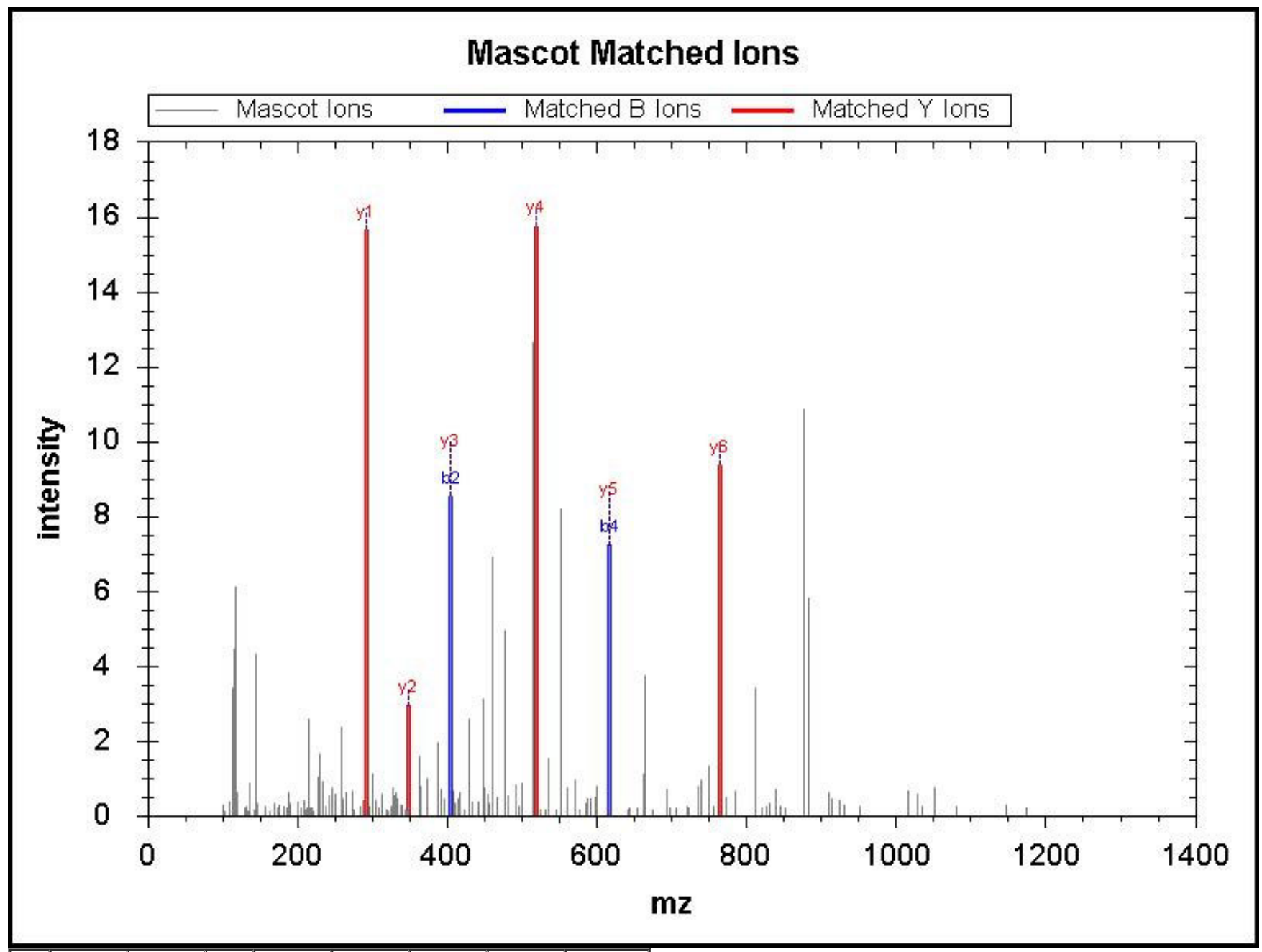

\begin{tabular}{|r|c|c|c|c|c|c|r|r|}
\hline \hline No & $\mathrm{b}$ & $\mathrm{b}++$ & Seq & $\mathrm{y}$ & $\mathrm{y}++$ & $\mathrm{y}^{*}$ & $\mathrm{y}^{*++}$ & RevNo \\
\hline 1 & 258.19 & 129.60 & $\mathrm{~L}$ & & & & & 7 \\
\hline 2 & 405.26 & 203.13 & $\mathrm{~F}$ & 764.48 & 382.74 & 747.45 & 374.23 & 6 \\
\hline 3 & 504.33 & 252.67 & $\mathrm{~V}$ & 617.41 & 309.21 & 600.38 & 300.70 & 5 \\
\hline 4 & 617.41 & 309.21 & $\mathrm{I}$ & 518.34 & 259.67 & 501.32 & 251.16 & 4 \\
\hline 5 & 674.44 & 337.72 & $\mathrm{G}$ & 405.26 & 203.13 & 388.23 & 194.62 & 3 \\
\hline 6 & 731.46 & 366.23 & $\mathrm{G}$ & 348.24 & 174.62 & 331.21 & 166.11 & 2 \\
\hline 7 & & & $\mathrm{~K}$ & 291.21 & 146.11 & 274.19 & 137.60 & 1 \\
\hline
\end{tabular}

Query 55320 Hit 1

MS/MS Fragmentation of EEEESESSTSDDKR

Found in sp|Q7Z6M4|MTER2_HUMAN, mTERF domain-containing protein 2 OS=Homo sapiens GN=MTERFD2 PE=1 SV=3 Match to Query 55320: 1914.856from(639.2927,3+)

Title: 4: Sum of 2 scans in range $101(\mathrm{rt}=11.8732, \mathrm{f}=2, \mathrm{i}=14)$ to $102(\mathrm{rt}=11.8986, \mathrm{f}=2, \mathrm{i}=15)$

[D:llab212 \membranelGrace JoyceliTRAQ_44_2.raw]

Data File:Submitted from 20120508-1(merge) by Mascot Daemon on JOYCE-VAIO

Monoisotopic mass of neutral peptide $\mathrm{Mr}$ (calc): 1914.856

Variable modifications:

K13 iTRAQ4plex (K)

Ions Score: 48.64 Expect: 0.007 


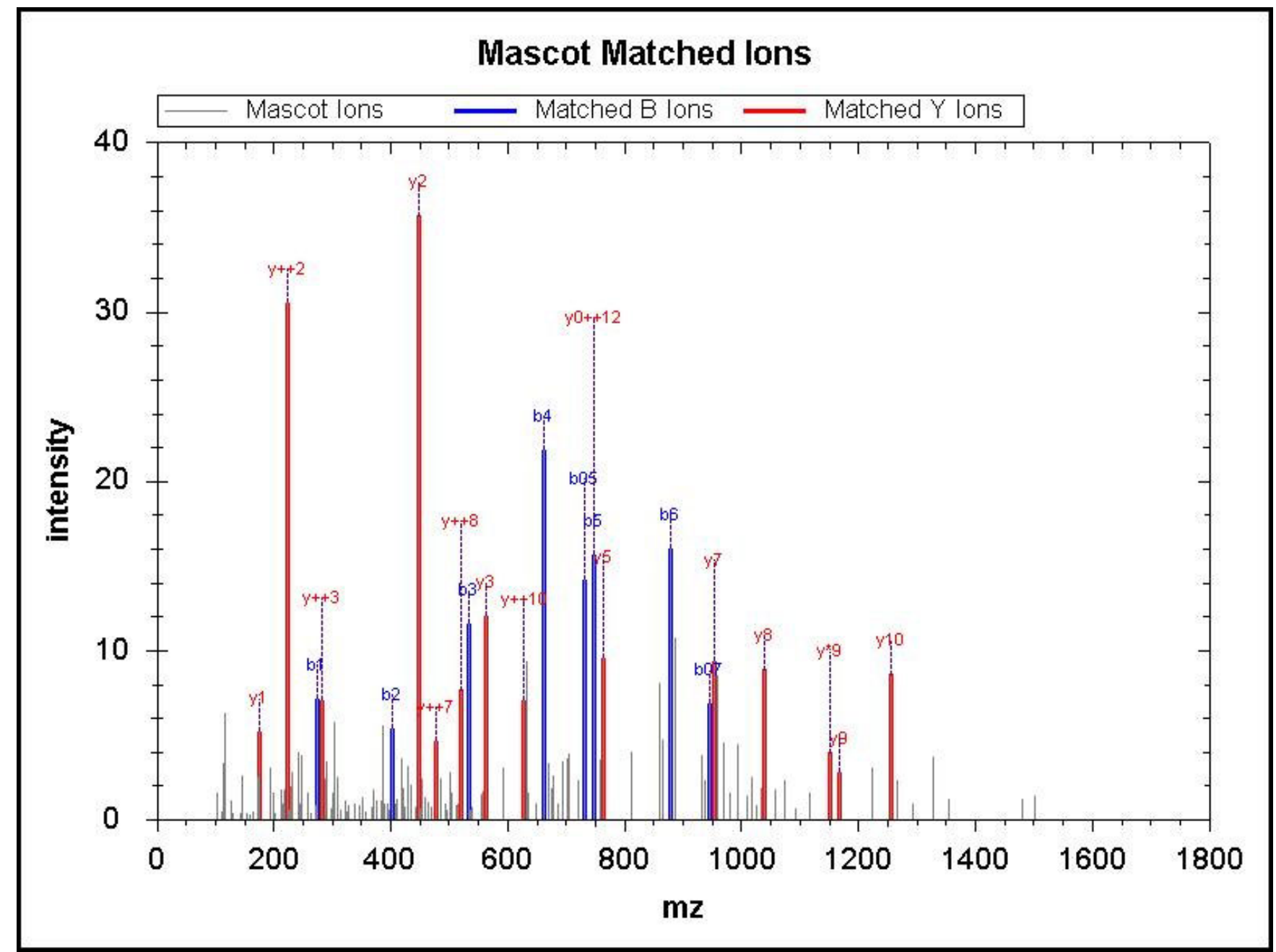

\begin{tabular}{|r|r|r|r|r|r|r|r|r|r|r|r|r|r|r|}
\hline \hline No & $\mathrm{b}$ & $\mathrm{b}++$ & $\mathrm{b}^{*}$ & $\mathrm{~b}{ }^{*++}$ & $\mathrm{b} 0$ & $\mathrm{~b} 0++$ & $\mathrm{Seq}$ & $\mathrm{y}$ & $\mathrm{y}++$ & $\mathrm{y}^{*}$ & $\mathrm{y}^{*++}$ & $\mathrm{y} 0$ & $\mathrm{y} 0++$ & $\mathrm{RevNo}$ \\
\hline 1 & 274.15 & 137.58 & & & 256.14 & 128.57 & $\mathrm{E}$ & & & & & & & 14 \\
\hline 2 & 403.19 & 202.10 & & & 385.18 & 193.10 & $\mathrm{E}$ & $1,642.72$ & 821.86 & $1,625.69$ & 813.35 & $1,624.71$ & 812.86 & 13 \\
\hline 3 & 532.24 & 266.62 & & & 514.23 & 257.62 & $\mathrm{E}$ & $1,513.67$ & 757.34 & $1,496.65$ & 748.83 & $1,495.66$ & 748.34 & 12 \\
\hline 4 & 661.28 & 331.14 & & & 643.27 & 322.14 & $\mathrm{E}$ & $1,384.63$ & 692.82 & $1,367.60$ & 684.31 & $1,366.62$ & 683.81 & 11 \\
\hline 5 & 748.31 & 374.66 & & & 730.30 & 365.65 & $\mathrm{~S}$ & $1,255.59$ & 628.30 & $1,238.56$ & 619.78 & $1,237.58$ & 619.29 & 10 \\
\hline 6 & 877.35 & 439.18 & & & 859.34 & 430.18 & $\mathrm{E}$ & $1,168.56$ & 584.78 & $1,151.53$ & 576.27 & $1,150.55$ & 575.78 & 9 \\
\hline 7 & 964.39 & 482.70 & & & 946.38 & 473.69 & $\mathrm{~S}$ & $1,039.51$ & 520.26 & $1,022.49$ & 511.75 & $1,021.50$ & 511.26 & 8 \\
\hline 8 & $1,051.42$ & 526.21 & & & $1,033.41$ & 517.21 & $\mathrm{~S}$ & 952.48 & 476.74 & 935.46 & 468.23 & 934.47 & 467.74 & 7 \\
\hline 9 & $1,152.47$ & 576.74 & & & $1,134.46$ & 567.73 & $\mathrm{~T}$ & 865.45 & 433.23 & 848.42 & 424.72 & 847.44 & 424.22 & 6 \\
\hline 10 & $1,239.50$ & 620.25 & & & $1,221.49$ & 611.25 & $\mathrm{~S}$ & 764.40 & 382.70 & 747.38 & 374.19 & 746.39 & 373.70 & 5 \\
\hline 11 & $1,354.53$ & 677.77 & & & $1,336.51$ & 668.76 & $\mathrm{D}$ & 677.37 & 339.19 & 660.34 & 330.68 & 659.36 & 330.18 & 4 \\
\hline 12 & $1,469.55$ & 735.28 & & & $1,451.54$ & 726.27 & $\mathrm{D}$ & 562.34 & 281.68 & 545.32 & 273.16 & 544.33 & 272.67 & 3 \\
\hline 13 & $1,741.75$ & 871.38 & $1,724.72$ & 862.86 & $1,723.74$ & 862.37 & $\mathrm{~K}$ & 447.32 & 224.16 & 430.29 & 215.65 & & & 2 \\
\hline 14 & & & & & & & $\mathrm{R}$ & 175.12 & 88.06 & 158.09 & 79.55 & & & 1 \\
\hline
\end{tabular}

Query 43782 Hit 1

MS/MS Fragmentation of NIVHNYSEAEIK

Found in sp|Q9Y6I3|EPN1_HUMAN, Epsin-1 OS=Homo sapiens GN=EPN1 PE=1 SV=2

Match to Query 43782: 1703.912from(568.9781,3+)

Title: 311 : Sum of 2 scans in range $713(\mathrm{rt}=25.8318, \mathrm{f}=4, \mathrm{f}=212)$ to $714(\mathrm{rt}=25.8572, \mathrm{f}=4, \mathrm{f}=213)$

[D:llab212\membranelGrace \oyceliTRAQ_42_2.raw]

Data File:Submitted from 20120508-1(merge) by Mascot Daemon on JOYCE-VAIO

Monoisotopic mass of neutral peptide $\mathrm{Mr}$ (calc): 1703.912

Variable modifications:

K12 :iTRAQ4plex (K)

Ions Score: 48.6 Expect: 0.012 


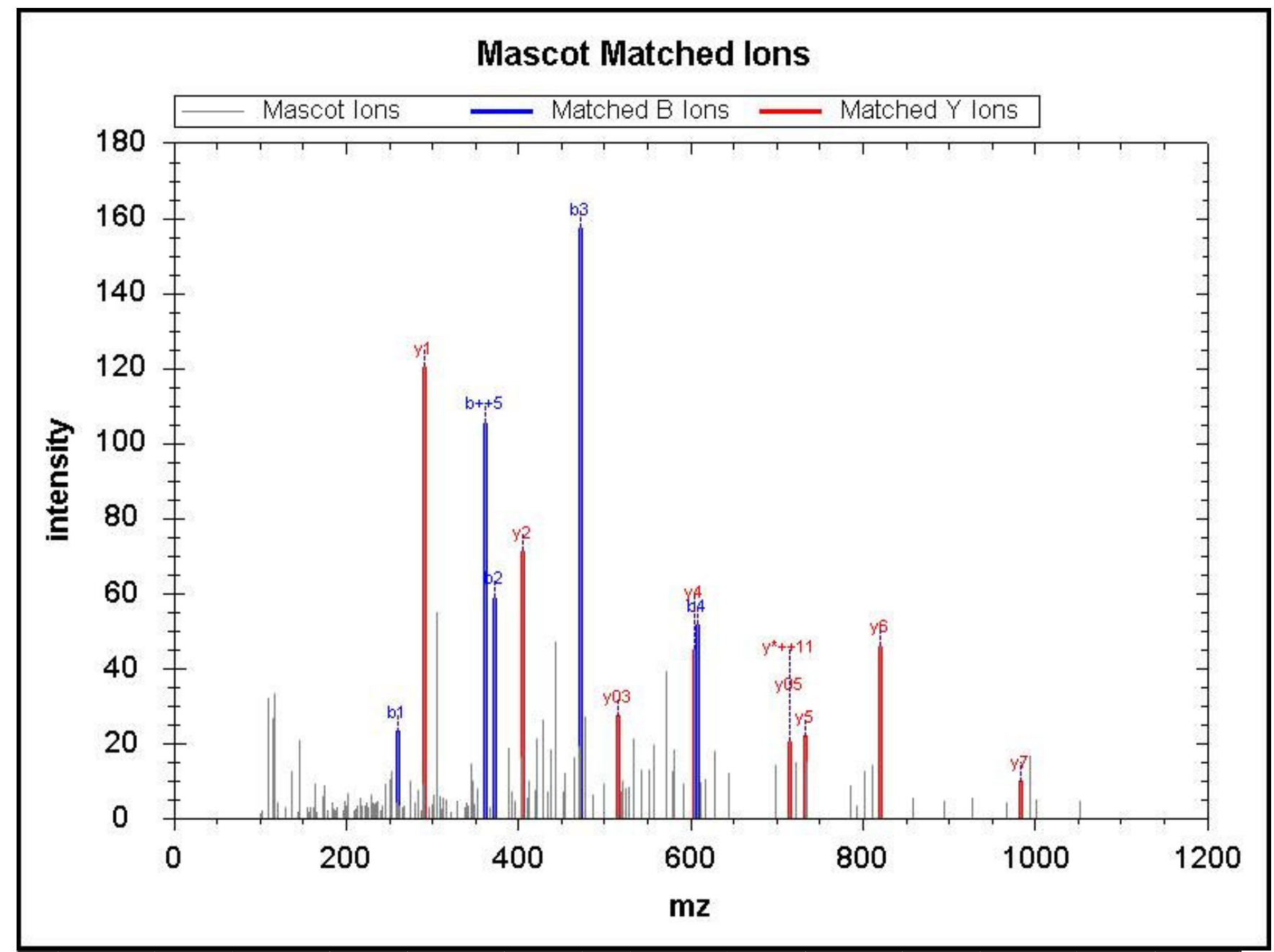

\begin{tabular}{|r|c|c|c|c|c|c|c|c|c|c|c|r|r|r|}
\hline $\mathrm{No}$ & $\mathrm{b}$ & $\mathrm{b}++$ & $\mathrm{b}^{*}$ & $\mathrm{~b}++$ & $\mathrm{b} 0$ & $\mathrm{~b} 0++$ & Seq & $\mathrm{y}$ & $\mathrm{y}++$ & $\mathrm{y}^{*}$ & $\mathrm{y}^{*++}$ & $\mathrm{y} 0$ & $\mathrm{y} 0++$ & $\mathrm{RevNo}$ \\
\hline 1 & 259.15 & 130.08 & 242.13 & 121.57 & & & $\mathrm{~N}$ & & & & & & & 12 \\
\hline 2 & 372.24 & 186.62 & 355.21 & 178.11 & & & $\mathrm{I}$ & $1,446.77$ & 723.89 & $1,429.74$ & 715.38 & $1,428.76$ & 714.88 & 11 \\
\hline 3 & 471.30 & 236.16 & 454.28 & 227.64 & & & $\mathrm{~V}$ & $1,333.69$ & 667.35 & $1,316.66$ & 658.83 & $1,315.68$ & 658.34 & 10 \\
\hline 4 & 608.36 & 304.69 & 591.34 & 296.17 & & & $\mathrm{H}$ & $1,234.62$ & 617.81 & $1,217.59$ & 609.30 & $1,216.61$ & 608.81 & 9 \\
\hline 5 & 722.41 & 361.71 & 705.38 & 353.19 & & & $\mathrm{~N}$ & $1,097.56$ & 549.28 & $1,080.53$ & 540.77 & $1,079.55$ & 540.28 & 8 \\
\hline 6 & 885.47 & 443.24 & 868.44 & 434.73 & & & $\mathrm{Y}$ & 983.52 & 492.26 & 966.49 & 483.75 & 965.51 & 483.26 & 7 \\
\hline 7 & 972.50 & 486.75 & 955.48 & 478.24 & 954.49 & 477.75 & $\mathrm{~S}$ & 820.45 & 410.73 & 803.43 & 402.22 & 802.44 & 401.72 & 6 \\
\hline 8 & $1,101.54$ & 551.28 & $1,084.52$ & 542.76 & $1,083.53$ & 542.27 & $\mathrm{E}$ & 733.42 & 367.21 & 716.39 & 358.70 & 715.41 & 358.21 & 5 \\
\hline 9 & $1,172.58$ & 586.79 & $1,155.56$ & 578.28 & $1,154.57$ & 577.79 & $\mathrm{~A}$ & 604.38 & 302.69 & 587.35 & 294.18 & 586.37 & 293.69 & 4 \\
\hline 10 & $1,301.62$ & 651.32 & $1,284.60$ & 642.80 & $1,283.61$ & 642.31 & $\mathrm{E}$ & 533.34 & 267.17 & 516.31 & 258.66 & 515.33 & 258.17 & 3 \\
\hline 11 & $1,414.71$ & 707.86 & $1,397.68$ & 699.34 & $1,396.70$ & 698.85 & $\mathrm{I}$ & 404.30 & 202.65 & 387.27 & 194.14 & & & 2 \\
\hline 12 & & & & & & & K & 291.21 & 146.11 & 274.19 & 137.60 & & & 1 \\
\hline
\end{tabular}

Query 90891 Hit 1

MS/MS Fragmentation of EIYLEVIHNLPDFELLSANTLEDR

Found in $\mathrm{sp}|\mathrm{Q} 8 \mathrm{IXB} 1| \mathrm{DJC} 10$ HUM AN, DnaJ homolog subfamily C member $10 \mathrm{OS}=$ Homo sapiens GN=DNAJC10 PE=1 SV=2 Match to Query 90891: 2986.542from(996.5215,3+)

Title: 971: Sum of 2 scans in range 2777 ( $\mathrm{rt}=68.6385, \mathrm{f}=4, \mathrm{i}=623)$ to 2778 ( $\mathrm{rt}=68.6638, \mathrm{f}=4, \mathrm{i}=624)$

[D:llab212\membranelGrace \JoyceliTRAQ_29_2.raw]

Data File:Submitted from 20120508-1(merge) by Mascot Daemon on JOYCE-VAIO

Monoisotopic mass of neutral peptide Mr(calc): 2986.542

Variable modifications:

Ions Score: 48.56 Expect: 0.012 
Mascot Matched lons

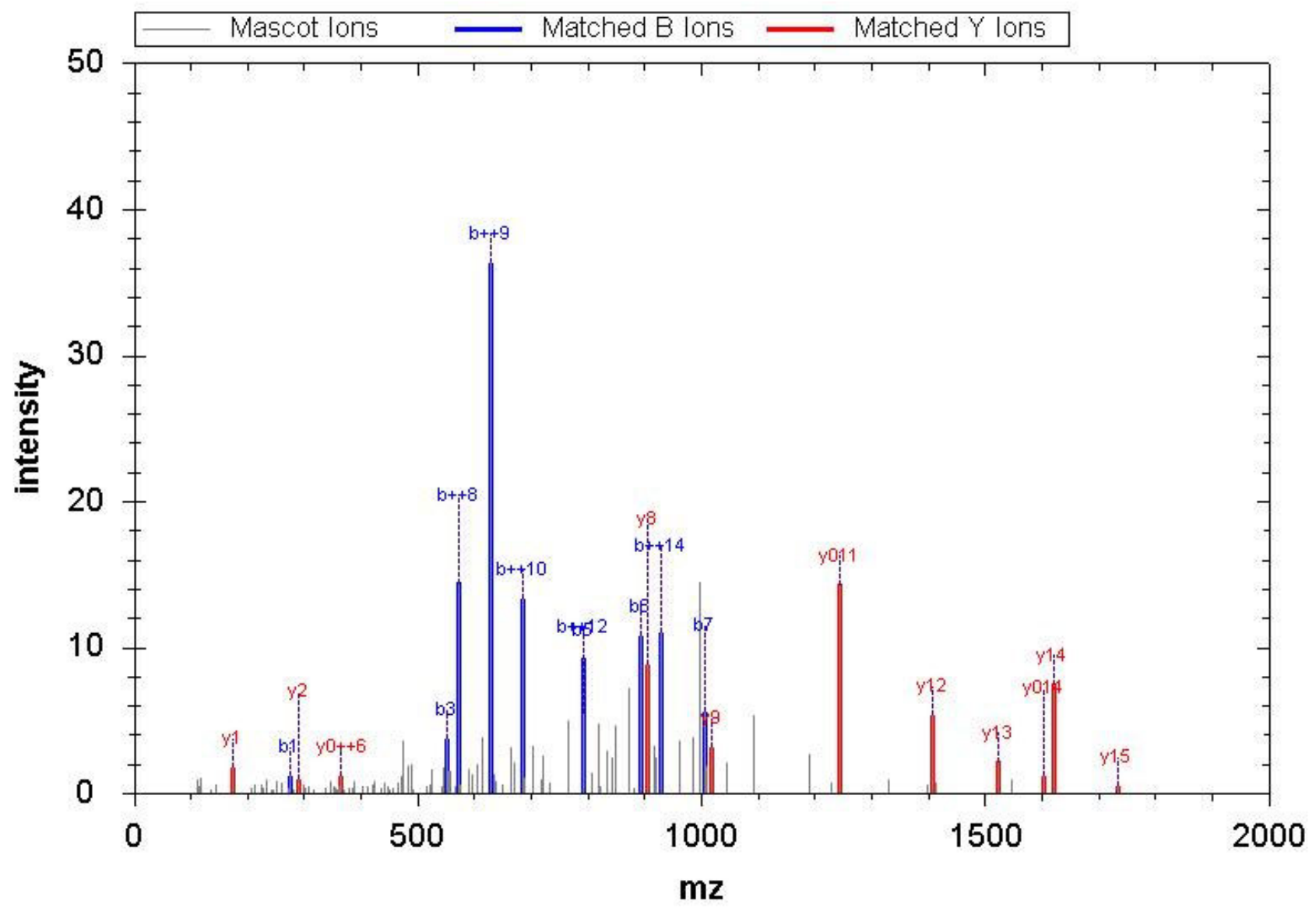

\begin{tabular}{|c|c|c|c|c|c|c|c|c|c|c|c|c|c|c|}
\hline No & $\mathrm{b}$ & $\mathrm{b}++$ & $b^{*}$ & $b^{*++}$ & b0 & $\mathrm{b} 0++$ & Seq & $\mathrm{y}$ & $\mathrm{y}++$ & $y^{*}$ & $y^{*++}$ & y0 & $\mathrm{y} 0++$ & RevNo \\
\hline 1 & 274.15 & 137.58 & & & 256.14 & 128.57 & $\mathrm{E}$ & & & & & & & 24 \\
\hline 2 & 387.24 & 194.12 & & & 369.23 & 185.12 & I & $2,714.40$ & $1,357.71$ & $2,697.38$ & $1,349.19$ & $2,696.39$ & $1,348.70$ & 23 \\
\hline 3 & 550.30 & 275.65 & & & 532.29 & 266.65 & $\mathrm{Y}$ & $2,601.32$ & $1,301.16$ & $2,584.29$ & $1,292.65$ & $2,583.31$ & $1,292.16$ & 22 \\
\hline 4 & 663.38 & 332.20 & & & 645.37 & 323.19 & $\mathrm{~L}$ & $2,438.26$ & $1,219.63$ & $2,421.23$ & $1,211.12$ & $2,420.25$ & $1,210.63$ & 21 \\
\hline 5 & 792.43 & 396.72 & & & 774.42 & 387.71 & $\mathrm{E}$ & $2,325.17$ & $1,163.09$ & $2,308.15$ & $1,154.58$ & $2,307.16$ & $1,154.08$ & 20 \\
\hline 6 & 891.49 & 446.25 & & & 873.48 & 437.25 & V & $2,196.13$ & $1,098.57$ & $2,179.10$ & $1,090.06$ & $2,178.12$ & $1,089.56$ & 19 \\
\hline 7 & $1,004.58$ & 502.79 & & & 986.57 & 493.79 & I & $2,097.06$ & $1,049.03$ & $2,080.03$ & $1,040.52$ & $2,079.05$ & $1,040.03$ & 18 \\
\hline 8 & $1,141.64$ & 571.32 & & & $1,123.63$ & 562.32 & $\mathrm{H}$ & $1,983.98$ & 992.49 & 1,9 & 983.98 & 5.97 & 983.49 & 17 \\
\hline 9 & $1,255.68$ & 628.34 & 1,23 & 3 & $1,237.67$ & 619.34 & $\mathrm{~N}$ & $1,846.92$ & 923.96 & $1,829.89$ & 915.45 & $1,828.91$ & 914.96 & 16 \\
\hline 10 & $1,368.76$ & 684.89 & $1,351.74$ & 676.37 & $1,350.75$ & 675.88 & $\mathrm{~L}$ & $1,732.88$ & 866.94 & $1,715.85$ & 858.43 & $1,714.86$ & 857.94 & 15 \\
\hline 11 & $1,465.82$ & 733.41 & $1,448.79$ & 724.90 & $1,447.81$ & 724.41 & $\mathrm{P}$ & $1,619.79$ & 810.40 & $1,602.76$ & 801.89 & $1,601.78$ & 801.39 & 14 \\
\hline 12 & $1,580.84$ & 790.93 & $1,563.82$ & 41 & $1,562.83$ & 781.92 & $\mathrm{D}$ & $1,522.74$ & 761.87 & $1,505.71$ & 753.36 & $1,504.73$ & 752.87 & 13 \\
\hline 13 & $1,727.91$ & 864.46 & $1,710.89$ & 855.95 & $1,709.90$ & 855.45 & $\mathrm{~F}$ & $1,407.71$ & 704.36 & $1,390.68$ & 695.85 & $1,389.70$ & 695.35 & 12 \\
\hline 14 & $1,856.96$ & 928.98 & $1,839.93$ & 920.47 & $1,838.94$ & 919.98 & $\mathrm{E}$ & $1,260.64$ & 630.83 & $1,243.62$ & 622.31 & $1,242.63$ & 621.82 & 11 \\
\hline 15 & $1,970.04$ & 985.52 & $1,953.01$ & 977.01 & $1,952.03$ & 976.52 & $\mathrm{~L}$ & $1,131.60$ & 566.30 & $1,114.57$ & 557.79 & $1,113.59$ & 557.30 & 10 \\
\hline 16 & $2,083.12$ & $1,042.07$ & $2,066.10$ & $1,033.55$ & $2,065.11$ & $1,033.06$ & $\mathrm{~L}$ & $1,018.52$ & 509.76 & $1,001.49$ & 501.25 & $1,000.51$ & 500.76 & 9 \\
\hline 17 & $2,170.16$ & $1,085.58$ & $2,153.13$ & $1,077.07$ & $2,152.14$ & $1,076.58$ & $\mathrm{~S}$ & 905.43 & 453.22 & 888.41 & .71 & 887.42 & 444.21 & 8 \\
\hline 18 & $2,241.19$ & $1,121.10$ & $2,224.17$ & $1,112.59$ & $2,223.18$ & $1,112.09$ & A & 818.40 & 409.70 & 801.37 & 401.19 & 800.39 & 400.70 & 7 \\
\hline 19 & $2,355.24$ & $1,178.12$ & $2,338.21$ & $1,169.61$ & $2,337.22$ & $1,169.12$ & $\mathrm{~N}$ & 747.36 & 374.19 & 730.34 & 365.67 & 729.35 & 365.18 & 6 \\
\hline 20 & $2,456.28$ & $1,228.65$ & $2,439.26$ & $1,220.13$ & $2,438.27$ & $1,219.64$ & $\mathrm{~T}$ & 633.32 & 317.16 & 616.29 & 308.65 & 615.31 & 308.16 & 5 \\
\hline 21 & $2,569.37$ & $1,285.19$ & $2,552.34$ & $1,276.67$ & $2,551.36$ & $1,276.18$ & $\mathrm{~L}$ & 532.27 & 266.64 & 515.25 & 258.13 & 514.26 & 257.63 & 4 \\
\hline 22 & $2,698.41$ & $1,349.71$ & $2,681.38$ & $1,341.20$ & $2,680.40$ & $1,340.70$ & $\mathrm{E}$ & 419.19 & 210.10 & 402.16 & 201.58 & 401.18 & 201.09 & 3 \\
\hline 23 & $2,813.44$ & $1,407.22$ & $2,796.41$ & $1,398.71$ & $2,795.43$ & $1,398.22$ & D & 290.15 & 145.58 & 273.12 & 137.06 & 272.14 & 136.57 & 2 \\
\hline
\end{tabular}


Query 89963 Hit 1

MS/MS Fragmentation of LGDYHFYGFGTDVDYETAFIHYR

Found in sp|Q9UBV2|SE1L1_HUM AN, Protein sel-1 homolog 1 OS=Homo sapiens GN=SEL1L PE=1 SV=3

Match to Query 89963: 2929.359from(733.3471,4+)

Title: 935: Scan 2023 ( $\mathrm{rt}=55.2646, \mathrm{f}=3, \mathrm{i}=320$ ) [D:llab212 $\backslash$ membranelGracelJoyceliTRAQ_45_1.raw]

Data File:Submitted from 20120508-1(merge) by Mascot Daemon on JOYCE-VAIO

Monoisotopic mass of neutral peptide $\mathrm{Mr}$ (calc): 2929.359

Variable modifications:

Ions Score: 48.52 Expect: 0.010

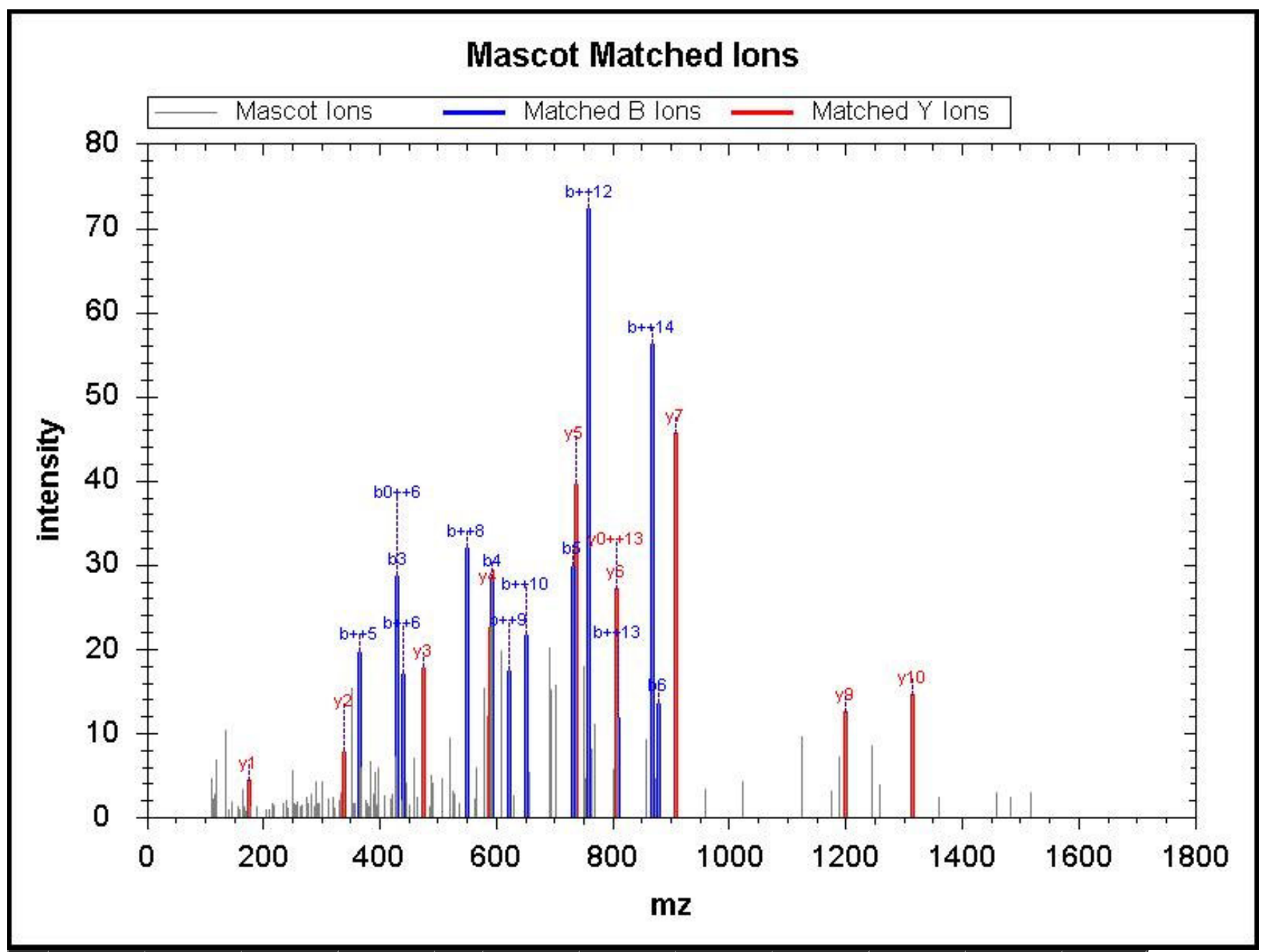

\begin{tabular}{|r|c|c|c|c|c|c|c|c|c|c|r|r|}
\hline \hline $\mathrm{No}$ & $\mathrm{b}$ & $\mathrm{b}++$ & $\mathrm{b} 0$ & $\mathrm{~b} 0++$ & $\mathrm{Seq}$ & $\mathrm{y}$ & $\mathrm{y}++$ & $\mathrm{y}^{*}$ & $\mathrm{y}^{*++}$ & $\mathrm{y} 0$ & $\mathrm{y} 0++$ & $\mathrm{RevNo}$ \\
\hline 1 & 258.19 & 129.60 & & & $\mathrm{~L}$ & & & & & & & 23 \\
\hline 2 & 315.21 & 158.11 & & & $\mathrm{G}$ & $2,673.17$ & $1,337.09$ & $2,656.14$ & $1,328.57$ & $2,655.16$ & $1,328.08$ & 22 \\
\hline 3 & 430.24 & 215.62 & 412.23 & 206.62 & $\mathrm{D}$ & $2,616.15$ & $1,308.58$ & $2,599.12$ & $1,300.06$ & $2,598.14$ & $1,299.57$ & 21 \\
\hline 4 & 593.31 & 297.16 & 575.29 & 288.15 & $\mathrm{Y}$ & $2,501.12$ & $1,251.06$ & $2,484.09$ & $1,242.55$ & $2,483.11$ & $1,242.06$ & 20 \\
\hline 5 & 730.36 & 365.69 & 712.35 & 356.68 & $\mathrm{H}$ & $2,338.06$ & $1,169.53$ & $2,321.03$ & $1,161.02$ & $2,320.05$ & $1,160.53$ & 19 \\
\hline 6 & 877.43 & 439.22 & 859.42 & 430.21 & $\mathrm{~F}$ & $2,201.00$ & $1,101.00$ & $2,183.97$ & $1,092.49$ & $2,182.99$ & $1,092.00$ & 18 \\
\hline 7 & $1,040.50$ & 520.75 & $1,022.49$ & 511.75 & $\mathrm{Y}$ & $2,053.93$ & $1,027.47$ & $2,036.90$ & $1,018.96$ & $2,035.92$ & $1,018.46$ & 17 \\
\hline 8 & $1,097.52$ & 549.26 & $1,079.51$ & 540.26 & $\mathrm{G}$ & $1,890.87$ & 945.94 & $1,873.84$ & 937.42 & $1,872.86$ & 936.93 & 16 \\
\hline 9 & $1,244.59$ & 622.80 & $1,226.58$ & 613.79 & $\mathrm{~F}$ & $1,833.84$ & 917.43 & $1,816.82$ & 908.91 & $1,815.83$ & 908.42 & 15 \\
\hline 10 & $1,301.61$ & 651.31 & $1,283.60$ & 642.30 & $\mathrm{G}$ & $1,686.78$ & 843.89 & $1,669.75$ & 835.38 & $1,668.77$ & 834.89 & 14 \\
\hline 11 & $1,402.65$ & 701.83 & $1,384.64$ & 692.83 & $\mathrm{~T}$ & $1,629.75$ & 815.38 & $1,612.73$ & 806.87 & $1,611.74$ & 806.38 & 13 \\
\hline 12 & $1,517.68$ & 759.34 & $1,499.67$ & 750.34 & $\mathrm{D}$ & $1,528.71$ & 764.86 & $1,511.68$ & 756.34 & $1,510.70$ & 755.85 & 12 \\
\hline 13 & $1,616.75$ & 808.88 & $1,598.74$ & 799.87 & $\mathrm{~V}$ & $1,413.68$ & 707.34 & $1,396.65$ & 698.83 & $1,395.67$ & 698.34 & 11 \\
\hline & & & & & & & & & & & &
\end{tabular}




\begin{tabular}{|r|r|r|r|r|r|r|r|r|r|r|r|r|r|}
14 & $1,731.78$ & 866.39 & $1,713.77$ & 857.39 & $\mathrm{D}$ & $1,314.61$ & 657.81 & $1,297.58$ & 649.30 & $1,296.60$ & 648.80 & 10 \\
\hline \hline 15 & $1,894.84$ & 947.92 & $1,876.83$ & 938.92 & $\mathrm{Y}$ & $1,199.58$ & 600.30 & $1,182.56$ & 591.78 & $1,181.57$ & 591.29 & 9 \\
\hline 16 & $2,023.88$ & $1,012.45$ & $2,005.87$ & $1,003.44$ & $\mathrm{E}$ & $1,036.52$ & 518.76 & $1,019.49$ & 510.25 & $1,018.51$ & 509.76 & 8 \\
\hline 17 & $2,124.93$ & $1,062.97$ & $2,106.92$ & $1,053.96$ & $\mathrm{~T}$ & 907.48 & 454.24 & 890.45 & 445.73 & 889.47 & 445.24 & 7 \\
\hline 18 & $2,195.97$ & $1,098.49$ & $2,177.96$ & $1,089.48$ & $\mathrm{~A}$ & 806.43 & 403.72 & 789.40 & 395.21 & & & 6 \\
\hline 19 & $2,343.04$ & $1,172.02$ & $2,325.03$ & $1,163.02$ & $\mathrm{~F}$ & 735.39 & 368.20 & 718.37 & 359.69 & & & 5 \\
\hline 20 & $2,456.12$ & $1,228.56$ & $2,438.11$ & $1,219.56$ & $\mathrm{I}$ & 588.33 & 294.67 & 571.30 & 286.15 & & & 4 \\
\hline 21 & $2,593.18$ & $1,297.09$ & $2,575.17$ & $1,288.09$ & $\mathrm{H}$ & 475.24 & 238.12 & 458.21 & 229.61 & & & 3 \\
\hline 22 & $2,756.24$ & $1,378.62$ & $2,738.23$ & $1,369.62$ & $\mathrm{Y}$ & 338.18 & 169.59 & 321.16 & 161.08 & & & 2 \\
\hline 23 & & & & & $\mathrm{R}$ & 175.12 & 88.06 & 158.09 & 79.55 & & & 1 \\
\hline
\end{tabular}

Query 81278 Hit 1

MS/MS Fragmentation of EVIQELEELGVGIGVVHAGYER

Found in sp|Q9Y2G8|DJC16_HUMAN, DnaJ homolog subfamily C member 16 OS=Homo sapiens GN=DNAJC16 PE=2 SV=3 Match to Query 81278: 2539.325from(847.4491,3+)

Title: 1088: Scan 2409 ( $\mathrm{rt}=63.581, \mathrm{f}=3, \mathrm{i}=373)$ [D:llab212 \membranelGrace JoyceliTRAQ_31_1.raw]

Data File:Submitted from 20120508-1(merge) by Mascot Daemon on JOYCE-VAIO

Monoisotopic mass of neutral peptide Mr(calc): 2539.325

Variable modifications:

Ions Score: 48.4 Expect: 0.013

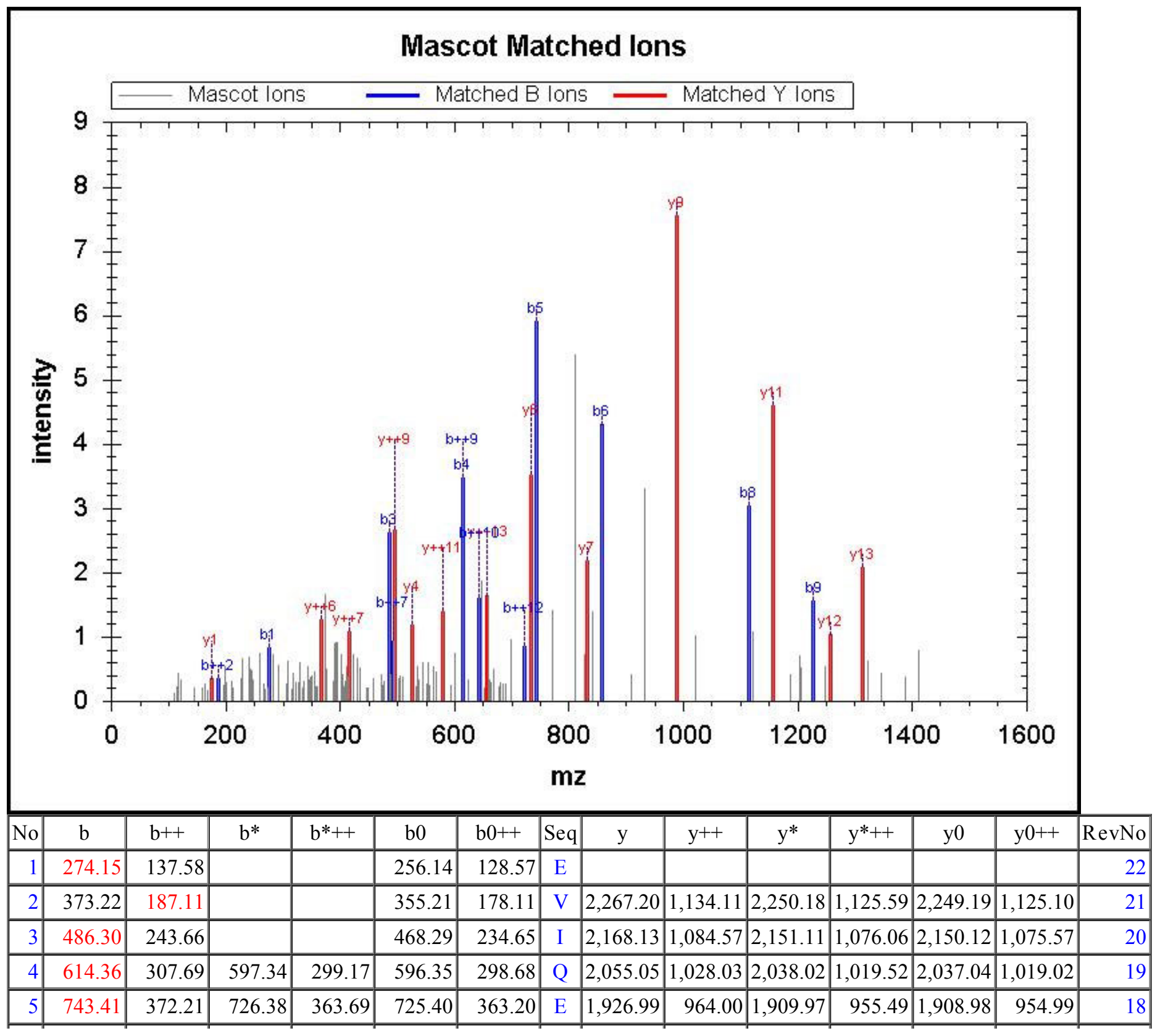




\begin{tabular}{|c|c|c|c|c|c|c|c|c|c|c|c|c|c|c|}
\hline 6 & 856.49 & 428.75 & 839.46 & 420.24 & 838.48 & 419.74 & $\mathrm{~L}$ & $1,797.95$ & 899.48 & $1,780.92$ & 890.97 & $|1,779.94|$ & 890.47 & 17 \\
\hline 7 & 985.53 & 493.27 & 968.51 & 48476 & 967.52 & 484.26 & $E$ & $1,684.87$ & 842.94 & $1,667.84$ & 34.42 & $1,666.85$ & 833.93 & 16 \\
\hline 8 & $1,114.57$ & 557.79 & $1,09^{\prime}$ & 549.28 & 1,09 & & $\mathrm{E}$ & & 42 & & 769.90 & & & \\
\hline 9 & $1,227.66$ & 614.33 & $1,210.63$ & 605.82 & 1,209 & & $\mathrm{~L}$ & & & 75 & 5.38 & 77 & & \\
\hline 10 & $1,284.68$ & 642.84 & $1,267.65$ & 634.33 & $1,266.67$ & 633.84 & $\mathrm{G}$ & $1,313.70$ & 657.35 & $1,296.67$ & 648.84 & $1,295.69$ & 648.35 & 13 \\
\hline 11 & $1,383.75$ & 692.38 & $1,366.72$ & 683.86 & $1,365.74$ & 683.37 & $\mathrm{~V}$ & 67 & .84 & .65 & .33 & .66 & .84 & 12 \\
\hline 12 & 7 & 7 & 1 & 8 & 1 & 711.88 & $G$ & $1,157.61$ & 1 & 1 & 9 & 1 & 570.30 & 11 \\
\hline 13 & 1 & 777.43 & 3 & 768.92 & 1, & 3 & I & $1,100.58$ & 80 & 1 , & 28 & 1, & 541.79 & 0 \\
\hline 14 & 610.88 & 805.94 & $1,593.85$ & 797.43 & $1,592.87$ & 796.94 & $\mathrm{G}$ & 7.50 & 494.25 & 970.47 & 485.74 & 969.49 & 485.25 & ) \\
\hline 15 & 1 & 855.48 & 1,69 & 846.96 & 1.93 & 846.47 & V & +0 & 46 & + & 457.23 & .47 & 5.74 & 8 \\
\hline 16 & $1,809.01$ & 05.01 & $1,791.99$ & 896.50 & $1,791.00$ & 896.00 & $\mathrm{~V}$ & 831.41 & 416.21 & 4.38 & 407.70 & 813.40 & 407.20 & 7 \\
\hline 17 & 946.07 & 973.54 & $1,929.05$ & 965.03 & $1,928.06$ & 964.53 & $\mathrm{H}$ & 732.34 & 366.67 & 715.32 & 358.16 & 714.33 & 357.67 & 6 \\
\hline 18 & $2,017.11$ & $1,009.06$ & 8 & 54 & 10 & 05 & A & 8 & 29 & 6 & 289.63 & .27 & 14 & P \\
\hline 19 & $2,074.13$ & $1,037.57$ & $2,057.10$ & $1,029.06$ & $2,056.12$ & $1,028.56$ & $\mathrm{G}$ & 524.25 & 262.63 & 507.22 & 254.11 & 506.24 & 253.62 & 4 \\
\hline 20 & $2,237.19$ & $1,119.10$ & $2,220.17$ & $1,110.59$ & $2,219.18$ & $1,110.10$ & $\mathrm{Y}$ & 467.22 & 234.12 & 450.20 & 225.60 & 449.21 & 225.11 & 3 \\
\hline 21 & $2,366.24$ & $1,183.62$ & $2,349.21$ & $1,175.11$ & $2,348.23$ & $1,174.62$ & $\mathrm{E}$ & 304.16 & 152.58 & 287.13 & 144.07 & 286.15 & 143.58 & 2 \\
\hline 22 & & & & & & & $\mathrm{R}$ & 175.12 & 88.06 & 158.09 & 79.55 & & & 1 \\
\hline
\end{tabular}

\section{Query 56433 Hit 1}

MS/MS Fragmentation of VAHSDKPGSTSTASFR

Found in sp|Q9P0L0|VAPA_HUMAN, Vesicle-associated membrane protein-associated protein A OS=Homo sapiens GN=VAPA PE=1 $\mathrm{SV}=3$

Match to Query 56433: 1935.007 from $(484.7591,4+)$

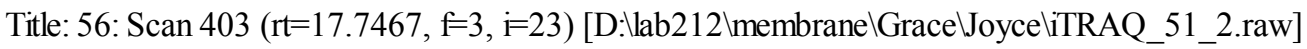

Data File:Submitted from 20120508-1(merge) by Mascot Daemon on JOYCE-VAIO

Monoisotopic mass of neutral peptide $\mathrm{Mr}$ (calc): 1935.007

Variable modifications:

K6 :iTRAQ4plex (K)

Ions Score: 48.3 Expect: 0.014 
Mascot Matched lons

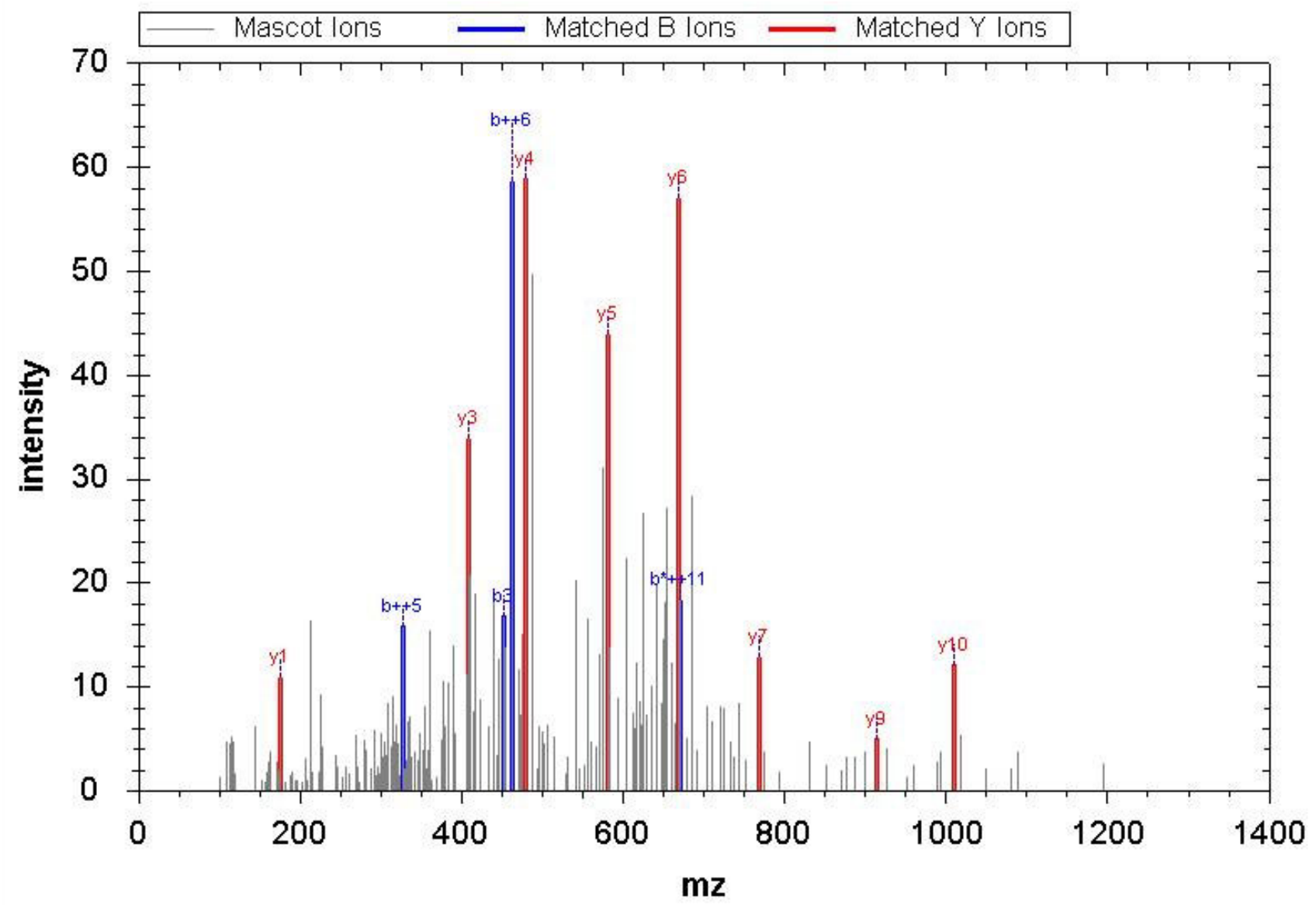

\begin{tabular}{|c|c|c|c|c|c|c|c|c|c|c|c|c|c|c|}
\hline No & $\mathrm{b}$ & $\mathrm{b}++$ & $b^{*}$ & $\mathrm{~b}^{*++}$ & b0 & b0++ & Seq & $\mathrm{y}$ & $\mathrm{y}++$ & $y^{*}$ & $y^{*++}$ & y0 & $\mathrm{y} 0++$ & RevNo \\
\hline 1 & 244.18 & 122.59 & & & & & V & & & & & & & 16 \\
\hline 2 & 315.21 & 158.11 & & & & & A & $1,692.84$ & 846.92 & $1,675.82$ & 838.41 & $1,674.83$ & 837.92 & 15 \\
\hline 3 & 452.27 & 226.64 & & & & & $\mathrm{H}$ & $1,621.81$ & 811.41 & $1,604.78$ & 802.89 & $1,603.79$ & 802.40 & 14 \\
\hline 4 & 539.31 & 270.16 & & & 521.30 & 261.15 & $\mathrm{~S}$ & $1,484.75$ & 742.88 & $1,467.72$ & 734.36 & $1,466.74$ & 733.87 & 13 \\
\hline 5 & 654.33 & 327.67 & & & 636.32 & 318.66 & D & $1,397.71$ & 699.36 & $1,380.69$ & 690.85 & $1,379.70$ & 690.36 & 12 \\
\hline 6 & 926.53 & 463.77 & 909.50 & 455.26 & 908.52 & 454.76 & K & $1,282.69$ & 641.85 & $1,265.66$ & 633.33 & $1,264.68$ & 632.84 & 11 \\
\hline 7 & $1,023.58$ & 512.29 & $1,006.56$ & 503.78 & $1,005.57$ & 503.29 & $\mathrm{P}$ & $1,010.49$ & 505.75 & 993.46 & 497.24 & 992.48 & 496.74 & 10 \\
\hline 8 & $1,080.60$ & 540.81 & $1,063.58$ & 532.29 & $1,062.59$ & 531.80 & G & 913.44 & 457.22 & 896.41 & 448.71 & 895.43 & 448.22 & 9 \\
\hline 9 & $1,167.64$ & 584.32 & $1,150.61$ & 575.81 & $1,149.63$ & 575.32 & $\mathrm{~S}$ & 856.42 & 428.71 & 839.39 & 420.20 & 838.41 & 419.71 & 8 \\
\hline 10 & $1,268.68$ & 634.85 & $1,251.66$ & 626.33 & $1,250.67$ & 625.84 & $\mathrm{~T}$ & 769.38 & 385.20 & 752.36 & 376.68 & 751.37 & 376.19 & 7 \\
\hline 11 & $1,355.72$ & 678.36 & $1,338.69$ & 669.85 & $1,337.71$ & 669.36 & $\mathrm{~S}$ & 668.34 & 334.67 & 651.31 & 326.16 & 650.33 & 325.67 & 6 \\
\hline 12 & $1,456.76$ & 728.89 & $1,439.74$ & 720.37 & $1,438.75$ & 719.88 & $\mathrm{~T}$ & 581.30 & 291.16 & 564.28 & 282.64 & 563.29 & 282.15 & 5 \\
\hline 13 & $1,527.80$ & 764.40 & $1,510.77$ & 755.89 & $1,509.79$ & 755.40 & A & 480.26 & 240.63 & 463.23 & 232.12 & 462.25 & 231.63 & 4 \\
\hline 14 & $1,614.83$ & 807.92 & $1,597.81$ & 799.41 & $1,596.82$ & 798.91 & $\mathrm{~S}$ & 409.22 & 205.11 & 392.19 & 196.60 & 391.21 & 196.11 & 3 \\
\hline 15 & $1,761.90$ & 881.45 & $1,744.87$ & 872.94 & $1,743.89$ & 872.45 & $\mathrm{~F}$ & 322.19 & 161.60 & 305.16 & 153.08 & & & 2 \\
\hline 16 & & & & & & & $\mathrm{R}$ & 175.12 & 88.06 & 158.09 & 79.55 & & & 1 \\
\hline
\end{tabular}

Query 84824 Hit 1

MS/MS Fragmentation of YVSEVVIGAPYAVTAELLSHFK

Found in sp|Q99447|PCY2_HUMAN, Ethanolamine-phosphate cytidylyltransferase OS=Homo sapiens GN=PCYT2 PE=1 SV=1 Match to Query 84824: 2680.469from(894.4969,3+)

Title: 1216: Sum of 2 scans in range $2852(\mathrm{rt}=72.9157, \mathrm{f}=2, \mathrm{i}=482)$ to $2853(\mathrm{rt}=72.9411, \mathrm{f}=2, \mathrm{i}=483)$

[D:llab212 \membranelGrace JoyceliTRAQ_32_2.raw]

Data File:Submitted from 20120508-1(merge) by Mascot Daemon on JOYCE-VAIO

Monoisotopic mass of neutral peptide $\mathrm{Mr}$ (calc): 2680.469 


\section{Mascot Matched lons}

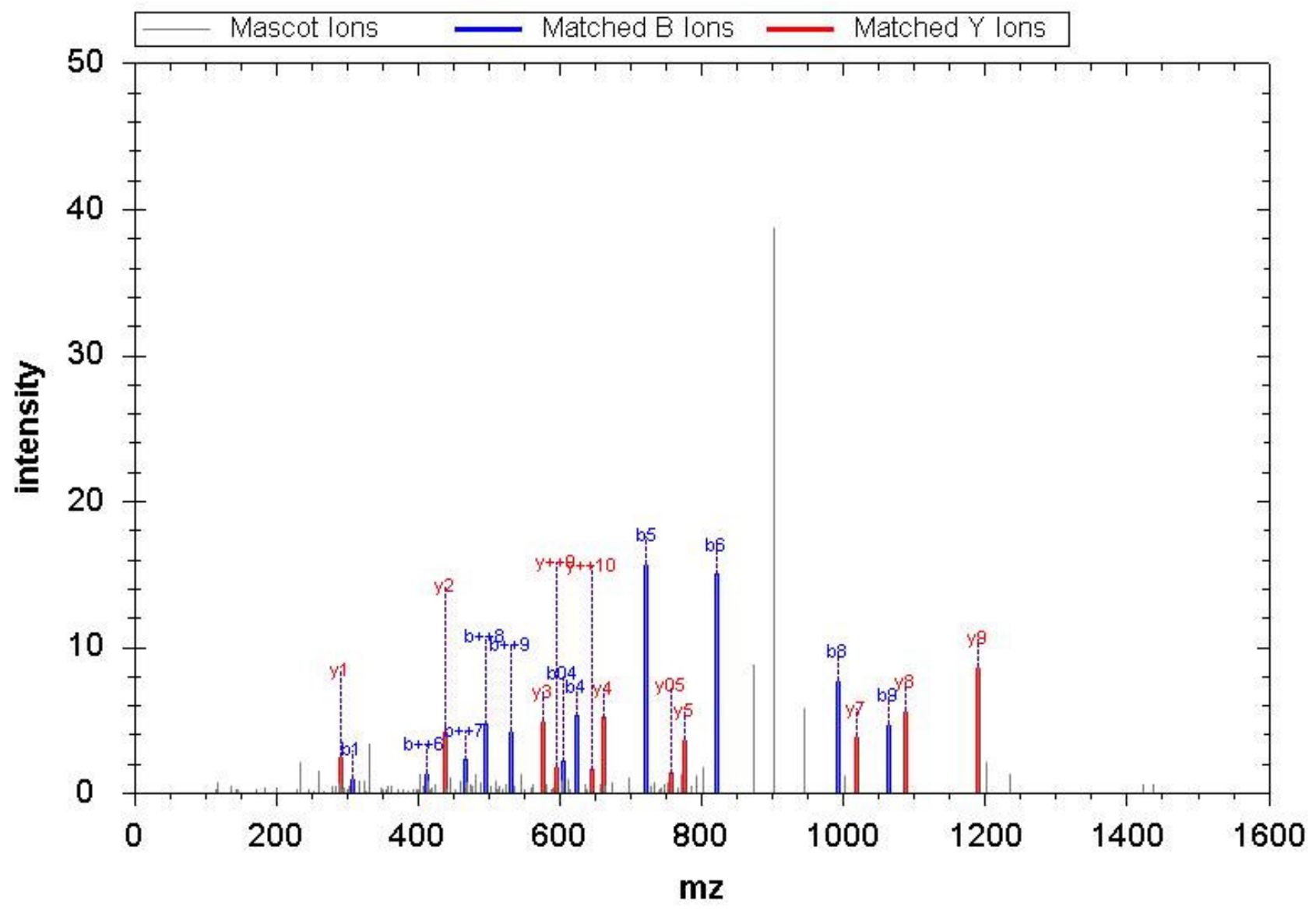

\begin{tabular}{|c|c|c|c|c|c|c|c|c|c|c|c|c|}
\hline No & b & ++ & b0 & + & $q$ & $\mathrm{y}$ & & $\mathrm{y}^{*}$ & & y0 & + & No \\
\hline 1 & 308.17 & & & & Y & & & & & & & 22 \\
\hline 2 & דים. וד & 204.12 & & & V & & & & & $2,356.30$ & & 21 \\
\hline 3 & 40427 & 4 & 6 & & $\mathrm{~S}$ & 25 & 3 & 22 & 61 & 23 & 1,1 & 20 \\
\hline 4 & 623.32 & 312.16 & 605.31 & 16 & $\mathrm{E}$ & 188.21 & .61 & 1.19 & 10 & 20 & 1,0 & 19 \\
\hline 5 & 722.38 & 361.70 & 704.37 & & V & & & & & $2,041.16$ & & 18 \\
\hline 6 & 821.45 & 411.23 & 803.44 & 22 & V & 10 & & .08 & .04 & .09 & 1.55 & 17 \\
\hline 7 & 934.54 & 467.77 & 16.53 & 77 & I & 03 & 02 & 1,8 & 51 & 02 & .02 & 16 \\
\hline 8 & 991.56 & 496.28 & 973.55 & 487.28 & G & .95 & 48 & .92 & 97 & 94 & +11 & 15 \\
\hline 9 & $1,062.60$ & 531.80 & 8 & 0 & A & 3 & 7 & 1, & 45 & 1,6 & 30 & 14 \\
\hline 10 & $1,159.65$ & 580.33 & $1,141.64$ & 571.32 & $\mathrm{P}$ & $1,619.89$ & 45 & 1,602 & 94 & 1,601 & 44 & 13 \\
\hline 11 & $1,322.71$ & 661.86 & $1,304.70$ & 652.85 & $\mathrm{Y}$ & $1,522.84$ & & $1,505.81$ & 753.41 & $1,504.83$ & 92 & 12 \\
\hline 12 & $1,393.75$ & 697.38 & $1,375.74$ & 688.37 & A & $1,359.78$ & 680.39 & $1,342.75$ & 671.88 & $1,341.76$ & .39 & 11 \\
\hline 13 & $1,492.82$ & 746.91 & $1,474.81$ & 737.91 & V & $1,288.74$ & 644.87 & $1,271.71$ & 636.36 & $1,270.73$ & 635.87 & 10 \\
\hline 14 & $1,593.86$ & 797.44 & $1,575.85$ & 788.43 & $\mathrm{~T}$ & $1,189.67$ & 595.34 & $1,172.64$ & 586.83 & $1,171.66$ & 586.33 & 9 \\
\hline 15 & $1,664.90$ & 832.95 & $1,646.89$ & 823.95 & A & $1,088.62$ & 81 & $1,071.60$ & .30 & $1,070.61$ & .81 & 0 \\
\hline 16 & 793.94 & 897.48 & $1,775.93$ & 888.47 & $\mathrm{E}$ & 1,0 & .30 & 1,0 & 78 & .57 & 29 & 7 \\
\hline 17 & $1,907.03$ & 954.02 & $1,889.02$ & 945.01 & $\mathrm{~L}$ & 888.54 & 444.77 & 871.52 & 436.26 & 870.53 & 435.77 & 6 \\
\hline 182 & $2,020.11$ & $1,010.56$ & $2,002.10$ & $1,001.55$ & $\mathrm{~L}$ & 775.46 & 388.23 & 758.43 & 379.72 & 757.45 & 379.23 & 5 \\
\hline 192 & $2,107.14$ & $1,054.08$ & $2,089.13$ & $1,045.07$ & S & 662.37 & 331.69 & 645.35 & 323.18 & 644.36 & 322.69 & 4 \\
\hline 202 & $2,244.20$ & $1,122.61$ & $2,226.19$ & $1,113.60$ & $\mathrm{H}$ & 575.34 & 288.17 & 558.32 & 279.66 & & & 3 \\
\hline $21 \mid 2$ & $2,391.27$ & $1,196.14$ & $2,373.26$ & $1,187.13$ & F & 438.28 & 219.65 & 421.26 & 211.13 & & & 2 \\
\hline
\end{tabular}




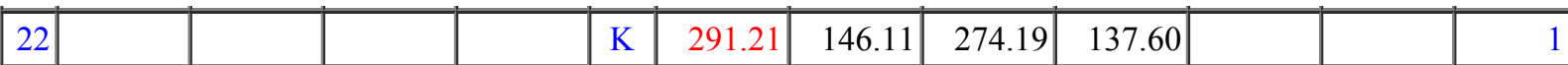

Query 50574 Hit 1

MS/MS Fragmentation of DLLALFNSFKPK

Found in sp|Q9H501|ESF1_HUMAN, ESF1 homolog OS=Homo sapiens GN=ESF1 PE=1 SV=1

Match to Query 50574: 1824.093from(609.0384,3+)

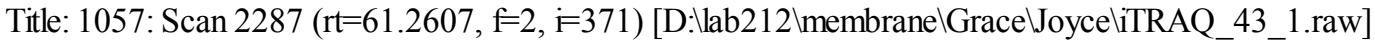

Data File:Submitted from 20120508-1(merge) by Mascot Daemon on JOYCE-VAIO

Monoisotopic mass of neutral peptide Mr(calc): 1824.093

Variable modifications:

K10 iTRAQ4plex (K)

K12 :TTRAQ4plex (K)

Ions Score: 48.26 Expect: 0.005

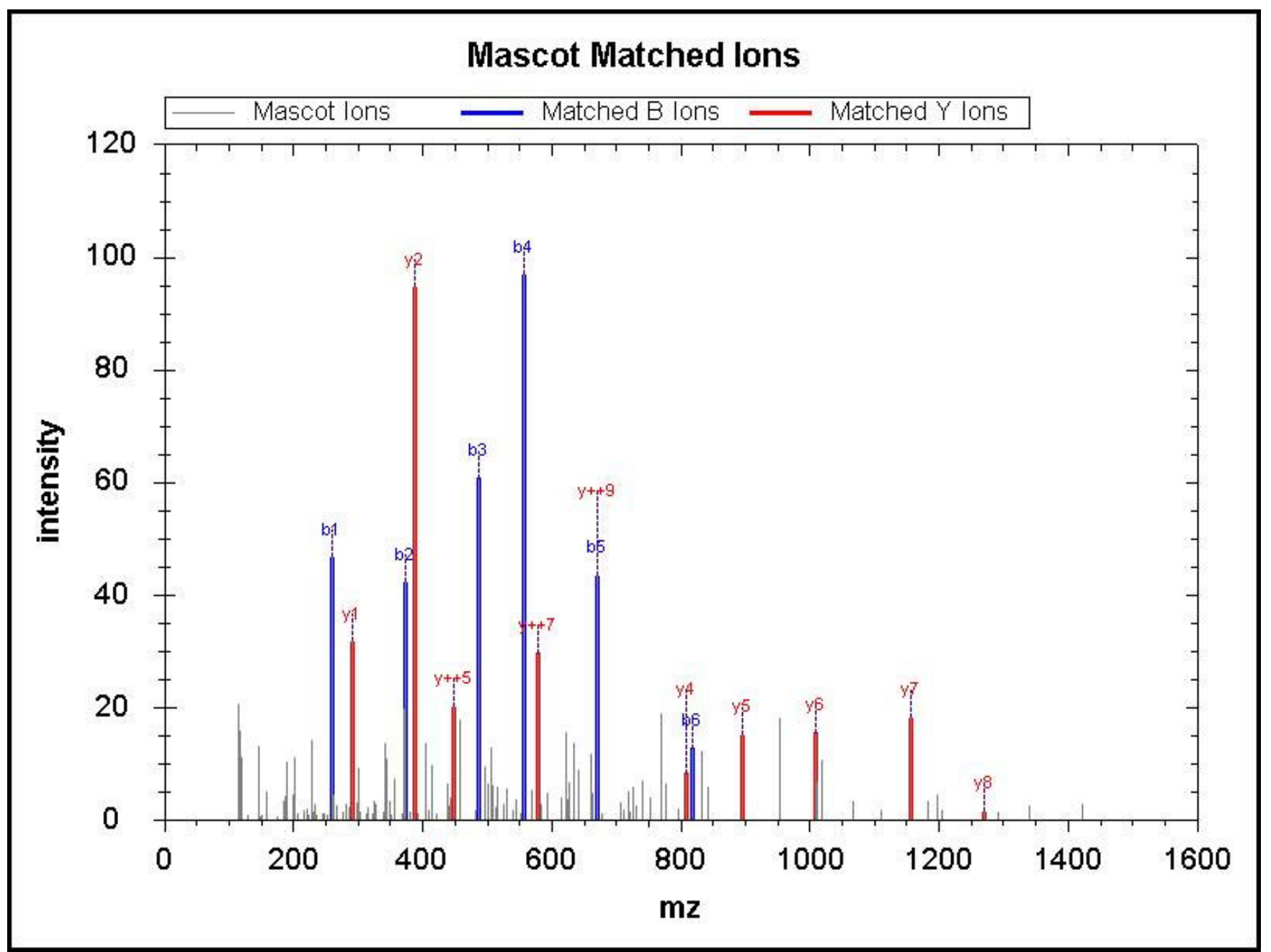

\begin{tabular}{|c|c|c|c|c|c|c|c|c|c|c|c|c|c|c|}
\hline No & $\mathrm{b}$ & $\mathrm{b}++$ & $b^{*}$ & $b^{*++}$ & b0 & $\mathrm{b} 0++$ & Seq & $\mathrm{y}$ & $\mathrm{y}++$ & $\mathrm{y}^{*}$ & $y^{*++}$ & y0 & $\mathrm{y} 0++$ & RevNo \\
\hline 1 & 260.14 & 130.57 & & & 242.13 & 121.57 & D & & & & & & & 12 \\
\hline 2 & 373.22 & 187.11 & & & 355.21 & 178.11 & $\mathrm{~L}$ & $1,565.97$ & 783.49 & $1,548.94$ & 774.97 & $1,547.96$ & 774.48 & 11 \\
\hline 3 & 486.30 & 243.66 & & & 468.29 & 234.65 & $\mathrm{~L}$ & $1,452.88$ & 726.94 & $1,435.86$ & 718.43 & $1,434.87$ & 717.94 & 10 \\
\hline 4 & 557.34 & 279.17 & & & 539.33 & 270.17 & A & $1,339.80$ & 670.40 & $1,322.77$ & 661.89 & $1,321.79$ & 661.40 & 9 \\
\hline 5 & 670.43 & 335.72 & & & 652.41 & 326.71 & $\mathrm{~L}$ & $1,268.76$ & 634.88 & $1,251.73$ & 626.37 & $1,250.75$ & 625.88 & 8 \\
\hline 6 & 817.49 & 409.25 & & & 799.48 & 400.25 & $\mathrm{~F}$ & $1,155.68$ & 578.34 & $1,138.65$ & 569.83 & $1,137.67$ & 569.34 & 7 \\
\hline 7 & 931.54 & 466.27 & 914.51 & 457.76 & 913.53 & 457.27 & $\mathrm{~N}$ & $1,008.61$ & 504.81 & 991.58 & 496.29 & 990.60 & 495.80 & 6 \\
\hline 8 & $1,018.57$ & 509.79 & $1,001.54$ & 501.27 & $1,000.56$ & 500.78 & $\mathrm{~S}$ & 894.57 & 447.79 & 877.54 & 439.27 & 876.55 & 438.78 & 5 \\
\hline 9 & $1,165.64$ & 583.32 & $1,148.61$ & 574.81 & $1,147.63$ & 574.32 & $\mathrm{~F}$ & 807.53 & 404.27 & 790.51 & 395.76 & & & 4 \\
\hline 10 & $1,437.83$ & 719.42 & $1,420.81$ & 710.91 & $1,419.82$ & 710.42 & K & 660.46 & 330.74 & 643.44 & 322.22 & & & 3 \\
\hline 11 & $1,534.89$ & 767.95 & $1,517.86$ & 759.43 & $1,516.88$ & 758.94 & $\mathrm{P}$ & 388.27 & 194.64 & 371.24 & 186.12 & & & 2 \\
\hline 12 & & & & & & & K & 291.21 & 146.11 & 274.19 & 137.60 & & & 1 \\
\hline
\end{tabular}


MS/MS Fragmentation of LLVFATDDGFHFAGDGK

Found in sp|P05107|ITB2_HUMAN, Integrin beta-2 OS=Homo sapiens GN=ITGB2 PE=1 SV=2

Match to Query 64308: 2097.082from(700.0344,3+)

Title: 863: Sum of 3 scans in range 1906 ( $\mathrm{rt}=52.3906, \mathrm{f}=3, \mathrm{i}=297)$ to 1908 ( $\mathrm{rt}=52.4414, \mathrm{f}=4, \mathrm{i}=574)$

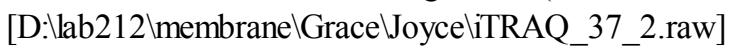

Data File:Submitted from 20120508-1(merge) by Mascot Daemon on JOYCE-VAIO

Monoisotopic mass of neutral peptide $\mathrm{Mr}$ (calc): 2097.082

Variable modifications:

K17 :iTRAQ4plex (K)

Ions Score: 48.25 Expect: 0.014

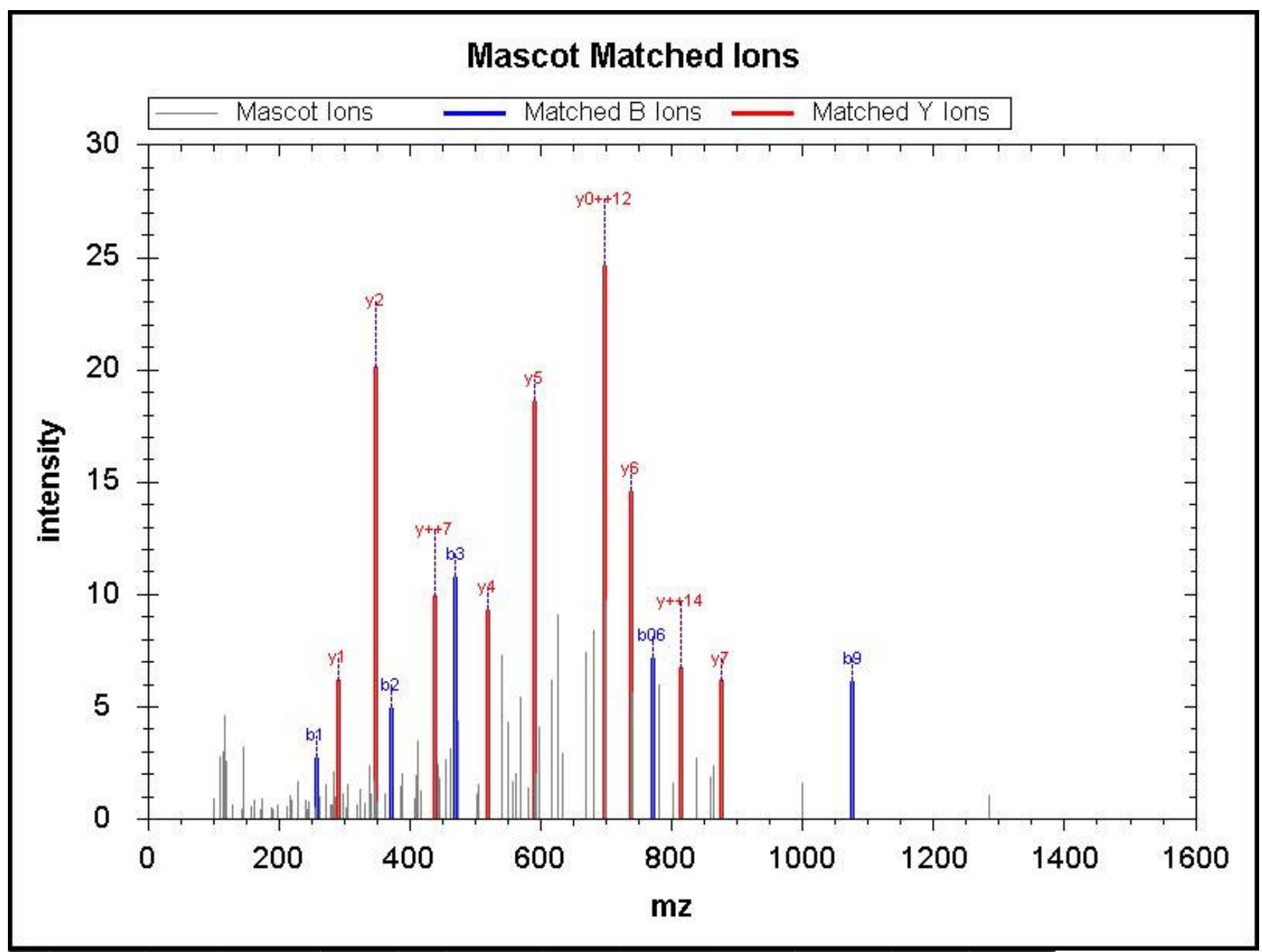

\begin{tabular}{|r|r|r|r|r|r|r|r|r|r|r|r|r|}
\hline \hline $\mathrm{No}$ & $\mathrm{b}$ & $\mathrm{b}++$ & $\mathrm{b} 0$ & $\mathrm{~b} 0++$ & $\mathrm{Seq}$ & $\mathrm{y}$ & $\mathrm{y}++$ & $\mathrm{y}^{*}$ & $\mathrm{y}++$ & $\mathrm{y} 0$ & $\mathrm{y} 0++$ & RevNo \\
\hline 1 & 258.19 & 129.60 & & & $\mathrm{~L}$ & & & & & & & 17 \\
\hline 2 & 371.28 & 186.14 & & & $\mathrm{~L}$ & $1,840.90$ & 920.95 & $1,823.87$ & 912.44 & $1,822.89$ & 911.95 & 16 \\
\hline 3 & 470.35 & 235.68 & & & $\mathrm{~V}$ & $1,727.81$ & 864.41 & $1,710.79$ & 855.90 & $1,709.80$ & 855.41 & 15 \\
\hline 4 & 617.41 & 309.21 & & & $\mathrm{~F}$ & $1,628.75$ & 814.88 & $1,611.72$ & 806.36 & $1,610.74$ & 805.87 & 14 \\
\hline 5 & 688.45 & 344.73 & & & $\mathrm{~A}$ & $1,481.68$ & 741.34 & $1,464.65$ & 732.83 & $1,463.67$ & 732.34 & 13 \\
\hline 6 & 789.50 & 395.25 & 771.49 & 386.25 & $\mathrm{~T}$ & $1,410.64$ & 705.82 & $1,393.61$ & 697.31 & $1,392.63$ & 696.82 & 12 \\
\hline 7 & 904.53 & 452.77 & 886.52 & 443.76 & $\mathrm{D}$ & $1,309.59$ & 655.30 & $1,292.57$ & 646.79 & $1,291.58$ & 646.29 & 11 \\
\hline 8 & $1,019.55$ & 510.28 & $1,001.54$ & 501.27 & $\mathrm{D}$ & $1,194.57$ & 597.79 & $1,177.54$ & 589.27 & $1,176.56$ & 588.78 & 10 \\
\hline 9 & $1,076.57$ & 538.79 & $1,058.56$ & 529.79 & $\mathrm{G}$ & $1,079.54$ & 540.27 & $1,062.51$ & 531.76 & $1,061.53$ & 531.27 & 9 \\
\hline 10 & $1,223.64$ & 612.33 & $1,205.63$ & 603.32 & $\mathrm{~F}$ & $1,022.52$ & 511.76 & $1,005.49$ & 503.25 & $1,004.51$ & 502.76 & 8 \\
\hline 11 & $1,360.70$ & 680.85 & $1,342.69$ & 671.85 & $\mathrm{H}$ & 875.45 & 438.23 & 858.42 & 429.71 & 857.44 & 429.22 & 7 \\
\hline 12 & $1,507.77$ & 754.39 & $1,489.76$ & 745.38 & $\mathrm{~F}$ & 738.39 & 369.70 & 721.36 & 361.19 & 720.38 & 360.69 & 6 \\
\hline 13 & $1,578.81$ & 789.91 & $1,560.80$ & 780.90 & A & 591.32 & 296.16 & 574.30 & 287.65 & 573.31 & 287.16 & 5 \\
\hline 14 & $1,635.83$ & 818.42 & $1,617.82$ & 809.41 & G & 520.28 & 260.65 & 503.26 & 252.13 & 502.27 & 251.64 & 4 \\
\hline
\end{tabular}




\begin{tabular}{|l|l|l|l|l|l|l|l|l|l|l|l|r|}
15 & $1,750.86$ & 875.93 & $1,732.85$ & 866.93 & $\mathrm{D}$ & 463.26 & 232.14 & 446.24 & 223.62 & 445.25 & 223.13 & 3 \\
\hline 16 & $1,807.88$ & 904.44 & $1,789.87$ & 895.44 & $\mathrm{G}$ & 348.24 & 174.62 & 331.21 & 166.11 & & & 2 \\
\hline 17 & & & & & $\mathrm{~K}$ & 291.21 & 146.11 & 274.19 & 137.60 & & & 1 \\
\hline
\end{tabular}

Query 7405 Hit 1

\section{MS/MS Fragmentation of EGNPYK}

Found in sp|Q9NZJ4|SACS_HUMAN, Sacsin OS=Homo sapiens GN=SACS PE=1 SV=2

Match to Query 7405: 995.5349from(498.7747,2+)

Title: 267 : Sum of 2 scans in range $848(\mathrm{rt}=27.5637, \mathrm{f}=4, \mathrm{f}=171)$ to $849(\mathrm{rt}=27.5892, \mathrm{f}=4, \mathrm{f}=172)$

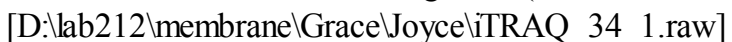

Data File:Submitted from 20120508-1(merge) by Mascot Daemon on JOYCE-VAIO

Monoisotopic mass of neutral peptide $\mathrm{Mr}$ (calc): 995.5349

Variable modifications:

N3 :Deamidated (NQ)

K6 :iTRAQ4plex (K)

Ions Score: 48.19 Expect: 0.009

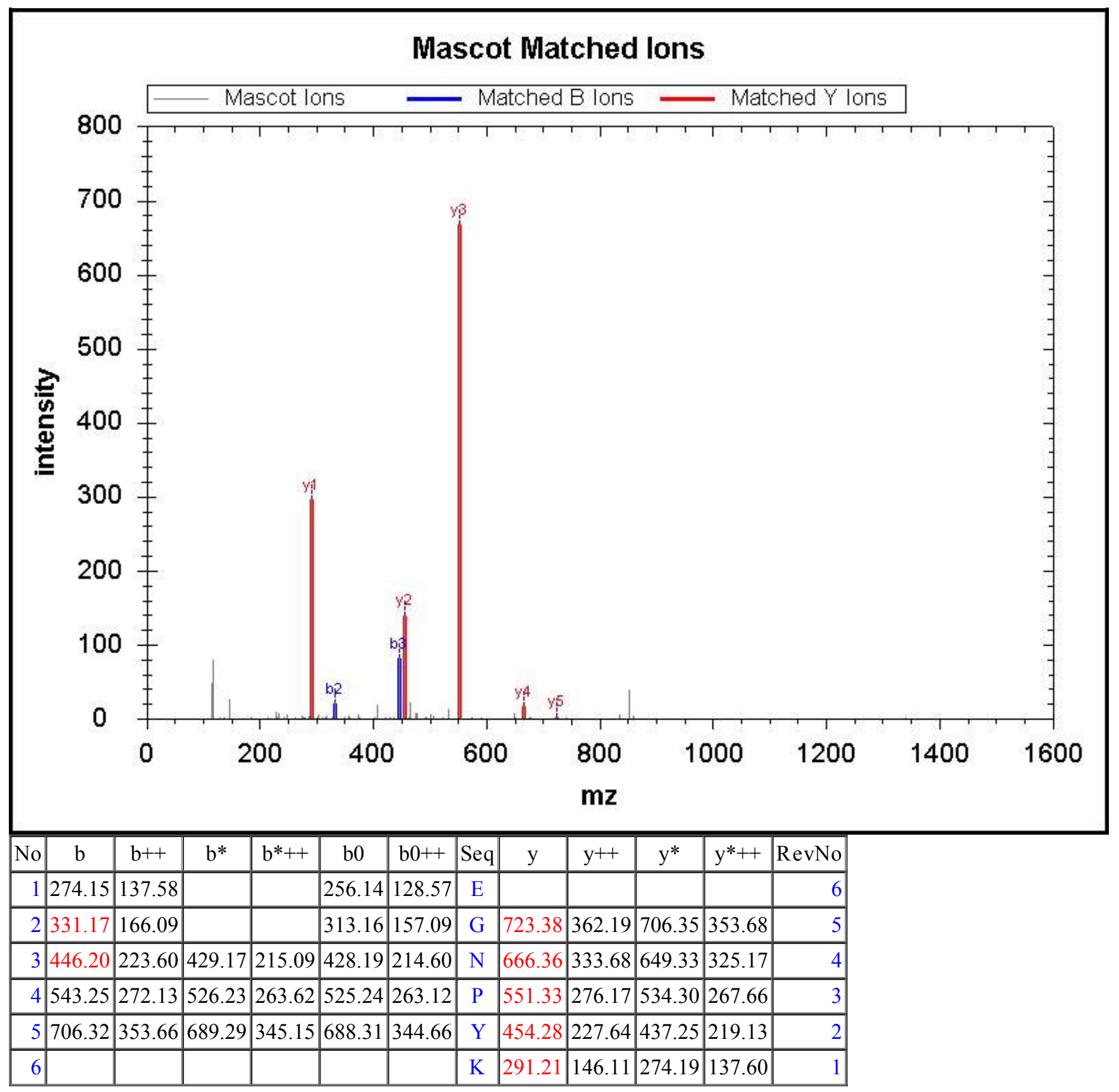

Query 93590 Hit 1 
Match to Query 93590: 3142.697from(786.6815,4+)

Title: 866: Scan 2495 ( $\mathrm{rt}=63.6754, \mathrm{f}=2, \mathrm{i}=436)$ [D:llab212 $\backslash$ membranelGrace JoyceliTRAQ_46_1.raw]

Data File:Submitted from 20120508-1(merge) by Mascot Daemon on JOYCE-VAIO

Monoisotopic mass of neutral peptide Mr(calc): 3142.697

Variable modifications:

K1 :iTRAQ4plex (K)

Ions Score: 48.16 Expect: 0.008

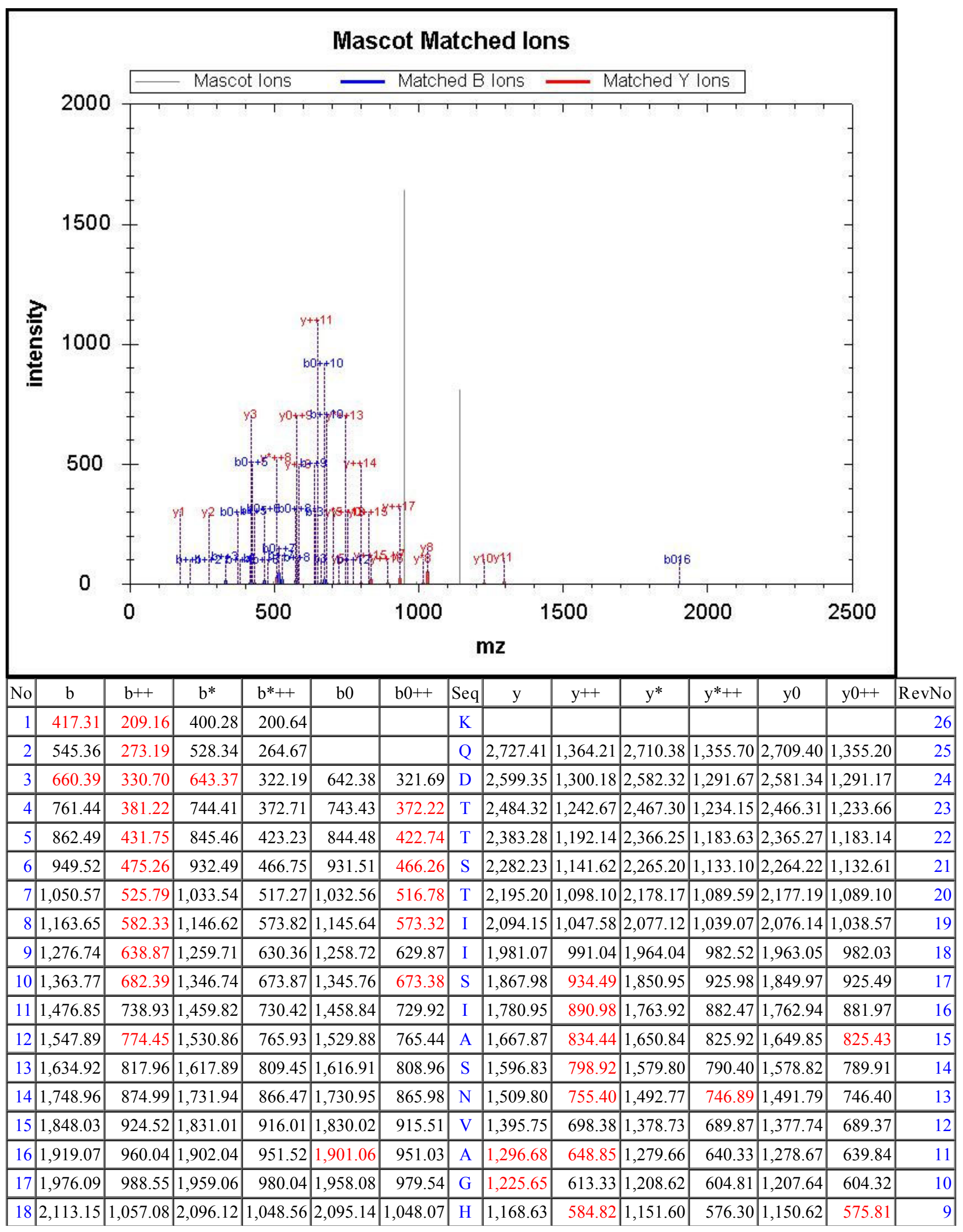




\begin{tabular}{|r|r|r|r|r|r|r|r|r|r|r|r|r|r|r|}
\hline 19 & $2,210.20$ & $1,105.60$ & $2,193.18$ & $1,097.09$ & $2,192.19$ & $1,096.60$ & $\mathrm{P}$ & $1,031.57$ & 516.29 & $1,014.54$ & 507.77 & $1,013.56$ & 507.28 & 8 \\
\hline 20 & $2,323.29$ & $1,162.15$ & $2,306.26$ & $1,153.63$ & $2,305.28$ & $1,153.14$ & $\mathrm{~L}$ & 934.51 & 467.76 & 917.49 & 459.25 & 916.50 & 458.76 & 7 \\
\hline 21 & $2,422.35$ & $1,211.68$ & $2,405.33$ & $1,203.17$ & $2,404.34$ & $1,202.68$ & $\mathrm{~V}$ & 821.43 & 411.22 & 804.40 & 402.71 & 803.42 & 402.21 & 6 \\
\hline 22 & $2,608.43$ & $1,304.72$ & $2,591.41$ & $1,296.21$ & $2,590.42$ & $1,295.72$ & $\mathrm{~W}$ & 722.36 & 361.68 & 705.34 & 353.17 & 704.35 & 352.68 & 5 \\
\hline 23 & $2,723.46$ & $1,362.23$ & $2,706.43$ & $1,353.72$ & $2,705.45$ & $1,353.23$ & $\mathrm{D}$ & 536.28 & 268.64 & 519.26 & 260.13 & 518.27 & 259.64 & 4 \\
\hline 24 & $2,870.53$ & $1,435.77$ & $2,853.50$ & $1,427.25$ & $2,852.52$ & $1,426.76$ & $\mathrm{~F}$ & 421.26 & 211.13 & 404.23 & 202.62 & & & 3 \\
\hline 25 & $2,969.60$ & $1,485.30$ & $2,952.57$ & $1,476.79$ & $2,951.59$ & $1,476.30$ & $\mathrm{~V}$ & 274.19 & 137.60 & 257.16 & 129.08 & & & 2 \\
\hline 26 & & & & & & & $\mathrm{R}$ & 175.12 & 88.06 & 158.09 & 79.55 & & & 1 \\
\hline
\end{tabular}

Query 6620 Hit 1

MS/MS Fragmentation of LELDALR

Found in sp|Q9ULX9|MAFF_HUMAN, Transcription factor MafF OS=Homo sapiens GN=MAFF PE=1 SV=2

Match to Query 6620: 972.6013from(487.3079,2+)

Title: 290: Sum of 2 scans in range 1384 ( $\mathrm{rt}=37.0885, \mathrm{f}=4, \mathrm{i}=171)$ to 1385 ( $\mathrm{rt}=37.1138, \mathrm{f}=4, \mathrm{i}=172$ )

[D:llab212 \membranelGrace JoyceliTRAQ_26_1.raw]

Data File:Submitted from 20120508-1(merge) by Mascot Daemon on JOYCE-VAIO

Monoisotopic mass of neutral peptide Mr(calc): 972.6013

Variable modifications:

Ions Score: 47.99 Expect: 0.009

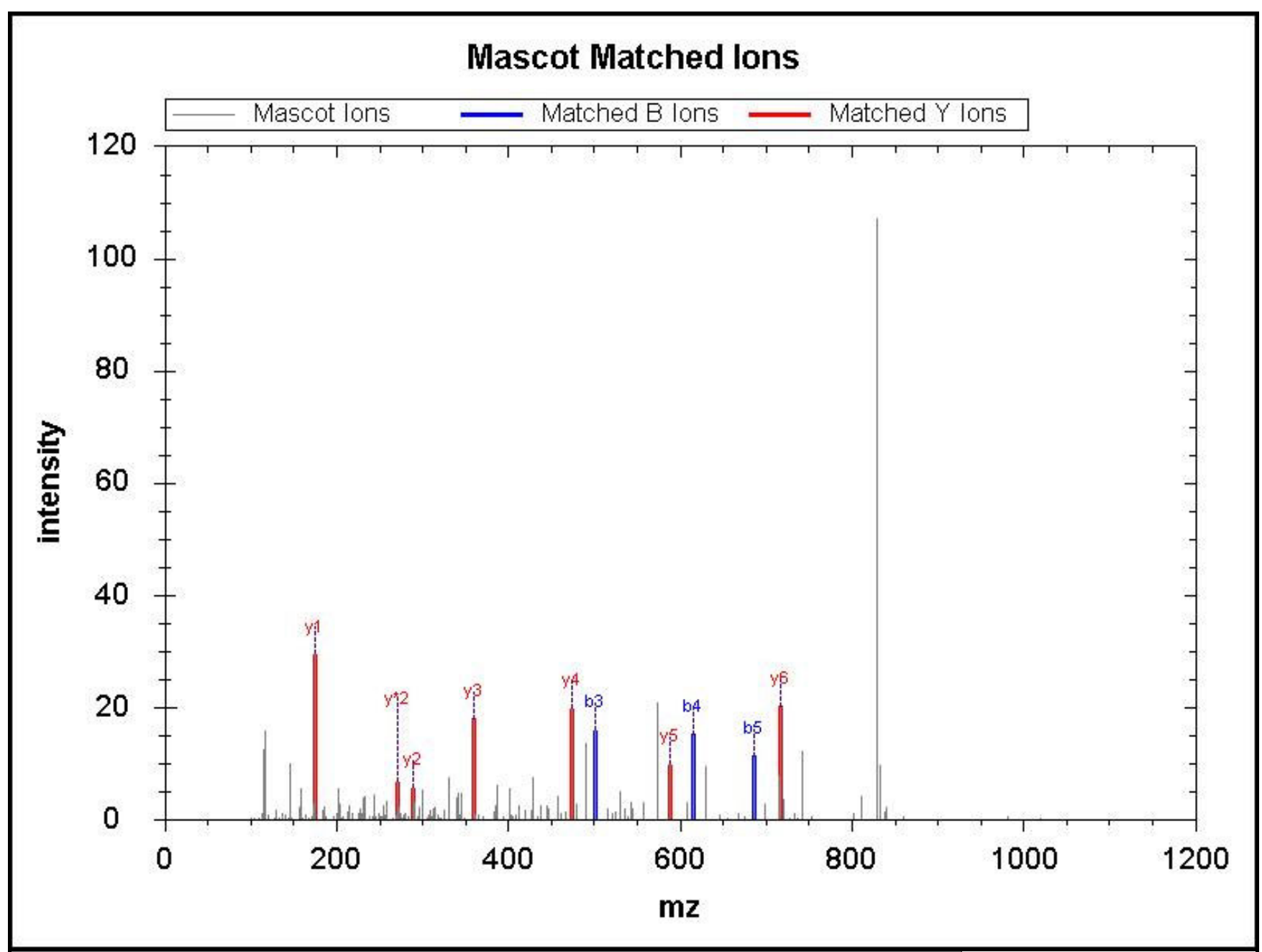

\begin{tabular}{|r|c|c|c|c|c|c|c|c|c|c|r|r|}
\hline No & $\mathrm{b}$ & $\mathrm{b}++$ & $\mathrm{b} 0$ & $\mathrm{~b} 0++$ & Seq & $\mathrm{y}$ & $\mathrm{y}++$ & $\mathrm{y} *$ & $\mathrm{y}++$ & $\mathrm{y} 0$ & $\mathrm{y} 0++$ & RevNo \\
\hline 1 & 258.19 & 129.60 & & & $\mathrm{~L}$ & & & & & & & 7 \\
\hline 2 & 387.24 & 194.12 & 369.23 & 185.12 & $\mathrm{E}$ & 716.39 & 358.70 & 699.37 & 350.19 & 698.38 & 349.70 & 6 \\
\hline 3 & 500.32 & 250.66 & 482.31 & 241.66 & $\mathrm{~L}$ & 587.35 & 294.18 & 570.32 & 285.67 & 569.34 & 285.17 & 5 \\
\hline 4 & 615.35 & 308.18 & 597.34 & 299.17 & $\mathrm{D}$ & 474.27 & 237.64 & 457.24 & 229.12 & 456.26 & 228.63 & 4 \\
\hline 5 & 686.38 & 343.70 & 668.37 & 334.69 & A & 359.24 & 180.12 & 342.21 & 171.61 & & & 3 \\
\hline 6 & 799.47 & 400.24 & 781.46 & 391.23 & L & 288.20 & 144.61 & 271.18 & 136.09 & & & 2 \\
\hline
\end{tabular}


Query 55792 Hit 1

MS/MS Fragmentation of STLVHSLFLTDLYK

Found in sp|Q99719|SEPT5_HUMAN, Septin-5 OS=Homo sapiens GN=SEPT5 PE=1 SV=1

Match to Query 55792: 1924.092from(642.3713,3+)

Title: 850: Scan $1894(\mathrm{rt}=52.1693, \mathrm{f}=2, \mathrm{i}=311)$ [D:lab212 $\backslash$ membranelGrace JoyceliTRAQ_39_1.raw]

Data File:Submitted from 20120508-1(merge) by Mascot Daemon on JOYCE-VAIO

Monoisotopic mass of neutral peptide Mr(calc): 1924.092

Variable modifications:

K14 :iTRAQ4plex (K)

Ions Score: 47.97 Expect: 0.009

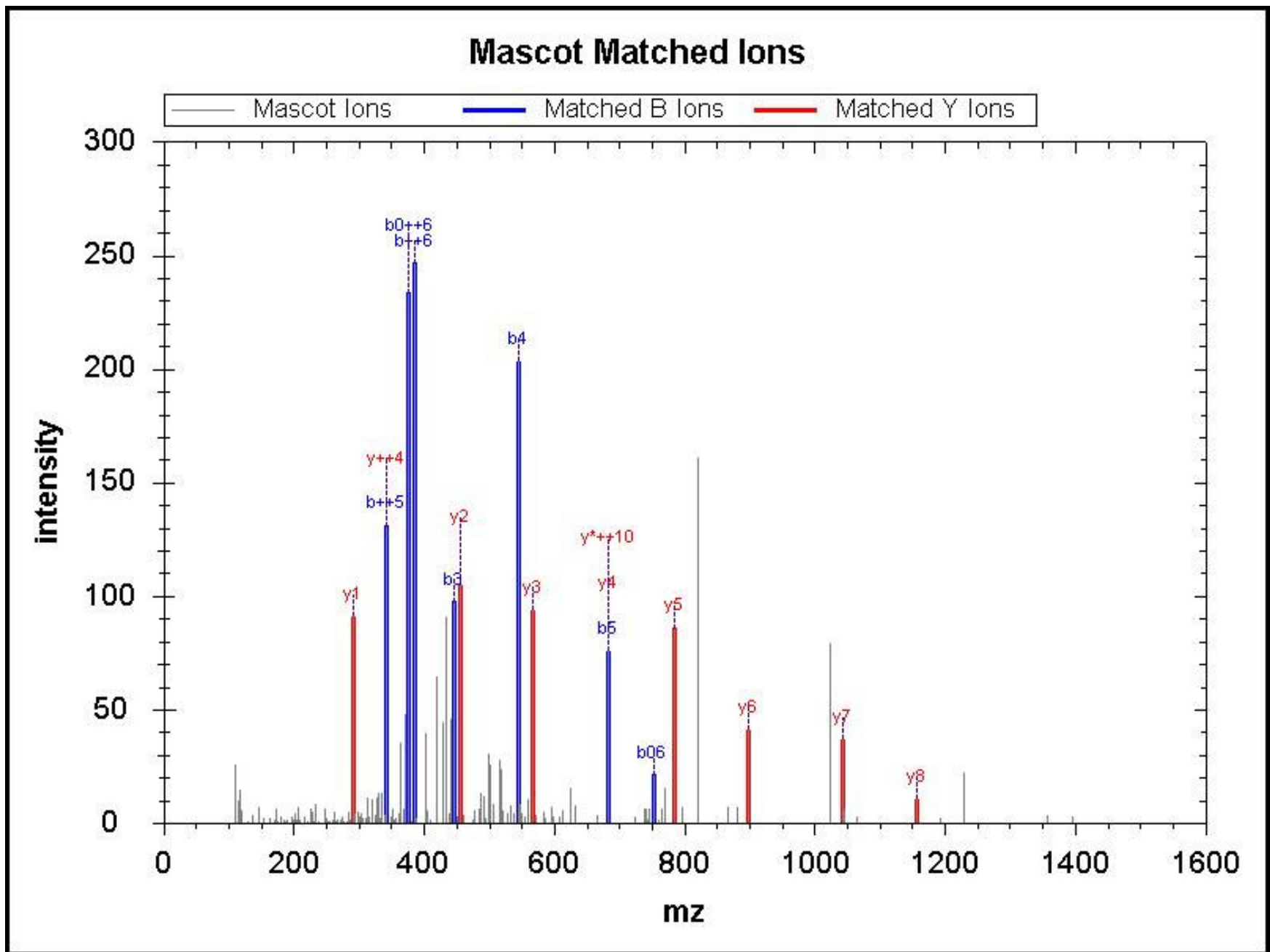

\begin{tabular}{|r|c|c|c|c|c|c|c|c|c|c|r|r|}
\hline $\mathrm{No}$ & $\mathrm{b}$ & $\mathrm{b}++$ & $\mathrm{b} 0$ & $\mathrm{~b} 0++$ & $\mathrm{Seq}$ & $\mathrm{y}$ & $\mathrm{y}++$ & $\mathrm{y}^{*}$ & $\mathrm{y} *++$ & $\mathrm{y} 0$ & $\mathrm{y} 0++$ & $\mathrm{RevNo}$ \\
\hline 1 & 232.14 & 116.57 & 214.13 & 107.57 & $\mathrm{~S}$ & & & & & & & 14 \\
\hline 2 & 333.19 & 167.10 & 315.18 & 158.09 & $\mathrm{~T}$ & $1,693.96$ & 847.49 & $1,676.94$ & 838.97 & $1,675.95$ & 838.48 & 13 \\
\hline 3 & 446.27 & 223.64 & 428.26 & 214.63 & $\mathrm{~L}$ & $1,592.92$ & 796.96 & $1,575.89$ & 788.45 & $1,574.91$ & 787.96 & 12 \\
\hline 4 & 545.34 & 273.17 & 527.33 & 264.17 & $\mathrm{~V}$ & $1,479.83$ & 740.42 & $1,462.81$ & 731.91 & $1,461.82$ & 731.41 & 11 \\
\hline 5 & 682.40 & 341.70 & 664.39 & 332.70 & $\mathrm{H}$ & $1,380.76$ & 690.89 & $1,363.74$ & 682.37 & $1,362.75$ & 681.88 & 10 \\
\hline 6 & 769.43 & 385.22 & 751.42 & 376.21 & $\mathrm{~S}$ & $1,243.71$ & 622.36 & $1,226.68$ & 613.84 & $1,225.69$ & 613.35 & 9 \\
\hline 7 & 882.52 & 441.76 & 864.51 & 432.76 & $\mathrm{~L}$ & $1,156.67$ & 578.84 & $1,139.65$ & 570.33 & $1,138.66$ & 569.84 & 8 \\
\hline 8 & $1,029.58$ & 515.30 & $1,011.57$ & 506.29 & $\mathrm{~F}$ & $1,043.59$ & 522.30 & $1,026.56$ & 513.79 & $1,025.58$ & 513.29 & 7 \\
\hline 9 & $1,142.67$ & 571.84 & $1,124.66$ & 562.83 & $\mathrm{~L}$ & 896.52 & 448.76 & 879.49 & 440.25 & 878.51 & 439.76 & 6 \\
\hline 10 & $1,243.72$ & 622.36 & $1,225.71$ & 613.36 & $\mathrm{~T}$ & 783.44 & 392.22 & 766.41 & 383.71 & 765.43 & 383.22 & 5 \\
\hline 11 & $1,358.74$ & 679.88 & $1,340.73$ & 670.87 & $\mathrm{D}$ & 682.39 & 341.70 & 665.36 & 333.18 & 664.38 & 332.69 & 4 \\
\hline 12 & $1,471.83$ & 736.42 & $1,453.82$ & 727.41 & $\mathrm{~L}$ & 567.36 & 284.18 & 550.34 & 275.67 & & & 3 \\
\hline & & & & & & & & & & & &
\end{tabular}




\begin{tabular}{|l|l|l|l|l|l|l|l|l|l|l|l|r|}
\hline 13 & $1,634.89$ & 817.95 & $1,616.88$ & 808.94 & $\mathrm{Y}$ & 454.28 & 227.64 & 437.25 & 219.13 & & & 2 \\
\hline 14 & & & & & $\mathrm{~K}$ & 291.21 & 146.11 & 274.19 & 137.60 & & & 1 \\
\hline
\end{tabular}

Query 76132 Hit 1

\section{MS/MS Fragmentation of FTDDTFDPELAATIGVDFK}

Found in sp|Q9NP72|RAB18_HUMAN, Ras-related protein Rab-18 OS=Homo sapiens GN=RAB18 PE=1 SV=1

Match to Query 76132: 2389.193from(797.4051,3+)

Title: 959: Sum of 2 scans in range 2240 ( $\mathrm{rt}=59.2722, \mathrm{f}=4, \mathrm{i}=625)$ to $2241(\mathrm{rt}=59.2976, \mathrm{f}=4, \mathrm{i}=626)$

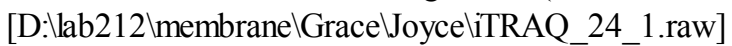

Data File:Submitted from 20120508-1(merge) by Mascot Daemon on JOYCE-VAIO

Monoisotopic mass of neutral peptide $\mathrm{Mr}$ (calc): 2389.193

Variable modifications:

K19 iTRAQ4plex (K)

Ions Score: 47.95 Expect: 0.016

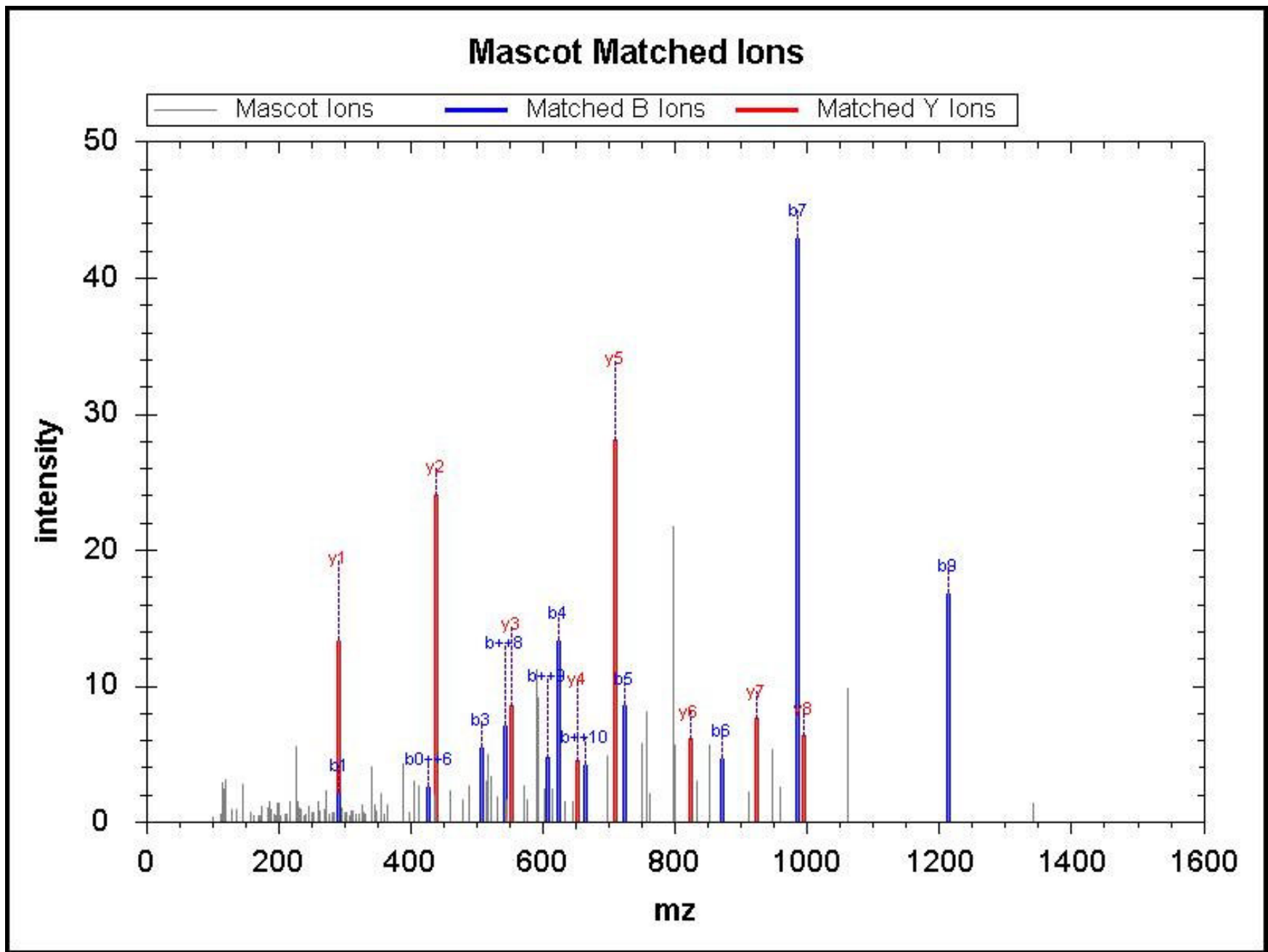

\begin{tabular}{|r|c|c|c|c|c|c|c|c|c|r|r|r|}
\hline \hline $\mathrm{No}$ & $\mathrm{b}$ & $\mathrm{b}++$ & $\mathrm{b} 0$ & $\mathrm{~b} 0++$ & $\mathrm{Seq}$ & $\mathrm{y}$ & $\mathrm{y}++$ & $\mathrm{y} *$ & $\mathrm{y}++$ & $\mathrm{y} 0$ & $\mathrm{y} 0++$ & $\mathrm{RevNo}$ \\
\hline 1 & 292.18 & 146.59 & & & $\mathrm{~F}$ & & & & & & & 19 \\
\hline 2 & 393.23 & 197.12 & 375.21 & 188.11 & $\mathrm{~T}$ & $2,099.03$ & $1,050.02$ & $2,082.00$ & $1,041.51$ & $2,081.02$ & $1,041.01$ & 18 \\
\hline 3 & 508.25 & 254.63 & 490.24 & 245.62 & $\mathrm{D}$ & $1,997.98$ & 999.49 & $1,980.96$ & 990.98 & $1,979.97$ & 990.49 & 17 \\
\hline 4 & 623.28 & 312.14 & 605.27 & 303.14 & $\mathrm{D}$ & $1,882.96$ & 941.98 & $1,865.93$ & 933.47 & $1,864.94$ & 932.98 & 16 \\
\hline 5 & 724.33 & 362.67 & 706.32 & 353.66 & $\mathrm{~T}$ & $1,767.93$ & 884.47 & $1,750.90$ & 875.95 & $1,749.92$ & 875.46 & 15 \\
\hline 6 & 871.40 & 436.20 & 853.38 & 427.20 & $\mathrm{~F}$ & $1,666.88$ & 833.94 & $1,649.85$ & 825.43 & $1,648.87$ & 824.94 & 14 \\
\hline 7 & 986.42 & 493.71 & 968.41 & 484.71 & $\mathrm{D}$ & $1,519.81$ & 760.41 & $1,502.79$ & 751.90 & $1,501.80$ & 751.40 & 13 \\
\hline 8 & $1,083.48$ & 542.24 & $1,065.46$ & 533.24 & $\mathrm{P}$ & $1,404.79$ & 702.90 & $1,387.76$ & 694.38 & $1,386.77$ & 693.89 & 12 \\
\hline 9 & $1,212.52$ & 606.76 & $1,194.51$ & 597.76 & $\mathrm{E}$ & $1,307.73$ & 654.37 & $1,290.71$ & 645.86 & $1,289.72$ & 645.36 & 11 \\
\hline 10 & $1,325.60$ & 663.30 & $1,307.59$ & 654.30 & $\mathrm{~L}$ & $1,178.69$ & 589.85 & $1,161.66$ & 581.34 & $1,160.68$ & 580.84 & 10 \\
\hline 11 & $1,396.64$ & 698.82 & $1,378.63$ & 689.82 & $\mathrm{~A}$ & $1,065.61$ & 533.31 & $1,048.58$ & 524.79 & $1,047.60$ & 524.30 & 9 \\
\hline
\end{tabular}




\begin{tabular}{|r|r|r|r|r|r|r|r|r|r|r|r|r|}
12 & $1,467.68$ & 734.34 & $1,449.67$ & 725.34 & $\mathrm{~A}$ & 994.57 & 497.79 & 977.54 & 489.27 & 976.56 & 488.78 & 8 \\
\hline 13 & $1,568.72$ & 784.87 & $1,550.71$ & 775.86 & $\mathrm{~T}$ & 923.53 & 462.27 & 906.51 & 453.76 & 905.52 & 453.26 & 7 \\
\hline 14 & $1,681.81$ & 841.41 & $1,663.80$ & 832.40 & $\mathrm{I}$ & 822.48 & 411.75 & 805.46 & 403.23 & 804.47 & 402.74 & 6 \\
\hline 15 & $1,738.83$ & 869.92 & $1,720.82$ & 860.91 & $\mathrm{G}$ & 709.40 & 355.20 & 692.37 & 346.69 & 691.39 & 346.20 & 5 \\
\hline 16 & $1,837.90$ & 919.45 & $1,819.89$ & 910.45 & $\mathrm{~V}$ & 652.38 & 326.69 & 635.35 & 318.18 & 634.37 & 317.69 & 4 \\
\hline 17 & $1,952.92$ & 976.97 & $1,934.91$ & 967.96 & $\mathrm{D}$ & 553.31 & 277.16 & 536.28 & 268.65 & 535.30 & 268.15 & 3 \\
\hline 18 & $2,099.99$ & $1,050.50$ & $2,081.98$ & $1,041.49$ & $\mathrm{~F}$ & 438.28 & 219.65 & 421.26 & 211.13 & & & 2 \\
\hline 19 & & & & & $\mathrm{~K}$ & 291.21 & 146.11 & 274.19 & 137.60 & & & 1 \\
\hline
\end{tabular}

Query 98108 Hit 1

MS/MS Fragmentation of LNLQSVTEQSSLDDFLATAELAGTEFVAEK

Found in sp|Q9H089|LSG1_HUM AN, Large subunit GTPase 1 homolog OS=Homo sapiens GN=LSG1 PE=1 SV=2

Match to Query 98108: 3513.79from(879.4548,4+)

Title: 1283: Sum of 2 scans in range $2999(\mathrm{rt}=75.9602, \mathrm{f}=4, \mathrm{i}=849)$ to $3000(\mathrm{rt}=75.9856, \mathrm{f}=4, \mathrm{i}=850)$

[D:llab212 \membranelGrace JoyceliTRAQ 23 2.raw]

Data File:Submitted from 20120508-1(merge) by Mascot Daemon on JOYCE-VAIO

Monoisotopic mass of neutral peptide $\operatorname{Mr}($ calc): 3513.79

Variable modifications:

K30 :TRAQ4plex (K)

Ions Score: 47.94 Expect: 0.013

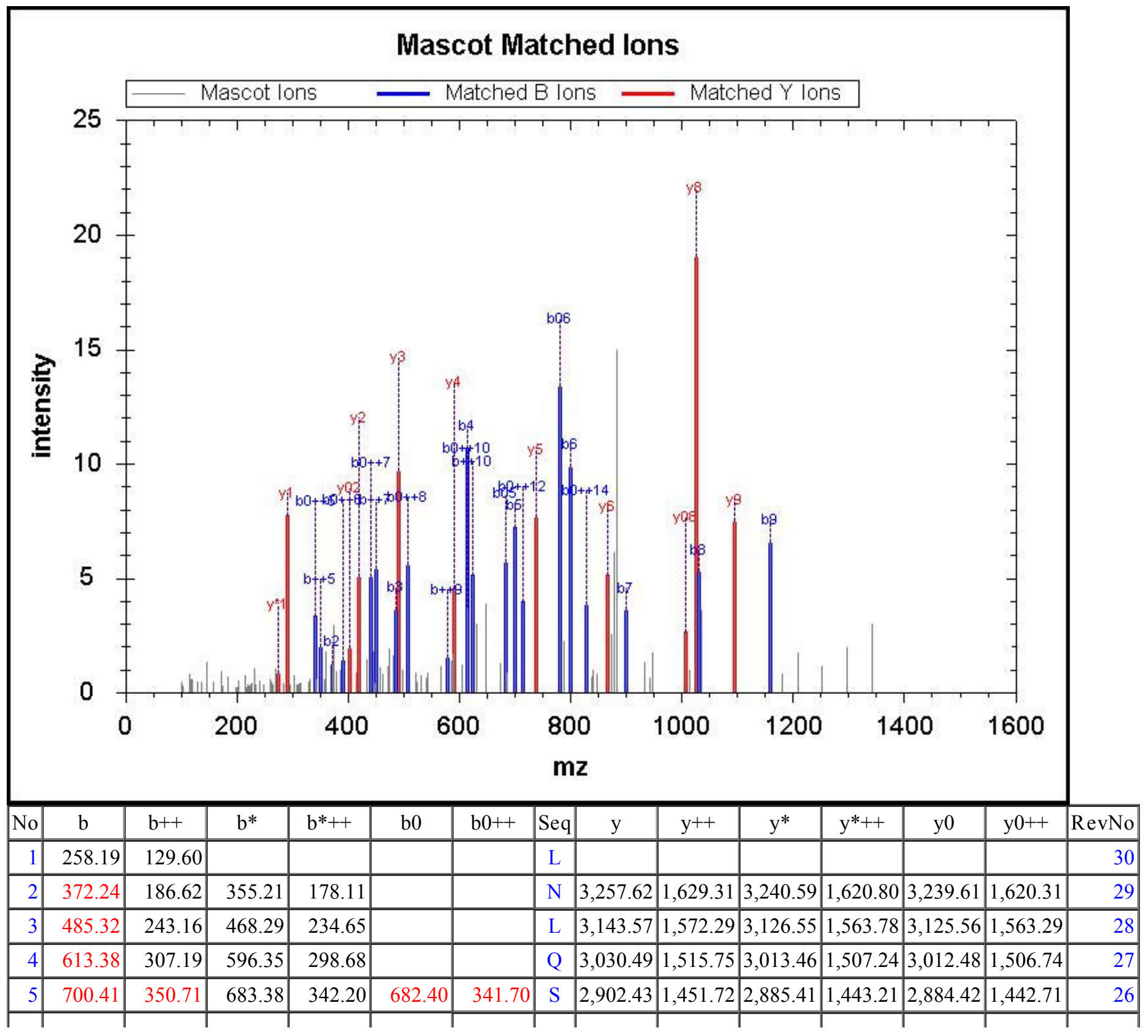




\begin{tabular}{|c|c|c|c|c|c|c|c|c|c|c|c|c|c|c|}
\hline 6 & 799.48 & 400.24 & 782.45 & 391.73 & $781.47 \mid$ & 391.24 & V & $2,815.40$ & $1,408.20 \mid$ & $|2,798.37|$ & $1,399.69$ & 2,7 & $\mid$ & \\
\hline 7 & 9 & 7 & 50 & & 2 & 6 & $\mathrm{~T}$ & 33 & 67 & 31 & 16 & 32 & 66 & 24 \\
\hline 8 & $1,029.57$ & 515.29 & $1,012.54$ & 506.78 & $1,011.56$ & 506.28 & $\mathrm{E}$ & $2,615.28$ & $1,308.15$ & $2,598.26$ & $1,299.63$ & $2,597.27$ & $\mid 1,299.14$ & 23 \\
\hline 9 & 763 & 579.32 & 4060 & 570.80 & 62 & 0.31 & Q & .24 & $1,243.62$ & $2,469.22$ & .11 & .23 & $1,234.62$ & 22 \\
\hline 10 & 1,24 & 3 & 1 & 61 & 1, & & $\mathrm{~S}$ & & & & & & & \\
\hline 11 & $1,331.69$ & 666.35 & $1,314.67$ & 657.84 & $1,313.68$ & & $\mathrm{~S}$ & & & 2,2 & 57 & .14 & .07 & \\
\hline 12 & $1,444.78$ & 2.89 & $1,427.75$ & 714.38 & .77 & .89 & $\mathrm{~L}$ & 12 & 56 & .09 & & .11 & .56 & \\
\hline 13 & 559.80 & 30.41 & 542.78 & 771.89 & .79 & 771.40 & $\mathrm{D}$ & 04 & 02 & 01 & 51 & .02 & .02 & \\
\hline 14 & 1 & 2 & 1 & 82 & 1, & 8 & $\mathrm{D}$ & 1 & 1 & 1 & 9 & & & \\
\hline 15 & 90 & 45 & 87 & 902.94 & 89 & 45 & $\mathrm{~F}$ & 98 & .99 & 1,8 & .48 & 1 , & .99 & \\
\hline 16 & ס & 68.00 & 917.90 & 959.48 & & & $\mathrm{~L}$ & & 847.46 & & & & & 15 \\
\hline 17 & 2 & 51 & 99 & 5.00 & 1 & 51 & A & 3 & 92 & 1 , & 40 & 82 & 781.91 & 14 \\
\hline 18 & $2,107.07$ & $1,054.04$ & $2,090.04$ & $1,045.52$ & $2,089.06$ & $1,045.03$ & $\mathrm{~T}$ & $1,509.79$ & 755.40 & $1,492.77$ & 746.89 & $1,491.78$ & 746.39 & \\
\hline 19 & $2,178.10$ & $1,089.56$ & $2,161.08$ & $1,081.04$ & .09 & 1,08 & A & 1,4 & 1.88 & .72 & 6.36 & .73 & 87 & \\
\hline 20 & 15 & .08 & $2,290.12$ & .56 & .14 & 07 & $\mathrm{E}$ & 1.11 & & 1,3 & .84 &.$/ 0$ & & 11 \\
\hline 21 & $2,420.23$ & $1,210.62$ & $2,403.20$ & $1,202.11$ & $2,402.22$ & $1,201.61$ & $\mathrm{~L}$ & $1,208.66$ & 604.84 & $1,191.64$ & 596.32 & $1,190.65$ & |.05| & 10 \\
\hline 22 & 491.27 & 246.14 & $2,474.24$ & $1,237.62$ & $2,473.26$ & $1,237.13$ & A & .58 & 3.29 & 1,0 & .78 & 7.57 & (x) & 9 \\
\hline 23 & 548.29 & $1,274.65$ & $2,531.26$ & $1,266.14$ & $2,530.28$ & $1,265.64$ & $\mathrm{G}$ & 1,02 & 512.78 & 1,00 & 26 & 1,0 & 77 & 8 \\
\hline 24 & 2,64 & 17 & 31 & 66 & 33 & 17 & $\mathrm{~T}$ & 52 & 26 & 50 & 75 & .51 & 26 & I \\
\hline 25 & $2,778.38$ & $1,389.69$ & $2,761.35$ & $1,381.18$ & $2,760.37$ & $1,380.69$ & $\mathrm{E}$ & 866.47 & 433.74 & 849.45 & 425.23 & 848.46 & 424.74 & D \\
\hline 26 & $2,925.45$ & $1,463.23$ & $2,908.42$ & $1,454.71$ & $2,907.44$ & $1,454.22$ & $\mathrm{~F}$ & 737.43 & 369.22 & 720.40 & 360.71 & 719.42 & 360.21 & 5 \\
\hline 27 & $3,024.52$ & $1,512.76$ & $3,007.49$ & $1,504.25$ & $3,006.51$ & $1,503.76$ & $\mathrm{~V}$ & 5 & 2 & 5 & 17 & 572.35 & \% & 4 \\
\hline 28 & $3,095.55$ & $1,548.28$ & 3,078 & $1,539.77$ & $3,07^{\prime}$ & $1,539.28$ & A & 9 & 2 & 27 & 4 & 473.28 & 7.15 & 3 \\
\hline 29 & $3,224.60$ & $1,612.80$ & $3,207.57$ & $1,604.29$ & $3,206.59$ & $1,603.80$ & $\mathrm{E}$ & 420.26 & 210.63 & 403.23 & 202.12 & 402.25 & 201.63 & 2 \\
\hline 30 & & & & & & & $\mathrm{~K}$ & 291.21 & 146.11 & 274.19 & 137.60 & & & 1 \\
\hline
\end{tabular}

Query 43237 Hit 1

MS/MS Fragmentation of EVTPVSSIPVETHR

Found in sp|Q9HD20|AT131_HUMAN, Probable cation-transporting ATPase 13A1 OS=Homo sapiens GN=ATP13A1 PE=1 SV=2 Match to Query 43237: 1693.913from(565.645,3+)

Title: 284 : Sum of 2 scans in range $886(\mathrm{rt}=28.4176, \mathrm{f}=4, \mathrm{f}=183)$ to $887(\mathrm{rt}=28.4431, \mathrm{f}=4, \mathrm{i}=184)$

[D:llab212 \membranelGrace JoyceliTRAQ_34_1.raw]

Data File:Submitted from 20120508-1(merge) by Mascot Daemon on JOYCE-VAIO

Monoisotopic mass of neutral peptide Mr(calc): 1693.913

Variable modifications:

Ions Score: 47.91 Expect: 0.014 


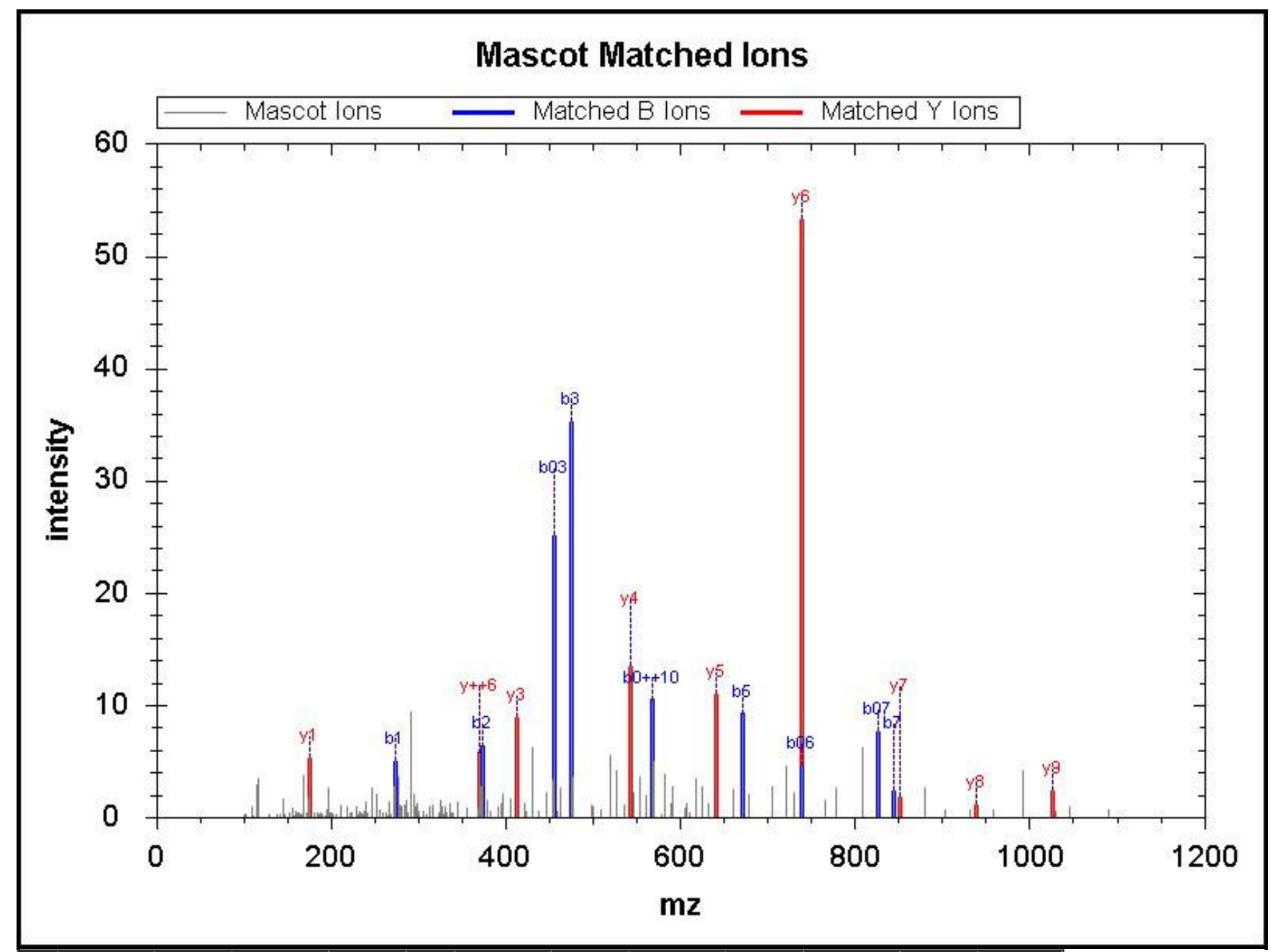

\begin{tabular}{|r|c|c|c|c|c|c|c|c|c|c|r|r|}
\hline \hline $\mathrm{No}$ & $\mathrm{b}$ & $\mathrm{b}++$ & $\mathrm{b} 0$ & $\mathrm{~b} 0++$ & $\mathrm{Seq}$ & $\mathrm{y}$ & $\mathrm{y}++$ & $\mathrm{y}^{*}$ & $\mathrm{y}{ }^{*++}$ & $\mathrm{y} 0$ & $\mathrm{y} 0++$ & $\mathrm{RevNo}$ \\
\hline 1 & 274.15 & 137.58 & 256.14 & 128.57 & $\mathrm{E}$ & & & & & & & 14 \\
\hline 2 & 373.22 & 187.11 & 355.21 & 178.11 & $\mathrm{~V}$ & $1,421.77$ & 711.39 & $1,404.75$ & 702.88 & $1,403.76$ & 702.39 & 13 \\
\hline 3 & 474.27 & 237.64 & 456.26 & 228.63 & $\mathrm{~T}$ & $1,322.71$ & 661.86 & $1,305.68$ & 653.34 & $1,304.70$ & 652.85 & 12 \\
\hline 4 & 571.32 & 286.16 & 553.31 & 277.16 & $\mathrm{P}$ & $1,221.66$ & 611.33 & $1,204.63$ & 602.82 & $1,203.65$ & 602.33 & 11 \\
\hline 5 & 670.39 & 335.70 & 652.38 & 326.69 & $\mathrm{~V}$ & $1,124.61$ & 562.81 & $1,107.58$ & 554.29 & $1,106.60$ & 553.80 & 10 \\
\hline 6 & 757.42 & 379.21 & 739.41 & 370.21 & $\mathrm{~S}$ & $1,025.54$ & 513.27 & $1,008.51$ & 504.76 & $1,007.53$ & 504.27 & 9 \\
\hline 7 & 844.45 & 422.73 & 826.44 & 413.72 & $\mathrm{~S}$ & 938.51 & 469.76 & 921.48 & 461.24 & 920.49 & 460.75 & 8 \\
\hline 8 & 957.54 & 479.27 & 939.53 & 470.27 & $\mathrm{I}$ & 851.47 & 426.24 & 834.45 & 417.73 & 833.46 & 417.24 & 7 \\
\hline 9 & $1,054.59$ & 527.80 & $1,036.58$ & 518.79 & $\mathrm{P}$ & 738.39 & 369.70 & 721.36 & 361.18 & 720.38 & 360.69 & 6 \\
\hline 10 & $1,153.66$ & 577.33 & $1,135.65$ & 568.33 & $\mathrm{~V}$ & 641.34 & 321.17 & 624.31 & 312.66 & 623.33 & 312.17 & 5 \\
\hline 11 & $1,282.70$ & 641.85 & $1,264.69$ & 632.85 & $\mathrm{E}$ & 542.27 & 271.64 & 525.24 & 263.12 & 524.26 & 262.63 & 4 \\
\hline 12 & $1,383.75$ & 692.38 & $1,365.74$ & 683.37 & $\mathrm{~T}$ & 413.23 & 207.12 & 396.20 & 198.60 & 395.21 & 198.11 & 3 \\
\hline 13 & $1,520.81$ & 760.91 & $1,502.80$ & 751.90 & $\mathrm{H}$ & 312.18 & 156.59 & 295.15 & 148.08 & & & 2 \\
\hline 14 & & & & & $\mathrm{R}$ & 175.12 & 88.06 & 158.09 & 79.55 & & & 1 \\
\hline
\end{tabular}

Query 64593 Hit 1

MS/MS Fragmentation of KFVFFNIPQIQYK

Found in sp|P82663|RT25_HUM AN, 28S ribosomal protein S25

Match to Query 64593: 2103.223from(702.0817,3+)

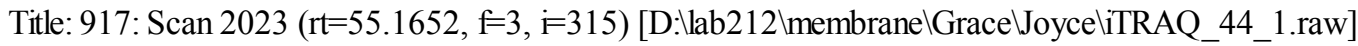

Data File:Submitted from 20120508-1(merge) by Mascot Daemon on JOYCE-VAIO

Monoisotopic mass of neutral peptide Mr(calc): 2103.223

Variable modifications:

$\mathrm{K} 1$ :iTRAQ4plex (K)

K13 :iTRAQ4plex (K)

Ions Score: 47.54 Expect: 0.007 


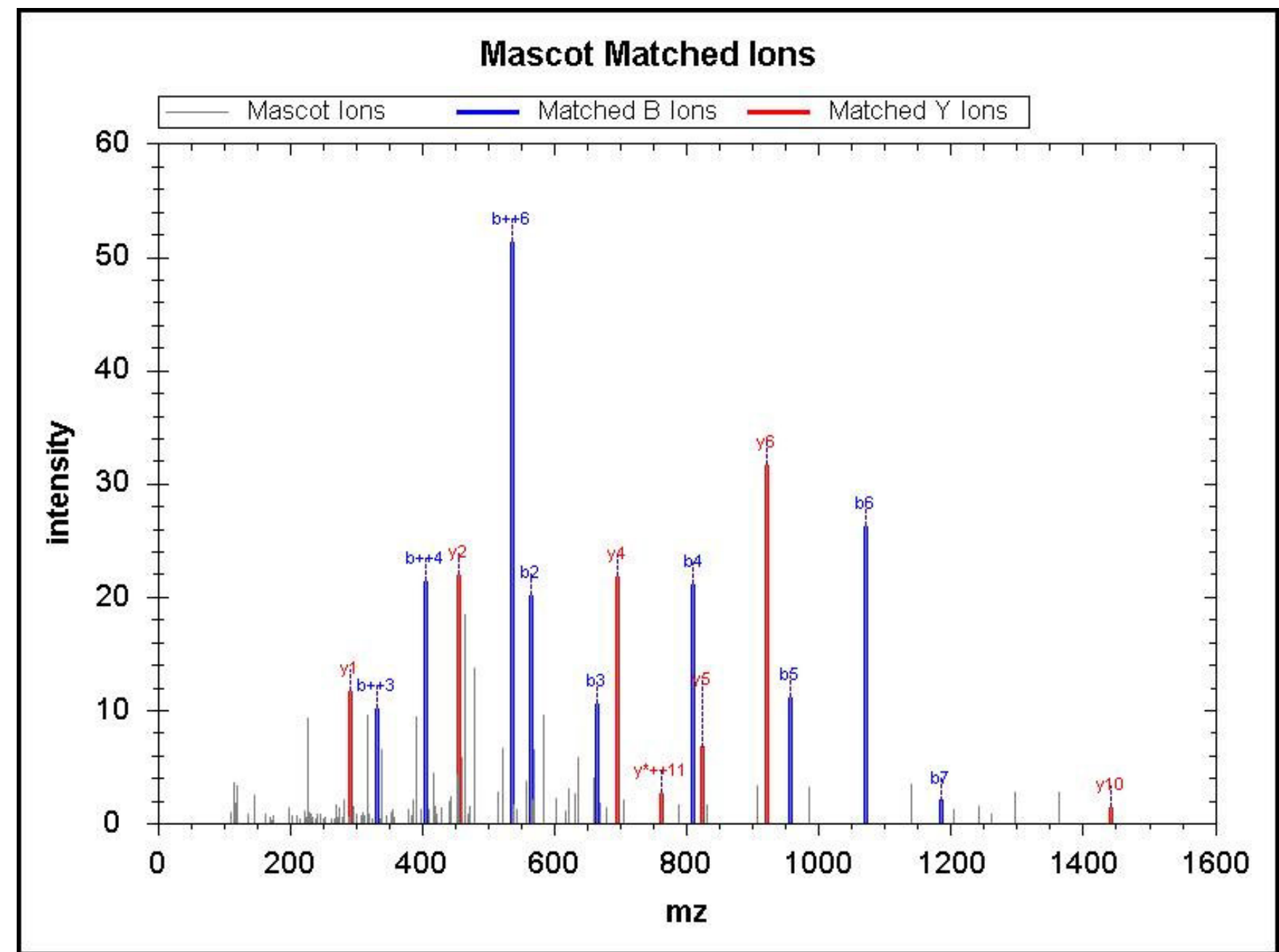

\begin{tabular}{|r|c|c|c|c|c|c|c|c|r|r|}
\hline No & $\mathrm{b}$ & $\mathrm{b}++$ & $\mathrm{b}^{*}$ & $\mathrm{~b}++$ & $\mathrm{Seq}$ & $\mathrm{y}$ & $\mathrm{y}++$ & $\mathrm{y}^{*}$ & $\mathrm{y}^{*++}$ & RevNo \\
\hline 1 & 417.31 & 209.16 & 400.28 & 200.64 & $\mathrm{~K}$ & & & & & 13 \\
\hline 2 & 564.37 & 282.69 & 547.35 & 274.18 & $\mathrm{~F}$ & $1,687.93$ & 844.47 & $1,670.91$ & 835.96 & 12 \\
\hline 3 & 663.44 & 332.23 & 646.42 & 323.71 & $\mathrm{~V}$ & $1,540.86$ & 770.94 & $1,523.84$ & 762.42 & 11 \\
\hline 4 & 810.51 & 405.76 & 793.49 & 397.25 & $\mathrm{~F}$ & $1,441.80$ & 721.40 & $1,424.77$ & 712.89 & 10 \\
\hline 5 & 957.58 & 479.29 & 940.55 & 470.78 & $\mathrm{~F}$ & $1,294.73$ & 647.87 & $1,277.70$ & 639.35 & 9 \\
\hline 6 & $1,071.62$ & 536.32 & $1,054.60$ & 527.80 & $\mathrm{~N}$ & $1,147.66$ & 574.33 & $1,130.63$ & 565.82 & 8 \\
\hline 7 & $1,184.71$ & 592.86 & $1,167.68$ & 584.34 & $\mathrm{I}$ & $1,033.62$ & 517.31 & $1,016.59$ & 508.80 & 7 \\
\hline 8 & $1,281.76$ & 641.38 & $1,264.73$ & 632.87 & $\mathrm{P}$ & 920.53 & 460.77 & 903.51 & 452.26 & 6 \\
\hline 9 & $1,409.82$ & 705.41 & $1,392.79$ & 696.90 & $\mathrm{Q}$ & 823.48 & 412.24 & 806.45 & 403.73 & 5 \\
\hline 10 & $1,522.90$ & 761.95 & $1,505.88$ & 753.44 & $\mathrm{I}$ & 695.42 & 348.21 & 678.39 & 339.70 & 4 \\
\hline 11 & $1,650.96$ & 825.98 & $1,633.93$ & 817.47 & $\mathrm{Q}$ & 582.34 & 291.67 & 565.31 & 283.16 & 3 \\
\hline 12 & $1,814.02$ & 907.52 & $1,797.00$ & 899.00 & $\mathrm{Y}$ & 454.28 & 227.64 & 437.25 & 219.13 & 2 \\
\hline 13 & & & & & $\mathrm{~K}$ & 291.21 & 146.11 & 274.19 & 137.60 & 1 \\
\hline
\end{tabular}

Query 45808 Hit 1

MS/MS Fragmentation of GM AFLHTLEPLIPR

Found in sp|Q13418|ILK_HUMAN, Integrin-linked protein kinase OS=Homo sapiens GN=ILK PE=1 SV=2

Match to Query 45808: 1737.972from(580.3312,3+)

Title: 845: Scan 2062 ( $\mathrm{rt}=54.911, \mathrm{f}=2, \mathrm{i}=297)$ [D:llab212 \membranelGracelJoyceliTRAQ_34_1.raw]

Data File:Submitted from 20120508-1(merge) by Mascot Daemon on JOYCE-VAIO

Monoisotopic mass of neutral peptide $\mathrm{Mr}$ (calc): 1737.972

Variable modifications:

Ions Score: 47.48 Expect: 0.013 


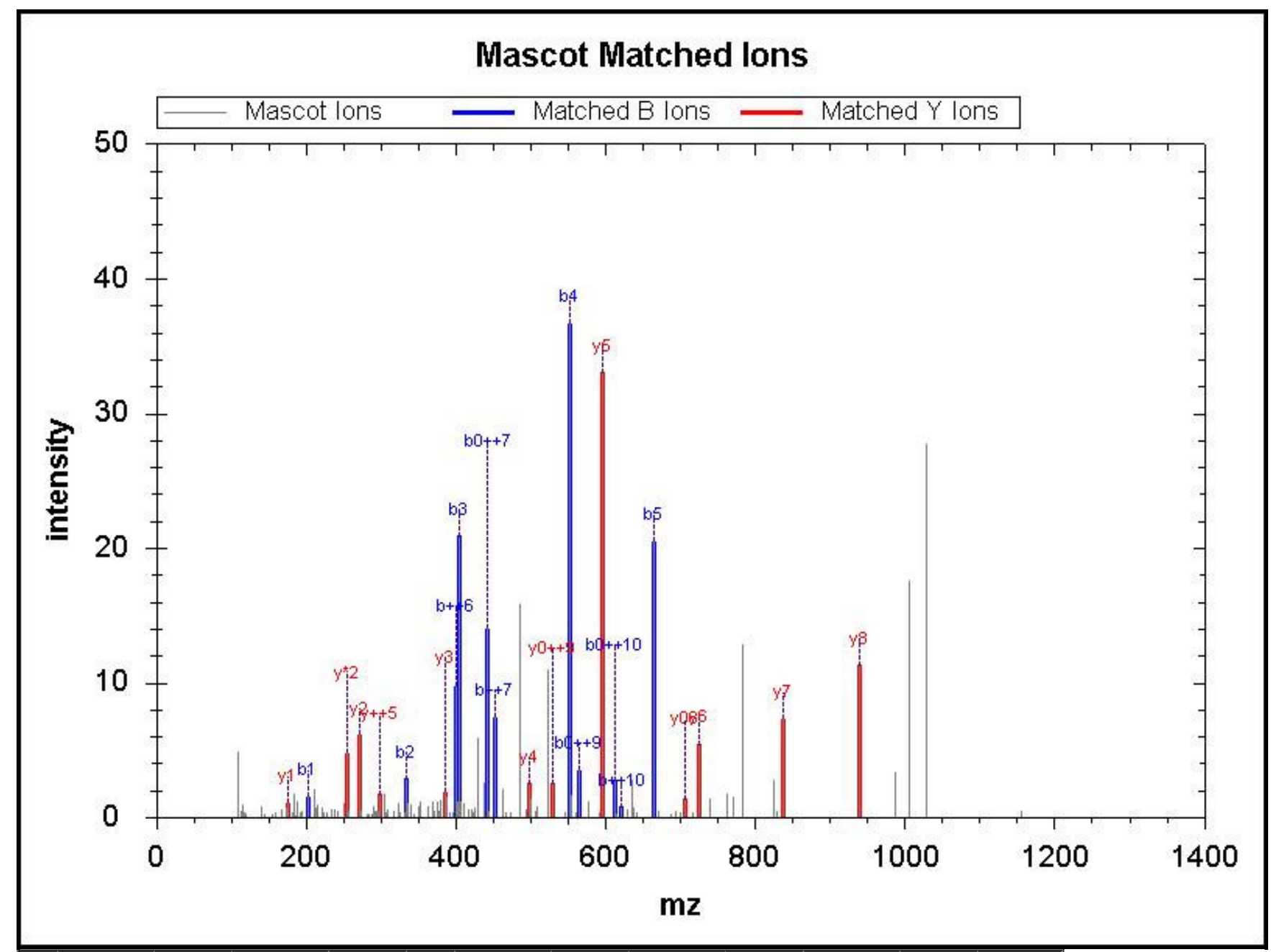

\begin{tabular}{|r|c|c|c|c|c|c|c|c|c|c|r|r|}
\hline \hline No & $\mathrm{b}$ & $\mathrm{b}++$ & $\mathrm{b} 0$ & $\mathrm{~b} 0++$ & $\mathrm{Seq}$ & $\mathrm{y}$ & $\mathrm{y}++$ & $\mathrm{y}^{*}$ & $\mathrm{y} *++$ & $\mathrm{y} 0$ & $\mathrm{y} 0++$ & RevNo \\
\hline 1 & 202.13 & 101.57 & & & $\mathrm{G}$ & & & & & & & 14 \\
\hline 2 & 333.17 & 167.09 & & & $\mathrm{M}$ & $1,537.86$ & 769.43 & $1,520.83$ & 760.92 & $1,519.85$ & 760.43 & 13 \\
\hline 3 & 404.21 & 202.61 & & & $\mathrm{~A}$ & $1,406.82$ & 703.91 & $1,389.79$ & 695.40 & $1,388.80$ & 694.91 & 12 \\
\hline 4 & 551.28 & 276.14 & & & $\mathrm{~F}$ & $1,335.78$ & 668.39 & $1,318.75$ & 659.88 & $1,317.77$ & 659.39 & 11 \\
\hline 5 & 664.36 & 332.68 & & & $\mathrm{~L}$ & $1,188.71$ & 594.86 & $1,171.68$ & 586.35 & $1,170.70$ & 585.85 & 10 \\
\hline 6 & 801.42 & 401.21 & & & $\mathrm{H}$ & $1,075.63$ & 538.32 & $1,058.60$ & 529.80 & $1,057.62$ & 529.31 & 9 \\
\hline 7 & 902.47 & 451.74 & 884.46 & 442.73 & $\mathrm{~T}$ & 938.57 & 469.79 & 921.54 & 461.27 & 920.56 & 460.78 & 8 \\
\hline 8 & $1,015.55$ & 508.28 & 997.54 & 499.27 & $\mathrm{~L}$ & 837.52 & 419.26 & 820.49 & 410.75 & 819.51 & 410.26 & 7 \\
\hline 9 & $1,144.59$ & 572.80 & $1,126.58$ & 563.80 & $\mathrm{E}$ & 724.44 & 362.72 & 707.41 & 354.21 & 706.42 & 353.72 & 6 \\
\hline 10 & $1,241.65$ & 621.33 & $1,223.64$ & 612.32 & $\mathrm{P}$ & 595.39 & 298.20 & 578.37 & 289.69 & & & 5 \\
\hline 11 & $1,354.73$ & 677.87 & $1,336.72$ & 668.86 & $\mathrm{~L}$ & 498.34 & 249.67 & 481.31 & 241.16 & & & 4 \\
\hline 12 & $1,467.82$ & 734.41 & $1,449.80$ & 725.41 & $\mathrm{I}$ & 385.26 & 193.13 & 368.23 & 184.62 & & & 3 \\
\hline 13 & $1,564.87$ & 782.94 & $1,546.86$ & 773.93 & $\mathrm{P}$ & 272.17 & 136.59 & 255.15 & 128.08 & & & 2 \\
\hline 14 & & & & & $\mathrm{R}$ & 175.12 & 88.06 & 158.09 & 79.55 & & & 1 \\
\hline
\end{tabular}

Query 49743 Hit 1

MS/MS Fragmentation of EFLDDLFSFMQK

Found in sp|Q99856|ARI3A_HUM AN, AT-rich interactive domain-containing protein 3A OS=Homo sapiens GN=ARID3A PE=1 SV=2 Match to Query 49743: 1806.914from(603.3121,3+)

Title: 1259: Scan 2793 ( $\mathrm{rt}=72.2436, \mathrm{f}=3, \mathrm{i}=435)$ [D:llab212 \membranelGrace JoyceliTRAQ_27_1.raw]

Data File:Submitted from 20120508-1(merge) by Mascot Daemon on JOYCE-VAIO

Monoisotopic mass of neutral peptide Mr(calc): 1806.914

Variable modifications:

K12 :iTRAQ4plex (K)

Ions Score: 47.46 Expect: 0.016 


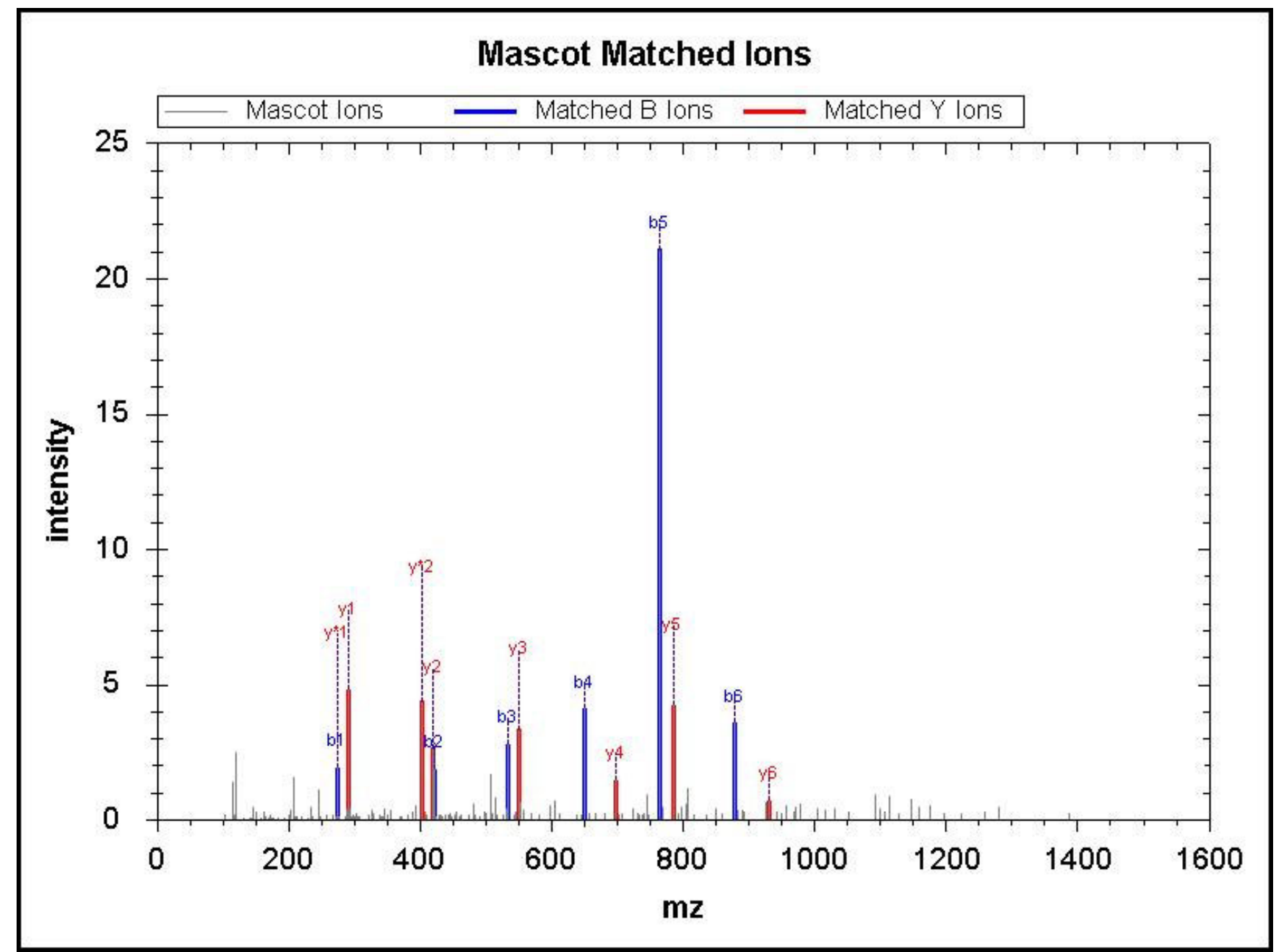

\begin{tabular}{|r|c|c|c|c|c|c|c|c|c|c|c|r|r|r|}
\hline \hline $\mathrm{No}$ & $\mathrm{b}$ & $\mathrm{b}++$ & $\mathrm{b}$ & $\mathrm{b}++$ & $\mathrm{b} 0$ & $\mathrm{~b} 0++$ & Seq & $\mathrm{y}$ & $\mathrm{y}++$ & $\mathrm{y}^{*}$ & $\mathrm{y}^{*++}$ & $\mathrm{y} 0$ & $\mathrm{y} 0++$ & $\mathrm{RevNo}$ \\
\hline 1 & 274.15 & 137.58 & & & 256.14 & 128.57 & $\mathrm{E}$ & & & & & & & 12 \\
\hline 2 & 421.22 & 211.11 & & & 403.21 & 202.11 & $\mathrm{~F}$ & $1,534.77$ & 767.89 & $1,517.75$ & 759.38 & $1,516.76$ & 758.89 & 11 \\
\hline 3 & 534.30 & 267.66 & & & 516.29 & 258.65 & $\mathrm{~L}$ & $1,387.70$ & 694.36 & $1,370.68$ & 685.84 & $1,369.69$ & 685.35 & 10 \\
\hline 4 & 649.33 & 325.17 & & & 631.32 & 316.16 & $\mathrm{D}$ & $1,274.62$ & 637.81 & $1,257.59$ & 629.30 & $1,256.61$ & 628.81 & 9 \\
\hline 5 & 764.36 & 382.68 & & & 746.35 & 373.68 & $\mathrm{D}$ & $1,159.59$ & 580.30 & $1,142.57$ & 571.79 & $1,141.58$ & 571.30 & 8 \\
\hline 6 & 877.44 & 439.22 & & & 859.43 & 430.22 & $\mathrm{~L}$ & $1,044.57$ & 522.79 & $1,027.54$ & 514.27 & $1,026.56$ & 513.78 & 7 \\
\hline 7 & $1,024.51$ & 512.76 & & & $1,006.50$ & 503.75 & $\mathrm{~F}$ & 931.48 & 466.25 & 914.46 & 457.73 & 913.47 & 457.24 & 6 \\
\hline 8 & $1,111.54$ & 556.28 & & & $1,093.53$ & 547.27 & S & 784.41 & 392.71 & 767.39 & 384.20 & 766.40 & 383.71 & 5 \\
\hline 9 & $1,258.61$ & 629.81 & & & $1,240.60$ & 620.80 & F & 697.38 & 349.19 & 680.36 & 340.68 & & & 4 \\
\hline 10 & $1,389.65$ & 695.33 & & & $1,371.64$ & 686.32 & M & 550.31 & 275.66 & 533.29 & 267.15 & & & 3 \\
\hline 11 & $1,517.71$ & 759.36 & $1,500.68$ & 750.85 & $1,499.70$ & 750.35 & Q & 419.27 & 210.14 & 402.25 & 201.63 & & & 2 \\
\hline 12 & & & & & & & K & 291.21 & 146.11 & 274.19 & 137.60 & & & 1 \\
\hline
\end{tabular}

Query 89673 Hit 1

\section{MS/MS Fragmentation of QEELGDLVDKEMAATSAAIETATAR}

Found in sp|O00291|HIP1_HUM AN, Huntingtin-interacting protein 1 OS=Homo sapiens GN=HIP1 PE=1 SV=5

Match to Query 89673: 2907.476from(727.8762,4+)

Title: 1002: Sum of 2 scans in range 2394 ( $\mathrm{rt}=62.3887, \mathrm{f}=4, \mathrm{i}=663)$ to $2395(\mathrm{rt}=62.4141, \mathrm{f}=4, \mathrm{i}=664)$

[D:llab212 \membranelGrace JoyceliTRAQ_34_1.raw]

Data File:Submitted from 20120508-1(merge) by Mascot Daemon on JOYCE-VAIO

Monoisotopic mass of neutral peptide Mr(calc): 2907.476

Variable modifications:

K10 :iTRAQ4plex (K)

Ions Score: 47.36 Expect: 0.017 
Mascot Matched lons

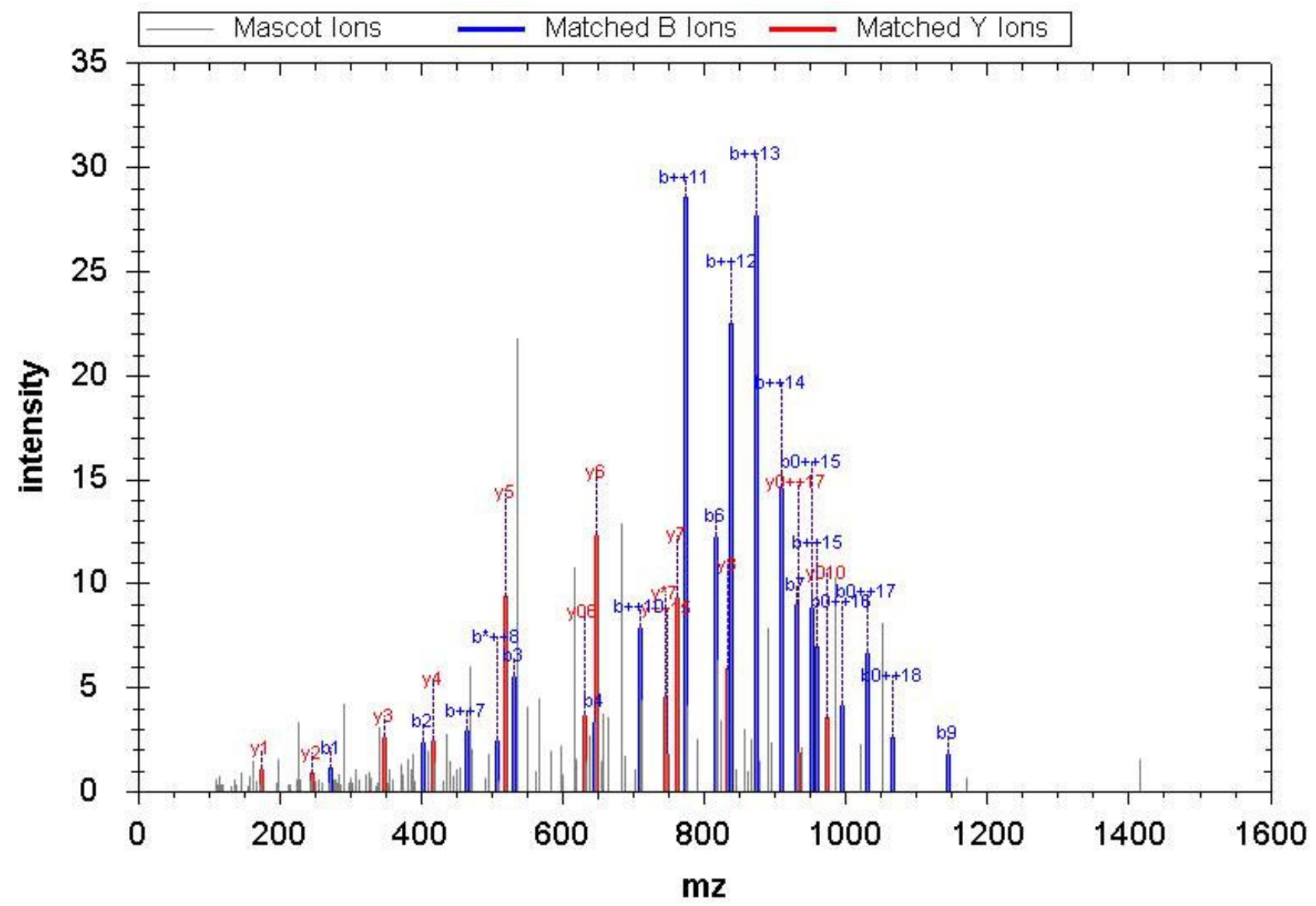

\begin{tabular}{|c|c|c|c|c|c|c|c|c|c|c|c|c|c|c|}
\hline No & $\mathrm{b}$ & $\mathrm{b}++$ & $b^{*}$ & $b^{*++}$ & b0 & $\mathrm{b} 0++$ & Seq & $\mathrm{y}$ & $\mathrm{y}++$ & $y^{*}$ & $y^{*++}$ & y0 & $\mathrm{y} 0++$ & RevNo \\
\hline 1 & 273.17 & 137.09 & 256.14 & 128.57 & & & Q & & & & & & & 2 \\
\hline 2 & 402.21 & 201.61 & 385.18 & 193.10 & 384.20 & 192.60 & $\mathrm{E}$ & $2,636.32$ & $1,318.66$ & $2,619.29$ & $1,310.15$ & $2,618.31$ & $1,309.66$ & 24 \\
\hline 3 & 531.25 & 266.13 & 514.23 & 257.62 & 513.24 & 257.12 & $\mathrm{E}$ & $2,507.28$ & $1,254.14$ & $2,490.25$ & $1,245.63$ & $2,489.27$ & $1,245.14$ & 23 \\
\hline 4 & 644.34 & 322.67 & 627.31 & 314.16 & 626.33 & 313.67 & $\mathrm{~L}$ & 8.24 & 9.62 & 21 & & .22 & 2 & 22 \\
\hline 5 & 701.36 & 351.18 & 684.33 & 342.67 & 683.35 & 342.18 & G & $2,265.15$ & $1,133.08$ & $2,248.12$ & $1,124.57$ & $2,247.14$ & $1,124.07$ & 21 \\
\hline 6 & 816.39 & 408.70 & 799.36 & 400.18 & 798.38 & 399.69 & $\mathrm{D}$ & $2,208.13$ & $1,104.57$ & $2,191.10$ & $1,096.06$ & $2,190.12$ & $1,095.56$ & 20 \\
\hline 7 & 929.47 & 465.24 & 912.44 & 456.73 & 911.46 & 456.23 & $\mathrm{~L}$ & $2,093.10$ & $1,047.06$ & $2,076.08$ & 8.54 & $2,075.09$ & $1,038.05$ & 19 \\
\hline 8 & $1,028.54$ & 514.77 & $1,011.51$ & 506.26 & $1,010.53$ & 505.77 & V & $1,980.02$ & 990.51 & 9 & 0 & 01 & .51 & 18 \\
\hline 9 & $1,143.57$ & 572.29 & $1,126.54$ & 563.77 & $1,125.55$ & 563.28 & $\mathrm{D}$ & $1,880.95$ & 940.98 & .92 & 932.47 & $1,862.94$ & 931.97 & 17 \\
\hline 10 & $1,415.76$ & 708.38 & $1,398.74$ & 699.87 & $1,397.75$ & 699.38 & K & $1,765.92$ & 883.47 & $1,748.90$ & 874.95 & $1,747.91$ & 874.46 & 16 \\
\hline 11 & $1,544.80$ & 772.91 & $1,527.78$ & 764.39 & $1,526.79$ & 763.90 & E & $1,493.73$ & 747.37 & $1,476.70$ & 738.85 & $1,475.72$ & 738.36 & 15 \\
\hline 12 & $1,675.85$ & 838.43 & $1,658.82$ & 829.91 & $1,657.83$ & 829.42 & $\mathrm{M}$ & $1,364.68$ & 682.85 & $1,347.66$ & 674.33 & $1,346.67$ & 673.84 & 14 \\
\hline 13 & $1,746.88$ & 873.94 & $1,729.86$ & 865.43 & $1,728.87$ & 864.94 & A & $1,233.64$ & 617.33 & $1,216.62$ & 608.81 & $1,215.63$ & 608.32 & 13 \\
\hline 14 & $1,817.92$ & 909.46 & $1,800.89$ & 900.95 & $1,799.91$ & 900.46 & A & $1,162.61$ & 581.81 & $1,145.58$ & 573.29 & $1,144.60$ & 572.80 & 12 \\
\hline 15 & $1,918.97$ & 959.99 & $1,901.94$ & 951.47 & $1,900.96$ & 950.98 & $\mathrm{~T}$ & $1,091.57$ & 546.29 & $1,074.54$ & 537.77 & $1,073.56$ & 537.28 & 11 \\
\hline 16 & $2,006.00$ & $1,003.50$ & $1,988.97$ & 994.99 & $1,987.99$ & 994.50 & $\mathrm{~S}$ & 990.52 & 495.76 & 973.49 & 487.25 & 972.51 & 486.76 & 10 \\
\hline 17 & $2,077.04$ & $1,039.02$ & $2,060.01$ & 30.51 & $2,059.03$ & $1,030.02$ & A & .49 & .25 & 886.46 & 74 & 885.48 & 443.24 & 9 \\
\hline 18 & $2,148.07$ & $1,074.54$ & $2,131.05$ & $1,066.03$ & $2,130.06$ & $1,065.54$ & A & 832.45 & 416.73 & 815.43 & 408.22 & 814.44 & 407.72 & 8 \\
\hline 19 & $2,261.16$ & $1,131.08$ & $2,244.13$ & $1,122.57$ & $2,243.15$ & $1,122.08$ & I & 761.42 & 381.21 & 744.39 & 372.70 & 743.40 & 372.21 & 7 \\
\hline 20 & $2,390.20$ & $1,195.60$ & $2,373.17$ & $1,187.09$ & $2,372.19$ & $1,186.60$ & $\mathrm{E}$ & 648.33 & 324.67 & 631.30 & 316.16 & 630.32 & 315.66 & 6 \\
\hline 21 & $2,491.25$ & $1,246.13$ & $2,474.22$ & $1,237.61$ & $2,473.24$ & $1,237.12$ & $\mathrm{~T}$ & 519.29 & 260.15 & 502.26 & 251.63 & 501.28 & 251.14 & 5 \\
\hline 22 & $2,562.28$ & $1,281.65$ & $2,545.26$ & $1,273.13$ & $2,544.27$ & $1,272.64$ & A & 418.24 & 209.62 & 401.21 & 201.11 & 400.23 & 200.62 & 4 \\
\hline 23 & $2,663.33$ & $1,332.17$ & $2,646.31$ & $1,323.66$ & $2,645.32$ & $1,323.16$ & $\mathrm{~T}$ & 347.20 & 174.11 & 330.18 & 165.59 & 329.19 & 165.10 & 3 \\
\hline
\end{tabular}




\begin{tabular}{|r|r|r|r|r|r|r|r|r|r|r|r|r|r|r|}
\hline 24 & $2,734.37$ & $1,367.69$ & $2,717.34$ & $1,359.18$ & $2,716.36$ & $1,358.68$ & $\mathrm{~A}$ & 246.16 & 123.58 & 229.13 & 115.07 & & & 2 \\
\hline 25 & & & & & & $\mathrm{R}$ & 175.12 & 88.06 & 158.09 & 79.55 & & & 1 \\
\hline
\end{tabular}

Query 75283 Hit 1

MS/MS Fragmentation of GR PITMFIPSDVDNYDDIR

Found in sp|Q9HC35|EM AL4_HUMAN, Echinoderm microtubule-associated protein-like 4 OS=Homo sapiens GN=EML4 PE=1 SV=3 Match to Query 75283: 2367.161 from(790.061,3+)

Title: 738: Sum of 2 scans in range $1668(\mathrm{rt}=46.9589, \mathrm{f}=4, \mathrm{i}=495)$ to $1669(\mathrm{rt}=46.9843, \mathrm{f}=4, \mathrm{i}=496)$

[D:llab212 \membranelGrace JoyceliTRAQ_35_1.raw]

Data File:Submitted from 20120508-1(merge) by Mascot Daemon on JOYCE-VAIO

Monoisotopic mass of neutral peptide $\operatorname{Mr}($ calc): 2367.161

Variable modifications:

Ions Score: 47.29 Expect: 0.017

\section{Mascot Matched lons}

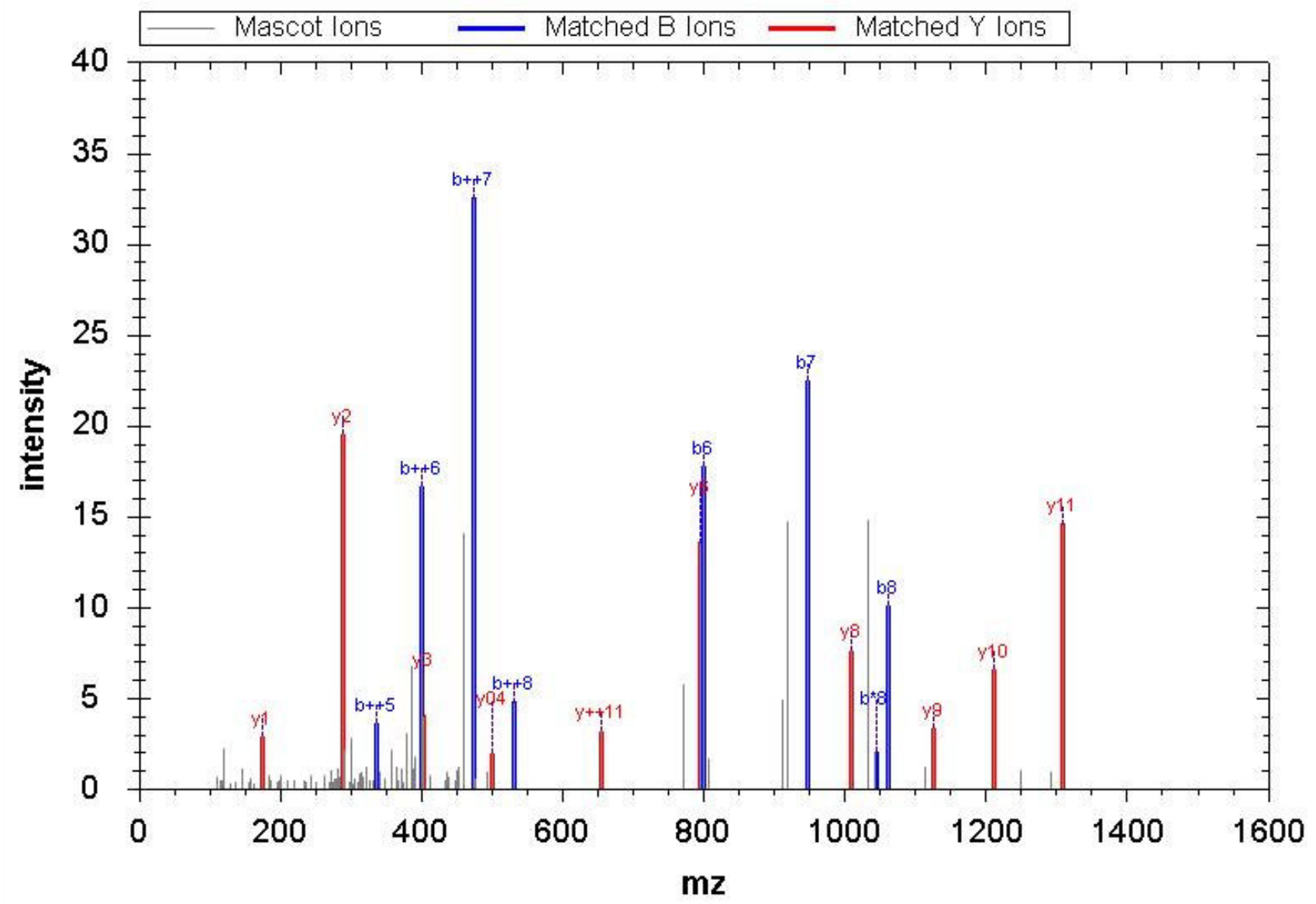

\begin{tabular}{|c|c|c|c|c|c|c|c|c|c|c|c|c|c|c|}
\hline No & $\mathrm{b}$ & $\mathrm{b}++$ & $b^{*}$ & $b^{*++}$ & b0 & $\mathrm{b} 0++$ & Seq & $\mathrm{y}$ & $\mathrm{y}++$ & $\mathrm{y}^{*}$ & $y^{*++}$ & y0 & $\mathrm{y} 0++$ & RevNo \\
\hline 1 & 202.13 & 101.57 & & & & & $\mathrm{G}$ & & & & & & & 19 \\
\hline 2 & 358.23 & 179.62 & 341.21 & 171.11 & & & $\mathrm{R}$ & $2,167.05$ & $1,084.03$ & $2,150.02$ & $1,075.51$ & $2,149.04$ & $1,075.02$ & 18 \\
\hline 3 & 455.28 & 228.15 & 438.26 & 219.63 & & & $\mathrm{P}$ & $2,010.95$ & $1,005.98$ & $1,993.92$ & 997.46 & $1,992.94$ & 996.97 & 17 \\
\hline 4 & 568.37 & 284.69 & 551.34 & 276.17 & & & I & $1,913.90$ & 957.45 & $1,896.87$ & 948.94 & $1,895.88$ & 948.45 & 16 \\
\hline 5 & 669.42 & 335.21 & 652.39 & 326.70 & 651.41 & 326.21 & $\mathrm{~T}$ & $1,800.81$ & 900.91 & $1,783.78$ & 892.40 & $1,782.80$ & 891.90 & 15 \\
\hline 6 & 800.46 & 400.73 & 783.43 & 392.22 & 782.45 & 391.73 & M & $1,699.76$ & 850.39 & $1,682.74$ & 841.87 & $1,681.75$ & 841.38 & 14 \\
\hline 7 & 947.53 & 474.27 & 930.50 & 465.75 & 929.51 & 465.26 & $\mathrm{~F}$ & $1,568.72$ & 784.86 & $1,551.70$ & 776.35 & $1,550.71$ & 775.86 & 13 \\
\hline 8 & $1,060.61$ & 530.81 & $1,043.58$ & 522.30 & $1,042.60$ & 521.80 & I & $1,421.65$ & 711.33 & $1,404.63$ & 702.82 & $1,403.64$ & 702.33 & 12 \\
\hline 9 & $1,157.66$ & 579.33 & $1,140.64$ & 570.82 & $1,139.65$ & 570.33 & $\mathrm{P}$ & $1,308.57$ & 654.79 & $1,291.54$ & 646.28 & $1,290.56$ & 645.78 & 11 \\
\hline 10 & $1,244.69$ & 622.85 & $1,227.67$ & 614.34 & $1,226.68$ & 613.85 & $\mathrm{~S}$ & $1,211.52$ & 606.26 & $1,194.49$ & 597.75 & $1,193.51$ & 597.26 & 10 \\
\hline 11 & $1,359.72$ & 680.36 & $1,342.69$ & 671.85 & $1,341.71$ & 671.36 & $\mathrm{D}$ & $1,124.49$ & 562.75 & $1,107.46$ & 554.23 & $1,106.47$ & 553.74 & 9 \\
\hline
\end{tabular}




\begin{tabular}{|r|r|r|r|r|r|r|r|r|r|r|r|r|r|r|}
\hline 12 & $1,458.79$ & 729.90 & $1,441.76$ & 721.39 & $1,440.78$ & 720.89 & $\mathrm{~V}$ & $1,009.46$ & 505.23 & 992.43 & 496.72 & 991.45 & 496.23 & 8 \\
\hline 13 & $1,573.82$ & 787.41 & $1,556.79$ & 778.90 & $1,555.81$ & 778.41 & $\mathrm{D}$ & 910.39 & 455.70 & 893.36 & 447.19 & 892.38 & 446.69 & 7 \\
\hline 14 & $1,687.86$ & 844.43 & $1,670.83$ & 835.92 & $1,669.85$ & 835.43 & $\mathrm{~N}$ & 795.36 & 398.19 & 778.34 & 389.67 & 777.35 & 389.18 & 6 \\
\hline 15 & $1,850.92$ & 925.97 & $1,833.90$ & 917.45 & $1,832.91$ & 916.96 & $\mathrm{Y}$ & 681.32 & 341.16 & 664.29 & 332.65 & 663.31 & 332.16 & 5 \\
\hline 16 & $1,965.95$ & 983.48 & $1,948.92$ & 974.97 & $1,947.94$ & 974.47 & $\mathrm{D}$ & 518.26 & 259.63 & 501.23 & 251.12 & 500.25 & 250.63 & 4 \\
\hline 17 & $2,080.98$ & $1,040.99$ & $2,063.95$ & $1,032.48$ & $2,062.97$ & $1,031.99$ & $\mathrm{D}$ & 403.23 & 202.12 & 386.20 & 193.61 & 385.22 & 193.11 & 3 \\
\hline 18 & $2,194.06$ & $1,097.53$ & $2,177.03$ & $1,089.02$ & $2,176.05$ & $1,088.53$ & $\mathrm{I}$ & 288.20 & 144.61 & 271.18 & 136.09 & & & 2 \\
\hline 19 & & & & & & & $\mathrm{R}$ & 175.12 & 88.06 & 158.09 & 79.55 & & & 1 \\
\hline
\end{tabular}

Query 62204 Hit 1

MS/MS Fragmentation of ELEELLESALDDFDK

Found in sp|P40855|PEX19_HUM AN, Peroxisomal biogenesis factor 19 OS=Homo sapiens GN=PEX19 PE=1 SV=1 Match to Query 62204: 2053.04from(685.354,3+)

Title: 1332: Sum of 2 scans in range 2953 ( $\mathrm{rt}=75.8217, \mathrm{f}=4, \mathrm{i}=893)$ to 2954 ( $\mathrm{rt}=75.8471, \mathrm{f}=4, \mathrm{i}=894)$

[D:llab212 \membranelGracelJoyceliTRAQ_27_1.raw]

Data File:Submitted from 20120508-1(merge) by Mascot Daemon on JOYCE-VAIO

Monoisotopic mass of neutral peptide $\mathrm{Mr}$ (calc): 2053.04

Variable modifications:

K15 :TRAQ4plex (K)

Ions Score: 47.27 Expect: 0.018

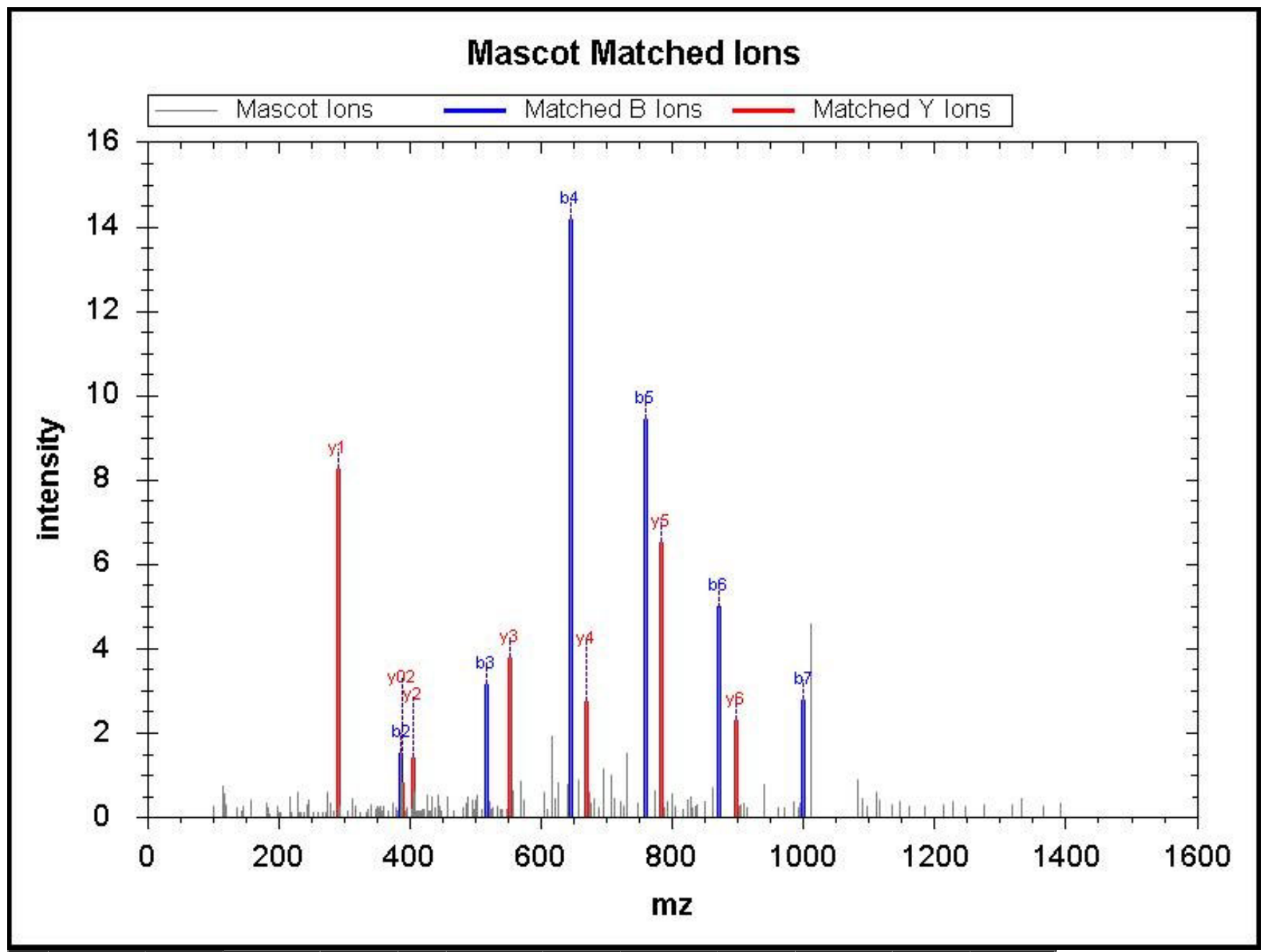

\begin{tabular}{|r|c|c|c|c|c|c|c|c|c|c|r|r|}
\hline No & $\mathrm{b}$ & $\mathrm{b}++$ & $\mathrm{b} 0$ & $\mathrm{~b} 0++$ & Seq & $\mathrm{y}$ & $\mathrm{y}++$ & $\mathrm{y}^{*}$ & $\mathrm{y}^{*++}$ & $\mathrm{y} 0$ & $\mathrm{y} 0++$ & RevNo \\
\hline 1 & 274.15 & 137.58 & 256.14 & 128.57 & $\mathrm{E}$ & & & & & & & 15 \\
\hline 2 & 387.24 & 194.12 & 369.23 & 185.12 & $\mathrm{~L}$ & $1,780.90$ & 890.95 & $1,763.87$ & 882.44 & $1,762.89$ & 881.95 & 14 \\
\hline 3 & 516.28 & 258.64 & 498.27 & 249.64 & $\mathrm{E}$ & $1,667.81$ & 834.41 & $1,650.79$ & 825.90 & $1,649.80$ & 825.40 & 13 \\
\hline 4 & 645.32 & 323.16 & 627.31 & 314.16 & $\mathrm{E}$ & $1,538.77$ & 769.89 & $1,521.74$ & 761.38 & $1,520.76$ & 760.88 & 12 \\
\hline & & & & & & & & & & & &
\end{tabular}




\begin{tabular}{|r|r|r|r|r|r|r|r|r|r|r|r|r|r|r|}
\hline 5 & 758.41 & 379.71 & 740.39 & 370.70 & $\mathrm{~L}$ & $1,409.73$ & 705.37 & $1,392.70$ & 696.85 & $1,391.72$ & 696.36 & 11 \\
\hline 6 & 871.49 & 436.25 & 853.48 & 427.24 & $\mathrm{~L}$ & $1,296.64$ & 648.83 & $1,279.62$ & 640.31 & $1,278.63$ & 639.82 & 10 \\
\hline 7 & $1,000.53$ & 500.77 & 982.52 & 491.76 & $\mathrm{E}$ & $1,183.56$ & 592.28 & $1,166.53$ & 583.77 & $1,165.55$ & 583.28 & 9 \\
\hline 8 & $1,087.56$ & 544.29 & $1,069.55$ & 535.28 & $\mathrm{~S}$ & $1,054.52$ & 527.76 & $1,037.49$ & 519.25 & $1,036.51$ & 518.76 & 8 \\
\hline 9 & $1,158.60$ & 579.80 & $1,140.59$ & 570.80 & $\mathrm{~A}$ & 967.49 & 484.25 & 950.46 & 475.73 & 949.47 & 475.24 & 7 \\
\hline 10 & $1,271.69$ & 636.35 & $1,253.67$ & 627.34 & $\mathrm{~L}$ & 896.45 & 448.73 & 879.42 & 440.21 & 878.44 & 439.72 & 6 \\
\hline 11 & $1,386.71$ & 693.86 & $1,368.70$ & 684.85 & $\mathrm{D}$ & 783.36 & 392.19 & 766.34 & 383.67 & 765.35 & 383.18 & 5 \\
\hline 12 & $1,501.74$ & 751.37 & $1,483.73$ & 742.37 & $\mathrm{D}$ & 668.34 & 334.67 & 651.31 & 326.16 & 650.33 & 325.67 & 4 \\
\hline 13 & $1,648.81$ & 824.91 & $1,630.80$ & 815.90 & $\mathrm{~F}$ & 553.31 & 277.16 & 536.28 & 268.65 & 535.30 & 268.15 & 3 \\
\hline 14 & $1,763.83$ & 882.42 & $1,745.82$ & 873.42 & $\mathrm{D}$ & 406.24 & 203.62 & 389.22 & 195.11 & 388.23 & 194.62 & 2 \\
\hline 15 & & & & & $\mathrm{~K}$ & 291.21 & 146.11 & 274.19 & 137.60 & & & 1 \\
\hline
\end{tabular}

Query 65671 Hit 1

MS/MS Fragmentation of VITSAWWVYHHQVSK

Found in sp|O60524|NEMF_HUMAN, Nuclear export mediator factor NEMF OS=Homo sapiens GN=NEMF PE=1 SV=4 Match to Query 65671:2128.071from(710.3641,3+)

Title: 603: Sum of 2 scans in range 1323 ( $\mathrm{rt}=39.496, \mathrm{f}=4, \mathrm{i}=403)$ to $1324(\mathrm{rt}=39.5214, \mathrm{f}=4, \mathrm{i}=404)$

[D:lab212 \membranelGrace JoyceliTRAQ_50_2.raw]

Data File:Submitted from 20120508-1(merge) by Mascot Daemon on JOYCE-VAIO

Monoisotopic mass of neutral peptide $\operatorname{Mr}(\mathrm{calc}): 2128.071$

Variable modifications:

K15 :TRAQ4plex (K)

Ions Score: 47.24 Expect: 0.018

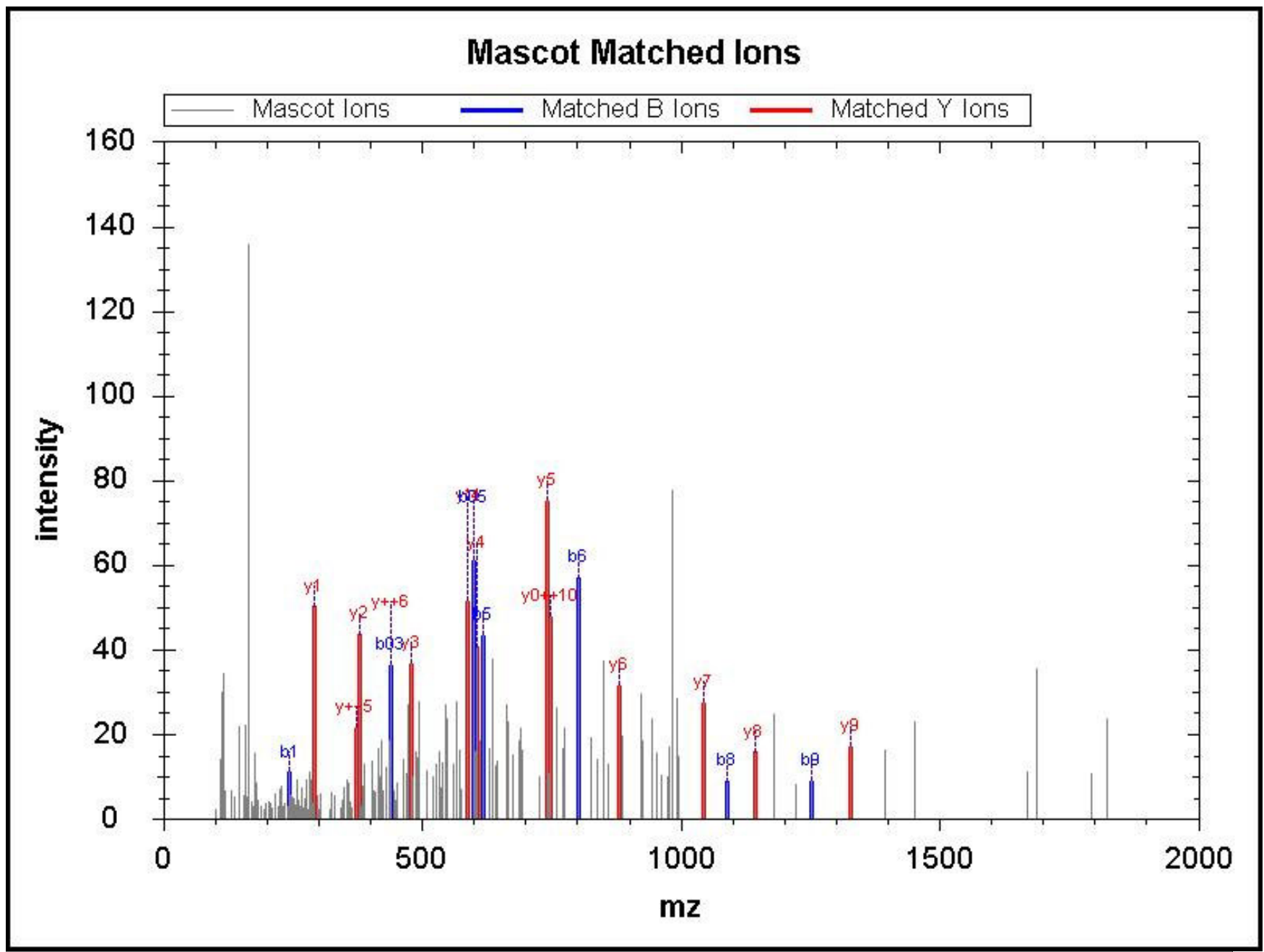

\begin{tabular}{|r|c|c|c|c|c|c|c|c|c|c|c|c|c|c|c|}
\hline \hline No & $\mathrm{b}$ & $\mathrm{b}++$ & $\mathrm{b}^{*}$ & $\mathrm{~b}^{*++}$ & $\mathrm{b} 0$ & $\mathrm{~b} 0++$ & Seq & $\mathrm{y}$ & $\mathrm{y}++$ & $\mathrm{y}^{*}$ & $\mathrm{y}^{*++}$ & $\mathrm{y} 0$ & $\mathrm{y} 0++$ & $\mathrm{RevNo}$ \\
\hline 1 & 244.18 & 122.59 & & & & & $\mathrm{~V}$ & & & & & & & 15 \\
\hline & & & & & & & & & & & & & &
\end{tabular}




\begin{tabular}{|r|r|r|r|r|r|r|r|r|r|r|r|r|r|r|r|}
\hline 2 & 357.26 & 179.13 & & & & & $\mathrm{I}$ & $1,885.98$ & 943.50 & $1,868.96$ & 934.98 & $1,867.97$ & 934.49 & 14 \\
\hline 3 & 458.31 & 229.66 & & & 440.30 & 220.65 & $\mathrm{~T}$ & $1,772.90$ & 886.95 & $1,755.87$ & 878.44 & $1,754.89$ & 877.95 & 13 \\
\hline 4 & 545.34 & 273.17 & & & 527.33 & 264.17 & $\mathrm{~S}$ & $1,671.85$ & 836.43 & $1,654.82$ & 827.92 & $1,653.84$ & 827.42 & 12 \\
\hline 5 & 616.38 & 308.69 & & & 598.37 & 299.69 & $\mathrm{~A}$ & $1,584.82$ & 792.91 & $1,567.79$ & 784.40 & $1,566.81$ & 783.91 & 11 \\
\hline 6 & 802.46 & 401.73 & & & 784.45 & 392.73 & $\mathrm{~W}$ & $1,513.78$ & 757.39 & $1,496.76$ & 748.88 & $1,495.77$ & 748.39 & 10 \\
\hline 7 & 988.54 & 494.77 & & & 970.53 & 485.77 & $\mathrm{~W}$ & $1,327.70$ & 664.36 & $1,310.68$ & 655.84 & $1,309.69$ & 655.35 & 9 \\
\hline 8 & $1,087.61$ & 544.31 & & & $1,069.60$ & 535.30 & $\mathrm{~V}$ & $1,141.62$ & 571.32 & $1,124.60$ & 562.80 & $1,123.61$ & 562.31 & 8 \\
\hline 9 & $1,250.67$ & 625.84 & & & $1,232.66$ & 616.83 & $\mathrm{Y}$ & $1,042.56$ & 521.78 & $1,025.53$ & 513.27 & $1,024.54$ & 512.78 & 7 \\
\hline 10 & $1,387.73$ & 694.37 & & & $1,369.72$ & 685.36 & $\mathrm{H}$ & 879.49 & 440.25 & 862.47 & 431.74 & 861.48 & 431.24 & 6 \\
\hline 11 & $1,524.79$ & 762.90 & & & $1,506.78$ & 753.89 & $\mathrm{H}$ & 742.43 & 371.72 & 725.41 & 363.21 & 724.42 & 362.71 & 5 \\
\hline 12 & $1,652.85$ & 826.93 & $1,635.82$ & 818.41 & $1,634.83$ & 817.92 & $\mathrm{Q}$ & 605.37 & 303.19 & 588.35 & 294.68 & 587.36 & 294.19 & 4 \\
\hline 13 & $1,751.91$ & 876.46 & $1,734.89$ & 867.95 & $1,733.90$ & 867.46 & $\mathrm{~V}$ & 477.32 & 239.16 & 460.29 & 230.65 & 459.30 & 230.16 & 3 \\
\hline 14 & $1,838.95$ & 919.98 & $1,821.92$ & 911.46 & $1,820.94$ & 910.97 & $\mathrm{~S}$ & 378.25 & 189.63 & 361.22 & 181.11 & 360.24 & 180.62 & 2 \\
\hline 15 & & & & & & & $\mathrm{~K}$ & 291.21 & 146.11 & 274.19 & 137.60 & & & 1 \\
\hline
\end{tabular}

Query 17394 Hit 1

MS/MS Fragmentation of LPLIFHLGR

Found in sp|P34059|GALNS_HUM AN, N-acetylgalactosamine-6-sulfatase OS=Homo sapiens GN=GALNS PE=1 SV=1

Match to Query 17394: 1208.752 from(403.9246,3+)

Title: 846 : Sum of 2 scans in range $1883(\mathrm{rt}=51.9289, \mathrm{f}=4, \mathrm{i}=565)$ to $1884(\mathrm{rt}=51.9543, \mathrm{f}=4, \mathrm{i}=566)$

[D:lab212 \membranelGracelJoyceliTRAQ_39_1.raw]

Data File:Submitted from 20120508-1(merge) by Mascot Daemon on JOYCE-VAIO

Monoisotopic mass of neutral peptide $\mathrm{Mr}(\mathrm{calc}): 1208.752$

Variable modifications:

Ions Score: 47.24 Expect: 0.008

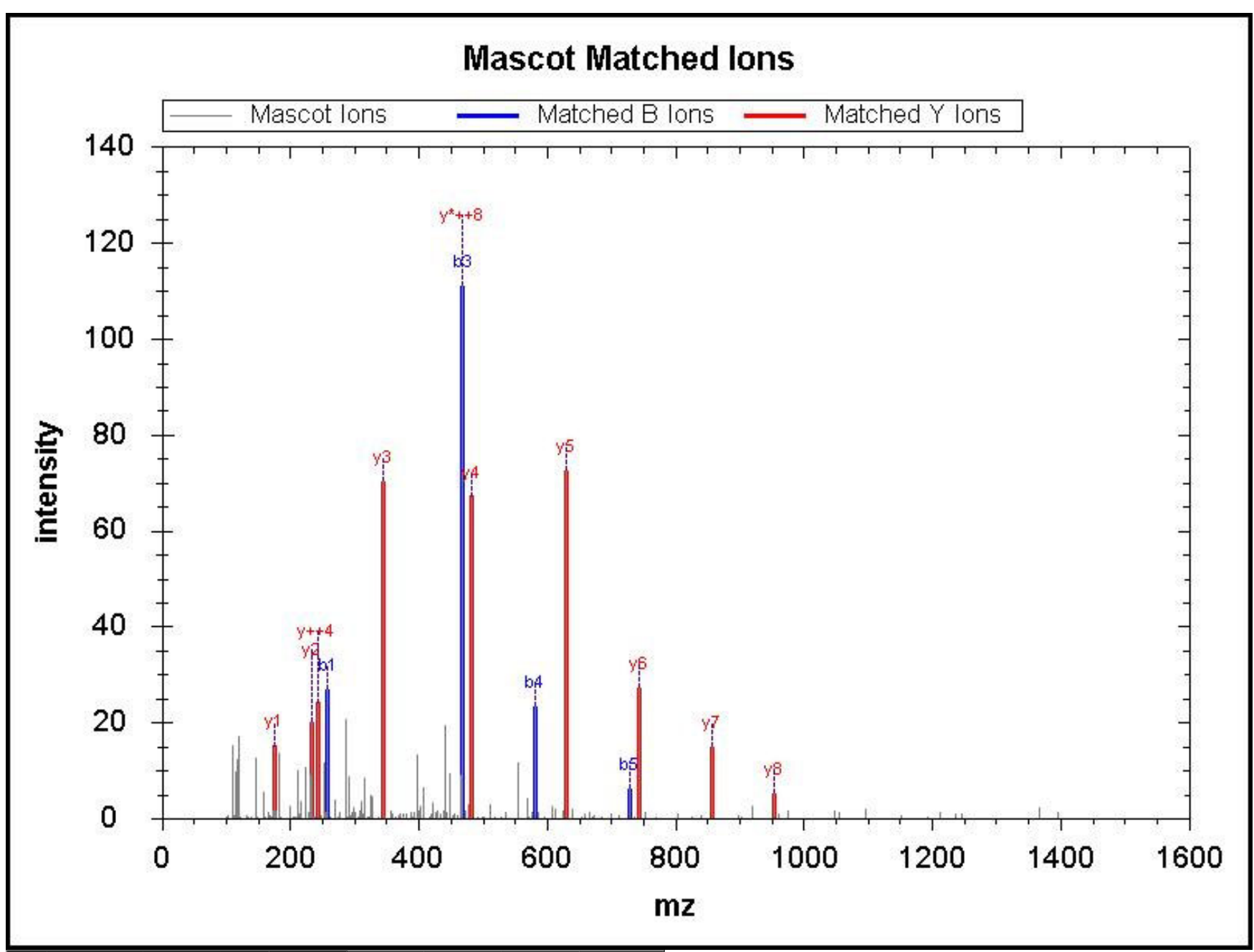

\begin{tabular}{|c|c|c|c|c|c|c|c|c|}
\hline No & $\mathrm{b}$ & $\mathrm{b}++$ & Seq & $\mathrm{y}$ & $\mathrm{y}++$ & $\mathrm{y}^{*}$ & $\mathrm{y}^{*++}$ & RevNo \\
\hline
\end{tabular}




\begin{tabular}{|r|r|r|r|r|r|r|r|r|}
\hline 1 & 258.19 & 129.60 & L & & & & & 9 \\
\hline 2 & 355.25 & 178.13 & P & 952.57 & 476.79 & 935.55 & 468.28 & 8 \\
\hline 3 & 468.33 & 234.67 & L & 855.52 & 428.26 & 838.49 & 419.75 & 7 \\
\hline 4 & 581.41 & 291.21 & I & 742.44 & 371.72 & 725.41 & 363.21 & 6 \\
\hline 5 & 728.48 & 364.74 & F & 629.35 & 315.18 & 612.33 & 306.67 & 5 \\
\hline 6 & 865.54 & 433.27 & H & 482.28 & 241.65 & 465.26 & 233.13 & 4 \\
\hline 7 & 978.63 & 489.82 & L & 345.22 & 173.12 & 328.20 & 164.60 & 3 \\
\hline 8 & $1,035.65$ & 518.33 & G & 232.14 & 116.57 & 215.11 & 108.06 & 2 \\
\hline 9 & & & R & 175.12 & 88.06 & 158.09 & 79.55 & 1 \\
\hline
\end{tabular}

Query 31050 Hit 1

MS/MS Fragmentation of FDLLEELVAK

Found in sp|Q8WWC4|CB047_HUM AN, Uncharacterized protein C2orf47

Match to Query 31050: 1463.844 from(732.9293,2+)

Title: 1033: Sum of 2 scans in range $2268(\mathrm{rt}=60.744, \mathrm{f}=4, \mathrm{i}=701)$ to $2269(\mathrm{rt}=60.7694, \mathrm{f}=4, \mathrm{i}=702)$

[D:lab212 \membranelGrace JoyceliTRAQ_27_2_327.raw]

Data File:Submitted from 20120508-1(merge) by Mascot Daemon on JOYCE-VAIO

Monoisotopic mass of neutral peptide $\operatorname{Mr}($ calc): 1463.844

Variable modifications:

K10 :TRAQ4plex (K)

Ions Score: 47.2 Expect: 0.013

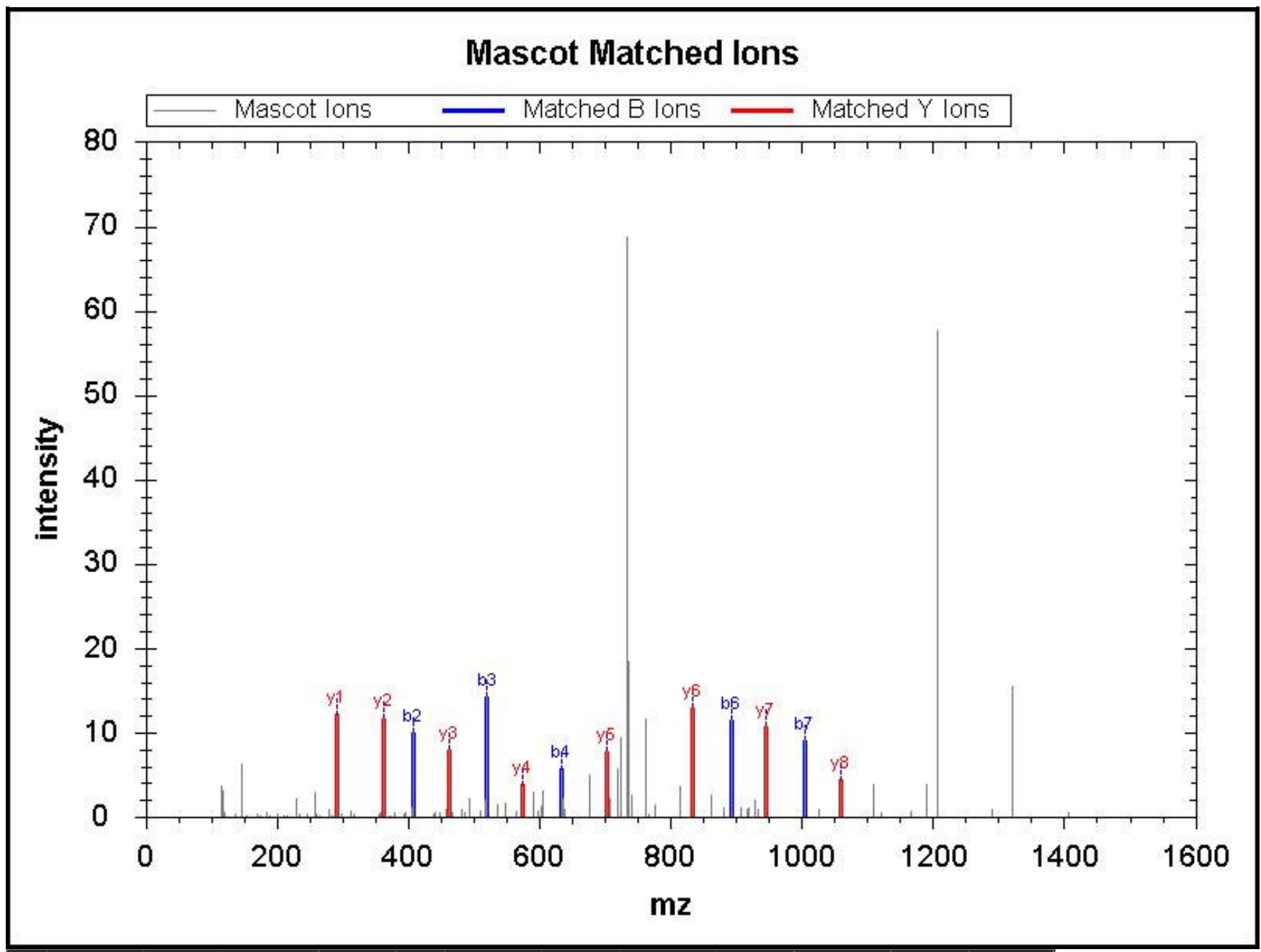

\begin{tabular}{|r|c|c|c|c|c|c|c|c|c|c|r|r|}
\hline \hline No & $\mathrm{b}$ & $\mathrm{b}++$ & $\mathrm{b} 0$ & $\mathrm{~b} 0++$ & Seq & $\mathrm{y}$ & $\mathrm{y}++$ & $\mathrm{y} *$ & $\mathrm{y} *++$ & $\mathrm{y} 0$ & $\mathrm{y} 0++$ & $\mathrm{RevNo}$ \\
\hline 1 & 292.18 & 146.59 & & & $\mathrm{~F}$ & & & & & & & 10 \\
\hline 2 & 407.20 & 204.11 & 389.19 & 195.10 & $\mathrm{D}$ & $1,173.68$ & 587.35 & $1,156.66$ & 578.83 & $1,155.67$ & 578.34 & 9 \\
\hline 3 & 520.29 & 260.65 & 502.28 & 251.64 & L & $1,058.66$ & 529.83 & $1,041.63$ & 521.32 & $1,040.65$ & 520.83 & 8 \\
\hline 4 & 633.37 & 317.19 & 615.36 & 308.18 & L & 945.57 & 473.29 & 928.55 & 464.78 & 927.56 & 464.29 & 7 \\
\hline & & & & & & & & & & & &
\end{tabular}




\begin{tabular}{|r|r|r|r|r|r|r|r|r|r|r|r|r|r|}
\hline 5 & 762.42 & 381.71 & 744.40 & 372.71 & $\mathrm{E}$ & 832.49 & 416.75 & 815.46 & 408.24 & 814.48 & 407.74 & 6 \\
\hline 6 & 891.46 & 446.23 & 873.45 & 437.23 & $\mathrm{E}$ & 703.45 & 352.23 & 686.42 & 343.71 & 685.44 & 343.22 & 5 \\
\hline 7 & $1,004.54$ & 502.77 & 986.53 & 493.77 & $\mathrm{~L}$ & 574.40 & 287.71 & 557.38 & 279.19 & & & 4 \\
\hline 8 & $1,103.61$ & 552.31 & $1,085.60$ & 543.30 & $\mathrm{~V}$ & 461.32 & 231.16 & 444.29 & 222.65 & & & 3 \\
\hline 9 & $1,174.65$ & 587.83 & $1,156.64$ & 578.82 & $\mathrm{~A}$ & 362.25 & 181.63 & 345.23 & 173.12 & & & 2 \\
\hline 10 & & & & & $\mathrm{~K}$ & 291.21 & 146.11 & 274.19 & 137.60 & & & 1 \\
\hline
\end{tabular}

Query 35982 Hit 1

MS/MS Fragmentation of RPEEVALGLHHR

Found in sp|Q96ER9|CCD51_HUMAN, Coiled-coil domain-containing protein $51 \mathrm{OS}=$ Homo sapiens GN=CCDC51 PE=1 SV=2 Match to Query 35982: 1556.863from(390.2231,4+)

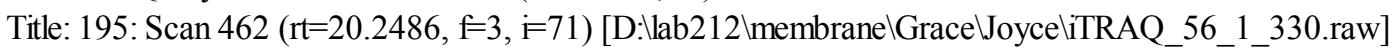

Data File:Submitted from 20120508-1(merge) by Mascot Daemon on JOYCE-VAIO

Monoisotopic mass of neutral peptide $\mathrm{Mr}$ (calc): 1556.863

Variable modifications:

Ions Score: 47.2 Expect: 0.014

\section{Mascot Matched lons}

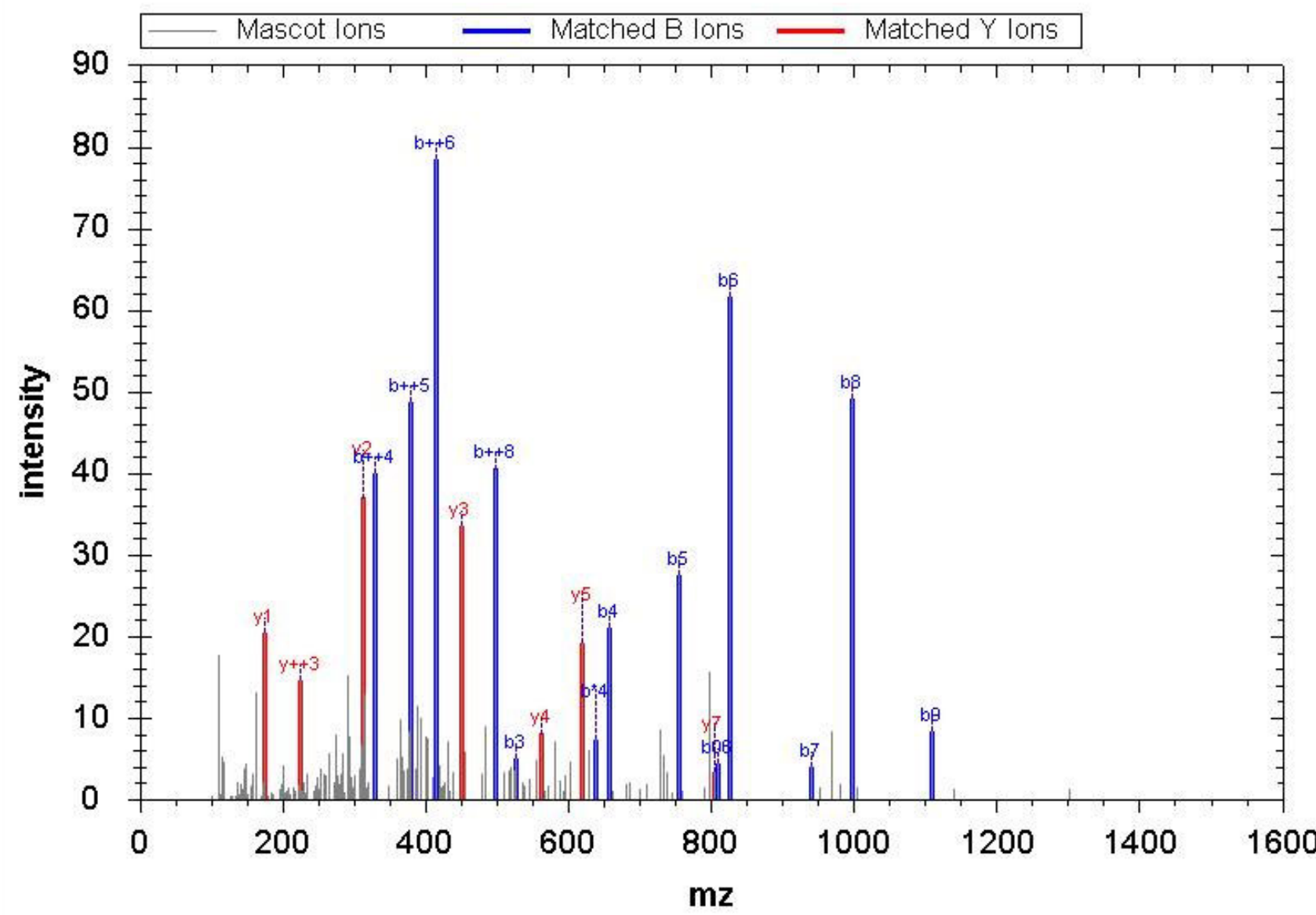

\begin{tabular}{|c|c|c|c|c|c|c|c|c|c|c|c|c|c|c|}
\hline No & $\mathrm{b}$ & $b++$ & $\mathrm{b}^{*}$ & $b^{*++}$ & $\mathrm{b} 0$ & $\mathrm{~b} 0++$ & Seq & $\mathrm{y}$ & $\mathrm{y}++$ & $\mathrm{y}^{*}$ & $\mathrm{y}^{*++}$ & y0 & $\mathrm{y} 0++$ & RevNo \\
\hline 1 & 301.21 & 151.11 & 284.18 & 142.60 & & & $\mathrm{R}$ & & & & & & & 12 \\
\hline 2 & 398.26 & 199.64 & 381.24 & 191.12 & & & $\mathrm{P}$ & $1,257.67$ & 629.34 & $1,240.64$ & 620.83 & $1,239.66$ & 620.33 & 11 \\
\hline 3 & 527.31 & 264.16 & 510.28 & 255.64 & 509.30 & 255.15 & $\mathrm{E}$ & $1,160.62$ & 580.81 & $1,143.59$ & 572.30 & $1,142.61$ & 571.81 & 10 \\
\hline 4 & 656.35 & 328.68 & 639.32 & 320.16 & 638.34 & 319.67 & $\mathrm{E}$ & $1,031.57$ & 516.29 & $1,014.55$ & 507.78 & $1,013.56$ & 507.29 & 9 \\
\hline 5 & 755.42 & 378.21 & 738.39 & 369.70 & 737.41 & 369.21 & V & 902.53 & 451.77 & 885.51 & 443.26 & & & 8 \\
\hline 6 & 826.45 & 413.73 & 809.43 & 405.22 & 808.44 & 404.73 & A & 803.46 & 402.24 & 786.44 & 393.72 & & & 7 \\
\hline 7 & 939.54 & 470.27 & 922.51 & 461.76 & 921.53 & 461.27 & $\mathrm{~L}$ & 732.43 & 366.72 & 715.40 & 358.20 & & & 6 \\
\hline 8 & 996.56 & 498.78 & 979.53 & 490.27 & 978.55 & 489.78 & G & 619.34 & 310.17 & 602.32 & $\mid 301.66$ & & & 5 \\
\hline
\end{tabular}




\begin{tabular}{|r|r|r|r|r|r|r|r|r|r|r|r|r|r|r|}
\hline 9 & $1,109.64$ & 555.33 & $1,092.62$ & 546.81 & $1,091.63$ & 546.32 & L & 562.32 & 281.66 & 545.29 & 273.15 & & & 4 \\
\hline 10 & $1,246.70$ & 623.85 & $1,229.68$ & 615.34 & $1,228.69$ & 614.85 & H & 449.24 & 225.12 & 432.21 & 216.61 & & & 3 \\
\hline 11 & $1,383.76$ & 692.38 & $1,366.73$ & 683.87 & $1,365.75$ & 683.38 & H & 312.18 & 156.59 & 295.15 & 148.08 & & & 2 \\
\hline 12 & & & & & & & R & 175.12 & 88.06 & 158.09 & 79.55 & & & 1 \\
\hline
\end{tabular}

Query 66724 Hit 1

MS/MS Fragmentation of NIFHLFHDVVPTYHK

Found in sp $|\mathrm{O} 43264| \mathrm{ZW} 10$ HUM AN, Centromere/kinetochore protein zw10 homolog OS=Homo sapiens GN=ZW10 PE=1 SV=3 Match to Query 66724:2154.167from(539.549,4+)

Title: 809: Scan 1727 ( $\mathrm{rt}=48.5562, \mathrm{f}=3$, $\mathrm{i}=268$ ) [D:llab212 $\backslash$ membranelGracelJoycel54-2.raw]

Data File:Submitted from 20120508-1(merge) by Mascot Daemon on JOYCE-VAIO

Monoisotopic mass of neutral peptide $\operatorname{Mr}($ calc): 2154.167

Variable modifications:

K15 :TRAQ4plex (K)

Ions Score: 47.2 Expect: 0.015

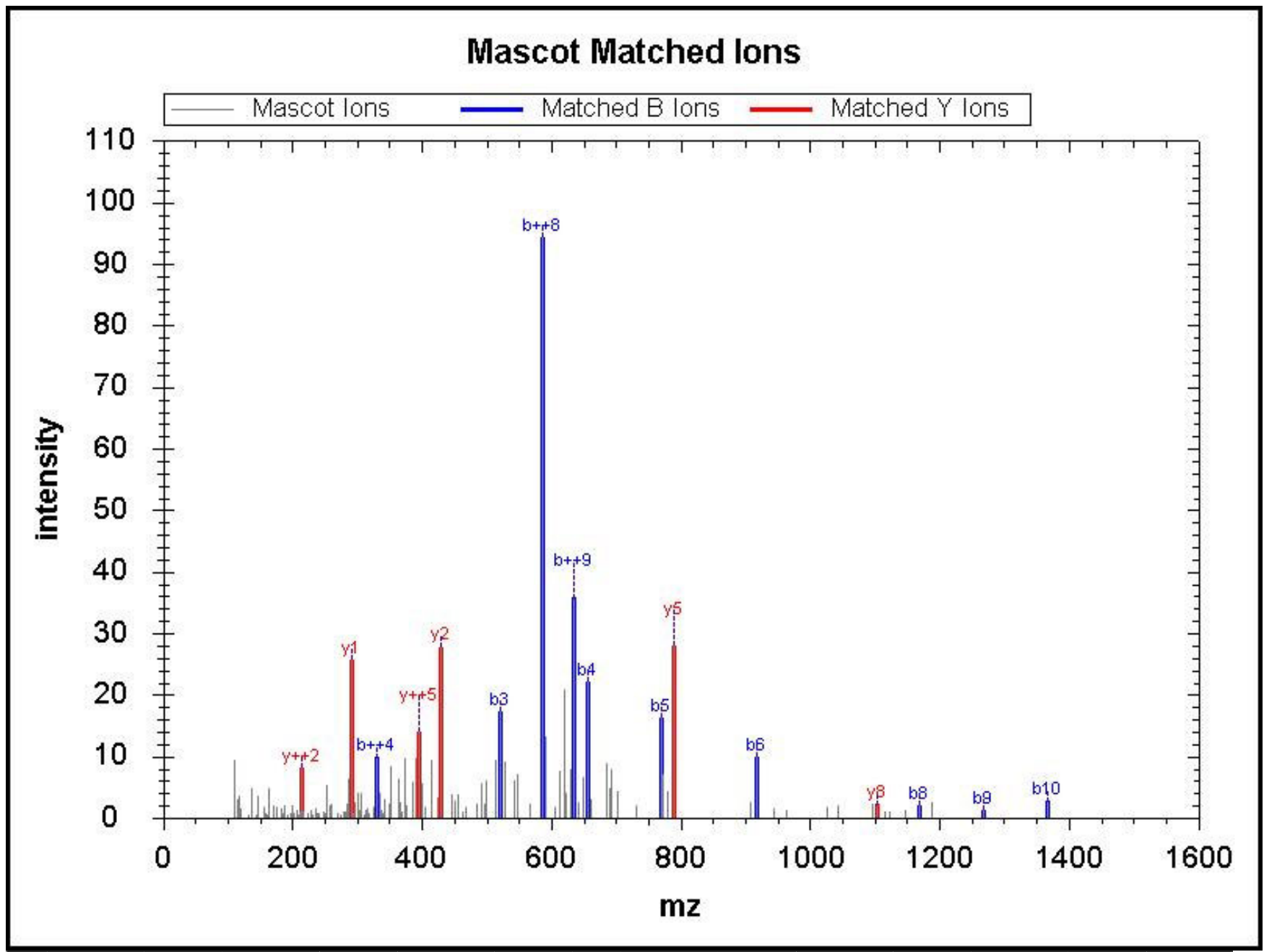

\begin{tabular}{|r|c|c|c|c|c|r|r|r|r|r|r|r|r|r|}
\hline \hline No & $\mathrm{b}$ & $\mathrm{b}++$ & $\mathrm{b} *$ & $\mathrm{~b} *++$ & $\mathrm{b} 0$ & $\mathrm{~b} 0++$ & Seq & $\mathrm{y}$ & $\mathrm{y}++$ & $\mathrm{y}^{*}$ & $\mathrm{y}^{*++}$ & $\mathrm{y} 0$ & $\mathrm{y} 0++$ & $\mathrm{RevNo}$ \\
\hline 1 & 259.15 & 130.08 & 242.13 & 121.57 & & & $\mathrm{~N}$ & & & & & & & 15 \\
\hline 2 & 372.24 & 186.62 & 355.21 & 178.11 & & & $\mathrm{I}$ & $1,897.02$ & 949.02 & $1,880.00$ & 940.50 & $1,879.01$ & 940.01 & 14 \\
\hline 3 & 519.30 & 260.16 & 502.28 & 251.64 & & & $\mathrm{~F}$ & $1,783.94$ & 892.47 & $1,766.91$ & 883.96 & $1,765.93$ & 883.47 & 13 \\
\hline 4 & 656.36 & 328.69 & 639.34 & 320.17 & & & $\mathrm{H}$ & $1,636.87$ & 818.94 & $1,619.85$ & 810.43 & $1,618.86$ & 809.93 & 12 \\
\hline 5 & 769.45 & 385.23 & 752.42 & 376.71 & & & $\mathrm{~L}$ & $1,499.81$ & 750.41 & $1,482.79$ & 741.90 & $1,481.80$ & 741.40 & 11 \\
\hline 6 & 916.52 & 458.76 & 899.49 & 450.25 & & & $\mathrm{~F}$ & $1,386.73$ & 693.87 & $1,369.70$ & 685.35 & $1,368.72$ & 684.86 & 10 \\
\hline 7 & $1,053.58$ & 527.29 & $1,036.55$ & 518.78 & & & H & $1,239.66$ & 620.33 & $1,222.63$ & 611.82 & $1,221.65$ & 611.33 & 9 \\
\hline 8 & $1,168.60$ & 584.80 & $1,151.58$ & 576.29 & $1,150.59$ & 575.80 & $\mathrm{D}$ & $1,102.60$ & 551.80 & $1,085.57$ & 543.29 & $1,084.59$ & 542.80 & 8 \\
\hline 9 & $1,267.67$ & 634.34 & $1,250.64$ & 625.83 & $1,249.66$ & 625.33 & $\mathrm{~V}$ & 987.57 & 494.29 & 970.55 & 485.78 & 969.56 & 485.29 & 7 \\
\hline 10 & $1,366.74$ & 683.87 & $1,349.71$ & 675.36 & $1,348.73$ & 674.87 & $\mathrm{~V}$ & 888.51 & 444.76 & 871.48 & 436.24 & 870.50 & 435.75 & 6 \\
\hline
\end{tabular}




\begin{tabular}{|l|l|l|l|l|l|l|l|l|l|l|l|l|l|l|}
11 & $1,463.79$ & 732.40 & $1,446.77$ & 723.89 & $1,445.78$ & 723.39 & $\mathrm{P}$ & 789.44 & 395.22 & 772.41 & 386.71 & 771.43 & 386.22 & 5 \\
\hline 12 & $1,564.84$ & 782.92 & $1,547.81$ & 774.41 & $1,546.83$ & 773.92 & $\mathrm{~T}$ & 692.38 & 346.70 & 675.36 & 338.18 & 674.37 & 337.69 & 4 \\
\hline 13 & $1,727.90$ & 864.45 & $1,710.88$ & 855.94 & $1,709.89$ & 855.45 & $\mathrm{Y}$ & 591.34 & 296.17 & 574.31 & 287.66 & & & 3 \\
\hline 14 & $1,864.96$ & 932.98 & $1,847.94$ & 924.47 & $1,846.95$ & 923.98 & $\mathrm{H}$ & 428.27 & 214.64 & 411.25 & 206.13 & & & 2 \\
\hline 15 & & & & & & & $\mathrm{~K}$ & 291.21 & 146.11 & 274.19 & 137.60 & & & 1 \\
\hline
\end{tabular}

Query 41953 Hit 1

MS/MS Fragmentation of TVHAGEVGSAEVVK

Found in sp|P00813|ADA_HUM AN, Adenosine deaminase OS=Homo sapiens GN=ADA PE=1 SV=3

Match to Query 41953: 1669.926from(557.6494,3+)

Title: 234: Sum of 2 scans in range $603(\mathrm{rt}=23.1192, \mathrm{f}=4, \mathrm{i}=153)$ to $604(\mathrm{rt}=23.1446, \mathrm{f}=4, \mathrm{i}=154)$

[D:lab212 \membranelGrace JoyceliTRAQ_41_2.raw]

Data File:Submitted from 20120508-1(merge) by Mascot Daemon on JOYCE-VAIO

Monoisotopic mass of neutral peptide $\operatorname{Mr}($ calc): 1669.926

Variable modifications:

K14 iTRAQ4plex (K)

Ions Score: 47.15 Expect: 0.014

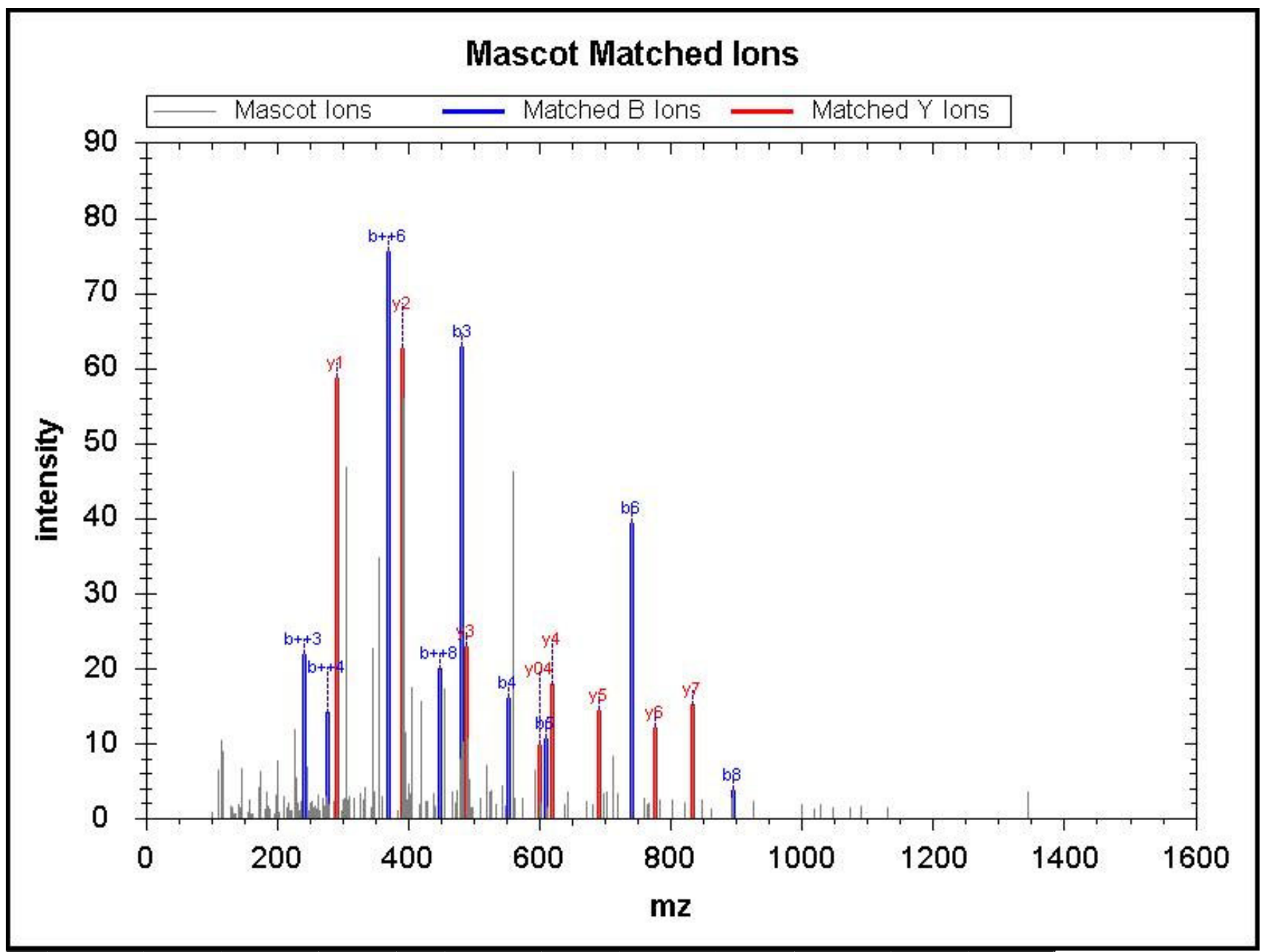

\begin{tabular}{|r|c|c|c|c|c|c|c|c|c|c|r|r|}
\hline \hline No & $\mathrm{b}$ & $\mathrm{b}++$ & $\mathrm{b} 0$ & $\mathrm{~b} 0++$ & $\mathrm{Seq}$ & $\mathrm{y}$ & $\mathrm{y}++$ & $\mathrm{y}^{*}$ & $\mathrm{y} *++$ & $\mathrm{y} 0$ & $\mathrm{y} 0++$ & RevNo \\
\hline 1 & 246.16 & 123.58 & 228.15 & 114.58 & $\mathrm{~T}$ & & & & & & & 14 \\
\hline 2 & 345.23 & 173.12 & 327.21 & 164.11 & $\mathrm{~V}$ & $1,425.78$ & 713.39 & $1,408.76$ & 704.88 & $1,407.77$ & 704.39 & 13 \\
\hline 3 & 482.28 & 241.65 & 464.27 & 232.64 & $\mathrm{H}$ & $1,326.71$ & 663.86 & $1,309.69$ & 655.35 & $1,308.70$ & 654.86 & 12 \\
\hline 4 & 553.32 & 277.16 & 535.31 & 268.16 & $\mathrm{~A}$ & $1,189.65$ & 595.33 & $1,172.63$ & 586.82 & $1,171.64$ & 586.33 & 11 \\
\hline 5 & 610.34 & 305.68 & 592.33 & 296.67 & $\mathrm{G}$ & $1,118.62$ & 559.81 & $1,101.59$ & 551.30 & $1,100.61$ & 550.81 & 10 \\
\hline 6 & 739.39 & 370.20 & 721.38 & 361.19 & $\mathrm{E}$ & $1,061.60$ & 531.30 & $1,044.57$ & 522.79 & $1,043.59$ & 522.30 & 9 \\
\hline 7 & 838.45 & 419.73 & 820.44 & 410.73 & $\mathrm{~V}$ & 932.55 & 466.78 & 915.53 & 458.27 & 914.54 & 457.78 & 8 \\
\hline 8 & 895.48 & 448.24 & 877.46 & 439.24 & $\mathrm{G}$ & 833.48 & 417.25 & 816.46 & 408.73 & 815.47 & 408.24 & 7 \\
\hline
\end{tabular}




\begin{tabular}{|r|r|r|r|r|r|r|r|r|r|r|r|r|}
9 & 982.51 & 491.76 & 964.50 & 482.75 & $\mathrm{~S}$ & 776.46 & 388.74 & 759.44 & 380.22 & 758.45 & 379.73 & 6 \\
\hline \hline 10 & $1,053.54$ & 527.28 & $1,035.53$ & 518.27 & $\mathrm{~A}$ & 689.43 & 345.22 & 672.40 & 336.71 & 671.42 & 336.21 & 5 \\
\hline 11 & $1,182.59$ & 591.80 & $1,164.58$ & 582.79 & $\mathrm{E}$ & 618.39 & 309.70 & 601.37 & 301.19 & 600.38 & 300.70 & 4 \\
\hline 12 & $1,281.66$ & 641.33 & $1,263.65$ & 632.33 & $\mathrm{~V}$ & 489.35 & 245.18 & 472.33 & 236.67 & & & 3 \\
\hline 13 & $1,380.72$ & 690.87 & $1,362.71$ & 681.86 & $\mathrm{~V}$ & 390.28 & 195.65 & 373.26 & 187.13 & & & 2 \\
\hline 14 & & & & & $\mathrm{~K}$ & 291.21 & 146.11 & 274.19 & 137.60 & & & 1 \\
\hline
\end{tabular}

Query 27043 Hit 1

MS/MS Fragmentation of DVINVFHHLR

Found in sp|Q9UK58|CCNL1_HUMAN, Cyclin-L1 OS=Homo sapiens GN=CCNL1 PE=1 SV=1

Match to Query 27043: 1392.782from(465.2679,3+)

Title: 450: Sum of 2 scans in range 1229 ( $\mathrm{rt}=36.361, \mathrm{f}=4, \mathrm{i}=295)$ to $1230(\mathrm{rt}=36.3864, \mathrm{f}=4, \mathrm{i}=296)$

[D:llab212\membranelGrace \oyceliTRAQ_51_2.raw]

Data File:Submitted from 20120508-1(merge) by Mascot Daemon on JOYCE-VAIO

Monoisotopic mass of neutral peptide $\mathrm{Mr}$ (calc): 1392.782

Variable modifications:

Ions Score: 47.02 Expect: 0.016

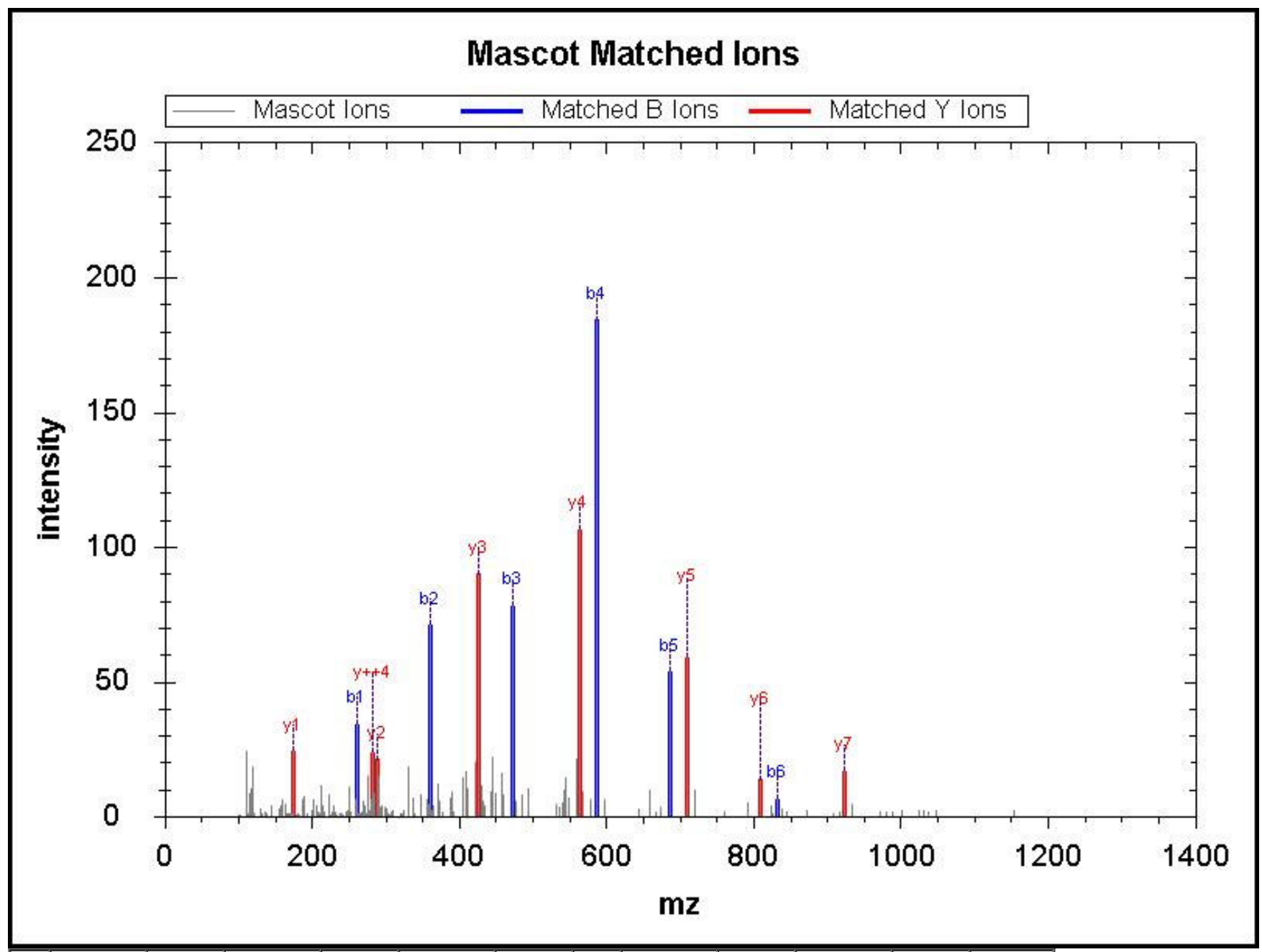

\begin{tabular}{|r|c|c|c|c|c|c|c|c|c|c|r|r|}
\hline \hline No & $\mathrm{b}$ & $\mathrm{b}++$ & $\mathrm{b}^{*}$ & $\mathrm{~b} *++$ & $\mathrm{b} 0$ & $\mathrm{~b} 0++$ & Seq & $\mathrm{y}$ & $\mathrm{y}++$ & $\mathrm{y}^{*}$ & $\mathrm{y}^{*++}$ & $\mathrm{RevNo}$ \\
\hline 1 & 260.14 & 130.57 & & & 242.13 & 121.57 & $\mathrm{D}$ & & & & & 10 \\
\hline 2 & 359.20 & 180.11 & & & 341.19 & 171.10 & $\mathrm{~V}$ & $1,134.65$ & 567.83 & $1,117.63$ & 559.32 & 9 \\
\hline 3 & 472.29 & 236.65 & & & 454.28 & 227.64 & $\mathrm{I}$ & $1,035.58$ & 518.30 & $1,018.56$ & 509.78 & 8 \\
\hline 4 & 586.33 & 293.67 & 569.31 & 285.16 & 568.32 & 284.66 & $\mathrm{~N}$ & 922.50 & 461.75 & 905.47 & 453.24 & 7 \\
\hline 5 & 685.40 & 343.20 & 668.37 & 334.69 & 667.39 & 334.20 & $\mathrm{~V}$ & 808.46 & 404.73 & 791.43 & 396.22 & 6 \\
\hline 6 & 832.47 & 416.74 & 815.44 & 408.22 & 814.46 & 407.73 & $\mathrm{~F}$ & 709.39 & 355.20 & 692.36 & 346.68 & 5 \\
\hline 7 & 969.53 & 485.27 & 952.50 & 476.75 & 951.52 & 476.26 & $\mathrm{H}$ & 562.32 & 281.66 & 545.29 & 273.15 & 4 \\
\hline 8 & $1,106.59$ & 553.80 & $1,089.56$ & 545.28 & $1,088.58$ & 544.79 & $\mathrm{H}$ & 425.26 & 213.13 & 408.24 & 204.62 & 3 \\
\hline
\end{tabular}




\begin{tabular}{|r|r|r|r|r|r|r|r|r|r|r|r|r|r|}
\hline 9 & $1,219.67$ & 610.34 & $1,202.64$ & 601.83 & $1,201.66$ & 601.33 & $\mathrm{~L}$ & 288.20 & 144.61 & 271.18 & 136.09 & 2 \\
\hline 10 & & & & & & & $\mathrm{R}$ & 175.12 & 88.06 & 158.09 & 79.55 & 1 \\
\hline
\end{tabular}

Query 38173 Hit 1

MS/MS Fragmentation of KEDFSEATLK

Found in sp $\mid$ Q53FA7|QORX_HUMAN, Quinone oxidoreductase PIG3 OS=Homo sapiens GN=TP53I3 PE=1 SV=2

Match to Query 38173: 1598.893from(533.9717,3+)

Title: 264: Sum of 2 scans in range $732(\mathrm{rt}=25.6894, \mathrm{f}=4, \mathrm{i}=171)$ to $733(\mathrm{rt}=25.7148, \mathrm{f}=4, \mathrm{i}=172)$

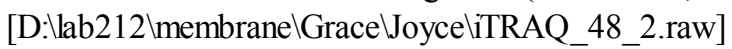

Data File:Submitted from 20120508-1(merge) by Mascot Daemon on JOYCE-VAIO

Monoisotopic mass of neutral peptide $\mathrm{Mr}$ (calc): 1598.893

Variable modifications:

$\mathrm{K} 1$ :iTRAQ4plex (K)

K10 :iTRAQ4plex (K)

Ions Score: 46.98 Expect: 0.014

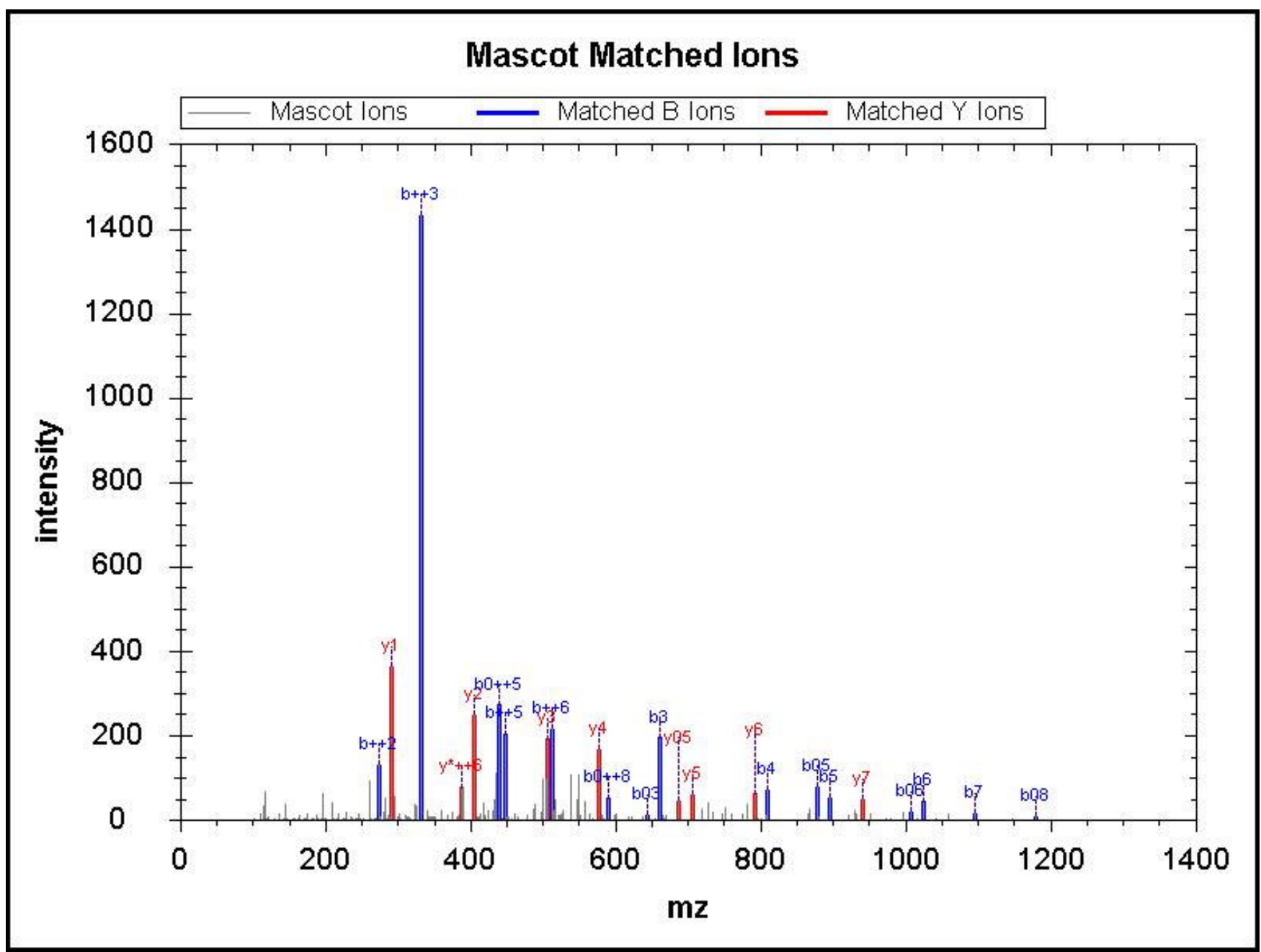

\begin{tabular}{|r|c|c|c|c|c|r|r|r|r|r|r|r|r|r|}
\hline $\mathrm{No}$ & $\mathrm{b}$ & $\mathrm{b}++$ & $\mathrm{b} *$ & $\mathrm{~b}++$ & $\mathrm{b} 0$ & $\mathrm{~b} 0++$ & $\mathrm{Seq}$ & $\mathrm{y}$ & $\mathrm{y}++$ & $\mathrm{y} *$ & $\mathrm{y}^{*++}$ & $\mathrm{y} 0$ & $\mathrm{y} 0++$ & $\mathrm{RevNo}$ \\
\hline 1 & 417.31 & 209.16 & 400.28 & 200.64 & & & $\mathrm{~K}$ & & & & & & & 10 \\
\hline 2 & 546.35 & 273.68 & 529.32 & 265.16 & 528.34 & 264.67 & $\mathrm{E}$ & $1,183.60$ & 592.30 & $1,166.57$ & 583.79 & $1,165.59$ & 583.30 & 9 \\
\hline 3 & 661.38 & 331.19 & 644.35 & 322.68 & 643.37 & 322.19 & $\mathrm{D}$ & $1,054.55$ & 527.78 & $1,037.53$ & 519.27 & $1,036.54$ & 518.78 & 8 \\
\hline 4 & 808.44 & 404.73 & 791.42 & 396.21 & 790.43 & 395.72 & $\mathrm{~F}$ & 939.53 & 470.27 & 922.50 & 461.75 & 921.52 & 461.26 & 7 \\
\hline 5 & 895.48 & 448.24 & 878.45 & 439.73 & 877.47 & 439.24 & $\mathrm{~S}$ & 792.46 & 396.73 & 775.43 & 388.22 & 774.45 & 387.73 & 6 \\
\hline 6 & $1,024.52$ & 512.76 & $1,007.49$ & 504.25 & $1,006.51$ & 503.76 & $\mathrm{E}$ & 705.43 & 353.22 & 688.40 & 344.70 & 687.42 & 344.21 & 5 \\
\hline 7 & $1,095.56$ & 548.28 & $1,078.53$ & 539.77 & $1,077.55$ & 539.28 & A & 576.38 & 288.70 & 559.36 & 280.18 & 558.37 & 279.69 & 4 \\
\hline 8 & $1,196.60$ & 598.81 & $1,179.58$ & 590.29 & $1,178.59$ & 589.80 & T & 505.35 & 253.18 & 488.32 & 244.66 & 487.34 & 244.17 & 3 \\
\hline 9 & $1,309.69$ & 655.35 & $1,292.66$ & 646.83 & $1,291.68$ & 646.34 & L & 404.30 & 202.65 & 387.27 & 194.14 & & & 2 \\
\hline 10 & & & & & & & K & 291.21 & 146.11 & 274.19 & 137.60 & & & \\
\hline
\end{tabular}


MS/MS Fragmentation of TWITNSPMADLFVVWAR

Found in sp|Q92947|GCDH_HUMAN, Glutaryl-CoA dehydrogenase

Match to Query 66530: 2150.119from(717.7136,3+)

Title: 1266: Sum of 2 scans in range $2957(\mathrm{rt}=75.5663, \mathrm{f}=4, \mathrm{i}=826)$ to 2958 ( $\mathrm{rt}=75.5917, \mathrm{f}=4, \mathrm{i}=827$ )

[D:llab212 \membranelGrace \Joycel20120320_iTRAQ_20-1.raw]

Data File:Submitted from 20120508-1(merge) by Mascot Daemon on JOYCE-VAIO

Monoisotopic mass of neutral peptide $\mathrm{Mr}$ (calc): 2150.119

Variable modifications:

Ions Score: 46.83 Expect: 0.019

\section{Mascot Matched lons}

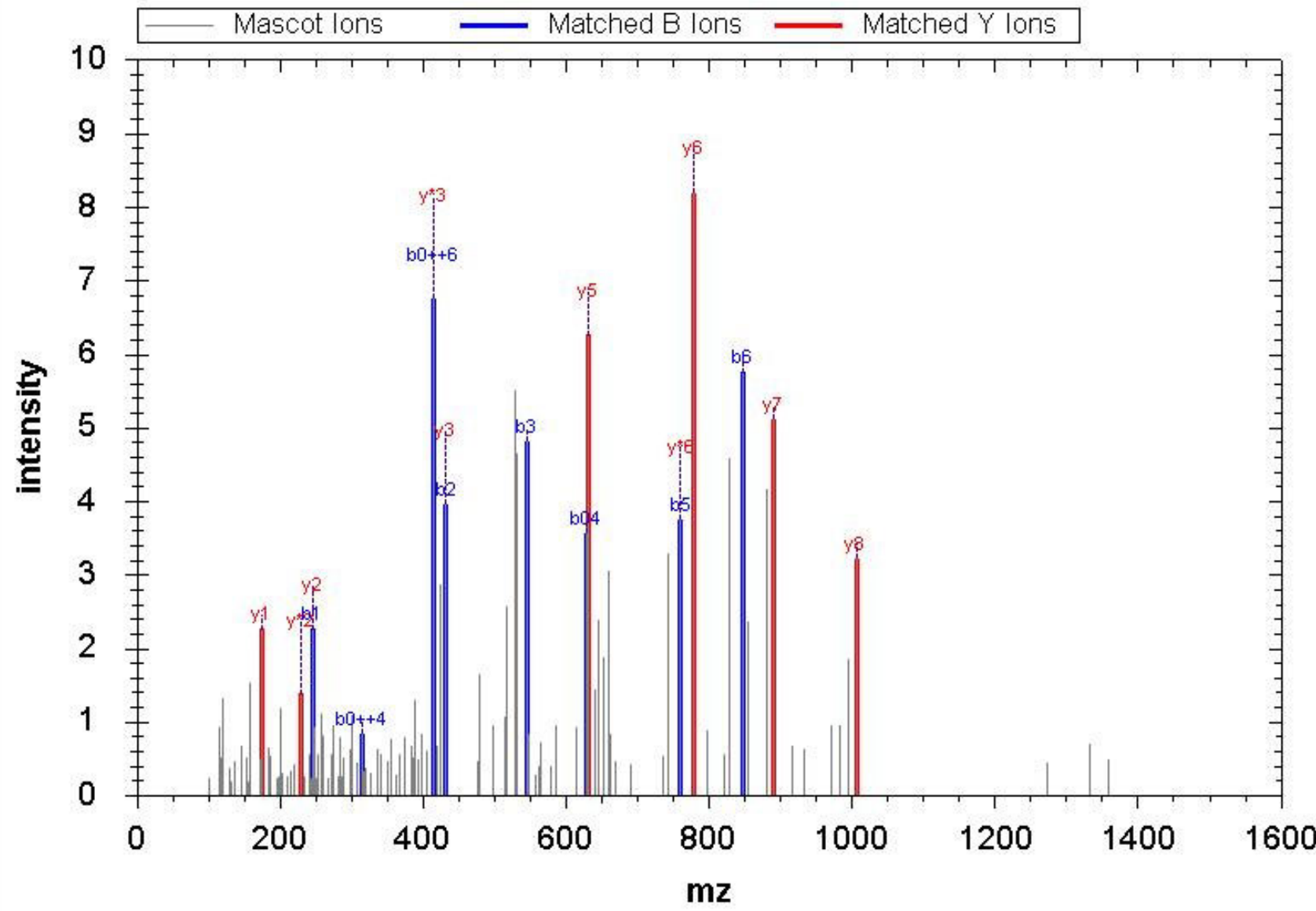

\begin{tabular}{|r|c|c|c|c|c|c|c|c|c|c|c|c|r|r|}
\hline $\mathrm{No}$ & $\mathrm{b}$ & $\mathrm{b}++$ & $\mathrm{b} *$ & $\mathrm{~b}^{*++}$ & $\mathrm{b} 0$ & $\mathrm{~b} 0++$ & $\mathrm{Seq}$ & $\mathrm{y}$ & $\mathrm{y}++$ & $\mathrm{y}^{*}$ & $\mathrm{y}^{*}++$ & $\mathrm{y} 0$ & $\mathrm{y} 0++$ & $\mathrm{RevNo}$ \\
\hline 1 & 246.16 & 123.58 & & & 228.15 & 114.58 & $\mathrm{~T}$ & & & & & & & 17 \\
\hline 2 & 432.24 & 216.62 & & & 414.23 & 207.62 & $\mathrm{~W}$ & $1,905.97$ & 953.49 & $1,888.94$ & 944.97 & $1,887.96$ & 944.48 & 16 \\
\hline 3 & 545.32 & 273.16 & & & 527.31 & 264.16 & $\mathrm{I}$ & $1,719.89$ & 860.45 & $1,702.86$ & 851.93 & $1,701.88$ & 851.44 & 15 \\
\hline 4 & 646.37 & 323.69 & & & 628.36 & 314.68 & $\mathrm{~T}$ & $1,606.80$ & 803.91 & $1,589.78$ & 795.39 & $1,588.79$ & 794.90 & 14 \\
\hline 5 & 760.41 & 380.71 & 743.38 & 372.20 & 742.40 & 371.70 & $\mathrm{~N}$ & $1,505.76$ & 753.38 & $1,488.73$ & 744.87 & $1,487.75$ & 744.38 & 13 \\
\hline 6 & 847.44 & 424.23 & 830.42 & 415.71 & 829.43 & 415.22 & $\mathrm{~S}$ & $1,391.71$ & 696.36 & $1,374.69$ & 687.85 & $1,373.70$ & 687.36 & 12 \\
\hline 7 & 944.50 & 472.75 & 927.47 & 464.24 & 926.49 & 463.75 & $\mathrm{P}$ & $1,304.68$ & 652.84 & $1,287.66$ & 644.33 & $1,286.67$ & 643.84 & 11 \\
\hline 8 & $1,075.54$ & 538.27 & $1,058.51$ & 529.76 & $1,057.53$ & 529.27 & $\mathrm{M}$ & $1,207.63$ & 604.32 & $1,190.60$ & 595.81 & $1,189.62$ & 595.31 & 10 \\
\hline 9 & $1,146.57$ & 573.79 & $1,129.55$ & 565.28 & $1,128.56$ & 564.79 & $\mathrm{~A}$ & $1,076.59$ & 538.80 & $1,059.56$ & 530.28 & $1,058.58$ & 529.79 & 9 \\
\hline 10 & $1,261.60$ & 631.30 & $1,244.57$ & 622.79 & $1,243.59$ & 622.30 & $\mathrm{D}$ & $1,005.55$ & 503.28 & 988.53 & 494.77 & 987.54 & 494.27 & 8 \\
\hline 11 & $1,374.68$ & 687.85 & $1,357.66$ & 679.33 & $1,356.67$ & 678.84 & $\mathrm{~L}$ & 890.52 & 445.77 & 873.50 & 437.25 & & & 7 \\
\hline 12 & $1,521.75$ & 761.38 & $1,504.73$ & 752.87 & $1,503.74$ & 752.37 & $\mathrm{~F}$ & 777.44 & 389.22 & 760.41 & 380.71 & & & 6 \\
\hline 13 & $1,620.82$ & 810.91 & $1,603.79$ & 802.40 & $1,602.81$ & 801.91 & $\mathrm{~V}$ & 630.37 & 315.69 & 613.35 & 307.18 & & & 5 \\
\hline 14 & $1,719.89$ & 860.45 & $1,702.86$ & 851.94 & $1,701.88$ & 851.44 & $\mathrm{~V}$ & 531.30 & 266.16 & 514.28 & 257.64 & & & 4 \\
\hline & & & & & & & & & & & & & &
\end{tabular}




\begin{tabular}{|r|r|r|r|r|r|r|r|r|r|r|r|r|r|r|r|}
\hline 15 & $1,905.97$ & 953.49 & $1,888.94$ & 944.97 & $1,887.96$ & 944.48 & $\mathrm{~W}$ & 432.24 & 216.62 & 415.21 & 208.11 & & & 3 \\
\hline 16 & $1,977.01$ & 989.01 & $1,959.98$ & 980.49 & $1,959.00$ & 980.00 & $\mathrm{~A}$ & 246.16 & 123.58 & 229.13 & 115.07 & & & 2 \\
\hline 17 & & & & & & & $\mathrm{R}$ & 175.12 & 88.06 & 158.09 & 79.55 & & & 1 \\
\hline
\end{tabular}

Query 22644 Hit 1

MS/MS Fragmentation of DTDSSVASEVR

Found in sp|Q86X29|LSR_HUMAN, Lipolysis-stimulated lipoprotein receptor OS=Homo sapiens GN=LSR PE=1 SV=4

Match to Query 22644: 1308.619from(655.3168,2+)

Title: 107: Scan 450 ( $\mathrm{rt}=19.2209, \mathrm{f}=3, \mathrm{i}=56)$ [D:llab212 membranelGrace JoyceliTRAQ_22_1.raw]

Data File:Submitted from 20120508-1(merge) by Mascot Daemon on JOYCE-VAIO

Monoisotopic mass of neutral peptide $\mathrm{Mr}$ (calc): 1308.619

Variable modifications:

Ions Score: 46.75 Expect: 0.013

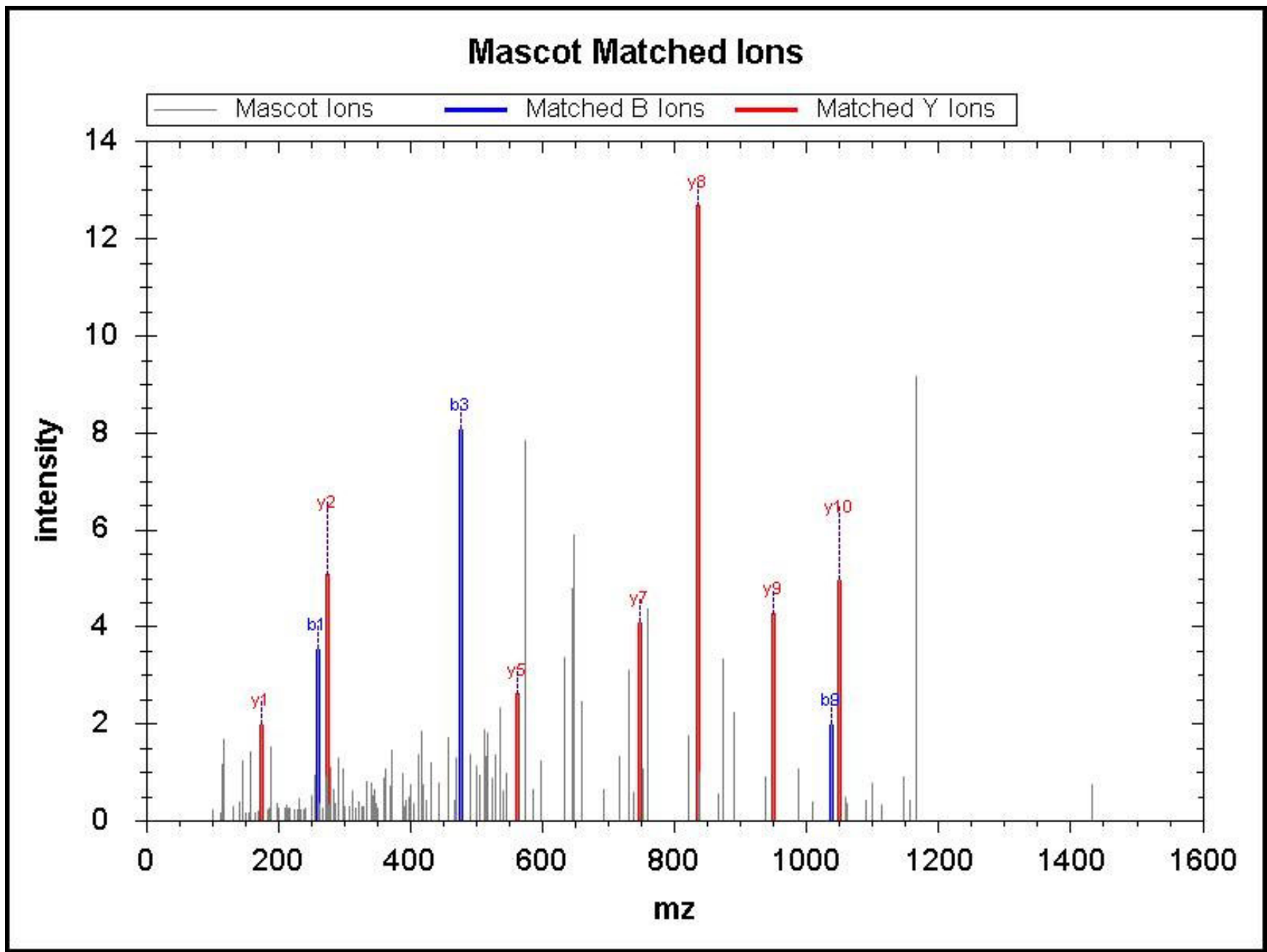

\begin{tabular}{|r|r|r|r|r|r|r|r|r|r|r|r|r|}
\hline No & $\mathrm{b}$ & $\mathrm{b}++$ & $\mathrm{b} 0$ & $\mathrm{~b} 0++$ & $\mathrm{Seq}$ & $\mathrm{y}$ & $\mathrm{y}++$ & $\mathrm{y} *$ & $\mathrm{y} *++$ & $\mathrm{y} 0$ & $\mathrm{y} 0++$ & RevNo \\
\hline 1 & 260.14 & 130.57 & 242.13 & 121.57 & $\mathrm{D}$ & & & & & & & 11 \\
\hline 2 & 361.18 & 181.10 & 343.17 & 172.09 & $\mathrm{~T}$ & $1,050.51$ & 525.76 & $1,033.48$ & 517.24 & $1,032.50$ & 516.75 & 10 \\
\hline 3 & 476.21 & 238.61 & 458.20 & 229.60 & $\mathrm{D}$ & 949.46 & 475.23 & 932.43 & 466.72 & 931.45 & 466.23 & 9 \\
\hline 4 & 563.24 & 282.13 & 545.23 & 273.12 & $\mathrm{~S}$ & 834.43 & 417.72 & 817.41 & 409.21 & 816.42 & 408.71 & 8 \\
\hline 5 & 650.27 & 325.64 & 632.26 & 316.64 & $\mathrm{~S}$ & 747.40 & 374.20 & 730.37 & 365.69 & 729.39 & 365.20 & 7 \\
\hline 6 & 749.34 & 375.18 & 731.33 & 366.17 & $\mathrm{~V}$ & 660.37 & 330.69 & 643.34 & 322.17 & 642.36 & 321.68 & 6 \\
\hline 7 & 820.38 & 410.69 & 802.37 & 401.69 & $\mathrm{~A}$ & 561.30 & 281.15 & 544.27 & 272.64 & 543.29 & 272.15 & 5 \\
\hline 8 & 907.41 & 454.21 & 889.40 & 445.20 & $\mathrm{~S}$ & 490.26 & 245.63 & 473.24 & 237.12 & 472.25 & 236.63 & 4 \\
\hline 9 & $1,036.46$ & 518.73 & $1,018.44$ & 509.73 & $\mathrm{E}$ & 403.23 & 202.12 & 386.20 & 193.61 & 385.22 & 193.11 & 3 \\
\hline 10 & $1,135.52$ & 568.27 & $1,117.51$ & 559.26 & $\mathrm{~V}$ & 274.19 & 137.60 & 257.16 & 129.08 & & & 2 \\
\hline 11 & & & & & $\mathrm{R}$ & 175.12 & 88.06 & 158.09 & 79.55 & & & 1
\end{tabular}


Query 52734 Hit 1

MS/MS Fragmentation of DLLVEGLAEILHQK

Found in sp|Q9Y3R5|DOP2_HUMAN, Protein dopey-2 OS=Homo sapiens GN=DOPEY2 PE=1 SV=5

Match to Query 52734: 1865.085from(622.7023,3+)

Title: 1103: Sum of 2 scans in range $2417(\mathrm{rt}=63.8596, \mathrm{f}=3, \mathrm{i}=378)$ to $2418(\mathrm{rt}=63.885, \mathrm{f}=3, \mathrm{i}=379)$

[D:llab212\membranelGrace JoyceliTRAQ_37_1.raw]

Data File:Submitted from 20120508-1(merge) by Mascot Daemon on JOYCE-VAIO

Monoisotopic mass of neutral peptide $\mathrm{Mr}$ (calc): 1865.085

Variable modifications:

K14 :iTRAQ4plex (K)

Ions Score: 46.74 Expect: 0.010

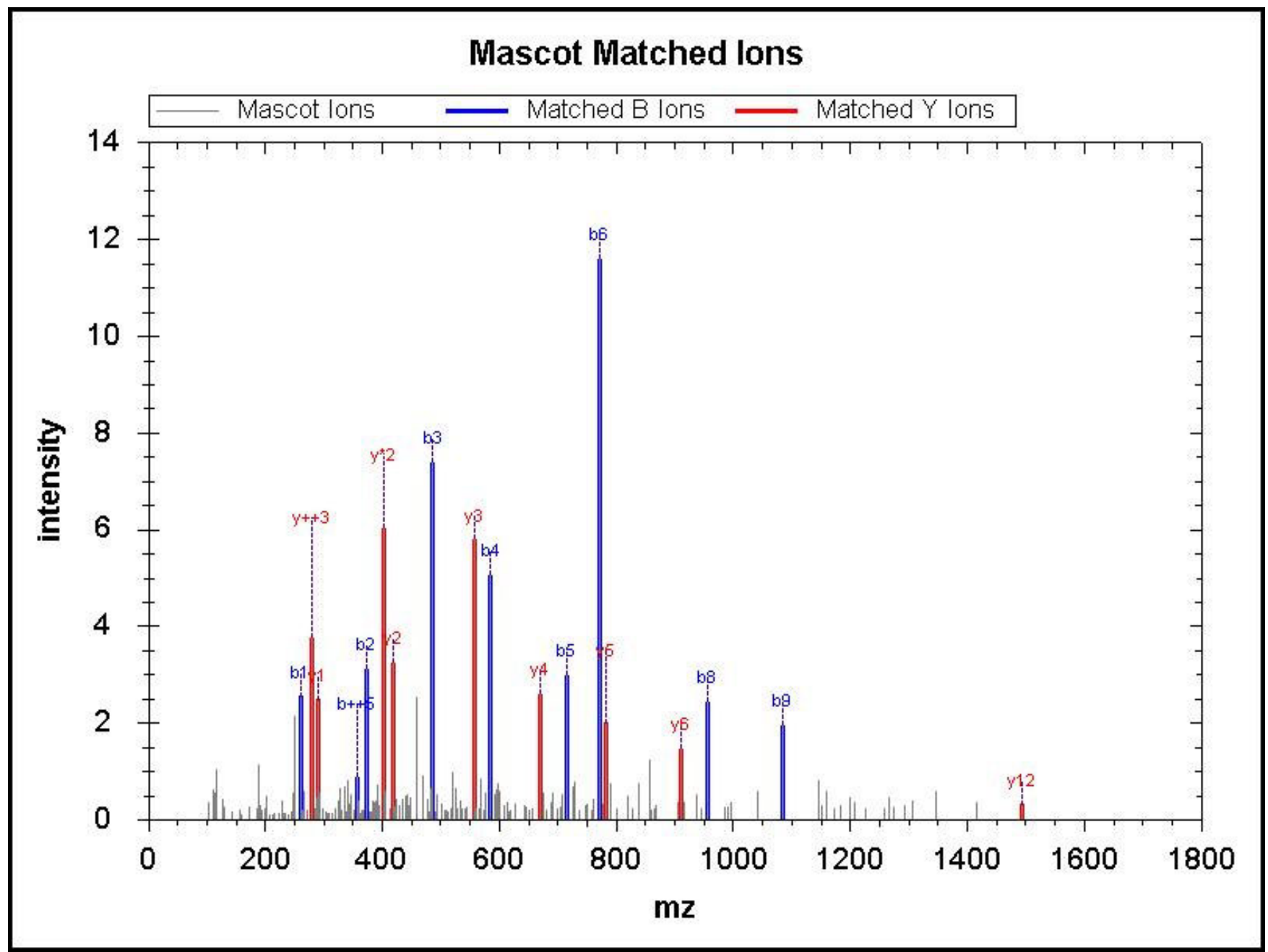

\begin{tabular}{|c|c|c|c|c|c|c|c|c|c|c|c|c|c|c|}
\hline No & $\mathrm{b}$ & $\mathrm{b}++$ & $b^{*}$ & $b^{*++}$ & b0 & $\mathrm{b} 0++$ & Seq & $\mathrm{y}$ & $\mathrm{y}++$ & $y^{*}$ & $\mathrm{y}^{*++}$ & y0 & y0++ & RevNo \\
\hline 1 & 260.14 & 130.57 & & & 242.13 & 121.57 & $\mathrm{D}$ & & & & & & & 14 \\
\hline 2 & 373.22 & 187.11 & & & 355.21 & 178.11 & $\mathrm{~L}$ & $1,606.96$ & 803.99 & $1,589.94$ & 795.47 & $1,588.95$ & 794.98 & 13 \\
\hline 3 & 486.30 & 243.66 & & & 468.29 & 234.65 & $\mathrm{~L}$ & $1,493.88$ & 747.44 & $1,476.85$ & 738.93 & $1,475.87$ & 738.44 & 12 \\
\hline 4 & 585.37 & 293.19 & & & 567.36 & 284.18 & V & $1,380.80$ & 690.90 & $1,363.77$ & 682.39 & $1,362.79$ & 681.90 & 11 \\
\hline 5 & 714.42 & 357.71 & & & 696.40 & 348.71 & $\mathrm{E}$ & $1,281.73$ & 641.37 & $1,264.70$ & 632.85 & $1,263.72$ & 632.36 & 10 \\
\hline 6 & 771.44 & 386.22 & & & 753.43 & 377.22 & G & $1,152.69$ & 576.85 & $1,135.66$ & 568.33 & $1,134.68$ & 567.84 & 9 \\
\hline 7 & 884.52 & 442.76 & & & 866.51 & 433.76 & L & $1,095.66$ & 548.34 & $1,078.64$ & 539.82 & $1,077.65$ & 539.33 & 8 \\
\hline 8 & 955.56 & 478.28 & & & 937.55 & 469.28 & A & 982.58 & 491.79 & 965.55 & 483.28 & 964.57 & 482.79 & 7 \\
\hline 9 & $1,084.60$ & 542.80 & & & $1,066.59$ & 533.80 & $\mathrm{E}$ & 911.54 & 456.28 & 894.52 & 447.76 & 893.53 & 447.27 & 6 \\
\hline 10 & $1,197.68$ & 599.35 & & & $1,179.67$ & 590.34 & I & 782.50 & 391.75 & 765.47 & 383.24 & & & 5 \\
\hline 11 & $1,310.77$ & 655.89 & & & $1,292.76$ & 646.88 & $\mathrm{~L}$ & 669.42 & 335.21 & 652.39 & 326.70 & & & 4 \\
\hline 12 & $1,447.83$ & 724.42 & & & $1,429.82$ & 715.41 & $\mathrm{H}$ & 556.33 & 278.67 & 539.31 & 270.16 & & & 3 \\
\hline 13 & $1,575.89$ & 788.45 & $1,558.86$ & 779.93 & $1,557.88$ & 779.44 & Q & 419.27 & 210.14 & 402.25 & 201.63 & & & 2 \\
\hline
\end{tabular}


Query 43479 Hit 1

MS/MS Fragmentation of AALEAQNALHNMK

Found in sp|Q92879|CELF1_HUMAN, CUGBP Elav-like family member 1 OS=Homo sapiens GN=CELF1 PE=1 SV=2

Match to Query 43479: 1697.91 from(566.9772,3+)

Title: 416: Scan 953 ( $\mathrm{rt}=31.2394, \mathrm{f}=3, \mathrm{i}=148)$ [D:llab212 \membranelGrace JoyceliTRAQ_42_2.raw]

Data File:Submitted from 20120508-1(merge) by Mascot Daemon on JOYCE-VAIO

Monoisotopic mass of neutral peptide $\mathrm{Mr}$ (calc): 1697.91

Variable modifications:

K13 :iTRAQ4plex (K)

Ions Score: 46.7 Expect: 0.017

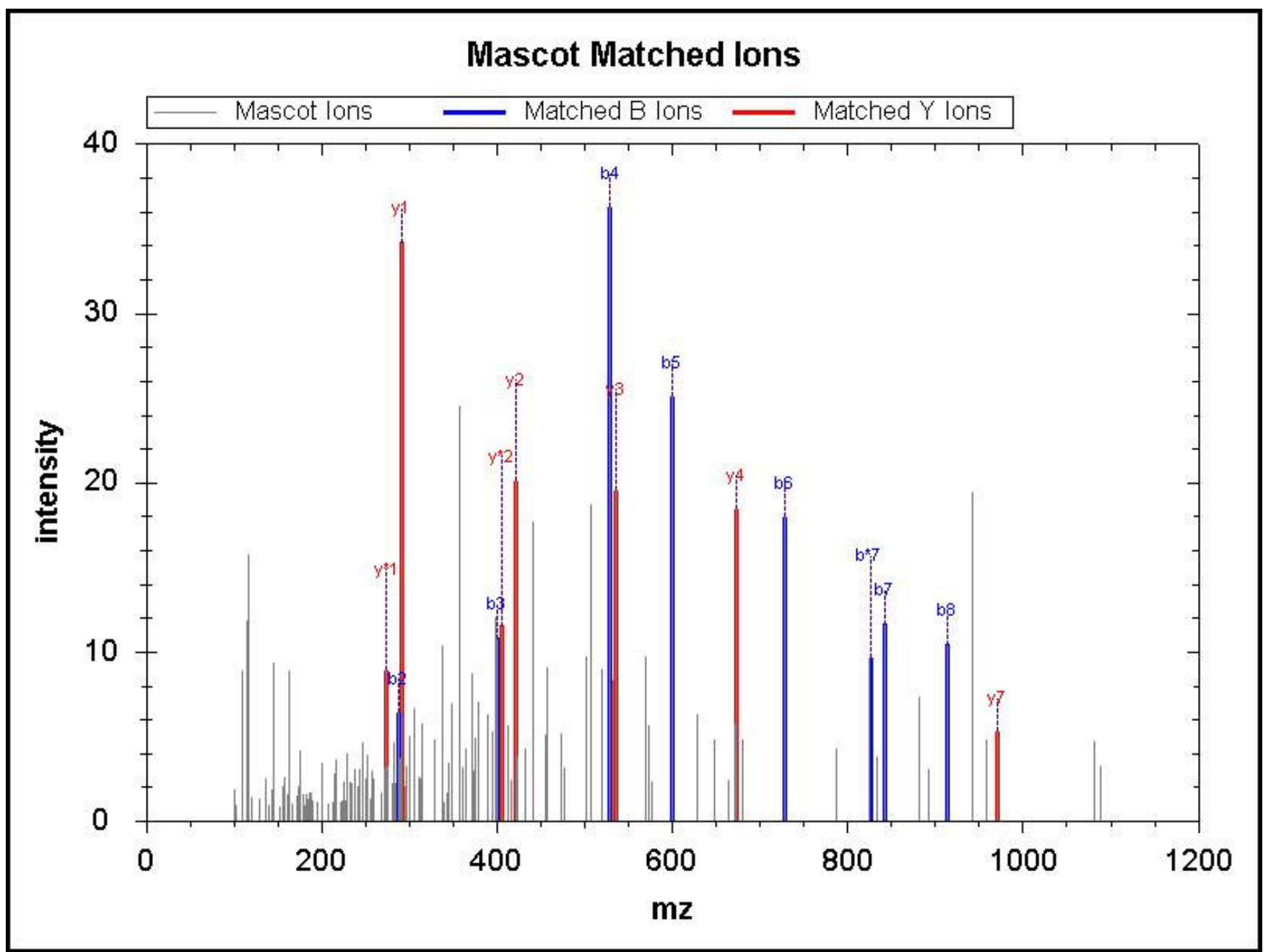

\begin{tabular}{|c|c|c|c|c|c|c|c|c|c|c|c|c|c|c|}
\hline No & $\mathrm{b}$ & $\mathrm{b}++$ & $b^{*}$ & $b^{*++}$ & b0 & b0++ & Seq & $\mathrm{y}$ & $\mathrm{y}++$ & $y^{*}$ & $y^{*++}$ & y0 & $\mathrm{y} 0++$ & RevNo \\
\hline 1 & 216.15 & 108.58 & & & & & A & & & & & & & 13 \\
\hline 2 & 287.18 & 144.10 & & & & & A & $1,483.78$ & 742.39 & $1,466.75$ & 733.88 & $1,465.77$ & 733.39 & 12 \\
\hline 3 & 400.27 & 200.64 & & & & & $\mathrm{~L}$ & $1,412.74$ & 706.88 & $1,395.72$ & 698.36 & $1,394.73$ & 697.87 & 11 \\
\hline 4 & 529.31 & 265.16 & & & 511.30 & 256.15 & $\mathrm{E}$ & $1,299.66$ & 650.33 & $1,282.63$ & 641.82 & $1,281.65$ & 641.33 & 10 \\
\hline 5 & 600.35 & 300.68 & & & 582.34 & 291.67 & A & $1,170.62$ & 585.81 & $1,153.59$ & 577.30 & & & 9 \\
\hline 6 & 728.41 & 364.71 & 711.38 & 356.19 & 710.40 & 355.70 & Q & $1,099.58$ & 550.29 & $1,082.55$ & 541.78 & & & 8 \\
\hline 7 & 842.45 & 421.73 & 825.42 & 413.21 & 824.44 & 412.72 & $\mathrm{~N}$ & 971.52 & 486.26 & 954.49 & 477.75 & & & 7 \\
\hline 8 & 913.49 & 457.25 & 896.46 & 448.73 & 895.48 & 448.24 & A & 857.48 & 429.24 & 840.45 & 420.73 & & & 6 \\
\hline 9 & $1,026.57$ & 513.79 & $1,009.54$ & 505.28 & $1,008.56$ & 504.78 & $\mathrm{~L}$ & 786.44 & 393.72 & 769.41 & 385.21 & & & 5 \\
\hline 10 & $1,163.63$ & 582.32 & $1,146.60$ & 573.80 & $1,145.62$ & 573.31 & $\mathrm{H}$ & 673.36 & 337.18 & 656.33 & 328.67 & & & 4 \\
\hline 11 & $1,277.67$ & 639.34 & $1,260.65$ & 630.83 & $1,259.66$ & 630.33 & $\mathrm{~N}$ & 536.30 & 268.65 & 519.27 & 260.14 & & & 3 \\
\hline 12 & $1,408.71$ & 704.86 & $1,391.69$ & 696.35 & $1,390.70$ & 695.85 & $\mathrm{M}$ & 422.26 & 211.63 & 405.23 & 203.12 & & & 2 \\
\hline 13 & & & & & & & $\mathrm{~K}$ & 291.21 & 146.11 & 274.19 & 137.60 & & & 1 \\
\hline
\end{tabular}


MS/MS Fragmentation of QVPDTSVQETDR

Found in sp|Q5J 8M3|TMM 85_HUM AN, Transmembrane protein 85 OS=Homo sapiens GN=TMEM85 PE=1 SV=2

Match to Query 33779: 1517.739 from(759.8768,2+)

Title: 86: Sum of 2 scans in range $494(\mathrm{rt}=19.5161, \mathrm{f}=4, \mathrm{i}=41)$ to $495(\mathrm{rt}=19.5415, \mathrm{f}=4, \mathrm{i}=42)$

[D:llab212 \membranelGrace JoyceliTRAQ_23_2.raw]

Data File:Submitted from 20120508-1(merge) by Mascot Daemon on JOYCE-VAIO

Monoisotopic mass of neutral peptide $\operatorname{Mr}($ calc): 1517.739

Variable modifications:

Ions Score: 46.57 Expect: 0.016

\section{Mascot Matched lons}

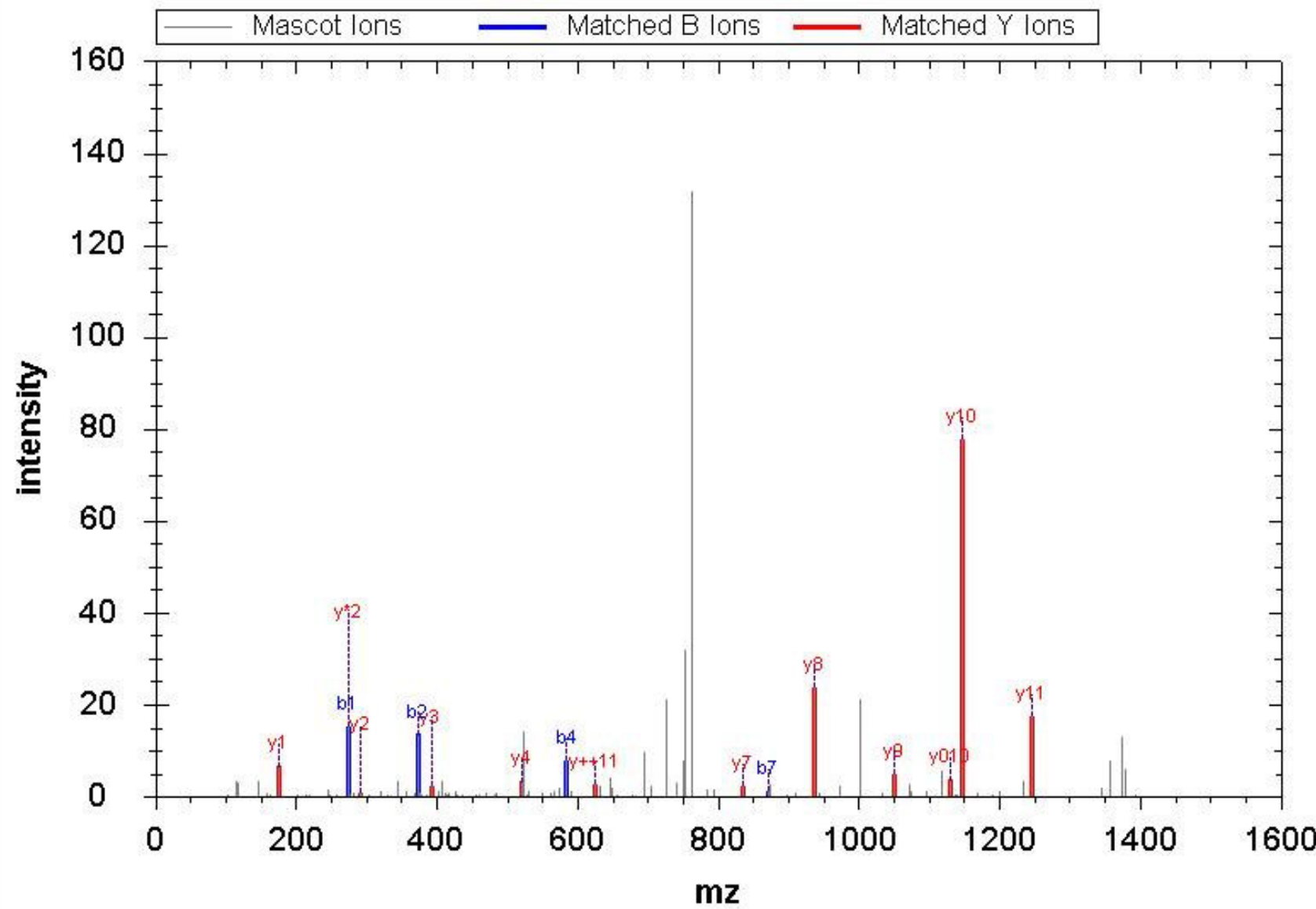

\begin{tabular}{|r|c|c|c|c|c|c|c|c|c|c|c|r|r|r|}
\hline $\mathrm{No}$ & $\mathrm{b}$ & $\mathrm{b}++$ & $\mathrm{b}^{*}$ & $\mathrm{~b}^{*++}$ & $\mathrm{b} 0$ & $\mathrm{~b} 0++$ & Seq & $\mathrm{y}$ & $\mathrm{y}++$ & $\mathrm{y}^{*}$ & $\mathrm{y}^{*++}$ & $\mathrm{y} 0$ & $\mathrm{y} 0++$ & $\mathrm{RevNo}$ \\
\hline 1 & 273.17 & 137.09 & 256.14 & 128.57 & & & $\mathrm{Q}$ & & & & & & & 12 \\
\hline 2 & 372.24 & 186.62 & 355.21 & 178.11 & & & $\mathrm{~V}$ & $1,246.59$ & 623.80 & $1,229.56$ & 615.29 & $1,228.58$ & 614.79 & 11 \\
\hline 3 & 469.29 & 235.15 & 452.26 & 226.63 & & & $\mathrm{P}$ & $1,147.52$ & 574.26 & $1,130.50$ & 565.75 & $1,129.51$ & 565.26 & 10 \\
\hline 4 & 584.32 & 292.66 & 567.29 & 284.15 & 566.31 & 283.66 & $\mathrm{D}$ & $1,050.47$ & 525.74 & $1,033.44$ & 517.23 & $1,032.46$ & 516.73 & 9 \\
\hline 5 & 685.36 & 343.19 & 668.34 & 334.67 & 667.35 & 334.18 & $\mathrm{~T}$ & 935.44 & 468.23 & 918.42 & 459.71 & 917.43 & 459.22 & 8 \\
\hline 6 & 772.40 & 386.70 & 755.37 & 378.19 & 754.39 & 377.70 & $\mathrm{~S}$ & 834.40 & 417.70 & 817.37 & 409.19 & 816.38 & 408.70 & 7 \\
\hline 7 & 871.46 & 436.24 & 854.44 & 427.72 & 853.45 & 427.23 & $\mathrm{~V}$ & 747.36 & 374.19 & 730.34 & 365.67 & 729.35 & 365.18 & 6 \\
\hline 8 & 999.52 & 500.27 & 982.50 & 491.75 & 981.51 & 491.26 & $\mathrm{Q}$ & 648.29 & 324.65 & 631.27 & 316.14 & 630.28 & 315.65 & 5 \\
\hline 9 & $1,128.57$ & 564.79 & $1,111.54$ & 556.27 & $1,110.55$ & 555.78 & $\mathrm{E}$ & 520.24 & 260.62 & 503.21 & 252.11 & 502.23 & 251.62 & 4 \\
\hline 10 & $1,229.61$ & 615.31 & $1,212.59$ & 606.80 & $1,211.60$ & 606.30 & $\mathrm{~T}$ & 391.19 & 196.10 & 374.17 & 187.59 & 373.18 & 187.10 & 3 \\
\hline 11 & $1,344.64$ & 672.82 & $1,327.61$ & 664.31 & $1,326.63$ & 663.82 & $\mathrm{D}$ & 290.15 & 145.58 & 273.12 & 137.06 & 272.14 & 136.57 & 2 \\
\hline 12 & & & & & & & $\mathrm{R}$ & 175.12 & 88.06 & 158.09 & 79.55 & & & 1 \\
\hline
\end{tabular}

Query 82374 Hit 1 
Found in sp|P18084|ITB5_HUMAN, Integrin beta-5 OS=Homo sapiens GN=ITGB5 PE=1 SV=1

Match to Query 82374: 2577.407 from(645.359,4+)

Title: 1001: Scan $2187(\mathrm{rt}=58.8698, \mathrm{f}=3, \mathrm{i}=340)$ [D:llab212 $\backslash$ membranelGrace JoyceliTRAQ_44_2.raw]

Data File:Submitted from 20120508-1(merge) by Mascot Daemon on JOYCE-VAIO

Monoisotopic mass of neutral peptide $\mathrm{Mr}$ (calc): 2577.407

Variable modifications:

K21 :iTRAQ4plex (K)

Ions Score: 46.55 Expect: 0.014

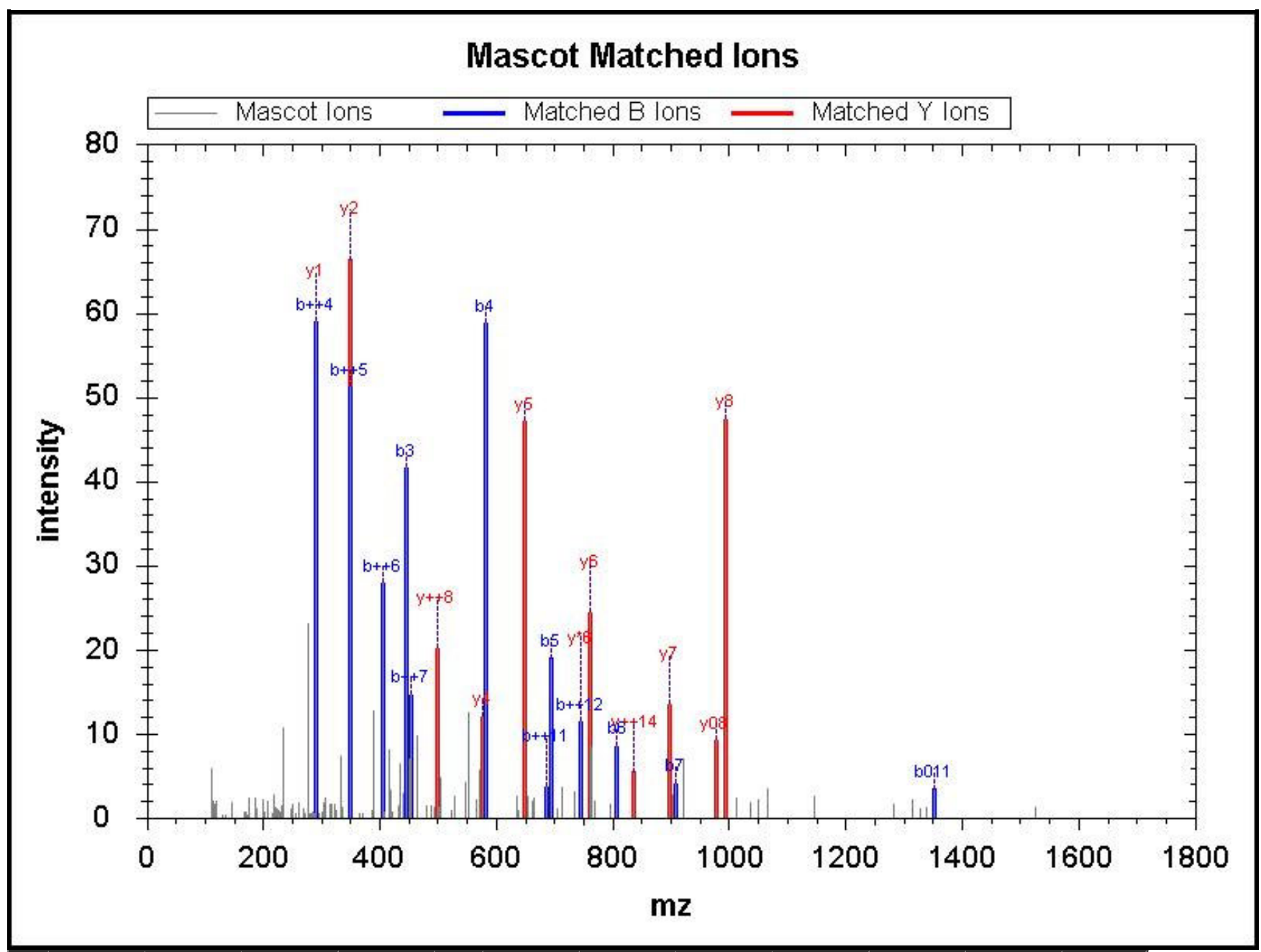

\begin{tabular}{|r|c|r|r|r|r|r|r|r|r|r|r|r|}
\hline $\mathrm{No}$ & $\mathrm{b}$ & $\mathrm{b}++$ & $\mathrm{b} 0$ & $\mathrm{~b} 0++$ & $\mathrm{Seq}$ & $\mathrm{y}$ & $\mathrm{y}++$ & $\mathrm{y} *$ & $\mathrm{y} *++$ & $\mathrm{y} 0$ & $\mathrm{y} 0++$ & $\mathrm{RevNo}$ \\
\hline 1 & 260.14 & 130.57 & 242.13 & 121.57 & $\mathrm{D}$ & & & & & & & 21 \\
\hline 2 & 331.17 & 166.09 & 313.16 & 157.09 & $\mathrm{~A}$ & $2,319.28$ & $1,160.15$ & $2,302.26$ & $1,151.63$ & $2,301.27$ & $1,151.14$ & 20 \\
\hline 3 & 444.26 & 222.63 & 426.25 & 213.63 & $\mathrm{~L}$ & $2,248.25$ & $1,124.63$ & $2,231.22$ & $1,116.11$ & $2,230.24$ & $1,115.62$ & 19 \\
\hline 4 & 581.32 & 291.16 & 563.31 & 282.16 & $\mathrm{H}$ & $2,135.16$ & $1,068.08$ & $2,118.14$ & $1,059.57$ & $2,117.15$ & $1,059.08$ & 18 \\
\hline 5 & 694.40 & 347.70 & 676.39 & 338.70 & $\mathrm{~L}$ & $1,998.10$ & 999.56 & $1,981.08$ & 991.04 & $1,980.09$ & 990.55 & 17 \\
\hline 6 & 807.48 & 404.25 & 789.47 & 395.24 & $\mathrm{~L}$ & $1,885.02$ & 943.01 & $1,867.99$ & 934.50 & $1,867.01$ & 934.01 & 16 \\
\hline 7 & 906.55 & 453.78 & 888.54 & 444.77 & $\mathrm{~V}$ & $1,771.93$ & 886.47 & $1,754.91$ & 877.96 & $1,753.92$ & 877.47 & 15 \\
\hline 8 & $1,053.62$ & 527.31 & $1,035.61$ & 518.31 & $\mathrm{~F}$ & $1,672.87$ & 836.94 & $1,655.84$ & 828.42 & $1,654.86$ & 827.93 & 14 \\
\hline 9 & $1,154.67$ & 577.84 & $1,136.66$ & 568.83 & $\mathrm{~T}$ & $1,525.80$ & 763.40 & $1,508.77$ & 754.89 & $1,507.79$ & 754.40 & 13 \\
\hline 10 & $1,255.72$ & 628.36 & $1,237.71$ & 619.36 & $\mathrm{~T}$ & $1,424.75$ & 712.88 & $1,407.72$ & 704.37 & $1,406.74$ & 703.87 & 12 \\
\hline 11 & $1,370.74$ & 685.88 & $1,352.73$ & 676.87 & $\mathrm{D}$ & $1,323.70$ & 662.35 & $1,306.68$ & 653.84 & $1,305.69$ & 653.35 & 11 \\
\hline 12 & $1,485.77$ & 743.39 & $1,467.76$ & 734.38 & $\mathrm{D}$ & $1,208.68$ & 604.84 & $1,191.65$ & 596.33 & $1,190.66$ & 595.84 & 10 \\
\hline 13 & $1,584.84$ & 792.92 & $1,566.83$ & 783.92 & $\mathrm{~V}$ & $1,093.65$ & 547.33 & $1,076.62$ & 538.81 & $1,075.64$ & 538.32 & 9 \\
\hline 14 & $1,681.89$ & 841.45 & $1,663.88$ & 832.44 & $\mathrm{P}$ & 994.58 & 497.79 & 977.55 & 489.28 & 976.57 & 488.79 & 8 \\
\hline 15 & $1,818.95$ & 909.98 & $1,800.94$ & 900.97 & $\mathrm{H}$ & 897.53 & 449.27 & 880.50 & 440.75 & 879.52 & 440.26 & 7 \\
\hline 16 & $1,932.03$ & 966.52 & $1,914.02$ & 957.52 & $\mathrm{I}$ & 760.47 & 380.74 & 743.44 & 372.22 & 742.46 & 371.73 & 6 \\
\hline 17 & $2,003.07$ & $1,002.04$ & $1,985.06$ & 993.03 & A & 647.38 & 324.20 & 630.36 & 315.68 & 629.37 & 315.19 & 5 \\
\hline
\end{tabular}




\begin{tabular}{|l|l|l|l|l|l|l|l|l|l|l|l|r|}
18 & $2,116.16$ & $1,058.58$ & $2,098.15$ & $1,049.58$ & $\mathrm{~L}$ & 576.35 & 288.68 & 559.32 & 280.16 & 558.34 & 279.67 & 4 \\
\hline \hline 19 & $2,231.18$ & $1,116.10$ & $2,213.17$ & $1,107.09$ & $\mathrm{D}$ & 463.26 & 232.14 & 446.24 & 223.62 & 445.25 & 223.13 & 3 \\
\hline 20 & $2,288.20$ & $1,144.61$ & $2,270.19$ & $1,135.60$ & $\mathrm{G}$ & 348.24 & 174.62 & 331.21 & 166.11 & & & 2 \\
\hline 21 & & & & & $\mathrm{~K}$ & 291.21 & 146.11 & 274.19 & 137.60 & & & 1 \\
\hline
\end{tabular}

Query 70127 Hit 1

MS/MS Fragmentation of ETTGTGPNVYHENDTIAK

Found in sp|Q4G0F5|VP26B_HUM AN, Vacuolar protein sorting-associated protein 26B OS=Homo sapiens GN=VPS26B PE=1 SV=2 Match to Query 70127:2234.11 from(745.7106,3+)

Title: 226: Scan 601 ( $\mathrm{rt}=22.8682, \mathrm{f}=3, \mathrm{i}=80)$ [D:llab212 \membranelGracelJoyceliTRAQ_38_1.raw]

Data File:Submitted from 20120508-1(merge) by Mascot Daemon on JOYCE-VAIO

Monoisotopic mass of neutral peptide $\mathrm{Mr}$ (calc): 2234.11

Variable modifications:

K18 :TTRAQ4plex (K)

Ions Score: 46.51 Expect: 0.021

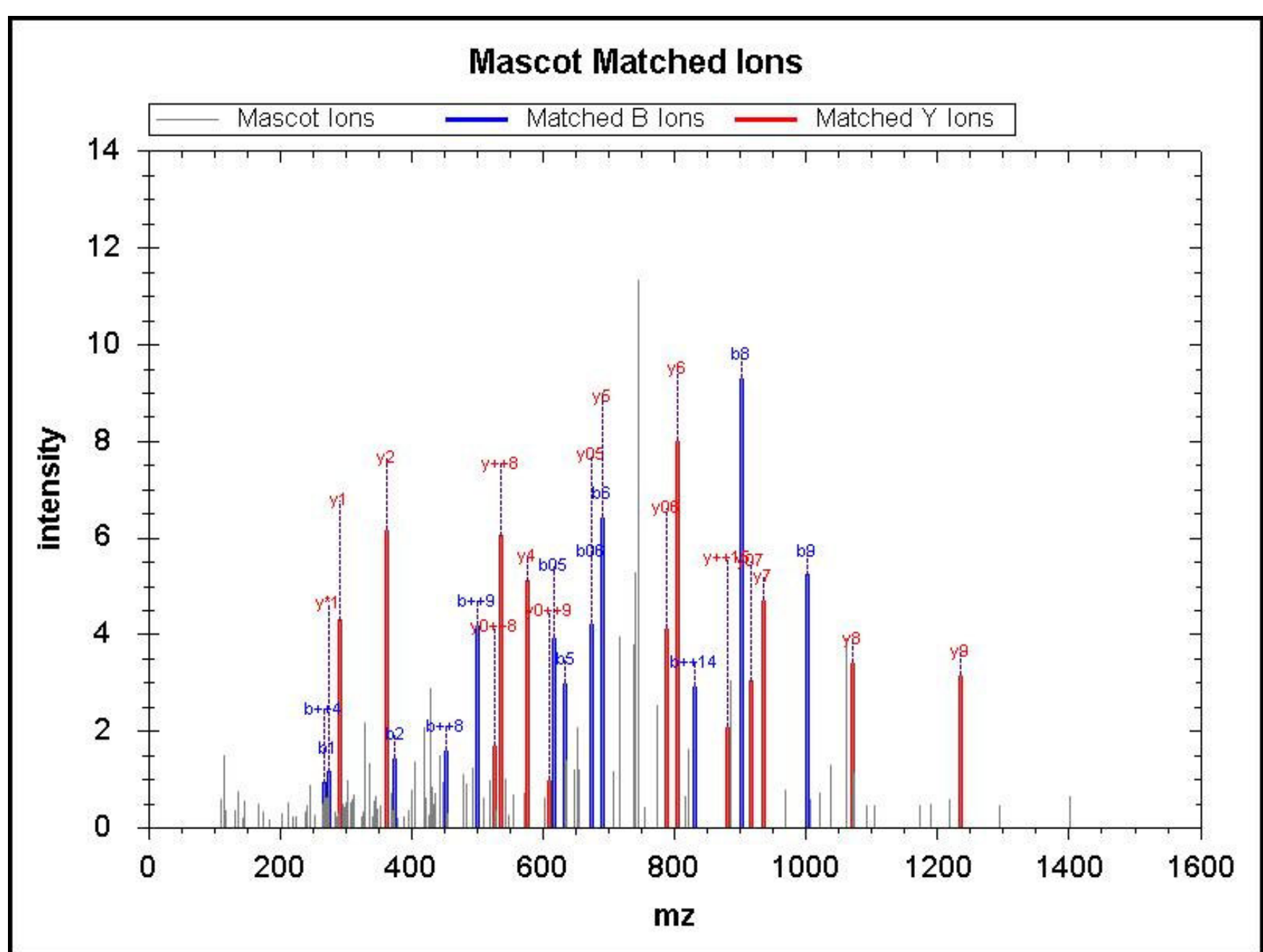

\begin{tabular}{|r|c|c|c|c|c|c|c|c|c|c|c|r|r|r|}
\hline \hline No & $\mathrm{b}$ & $\mathrm{b}++$ & $\mathrm{b} *$ & $\mathrm{~b} *++$ & $\mathrm{b} 0$ & $\mathrm{~b} 0++$ & $\mathrm{Seq}$ & $\mathrm{y}$ & $\mathrm{y}++$ & $\mathrm{y}^{*}$ & $\mathrm{y}^{*++}$ & $\mathrm{y} 0$ & $\mathrm{y} 0++$ & $\mathrm{RevNo}$ \\
\hline 1 & 274.15 & 137.58 & & & 256.14 & 128.57 & $\mathrm{E}$ & & & & & & & 18 \\
\hline 2 & 375.20 & 188.10 & & & 357.19 & 179.10 & $\mathrm{~T}$ & $1,961.97$ & 981.49 & $1,944.94$ & 972.97 & $1,943.96$ & 972.48 & 17 \\
\hline 3 & 476.25 & 238.63 & & & 458.24 & 229.62 & $\mathrm{~T}$ & $1,860.92$ & 930.96 & $1,843.89$ & 922.45 & $1,842.91$ & 921.96 & 16 \\
\hline 4 & 533.27 & 267.14 & & & 515.26 & 258.13 & $\mathrm{G}$ & $1,759.87$ & 880.44 & $1,742.85$ & 871.93 & $1,741.86$ & 871.43 & 15 \\
\hline 5 & 634.32 & 317.66 & & & 616.31 & 308.66 & $\mathrm{~T}$ & $1,702.85$ & 851.93 & $1,685.83$ & 843.42 & $1,684.84$ & 842.92 & 14 \\
\hline 6 & 691.34 & 346.17 & & & 673.33 & 337.17 & $\mathrm{G}$ & $1,601.80$ & 801.41 & $1,584.78$ & 792.89 & $1,583.79$ & 792.40 & 13 \\
\hline 7 & 788.39 & 394.70 & & & 770.38 & 385.69 & $\mathrm{P}$ & $1,544.78$ & 772.89 & $1,527.76$ & 764.38 & $1,526.77$ & 763.89 & 12 \\
\hline 8 & 902.43 & 451.72 & 885.41 & 443.21 & 884.42 & 442.72 & $\mathrm{~N}$ & $1,447.73$ & 724.37 & $1,430.70$ & 715.86 & $1,429.72$ & 715.36 & 11 \\
\hline 9 & $1,001.50$ & 501.25 & 984.48 & 492.74 & 983.49 & 492.25 & $\mathrm{~V}$ & $1,333.69$ & 667.35 & $1,316.66$ & 658.83 & $1,315.68$ & 658.34 & 10 \\
\hline 10 & $1,164.57$ & 582.79 & $1,147.54$ & 574.27 & $1,146.55$ & 573.78 & $\mathrm{Y}$ & $1,234.62$ & 617.81 & $1,217.59$ & 609.30 & $1,216.61$ & 608.81 & 9 \\
\hline
\end{tabular}




\begin{tabular}{|r|r|r|r|r|r|r|r|r|r|r|r|r|r|r|r|}
111 & $1,301.62$ & 651.32 & $1,284.60$ & 642.80 & $1,283.61$ & 642.31 & $\mathrm{H}$ & $1,071.56$ & 536.28 & $1,054.53$ & 527.77 & $1,053.54$ & 527.28 & 8 \\
\hline 12 & $1,430.67$ & 715.84 & $1,413.64$ & 707.32 & $1,412.66$ & 706.83 & $\mathrm{E}$ & 934.50 & 467.75 & 917.47 & 459.24 & 916.49 & 458.75 & 7 \\
\hline 13 & $1,544.71$ & 772.86 & $1,527.68$ & 764.35 & $1,526.70$ & 763.85 & $\mathrm{~N}$ & 805.45 & 403.23 & 788.43 & 394.72 & 787.44 & 394.23 & 6 \\
\hline 14 & $1,659.74$ & 830.37 & $1,642.71$ & 821.86 & $1,641.73$ & 821.37 & $\mathrm{D}$ & 691.41 & 346.21 & 674.38 & 337.70 & 673.40 & 337.20 & 5 \\
\hline 15 & $1,760.78$ & 880.90 & $1,743.76$ & 872.38 & $1,742.77$ & 871.89 & $\mathrm{~T}$ & 576.38 & 288.70 & 559.36 & 280.18 & 558.37 & 279.69 & 4 \\
\hline 16 & $1,873.87$ & 937.44 & $1,856.84$ & 928.92 & $1,855.86$ & 928.43 & $\mathrm{I}$ & 475.34 & 238.17 & 458.31 & 229.66 & & & 3 \\
\hline 17 & $1,944.91$ & 972.96 & $1,927.88$ & 964.44 & $1,926.90$ & 963.95 & $\mathrm{~A}$ & 362.25 & 181.63 & 345.23 & 173.12 & & & 2 \\
\hline 18 & & & & & & & $\mathrm{~K}$ & 291.21 & 146.11 & 274.19 & 137.60 & & & 1 \\
\hline
\end{tabular}

Query 70293 Hit 1

MS/MS Fragmentation of ITELR PFNSWEALFTK

Found in sp|Q9H4L7|SMRCD_HUM AN, SWI/SNF-related matrix-associated actin-dependent regulator of chromatin subfamily A containing $\mathrm{DEAD} / \mathrm{H}$ box $1 \mathrm{OS}=$ Homo sapiens $\mathrm{GN}=\mathrm{SMARCAD} 1 \mathrm{PE}=1 \mathrm{SV}=2$

Match to Query 70293: 2239.225from(747.4157,3+)

Title: 1049: Scan $2291(\mathrm{rt}=61.2141, \mathrm{f}=3, \mathrm{i}=360)$ [D:llab212 $\mathrm{membrane} /$ GracelJoyceliTRAQ_39_2.raw]

Data File:Submitted from 20120508-1(merge) by Mascot Daemon on JOYCE-VAIO

Monoisotopic mass of neutral peptide $\mathrm{Mr}$ (calc): 2239.225

Variable modifications:

K16 :TRAQ4plex (K)

Ions Score: 46.47 Expect: 0.016

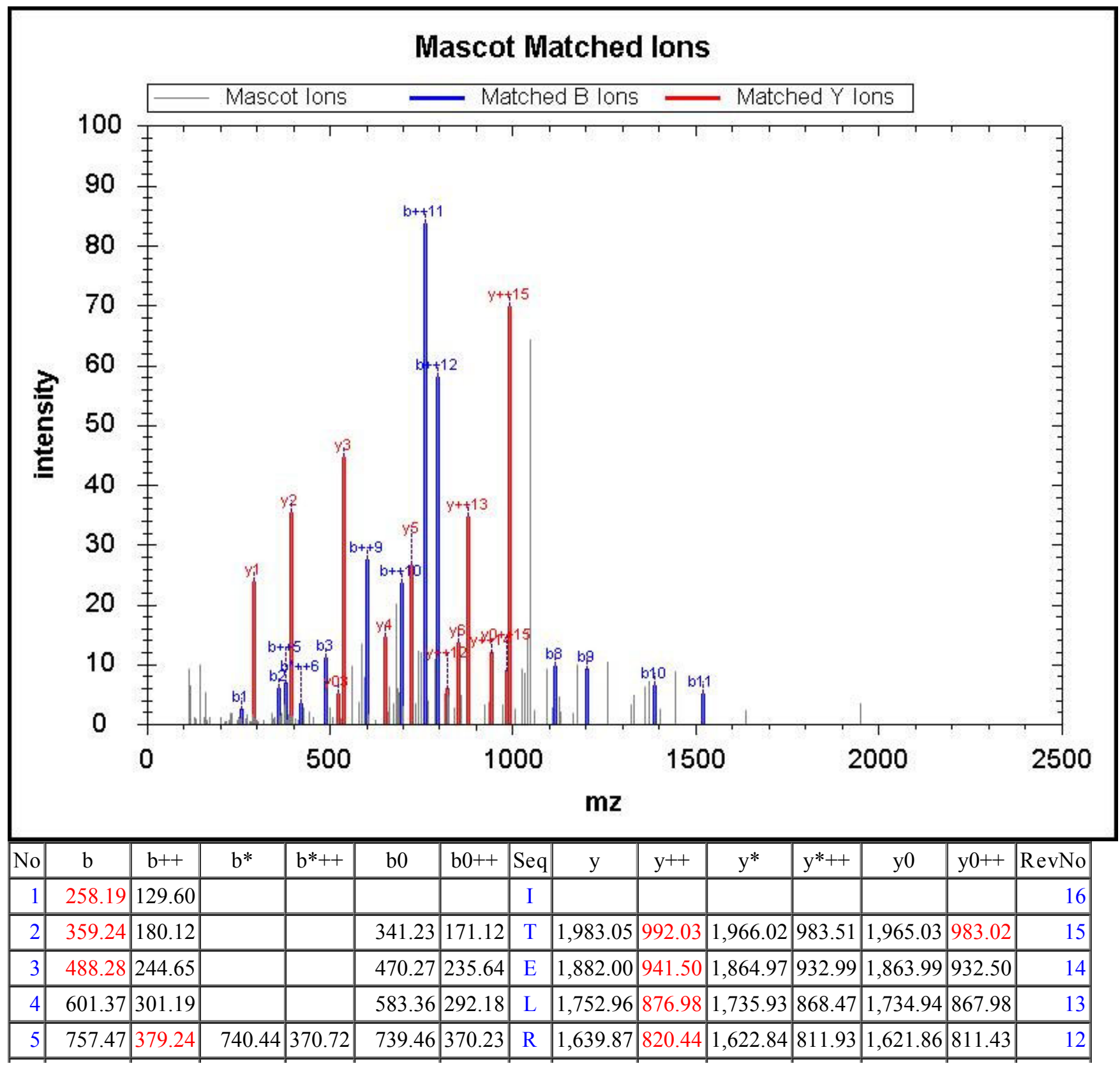




\begin{tabular}{|r|r|r|r|r|r|r|r|r|r|r|r|r|r|r|r|}
6 & 854.52 & 427.76 & 837.49 & 419.25 & 836.51 & 418.76 & $\mathrm{P}$ & $1,483.77$ & 742.39 & $1,466.74$ & 733.88 & $1,465.76$ & 733.38 & 11 \\
\hline \hline 7 & $1,001.59$ & 501.30 & 984.56 & 492.79 & 983.58 & 492.29 & $\mathrm{~F}$ & $1,386.72$ & 693.86 & $1,369.69$ & 685.35 & $1,368.71$ & 684.86 & 10 \\
\hline 8 & $1,115.63$ & 558.32 & $1,098.61$ & 549.81 & $1,097.62$ & 549.31 & $\mathrm{~N}$ & $1,239.65$ & 620.33 & $1,222.62$ & 611.81 & $1,221.64$ & 611.32 & 9 \\
\hline 9 & $1,202.66$ & 601.84 & $1,185.64$ & 593.32 & $1,184.65$ & 592.83 & $\mathrm{~S}$ & $1,125.61$ & 563.31 & $1,108.58$ & 554.79 & $1,107.60$ & 554.30 & 8 \\
\hline 10 & $1,388.74$ & 694.88 & $1,371.72$ & 686.36 & $1,370.73$ & 685.87 & $\mathrm{~W}$ & $1,038.57$ & 519.79 & $1,021.55$ & 511.28 & $1,020.56$ & 510.79 & 7 \\
\hline 11 & $1,517.79$ & 759.40 & $1,500.76$ & 750.88 & $1,499.78$ & 750.39 & $\mathrm{E}$ & 852.49 & 426.75 & 835.47 & 418.24 & 834.48 & 417.75 & 6 \\
\hline 12 & $1,588.82$ & 794.92 & $1,571.80$ & 786.40 & $1,570.81$ & 785.91 & $\mathrm{~A}$ & 723.45 & 362.23 & 706.43 & 353.72 & 705.44 & 353.22 & 5 \\
\hline 13 & $1,701.91$ & 851.46 & $1,684.88$ & 842.94 & $1,683.90$ & 842.45 & $\mathrm{~L}$ & 652.41 & 326.71 & 635.39 & 318.20 & 634.40 & 317.71 & 4 \\
\hline 14 & $1,848.98$ & 924.99 & $1,831.95$ & 916.48 & $1,830.97$ & 915.99 & $\mathrm{~F}$ & 539.33 & 270.17 & 522.30 & 261.66 & 521.32 & 261.16 & 3 \\
\hline 15 & $1,950.02$ & 975.52 & $1,933.00$ & 967.00 & $1,932.01$ & 966.51 & $\mathrm{~T}$ & 392.26 & 196.63 & 375.24 & 188.12 & 374.25 & 187.63 & 2 \\
\hline 16 & & & & & & & $\mathrm{~K}$ & 291.21 & 146.11 & 274.19 & 137.60 & & & 1 \\
\hline
\end{tabular}

Query 74619 Hit 1

MS/MS Fragmentation of DTPEDIVLEAPASGLAFHPAR

Found in sp|Q9H6Y2|WDR55_HUMAN, WD repeat-containing protein 55 OS=Homo sapiens GN=WDR55 PE=1 SV=2

Match to Query 74619: 2349.209from(784.077,3+)

Title: 762: Sum of 2 scans in range 1769 ( $\mathrm{rt}=49.0159, \mathrm{f}=4, \mathrm{i}=511)$ to 1770 ( $\mathrm{rt}=49.0413, \mathrm{f}=4, \mathrm{i}=512$ )

[D:llab212\membranelGrace JoyceliTRAQ_30_1.raw]

Data File:Submitted from 20120508-1(merge) by Mascot Daemon on JOYCE-VAIO

Monoisotopic mass of neutral peptide Mr(calc): 2349.209

Variable modifications:

Ions Score: 46.44 Expect: 0.022

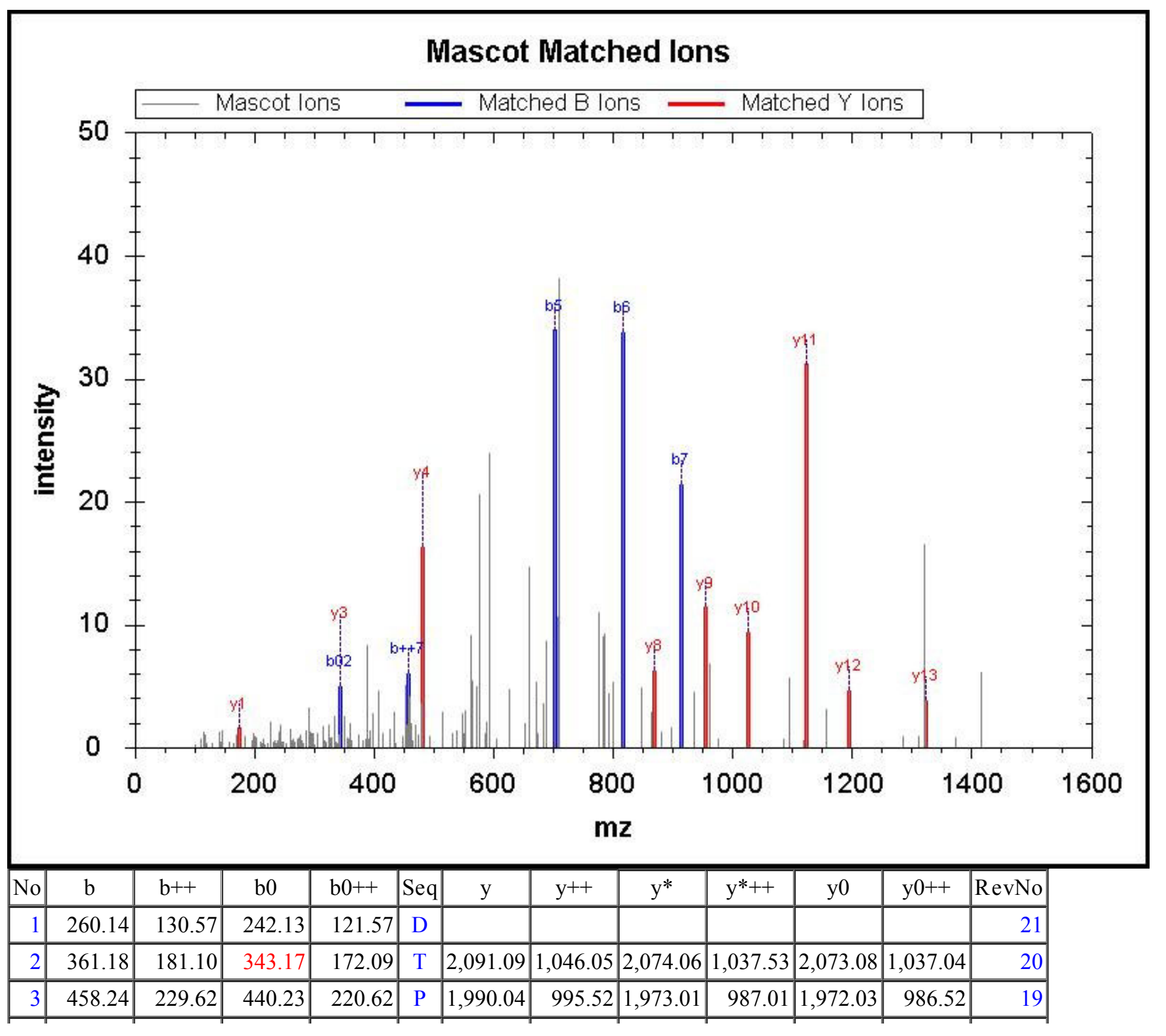




\begin{tabular}{|r|r|r|r|r|r|r|r|r|r|r|r|r|}
\hline 4 & 587.28 & 294.14 & 569.27 & 285.14 & $\mathrm{E}$ & $1,892.99$ & 947.00 & $1,875.96$ & 938.48 & $1,874.98$ & 937.99 & 18 \\
\hline 5 & 702.31 & 351.66 & 684.30 & 342.65 & $\mathrm{D}$ & $1,763.94$ & 882.48 & $1,746.92$ & 873.96 & $1,745.93$ & 873.47 & 17 \\
\hline 6 & 815.39 & 408.20 & 797.38 & 399.19 & $\mathrm{I}$ & $1,648.92$ & 824.96 & $1,631.89$ & 816.45 & $1,630.91$ & 815.96 & 16 \\
\hline 7 & 914.46 & 457.73 & 896.45 & 448.73 & $\mathrm{~V}$ & $1,535.83$ & 768.42 & $1,518.81$ & 759.91 & $1,517.82$ & 759.41 & 15 \\
\hline 8 & $1,027.54$ & 514.28 & $1,009.53$ & 505.27 & $\mathrm{~L}$ & $1,436.76$ & 718.89 & $1,419.74$ & 710.37 & $1,418.75$ & 709.88 & 14 \\
\hline 9 & $1,156.59$ & 578.80 & $1,138.57$ & 569.79 & $\mathrm{E}$ & $1,323.68$ & 662.34 & $1,306.65$ & 653.83 & $1,305.67$ & 653.34 & 13 \\
\hline 10 & $1,227.62$ & 614.31 & $1,209.61$ & 605.31 & $\mathrm{~A}$ & $1,194.64$ & 597.82 & $1,177.61$ & 589.31 & $1,176.63$ & 588.82 & 12 \\
\hline 11 & $1,324.68$ & 662.84 & $1,306.66$ & 653.84 & $\mathrm{P}$ & $1,123.60$ & 562.30 & $1,106.57$ & 553.79 & $1,105.59$ & 553.30 & 11 \\
\hline 12 & $1,395.71$ & 698.36 & $1,377.70$ & 689.35 & $\mathrm{~A}$ & $1,026.55$ & 513.78 & $1,009.52$ & 505.26 & $1,008.54$ & 504.77 & 10 \\
\hline 13 & $1,482.74$ & 741.88 & $1,464.73$ & 732.87 & $\mathrm{~S}$ & 955.51 & 478.26 & 938.48 & 469.75 & 937.50 & 469.25 & 9 \\
\hline 14 & $1,539.77$ & 770.39 & $1,521.76$ & 761.38 & $\mathrm{G}$ & 868.48 & 434.74 & 851.45 & 426.23 & & & 8 \\
\hline 15 & $1,652.85$ & 826.93 & $1,634.84$ & 817.92 & $\mathrm{~L}$ & 811.46 & 406.23 & 794.43 & 397.72 & & & 7 \\
\hline 16 & $1,723.89$ & 862.45 & $1,705.88$ & 853.44 & $\mathrm{~A}$ & 698.37 & 349.69 & 681.35 & 341.18 & & & 6 \\
\hline 17 & $1,870.96$ & 935.98 & $1,852.94$ & 926.98 & $\mathrm{~F}$ & 627.34 & 314.17 & 610.31 & 305.66 & & & 5 \\
\hline 18 & $2,008.01$ & $1,004.51$ & $1,990.00$ & 995.51 & $\mathrm{H}$ & 480.27 & 240.64 & 463.24 & 232.12 & & & 4 \\
\hline 19 & $2,105.07$ & $1,053.04$ & $2,087.06$ & $1,044.03$ & $\mathrm{P}$ & 343.21 & 172.11 & 326.18 & 163.59 & & & 3 \\
\hline 20 & $2,176.10$ & $1,088.56$ & $2,158.09$ & $1,079.55$ & $\mathrm{~A}$ & 246.16 & 123.58 & 229.13 & 115.07 & & & 2 \\
\hline 21 & & & & & $\mathrm{R}$ & 175.12 & 88.06 & 158.09 & 79.55 & & & 1 \\
\hline
\end{tabular}

Query 46595 Hit 1

MS/MS Fragmentation of EYQDIEEMIFHR

Found in sp|O70423|AOC3_MOUSE, Membrane primary amine oxidase OS=Mus musculus GN=Aoc3 PE=2 SV=3

Match to Query 46595: 1752.831 from(585.2843,3+)

Title: 839: Sum of 2 scans in range $1866(\mathrm{rt}=51.4549, \mathrm{f}=4, \mathrm{i}=559)$ to $1867(\mathrm{rt}=51.4803, \mathrm{f}=4, \mathrm{i}=560)$

[D:lab212 \membranelGrace JoyceliTRAQ_36_2.raw]

Data File:Submitted from 20120508-1(merge) by Mascot Daemon on JOYCE-VAIO

Monoisotopic mass of neutral peptide $\mathrm{Mr}$ (calc): 1752.831

Variable modifications:

Ions Score: 46.32 Expect: 0.017 


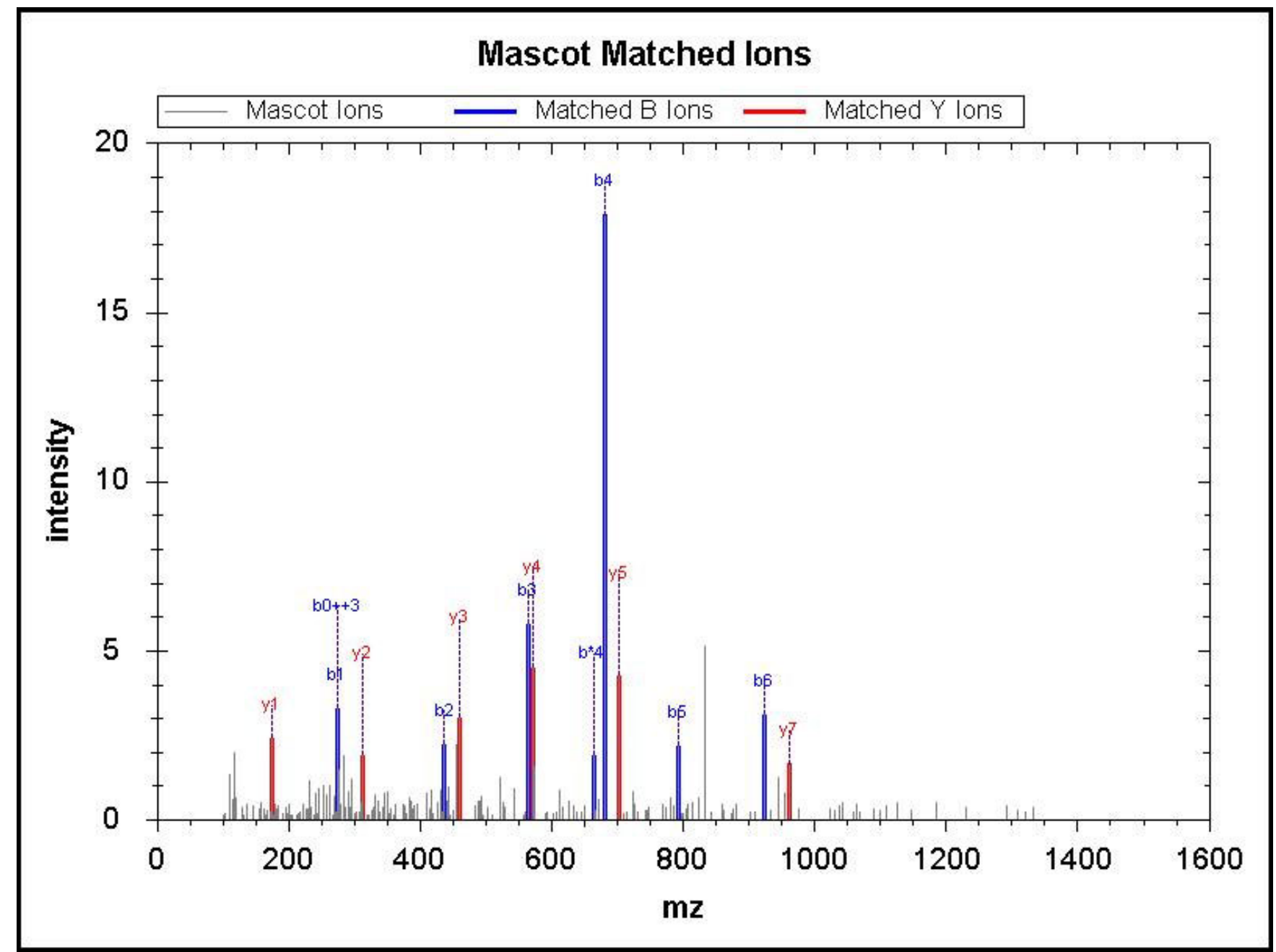

\begin{tabular}{|r|c|c|c|c|c|c|c|c|c|c|c|r|r|r|}
\hline \hline $\mathrm{No}$ & $\mathrm{b}$ & $\mathrm{b}++$ & $\mathrm{b}^{*}$ & $\mathrm{~b}++$ & $\mathrm{b} 0$ & $\mathrm{~b} 0++$ & Seq & $\mathrm{y}$ & $\mathrm{y}++$ & $\mathrm{y}^{*}$ & $\mathrm{y}^{*++}$ & $\mathrm{y} 0$ & $\mathrm{y} 0++$ & $\mathrm{RevNo}$ \\
\hline 1 & 274.15 & 137.58 & & & 256.14 & 128.57 & $\mathrm{E}$ & & & & & & & 12 \\
\hline 2 & 437.22 & 219.11 & & & 419.20 & 210.11 & $\mathrm{Y}$ & $1,480.69$ & 740.85 & $1,463.66$ & 732.33 & $1,462.68$ & 731.84 & 11 \\
\hline 3 & 565.27 & 283.14 & 548.25 & 274.63 & 547.26 & 274.14 & $\mathrm{Q}$ & $1,317.63$ & 659.32 & $1,300.60$ & 650.80 & $1,299.61$ & 650.31 & 10 \\
\hline 4 & 680.30 & 340.65 & 663.27 & 332.14 & 662.29 & 331.65 & $\mathrm{D}$ & $1,189.57$ & 595.29 & $1,172.54$ & 586.77 & $1,171.56$ & 586.28 & 9 \\
\hline 5 & 793.38 & 397.20 & 776.36 & 388.68 & 775.37 & 388.19 & $\mathrm{I}$ & $1,074.54$ & 537.77 & $1,057.51$ & 529.26 & $1,056.53$ & 528.77 & 8 \\
\hline 6 & 922.43 & 461.72 & 905.40 & 453.20 & 904.42 & 452.71 & $\mathrm{E}$ & 961.46 & 481.23 & 944.43 & 472.72 & 943.45 & 472.23 & 7 \\
\hline 7 & $1,051.47$ & 526.24 & $1,034.44$ & 517.73 & $1,033.46$ & 517.23 & $\mathrm{E}$ & 832.41 & 416.71 & 815.39 & 408.20 & 814.40 & 407.71 & 6 \\
\hline 8 & $1,182.51$ & 591.76 & $1,165.48$ & 583.25 & $1,164.50$ & 582.75 & $\mathrm{M}$ & 703.37 & 352.19 & 686.34 & 343.68 & & & 5 \\
\hline 9 & $1,295.59$ & 648.30 & $1,278.57$ & 639.79 & $1,277.58$ & 639.30 & $\mathrm{I}$ & 572.33 & 286.67 & 555.30 & 278.16 & & & 4 \\
\hline 10 & $1,442.66$ & 721.84 & $1,425.64$ & 713.32 & $1,424.65$ & 712.83 & $\mathrm{~F}$ & 459.25 & 230.13 & 442.22 & 221.61 & & & 3 \\
\hline 11 & $1,579.72$ & 790.36 & $1,562.70$ & 781.85 & $1,561.71$ & 781.36 & $\mathrm{H}$ & 312.18 & 156.59 & 295.15 & 148.08 & & & 2 \\
\hline 12 & & & & & & & $\mathrm{R}$ & 175.12 & 88.06 & 158.09 & 79.55 & & & 1 \\
\hline
\end{tabular}

Query 43336 Hit 1

\section{MS/MS Fragmentation of QGNVLVISHQAVMR}

Found in sp $|\mathrm{O} 60825| \mathrm{F} 262$ HUM AN, 6-phosphofructo-2-kinase/fructose-2

Match to Query 43336: 1694.925 from $(565.9824,3+)$

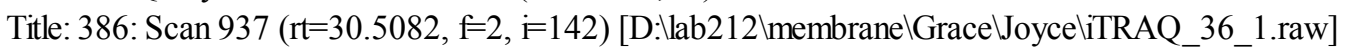

Data File:Submitted from 20120508-1(merge) by Mascot Daemon on JOYCE-VAIO

Monoisotopic mass of neutral peptide Mr(calc): 1694.925

Variable modifications:

Ions Score: 46.17 Expect: 0.019 


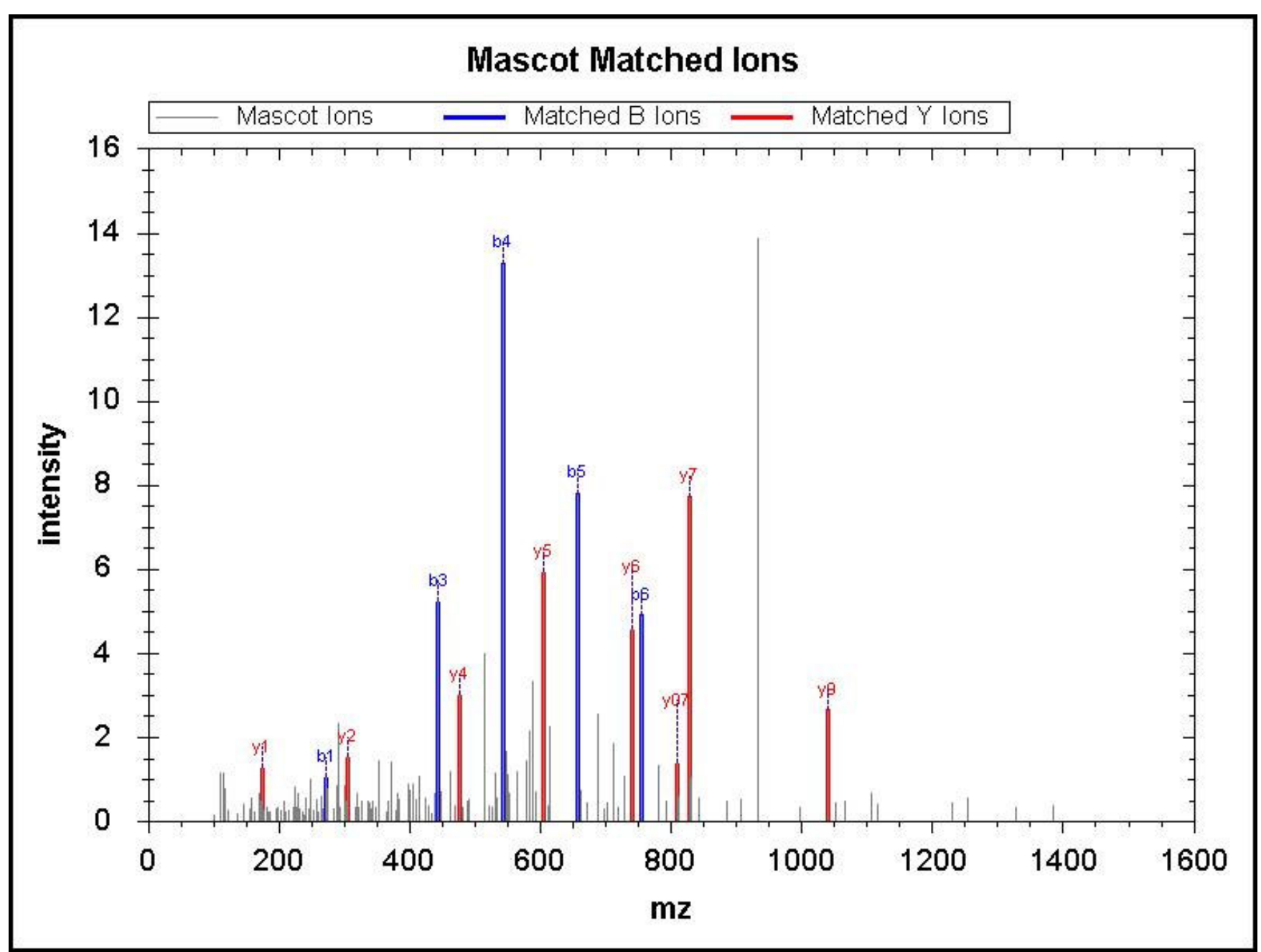

\begin{tabular}{|c|c|c|c|c|c|c|c|c|c|c|c|c|c|c|}
\hline No & $\mathrm{b}$ & $\mathrm{b}++$ & $b^{*}$ & $\mathrm{~b}^{*++}$ & b0 & b0++ & Seq & $\mathrm{y}$ & $\mathrm{y}++$ & $y^{*}$ & $\mathrm{y}^{*++}$ & y0 & $\mathrm{y} 0++$ & RevNo \\
\hline 1 & 273.17 & 137.09 & 256.14 & 128.57 & & & Q & & & & & & & 14 \\
\hline 2 & 330.19 & 165.60 & 313.16 & 157.09 & & & G & $1,423.78$ & 712.40 & $1,406.76$ & 703.88 & $1,405.77$ & 703.39 & 13 \\
\hline 3 & 444.23 & 222.62 & 427.21 & 214.11 & & & $\mathrm{~N}$ & $1,366.76$ & 683.88 & $1,349.74$ & 675.37 & $1,348.75$ & 674.88 & 12 \\
\hline 4 & 543.30 & 272.15 & 526.27 & 263.64 & & & V & $1,252.72$ & 626.86 & $1,235.69$ & 618.35 & $1,234.71$ & 617.86 & 11 \\
\hline 5 & 656.38 & 328.70 & 639.36 & 320.18 & & & $\mathrm{~L}$ & $1,153.65$ & 577.33 & $1,136.62$ & 568.82 & $1,135.64$ & 568.32 & 10 \\
\hline 6 & 755.45 & 378.23 & 738.43 & 369.72 & & & V & $1,040.57$ & 520.79 & $1,023.54$ & 512.27 & $1,022.56$ & 511.78 & 9 \\
\hline 7 & 868.54 & 434.77 & 851.51 & 426.26 & & & I & 941.50 & 471.25 & 924.47 & 462.74 & 923.49 & 462.25 & 8 \\
\hline 8 & 955.57 & 478.29 & 938.54 & 469.77 & 937.56 & 469.28 & $\mathrm{~S}$ & 828.41 & 414.71 & 811.39 & 406.20 & 810.40 & 405.71 & 7 \\
\hline 9 & $1,092.63$ & 546.82 & $1,075.60$ & 538.30 & $1,074.62$ & 537.81 & $\mathrm{H}$ & 741.38 & 371.19 & 724.36 & 362.68 & & & 6 \\
\hline 10 & $1,220.69$ & 610.85 & $1,203.66$ & 602.33 & $1,202.68$ & 601.84 & Q & 604.32 & 302.67 & 587.30 & 294.15 & & & 5 \\
\hline 11 & $1,291.72$ & 646.37 & $1,274.70$ & 637.85 & $1,273.71$ & 637.36 & A & 476.26 & 238.64 & 459.24 & 230.12 & & & 4 \\
\hline 12 & $1,390.79$ & 695.90 & $1,373.77$ & 687.39 & $1,372.78$ & 686.89 & V & 405.23 & 203.12 & 388.20 & 194.60 & & & 3 \\
\hline 13 & $1,521.83$ & 761.42 & $1,504.81$ & 752.91 & $1,503.82$ & 752.41 & $\mathrm{M}$ & 306.16 & 153.58 & 289.13 & 145.07 & & & 2 \\
\hline 14 & & & & & & & $\mathrm{R}$ & 175.12 & 88.06 & 158.09 & 79.55 & & & 1 \\
\hline
\end{tabular}

Query 56454 Hit 1

MS/MS Fragmentation of WKDTDEADLVLAK

Found in sp|P45973|CBX5_HUMAN, Chromobox protein homolog 5 OS=Homo sapiens GN=CBX5 PE=1 SV=1

Match to Query 56454: 1935.069from(646.0303,3+)

Title: 567: Sum of 2 scans in range 1255 ( $\mathrm{rt}=37.9625, \mathrm{f}=4, \mathrm{i}=381)$ to 1256 ( $\mathrm{rt}=37.9879, \mathrm{f}=4, \mathrm{i}=382$ )

[D:llab212 \membranelGrace JoyceliTRAQ_45_1.raw]

Data File:Submitted from 20120508-1(merge) by Mascot Daemon on JOYCE-VAIO

Monoisotopic mass of neutral peptide $\mathrm{Mr}$ (calc): 1935.069

Variable modifications:

K2 :iTRAQ4plex (K)

$\mathrm{K} 13$ :iTRAQ4plex (K) 


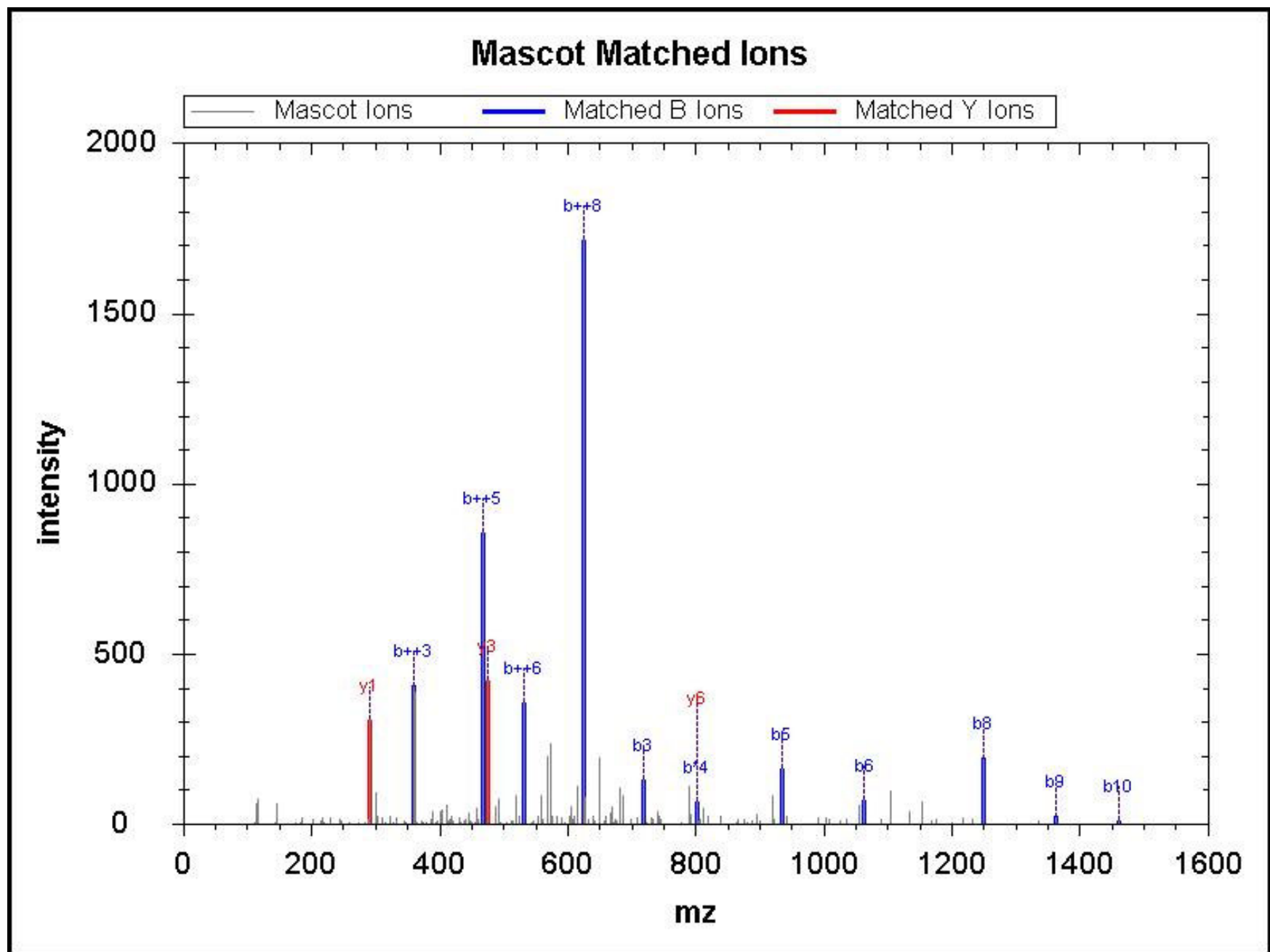

\begin{tabular}{|r|c|c|c|c|c|c|c|c|c|c|c|r|r|r|}
\hline \hline $\mathrm{No}$ & $\mathrm{b}$ & $\mathrm{b}++$ & $\mathrm{b} *$ & $\mathrm{~b}^{*++}$ & $\mathrm{b} 0$ & $\mathrm{~b} 0++$ & $\mathrm{Seq}$ & $\mathrm{y}$ & $\mathrm{y}++$ & $\mathrm{y} *$ & $\mathrm{y}^{*++}$ & $\mathrm{y} 0$ & $\mathrm{y} 0++$ & $\mathrm{RevNo}$ \\
\hline 1 & 331.19 & 166.10 & & & & & $\mathrm{~W}$ & & & & & & & 13 \\
\hline 2 & 603.39 & 302.20 & 586.36 & 293.68 & & & $\mathrm{~K}$ & $1,605.89$ & 803.45 & $1,588.87$ & 794.94 & $1,587.88$ & 794.45 & 12 \\
\hline 3 & 718.41 & 359.71 & 701.39 & 351.20 & 700.40 & 350.70 & $\mathrm{D}$ & $1,333.70$ & 667.35 & $1,316.67$ & 658.84 & $1,315.69$ & 658.35 & 11 \\
\hline 4 & 819.46 & 410.23 & 802.43 & 401.72 & 801.45 & 401.23 & $\mathrm{~T}$ & $1,218.67$ & 609.84 & $1,201.64$ & 601.33 & $1,200.66$ & 600.83 & 10 \\
\hline 5 & 934.49 & 467.75 & 917.46 & 459.23 & 916.48 & 458.74 & $\mathrm{D}$ & $1,117.62$ & 559.31 & $1,100.60$ & 550.80 & $1,099.61$ & 550.31 & 9 \\
\hline 6 & $1,063.53$ & 532.27 & $1,046.50$ & 523.76 & $1,045.52$ & 523.26 & $\mathrm{E}$ & $1,002.60$ & 501.80 & 985.57 & 493.29 & 984.58 & 492.80 & 8 \\
\hline 7 & $1,134.57$ & 567.79 & $1,117.54$ & 559.27 & $1,116.56$ & 558.78 & A & 873.55 & 437.28 & 856.53 & 428.77 & 855.54 & 428.27 & 7 \\
\hline 8 & $1,249.59$ & 625.30 & $1,232.57$ & 616.79 & $1,231.58$ & 616.30 & D & 802.52 & 401.76 & 785.49 & 393.25 & 784.50 & 392.76 & 6 \\
\hline 9 & $1,362.68$ & 681.84 & $1,345.65$ & 673.33 & $1,344.67$ & 672.84 & L & 687.49 & 344.25 & 670.46 & 335.73 & & & 5 \\
\hline 10 & $1,461.75$ & 731.38 & $1,444.72$ & 722.86 & $1,443.74$ & 722.37 & V & 574.40 & 287.71 & 557.38 & 279.19 & & & 4 \\
\hline 11 & $1,574.83$ & 787.92 & $1,557.80$ & 779.41 & $1,556.82$ & 778.91 & L & 475.34 & 238.17 & 458.31 & 229.66 & & & 3 \\
\hline 12 & $1,645.87$ & 823.44 & $1,628.84$ & 814.92 & $1,627.86$ & 814.43 & A & 362.25 & 181.63 & 345.23 & 173.12 & & & 2 \\
\hline 13 & & & & & & & K & 291.21 & 146.11 & 274.19 & 137.60 & & & 1 \\
\hline
\end{tabular}

Query 69344 Hit 1

MS/MS Fragmentation of SLEDLIFESLPENASHK

Found in sp $\mid$ O95260|ATE1_HUM AN, Arginyl-tRNA--protein transferase 1 OS=Homo sapiens GN=ATE1 PE=1 SV=2

Match to Query 69344: 2216.161 from(739.7275,3+)

Title: 975: Sum of 2 scans in range $2140(\mathrm{rt}=57.6488, \mathrm{f}=4, \mathrm{i}=649)$ to $2141(\mathrm{rt}=57.6742, \mathrm{f}=4, \mathrm{i}=650)$

[D:llab212 \membranelGrace JoyceliTRAQ_37_1.raw]

Data File:Submitted from 20120508-1(merge) by Mascot Daemon on JOYCE-VAIO

Monoisotopic mass of neutral peptide $\mathrm{Mr}$ (calc): 2216.161

Variable modifications:

K17 :iTRAQ4plex $(\mathrm{K})$ 


\section{Mascot Matched lons}

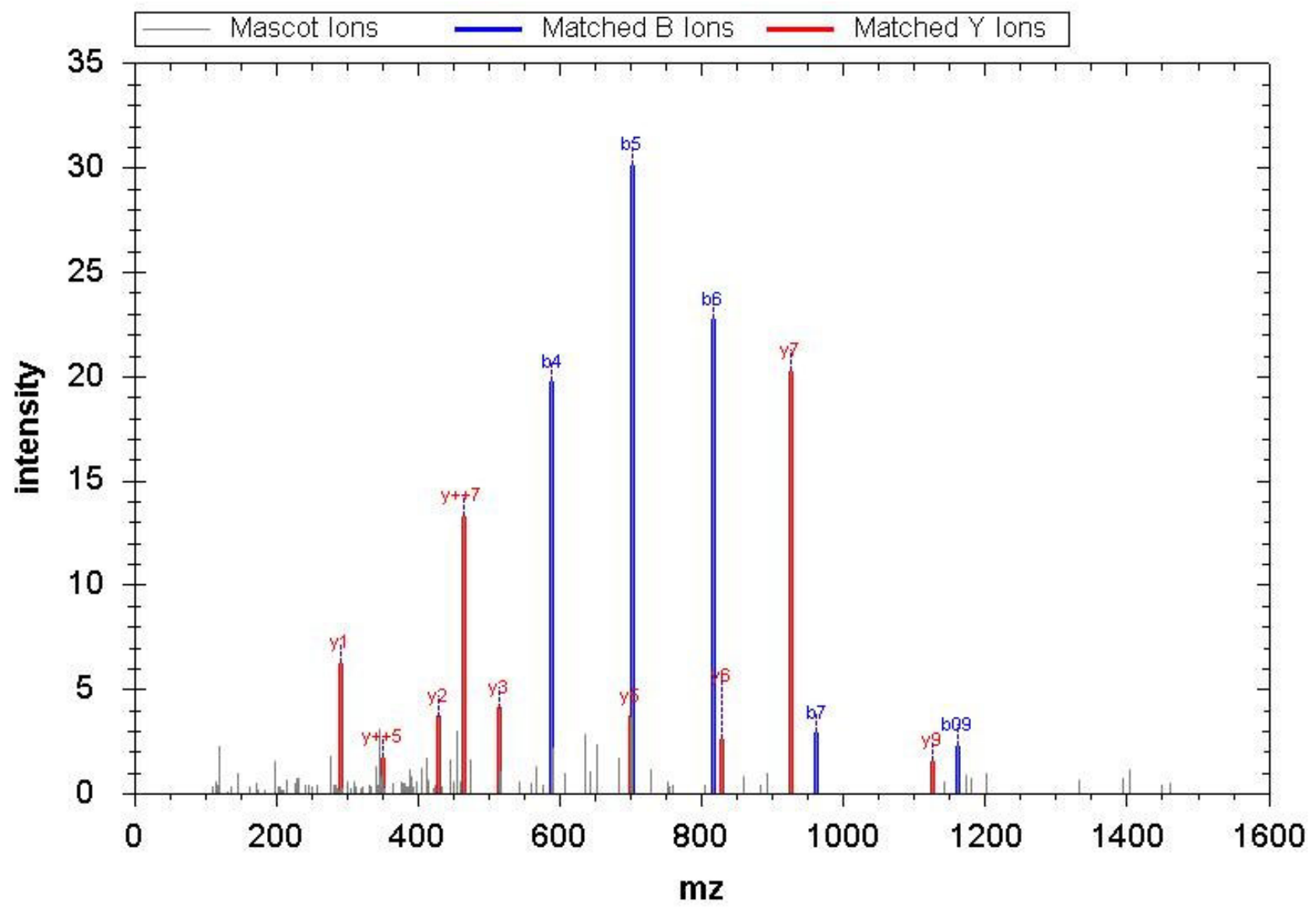

\begin{tabular}{|c|c|c|c|c|c|c|c|c|c|c|c|c|c|c|}
\hline No & b & $b++$ & $b^{*}$ & $\mathrm{~b}^{*++}$ & b0 & $\mathrm{b} 0++$ & Seq & $\mathrm{y}$ & $\mathrm{y}++$ & $\mathrm{y}^{*}$ & $\mathrm{y}^{*++}$ & y0 & $\mathrm{y} 0++$ & RevNo \\
\hline 1 & 232.14 & 116.57 & & & 214.13 & 107.57 & $\mathrm{~S}$ & & & & & & & 17 \\
\hline 2 & 345.23 & 173.12 & & & 327.21 & 164.11 & $\mathrm{~L}$ & $1,986.03$ & 993.52 & $1,969.00$ & 985.01 & $1,968.02$ & 984.51 & 16 \\
\hline 3 & 474.27 & 237.64 & & & 456.26 & 228.63 & E & $1,872.95$ & 936.98 & $1,855.92$ & 928.46 & $1,854.94$ & 927.97 & 15 \\
\hline 4 & 589.29 & 295.15 & & & 571.28 & 286.15 & D & $1,743.90$ & 872.46 & $1,726.88$ & 863.94 & $1,725.89$ & 863.45 & 14 \\
\hline 5 & 702.38 & 351.69 & & & 684.37 & 342.69 & $\mathrm{~L}$ & $1,628.88$ & 814.94 & $1,611.85$ & 806.43 & $1,610.87$ & 805.94 & 13 \\
\hline 6 & 815.46 & 408.24 & & & 797.45 & 399.23 & I & $1,515.79$ & 758.40 & $1,498.77$ & 749.89 & $1,497.78$ & 749.39 & 12 \\
\hline 7 & 962.53 & 481.77 & & & 944.52 & 472.76 & $\mathrm{~F}$ & $1,402.71$ & 701.86 & $1,385.68$ & 693.34 & $1,384.70$ & 692.85 & 11 \\
\hline 8 & $1,091.57$ & 546.29 & & & $1,073.56$ & 537.29 & $\mathrm{E}$ & $1,255.64$ & 628.32 & $1,238.61$ & 619.81 & $1,237.63$ & 619.32 & 10 \\
\hline 9 & $1,178.61$ & 589.81 & & & $1,160.60$ & 580.80 & $\mathrm{~S}$ & $1,126.60$ & 563.80 & $1,109.57$ & 555.29 & $1,108.59$ & 554.80 & 9 \\
\hline 10 & $1,291.69$ & 646.35 & & & $1,273.68$ & 637.34 & $\mathrm{~L}$ & $1,039.57$ & 520.29 & $1,022.54$ & 511.77 & $1,021.55$ & 511.28 & 8 \\
\hline 11 & $1,388.74$ & 694.88 & & & $1,370.73$ & 685.87 & $\mathrm{P}$ & 926.48 & 463.74 & 909.45 & 455.23 & 908.47 & 454.74 & 7 \\
\hline 12 & $1,517.79$ & 759.40 & & & $1,499.77$ & 750.39 & E & 829.43 & 415.22 & 812.40 & 406.70 & 811.42 & 406.21 & 6 \\
\hline 13 & $1,631.83$ & 816.42 & $1,614.80$ & 807.90 & $1,613.82$ & 807.41 & $\mathrm{~N}$ & 700.39 & 350.70 & 683.36 & 342.18 & 682.38 & 341.69 & 5 \\
\hline 14 & $1,702.87$ & 851.94 & $1,685.84$ & 843.42 & $1,684.85$ & 842.93 & A & 586.34 & 293.68 & 569.32 & 285.16 & 568.33 & 284.67 & 4 \\
\hline 15 & $1,789.90$ & 895.45 & $1,772.87$ & 886.94 & $1,771.89$ & 886.45 & $\mathrm{~S}$ & 515.31 & 258.16 & 498.28 & 249.64 & 497.30 & 249.15 & 3 \\
\hline 16 & $1,926.96$ & 963.98 & $1,909.93$ & 955.47 & $1,908.95$ & 954.98 & $\mathrm{H}$ & 428.27 & 214.64 & 411.25 & 206.13 & & & 2 \\
\hline 17 & & & & & & & $\mathrm{~K}$ & 291.21 & 146.11 & 274.19 & 137.60 & & & 1 \\
\hline
\end{tabular}

Query 42907 Hit 1

MS/MS Fragmentation of LGVTNTIISHYDGR

Found in sp|P46087|NOP2 HUMAN, Putative ribosomal RNA methyltransferase NOP2 OS=Homo sapiens GN=NOP2 PE=1 SV=2 Match to Query 42907: 1688.894from(563.9719,3+) 
Title: 513: Scan $1201(\mathrm{rt}=36.4637, \mathrm{f}=2, \mathrm{i}=185)$ [D:lab212 \membranelGrace JoyceliTRAQ_36_2.raw]

Data File:Submitted from 20120508-1(merge) by Mascot Daemon on JOYCE-VAIO

Monoisotopic mass of neutral peptide $\mathrm{Mr}$ (calc): 1688.894

Variable modifications:

Ions Score: 45.99 Expect: 0.022

\section{Mascot Matched lons}

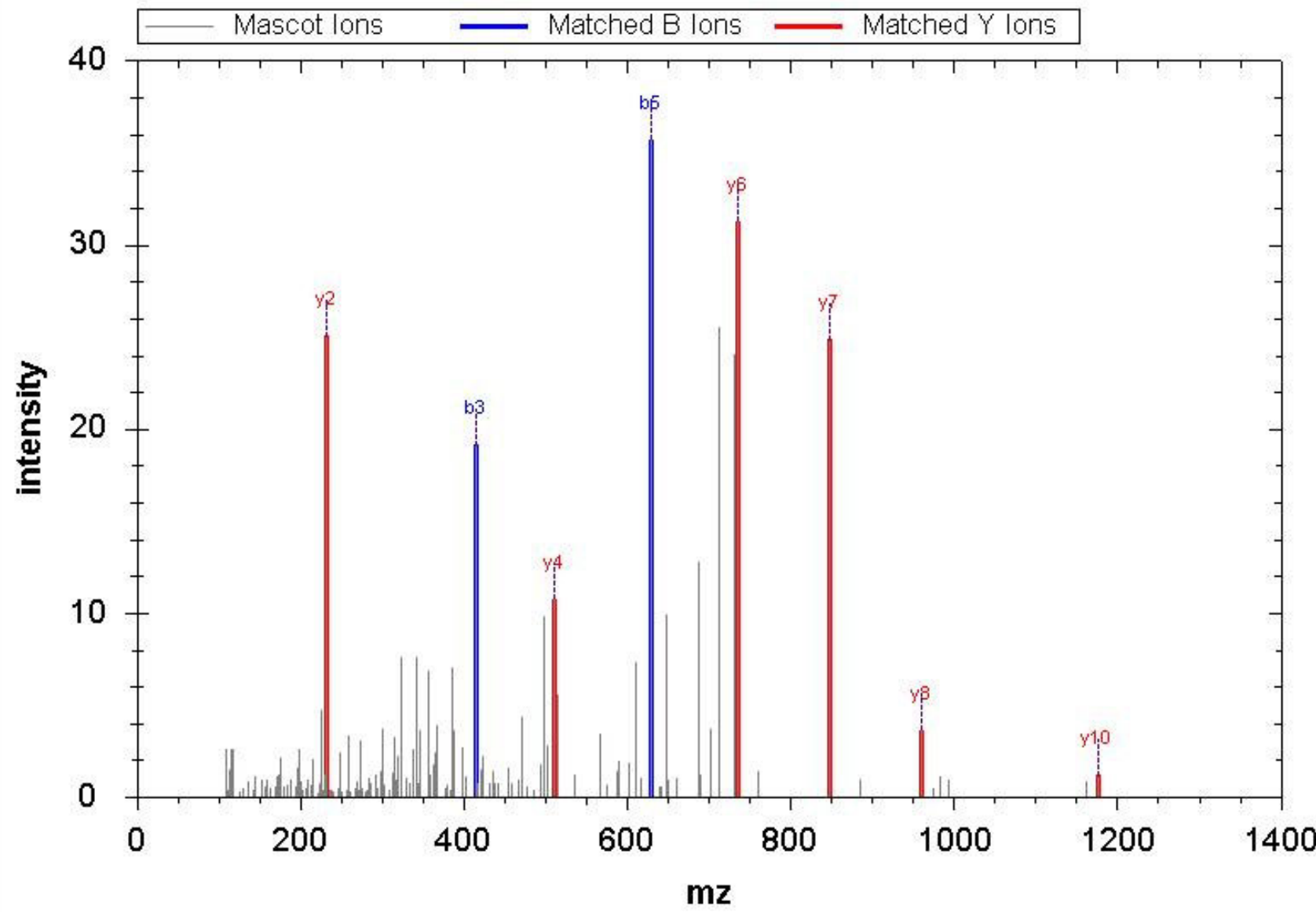

\begin{tabular}{|c|c|c|c|c|c|c|c|c|c|c|c|c|c|c|}
\hline No & $\mathrm{b}$ & $\mathrm{b}++$ & $b^{*}$ & $b^{*++}$ & b0 & $\mathrm{b} 0++$ & Seq & $\mathrm{y}$ & $\mathrm{y}++$ & $y^{*}$ & $y^{*++}$ & y0 & $\mathrm{y} 0++$ & RevNo \\
\hline 1 & 258.19 & 129.60 & & & & & $\mathrm{~L}$ & & & & & & & 14 \\
\hline 2 & 315.21 & 158.11 & & & & & G & $1,432.72$ & 716.86 & $1,415.69$ & 708.35 & $1,414.71$ & 707.86 & 13 \\
\hline 3 & 414.28 & 207.65 & & & & & V & $1,375.70$ & 688.35 & $1,358.67$ & 679.84 & $1,357.69$ & 679.35 & 12 \\
\hline 4 & 515.33 & 258.17 & & & 497.32 & 249.16 & $\mathrm{~T}$ & $1,276.63$ & 638.82 & $1,259.60$ & 630.30 & $1,258.62$ & 629.81 & 11 \\
\hline 5 & 629.37 & 315.19 & 612.35 & 306.68 & 611.36 & 306.19 & $\mathrm{~N}$ & $1,175.58$ & 588.29 & $1,158.55$ & 579.78 & $1,157.57$ & 579.29 & 10 \\
\hline 6 & 730.42 & 365.71 & 713.39 & 357.20 & 712.41 & 356.71 & $\mathrm{~T}$ & $1,061.54$ & 531.27 & $1,044.51$ & 522.76 & $1,043.53$ & 522.27 & 9 \\
\hline 7 & 843.51 & 422.26 & 826.48 & 413.74 & 825.49 & 413.25 & I & 960.49 & 480.75 & 943.46 & 472.24 & 942.48 & 471.74 & 8 \\
\hline 8 & 956.59 & 478.80 & 939.56 & 470.29 & 938.58 & 469.79 & I & 847.41 & 424.21 & 830.38 & 415.69 & 829.40 & 415.20 & 7 \\
\hline 9 & $1,043.62$ & 522.31 & $1,026.60$ & 513.80 & $1,025.61$ & 513.31 & $\mathrm{~S}$ & 734.32 & 367.66 & 717.30 & 359.15 & 716.31 & 358.66 & 6 \\
\hline 10 & $1,180.68$ & 590.84 & $1,163.65$ & 582.33 & $1,162.67$ & 581.84 & $\mathrm{H}$ & 647.29 & 324.15 & 630.26 & 315.64 & 629.28 & 315.14 & 5 \\
\hline 11 & $1,343.74$ & 672.38 & $1,326.72$ & 663.86 & $1,325.73$ & 663.37 & Y & 510.23 & 255.62 & 493.20 & 247.11 & 492.22 & 246.61 & 4 \\
\hline 12 & $1,458.77$ & 729.89 & $1,441.74$ & 721.38 & $1,440.76$ & 720.88 & D & 347.17 & 174.09 & 330.14 & 165.57 & 329.16 & 165.08 & 3 \\
\hline 13 & $1,515.79$ & 758.40 & $1,498.77$ & 749.89 & $1,497.78$ & 749.39 & G & 232.14 & 116.57 & 215.11 & 108.06 & & & 2 \\
\hline 14 & & & & & & & $\mathrm{R}$ & 175.12 & 88.06 & 158.09 & 79.55 & & & 1 \\
\hline
\end{tabular}

Query 51389 Hit 1

Found in sp|Q13442|HAP28_HUM AN, 28 kDa heat- and acid-stable phosphoprotein OS=Homo sapiens GN=PDAP1 PE=1 SV=1 Match to Query 51389: 1841.03from(614.6841,3+)

Title: 560: Scan $1276(\mathrm{rt}=38.3412, \mathrm{f}=3, \mathrm{i}=193)$ [D:lab212 $\backslash$ membranelGrace JoyceliTRAQ_44_2.raw] 
Data File:Submitted from 20120508-1(merge) by Mascot Daemon on JOYCE-VAIO

Monoisotopic mass of neutral peptide $\mathrm{Mr}$ (calc): 1841.03

Variable modifications:

K1 :iTRAQ4plex (K)

Ions Score: 45.92 Expect: 0.017

\section{Mascot Matched lons}

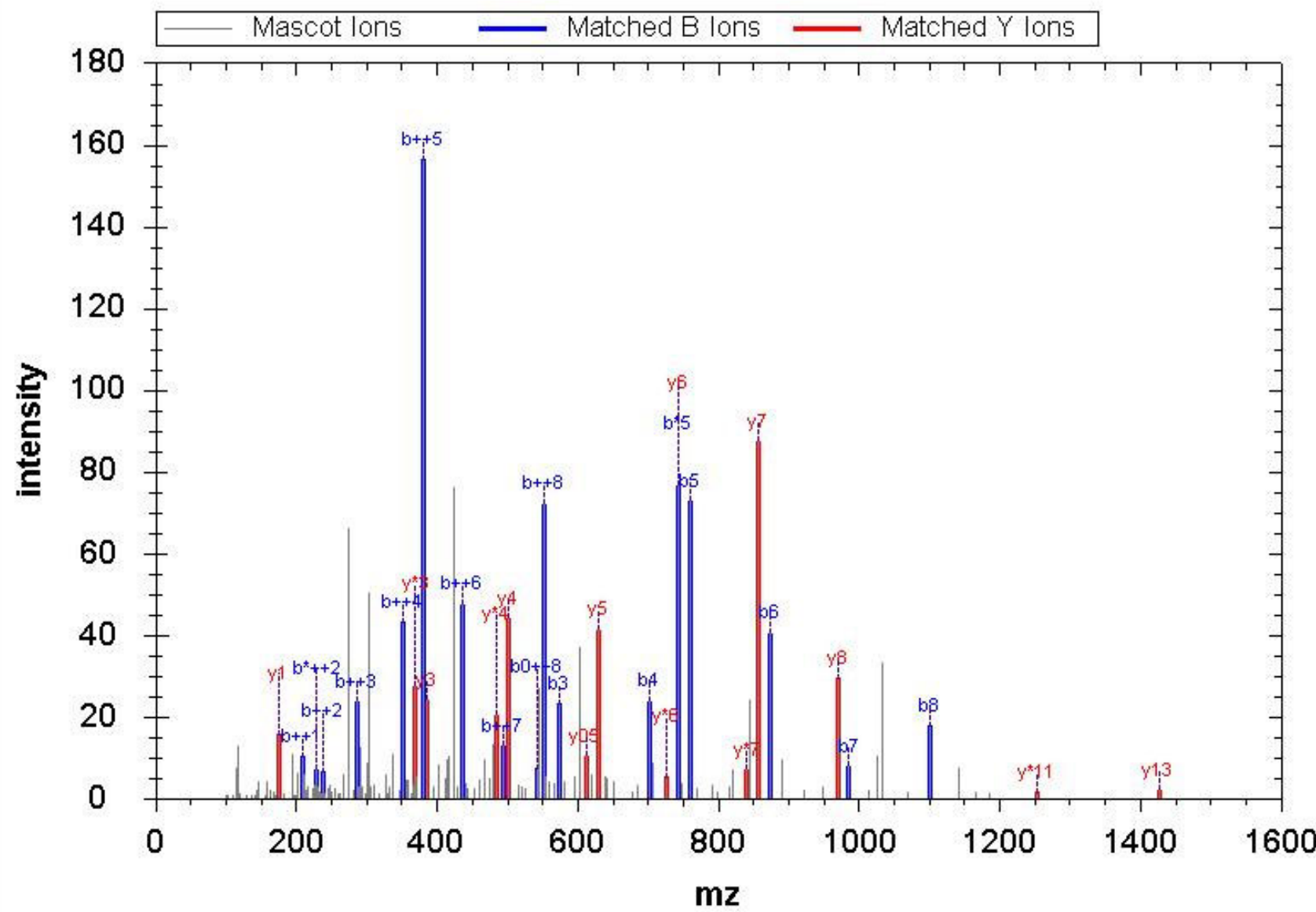

\begin{tabular}{|r|c|c|c|c|c|c|c|c|c|c|c|r|r|r|}
\hline \hline $\mathrm{No}$ & $\mathrm{b}$ & $\mathrm{b}++$ & $\mathrm{b} *$ & $\mathrm{~b}^{*++}$ & $\mathrm{b} 0$ & $\mathrm{~b} 0++$ & $\mathrm{Seq}$ & $\mathrm{y}$ & $\mathrm{y}++$ & $\mathrm{y}^{*}$ & $\mathrm{y}^{*++}$ & $\mathrm{y} 0$ & $\mathrm{y} 0++$ & $\mathrm{RevNo}$ \\
\hline 1 & 417.31 & 209.16 & 400.28 & 200.64 & & & $\mathrm{~K}$ & & & & & & & 14 \\
\hline 2 & 474.33 & 237.67 & 457.30 & 229.15 & & & $\mathrm{G}$ & $1,425.73$ & 713.37 & $1,408.71$ & 704.86 & $1,407.72$ & 704.36 & 13 \\
\hline 3 & 573.40 & 287.20 & 556.37 & 278.69 & & & $\mathrm{~V}$ & $1,368.71$ & 684.86 & $1,351.69$ & 676.35 & $1,350.70$ & 675.85 & 12 \\
\hline 4 & 702.44 & 351.72 & 685.41 & 343.21 & 684.43 & 342.72 & $\mathrm{E}$ & $1,269.64$ & 635.33 & $1,252.62$ & 626.81 & $1,251.63$ & 626.32 & 11 \\
\hline 5 & 759.46 & 380.23 & 742.43 & 371.72 & 741.45 & 371.23 & $\mathrm{G}$ & $1,140.60$ & 570.80 & $1,123.57$ & 562.29 & $1,122.59$ & 561.80 & 10 \\
\hline 6 & 872.54 & 436.78 & 855.52 & 428.26 & 854.53 & 427.77 & $\mathrm{~L}$ & $1,083.58$ & 542.29 & $1,066.55$ & 533.78 & $1,065.57$ & 533.29 & 9 \\
\hline 7 & 985.63 & 493.32 & 968.60 & 484.80 & 967.62 & 484.31 & $\mathrm{I}$ & 970.50 & 485.75 & 953.47 & 477.24 & 952.48 & 476.75 & 8 \\
\hline 8 & $1,100.66$ & 550.83 & $1,083.63$ & 542.32 & $1,082.64$ & 541.83 & $\mathrm{D}$ & 857.41 & 429.21 & 840.38 & 420.70 & 839.40 & 420.20 & 7 \\
\hline 9 & $1,213.74$ & 607.37 & $1,196.71$ & 598.86 & $1,195.73$ & 598.37 & $\mathrm{I}$ & 742.38 & 371.70 & 725.36 & 363.18 & 724.37 & 362.69 & 6 \\
\hline 10 & $1,342.78$ & 671.89 & $1,325.76$ & 663.38 & $1,324.77$ & 662.89 & $\mathrm{E}$ & 629.30 & 315.15 & 612.27 & 306.64 & 611.29 & 306.15 & 5 \\
\hline 11 & $1,456.83$ & 728.92 & $1,439.80$ & 720.40 & $1,438.81$ & 719.91 & $\mathrm{~N}$ & 500.26 & 250.63 & 483.23 & 242.12 & & & 4 \\
\hline 12 & $1,553.88$ & 777.44 & $1,536.85$ & 768.93 & $1,535.87$ & 768.44 & $\mathrm{P}$ & 386.21 & 193.61 & 369.19 & 185.10 & & & 3 \\
\hline 13 & $1,667.92$ & 834.46 & $1,650.89$ & 825.95 & $1,649.91$ & 825.46 & $\mathrm{~N}$ & 289.16 & 145.08 & 272.14 & 136.57 & & & 2 \\
\hline 14 & & & & & & & $\mathrm{R}$ & 175.12 & 88.06 & 158.09 & 79.55 & & & 1 \\
\hline
\end{tabular}

Query 56949 Hit 1

MS/MS Fragmentation of DILLELVEHHLTPK

Found in sp|Q9D8Y7|TP8L2_MOUSE, Tumor necrosis factor alpha-induced protein 8-like protein 2 OS=Mus musculus GN=Tnfaip812 $\mathrm{PE}=1 \mathrm{SV}=1$

Match to Query 56949: 1944.131 from(487.04,4+) 
Title: 952: Sum of 2 scans in range 2158 ( $\mathrm{rt}=58.0805, \mathrm{f}=4, \mathrm{i}=656)$ to $2159(\mathrm{rt}=58.1059, \mathrm{f}=4, \mathrm{i}=657$ )

[D:\lab212 \membrane $\backslash$ Grace $\backslash J o y c e \backslash 47$ 1.raw]

Data File:Submitted from 20120508-1(merge) by Mascot Daemon on JOYCE-VAIO

Monoisotopic mass of neutral peptide $\mathrm{Mr}$ (calc): 1944.131

Variable modifications:

K14 :iTRAQ4plex (K)

Ions Score: 45.89 Expect: 0.012

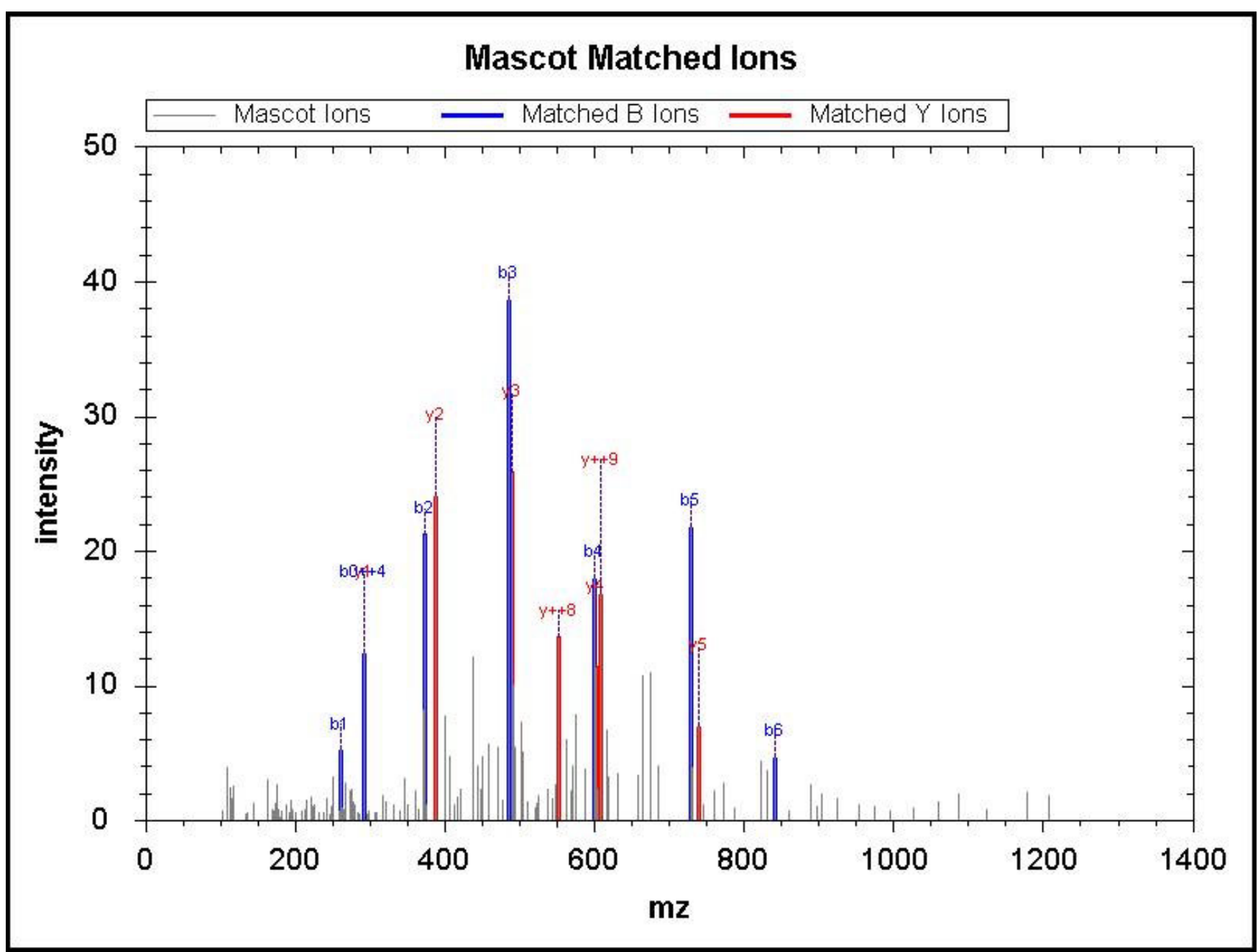

\begin{tabular}{|r|c|c|c|c|c|c|c|c|c|c|r|r|}
\hline No & $\mathrm{b}$ & $\mathrm{b}++$ & $\mathrm{b} 0$ & $\mathrm{~b} 0++$ & $\mathrm{Seq}$ & $\mathrm{y}$ & $\mathrm{y}++$ & $\mathrm{y}^{*}$ & $\mathrm{y} *++$ & $\mathrm{y} 0$ & $\mathrm{y} 0++$ & RevNo \\
\hline 1 & 260.14 & 130.57 & 242.13 & 121.57 & $\mathrm{D}$ & & & & & & & 14 \\
\hline 2 & 373.22 & 187.11 & 355.21 & 178.11 & $\mathrm{I}$ & $1,686.01$ & 843.51 & $1,668.98$ & 834.99 & $1,668.00$ & 834.50 & 13 \\
\hline 3 & 486.30 & 243.66 & 468.29 & 234.65 & $\mathrm{~L}$ & $1,572.92$ & 786.97 & $1,555.90$ & 778.45 & $1,554.91$ & 777.96 & 12 \\
\hline 4 & 599.39 & 300.20 & 581.38 & 291.19 & $\mathrm{~L}$ & $1,459.84$ & 730.42 & $1,442.81$ & 721.91 & $1,441.83$ & 721.42 & 11 \\
\hline 5 & 728.43 & 364.72 & 710.42 & 355.71 & $\mathrm{E}$ & $1,346.75$ & 673.88 & $1,329.73$ & 665.37 & $1,328.74$ & 664.88 & 10 \\
\hline 6 & 841.52 & 421.26 & 823.50 & 412.26 & $\mathrm{~L}$ & $1,217.71$ & 609.36 & $1,200.69$ & 600.85 & $1,199.70$ & 600.35 & 9 \\
\hline 7 & 940.58 & 470.80 & 922.57 & 461.79 & $\mathrm{~V}$ & $1,104.63$ & 552.82 & $1,087.60$ & 544.30 & $1,086.62$ & 543.81 & 8 \\
\hline 8 & $1,069.63$ & 535.32 & $1,051.62$ & 526.31 & $\mathrm{E}$ & $1,005.56$ & 503.28 & 988.53 & 494.77 & 987.55 & 494.28 & 7 \\
\hline 9 & $1,206.69$ & 603.85 & $1,188.67$ & 594.84 & $\mathrm{H}$ & 876.52 & 438.76 & 859.49 & 430.25 & 858.51 & 429.76 & 6 \\
\hline 10 & $1,343.74$ & 672.38 & $1,325.73$ & 663.37 & $\mathrm{H}$ & 739.46 & 370.23 & 722.43 & 361.72 & 721.45 & 361.23 & 5 \\
\hline 11 & $1,456.83$ & 728.92 & $1,438.82$ & 719.91 & $\mathrm{~L}$ & 602.40 & 301.70 & 585.37 & 293.19 & 584.39 & 292.70 & 4 \\
\hline 12 & $1,557.88$ & 779.44 & $1,539.87$ & 770.44 & $\mathrm{~T}$ & 489.32 & 245.16 & 472.29 & 236.65 & 471.30 & 236.16 & 3 \\
\hline 13 & $1,654.93$ & 827.97 & $1,636.92$ & 818.96 & $\mathrm{P}$ & 388.27 & 194.64 & 371.24 & 186.12 & & & 2 \\
\hline 14 & & & & & $\mathrm{~K}$ & 291.21 & 146.11 & 274.19 & 137.60 & & & 1 \\
\hline
\end{tabular}

Query 61577 Hit 1 
Match to Query 61577: 2043.223from(682.0815,3+)

Title: 1302: Scan 2963 ( $\mathrm{rt}=75.553, \mathrm{f}=3, \mathrm{i}=444)$ [D:llab212 $\backslash$ membranelGrace JoyceliTRAQ_24_1.raw]

Data File:Submitted from 20120508-1(merge) by Mascot Daemon on JOYCE-VAIO

Monoisotopic mass of neutral peptide Mr(calc): 2043.223

Variable modifications:

K17 :iTRAQ4plex (K)

Ions Score: 45.78 Expect: 0.006

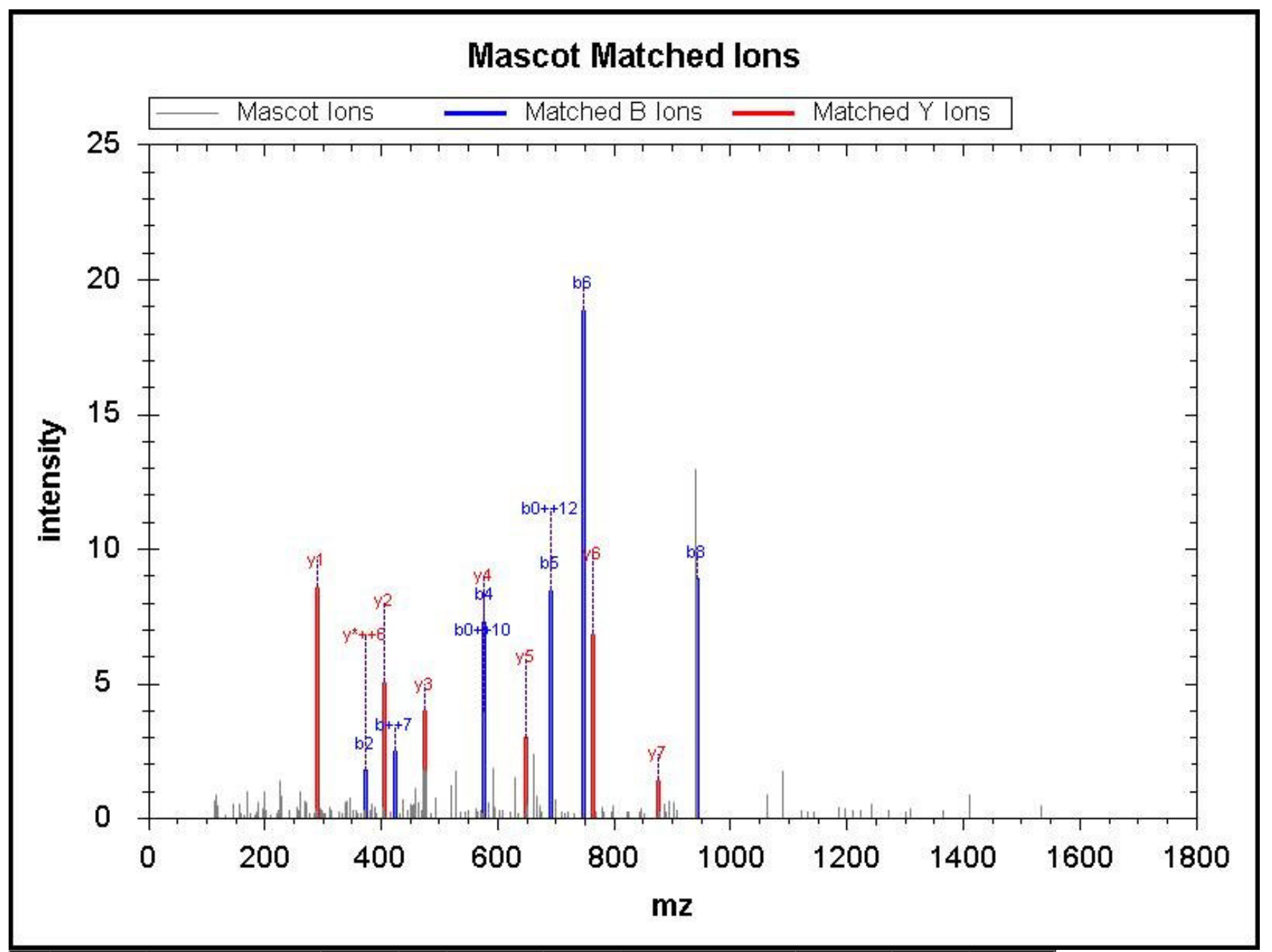

\begin{tabular}{|r|c|c|c|c|c|c|c|c|c|c|r|r|}
\hline \hline No & $\mathrm{b}$ & $\mathrm{b}++$ & $\mathrm{b} 0$ & $\mathrm{~b} 0++$ & $\mathrm{Seq}$ & $\mathrm{y}$ & $\mathrm{y}++$ & $\mathrm{y}^{*}$ & $\mathrm{y} *++$ & $\mathrm{y} 0$ & $\mathrm{y} 0++$ & $\mathrm{RevNo}$ \\
\hline 1 & 260.14 & 130.57 & 242.13 & 121.57 & $\mathrm{D}$ & & & & & & & 17 \\
\hline 2 & 373.22 & 187.11 & 355.21 & 178.11 & $\mathrm{I}$ & $1,785.10$ & 893.05 & $1,768.07$ & 884.54 & $1,767.09$ & 884.05 & 16 \\
\hline 3 & 520.29 & 260.65 & 502.28 & 251.64 & $\mathrm{~F}$ & $1,672.02$ & 836.51 & $1,654.99$ & 828.00 & $1,654.01$ & 827.51 & 15 \\
\hline 4 & 577.31 & 289.16 & 559.30 & 280.15 & $\mathrm{G}$ & $1,524.95$ & 762.98 & $1,507.92$ & 754.46 & $1,506.94$ & 753.97 & 14 \\
\hline 5 & 690.39 & 345.70 & 672.38 & 336.70 & $\mathrm{~L}$ & $1,467.93$ & 734.47 & $1,450.90$ & 725.95 & $1,449.92$ & 725.46 & 13 \\
\hline 6 & 747.42 & 374.21 & 729.41 & 365.21 & $\mathrm{G}$ & $1,354.84$ & 677.92 & $1,337.82$ & 669.41 & $1,336.83$ & 668.92 & 12 \\
\hline 7 & 844.47 & 422.74 & 826.46 & 413.73 & $\mathrm{P}$ & $1,297.82$ & 649.41 & $1,280.79$ & 640.90 & $1,279.81$ & 640.41 & 11 \\
\hline 8 & 943.54 & 472.27 & 925.53 & 463.27 & $\mathrm{~V}$ & $1,200.77$ & 600.89 & $1,183.74$ & 592.37 & $1,182.76$ & 591.88 & 10 \\
\hline 9 & $1,056.62$ & 528.81 & $1,038.61$ & 519.81 & $\mathrm{~L}$ & $1,101.70$ & 551.35 & $1,084.67$ & 542.84 & $1,083.69$ & 542.35 & 9 \\
\hline 10 & $1,169.71$ & 585.36 & $1,151.69$ & 576.35 & $\mathrm{~L}$ & 988.62 & 494.81 & 971.59 & 486.30 & 970.61 & 485.81 & 8 \\
\hline 11 & $1,282.79$ & 641.90 & $1,264.78$ & 632.89 & $\mathrm{~L}$ & 875.53 & 438.27 & 858.51 & 429.76 & 857.52 & 429.26 & 7 \\
\hline 12 & $1,397.82$ & 699.41 & $1,379.81$ & 690.41 & $\mathrm{D}$ & 762.45 & 381.73 & 745.42 & 373.21 & 744.44 & 372.72 & 6 \\
\hline 13 & $1,468.85$ & 734.93 & $1,450.84$ & 725.93 & A & 647.42 & 324.21 & 630.39 & 315.70 & 629.41 & 315.21 & 5 \\
\hline 14 & $1,569.90$ & 785.45 & $1,551.89$ & 776.45 & T & 576.38 & 288.70 & 559.36 & 280.18 & 558.37 & 279.69 & 4 \\
\hline 15 & $1,640.94$ & 820.97 & $1,622.93$ & 811.97 & A & 475.34 & 238.17 & 458.31 & 229.66 & & & 3 \\
\hline 16 & $1,754.02$ & 877.51 & $1,736.01$ & 868.51 & L & 404.30 & 202.65 & 387.27 & 194.14 & & & 2 \\
\hline 17 & & & & & K & 291.21 & 146.11 & 274.19 & 137.60 & & & 1 \\
\hline
\end{tabular}


MS/MS Fragmentation of NVVALDTEVASNR

Found in sp|P01130|LDLR_HUMAN, Low-density lipoprotein receptor OS=Homo sapiens GN=LDLR PE=1 SV=1

Match to Query 34518: 1530.807from(766.4107,2+)

Title: 247: Sum of 2 scans in range 1104 ( $\mathrm{rt}=32.1989, \mathrm{f}=4, \mathrm{i}=159)$ to $1105(\mathrm{rt}=32.2243, \mathrm{f}=4, \mathrm{i}=160)$

[D:lab212 \membrane \Grace \oyce \20120320_iTRAQ_20-2.raw]

Data File:Submitted from 20120508-1(merge) by Mascot Daemon on JOYCE-VAIO

Monoisotopic mass of neutral peptide $\mathrm{Mr}$ (calc): 1530.807

Variable modifications:

Ions Score: 45.77 Expect: 0.022

\section{Mascot Matched lons}

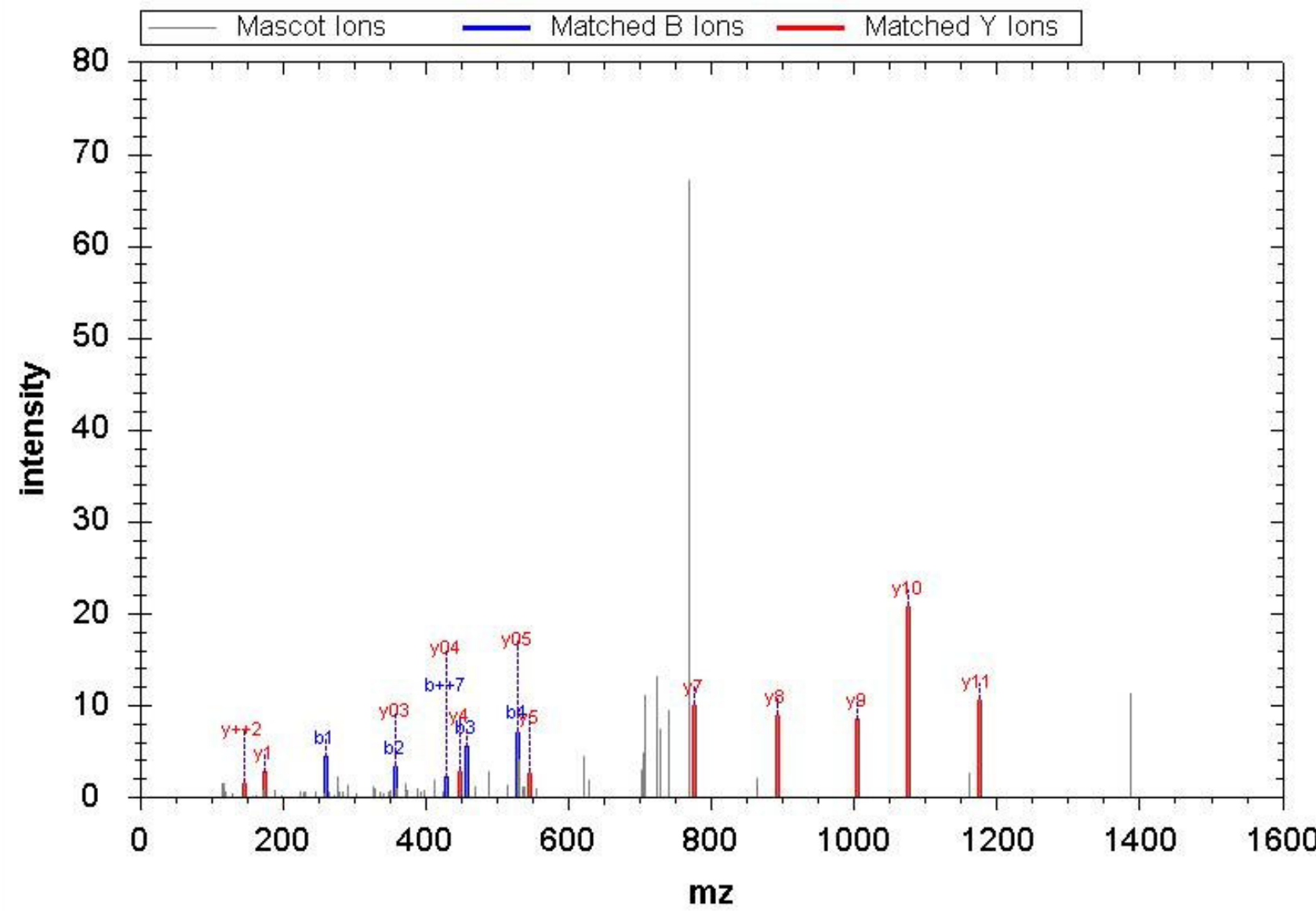

\begin{tabular}{|r|c|c|c|c|c|c|c|c|c|c|r|r|r|r|}
\hline \hline $\mathrm{No}$ & $\mathrm{b}$ & $\mathrm{b}++$ & $\mathrm{b}^{*}$ & $\mathrm{~b}^{*++}$ & $\mathrm{b} 0$ & $\mathrm{~b} 0++$ & $\mathrm{Seq}$ & $\mathrm{y}$ & $\mathrm{y}++$ & $\mathrm{y}^{*}$ & $\mathrm{y}^{*++}$ & $\mathrm{y} 0$ & $\mathrm{y} 0++$ & $\mathrm{RevNo}$ \\
\hline 1 & 259.15 & 130.08 & 242.13 & 121.57 & & & $\mathrm{~N}$ & & & & & & & 13 \\
\hline 2 & 358.22 & 179.61 & 341.19 & 171.10 & & & $\mathrm{~V}$ & $1,273.67$ & 637.34 & $1,256.65$ & 628.83 & $1,255.66$ & 628.34 & 12 \\
\hline 3 & 457.29 & 229.15 & 440.26 & 220.63 & & & $\mathrm{~V}$ & $1,174.61$ & 587.81 & $1,157.58$ & 579.29 & $1,156.60$ & 578.80 & 11 \\
\hline 4 & 528.33 & 264.67 & 511.30 & 256.15 & & & $\mathrm{~A}$ & $1,075.54$ & 538.27 & $1,058.51$ & 529.76 & $1,057.53$ & 529.27 & 10 \\
\hline 5 & 641.41 & 321.21 & 624.38 & 312.70 & & & $\mathrm{~L}$ & $1,004.50$ & 502.75 & 987.47 & 494.24 & 986.49 & 493.75 & 9 \\
\hline 6 & 756.44 & 378.72 & 739.41 & 370.21 & 738.43 & 369.72 & $\mathrm{D}$ & 891.42 & 446.21 & 874.39 & 437.70 & 873.41 & 437.21 & 8 \\
\hline 7 & 857.48 & 429.25 & 840.46 & 420.73 & 839.47 & 420.24 & $\mathrm{~T}$ & 776.39 & 388.70 & 759.36 & 380.19 & 758.38 & 379.69 & 7 \\
\hline 8 & 986.53 & 493.77 & 969.50 & 485.25 & 968.52 & 484.76 & $\mathrm{E}$ & 675.34 & 338.17 & 658.32 & 329.66 & 657.33 & 329.17 & 6 \\
\hline 9 & $1,085.60$ & 543.30 & $1,068.57$ & 534.79 & $1,067.59$ & 534.30 & $\mathrm{~V}$ & 546.30 & 273.65 & 529.27 & 265.14 & 528.29 & 264.65 & 5 \\
\hline 10 & $1,156.63$ & 578.82 & $1,139.61$ & 570.31 & $1,138.62$ & 569.81 & A & 447.23 & 224.12 & 430.20 & 215.61 & 429.22 & 215.11 & 4 \\
\hline 11 & $1,243.67$ & 622.34 & $1,226.64$ & 613.82 & $1,225.65$ & 613.33 & S & 376.19 & 188.60 & 359.17 & 180.09 & 358.18 & 179.60 & 3 \\
\hline 12 & $1,357.71$ & 679.36 & $1,340.68$ & 670.84 & $1,339.70$ & 670.35 & N & 289.16 & 145.08 & 272.14 & 136.57 & & & 2 \\
\hline 13 & & & & & & & R & 175.12 & 88.06 & 158.09 & 79.55 & & & 1 \\
\hline
\end{tabular}


MS/MS Fragmentation of SIFNSAMQEMEVYVENIR

Found in sp|Q9ULU4|PKCB1_HUM AN, Protein kinase C-binding protein 1 OS=Homo sapiens GN=ZMYND8 PE=1 SV=2

Match to Query 72827:2303.105from(768.709,3+)

Title: 1233: Sum of 2 scans in range $2894(\mathrm{rt}=73.5931, \mathrm{f}=4, \mathrm{i}=815)$ to $2895(\mathrm{rt}=73.6185, \mathrm{f}=4, \mathrm{i}=816)$

[D:lab212 \membranelGracelJoyceliTRAQ_23_2.raw]

Data File:Submitted from 20120508-1(merge) by Mascot Daemon on JOYCE-VAIO

Monoisotopic mass of neutral peptide $\mathrm{Mr}$ (calc): 2303.105

Variable modifications:

Ions Score: 45.66 Expect: 0.023

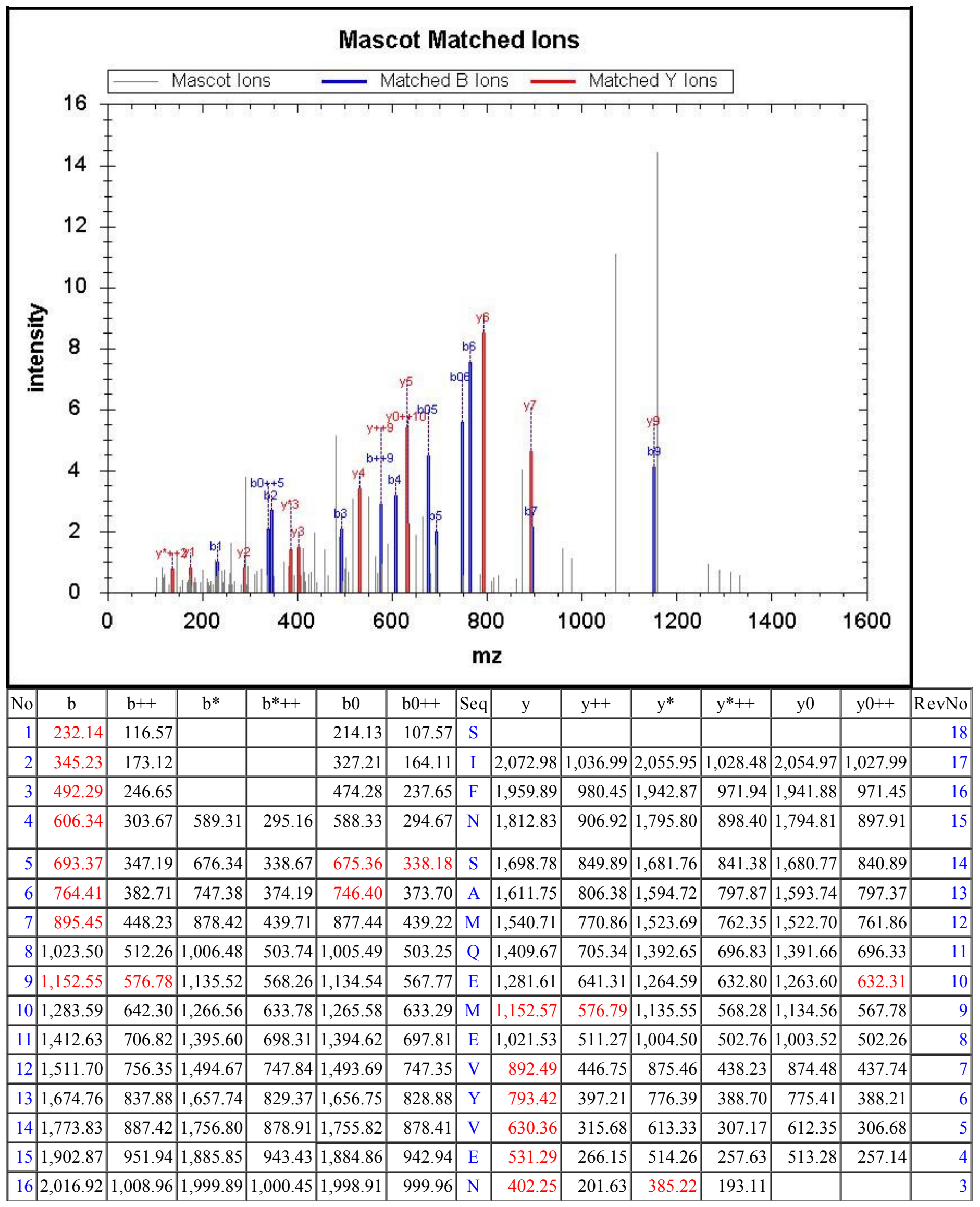




\begin{tabular}{|r|r|r|r|r|r|r|r|r|r|r|r|r|r|r|}
\hline 17 & $2,130.00$ & $1,065.50$ & $2,112.97$ & $1,056.99$ & $2,111.99$ & $1,056.50$ & $\mathrm{I}$ & 288.20 & 144.61 & 271.18 & 136.09 & & & 2 \\
\hline 18 & & & & & & & $\mathrm{R}$ & 175.12 & 88.06 & 158.09 & 79.55 & & & 1 \\
\hline
\end{tabular}

Query 46275 Hit 1

MS/MS Fragmentation of R VPVLGSLLNLPGIR

Found in sp|Q9Y3E0|GOT1B HUM AN, Vesicle transport protein GOT1B OS=Homo sapiens GN=GOLT1B PE=1 SV=1 Match to Query 46275: 1747.097from(583.3729,3+)

Title: 983 : Sum of 2 scans in range $2169(\mathrm{rt}=58.3711, \mathrm{f}=4, \mathrm{i}=657)$ to $2170(\mathrm{rt}=58.3965, \mathrm{f}=4, \mathrm{i}=658)$

[D:llab212 \membranelGrace JoyceliTRAQ_39_1.raw]

Data File:Submitted from 20120508-1(merge) by Mascot Daemon on JOYCE-VAIO

Monoisotopic mass of neutral peptide $\mathrm{Mr}$ (calc): 1747.097

Variable modifications:

Ions Score: 45.65 Expect: 0.005

\section{Mascot Matched lons}

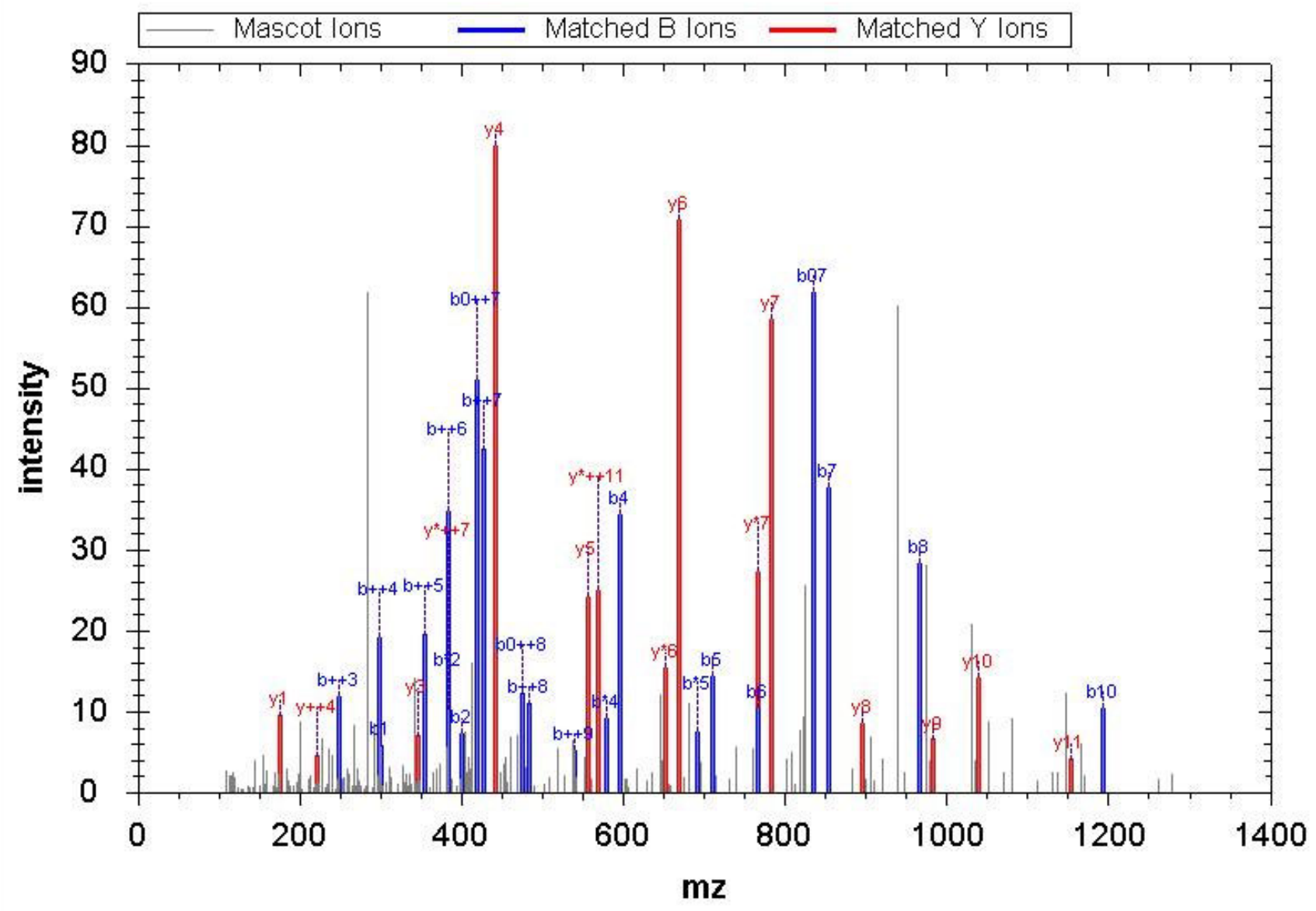

\begin{tabular}{|r|c|c|c|c|c|r|r|r|r|r|r|r|r|r|}
\hline \hline No & $\mathrm{b}$ & $\mathrm{b}++$ & $\mathrm{b} *$ & $\mathrm{~b} *++$ & $\mathrm{b} 0$ & $\mathrm{~b} 0++$ & $\mathrm{Seq}$ & $\mathrm{y}$ & $\mathrm{y}++$ & $\mathrm{y} *$ & $\mathrm{y}^{*++}$ & $\mathrm{y} 0$ & $\mathrm{y} 0++$ & $\mathrm{RevNo}$ \\
\hline 1 & 301.21 & 151.11 & 284.18 & 142.60 & & & $\mathrm{R}$ & & & & & & & 15 \\
\hline 2 & 400.28 & 200.64 & 383.25 & 192.13 & & & $\mathrm{~V}$ & $1,447.90$ & 724.45 & $1,430.87$ & 715.94 & $1,429.89$ & 715.45 & 14 \\
\hline 3 & 497.33 & 249.17 & 480.31 & 240.66 & & & $\mathrm{P}$ & $1,348.83$ & 674.92 & $1,331.80$ & 666.41 & $1,330.82$ & 665.91 & 13 \\
\hline 4 & 596.40 & 298.70 & 579.37 & 290.19 & & & $\mathrm{~V}$ & $1,251.78$ & 626.39 & $1,234.75$ & 617.88 & $1,233.77$ & 617.39 & 12 \\
\hline 5 & 709.48 & 355.25 & 692.46 & 346.73 & & & $\mathrm{~L}$ & $1,152.71$ & 576.86 & $1,135.68$ & 568.35 & $1,134.70$ & 567.85 & 11 \\
\hline 6 & 766.51 & 383.76 & 749.48 & 375.24 & & & $\mathrm{G}$ & $1,039.63$ & 520.32 & $1,022.60$ & 511.80 & $1,021.62$ & 511.31 & 10 \\
\hline 7 & 853.54 & 427.27 & 836.51 & 418.76 & 835.53 & 418.27 & S & 982.60 & 491.81 & 965.58 & 483.29 & 964.59 & 482.80 & 9 \\
\hline 8 & 966.62 & 483.81 & 949.60 & 475.30 & 948.61 & 474.81 & L & 895.57 & 448.29 & 878.55 & 439.78 & & & 8 \\
\hline 9 & $1,079.71$ & 540.36 & $1,062.68$ & 531.84 & $1,061.70$ & 531.35 & L & 782.49 & 391.75 & 765.46 & 383.23 & & & 7 \\
\hline 10 & $1,193.75$ & 597.38 & $1,176.72$ & 588.86 & $1,175.74$ & 588.37 & N & 669.40 & 335.21 & 652.38 & 326.69 & & & 6 \\
\hline 11 & $1,306.83$ & 653.92 & $1,289.81$ & 645.41 & $1,288.82$ & 644.91 & L & 555.36 & 278.18 & 538.33 & 269.67 & & & 5 \\
\hline 12 & $1,403.89$ & 702.45 & $1,386.86$ & 693.93 & $1,385.88$ & 693.44 & P & 442.28 & 221.64 & 425.25 & 213.13 & & & 4 \\
\hline
\end{tabular}




\begin{tabular}{|l|l|l|l|l|l|l|l|l|l|l|l|l|l|l|r|}
\hline 13 & $1,460.91$ & 730.96 & $1,443.88$ & 722.44 & $1,442.90$ & 721.95 & $\mathrm{G}$ & 345.22 & 173.12 & 328.20 & 164.60 & & & 3 \\
\hline 14 & $1,573.99$ & 787.50 & $1,556.96$ & 778.99 & $1,555.98$ & 778.49 & $\mathrm{I}$ & 288.20 & 144.61 & 271.18 & 136.09 & & & 2 \\
\hline 15 & & & & & & & $\mathrm{R}$ & 175.12 & 88.06 & 158.09 & 79.55 & & & 1 \\
\hline
\end{tabular}

Query 67781 Hit 1

MS/MS Fragmentation of VLGHVNNILISAVLPTAFR

Found in sp|Q8TB36|GDAP1_HUMAN, Ganglioside-induced differentiation-associated protein 1 OS=Homo sapiens GN=GDAP1 PE=1 $\mathrm{SV}=3$

Match to Query 67781:2177.28from(726.7671,3+)

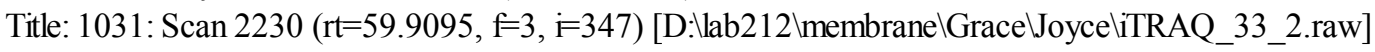

Data File:Submitted from 20120508-1(merge) by Mascot Daemon on JOYCE-VAIO

Monoisotopic mass of neutral peptide $\mathrm{Mr}$ (calc): 2177.28

Variable modifications:

Ions Score: 45.63 Expect: 0.008

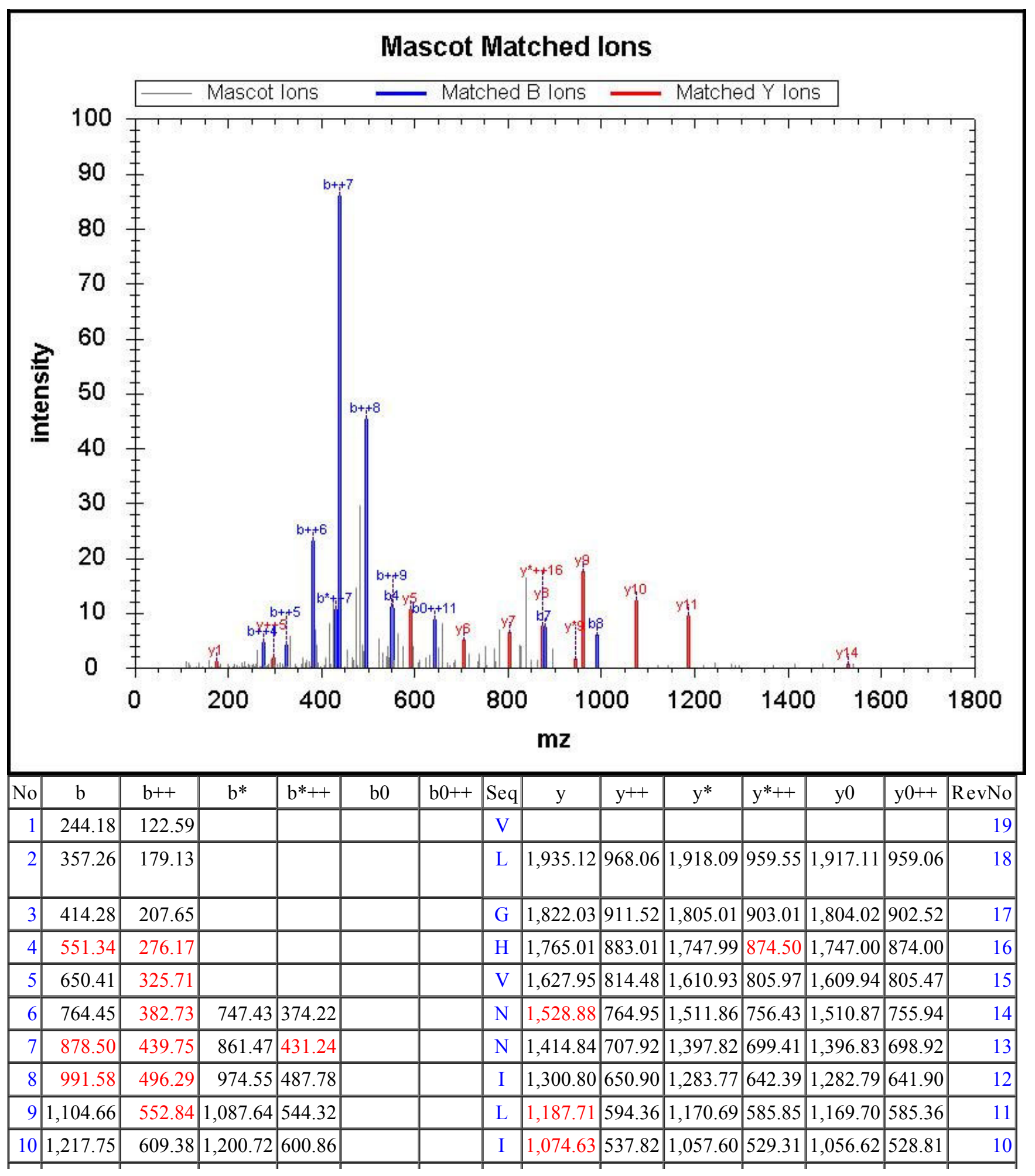




\begin{tabular}{|l|l|r|r|r|r|r|r|r|r|r|r|r|r|r|}
11 & $1,304.78$ & 652.89 & $1,287.75$ & 644.38 & $1,286.77$ & 643.89 & $\mathrm{~S}$ & 961.55 & 481.28 & 944.52 & 472.76 & 943.54 & 472.27 & 9 \\
\hline \hline 12 & $1,375.82$ & 688.41 & $1,358.79$ & 679.90 & $1,357.81$ & 679.41 & $\mathrm{~A}$ & 874.51 & 437.76 & 857.49 & 429.25 & 856.50 & 428.76 & 8 \\
\hline 13 & $1,474.89$ & 737.95 & $1,457.86$ & 729.43 & $1,456.88$ & 728.94 & $\mathrm{~V}$ & 803.48 & 402.24 & 786.45 & 393.73 & 785.47 & 393.24 & 7 \\
\hline 14 & $1,587.97$ & 794.49 & $1,570.94$ & 785.98 & $1,569.96$ & 785.48 & $\mathrm{~L}$ & 704.41 & 352.71 & 687.38 & 344.19 & 686.40 & 343.70 & 6 \\
\hline 15 & $1,685.02$ & 843.02 & $1,668.00$ & 834.50 & $1,667.01$ & 834.01 & $\mathrm{P}$ & 591.32 & 296.17 & 574.30 & 287.65 & 573.31 & 287.16 & 5 \\
\hline 16 & $1,786.07$ & 893.54 & $1,769.04$ & 885.03 & $1,768.06$ & 884.53 & $\mathrm{~T}$ & 494.27 & 247.64 & 477.25 & 239.13 & 476.26 & 238.63 & 4 \\
\hline 17 & $1,857.11$ & 929.06 & $1,840.08$ & 920.54 & $1,839.10$ & 920.05 & $\mathrm{~A}$ & 393.22 & 197.12 & 376.20 & 188.60 & & & 3 \\
\hline 18 & $2,004.18$ & $1,002.59$ & $1,987.15$ & 994.08 & $1,986.17$ & 993.59 & $\mathrm{~F}$ & 322.19 & 161.60 & 305.16 & 153.08 & & & 2 \\
\hline 19 & & & & & & & $\mathrm{R}$ & 175.12 & 88.06 & 158.09 & 79.55 & & & 1 \\
\hline
\end{tabular}

Query 77791 Hit 1

MS/MS Fragmentation of LWNKDPLTGILYQINSK

Found in sp|Q61830|MRC1_MOUSE, Macrophage mannose receptor 1 OS=Mus musculus GN=Mrc1 PE=1 SV=2

Match to Query 77791: 2434.392from(812.4713,3+)

Title: 738: Sum of 2 scans in range 2282 ( $\mathrm{rt}=58.4707, \mathrm{f}=4, \mathrm{i}=479)$ to $2283(\mathrm{rt}=58.4962, \mathrm{f}=4, \mathrm{i}=480$ )

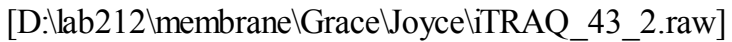

Data File:Submitted from 20120508-1(merge) by Mascot Daemon on JOYCE-VAIO

Monoisotopic mass of neutral peptide $\mathrm{Mr}$ (calc): 2434.392

Variable modifications:

K4 :iTRAQ4plex (K)

$\mathrm{K} 17$ :iTRAQ4plex $(\mathrm{K})$

Ions Score: 45.6 Expect: 0.010

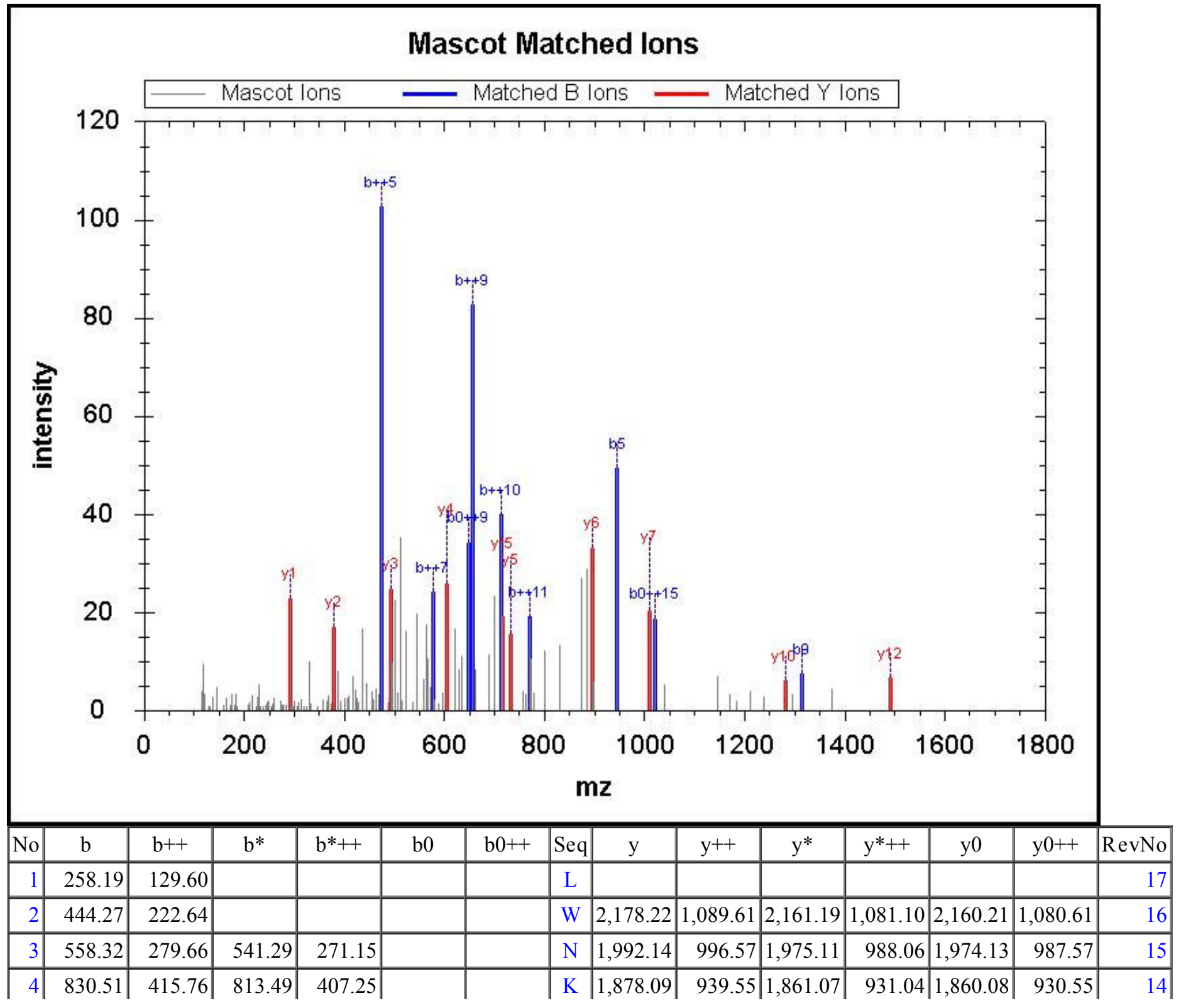




\begin{tabular}{|r|r|r|r|r|r|r|r|r|r|r|r|r|r|r|}
\hline 5 & 945.54 & 473.27 & 928.51 & 464.76 & 927.53 & 464.27 & $\mathrm{D}$ & $1,605.90$ & 803.45 & $1,588.87$ & 794.94 & $1,587.89$ & 794.45 & 13 \\
\hline 6 & $1,042.59$ & 521.80 & $1,025.57$ & 513.29 & $1,024.58$ & 512.79 & $\mathrm{P}$ & $1,490.87$ & 745.94 & $1,473.84$ & 737.43 & $1,472.86$ & 736.93 & 12 \\
\hline 7 & $1,155.68$ & 578.34 & $1,138.65$ & 569.83 & $1,137.67$ & 569.34 & $\mathrm{~L}$ & $1,393.82$ & 697.41 & $1,376.79$ & 688.90 & $1,375.81$ & 688.41 & 11 \\
\hline 8 & $1,256.72$ & 628.87 & $1,239.70$ & 620.35 & $1,238.71$ & 619.86 & $\mathrm{~T}$ & $1,280.73$ & 640.87 & $1,263.71$ & 632.36 & $1,262.72$ & 631.86 & 10 \\
\hline 9 & $1,313.75$ & 657.38 & $1,296.72$ & 648.86 & $1,295.74$ & 648.37 & $\mathrm{G}$ & $1,179.69$ & 590.35 & $1,162.66$ & 581.83 & $1,161.67$ & 581.34 & 9 \\
\hline 10 & $1,426.83$ & 713.92 & $1,409.80$ & 705.41 & $1,408.82$ & 704.91 & $\mathrm{I}$ & $1,122.66$ & 561.84 & $1,105.64$ & 553.32 & $1,104.65$ & 552.83 & 8 \\
\hline 11 & $1,539.91$ & 770.46 & $1,522.89$ & 761.95 & $1,521.90$ & 761.46 & $\mathrm{~L}$ & $1,009.58$ & 505.29 & 992.55 & 496.78 & 991.57 & 496.29 & 7 \\
\hline 12 & $1,702.98$ & 851.99 & $1,685.95$ & 843.48 & $1,684.97$ & 842.99 & $\mathrm{Y}$ & 896.50 & 448.75 & 879.47 & 440.24 & 878.49 & 439.75 & 6 \\
\hline 13 & $1,831.04$ & 916.02 & $1,814.01$ & 907.51 & $1,813.03$ & 907.02 & $\mathrm{Q}$ & 733.43 & 367.22 & 716.41 & 358.71 & 715.42 & 358.21 & 5 \\
\hline 14 & $1,944.12$ & 972.56 & $1,927.09$ & 964.05 & $1,926.11$ & 963.56 & $\mathrm{I}$ & 605.37 & 303.19 & 588.35 & 294.68 & 587.36 & 294.19 & 4 \\
\hline 15 & $2,058.16$ & $1,029.59$ & $2,041.14$ & $1,021.07$ & $2,040.15$ & $1,020.58$ & $\mathrm{~N}$ & 492.29 & 246.65 & 475.26 & 238.14 & 474.28 & 237.64 & 3 \\
\hline 16 & $2,145.19$ & $1,073.10$ & $2,128.17$ & $1,064.59$ & $2,127.18$ & $1,064.10$ & $\mathrm{~S}$ & 378.25 & 189.63 & 361.22 & 181.11 & 360.24 & 180.62 & 2 \\
\hline 17 & & & & & & & $\mathrm{~K}$ & 291.21 & 146.11 & 274.19 & 137.60 & & & 1 \\
\hline
\end{tabular}

Query 14134 Hit 1

MS/MS Fragmentation of SGLSDIILGK

Found in sp|Q9UBC2|EP15R_HUM AN, Epidermal growth factor receptor substrate 15-like 1 OS=Homo sapiens GN=EPS15L1 PE=1 $\mathrm{SV}=1$

Match to Query 14134: 1145.654from(573.834,2+)

Title: 257: Scan 659 (rt=24.2052, f=3, i=90) [D:llab212 \membranelGracelJoyceliTRAQ_31_1.raw]

Data File:Submitted from 20120508-1(merge) by Mascot Daemon on JOYCE-VAIO

Monoisotopic mass of neutral peptide Mr(calc): 1145.654

Variable modifications:

K10 :TRAQ4plex (K)

Ions Score: 45.56 Expect: 0.023

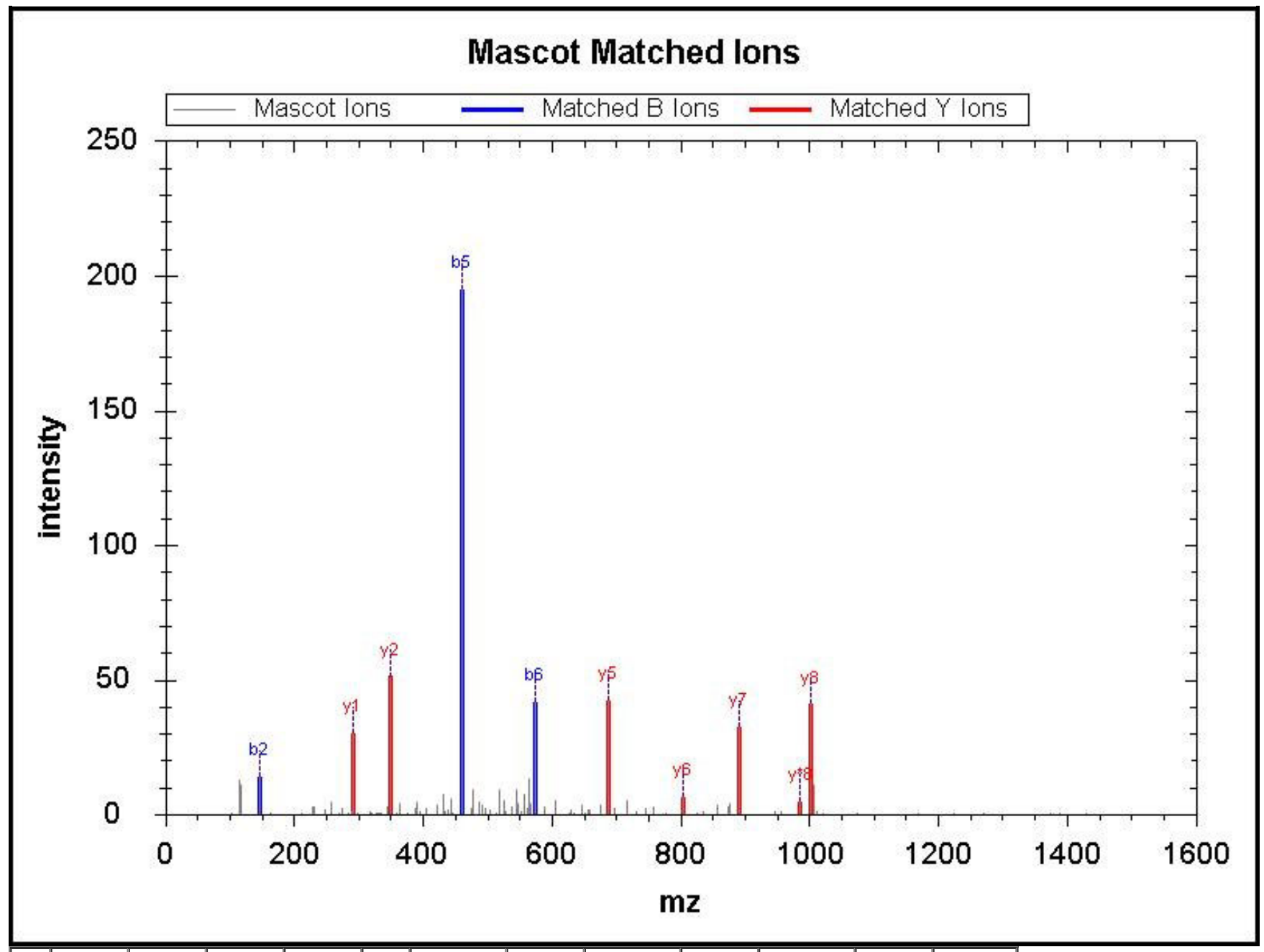

\begin{tabular}{|c|c|c|c|c|c|c|c|c|c|c|c|c|}
\hline \hline No & $\mathrm{b}$ & $\mathrm{b}++$ & $\mathrm{b} 0$ & $\mathrm{~b} 0++$ & Seq & $\mathrm{y}$ & $\mathrm{y}++$ & $\mathrm{y}^{*}$ & $\mathrm{y}^{*++}$ & $\mathrm{y} 0$ & $\mathrm{y} 0++$ & RevNo \\
\hline
\end{tabular}




\begin{tabular}{|r|r|r|r|r|r|r|r|r|r|r|r|r|}
11 & 88.04 & 44.52 & 70.03 & 35.52 & $\mathrm{~S}$ & & & & & & & 10 \\
\hline \hline 2 & 145.06 & 73.03 & 127.05 & 64.03 & $\mathrm{G}$ & $1,059.65$ & 530.33 & $1,042.63$ & 521.82 & $1,041.64$ & 521.32 & 9 \\
\hline 3 & 258.14 & 129.58 & 240.13 & 120.57 & $\mathrm{~L}$ & $1,002.63$ & 501.82 & 985.60 & 493.31 & 984.62 & 492.81 & 8 \\
\hline 4 & 345.18 & 173.09 & 327.17 & 164.09 & $\mathrm{~S}$ & 889.55 & 445.28 & 872.52 & 436.76 & 871.54 & 436.27 & 7 \\
\hline 5 & 460.20 & 230.61 & 442.19 & 221.60 & $\mathrm{D}$ & 802.52 & 401.76 & 785.49 & 393.25 & 784.50 & 392.76 & 6 \\
\hline 6 & 573.29 & 287.15 & 555.28 & 278.14 & $\mathrm{I}$ & 687.49 & 344.25 & 670.46 & 335.73 & & & 5 \\
\hline 7 & 686.37 & 343.69 & 668.36 & 334.68 & $\mathrm{I}$ & 574.40 & 287.71 & 557.38 & 279.19 & & & 4 \\
\hline 8 & 799.46 & 400.23 & 781.45 & 391.23 & $\mathrm{~L}$ & 461.32 & 231.16 & 444.29 & 222.65 & & & 3 \\
\hline 9 & 856.48 & 428.74 & 838.47 & 419.74 & $\mathrm{G}$ & 348.24 & 174.62 & 331.21 & 166.11 & & & 2 \\
\hline 10 & & & & & $\mathrm{~K}$ & 291.21 & 146.11 & 274.19 & 137.60 & & & 1 \\
\hline
\end{tabular}

Query 57686 Hit 1

MS/MS Fragmentation of GM GLVNHVFTEDNLK

Found in sp|Q06203|PUR 1_HUMAN, Amidophosphoribosyltransferase OS=Homo sapiens GN=PPAT PE=1 SV=1 Match to Query 57686: 1961.028from(654.6834,3+)

Title: 646: Scan $1431(\mathrm{rt}=41.9167, \mathrm{f}=3, \mathrm{i}=224)$ [D:lab212 \membranelGrace JoyceliTRAQ_40_1.raw]

Data File:Submitted from 20120508-1(merge) by Mascot Daemon on JOYCE-VAIO

Monoisotopic mass of neutral peptide $\mathrm{Mr}$ (calc): 1961.028

Variable modifications:

K15 :iTRAQ4plex (K)

Ions Score: 45.52 Expect: 0.026

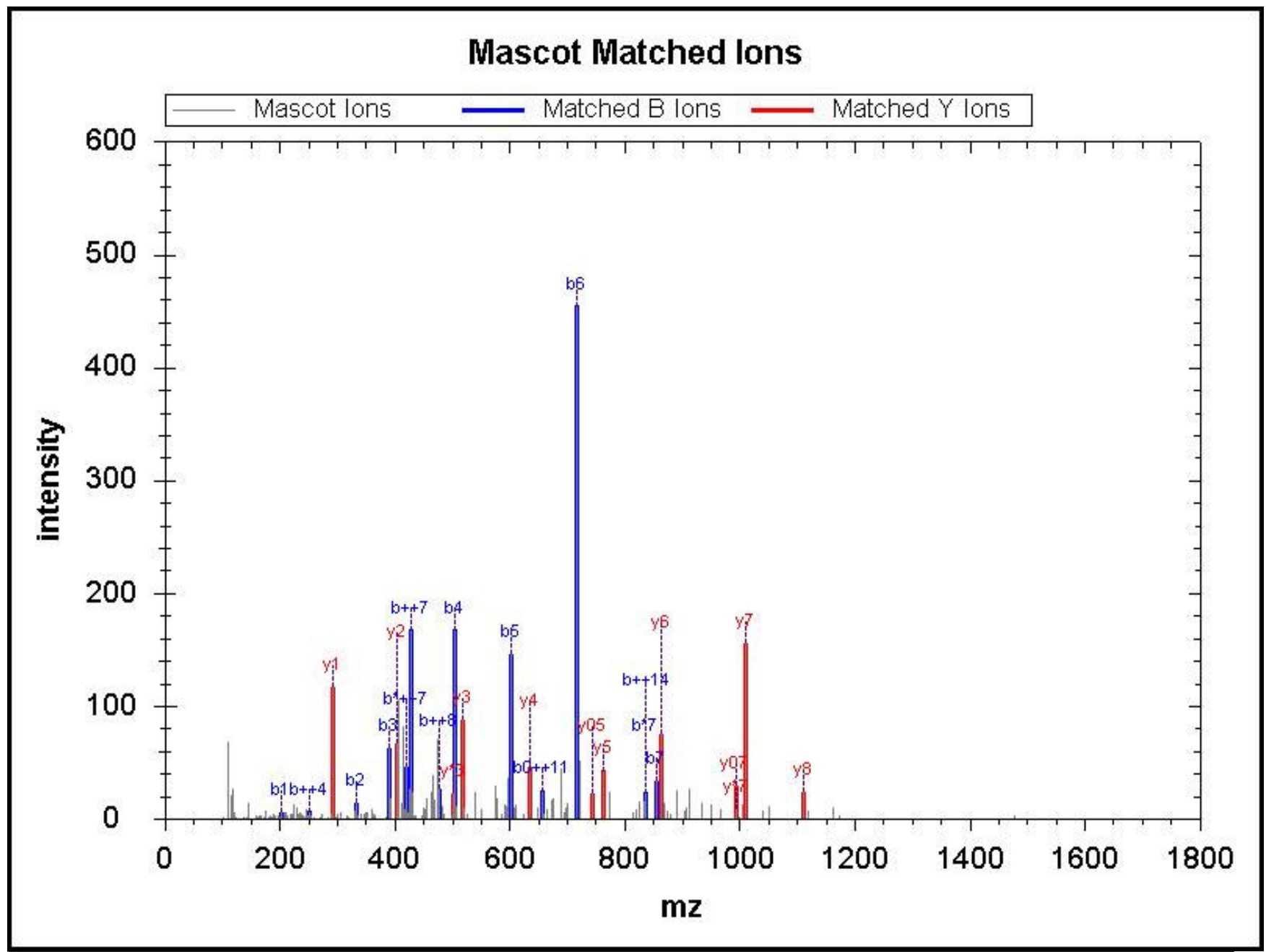

\begin{tabular}{|r|c|c|c|c|c|c|c|c|c|c|c|r|r|r|r|}
\hline \hline No & $\mathrm{b}$ & $\mathrm{b}++$ & $\mathrm{b}^{*}$ & $\mathrm{~b}+++$ & $\mathrm{b} 0$ & $\mathrm{~b} 0++$ & Seq & $\mathrm{y}$ & $\mathrm{y}++$ & $\mathrm{y}^{*}$ & $\mathrm{y}^{*++}$ & $\mathrm{y} 0$ & $\mathrm{y} 0++$ & $\mathrm{RevNo}$ \\
\hline 1 & 202.13 & 101.57 & & & & & $\mathrm{G}$ & & & & & & & 15 \\
\hline 2 & 333.17 & 167.09 & & & & & $\mathrm{M}$ & $1,760.91$ & 880.96 & $1,743.89$ & 872.45 & $1,742.90$ & 871.95 & 14 \\
\hline 3 & 390.19 & 195.60 & & & & & G & $1,629.87$ & 815.44 & $1,612.85$ & 806.93 & $1,611.86$ & 806.43 & 13 \\
\hline 4 & 503.28 & 252.14 & & & & & L & $1,572.85$ & 786.93 & $1,555.82$ & 778.42 & $1,554.84$ & 777.92 & 12 \\
\hline
\end{tabular}




\begin{tabular}{|r|r|r|r|r|r|r|r|r|r|r|r|r|r|r|r|}
\hline 5 & 602.35 & 301.68 & & & & & $\mathrm{~V}$ & $1,459.77$ & 730.39 & $1,442.74$ & 721.87 & $1,441.76$ & 721.38 & 11 \\
\hline 6 & 716.39 & 358.70 & 699.36 & 350.18 & & & $\mathrm{~N}$ & $1,360.70$ & 680.85 & $1,343.67$ & 672.34 & $1,342.69$ & 671.85 & 10 \\
\hline 7 & 853.45 & 427.23 & 836.42 & 418.71 & & & $\mathrm{H}$ & $1,246.65$ & 623.83 & $1,229.63$ & 615.32 & $1,228.64$ & 614.83 & 9 \\
\hline 8 & 952.52 & 476.76 & 935.49 & 468.25 & & & $\mathrm{~V}$ & $1,109.60$ & 555.30 & $1,092.57$ & 546.79 & $1,091.59$ & 546.30 & 8 \\
\hline 9 & $1,099.58$ & 550.30 & $1,082.56$ & 541.78 & & & $\mathrm{~F}$ & $1,010.53$ & 505.77 & 993.50 & 497.25 & 992.52 & 496.76 & 7 \\
\hline 10 & $1,200.63$ & 600.82 & $1,183.61$ & 592.31 & $1,182.62$ & 591.81 & $\mathrm{~T}$ & 863.46 & 432.23 & 846.43 & 423.72 & 845.45 & 423.23 & 6 \\
\hline 11 & $1,329.67$ & 665.34 & $1,312.65$ & 656.83 & $1,311.66$ & 656.34 & $\mathrm{E}$ & 762.41 & 381.71 & 745.38 & 373.20 & 744.40 & 372.70 & 5 \\
\hline 12 & $1,444.70$ & 722.85 & $1,427.67$ & 714.34 & $1,426.69$ & 713.85 & $\mathrm{D}$ & 633.37 & 317.19 & 616.34 & 308.67 & 615.36 & 308.18 & 4 \\
\hline 13 & $1,558.74$ & 779.88 & $1,541.72$ & 771.36 & $1,540.73$ & 770.87 & $\mathrm{~N}$ & 518.34 & 259.67 & 501.32 & 251.16 & & & 3 \\
\hline 14 & $1,671.83$ & 836.42 & $1,654.80$ & 827.90 & $1,653.82$ & 827.41 & $\mathrm{~L}$ & 404.30 & 202.65 & 387.27 & 194.14 & & & 2 \\
\hline 15 & & & & & & & $\mathrm{~K}$ & 291.21 & 146.11 & 274.19 & 137.60 & & & 1 \\
\hline
\end{tabular}

Query 79330 Hit 1

\section{MS/MS Fragmentation of QMNAFLEGFTELLPIDLIK}

Found in sp|Q96PU5|NED4L_HUMAN, E3 ubiquitin-protein ligase NEDD4-like OS=Homo sapiens GN=NEDD4L PE=1 SV=2 Match to Query 79330: 2479.369from(827.4637,3+)

Title: 1216: Sum of 2 scans in range $3390(\mathrm{rt}=82.1473, \mathrm{f}=2, \mathrm{i}=467)$ to $3391(\mathrm{rt}=82.1727, \mathrm{f}=2, \mathrm{i}=468)$

[D:llab212 \membranelGrace JoyceliTRAQ_25_1.raw]

Data File:Submitted from 20120508-1(merge) by Mascot Daemon on JOYCE-VAIO

Monoisotopic mass of neutral peptide $\operatorname{Mr}($ calc): 2479.369

Variable modifications:

K19 :TRAQ4plex (K)

Ions Score: 45.51 Expect: 0.016

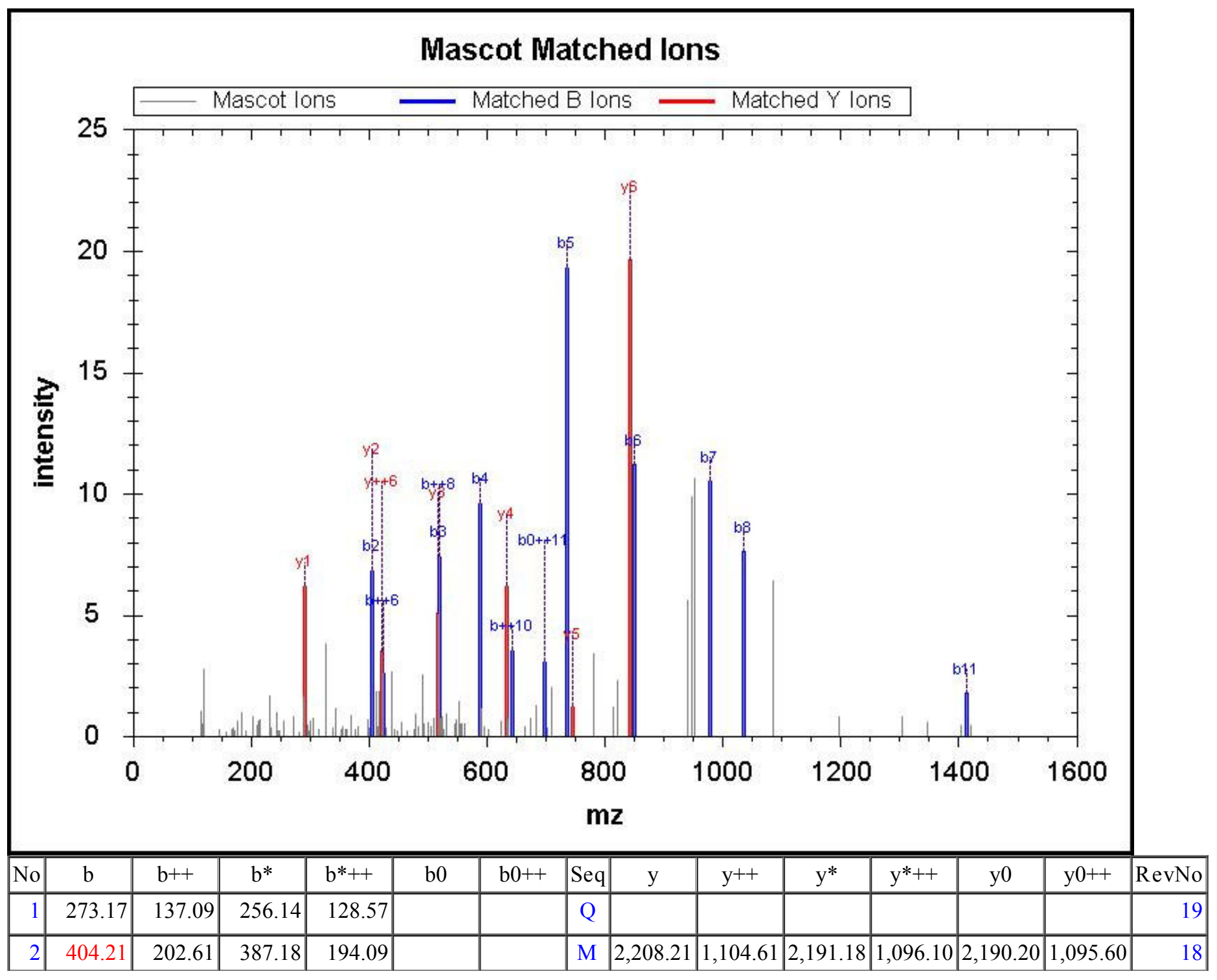




\begin{tabular}{|r|r|r|r|r|r|r|r|r|r|r|r|r|r|r|}
\hline 3 & 518.25 & 259.63 & 501.22 & 251.12 & & & $\mathrm{~N}$ & $2,077.17$ & $1,039.09$ & $2,060.14$ & $1,030.58$ & $2,059.16$ & $1,030.08$ & 17 \\
\hline 4 & 589.29 & 295.15 & 572.26 & 286.63 & & & $\mathrm{~A}$ & $1,963.13$ & 982.07 & $1,946.10$ & 973.55 & $1,945.12$ & 973.06 & 16 \\
\hline 5 & 736.36 & 368.68 & 719.33 & 360.17 & & & $\mathrm{~F}$ & $1,892.09$ & 946.55 & $1,875.06$ & 938.04 & $1,874.08$ & 937.54 & 15 \\
\hline 6 & 849.44 & 425.22 & 832.41 & 416.71 & & & $\mathrm{~L}$ & $1,745.02$ & 873.01 & $1,728.00$ & 864.50 & $1,727.01$ & 864.01 & 14 \\
\hline 7 & 978.48 & 489.75 & 961.46 & 481.23 & 960.47 & 480.74 & $\mathrm{E}$ & $1,631.94$ & 816.47 & $1,614.91$ & 807.96 & $1,613.93$ & 807.47 & 13 \\
\hline 8 & $1,035.51$ & 518.26 & $1,018.48$ & 509.74 & $1,017.49$ & 509.25 & $\mathrm{G}$ & $1,502.90$ & 751.95 & $1,485.87$ & 743.44 & $1,484.88$ & 742.95 & 12 \\
\hline 9 & $1,182.57$ & 591.79 & $1,165.55$ & 583.28 & $1,164.56$ & 582.79 & $\mathrm{~F}$ & $1,445.87$ & 723.44 & $1,428.85$ & 714.93 & $1,427.86$ & 714.44 & 11 \\
\hline 10 & $1,283.62$ & 642.31 & $1,266.59$ & 633.80 & $1,265.61$ & 633.31 & $\mathrm{~T}$ & $1,298.81$ & 649.91 & $1,281.78$ & 641.39 & $1,280.79$ & 640.90 & 10 \\
\hline 11 & $1,412.66$ & 706.84 & $1,395.64$ & 698.32 & $1,394.65$ & 697.83 & $\mathrm{E}$ & $1,197.76$ & 599.38 & $1,180.73$ & 590.87 & $1,179.75$ & 590.38 & 9 \\
\hline 12 & $1,525.75$ & 763.38 & $1,508.72$ & 754.86 & $1,507.74$ & 754.37 & $\mathrm{~L}$ & $1,068.71$ & 534.86 & $1,051.69$ & 526.35 & $1,050.70$ & 525.86 & 8 \\
\hline 13 & $1,638.83$ & 819.92 & $1,621.81$ & 811.41 & $1,620.82$ & 810.91 & $\mathrm{~L}$ & 955.63 & 478.32 & 938.60 & 469.81 & 937.62 & 469.31 & 7 \\
\hline 14 & $1,735.88$ & 868.45 & $1,718.86$ & 859.93 & $1,717.87$ & 859.44 & $\mathrm{P}$ & 842.55 & 421.78 & 825.52 & 413.26 & 824.54 & 412.77 & 6 \\
\hline 15 & $1,848.97$ & 924.99 & $1,831.94$ & 916.47 & $1,830.96$ & 915.98 & $\mathrm{I}$ & 745.49 & 373.25 & 728.47 & 364.74 & 727.48 & 364.25 & 5 \\
\hline 16 & $1,964.00$ & 982.50 & $1,946.97$ & 973.99 & $1,945.99$ & 973.50 & $\mathrm{D}$ & 632.41 & 316.71 & 615.38 & 308.20 & 614.40 & 307.70 & 4 \\
\hline 17 & $2,077.08$ & $1,039.04$ & $2,060.05$ & $1,030.53$ & $2,059.07$ & $1,030.04$ & $\mathrm{~L}$ & 517.38 & 259.20 & 500.36 & 250.68 & & & 3 \\
\hline 18 & $2,190.16$ & $1,095.59$ & $2,173.14$ & $1,087.07$ & $2,172.15$ & $1,086.58$ & $\mathrm{I}$ & 404.30 & 202.65 & 387.27 & 194.14 & & & 2 \\
\hline 19 & & & & & & & $\mathrm{~K}$ & 291.21 & 146.11 & 274.19 & 137.60 & & & 1 \\
\hline
\end{tabular}

Query 27463 Hit 1

\section{MS/MS Fragmentation of YIFHNFMER}

Found in sp|Q14624|ITIH4_HUM AN, Inter-alpha-trypsin inhibitor heavy chain H4 OS=Homo sapiens GN=ITIH4 PE=1 SV=4 Match to Query 27463: 1399.688 from(467.5699,3+)

Title: 635: Scan 1422 ( $\mathrm{rt}=41.6552, \mathrm{f}=2, \mathrm{i}=231)$ [D:lab212 $\backslash$ membranelGracelJoyceliTRAQ_41_1.raw]

Data File:Submitted from 20120508-1(merge) by Mascot Daemon on JOYCE-VAIO

Monoisotopic mass of neutral peptide $\mathrm{Mr}$ (calc): 1399.688

Variable modifications:

Ions Score: 45.51 Expect: 0.018 


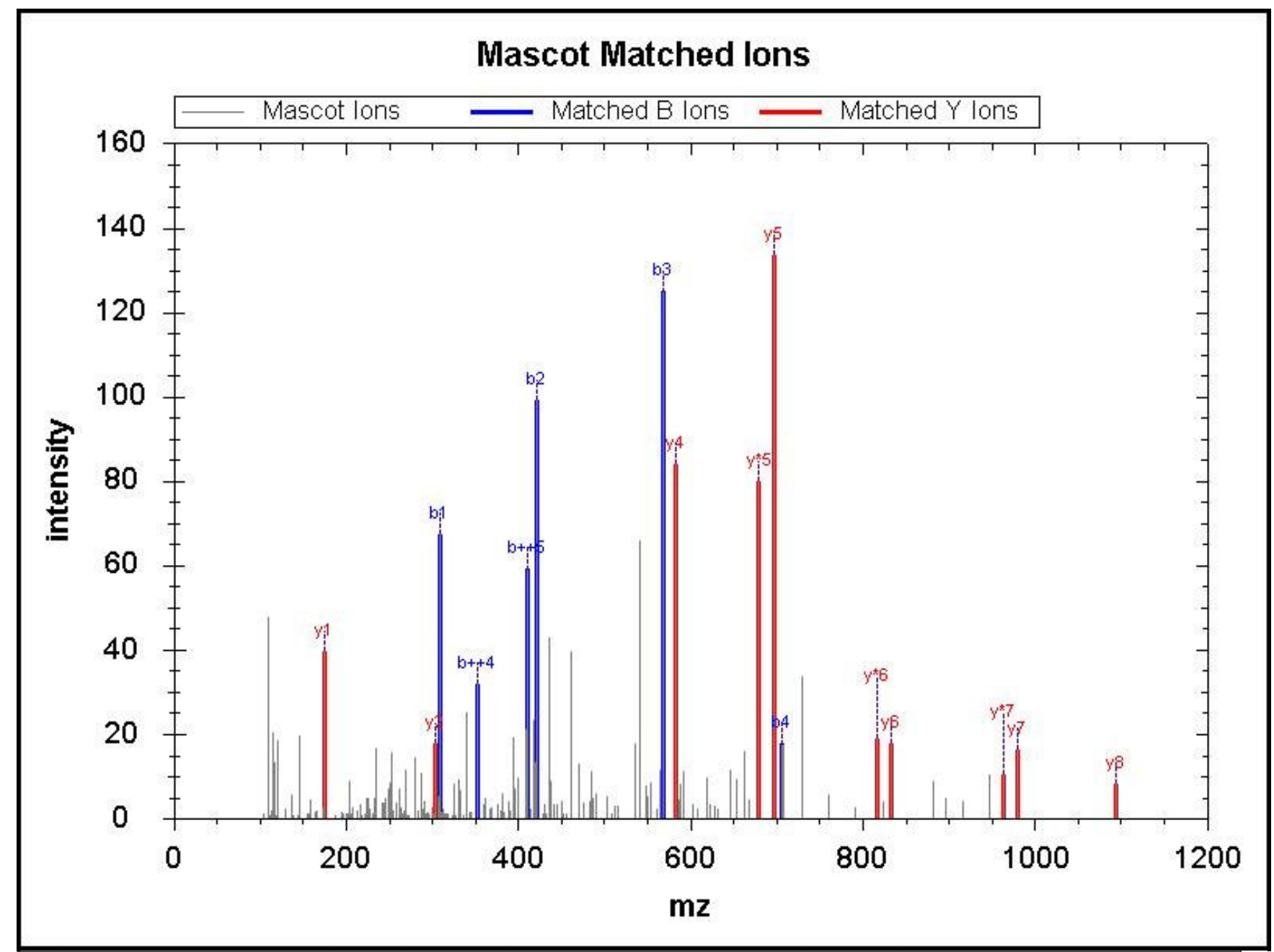

\begin{tabular}{|r|c|c|c|c|c|r|r|r|r|r|r|r|r|r|}
\hline \hline No & $\mathrm{b}$ & $\mathrm{b}++$ & $\mathrm{b} *$ & $\mathrm{~b} *++$ & $\mathrm{b} 0$ & $\mathrm{~b} 0++$ & Seq & $\mathrm{y}$ & $\mathrm{y}++$ & $\mathrm{y}^{*}$ & $\mathrm{y}^{*++}$ & $\mathrm{y} 0$ & $\mathrm{y} 0++$ & $\mathrm{RevNo}$ \\
\hline 1 & 308.17 & 154.59 & & & & & $\mathrm{Y}$ & & & & & & & 9 \\
\hline 2 & 421.26 & 211.13 & & & & & $\mathrm{I}$ & $1,093.52$ & 547.27 & $1,076.50$ & 538.75 & $1,075.51$ & 538.26 & 8 \\
\hline 3 & 568.33 & 284.67 & & & & & $\mathrm{~F}$ & 980.44 & 490.72 & 963.41 & 482.21 & 962.43 & 481.72 & 7 \\
\hline 4 & 705.38 & 353.20 & & & & & $\mathrm{H}$ & 833.37 & 417.19 & 816.35 & 408.68 & 815.36 & 408.18 & 6 \\
\hline 5 & 819.43 & 410.22 & 802.40 & 401.70 & & & $\mathrm{~N}$ & 696.31 & 348.66 & 679.29 & 340.15 & 678.30 & 339.65 & 5 \\
\hline 6 & 966.50 & 483.75 & 949.47 & 475.24 & & & $\mathrm{~F}$ & 582.27 & 291.64 & 565.24 & 283.13 & 564.26 & 282.63 & 4 \\
\hline 7 & $1,097.54$ & 549.27 & $1,080.51$ & 540.76 & & & $\mathrm{M}$ & 435.20 & 218.10 & 418.18 & 209.59 & 417.19 & 209.10 & 3 \\
\hline 8 & $1,226.58$ & 613.79 & $1,209.55$ & 605.28 & $1,208.57$ & 604.79 & $\mathrm{E}$ & 304.16 & 152.58 & 287.13 & 144.07 & 286.15 & 143.58 & 2 \\
\hline 9 & & & & & & & $\mathrm{R}$ & 175.12 & 88.06 & 158.09 & 79.55 & & & 1 \\
\hline
\end{tabular}

Query 58400 Hit 1

MS/MS Fragmentation of IKDLLQQMEEGLK

Found in sp|Q9NS86|LANC2_HUMAN, LanC-like protein 2 OS=Homo sapiens GN=LANCL2 PE=1 SV=1

Match to Query 58400: 1976.141 from(659.7211,3+)

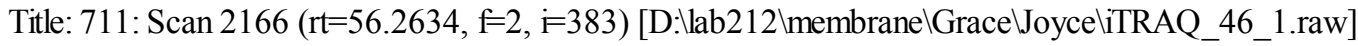

Data File:Submitted from 20120508-1(merge) by Mascot Daemon on JOYCE-VAIO

Monoisotopic mass of neutral peptide Mr(calc): 1976.141

Variable modifications:

K2 :iTRAQ4plex (K)

$\mathrm{K} 13$ :iTRAQ4plex $(\mathrm{K})$

Ions Score: 45.47 Expect: 0.014 


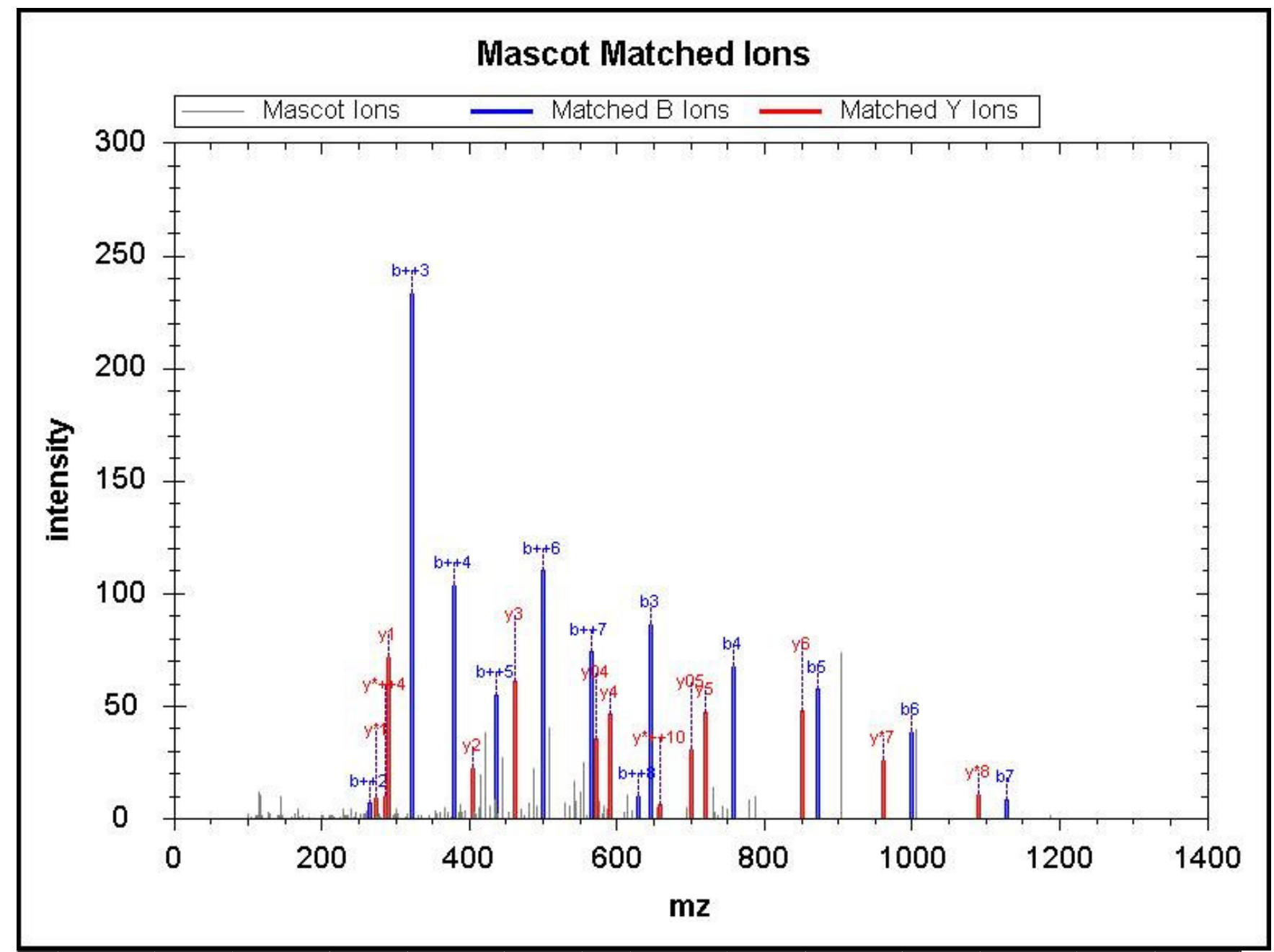

\begin{tabular}{|c|c|c|c|c|c|c|c|c|c|c|c|c|c|c|}
\hline No & $\mathrm{b}$ & $\mathrm{b}++$ & $b^{*}$ & $b^{*++}$ & b0 & $\mathrm{b} 0++$ & Seq & $\mathrm{y}$ & $\mathrm{y}++$ & $y^{*}$ & $y^{*++}$ & y0 & $\mathrm{y} 0++$ & RevNo \\
\hline 1 & 258.19 & 129.60 & & & & & I & & & & & & & 13 \\
\hline 2 & 530.39 & 265.70 & 513.36 & 257.19 & & & $\mathrm{~K}$ & $1,719.96$ & 860.48 & $1,702.93$ & 851.97 & $1,701.94$ & 851.48 & 12 \\
\hline 3 & 645.42 & 323.21 & 628.39 & 314.70 & 627.41 & 314.21 & $\mathrm{D}$ & $1,447.76$ & 724.38 & $1,430.73$ & 715.87 & $1,429.75$ & 715.38 & 11 \\
\hline 4 & 758.50 & 379.75 & 741.47 & 371.24 & 740.49 & 370.75 & $\mathrm{~L}$ & $1,332.73$ & 666.87 & $1,315.70$ & 658.36 & $1,314.72$ & 657.86 & 10 \\
\hline 5 & 871.59 & 436.30 & 854.56 & 427.78 & 853.57 & 427.29 & $\mathrm{~L}$ & $1,219.65$ & 610.33 & $1,202.62$ & 601.81 & $1,201.64$ & 601.32 & 9 \\
\hline 6 & 999.64 & 500.33 & 982.62 & 491.81 & 981.63 & 491.32 & Q & $1,106.56$ & 553.79 & $1,089.54$ & 545.27 & $1,088.55$ & 544.78 & 8 \\
\hline 7 & $1,127.70$ & 564.35 & $1,110.68$ & 555.84 & $1,109.69$ & 555.35 & Q & 978.50 & 489.76 & 961.48 & 481.24 & 960.49 & 480.75 & 7 \\
\hline 8 & $1,258.74$ & 629.88 & $1,241.72$ & 621.36 & $1,240.73$ & 620.87 & $M$ & 850.45 & 425.73 & 833.42 & 417.21 & 832.44 & 416.72 & 6 \\
\hline 9 & $1,387.79$ & 694.40 & $1,370.76$ & 685.88 & $1,369.78$ & 685.39 & $\mathrm{E}$ & 719.41 & 360.21 & 702.38 & 351.69 & 701.39 & 351.20 & 5 \\
\hline 10 & $1,516.83$ & 758.92 & $1,499.80$ & 750.40 & $1,498.82$ & 749.91 & $\mathrm{E}$ & 590.36 & 295.69 & 573.34 & 287.17 & 572.35 & 286.68 & 4 \\
\hline 11 & $1,573.85$ & 787.43 & $1,556.82$ & 778.92 & $1,555.84$ & \begin{tabular}{|l|}
778.42 \\
\end{tabular} & G & 461.32 & 231.16 & 444.29 & 222.65 & & & 3 \\
\hline 12 & $1,686.93$ & 843.97 & $1,669.91$ & 835.46 & $1,668.92$ & 834.97 & $\mathrm{~L}$ & 404.30 & 202.65 & 387.27 & 194.14 & & & 2 \\
\hline 13 & & & & & & & $\mathrm{~K}$ & 291.21 & 146.11 & 274.19 & 137.60 & & & 1 \\
\hline
\end{tabular}

Query 36916 Hit 1

MS/MS Fragmentation of AFVRPSGTEDVVR

Found in sp|O95394|AGM 1_HUMAN, Phosphoacetylglucosamine mutase OS=Homo sapiens GN=PGM3 PE=1 SV=1

Match to Query 36916: 1575.844from(526.2888,3+)

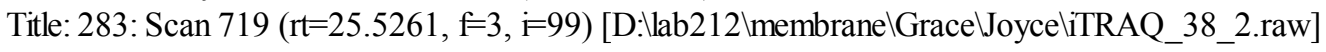

Data File:Submitted from 20120508-1(merge) by Mascot Daemon on JOYCE-VAIO

Monoisotopic mass of neutral peptide $\mathrm{Mr}$ (calc): 1575.844

Variable modifications:

Ions Score: 45.47 Expect: 0.025 


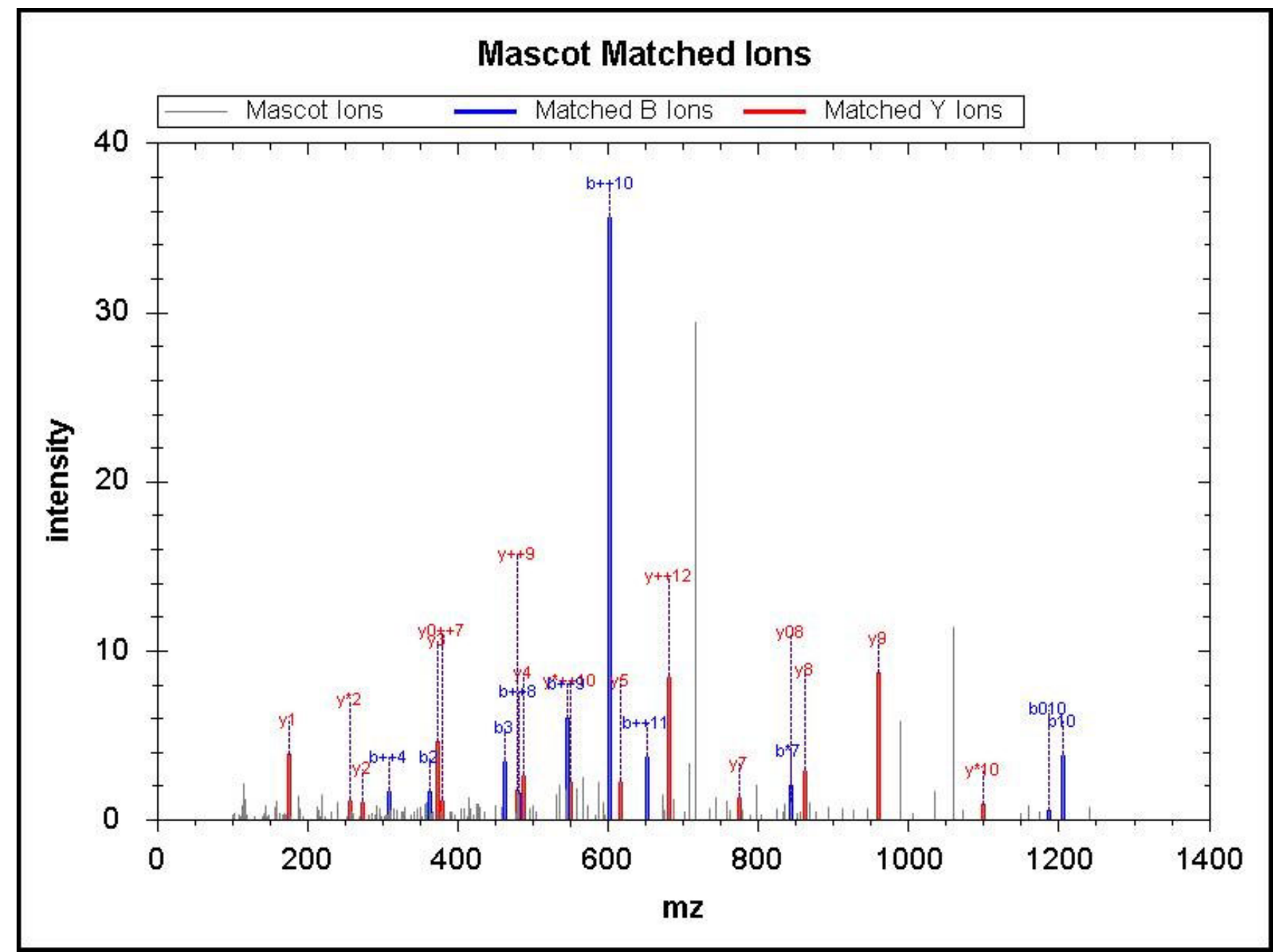

\begin{tabular}{|c|c|c|c|c|c|c|c|c|c|c|c|c|c|c|}
\hline No & $\mathrm{b}$ & $\mathrm{b}++$ & $b^{*}$ & $b^{*++}$ & b0 & $\mathrm{b} 0++$ & Seq & $\mathrm{y}$ & $\mathrm{y}++$ & $y^{*}$ & $y^{*++}$ & y0 & $\mathrm{y} 0++$ & RevNo \\
\hline 1 & 216.15 & 108.58 & & & & & A & & & & & & & 13 \\
\hline 2 & 363.21 & 182.11 & & & & & $\mathrm{~F}$ & $1,361.72$ & 681.36 & $1,344.69$ & 672.85 & $1,343.71$ & 672.36 & 12 \\
\hline 3 & 462.28 & 231.65 & & & & & $\mathrm{~V}$ & $1,214.65$ & 607.83 & $1,197.62$ & 599.31 & $1,196.64$ & 598.82 & 11 \\
\hline 4 & 618.38 & 309.70 & 601.36 & 301.18 & & & $\mathrm{R}$ & $1,115.58$ & 558.29 & $1,098.55$ & 549.78 & $1,097.57$ & 549.29 & 10 \\
\hline 5 & 715.44 & 358.22 & 698.41 & 349.71 & & & $\mathrm{P}$ & 959.48 & 480.24 & 942.45 & 471.73 & 941.47 & 471.24 & 9 \\
\hline 6 & 802.47 & 401.74 & 785.44 & 393.22 & 784.46 & 392.73 & $\mathrm{~S}$ & 862.43 & 431.72 & 845.40 & 423.20 & 844.42 & 422.71 & 8 \\
\hline 7 & 859.49 & 430.25 & 842.46 & 421.74 & 841.48 & 421.24 & G & 775.39 & 388.20 & 758.37 & 379.69 & 757.38 & 379.20 & 7 \\
\hline 8 & 960.54 & 480.77 & 943.51 & 472.26 & 942.53 & 471.77 & $\mathrm{~T}$ & 718.37 & 359.69 & 701.35 & 351.18 & 700.36 & 350.68 & 6 \\
\hline 9 & $1,089.58$ & 545.29 & $1,072.55$ & 536.78 & $1,071.57$ & 536.29 & $E$ & 617.33 & 309.17 & 600.30 & 300.65 & 599.31 & 300.16 & 5 \\
\hline 10 & $1,204.61$ & 602.81 & $1,187.58$ & 594.29 & $1,186.60$ & 593.80 & $\mathrm{D}$ & 488.28 & 244.65 & 471.26 & 236.13 & 470.27 & 235.64 & 4 \\
\hline 11 & $1,303.68$ & 652.34 & $1,286.65$ & 643.83 & $1,285.67$ & 643.34 & $\mathrm{~V}$ & 373.26 & 187.13 & 356.23 & 178.62 & & & 3 \\
\hline 12 & $1,402.74$ & 701.88 & $1,385.72$ & 693.36 & $1,384.73$ & 692.87 & V & 274.19 & 137.60 & 257.16 & 129.08 & & & 2 \\
\hline 13 & & & & & & & $\mathrm{R}$ & 175.12 & 88.06 & 158.09 & 79.55 & & & 1 \\
\hline
\end{tabular}

Query 46054 Hit 1

MS/MS Fragmentation of EAFEEAGVLLLRPR

Found in sp|A8M XV4|NUD19_HUMAN, Nucleoside diphosphate-linked moiety X motif 19

Match to Query 46054: 1742.979from(582.0001,3+)

Title: 741: Sum of 2 scans in range $1674(\mathrm{rt}=47.0946, \mathrm{f}=4, \mathrm{r}=497)$ to $1675(\mathrm{rt}=47.12, \mathrm{f}=4, \mathrm{r}=498)$

[D:llab212\membranelGrace JoyceliTRAQ_35_1.raw]

Data File:Submitted from 20120508-1(merge) by Mascot Daemon on JOYCE-VAIO

Monoisotopic mass of neutral peptide Mr(calc): 1742.979

Variable modifications:

Ions Score: 45.41 Expect: 0.020 


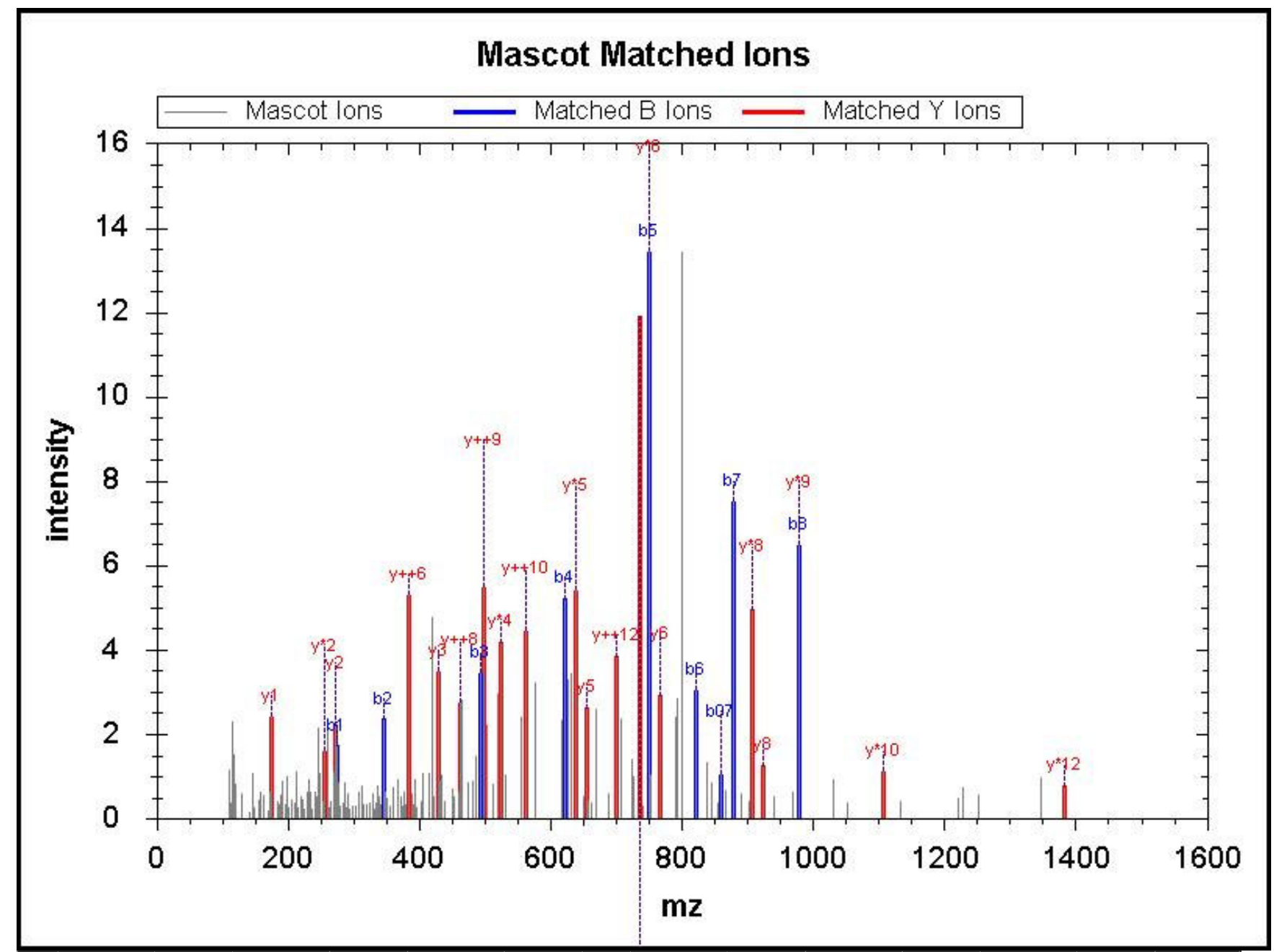

\begin{tabular}{|c|c|c|c|c|c|c|c|c|c|c|c|c|c|c|}
\hline No & $\mathrm{b}$ & $\mathrm{b}++$ & $b^{*}$ & $\mathrm{~b}^{*++}$ & b0 & $\mathrm{b} 0++$ & Seq & $\mathrm{y}$ & $\mathrm{y}^{++}$ & $y^{*}$ & $y^{*++}$ & y0 & $\mathrm{y} 0++$ & RevNo \\
\hline 1 & 274.15 & 137.58 & & & 256.14 & 128.57 & $\mathrm{E}$ & & & & & & & 14 \\
\hline 2 & 345.19 & 173.10 & & & 327.18 & 164.09 & A & $1,470.84$ & 735.93 & $1,453.82$ & 727.41 & $1,452.83$ & 726.92 & 13 \\
\hline 3 & 492.26 & 246.63 & & & 474.25 & 237.63 & $\mathrm{~F}$ & $1,399.81$ & 700.41 & $1,382.78$ & 691.89 & $1,381.80$ & 691.40 & 12 \\
\hline 4 & 621.30 & 311.15 & & & 603.29 & 302.15 & $\mathrm{E}$ & $1,252.74$ & 626.87 & $1,235.71$ & 618.36 & $1,234.73$ & 617.87 & 11 \\
\hline 5 & 750.34 & 375.67 & & & 732.33 & 366.67 & $\mathrm{E}$ & $1,123.69$ & 562.35 & $1,106.67$ & 553.84 & $1,105.68$ & 553.35 & 10 \\
\hline 6 & 821.38 & 411.19 & & & 803.37 & 402.19 & A & 994.65 & 497.83 & 977.63 & 489.32 & & & 9 \\
\hline 7 & 878.40 & 439.70 & & & 860.39 & 430.70 & $\mathrm{G}$ & 923.61 & 462.31 & 906.59 & 453.80 & & & 8 \\
\hline 8 & 977.47 & 489.24 & & & 959.46 & 480.23 & $\mathrm{~V}$ & 866.59 & 433.80 & 849.57 & 425.29 & & & 7 \\
\hline 9 & $1,090.55$ & 545.78 & & & $1,072.54$ & 536.78 & $\mathrm{~L}$ & 767.52 & 384.27 & 750.50 & 375.75 & & & 6 \\
\hline 10 & $1,203.64$ & 602.32 & & & $1,185.63$ & 593.32 & $\mathrm{~L}$ & 654.44 & 327.72 & 637.41 & 319.21 & & & 5 \\
\hline 11 & $1,316.72$ & 658.86 & & & $1,298.71$ & 649.86 & $\mathrm{~L}$ & 541.36 & 271.18 & 524.33 & 262.67 & & & 4 \\
\hline 12 & $1,472.82$ & 736.92 & $1,455.80$ & 728.40 & $1,454.81$ & 727.91 & $\mathrm{R}$ & 428.27 & 214.64 & 411.25 & 206.13 & & & 3 \\
\hline 13 & $1,569.88$ & 785.44 & $1,552.85$ & 776.93 & $1,551.87$ & \begin{tabular}{|l|}
776.44 \\
\end{tabular} & $\mathrm{P}$ & 272.17 & 136.59 & 255.15 & 128.08 & & & 2 \\
\hline 14 & & & & & & & $\mathrm{R}$ & 175.12 & 88.06 & 158.09 & 79.55 & & & 1 \\
\hline
\end{tabular}


MS/MS Fragmentation of EVAQQAVDADVHAVGVSTLAAGHK

Found in sp|P16332|MUTA_MOUSE, Methylmalonyl-CoA mutase

Match to Query 84451:2660.409from(666.1095,4+)

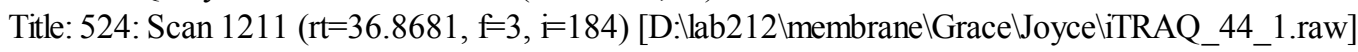

Data File:Submitted from 20120508-1(merge) by Mascot Daemon on JOYCE-VAIO

Monoisotopic mass of neutral peptide $\mathrm{Mr}$ (calc): 2660.409

Variable modifications:

K24 :iTRAQ4plex (K)

Ions Score: 136.01 Expect: 0.000

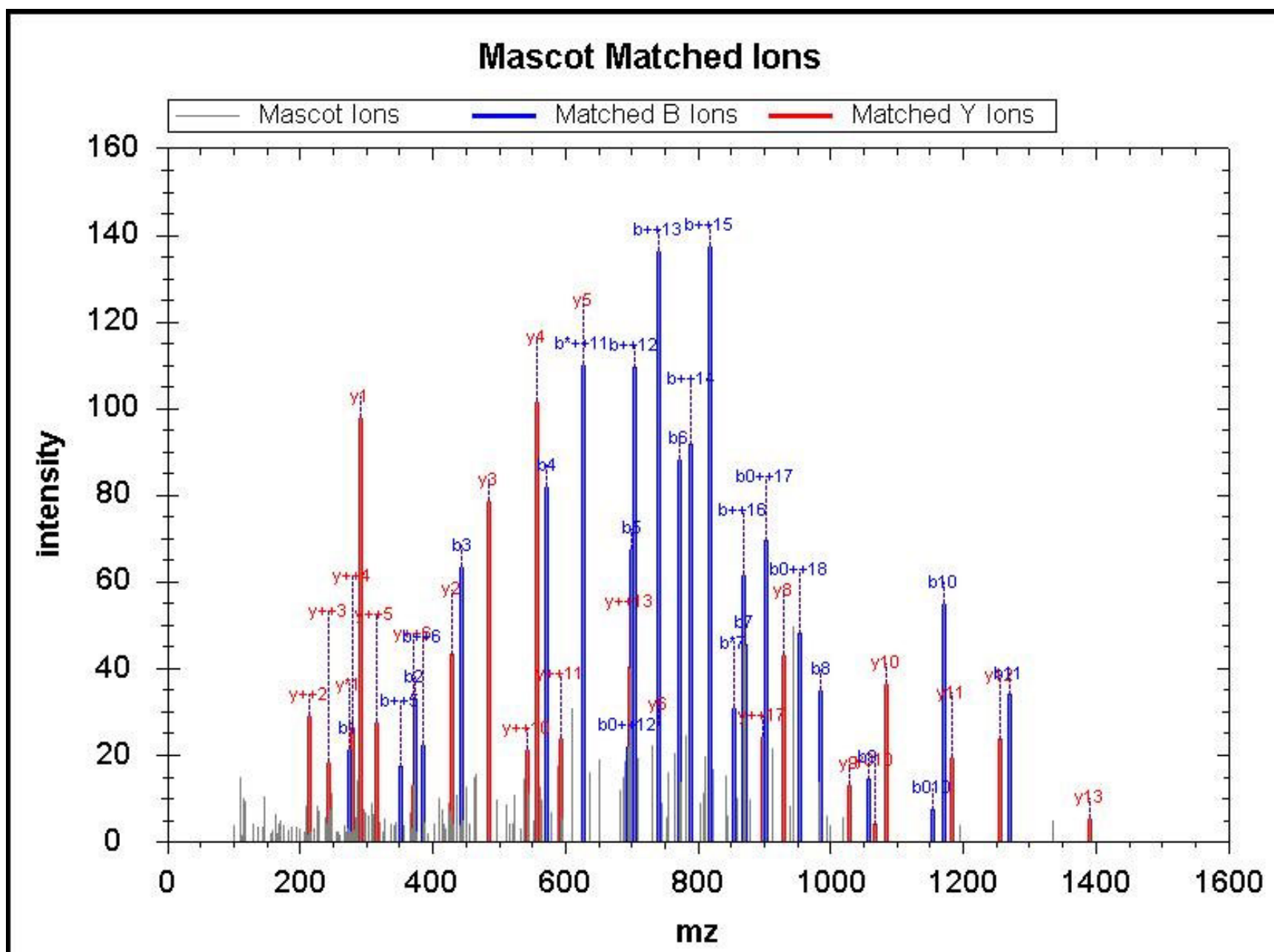

\begin{tabular}{|c|c|c|c|c|c|c|c|c|c|c|c|c|c|c|}
\hline No & $\mathrm{b}$ & $b++$ & $b^{*}$ & $b^{*++}$ & b0 & $\mathrm{b} 0++$ & Seq & $\mathrm{y}$ & $\mathrm{y}^{++}$ & $y^{*}$ & $y^{*++}$ & y0 & $\mathrm{y} 0++$ & RevNo \\
\hline 1 & 274.15 & 137.58 & & & 256.14 & 128.57 & $\mathrm{E}$ & & & & & & & 24 \\
\hline 2 & 373.22 & 187.11 & & & 355.21 & 178.11 & V & $2,388.27$ & $1,194.64$ & $2,371.25$ & $1,186.13$ & $2,370.26$ & $1,185.64$ & 23 \\
\hline 3 & 444.26 & 222.63 & & & 426.25 & 213.63 & A & $2,289.21$ & .11 & $2,272.18$ & $1,136.59$ & $2,271.20$ & $1,136.10$ & 22 \\
\hline 4 & 572.32 & 286.66 & 5 & 5 & 554.31 & 277.66 & Q & $2,218.17$ & .59 & $2,201.14$ & 1.08 & $2,200.16$ & 100.58 & 21 \\
\hline 5 & 700.37 & 350.69 & 683.35 & 342.18 & 682.36 & 341.69 & Q & $2,090.11$ & $1,045.56$ & $2,073.08$ & $1,037.05$ & $2,072.10$ & $1,036.55$ & 20 \\
\hline 6 & 771.41 & 386.21 & 754.39 & 377.70 & 753.40 & 377.20 & A & $1,962.05$ & 981.53 & $1,945.03$ & 973.02 & $1,944.04$ & 972.52 & 19 \\
\hline 7 & 870.48 & 435.74 & 853.45 & 427.23 & 852.47 & 426.74 & V & $1,891.02$ & 946.01 & $1,873.99$ & 937.50 & $1,873.00$ & 937.01 & 18 \\
\hline 8 & 985.51 & 493.26 & 968.48 & 484.74 & 967.50 & 484.25 & D & $1,791.95$ & 896.48 & $1,774.92$ & 887.96 & $1,773.94$ & 887.47 & 17 \\
\hline 9 & $1,056.54$ & 528.78 & $1,039.52$ & 520.26 & $1,038.53$ & 519.77 & A & $1,676.92$ & 838.96 & $1,659.89$ & 830.45 & $1,658.91$ & 829.96 & 16 \\
\hline 10 & $1,171.57$ & 586.29 & $1,154.54$ & 577.78 & $1,153.56$ & 577.28 & D & $1,605.88$ & 803.45 & $1,588.86$ & 794.93 & $1,587.87$ & 794.44 & 15 \\
\hline 11 & $1,270.64$ & 635.82 & $1,253.61$ & 627.31 & $1,252.63$ & 626.82 & V & $1,490.86$ & 745.93 & $1,473.83$ & 737.42 & $1,472.85$ & 736.93 & 14 \\
\hline 12 & $1,407.70$ & 704.35 & $1,390.67$ & 695.84 & $1,389.69$ & 695.35 & $\mathrm{H}$ & $1,391.79$ & 696.40 & $1,374.76$ & 687.88 & $1,373.78$ & 687.39 & 13 \\
\hline 13 & $1,478.74$ & 739.87 & $1,461.71$ & 731.36 & $1,460.73$ & 730.87 & A & $1,254.73$ & 627.87 & $1,237.70$ & 619.35 & $1,236.72$ & 618.86 & 12 \\
\hline 14 & $1,577.80$ & 789.41 & $1,560.78$ & 780.89 & $1,559.79$ & 780.40 & $\mathrm{~V}$ & $1,183.69$ & 592.35 & $1,166.66$ & 583.84 & $1,165.68$ & 583.34 & 11 \\
\hline 15 & $1,634.83$ & 817.92 & $|1,617.80|$ & 809.40 & $|1,616.81|$ & 808.91 & G & $1,084.62$ & 542.82 & $|1,067.60|$ & 534.30 & $|1,066.61|$ & 533.81 & 10 \\
\hline
\end{tabular}




\begin{tabular}{|r|r|r|r|r|r|r|r|r|r|r|r|r|r|r|}
\hline 16 & $1,733.89$ & 867.45 & $1,716.87$ & 858.94 & $1,715.88$ & 858.45 & $\mathrm{~V}$ & $1,027.60$ & 514.30 & $1,010.58$ & 505.79 & $1,009.59$ & 505.30 & 9 \\
\hline 17 & $1,820.93$ & 910.97 & $1,803.90$ & 902.45 & $1,802.92$ & 901.96 & $\mathrm{~S}$ & 928.53 & 464.77 & 911.51 & 456.26 & 910.52 & 455.76 & 8 \\
\hline 18 & $1,921.97$ & 961.49 & $1,904.95$ & 952.98 & $1,903.96$ & 952.49 & $\mathrm{~T}$ & 841.50 & 421.25 & 824.47 & 412.74 & 823.49 & 412.25 & 7 \\
\hline 19 & $2,035.06$ & $1,018.03$ & $2,018.03$ & $1,009.52$ & $2,017.05$ & $1,009.03$ & $\mathrm{~L}$ & 740.45 & 370.73 & 723.43 & 362.22 & & & 6 \\
\hline 20 & $2,106.09$ & $1,053.55$ & $2,089.07$ & $1,045.04$ & $2,088.08$ & $1,044.55$ & $\mathrm{~A}$ & 627.37 & 314.19 & 610.34 & 305.68 & & & 5 \\
\hline 21 & $2,177.13$ & $1,089.07$ & $2,160.11$ & $1,080.56$ & $2,159.12$ & $1,080.06$ & $\mathrm{~A}$ & 556.33 & 278.67 & 539.31 & 270.16 & & & 4 \\
\hline 22 & $2,234.15$ & $1,117.58$ & $2,217.13$ & $1,109.07$ & $2,216.14$ & $1,108.58$ & $\mathrm{G}$ & 485.30 & 243.15 & 468.27 & 234.64 & & & 3 \\
\hline 23 & $2,371.21$ & $1,186.11$ & $2,354.19$ & $1,177.60$ & $2,353.20$ & $1,177.10$ & $\mathrm{H}$ & 428.27 & 214.64 & 411.25 & 206.13 & & & 2 \\
\hline 24 & & & & & & & $\mathrm{~K}$ & 291.21 & 146.11 & 274.19 & 137.60 & & & 1 \\
\hline
\end{tabular}

Query 38682 Hit 1

MS/MS Fragmentation of DESTNVDMSLAQR

Found in sp|P50995|ANX11_HUM AN, Annexin A11 OS=Homo sapiens GN=ANXA11 PE=1 SV=1

Match to Query 38682: 1608.755 from(805.3846,2+)

Title: 163: Scan 930 ( $\mathrm{rt}=28.0072, \mathrm{f}=3, \mathrm{i}=57)$ [D:llab212 $\backslash$ membranelGrace JoyceliTRAQ_22_2.raw]

Data File:Submitted from 20120508-1(merge) by Mascot Daemon on JOYCE-VAIO

Monoisotopic mass of neutral peptide $\mathrm{Mr}$ (calc): 1608.755

Variable modifications:

Ions Score: 106.56 Expect: 0.000

\section{Mascot Matched lons}

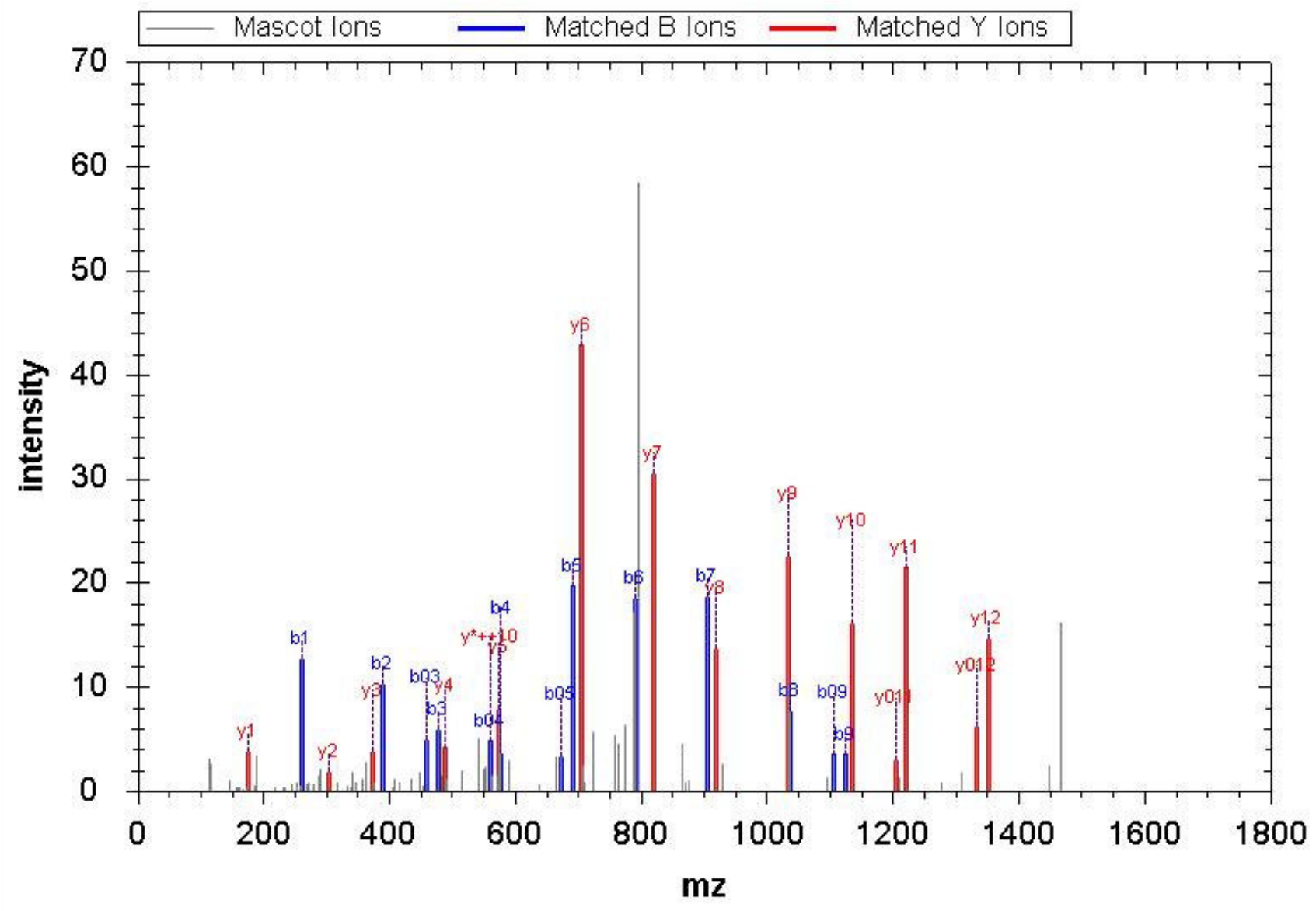

\begin{tabular}{|r|c|c|c|c|c|c|c|c|c|c|c|r|r|r|}
\hline \hline No & $\mathrm{b}$ & $\mathrm{b}++$ & $\mathrm{b}^{*}$ & $\mathrm{~b} *++$ & $\mathrm{b} 0$ & $\mathrm{~b} 0++$ & Seq & $\mathrm{y}$ & $\mathrm{y}++$ & $\mathrm{y}^{*}$ & $\mathrm{y}^{*++}$ & $\mathrm{y} 0$ & $\mathrm{y} 0++$ & $\mathrm{RevNo}$ \\
\hline 1 & 260.14 & 130.57 & & & 242.13 & 121.57 & $\mathrm{D}$ & & & & & & & 13 \\
\hline 2 & 389.18 & 195.09 & & & 371.17 & 186.09 & $\mathrm{E}$ & $1,350.63$ & 675.82 & $1,333.61$ & 667.31 & $1,332.62$ & 666.81 & 12 \\
\hline 3 & 476.21 & 238.61 & & & 458.20 & 229.60 & $\mathrm{~S}$ & $1,221.59$ & 611.30 & $1,204.56$ & 602.78 & $1,203.58$ & 602.29 & 11 \\
\hline 4 & 577.26 & 289.13 & & & 559.25 & 280.13 & $\mathrm{~T}$ & $1,134.56$ & 567.78 & $1,117.53$ & 559.27 & $1,116.55$ & 558.78 & 10 \\
\hline 5 & 691.30 & 346.15 & 674.27 & 337.64 & 673.29 & 337.15 & $\mathrm{~N}$ & $1,033.51$ & 517.26 & $1,016.48$ & 508.75 & $1,015.50$ & 508.25 & 9 \\
\hline 6 & 790.37 & 395.69 & 773.34 & 387.18 & 772.36 & 386.68 & $\mathrm{~V}$ & 919.47 & 460.24 & 902.44 & 451.72 & 901.46 & 451.23 & 8
\end{tabular}




\begin{tabular}{|r|r|r|r|r|r|r|r|r|r|r|r|r|r|r|}
\hline 7 & 905.40 & 453.20 & 888.37 & 444.69 & 887.39 & 444.20 & $\mathrm{D}$ & 820.40 & 410.70 & 803.37 & 402.19 & 802.39 & 401.70 & 7 \\
\hline 8 & $1,036.44$ & 518.72 & $1,019.41$ & 510.21 & $1,018.43$ & 509.72 & $\mathrm{M}$ & 705.37 & 353.19 & 688.34 & 344.68 & 687.36 & 344.18 & 6 \\
\hline 9 & $1,123.47$ & 562.24 & $1,106.44$ & 553.73 & $1,105.46$ & 553.23 & $\mathrm{~S}$ & 574.33 & 287.67 & 557.30 & 279.16 & 556.32 & 278.66 & 5 \\
\hline 10 & $1,236.55$ & 618.78 & $1,219.53$ & 610.27 & $1,218.54$ & 609.78 & $\mathrm{~L}$ & 487.30 & 244.15 & 470.27 & 235.64 & & & 4 \\
\hline 11 & $1,307.59$ & 654.30 & $1,290.56$ & 645.79 & $1,289.58$ & 645.29 & $\mathrm{~A}$ & 374.21 & 187.61 & 357.19 & 179.10 & & & 3 \\
\hline 12 & $1,435.65$ & 718.33 & $1,418.62$ & 709.82 & $1,417.64$ & 709.32 & $\mathrm{Q}$ & 303.18 & 152.09 & 286.15 & 143.58 & & & 2 \\
\hline 13 & & & & & & & $\mathrm{R}$ & 175.12 & 88.06 & 158.09 & 79.55 & & & 1 \\
\hline
\end{tabular}

Query 85745 Hit 1

MS/MS Fragmentation of IHSEVVEDTEAVSAVQQLLDDER

Found in sp|Q96GA7|SDSL_HUMAN, Serine dehydratase-like OS=Homo sapiens GN=SDSL PE=1 SV=1

Match to Query 85745: 2725.35from(909.4572,3+)

Title: 908: Scan $2000(\mathrm{rt}=54.5787, \mathrm{f}=2, \mathrm{i}=316)$ [D:lab212 \membranelGrace JoyceliTRAQ_34_2.raw]

Data File:Submitted from 20120508-1(merge) by Mascot Daemon on JOYCE-VAIO

Monoisotopic mass of neutral peptide $\mathrm{Mr}$ (calc): 2725.35

Variable modifications:

Ions Score: 105.8 Expect: 0.000

\section{Mascot Matched lons}

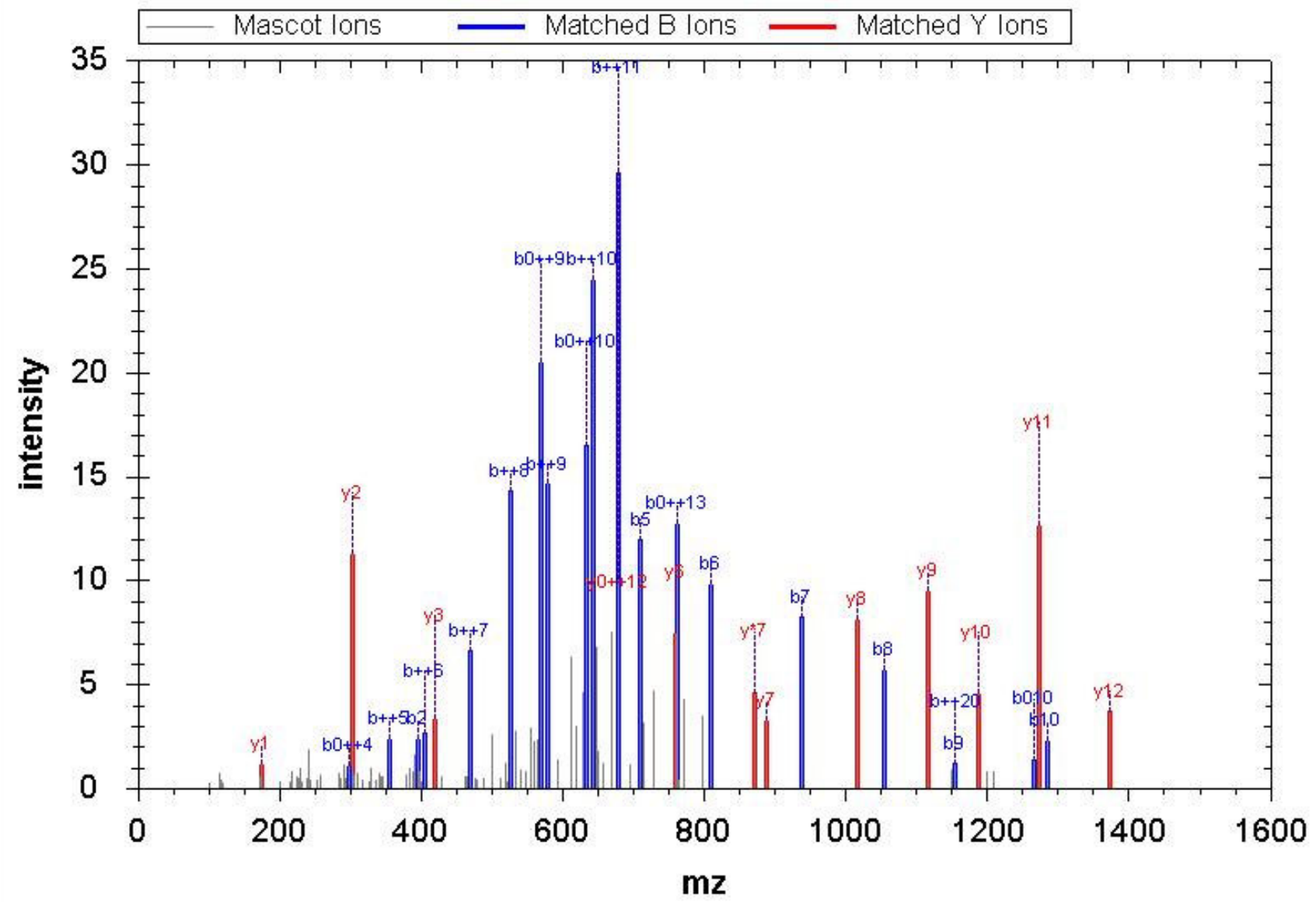

\begin{tabular}{|c|c|c|c|c|c|c|c|c|c|c|c|c|c|c|}
\hline No & $\mathrm{b}$ & $\mathrm{b}++$ & $b^{*}$ & $b^{*++}$ & b0 & $\mathrm{b} 0++$ & Seq & $\mathrm{y}$ & $\mathrm{y}^{++}$ & $y^{*}$ & $y^{*++}$ & y0 & $\mathrm{y} 0++$ & RevNo \\
\hline 1 & 258.19 & 129.60 & & & & & I & & & & & & & 23 \\
\hline 2 & 395.25 & 198.13 & & & & & $\mathrm{H}$ & $2,469.17$ & $1,235.09$ & $2,452.15$ & $1,226.58$ & $2,451.16$ & $1,226.09$ & 22 \\
\hline 3 & 482.28 & 241.65 & & & 464.27 & 232.64 & $\mathrm{~S}$ & $2,332.11$ & $1,166.56$ & $2,315.09$ & $1,158.05$ & $2,314.10$ & $1,157.56$ & 21 \\
\hline 4 & 611.33 & 306.17 & & & 593.32 & 297.16 & E & $2,245.08$ & $1,123.05$ & $2,228.06$ & $1,114.53$ & $2,227.07$ & $1,114.04$ & 20 \\
\hline 5 & 710.40 & 355.70 & & & 692.38 & 346.70 & V & $2,116.04$ & $1,058.52$ & $2,099.01$ & $1,050.01$ & $2,098.03$ & $1,049.52$ & 19 \\
\hline 6 & 809.46 & 405.24 & & & 791.45 & 396.23 & V & $2,016.97$ & $1,008.99$ & $1,999.95$ & $1,000.48$ & $1,998.96$ & 999.98 & 18 \\
\hline 7 & 938.51 & 469.76 & & & 920.50 & 460.75 & $\mathrm{E}$ & $1,917.90$ & 959.46 & $1,900.88$ & 950.94 & $1,899.89$ & 950.45 & 17 \\
\hline 8 & $1,053.53$ & 527.27 & & & $1,035.52$ & 518.27 & $\mathrm{D}$ & $1,788.86$ & 894.93 & $1,771.83$ & 886.42 & $1,770.85$ & 885.93 & 16 \\
\hline
\end{tabular}




\begin{tabular}{|r|r|r|l|r|r|r|r|r|r|r|r|r|r|r|}
\hline 9 & $1,154.58$ & 577.79 & & & $1,136.57$ & 568.79 & $\mathrm{~T}$ & $1,673.83$ & 837.42 & $1,656.81$ & 828.91 & $1,655.82$ & 828.42 & 15 \\
\hline 10 & $1,283.62$ & 642.32 & & & $1,265.61$ & 633.31 & $\mathrm{E}$ & $1,572.79$ & 786.90 & $1,555.76$ & 778.38 & $1,554.78$ & 777.89 & 14 \\
\hline 11 & $1,354.66$ & 677.83 & & & $1,336.65$ & 668.83 & $\mathrm{~A}$ & $1,443.74$ & 722.38 & $1,426.72$ & 713.86 & $1,425.73$ & 713.37 & 13 \\
\hline 12 & $1,453.73$ & 727.37 & & & $1,435.72$ & 718.36 & $\mathrm{~V}$ & $1,372.71$ & 686.86 & $1,355.68$ & 678.34 & $1,354.70$ & 677.85 & 12 \\
\hline 13 & $1,540.76$ & 770.88 & & & $1,522.75$ & 761.88 & $\mathrm{~S}$ & $1,273.64$ & 637.32 & $1,256.61$ & 628.81 & $1,255.63$ & 628.32 & 11 \\
\hline 14 & $1,611.80$ & 806.40 & & & $1,593.79$ & 797.40 & $\mathrm{~A}$ & $1,186.61$ & 593.81 & $1,169.58$ & 585.29 & $1,168.60$ & 584.80 & 10 \\
\hline 15 & $1,710.87$ & 855.94 & & & $1,692.86$ & 846.93 & $\mathrm{~V}$ & $1,115.57$ & 558.29 & $1,098.54$ & 549.77 & $1,097.56$ & 549.28 & 9 \\
\hline 16 & $1,838.93$ & 919.97 & $1,821.90$ & 911.45 & $1,820.91$ & 910.96 & $\mathrm{Q}$ & $1,016.50$ & 508.75 & 999.47 & 500.24 & 998.49 & 499.75 & 8 \\
\hline 17 & $1,966.98$ & 984.00 & $1,949.96$ & 975.48 & $1,948.97$ & 974.99 & $\mathrm{Q}$ & 888.44 & 444.72 & 871.42 & 436.21 & 870.43 & 435.72 & 7 \\
\hline 18 & $2,080.07$ & $1,040.54$ & $2,063.04$ & $1,032.02$ & $2,062.06$ & $1,031.53$ & $\mathrm{~L}$ & 760.38 & 380.70 & 743.36 & 372.18 & 742.37 & 371.69 & 6 \\
\hline 19 & $2,193.15$ & $1,097.08$ & $2,176.13$ & $1,088.57$ & $2,175.14$ & $1,088.07$ & $\mathrm{~L}$ & 647.30 & 324.15 & 630.27 & 315.64 & 629.29 & 315.15 & 5 \\
\hline 20 & $2,308.18$ & $1,154.59$ & $2,291.15$ & $1,146.08$ & $2,290.17$ & $1,145.59$ & $\mathrm{D}$ & 534.22 & 267.61 & 517.19 & 259.10 & 516.20 & 258.61 & 4 \\
\hline 21 & $2,423.21$ & $1,212.11$ & $2,406.18$ & $1,203.59$ & $2,405.20$ & $1,203.10$ & $\mathrm{D}$ & 419.19 & 210.10 & 402.16 & 201.58 & 401.18 & 201.09 & 3 \\
\hline 22 & $2,552.25$ & $1,276.63$ & $2,535.22$ & $1,268.11$ & $2,534.24$ & $1,267.62$ & $\mathrm{E}$ & 304.16 & 152.58 & 287.13 & 144.07 & 286.15 & 143.58 & 2 \\
\hline 23 & & & & & & & $\mathrm{R}$ & 175.12 & 88.06 & 158.09 & 79.55 & & & 1 \\
\hline
\end{tabular}

Query 53024 Hit 1

MS/MS Fragmentation of SELEEQLTPVAEETR

Found in sp|P02649|APOE_HUM AN, Apolipoprotein E OS=Homo sapiens GN=APOE PE=1 SV=1

Match to Query 53024: 1873.943 from(937.9787,2+)

Title: 198: Sum of 2 scans in range $1367(\mathrm{rt}=37.0315, \mathrm{f}=4, \mathrm{i}=160)$ to $1368(\mathrm{rt}=37.0569, \mathrm{f}=4, \mathrm{i}=161)$

[D:lab212 \membranelGracelJoyceliTRAQ_19_2_330.raw]

Data File:Submitted from 20120508-1(merge) by Mascot Daemon on JOYCE-VAIO

Monoisotopic mass of neutral peptide $\operatorname{Mr}($ calc): 1873.943

Variable modifications:

Ions Score: 103.35 Expect: 0.000

Mascot Matched lons

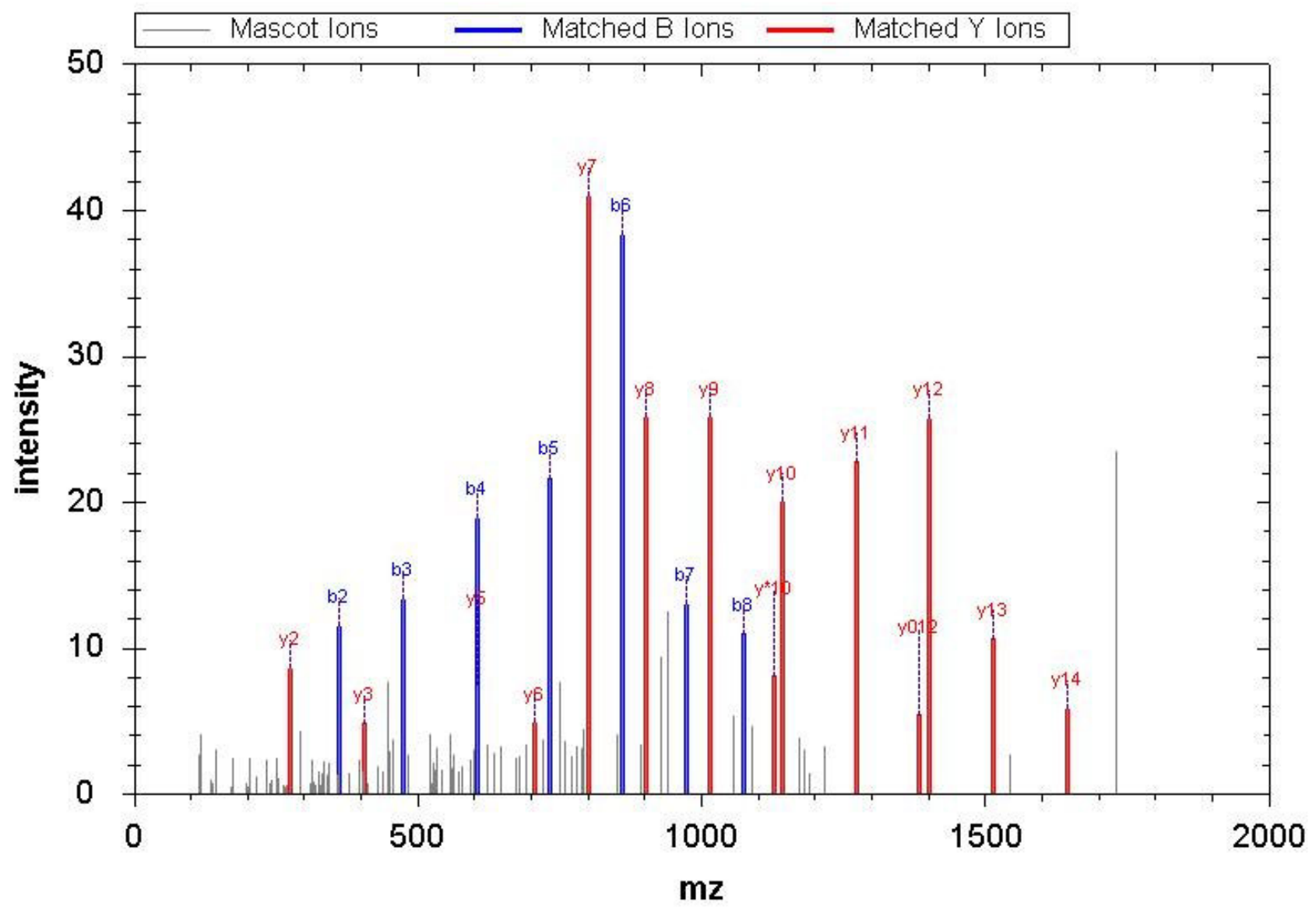




\begin{tabular}{|c|c|c|c|c|c|c|c|c|c|c|c|c|c|c|}
\hline No & $\mathrm{b}$ & $\mathrm{b}++$ & $b^{*}$ & $b^{*++}$ & b0 & $\mathrm{b} 0++$ & Seq & $\mathrm{y}$ & $\mathrm{y}^{++}$ & $y^{*}$ & $\mathrm{y}^{*++}$ & y0 & $\mathrm{y} 0++$ & RevNo \\
\hline 1 & 232.14 & 116.57 & & & 214.13 & 107.57 & $\mathrm{~S}$ & & & & & & & 15 \\
\hline 2 & 361.18 & 181.10 & & & 343.17 & 172.09 & $\mathrm{E}$ & $1,643.81$ & 822.41 & $1,626.79$ & 813.90 & $1,625.80$ & 813.40 & 14 \\
\hline 3 & 474.27 & 237.64 & & & 456.26 & 228.63 & $\mathrm{~L}$ & $1,514.77$ & 757.89 & $1,497.74$ & 749.38 & $1,496.76$ & 748.88 & 13 \\
\hline 4 & 603.31 & 302.16 & & & 585.30 & 293.15 & $\mathrm{E}$ & $1,401.69$ & 701.35 & $1,384.66$ & 692.83 & $1,383.67$ & 692.34 & 12 \\
\hline 5 & 732.35 & 366.68 & & & 714.34 & 357.67 & $\mathrm{E}$ & $1,272.64$ & 636.83 & $1,255.62$ & 628.31 & $1,254.63$ & 627.82 & 11 \\
\hline 6 & 860.41 & 430.71 & 843.39 & 422.20 & 842.40 & 421.70 & Q & $1,143.60$ & 572.30 & $1,126.57$ & 563.79 & $1,125.59$ & 563.30 & 10 \\
\hline 7 & 973.50 & 487.25 & 956.47 & 478.74 & 955.49 & 478.25 & $\mathrm{~L}$ & $1,015.54$ & 508.27 & 998.52 & 499.76 & 997.53 & 499.27 & 9 \\
\hline 8 & $1,074.54$ & 537.78 & $1,057.52$ & 529.26 & $1,056.53$ & 528.77 & $\mathrm{~T}$ & 902.46 & 451.73 & 885.43 & 443.22 & 884.45 & 442.73 & 8 \\
\hline 9 & $1,171.60$ & 586.30 & $1,154.57$ & 577.79 & $1,153.59$ & 577.30 & $\mathrm{P}$ & 801.41 & 401.21 & 784.38 & 392.70 & 783.40 & 392.20 & 7 \\
\hline 10 & $1,270.66$ & 635.84 & $1,253.64$ & 627.32 & $1,252.65$ & 626.83 & V & 704.36 & 352.68 & 687.33 & 344.17 & 686.35 & 343.68 & 6 \\
\hline 11 & $1,341.70$ & 671.35 & $1,324.68$ & 662.84 & $1,323.69$ & 662.35 & A & 605.29 & 303.15 & 588.26 & 294.63 & 587.28 & 294.14 & 5 \\
\hline 12 & $1,470.74$ & 735.88 & $1,453.72$ & 727.36 & $1,452.73$ & 726.87 & $\mathrm{E}$ & 534.25 & 267.63 & 517.23 & 259.12 & 516.24 & 258.62 & 4 \\
\hline 13 & $1,599.79$ & 800.40 & $1,582.76$ & 791.88 & $1,581.78$ & 791.39 & $\mathrm{E}$ & 405.21 & 203.11 & 388.18 & 194.59 & 387.20 & 194.10 & 3 \\
\hline 14 & $1,700.83$ & 850.92 & $1,683.81$ & 842.41 & $1,682.82$ & 841.92 & $\mathrm{~T}$ & 276.17 & 138.59 & 259.14 & 130.07 & 258.16 & 129.58 & 2 \\
\hline 15 & & & & & & & $\mathrm{R}$ & 175.12 & 88.06 & 158.09 & 79.55 & & & 1 \\
\hline
\end{tabular}

Query 32852 Hit 1

MS/MS Fragmentation of TLGDQLSLLLGAR

Found in sp|Q9UBG0|MRC2_HUMAN, C-type mannose receptor 2 OS=Homo sapiens GN=MRC2 PE=1 SV=2

Match to Query 32852: 1499.875from(750.9446,2+)

Title: 972: Sum of 2 scans in range 2227 ( $\mathrm{rt}=59.2248, \mathrm{f}=4, \mathrm{i}=633$ ) to 2228 ( $\mathrm{rt}=59.2503, \mathrm{f}=4, \mathrm{i}=634$ )

[D:llab212\membranelGrace JoyceliTRAQ_23_1.raw]

Data File:Submitted from 20120508-1(merge) by Mascot Daemon on JOYCE-VAIO

Monoisotopic mass of neutral peptide $\mathrm{Mr}$ (calc): 1499.875

Variable modifications:

Ions Score: 98.7 Expect: 0.000 


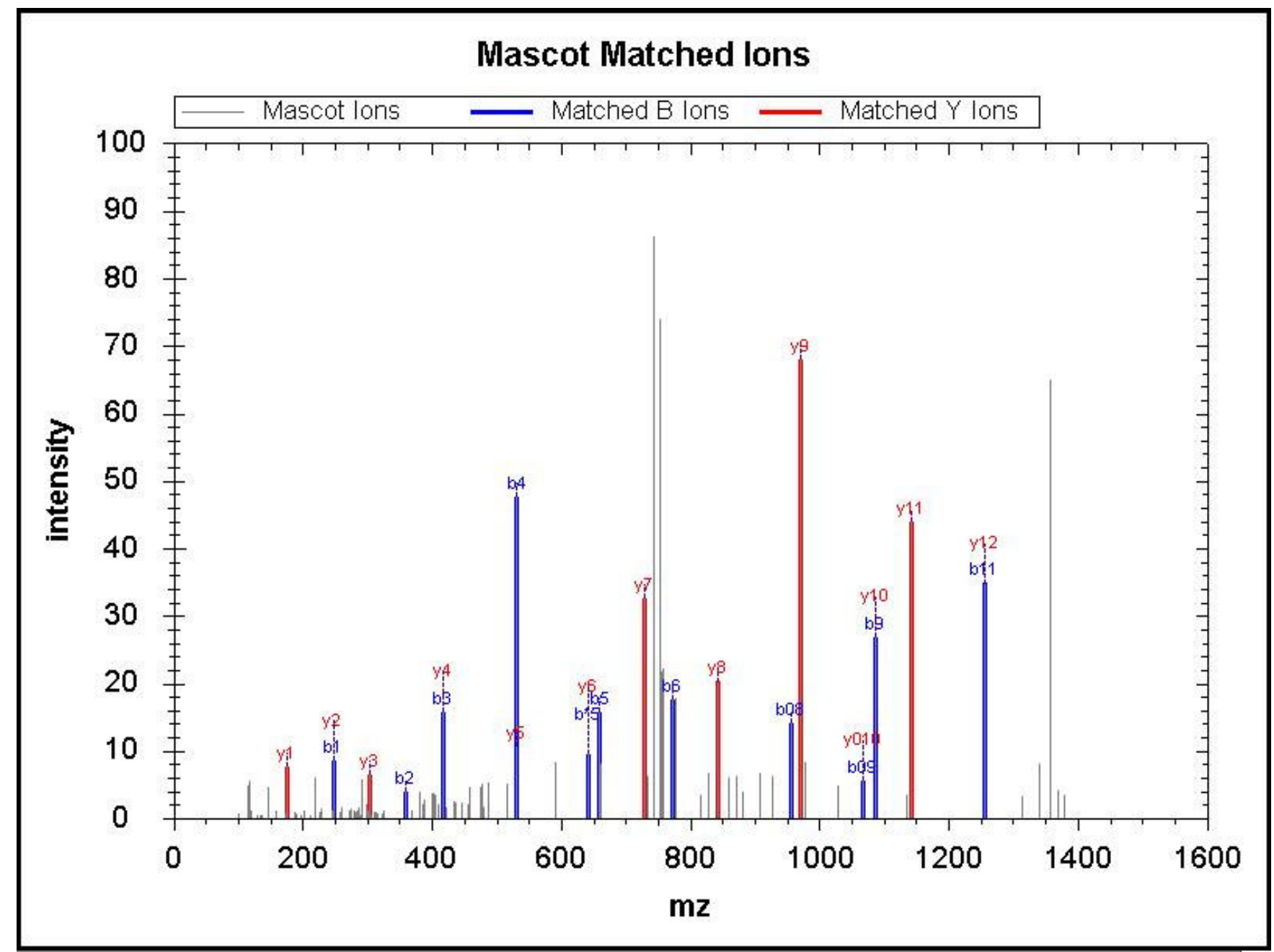

\begin{tabular}{|c|c|c|c|c|c|c|c|c|c|c|c|c|c|c|}
\hline No & $\mathrm{b}$ & $\mathrm{b}++$ & $b^{*}$ & $b^{*++}$ & b0 & $\mathrm{b} 0++$ & Seq & $\mathrm{y}$ & $\mathrm{y}++$ & $y^{*}$ & $y^{*++}$ & y0 & $\mathrm{y} 0++$ & RevNo \\
\hline 1 & 246.16 & 123.58 & & & 228.15 & 114.58 & $\mathrm{~T}$ & & & & & & & 13 \\
\hline 2 & 359.24 & 180.12 & & & 341.23 & 171.12 & $\mathrm{~L}$ & $1,255.74$ & 628.37 & $1,238.71$ & 619.86 & $1,237.73$ & 619.37 & 12 \\
\hline 3 & 416.26 & 208.63 & & & 398.25 & 199.63 & G & $1,142.65$ & 571.83 & $1,125.63$ & 563.32 & $1,124.64$ & 562.82 & 11 \\
\hline 4 & 531.29 & 266.15 & & & 513.28 & 257.14 & $\mathrm{D}$ & $1,085.63$ & 543.32 & $1,068.60$ & 534.81 & $1,067.62$ & 534.31 & 10 \\
\hline 5 & 659.35 & 330.18 & 642.32 & 321.66 & 641.34 & 321.17 & Q & 970.60 & 485.81 & 953.58 & 477.29 & 952.59 & 476.80 & 9 \\
\hline 6 & 772.43 & 386.72 & 755.41 & 378.21 & 754.42 & 377.71 & $\mathrm{~L}$ & 842.55 & 421.78 & 825.52 & 413.26 & 824.54 & 412.77 & 8 \\
\hline 7 & 859.46 & 430.24 & 842.44 & 421.72 & 841.45 & 421.23 & $\mathrm{~S}$ & 729.46 & 365.23 & 712.44 & 356.72 & 711.45 & 356.23 & 7 \\
\hline 8 & 972.55 & 486.78 & 955.52 & 478.26 & 954.54 & 477.77 & $\mathrm{~L}$ & 642.43 & 321.72 & 625.40 & 313.21 & & & 6 \\
\hline 9 & $1,085.63$ & 543.32 & $1,068.61$ & 534.81 & $1,067.62$ & 534.31 & $\mathrm{~L}$ & 529.35 & 265.18 & 512.32 & 256.66 & & & 5 \\
\hline 10 & $1,198.72$ & 599.86 & $1,181.69$ & 591.35 & $1,180.71$ & 590.86 & $\mathrm{~L}$ & 416.26 & 208.63 & 399.24 & 200.12 & & & 4 \\
\hline 11 & $1,255.74$ & 628.37 & $1,238.71$ & 619.86 & $1,237.73$ & 619.37 & G & 303.18 & 152.09 & 286.15 & 143.58 & & & 3 \\
\hline 12 & $1,326.78$ & 663.89 & $1,309.75$ & 655.38 & $1,308.76$ & 654.89 & A & 246.16 & 123.58 & 229.13 & 115.07 & & & 2 \\
\hline 13 & & & & & & & $\mathrm{R}$ & 175.12 & 88.06 & 158.09 & 79.55 & & & 1 \\
\hline
\end{tabular}

Query 82496 Hit 1

MS/MS Fragmentation of LVVTQEDVELAYQEAMMNMAR

Found in sp|P49903|SPS1 HUMAN, Selenide

Match to Query 82496: 2584.254from(862.4254,3+)

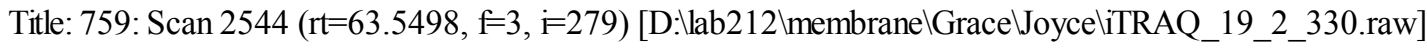

Data File:Submitted from 20120508-1(merge) by Mascot Daemon on JOYCE-VAIO

Monoisotopic mass of neutral peptide $\mathrm{Mr}$ (calc): 2584.254

Variable modifications:

Ions Score: 95.44 Expect: 0.000 


\section{Mascot Matched Ions}

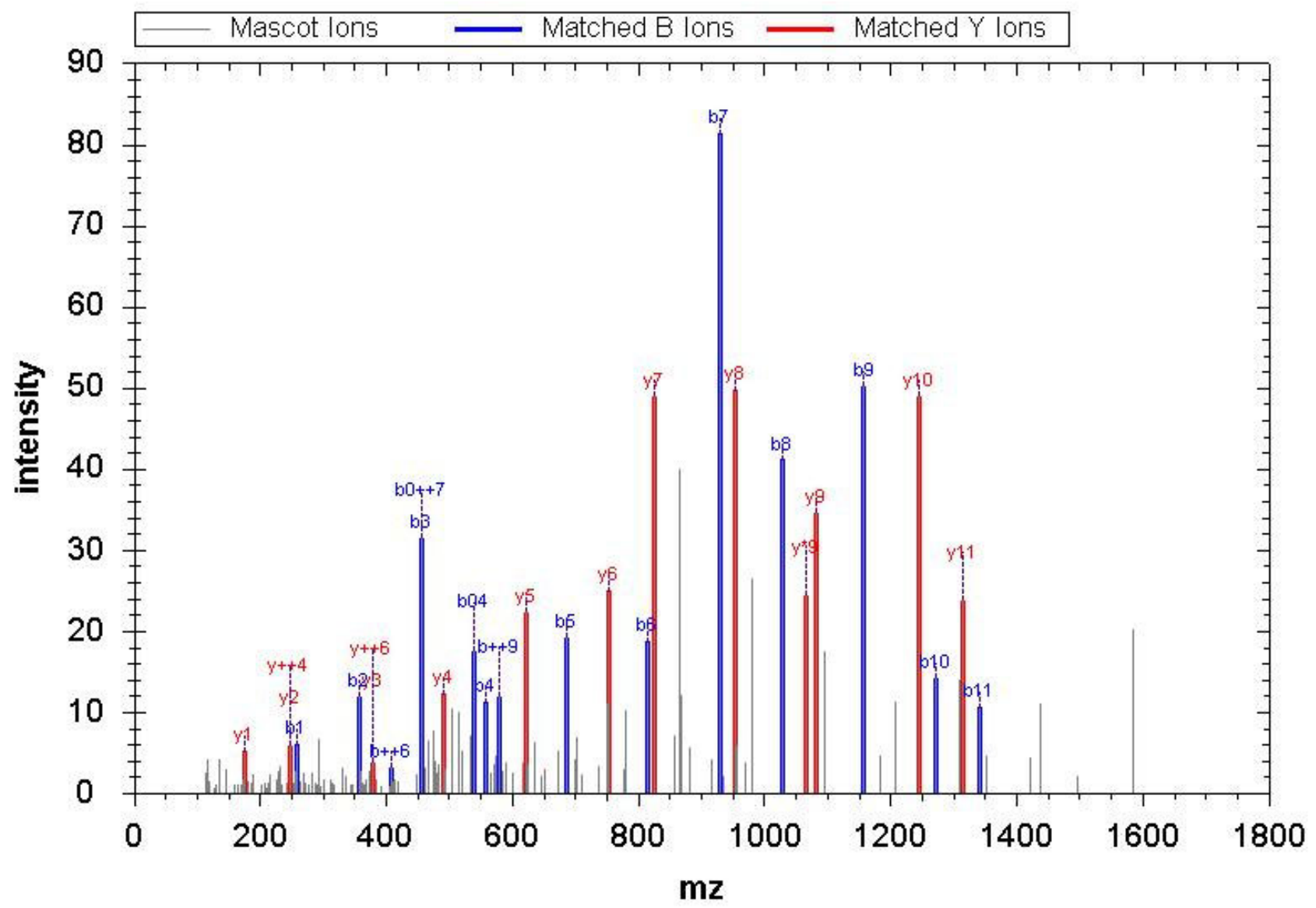

\begin{tabular}{|c|c|c|c|c|c|c|c|c|c|c|c|c|c|c|}
\hline No & $\mathrm{b}$ & $\mathrm{b}++$ & $b^{*}$ & $b^{*++}$ & b0 & $\mathrm{b} 0++$ & Seq & $\mathrm{y}$ & $\mathrm{y}++$ & $\mathrm{y}^{*}$ & $\mathrm{y}^{*++}$ & y0 & $\mathrm{y} 0++$ & RevNo \\
\hline 1 & 258.19 & 129.60 & & & & & $\mathrm{~L}$ & & & & & & & 21 \\
\hline 2 & 357.26 & 179.13 & & & & & V & $2,328.07$ & $1,164.54$ & $2,311.04$ & $1,156.02$ & $2,310.06$ & $1,155.53$ & 20 \\
\hline 3 & 456.33 & 228.67 & & & & & V & $2,229.00$ & $1,115.00$ & $2,211.97$ & $1,106.49$ & $2,210.99$ & $1,106.00$ & 19 \\
\hline 4 & 557.38 & 279.19 & & & 539.37 & 270.19 & $\mathrm{~T}$ & $2,129.93$ & $1,065.47$ & $2,112.90$ & $1,056.96$ & $2,111.92$ & $1,056.46$ & 18 \\
\hline 5 & 685.44 & 343.22 & 668.41 & 334.71 & 667.43 & 334.22 & Q & $2,028.88$ & $1,014.94$ & $2,011.86$ & $1,006.43$ & $2,010.87$ & $1,005.94$ & 17 \\
\hline 6 & 814.48 & 407.74 & 797.45 & 399.23 & 796.47 & 398.74 & $\mathrm{E}$ & $1,900.82$ & 950.92 & $1,883.80$ & 942.40 & $1,882.81$ & 941.91 & 16 \\
\hline 7 & 929.51 & 465.26 & 912.48 & 456.74 & 911.50 & 456.25 & $\mathrm{D}$ & $1,771.78$ & 886.39 & $1,754.75$ & 877.88 & $1,753.77$ & 877.39 & 15 \\
\hline 8 & $1,028.57$ & 514.79 & $1,011.55$ & 506.28 & $1,010.56$ & 505.79 & V & $1,656.75$ & 828.88 & $1,639.73$ & 820.37 & $1,638.74$ & 819.88 & 14 \\
\hline 9 & $1,157.62$ & 579.31 & $1,140.59$ & 570.80 & $1,139.61$ & 570.31 & $\mathrm{E}$ & $1,557.69$ & 779.35 & $1,540.66$ & 770.83 & $1,539.68$ & 770.34 & 13 \\
\hline 10 & $1,270.70$ & 635.85 & $1,253.67$ & 627.34 & $1,252.69$ & 626.85 & L & $1,428.64$ & 714.83 & $1,411.62$ & 706.31 & $1,410.63$ & 705.82 & 12 \\
\hline 11 & $1,341.74$ & 671.37 & $1,324.71$ & 662.86 & $1,323.73$ & 662.37 & A & $1,315.56$ & 658.28 & $1,298.53$ & 649.77 & $1,297.55$ & 649.28 & 11 \\
\hline 12 & $1,504.80$ & 752.90 & $1,487.78$ & 744.39 & $1,486.79$ & 743.90 & Y & $1,244.52$ & 622.76 & $1,227.50$ & 614.25 & $1,226.51$ & 613.76 & 10 \\
\hline 13 & $1,632.86$ & 816.93 & $1,615.83$ & 808.42 & $1,614.85$ & 807.93 & Q & $1,081.46$ & 541.23 & $1,064.43$ & 532.72 & $1,063.45$ & 532.23 & 9 \\
\hline 14 & $1,761.90$ & 881.46 & $1,744.88$ & 872.94 & $1,743.89$ & 872.45 & $\mathrm{E}$ & 953.40 & 477.20 & 936.37 & 468.69 & 935.39 & 468.20 & 8 \\
\hline 15 & $1,832.94$ & 916.97 & $1,815.91$ & 908.46 & $1,814.93$ & 907.97 & A & 824.36 & 412.68 & 807.33 & 404.17 & & & 7 \\
\hline 16 & $1,963.98$ & 982.49 & $1,946.95$ & 973.98 & $1,945.97$ & 973.49 & M & 753.32 & 377.16 & 736.29 & 368.65 & & & 6 \\
\hline 17 & $2,095.02$ & $1,048.01$ & $2,077.99$ & $1,039.50$ & $2,077.01$ & $1,039.01$ & M & 622.28 & 311.64 & 605.25 & 303.13 & & & 5 \\
\hline 18 & $2,209.06$ & $1,105.04$ & $2,192.04$ & $1,096.52$ & $2,191.05$ & $1,096.03$ & $\mathrm{~N}$ & 491.24 & 246.12 & 474.21 & 237.61 & & & 4 \\
\hline 19 & $2,340.10$ & $1,170.56$ & $2,323.08$ & $1,162.04$ & $2,322.09$ & $1,161.55$ & $\mathrm{M}$ & 377.20 & 189.10 & 360.17 & 180.59 & & & 3 \\
\hline 20 & $2,411.14$ & $1,206.07$ & $2,394.11$ & $1,197.56$ & $2,393.13$ & $1,197.07$ & A & 246.16 & 123.58 & 229.13 & 115.07 & & & 2 \\
\hline 21 & & & & & & & $\mathrm{R}$ & 175.12 & 88.06 & 158.09 & 79.55 & & & 1 \\
\hline
\end{tabular}

Query 37987 Hit 1 
Found in sp|P33240|CSTF2_HUMAN, Cleavage stimulation factor subunit 2 OS=Homo sapiens GN=CSTF2 PE=1 SV=1 Match to Query 37987: 1594.846 from(798.4301,2+)

Title: 843: Scan $2012(\mathrm{rt}=54.5108, \mathrm{f}=3, \mathrm{i}=304)$ [D:lab212 \membranelGrace JoyceliTRAQ_21_2.raw]

Data File:Submitted from 20120508-1(merge) by Mascot Daemon on JOYCE-VAIO

Monoisotopic mass of neutral peptide $\mathrm{Mr}$ (calc): 1594.846

Variable modifications:

Ions Score: 92.95 Expect: 0.000

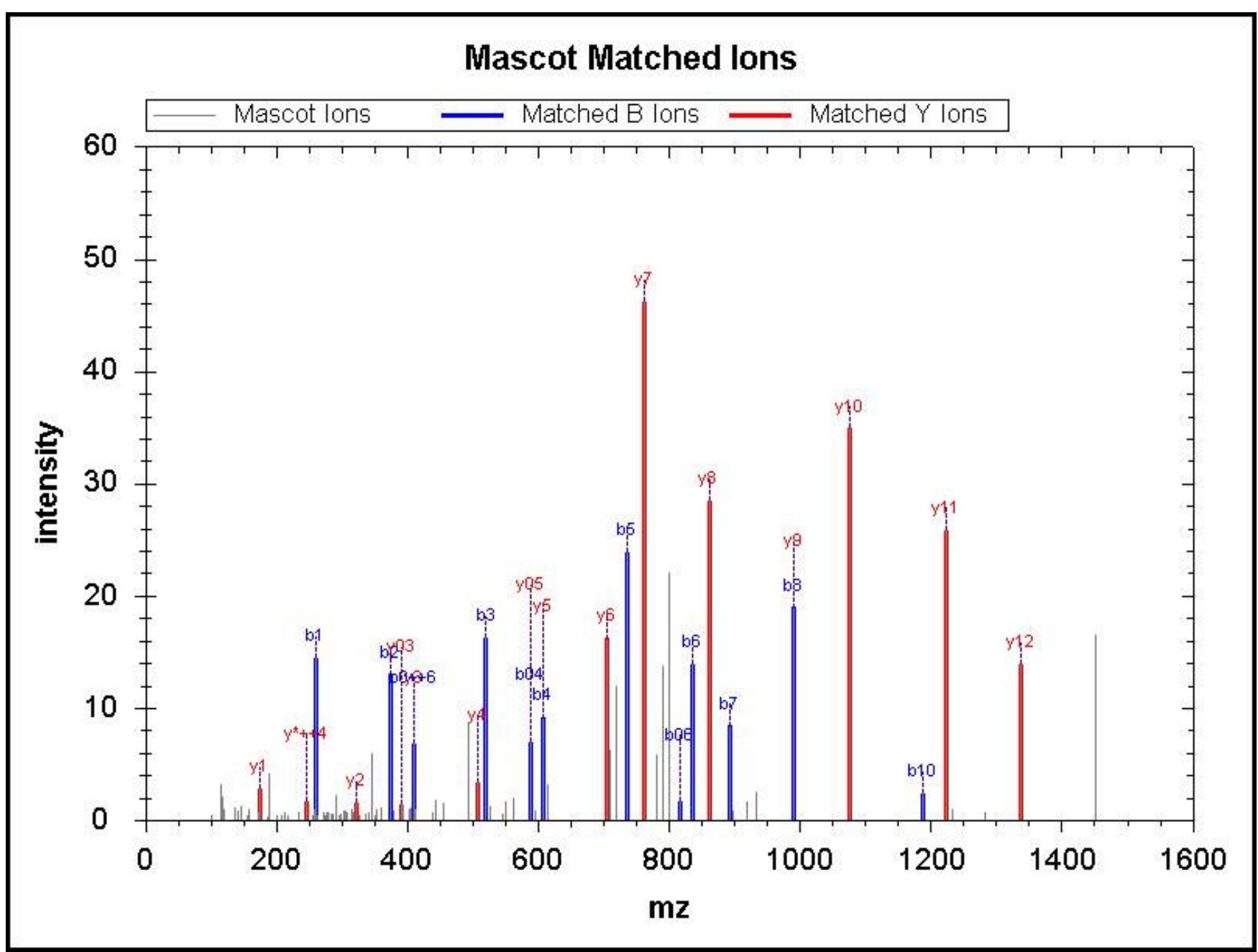

\begin{tabular}{|r|c|c|c|c|c|c|c|c|c|c|r|r|}
\hline $\mathrm{No}$ & $\mathrm{b}$ & $\mathrm{b}++$ & $\mathrm{b} 0$ & $\mathrm{~b} 0++$ & $\mathrm{Seq}$ & $\mathrm{y}$ & $\mathrm{y}++$ & $\mathrm{y} *$ & $\mathrm{y} *++$ & $\mathrm{y} 0$ & $\mathrm{y} 0++$ & $\mathrm{RevNo}$ \\
\hline 1 & 260.14 & 130.57 & 242.13 & 121.57 & $\mathrm{D}$ & & & & & & & 13 \\
\hline 2 & 373.22 & 187.11 & 355.21 & 178.11 & $\mathrm{I}$ & $1,336.73$ & 668.87 & $1,319.70$ & 660.35 & $1,318.72$ & 659.86 & 12 \\
\hline 3 & 520.29 & 260.65 & 502.28 & 251.64 & $\mathrm{~F}$ & $1,223.64$ & 612.32 & $1,206.62$ & 603.81 & $1,205.63$ & 603.32 & 11 \\
\hline 4 & 607.32 & 304.16 & 589.31 & 295.16 & $\mathrm{~S}$ & $1,076.57$ & 538.79 & $1,059.55$ & 530.28 & $1,058.56$ & 529.79 & 10 \\
\hline 5 & 736.36 & 368.69 & 718.35 & 359.68 & $\mathrm{E}$ & 989.54 & 495.27 & 972.51 & 486.76 & 971.53 & 486.27 & 9 \\
\hline 6 & 835.43 & 418.22 & 817.42 & 409.21 & $\mathrm{~V}$ & 860.50 & 430.75 & 843.47 & 422.24 & 842.49 & 421.75 & 8 \\
\hline 7 & 892.45 & 446.73 & 874.44 & 437.73 & $\mathrm{G}$ & 761.43 & 381.22 & 744.40 & 372.71 & 743.42 & 372.21 & 7 \\
\hline 8 & 989.51 & 495.26 & 971.50 & 486.25 & $\mathrm{P}$ & 704.41 & 352.71 & 687.38 & 344.19 & 686.40 & 343.70 & 6 \\
\hline 9 & $1,088.57$ & 544.79 & $1,070.56$ & 535.79 & $\mathrm{~V}$ & 607.36 & 304.18 & 590.33 & 295.67 & 589.35 & 295.18 & 5 \\
\hline 10 & $1,187.64$ & 594.33 & $1,169.63$ & 585.32 & $\mathrm{~V}$ & 508.29 & 254.65 & 491.26 & 246.13 & 490.28 & 245.64 & 4 \\
\hline 11 & $1,274.67$ & 637.84 & $1,256.66$ & 628.84 & $\mathrm{~S}$ & 409.22 & 205.11 & 392.19 & 196.60 & 391.21 & 196.11 & 3 \\
\hline 12 & $1,421.74$ & 711.38 & $1,403.73$ & 702.37 & $\mathrm{~F}$ & 322.19 & 161.60 & 305.16 & 153.08 & & & 2 \\
\hline 13 & & & & & $\mathrm{R}$ & 175.12 & 88.06 & 158.09 & 79.55 & & & 1 \\
\hline
\end{tabular}

Query 49554 Hit 1

MS/MS Fragmentation of SAAMLGNSEDHTALSR

Found in sp|O60749|SNX2_HUMAN, Sorting nexin-2 OS=Homo sapiens GN=SNX2 PE=1 SV=2

Match to Query 49554: 1802.871 from(601.9642,3+) 
Title: 262 : Sum of 2 scans in range $672(\mathrm{rt}=24.5437, \mathrm{f}=4, \mathrm{f}=173)$ to $673(\mathrm{rt}=24.5691, \mathrm{f}=4, \mathrm{i}=174)$

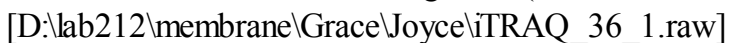

Data File:Submitted from 20120508-1(merge) by Mascot Daemon on JOYCE-VAIO

Monoisotopic mass of neutral peptide Mr(calc): 1802.871

Variable modifications:

Ions Score: 92.22 Expect: 0.000

\section{Mascot Matched lons}

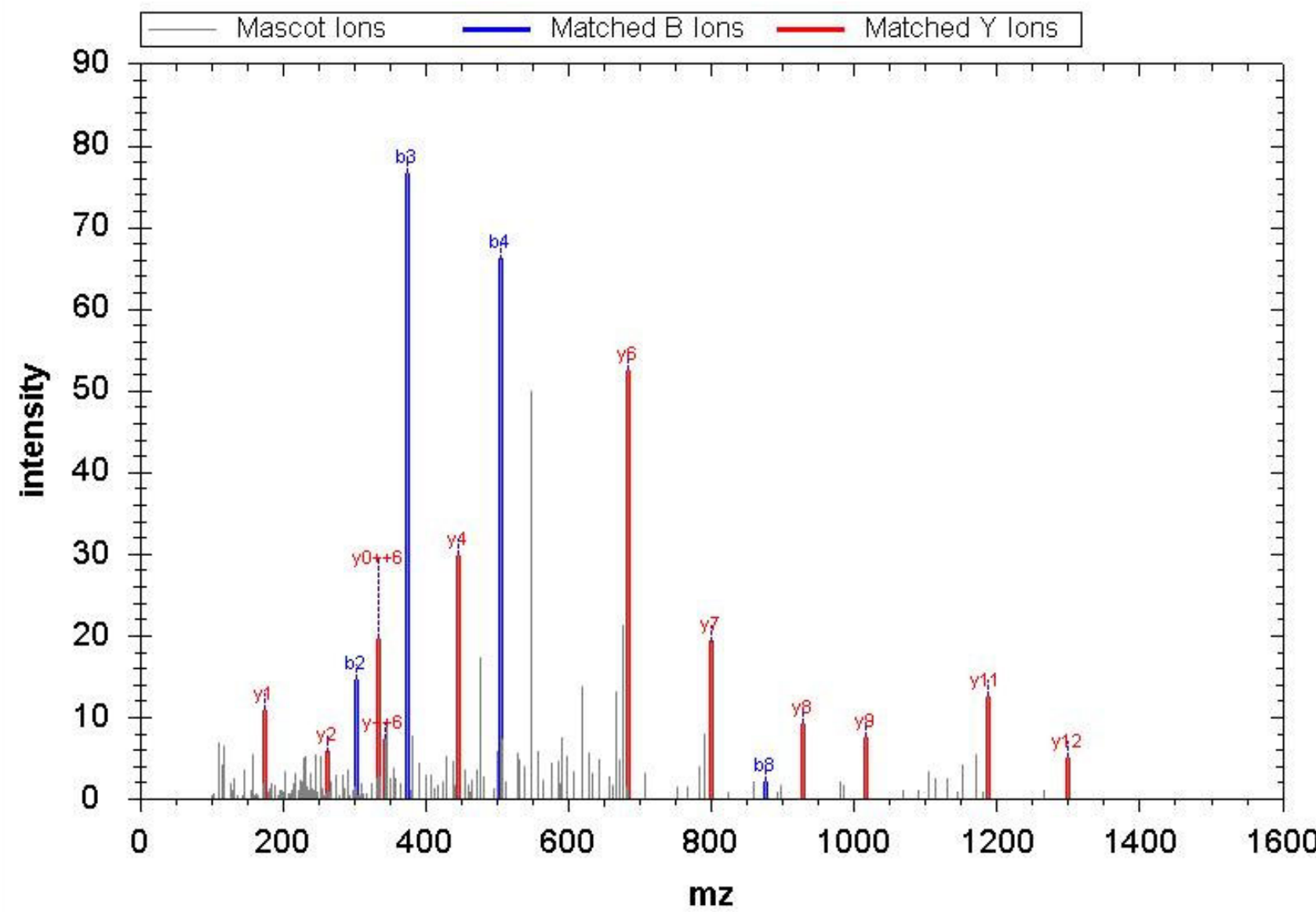

\begin{tabular}{|r|r|r|r|r|r|r|r|r|r|r|r|r|r|r|}
\hline \hline $\mathrm{No}$ & $\mathrm{b}$ & $\mathrm{b}++$ & $\mathrm{b} *$ & $\mathrm{~b}++$ & $\mathrm{b} 0$ & $\mathrm{~b} 0++$ & $\mathrm{Seq}$ & $\mathrm{y}$ & $\mathrm{y}++$ & $\mathrm{y}^{*}$ & $\mathrm{y}^{*++}$ & $\mathrm{y} 0$ & $\mathrm{y} 0++$ & $\mathrm{RevNo}$ \\
\hline 1 & 232.14 & 116.57 & & & 214.13 & 107.57 & $\mathrm{~S}$ & & & & & & & 16 \\
\hline 2 & 303.18 & 152.09 & & & 285.17 & 143.09 & $\mathrm{~A}$ & $1,572.74$ & 786.88 & $1,555.72$ & 778.36 & $1,554.73$ & 777.87 & 15 \\
\hline 3 & 374.22 & 187.61 & & & 356.21 & 178.61 & $\mathrm{~A}$ & $1,501.71$ & 751.36 & $1,484.68$ & 742.84 & $1,483.70$ & 742.35 & 14 \\
\hline 4 & 505.26 & 253.13 & & & 487.25 & 244.13 & $\mathrm{M}$ & $1,430.67$ & 715.84 & $1,413.64$ & 707.33 & $1,412.66$ & 706.83 & 13 \\
\hline 5 & 618.34 & 309.67 & & & 600.33 & 300.67 & $\mathrm{~L}$ & $1,299.63$ & 650.32 & $1,282.60$ & 641.80 & $1,281.62$ & 641.31 & 12 \\
\hline 6 & 675.36 & 338.18 & & & 657.35 & 329.18 & $\mathrm{G}$ & $1,186.54$ & 593.78 & $1,169.52$ & 585.26 & $1,168.53$ & 584.77 & 11 \\
\hline 7 & 789.40 & 395.21 & 772.38 & 386.69 & 771.39 & 386.20 & $\mathrm{~N}$ & $1,129.52$ & 565.27 & $1,112.50$ & 556.75 & $1,111.51$ & 556.26 & 10 \\
\hline 8 & 876.44 & 438.72 & 859.41 & 430.21 & 858.43 & 429.72 & $\mathrm{~S}$ & $1,015.48$ & 508.24 & 998.45 & 499.73 & 997.47 & 499.24 & 9 \\
\hline 9 & $1,005.48$ & 503.24 & 988.45 & 494.73 & 987.47 & 494.24 & $\mathrm{E}$ & 928.45 & 464.73 & 911.42 & 456.21 & 910.44 & 455.72 & 8 \\
\hline 10 & $1,120.51$ & 560.76 & $1,103.48$ & 552.24 & $1,102.50$ & 551.75 & $\mathrm{D}$ & 799.41 & 400.21 & 782.38 & 391.69 & 781.40 & 391.20 & 7 \\
\hline 11 & $1,257.57$ & 629.29 & $1,240.54$ & 620.77 & $1,239.55$ & 620.28 & $\mathrm{H}$ & 684.38 & 342.69 & 667.35 & 334.18 & 666.37 & 333.69 & 6 \\
\hline 12 & $1,358.61$ & 679.81 & $1,341.59$ & 671.30 & $1,340.60$ & 670.80 & $\mathrm{~T}$ & 547.32 & 274.16 & 530.29 & 265.65 & 529.31 & 265.16 & 5 \\
\hline 13 & $1,429.65$ & 715.33 & $1,412.62$ & 706.82 & $1,411.64$ & 706.32 & A & 446.27 & 223.64 & 429.25 & 215.13 & 428.26 & 214.63 & 4 \\
\hline 14 & $1,542.73$ & 771.87 & $1,525.71$ & 763.36 & $1,524.72$ & 762.87 & L & 375.24 & 188.12 & 358.21 & 179.61 & 357.22 & 179.12 & 3 \\
\hline 15 & $1,629.77$ & 815.39 & $1,612.74$ & 806.87 & $1,611.76$ & 806.38 & S & 262.15 & 131.58 & 245.12 & 123.07 & 244.14 & 122.57 & 2 \\
\hline 16 & & & & & & & R & 175.12 & 88.06 & 158.09 & 79.55 & & & 1 \\
\hline
\end{tabular}

Query 89469 Hit 1 
MS/MS Fragmentation of RPDVENQPDAASQLNVDASGNLAK

Found in sp|Q9NZL9|MAT2B_HUM AN, Methionine adenosyltransferase 2 subunit beta OS=Homo sapiens GN=MAT2B PE=1 SV=1 Match to Query 89469: 2895.495 from(724.881,4+)

Title: 411: Scan $986(\mathrm{rt}=31.5413, \mathrm{f}=3, \mathrm{i}=142)$ [D:llab212 \membranelGrace JoyceliTRAQ_38_2.raw]

Data File:Submitted from 20120508-1(merge) by Mascot Daemon on JOYCE-VAIO

Monoisotopic mass of neutral peptide Mr(calc): 2895.495

Variable modifications:

K25 :iTRAQ4plex (K)

Ions Score: 91.66 Expect: 0.000

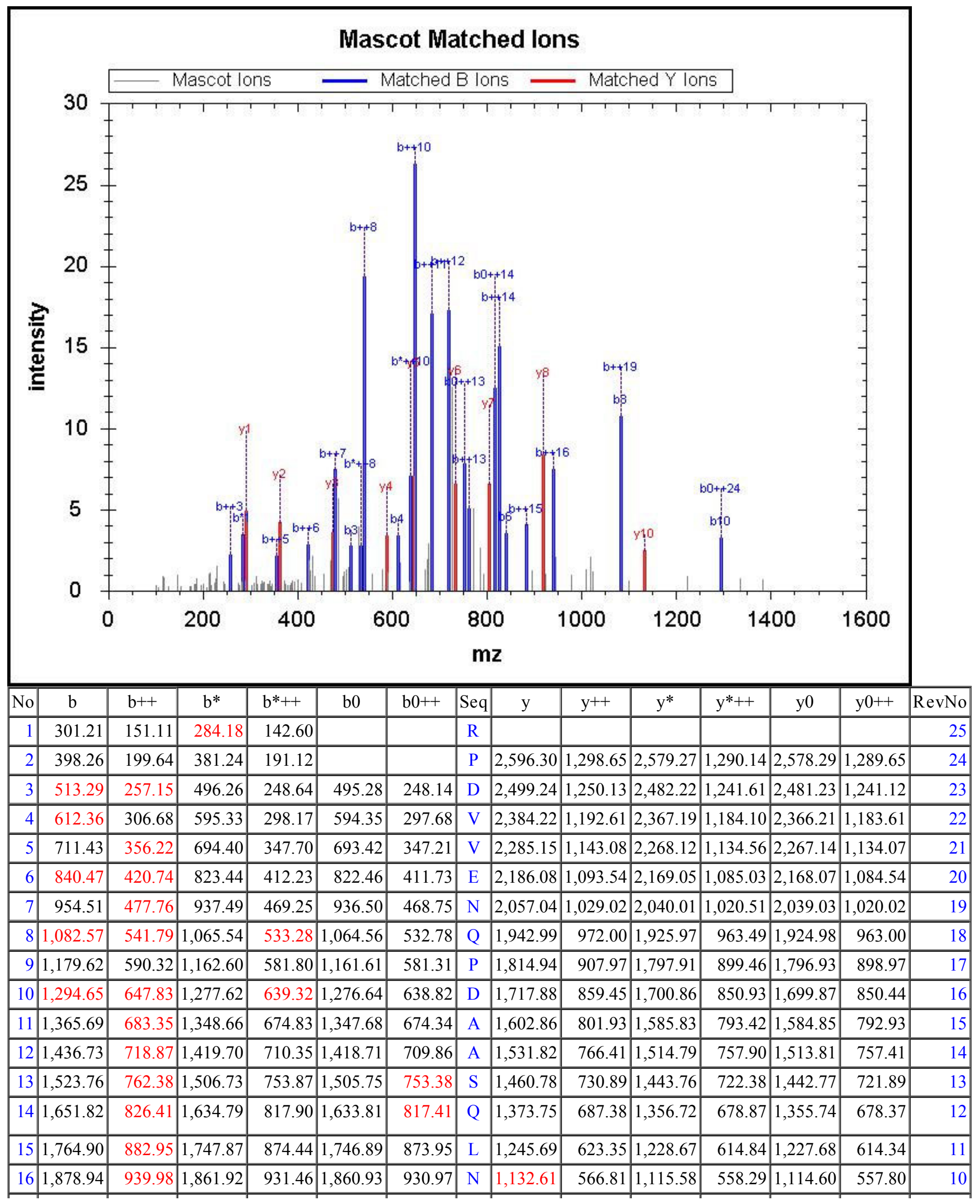




\begin{tabular}{|r|r|r|r|r|r|r|r|r|r|r|r|r|r|r|r|}
17 & $1,978.01$ & 989.51 & $1,960.98$ & 981.00 & $1,960.00$ & 980.50 & $\mathrm{~V}$ & $1,018.56$ & 509.79 & $1,001.54$ & 501.27 & $1,000.55$ & 500.78 & 9 \\
\hline \hline 18 & $2,093.04$ & $1,047.02$ & $2,076.01$ & $1,038.51$ & $2,075.03$ & $1,038.02$ & $\mathrm{D}$ & 919.50 & 460.25 & 902.47 & 451.74 & 901.49 & 451.25 & 8 \\
\hline 19 & $2,164.08$ & $1,082.54$ & $2,147.05$ & $1,074.03$ & $2,146.06$ & $1,073.54$ & $\mathrm{~A}$ & 804.47 & 402.74 & 787.44 & 394.23 & 786.46 & 393.73 & 7 \\
\hline 20 & $2,251.11$ & $1,126.06$ & $2,234.08$ & $1,117.54$ & $2,233.10$ & $1,117.05$ & $\mathrm{~S}$ & 733.43 & 367.22 & 716.41 & 358.71 & 715.42 & 358.21 & 6 \\
\hline 21 & $2,308.13$ & $1,154.57$ & $2,291.10$ & $1,146.05$ & $2,290.12$ & $1,145.56$ & $\mathrm{G}$ & 646.40 & 323.70 & 629.37 & 315.19 & & & 5 \\
\hline 22 & $2,422.17$ & $1,211.59$ & $2,405.15$ & $1,203.08$ & $2,404.16$ & $1,202.58$ & $\mathrm{~N}$ & 589.38 & 295.19 & 572.35 & 286.68 & & & 4 \\
\hline 23 & $2,535.26$ & $1,268.13$ & $2,518.23$ & $1,259.62$ & $2,517.25$ & $1,259.13$ & $\mathrm{~L}$ & 475.34 & 238.17 & 458.31 & 229.66 & & & 3 \\
\hline 24 & $2,606.29$ & $1,303.65$ & $2,589.27$ & $1,295.14$ & $2,588.28$ & $1,294.64$ & $\mathrm{~A}$ & 362.25 & 181.63 & 345.23 & 173.12 & & & 2 \\
\hline 25 & & & & & & & $\mathrm{~K}$ & 291.21 & 146.11 & 274.19 & 137.60 & & & 1 \\
\hline
\end{tabular}

Query 62397 Hit 1

MS/MS Fragmentation of ENLELILTQSVENVGVR

Found in sp|Q9BYD2|RM09 HUM AN, 39S ribosomal protein L9

Match to Query 62397: 2056.126from(686.3827,3+)

Title: 829: Sum of 2 scans in range $2320(\mathrm{rt}=59.597, \mathrm{f}=4, \mathrm{i}=551)$ to $2321(\mathrm{rt}=59.6224, \mathrm{f}=4, \mathrm{i}=552)$

[D:lab212 \membranelGrace Joycel20120320_iTRAQ_20-2.raw]

Data File:Submitted from 20120508-1(merge) by Mascot Daemon on JOYCE-VAIO

Monoisotopic mass of neutral peptide $\mathrm{Mr}$ (calc): 2056.126

Variable modifications:

Ions Score: 90.38 Expect: 0.000

\section{Mascot Matched lons}

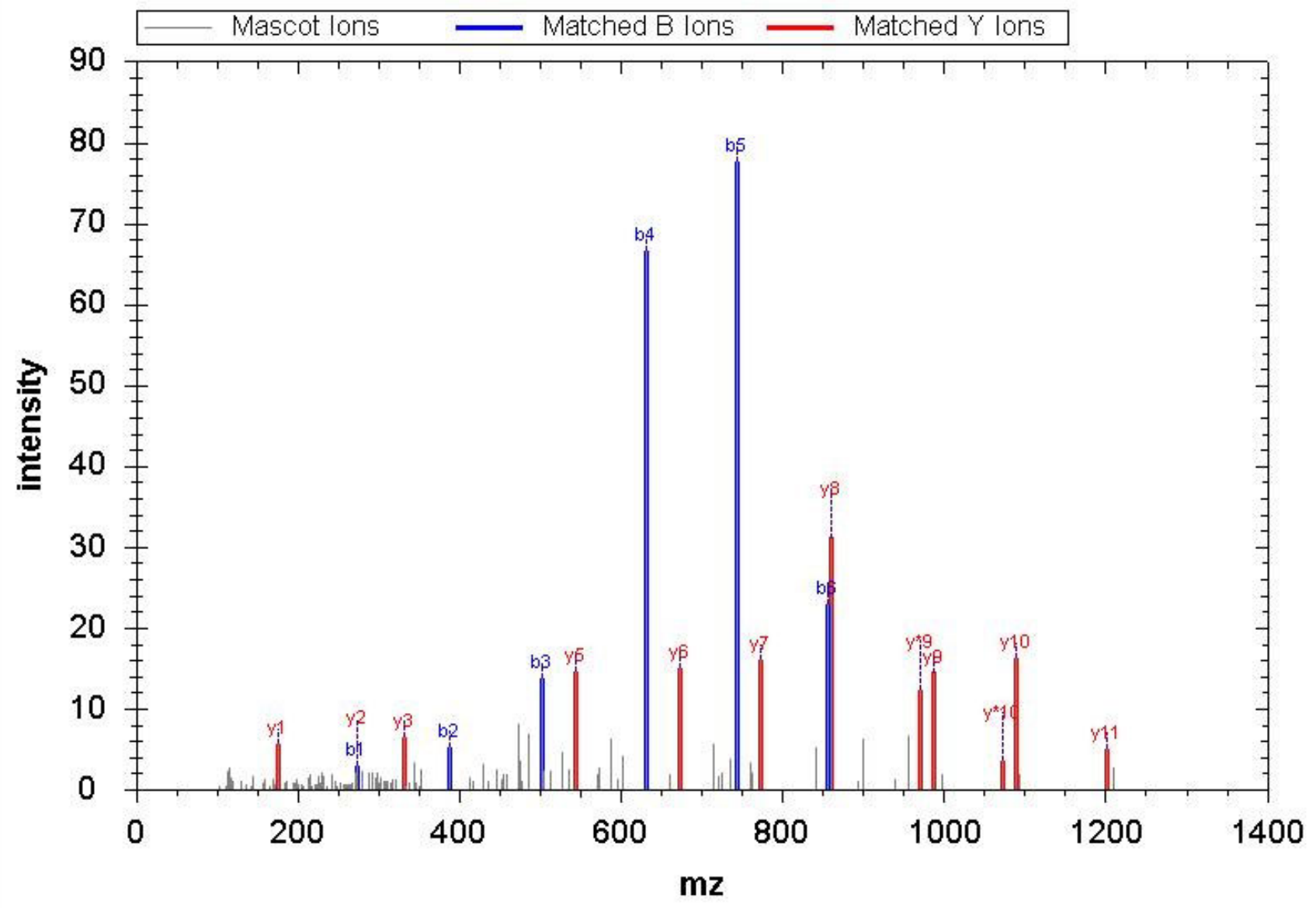

\begin{tabular}{|r|c|c|c|c|c|c|c|c|c|c|c|r|r|r|}
\hline \hline No & $\mathrm{b}$ & $\mathrm{b}++$ & $\mathrm{b}^{*}$ & $\mathrm{~b} *++$ & $\mathrm{b} 0$ & $\mathrm{~b} 0++$ & Seq & $\mathrm{y}$ & $\mathrm{y}++$ & $\mathrm{y}^{*}$ & $\mathrm{y}^{*++}$ & $\mathrm{y} 0$ & $\mathrm{y} 0++$ & $\mathrm{RevNo}$ \\
\hline 1 & 274.15 & 137.58 & & & 256.14 & 128.57 & $\mathrm{E}$ & & & & & & & 17 \\
\hline 2 & 388.19 & 194.60 & 371.17 & 186.09 & 370.18 & 185.60 & $\mathrm{~N}$ & $1,783.99$ & 892.50 & $1,766.96$ & 883.99 & $1,765.98$ & 883.49 & 16 \\
\hline 3 & 501.28 & 251.14 & 484.25 & 242.63 & 483.27 & 242.14 & $\mathrm{~L}$ & $1,669.95$ & 835.48 & $1,652.92$ & 826.96 & $1,651.94$ & 826.47 & 15 \\
\hline 4 & 630.32 & 315.66 & 613.29 & 307.15 & 612.31 & 306.66 & $\mathrm{E}$ & $1,556.86$ & 778.94 & $1,539.84$ & 770.42 & $1,538.85$ & 769.93 & 14 \\
\hline 5 & 743.41 & 372.21 & 726.38 & 363.69 & 725.40 & 363.20 & $\mathrm{~L}$ & $1,427.82$ & 714.41 & $1,410.80$ & 705.90 & $1,409.81$ & 705.41 & 13 \\
\hline
\end{tabular}




\begin{tabular}{|r|r|r|r|r|r|r|r|r|r|r|r|r|r|r|r|r|}
\hline 6 & 856.49 & 428.75 & 839.46 & 420.24 & 838.48 & 419.74 & $\mathrm{I}$ & $1,314.74$ & 657.87 & $1,297.71$ & 649.36 & $1,296.73$ & 648.87 & 12 \\
\hline 7 & 969.57 & 485.29 & 952.55 & 476.78 & 951.56 & 476.29 & $\mathrm{~L}$ & $1,201.65$ & 601.33 & $1,184.63$ & 592.82 & $1,183.64$ & 592.33 & 11 \\
\hline 8 & $1,070.62$ & 535.81 & $1,053.59$ & 527.30 & $1,052.61$ & 526.81 & $\mathrm{~T}$ & $1,088.57$ & 544.79 & $1,071.54$ & 536.28 & $1,070.56$ & 535.78 & 10 \\
\hline 9 & $1,198.68$ & 599.84 & $1,181.65$ & 591.33 & $1,180.67$ & 590.84 & $\mathrm{Q}$ & 987.52 & 494.26 & 970.50 & 485.75 & 969.51 & 485.26 & 9 \\
\hline 10 & $1,285.71$ & 643.36 & $1,268.69$ & 634.85 & $1,267.70$ & 634.35 & $\mathrm{~S}$ & 859.46 & 430.24 & 842.44 & 421.72 & 841.45 & 421.23 & 8 \\
\hline 11 & $1,384.78$ & 692.89 & $1,367.75$ & 684.38 & $1,366.77$ & 683.89 & $\mathrm{~V}$ & 772.43 & 386.72 & 755.40 & 378.21 & 754.42 & 377.71 & 7 \\
\hline 12 & $1,513.82$ & 757.42 & $1,496.80$ & 748.90 & $1,495.81$ & 748.41 & $\mathrm{E}$ & 673.36 & 337.18 & 656.34 & 328.67 & 655.35 & 328.18 & 6 \\
\hline 13 & $1,627.87$ & 814.44 & $1,610.84$ & 805.92 & $1,609.86$ & 805.43 & $\mathrm{~N}$ & 544.32 & 272.66 & 527.29 & 264.15 & & & 5 \\
\hline 14 & $1,726.93$ & 863.97 & $1,709.91$ & 855.46 & $1,708.92$ & 854.97 & $\mathrm{~V}$ & 430.28 & 215.64 & 413.25 & 207.13 & & & 4 \\
\hline 15 & $1,783.96$ & 892.48 & $1,766.93$ & 883.97 & $1,765.95$ & 883.48 & $\mathrm{G}$ & 331.21 & 166.11 & 314.18 & 157.59 & & & 3 \\
\hline 16 & $1,883.02$ & 942.02 & $1,866.00$ & 933.50 & $1,865.01$ & 933.01 & $\mathrm{~V}$ & 274.19 & 137.60 & 257.16 & 129.08 & & & 2 \\
\hline 17 & & & & & & & $\mathrm{R}$ & 175.12 & 88.06 & 158.09 & 79.55 & & & 1 \\
\hline
\end{tabular}

Query 97263 Hit 1

MS/MS Fragmentation of GNKPDFHLAM PTEQAEGFYNSFLEQLR

Found in sp|Q8TEA8|DTD1_HUM AN, D-tyrosyl-tRNA(Tyr) deacylase 1 OS=Homo sapiens GN=DTD1 PE=1 SV=2 Match to Query $97263: 3426.685$ from(857.6785,4+)

Title: 963: Sum of 2 scans in range 2316 ( $\mathrm{rt}=60.901, \mathrm{f}=4, \mathrm{i}=643)$ to $2317(\mathrm{rt}=60.9264, \mathrm{f}=4, \mathrm{i}=644)$

[D:llab212 \membranelGrace JoyceliTRAQ_46_2.raw]

Data File:Submitted from 20120508-1(merge) by Mascot Daemon on JOYCE-VAIO

Monoisotopic mass of neutral peptide $\mathrm{Mr}$ (calc): 3426.685

Variable modifications:

K3 :TRAQ4plex (K)

Ions Score: 88.98 Expect: 0.000

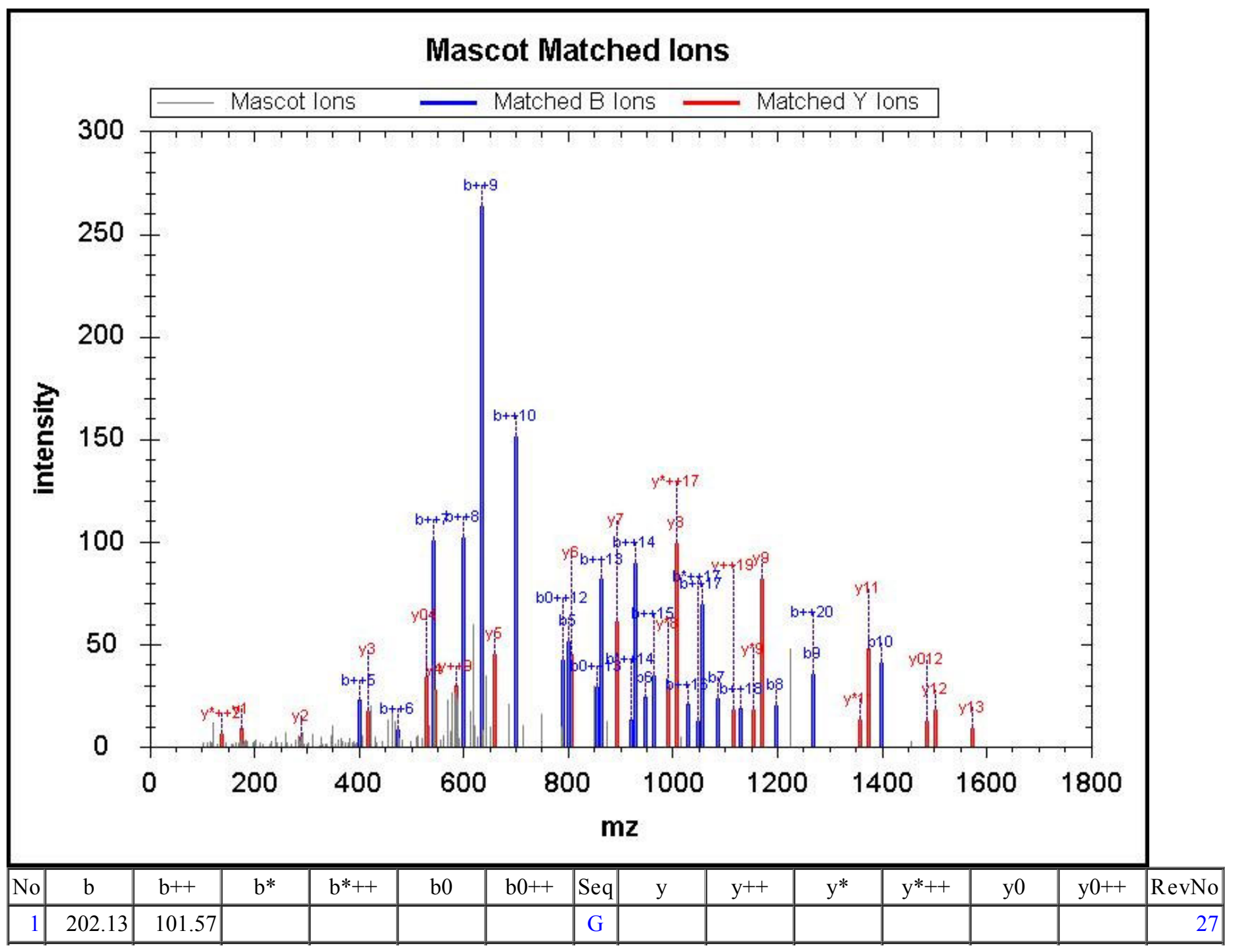




\begin{tabular}{|c|c|c|c|c|c|c|c|c|c|c|c|c|c|c|}
\hline 2 & 316.17 & 158.59 & 299.15 & 150.08 & & & $\mathrm{~N}$ & $|3,226.57|$ & $|1,613.79|$ & $|3,209.55|$ & $1,605.28$ & $3,208.56 \mid$ & $|1,604.79|$ & 26 \\
\hline 3 & 588.37 & 294.69 & 571.34 & 286.18 & & & $\mathrm{~K}$ & $3,112.53$ & $1,556.77$ & $3,095.50$ & $1,548.26$ & $3,094.52$ & $1,547.76$ & 25 \\
\hline 4 & 685.42 & 343.22 & 668.40 & 334.70 & & & $\mathrm{P}$ & $2,840.33$ & $1,420.67$ & $2,823.31$ & $1,412.16$ & $2,822.32$ & $1,411.67$ & \\
\hline 5 & 800.45 & 400.73 & 783.42 & 392.22 & 782.44 & 1.72 & $\mathrm{D}$ & $2,743.28$ & $1,372.14$ & $2,726.26$ & $1,363.63$ & .27 & 3.14 & \\
\hline 6 & 947.52 & 474.26 & 930.49 & 465.75 & 929.51 & 46 & $\mathrm{~F}$ & 2,6 & 63 & $2,611.23$ & 5.12 & .24 & 5.63 & \\
\hline 7 & $1,084.58$ & 542.79 & $1,067.55$ & 534.28 & $1,066.57$ & 533.79 & $\mathrm{H}$ & $2,481.19$ & $1,241.10$ & $2,464.16$ & $1,232.58$ & $2,463.18$ & $1,232.09$ & 21 \\
\hline 8 & $1,197.66$ & 599.33 & $1,180.64$ & 590.82 & .65 & 590.33 & $\mathrm{~L}$ & 2,3 & .57 & $2,327.10$ & .05 & .12 & 3.56 & \\
\hline 9 & $1,268.70$ & 634.85 & $1,251.67$ & 626.34 & 69 & 85 & A & $2,231.04$ & $1,116.03$ & $2,214.02$ & $1,107.51$ & $2,213.03$ & $1,107.02$ & \\
\hline 10 & $1,399.74$ & 700.37 & $1,382.71$ & 691.86 & 73 & 37 & M & 2,1 & .51 & .98 & .99 & .00 & .50 & \\
\hline 11 & $1,496.79$ & 748.90 & $1,479.77$ & 740.39 & $1,478.78$ & 739.89 & $\mathrm{P}$ & $2,028.97$ & .99 & $2,011.94$ & $1,006.47$ & 0.96 & $1,005.98$ & \\
\hline 12 & $1,597.84$ & 799.42 & $1,580.81$ & 790.91 & $1,579.83$ & 790.42 & $\mathrm{~T}$ & $1,931.91$ & 966.46 & $1,914.89$ & 957.95 & $1,913.90$ & 957.46 & \\
\hline 13 & $1,726.88$ & 863.95 & $1,709.86$ & 855.43 & 87 & 854.94 & $\mathrm{E}$ & $1,830.87$ & 915.94 & $1,813.84$ & 907.42 & $1,812.86$ & 900.93 & 15 \\
\hline 14 & $1,854.94$ & 927.97 & 1,8 & 919.46 & 1,8 & 918 & Q & 1,7 & 851.42 & 1,68 & 842.90 & 3.81 & 842.41 & 14 \\
\hline 15 & $1,925.98$ & 963.49 & $1,908.95$ & 954.98 & $1,907.97$ & 954.49 & A & $1,573.76$ & 787.39 & $1,556.74$ & 778.87 & .75 & 778.38 & 13 \\
\hline 16 & $2,055.02$ & 28.01 & $2,037.99$ & $1,019.50$ & 2,03 & 1,01 & $\mathrm{E}$ & 1,5 & .87 & 1,4 & .35 & 72 & 2.00 & 12 \\
\hline 17 & $2,112.04$ & $1,056.52$ & $2,095.02$ & $1,048.01$ & $2,094.03$ & $1,047.52$ & $\mathrm{G}$ & $1,373.68$ & 687.35 & $1,356.66$ & 678.83 & 67 & 678.34 & 11 \\
\hline 18 & $2,259.11$ & $1,130.06$ & $2,242.08$ & $1,121.55$ & $2,241.10$ & $1,121.05$ & $\mathrm{~F}$ & $1,316.66$ & 658.84 & $1,299.64$ & 650.32 & 3.65 & 649.83 & 10 \\
\hline 19 & $2,422.17$ & $1,211.59$ & $2,405.15$ & $1,203.08$ & $2,404.16$ & $1,202.59$ & $\mathrm{Y}$ & $1,169.59$ & 585.30 & $1,152.57$ & 576.79 & $1,151.58$ & 576.30 & 9 \\
\hline 20 & $2,536.22$ & $1,268.61$ & $2,519.19$ & $1,260.10$ & $2,518.21$ & $1,259.61$ & $\mathrm{~N}$ & $1,006.53$ & 503.77 & 989.51 & 495.26 & 988.52 & 494.76 & 8 \\
\hline 21 & $2,623.25$ & $1,312.13$ & $2,606.22$ & $1,303.61$ & $2,605.24$ & $1,303.12$ & $\mathrm{~S}$ & 892.49 & 446.75 & 875.46 & 438.23 & 874.48 & 437.74 & 7 \\
\hline 22 & $2,770.32$ & $1,385.66$ & $2,753.29$ & $1,377.15$ & $2,752.31$ & $1,376.66$ & $\mathrm{~F}$ & 46 & .23 & .43 & .72 & .45 & 23 & 6 \\
\hline 23 & $2,883.40$ & $1,442.20$ & $2,866.37$ & $1,433.69$ & $2,865.39$ & $1,433.20$ & $\mathrm{~L}$ & 658.39 & 329.70 & 641.36 & 321.18 & 640.38 & 320.69 & 5 \\
\hline 24 & $3,012.44$ & $1,506.73$ & $2,995.42$ & $1,498.21$ & $2,994.43$ & $1,497.72$ & $\mathrm{E}$ & 545.30 & 273.16 & 528.28 & 264.64 & 527.29 & 264.15 & 4 \\
\hline 25 & $3,140.50$ & $1,570.75$ & $3,123.48$ & $1,562.24$ & $3,122.49$ & $1,561.75$ & Q & 416.26 & 208.63 & 399.24 & 200.12 & & & 3 \\
\hline 26 & $3,253.59$ & $1,627.30$ & $3,236.56$ & $1,618.78$ & $3,235.58$ & $1,618.29$ & $\mathrm{~L}$ & 288.20 & 144.61 & 271.18 & 136.09 & & & 2 \\
\hline 27 & & & & & & & $\mathrm{R}$ & 175.12 & 88.06 & 158.09 & 79.55 & & & 1 \\
\hline
\end{tabular}

Query 77140 Hit 1

MS/MS Fragmentation of DLLEQMM AEMIGEFPDLHR

Found in sp|Q9H4I3|TRABD_HUMAN, TraB domain-containing protein OS=Homo sapiens GN=TRABD PE=2 SV=1

Match to Query 77140: 2418.153from(807.0583,3+)

Title: 1243: Sum of 2 scans in range $3182(\mathrm{rt}=78.9956, \mathrm{f}=2, \mathrm{i}=555)$ to $3183(\mathrm{rt}=79.021, \mathrm{f}=2, \mathrm{i}=556)$

[D:llab212 \membranelGrace JoyceliTRAQ_31_1.raw]

Data File:Submitted from 20120508-1(merge) by Mascot Daemon on JOYCE-VAIO

Monoisotopic mass of neutral peptide Mr(calc): 2418.153

Variable modifications:

Ions Score: 88.71 Expect: 0.000 


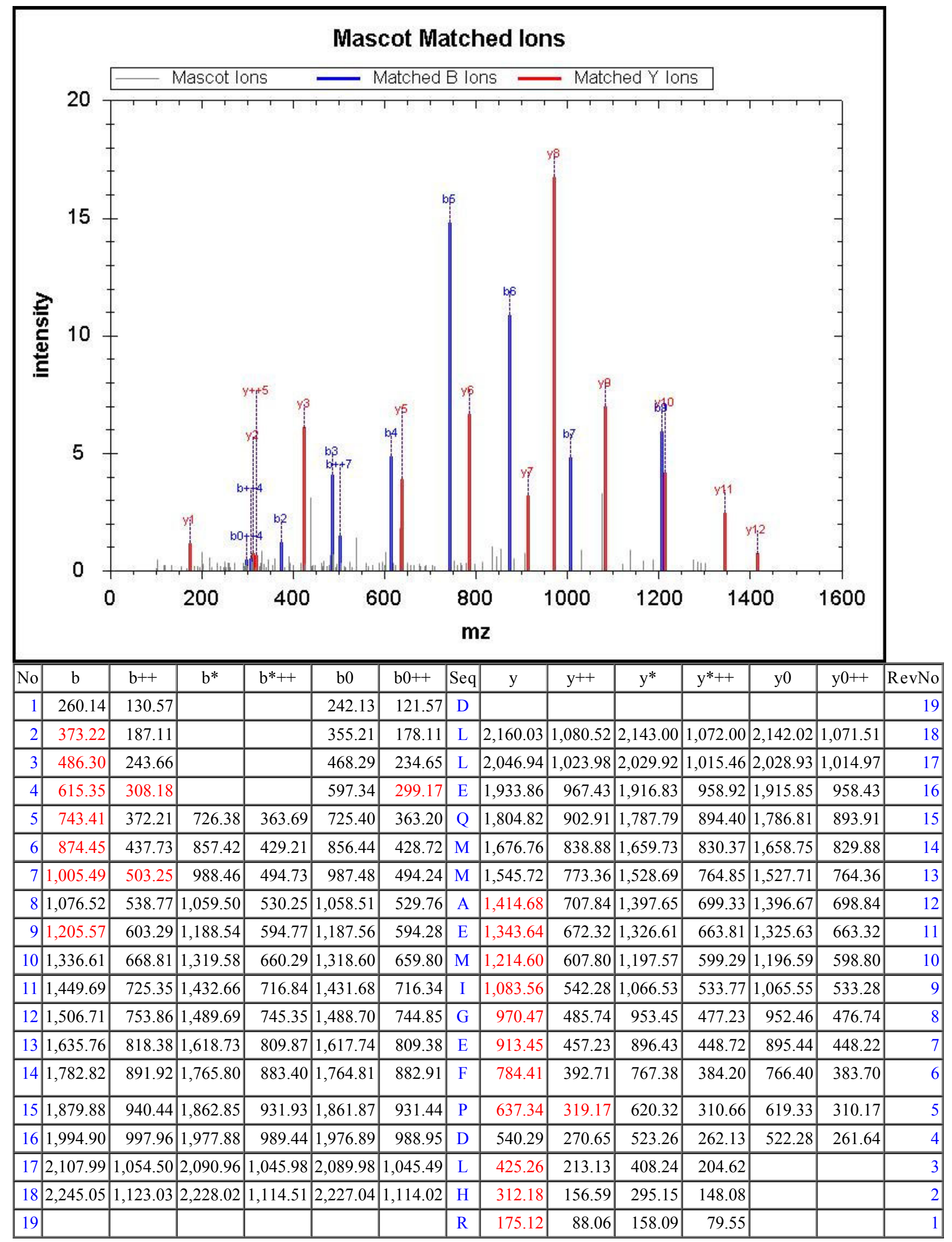

Query 60468 Hit 1 
Title: 575: Sum of 2 scans in range 1275 ( $\mathrm{rt}=38.4732, \mathrm{f}=4, \mathrm{i}=391)$ to $1276(\mathrm{rt}=38.4986, \mathrm{f}=4, \mathrm{~F}=392$ )

[D:lab212 \membranelGrace \oyceliTRAQ40.raw]

Data File:Submitted from 20120508-1(merge) by Mascot Daemon on JOYCE-VAIO

Monoisotopic mass of neutral peptide Mr(calc): 2021.006

Variable modifications:

K15 :iTRAQ4plex (K)

Ions Score: 87.95 Expect: 0.000

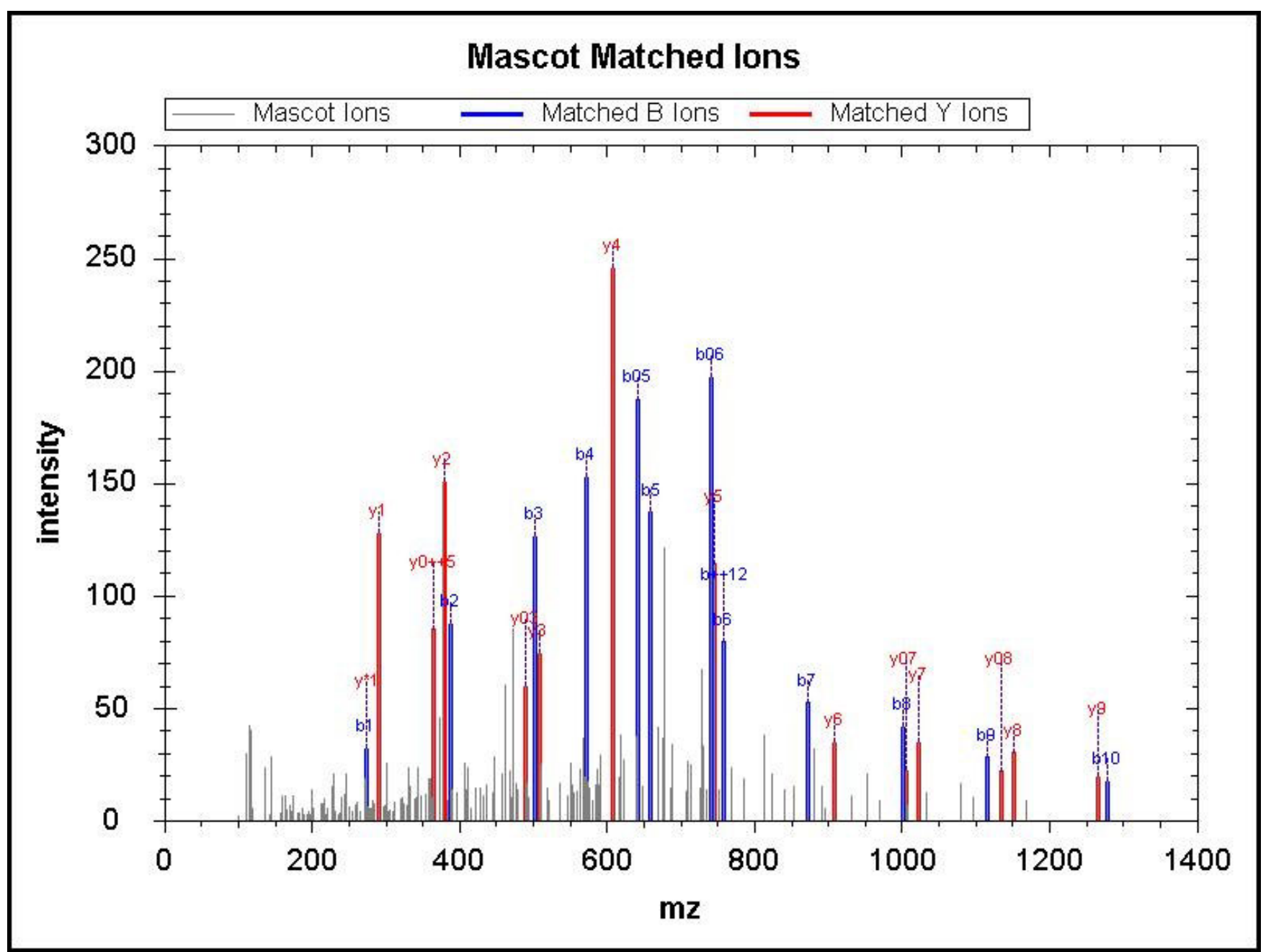

\begin{tabular}{|r|c|c|c|c|c|c|c|c|c|c|r|r|r|r|}
\hline \hline $\mathrm{No}$ & $\mathrm{b}$ & $\mathrm{b}++$ & $\mathrm{b}^{*}$ & $\mathrm{~b}++$ & $\mathrm{b} 0$ & $\mathrm{~b} 0++$ & $\mathrm{Seq}$ & $\mathrm{y}$ & $\mathrm{y}++$ & $\mathrm{y}^{*}$ & $\mathrm{y}^{*++}$ & $\mathrm{y} 0$ & $\mathrm{y} 0++$ & $\mathrm{RevNo}$ \\
\hline 1 & 274.15 & 137.58 & & & 256.14 & 128.57 & $\mathrm{E}$ & & & & & & & 15 \\
\hline 2 & 388.19 & 194.60 & 371.17 & 186.09 & 370.18 & 185.60 & $\mathrm{~N}$ & $1,748.89$ & 874.95 & $1,731.87$ & 866.44 & $1,730.88$ & 865.95 & 14 \\
\hline 3 & 501.28 & 251.14 & 484.25 & 242.63 & 483.27 & 242.14 & $\mathrm{I}$ & $1,634.85$ & 817.93 & $1,617.82$ & 809.42 & $1,616.84$ & 808.92 & 13 \\
\hline 4 & 572.32 & 286.66 & 555.29 & 278.15 & 554.31 & 277.66 & $\mathrm{~A}$ & $1,521.77$ & 761.39 & $1,504.74$ & 752.87 & $1,503.76$ & 752.38 & 12 \\
\hline 5 & 659.35 & 330.18 & 642.32 & 321.66 & 641.34 & 321.17 & $\mathrm{~S}$ & $1,450.73$ & 725.87 & $1,433.70$ & 717.36 & $1,432.72$ & 716.86 & 11 \\
\hline 6 & 758.42 & 379.71 & 741.39 & 371.20 & 740.41 & 370.71 & $\mathrm{~V}$ & $1,363.70$ & 682.35 & $1,346.67$ & 673.84 & $1,345.69$ & 673.35 & 10 \\
\hline 7 & 871.50 & 436.25 & 854.47 & 427.74 & 853.49 & 427.25 & $\mathrm{~L}$ & $1,264.63$ & 632.82 & $1,247.60$ & 624.30 & $1,246.62$ & 623.81 & 9 \\
\hline 8 & $1,000.54$ & 500.78 & 983.52 & 492.26 & 982.53 & 491.77 & $\mathrm{E}$ & $1,151.54$ & 576.28 & $1,134.52$ & 567.76 & $1,133.53$ & 567.27 & 8 \\
\hline 9 & $1,114.59$ & 557.80 & $1,097.56$ & 549.28 & $1,096.58$ & 548.79 & $\mathrm{~N}$ & $1,022.50$ & 511.75 & $1,005.48$ & 503.24 & $1,004.49$ & 502.75 & 7 \\
\hline 10 & $1,277.65$ & 639.33 & $1,260.62$ & 630.82 & $1,259.64$ & 630.32 & $\mathrm{Y}$ & 908.46 & 454.73 & 891.43 & 446.22 & 890.45 & 445.73 & 6 \\
\hline 11 & $1,414.71$ & 707.86 & $1,397.68$ & 699.34 & $1,396.70$ & 698.85 & H & 745.40 & 373.20 & 728.37 & 364.69 & 727.39 & 364.20 & 5 \\
\hline 12 & $1,515.76$ & 758.38 & $1,498.73$ & 749.87 & $1,497.75$ & 749.38 & T & 608.34 & 304.67 & 591.31 & 296.16 & 590.33 & 295.67 & 4 \\
\hline 13 & $1,644.80$ & 822.90 & $1,627.77$ & 814.39 & $1,626.79$ & 813.90 & E & 507.29 & 254.15 & 490.26 & 245.64 & 489.28 & 245.14 & 3 \\
\hline 14 & $1,731.83$ & 866.42 & $1,714.80$ & 857.91 & $1,713.82$ & 857.41 & S & 378.25 & 189.63 & 361.22 & 181.11 & 360.24 & 180.62 & 2 \\
\hline 15 & & & & & & & K & 291.21 & 146.11 & 274.19 & 137.60 & & & 1 \\
\hline
\end{tabular}


MS/MS Fragmentation of FYPGQAPSLAENFAEHVLR

Found in sp|Q9Y276|BCS1_HUM AN, Mitochondrial chaperone BCS1 OS=Homo sapiens GN=BCS1L PE=1 SV=1

Match to Query 72333: 2289.163from(764.0615,3+)

Title: 974: Sum of 2 scans in range $2160(\mathrm{rt}=58.0317, \mathrm{f}=4, \mathrm{i}=647)$ to $2161(\mathrm{rt}=58.0571, \mathrm{f}=4, \mathrm{i}=648)$

[D:llab212 \membranelGrace JoyceliTRAQ 31_1.raw]

Data File:Submitted from 20120508-1(merge) by Mascot Daemon on JOYCE-VAIO

Monoisotopic mass of neutral peptide $\mathrm{Mr}$ (calc): 2289.163

Variable modifications:

Ions Score: 87.95 Expect: 0.000

\section{Mascot Matched lons}

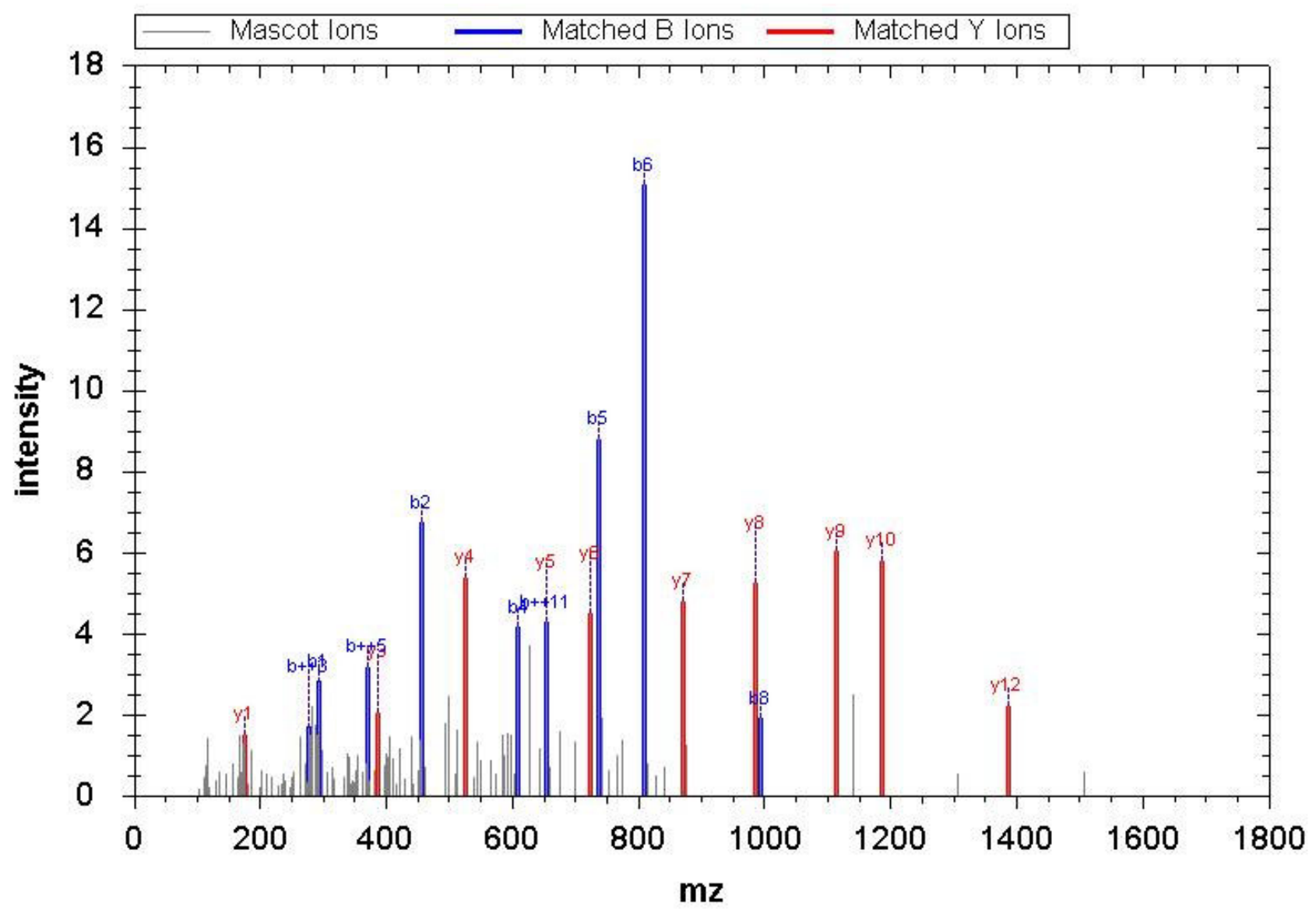

\begin{tabular}{|c|c|c|c|c|c|c|c|c|c|c|c|c|c|c|}
\hline No & $\mathrm{b}$ & $\mathrm{b}++$ & $b^{*}$ & $\mathrm{~b}^{*++}$ & b0 & b0++ & Seq & $\mathrm{y}$ & $\mathrm{y}++$ & $y^{*}$ & $\mathrm{y}^{*++}$ & y0 & $\mathrm{y} 0++$ & RevNo \\
\hline 1 & 292.18 & 146.59 & & & & & $\mathrm{~F}$ & & & & & & & 19 \\
\hline 2 & 455.24 & 228.12 & & & & & $\mathrm{Y}$ & $1,999.00$ & $1,000.01$ & $1,981.98$ & 991.49 & $1,980.99$ & 991.00 & 18 \\
\hline 3 & 552.29 & 276.65 & & & & & $\mathrm{P}$ & $1,835.94$ & 918.47 & $1,818.91$ & 909.96 & $1,817.93$ & 909.47 & 17 \\
\hline 4 & 609.32 & 305.16 & & & & & G & $1,738.89$ & 869.95 & $1,721.86$ & 861.43 & $1,720.88$ & 860.94 & 16 \\
\hline 5 & 737.37 & 369.19 & 720.35 & 360.68 & & & Q & $1,681.87$ & 841.44 & $1,664.84$ & 832.92 & $1,663.85$ & 832.43 & 15 \\
\hline 6 & 808.41 & 404.71 & 791.38 & 396.20 & & & A & $1,553.81$ & 777.41 & $1,536.78$ & 768.89 & $1,535.80$ & 768.40 & 14 \\
\hline 7 & 905.46 & 453.24 & 888.44 & 444.72 & & & $\mathrm{P}$ & $1,482.77$ & 741.89 & $1,465.74$ & 733.38 & $1,464.76$ & 732.88 & 13 \\
\hline 8 & 992.50 & 496.75 & 975.47 & 488.24 & 974.49 & 487.75 & $\mathrm{~S}$ & $1,385.72$ & 693.36 & $1,368.69$ & 684.85 & $1,367.71$ & 684.36 & 12 \\
\hline 9 & $1,105.58$ & 553.29 & $1,088.55$ & 544.78 & $1,087.57$ & 544.29 & $\mathrm{~L}$ & $1,298.69$ & 649.85 & $1,281.66$ & 641.33 & $1,280.67$ & 640.84 & 11 \\
\hline 10 & $1,176.62$ & 588.81 & $1,159.59$ & 580.30 & $1,158.61$ & 579.81 & A & $1,185.60$ & 593.30 & $1,168.57$ & 584.79 & $1,167.59$ & 584.30 & 10 \\
\hline 11 & $1,305.66$ & 653.33 & $1,288.63$ & 644.82 & $1,287.65$ & 644.33 & E & $1,114.56$ & 557.79 & $1,097.54$ & 549.27 & $1,096.55$ & 548.78 & 9 \\
\hline 12 & $1,419.70$ & 710.35 & $1,402.68$ & 701.84 & $1,401.69$ & 701.35 & $\mathrm{~N}$ & 985.52 & 493.26 & 968.49 & 484.75 & 967.51 & 484.26 & 8 \\
\hline 13 & $1,566.77$ & 783.89 & $1,549.74$ & 775.38 & $1,548.76$ & 774.88 & $\mathrm{~F}$ & 871.48 & 436.24 & 854.45 & 427.73 & 853.47 & 427.24 & 7 \\
\hline 14 & $1,637.81$ & 819.41 & $1,620.78$ & 810.89 & $1,619.80$ & 810.40 & A & 724.41 & 362.71 & 707.38 & 354.20 & 706.40 & 353.70 & 6 \\
\hline 15 & $1,766.85$ & 883.93 & $1,749.82$ & 875.42 & $1,748.84$ & 874.92 & E & 653.37 & 327.19 & 636.35 & 318.68 & 635.36 & 318.18 & 5 \\
\hline 16 & $1,903.91$ & 952.46 & $1,886.88$ & 943.95 & $1,885.90$ & 943.45 & $\mathrm{H}$ & 524.33 & 262.67 & 507.30 & 254.16 & & & 4 \\
\hline
\end{tabular}




\begin{tabular}{|r|r|r|r|r|r|r|r|r|r|r|r|r|r|r|}
17 & $2,002.98$ & $1,001.99$ & $1,985.95$ & 993.48 & $1,984.97$ & 992.99 & $\mathrm{~V}$ & 387.27 & 194.14 & 370.24 & 185.63 & & & 3 \\
\hline 18 & $2,116.06$ & $1,058.53$ & $2,099.04$ & $1,050.02$ & $2,098.05$ & $1,049.53$ & $\mathrm{~L}$ & 288.20 & 144.61 & 271.18 & 136.09 & & & 2 \\
\hline 19 & & & & & & & $\mathrm{R}$ & 175.12 & 88.06 & 158.09 & 79.55 & & & 1 \\
\hline
\end{tabular}

Query 85886 Hit 1

MS/MS Fragmentation of LLFGHSTEGDILELVDGHFDTK

Found in sp|Q9UHY7|ENOPH_HUMAN, Enolase-phosphatase E1 OS=Homo sapiens GN=ENOPH1 PE=1 SV=1

Match to Query 85886: 2730.415from(911.1454,3+)

Title: 770: Scan 2291 ( $\mathrm{r}=59.0828, \mathrm{f}=3, \mathrm{i}=288)$ [D:lab212 $\backslash$ membranelGracelJoyceliTRAQ_46_1.raw]

Data File:Submitted from 20120508-1(merge) by Mascot Daemon on JOYCE-VAIO

Monoisotopic mass of neutral peptide $\mathrm{Mr}$ (calc): 2730.415

Variable modifications:

K22 :TRAQ4plex (K)

Ions Score: 85.64 Expect: 0.000

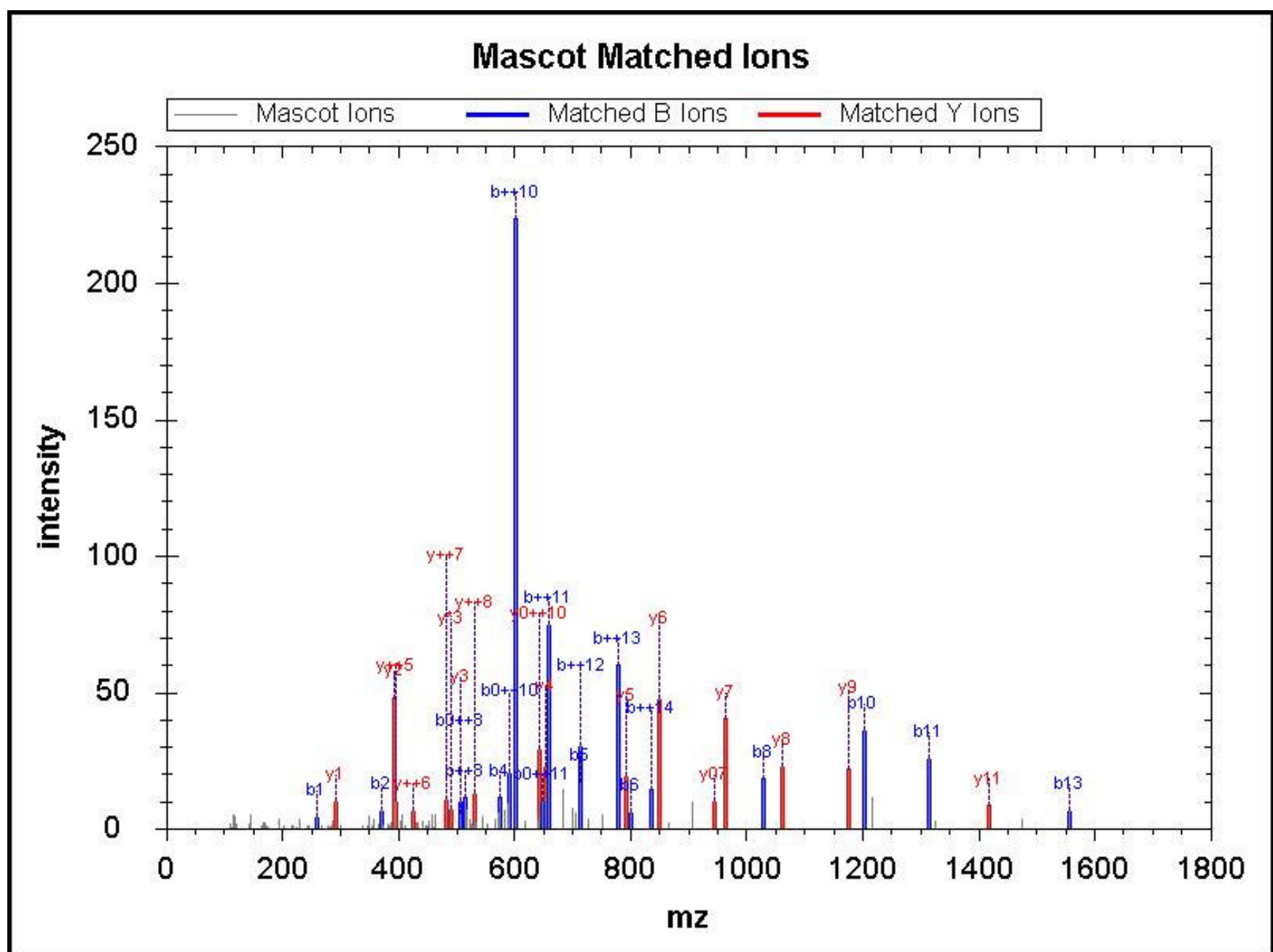

\begin{tabular}{|r|c|c|c|c|c|c|c|c|c|c|r|r|}
\hline \hline $\mathrm{No}$ & $\mathrm{b}$ & $\mathrm{b}++$ & $\mathrm{b} 0$ & $\mathrm{~b} 0++$ & $\mathrm{Seq}$ & $\mathrm{y}$ & $\mathrm{y}++$ & $\mathrm{y}^{*}$ & $\mathrm{y}^{*++}$ & $\mathrm{y} 0$ & $\mathrm{y} 0++$ & $\mathrm{RevNo}$ \\
\hline 1 & 258.19 & 129.60 & & & $\mathrm{~L}$ & & & & & & & 22 \\
\hline 2 & 371.28 & 186.14 & & & $\mathrm{~L}$ & $2,474.23$ & $1,237.62$ & $2,457.21$ & $1,229.11$ & $2,456.22$ & $1,228.61$ & 21 \\
\hline 3 & 518.35 & 259.68 & & & $\mathrm{~F}$ & $2,361.15$ & $1,181.08$ & $2,344.12$ & $1,172.56$ & $2,343.14$ & $1,172.07$ & 20 \\
\hline 4 & 575.37 & 288.19 & & & $\mathrm{G}$ & $2,214.08$ & $1,107.54$ & $2,197.05$ & $1,099.03$ & $2,196.07$ & $1,098.54$ & 19 \\
\hline 5 & 712.43 & 356.72 & & & $\mathrm{H}$ & $2,157.06$ & $1,079.03$ & $2,140.03$ & $1,070.52$ & $2,139.05$ & $1,070.03$ & 18 \\
\hline 6 & 799.46 & 400.23 & 781.45 & 391.23 & $\mathrm{~S}$ & $2,020.00$ & $1,010.50$ & $2,002.97$ & $1,001.99$ & $2,001.99$ & $1,001.50$ & 17 \\
\hline 7 & 900.51 & 450.76 & 882.50 & 441.75 & $\mathrm{~T}$ & $1,932.97$ & 966.99 & $1,915.94$ & 958.47 & $1,914.96$ & 957.98 & 16 \\
\hline 8 & $1,029.55$ & 515.28 & $1,011.54$ & 506.27 & $\mathrm{E}$ & $1,831.92$ & 916.46 & $1,814.89$ & 907.95 & $1,813.91$ & 907.46 & 15 \\
\hline 9 & $1,086.57$ & 543.79 & $1,068.56$ & 534.78 & $\mathrm{G}$ & $1,702.88$ & 851.94 & $1,685.85$ & 843.43 & $1,684.87$ & 842.94 & 14 \\
\hline 10 & $1,201.60$ & 601.30 & $1,183.59$ & 592.30 & $\mathrm{D}$ & $1,645.86$ & 823.43 & $1,628.83$ & 814.92 & $1,627.84$ & 814.43 & 13 \\
\hline 11 & $1,314.68$ & 657.84 & $1,296.67$ & 648.84 & $\mathrm{I}$ & $1,530.83$ & 765.92 & $1,513.80$ & 757.40 & $1,512.82$ & 756.91 & 12 \\
\hline
\end{tabular}




\begin{tabular}{|r|r|r|r|r|r|r|r|r|r|r|r|r|}
12 & $1,427.77$ & 714.39 & $1,409.75$ & 705.38 & $\mathrm{~L}$ & $1,417.74$ & 709.38 & $1,400.72$ & 700.86 & $1,399.73$ & 700.37 & 11 \\
\hline \hline 13 & $1,556.81$ & 778.91 & $1,538.80$ & 769.90 & $\mathrm{E}$ & $1,304.66$ & 652.83 & $1,287.63$ & 644.32 & $1,286.65$ & 643.83 & 10 \\
\hline 14 & $1,669.89$ & 835.45 & $1,651.88$ & 826.44 & $\mathrm{~L}$ & $1,175.62$ & 588.31 & $1,158.59$ & 579.80 & $1,157.61$ & 579.31 & 9 \\
\hline 15 & $1,768.96$ & 884.98 & $1,750.95$ & 875.98 & $\mathrm{~V}$ & $1,062.53$ & 531.77 & $1,045.51$ & 523.26 & $1,044.52$ & 522.77 & 8 \\
\hline 16 & $1,883.99$ & 942.50 & $1,865.98$ & 933.49 & $\mathrm{D}$ & 963.47 & 482.24 & 946.44 & 473.72 & 945.45 & 473.23 & 7 \\
\hline 17 & $1,941.01$ & 971.01 & $1,923.00$ & 962.00 & $\mathrm{G}$ & 848.44 & 424.72 & 831.41 & 416.21 & 830.43 & 415.72 & 6 \\
\hline 18 & $2,078.07$ & $1,039.54$ & $2,060.06$ & $1,030.53$ & $\mathrm{H}$ & 791.42 & 396.21 & 774.39 & 387.70 & 773.41 & 387.21 & 5 \\
\hline 19 & $2,225.14$ & $1,113.07$ & $2,207.13$ & $1,104.07$ & $\mathrm{~F}$ & 654.36 & 327.68 & 637.33 & 319.17 & 636.35 & 318.68 & 4 \\
\hline 20 & $2,340.16$ & $1,170.59$ & $2,322.15$ & $1,161.58$ & $\mathrm{D}$ & 507.29 & 254.15 & 490.26 & 245.64 & 489.28 & 245.14 & 3 \\
\hline 21 & $2,441.21$ & $1,221.11$ & $2,423.20$ & $1,212.10$ & $\mathrm{~T}$ & 392.26 & 196.63 & 375.24 & 188.12 & 374.25 & 187.63 & 2 \\
\hline 22 & & & & & $\mathrm{~K}$ & 291.21 & 146.11 & 274.19 & 137.60 & & & 1 \\
\hline
\end{tabular}

Query 91440 Hit 1

MS/MS Fragmentation of TKEEVAGTLEAVQTIQSITQALQK

Found in sp|Q0JRZ9|FCHO2_HUMAN, FCH domain only protein 2 OS=Homo sapiens GN=FCHO2 PE=1 SV=2

Match to Query 91440: 3017.702from(755.4329,4+)

Title: 1095: Scan 2403 ( $\mathrm{rt}=63.6431, \mathrm{f}=3, \mathrm{i}=367)$ [D:lab212 \membranelGrace JoyceliTRAQ_39_1.raw]

Data File:Submitted from 20120508-1(merge) by Mascot Daemon on JOYCE-VAIO

Monoisotopic mass of neutral peptide $\mathrm{Mr}$ (calc): 3017.702

Variable modifications:

K2 :iTRAQ4plex (K)

K24 :iTRAQ4plex (K)

Ions Score: 85.42 Expect: 0.000

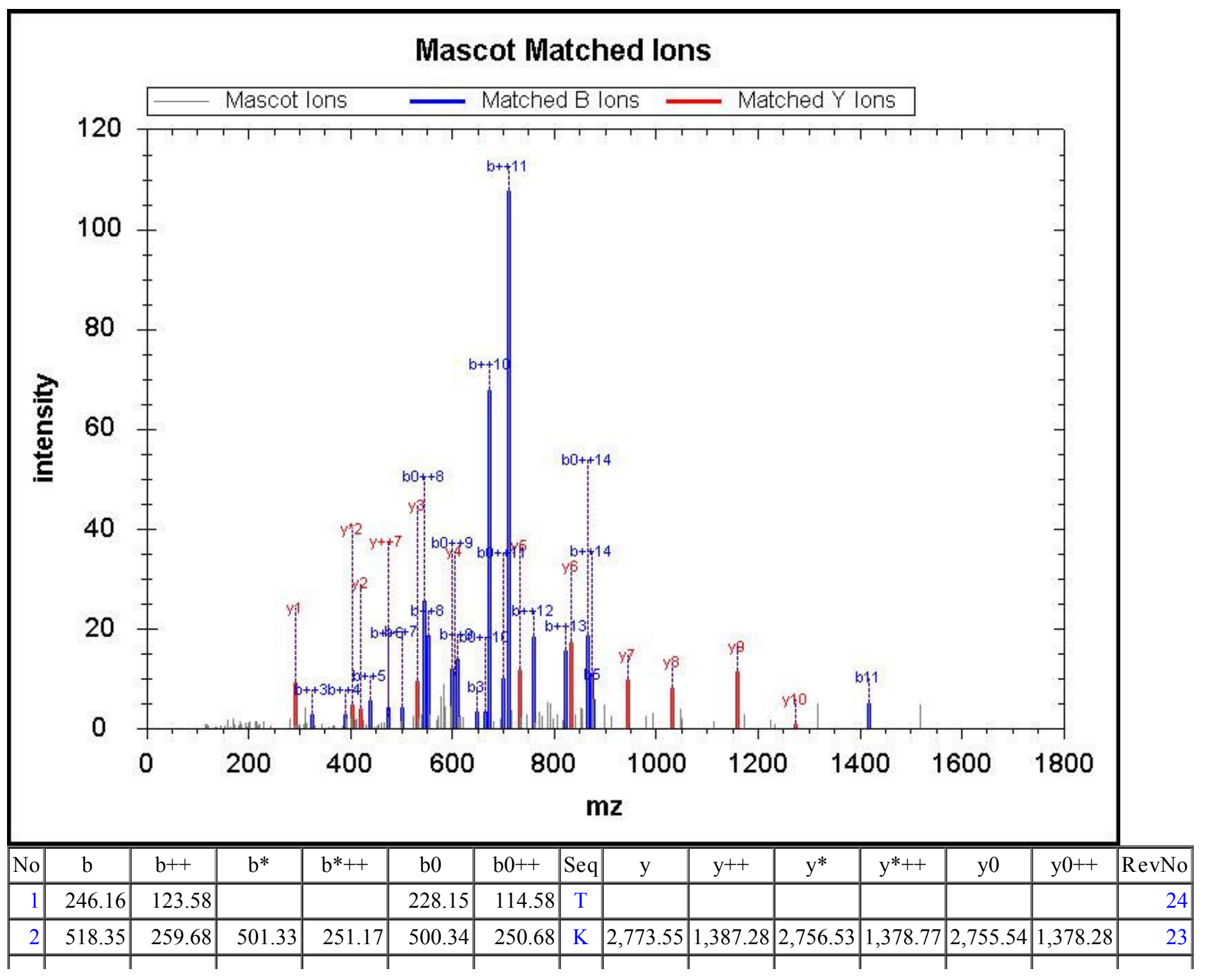




\begin{tabular}{|c|c|c|c|c|c|c|c|c|c|c|c|c|c|c|}
\hline 3 & 647.40 & 324.20 & 630.37 & 315.69 & 629.39 & 315.20 & $\mathrm{E}$ & $|2,501.36|$ & $1,251.18$ & $|2,484.33|$ & $1,242.67$ & $2,483.35 \mid$ & $|1,242.18|$ & 22 \\
\hline 4 & 776.44 & 388.72 & 759.41 & 380.21 & 758.43 & 379.72 & $\mathrm{E}$ & $2,372.32$ & $1,186.66$ & $2,355.29$ & $1,178.15$ & $2,354.30$ & $1,177.66$ & 21 \\
\hline 5 & 875.51 & 438.26 & 858.48 & 429.74 & 857.50 & 429.25 & $\mathrm{~V}$ & $2,243.27$ & $1,122.14$ & $2,226.25$ & $1,113.63$ & $2,225.26$ & $1,113.13$ & 20 \\
\hline 6 & 946.54 & 473.78 & 929.52 & 465.26 & 928.53 & 464.77 & A & $2,144.20$ & $1,072.61$ & $2,127.18$ & $1,064.09$ & $2,126.19$ & $1,063.60$ & \\
\hline 7 & $1,003.57$ & 502.29 & 986.54 & 493.77 & 985.56 & 493.28 & G & $2,073.17$ & $1,037.09$ & $2,056.14$ & $1,028.57$ & $2,055.16$ & $1,028.08$ & 18 \\
\hline 8 & $1,104.61$ & 552.81 & $1,087.59$ & 544.30 & $1,086.60$ & 543.81 & $\mathrm{~T}$ & $2,016.15$ & $1,008.58$ & $1,999.12$ & $1,000.06$ & $1,998.14$ & 999.57 & 17 \\
\hline 9 & $1,217.70$ & 609.35 & $1,200.67$ & 600.84 & $1,199.69$ & 600.35 & $\mathrm{~L}$ & $1,915.10$ & 958.05 & $1,898.07$ & 949.54 & $1,897.09$ & 949.05 & 16 \\
\hline 10 & $1,346.74$ & 673.87 & $1,329.71$ & 665.36 & $1,328.73$ & 664.87 & $\mathrm{E}$ & $1,802.01$ & 901.51 & $1,784.99$ & 893.00 & $1,784.00$ & 892.51 & 15 \\
\hline 11 & $1,417.78$ & 709.39 & $1,400.75$ & 700.88 & $1,399.77$ & 700.39 & A & $1,672.97$ & 836.99 & $1,655.94$ & 828.48 & $1,654.96$ & 827.98 & 4 \\
\hline 12 & $1,516.85$ & 758.93 & $1,499.82$ & 750.41 & $1,498.84$ & 749.92 & V & $1,601.93$ & 801.47 & $1,584.91$ & 792.96 & $1,583.92$ & 792.47 & 13 \\
\hline 13 & $1,644.90$ & 822.96 & $1,627.88$ & 814.44 & $1,626.89$ & 813.95 & Q & $1,502.87$ & 751.94 & $1,485.84$ & 743.42 & $1,484.86$ & 742.93 & 12 \\
\hline 14 & $1,745.95$ & 873.48 & $1,728.93$ & 864.97 & $1,727.94$ & 864.47 & $\mathrm{~T}$ & $1,374.81$ & 687.91 & $1,357.78$ & 679.39 & $1,356.80$ & 678.90 & 11 \\
\hline 15 & $1,859.04$ & 930.02 & $1,842.01$ & 921.51 & $1,841.03$ & 921.02 & $\mathrm{I}$ & $1,273.76$ & 637.38 & $1,256.73$ & 628.87 & $1,255.75$ & 628.38 & 10 \\
\hline 16 & $1,987.10$ & 994.05 & $1,970.07$ & 985.54 & $1,969.08$ & 985.05 & Q & $1,160.68$ & 580.84 & $1,143.65$ & 572.33 & $1,142.66$ & 571.84 & ? \\
\hline 17 & $2,074.13$ & $1,037.57$ & $2,057.10$ & $1,029.05$ & $2,056.12$ & $1,028.56$ & $\mathrm{~S}$ & $1,032.62$ & 516.81 & $1,015.59$ & 508.30 & $1,014.61$ & 507.81 & 8 \\
\hline 18 & $2,187.21$ & $1,094.11$ & $2,170.18$ & $1,085.60$ & $2,169.20$ & $1,085.10$ & I & 945.58 & 473.30 & 928.56 & 464.78 & 927.57 & 464.29 & 7 \\
\hline 19 & $2,288.26$ & $1,144.63$ & $2,271.23$ & $1,136.12$ & $2,270.25$ & $1,135.63$ & $\mathrm{~T}$ & 832.50 & 416.75 & 815.47 & 408.24 & 814.49 & 407.75 & 5 \\
\hline 20 & $2,416.32$ & $1,208.66$ & $2,399.29$ & $1,200.15$ & $2,398.31$ & $1,199.66$ & Q & 731.45 & 366.23 & 714.43 & 357.72 & & & 5 \\
\hline 21 & $2,487.35$ & $1,244.18$ & $2,470.33$ & $1,235.67$ & $2,469.34$ & $1,235.18$ & A & 603.39 & 302.20 & 586.37 & 293.69 & & & 4 \\
\hline 22 & $2,600.44$ & $1,300.72$ & $2,583.41$ & $1,292.21$ & $2,582.43$ & $1,291.72$ & $\mathrm{~L}$ & 532.36 & 266.68 & 515.33 & 258.17 & & & 3 \\
\hline 23 & $2,728.50$ & $1,364.75$ & $2,711.47$ & $1,356.24$ & $2,710.49$ & $1,355.75$ & Q & 419.27 & 210.14 & 402.25 & 201.63 & & & 2 \\
\hline 24 & & & & & & & $\mathrm{~K}$ & 291.21 & 146.11 & 274.19 & 137.60 & & & 1 \\
\hline
\end{tabular}

Query 85432 Hit 1

\section{MS/MS Fragmentation of DLNLAGTAEVGLAGYFMDHTVAFR}

Found in sp|Q9NP81|SYSM_HUMAN, Serine--tRNA ligase

Match to Query 85432: 2711.352from(904.7912,3+)

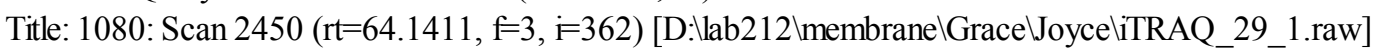

Data File:Submitted from 20120508-1(merge) by Mascot Daemon on JOYCE-VAIO

Monoisotopic mass of neutral peptide $\mathrm{Mr}$ (calc): 2711.352

Variable modifications:

Ions Score: 85.27 Expect: 0.000 
Mascot Matched lons

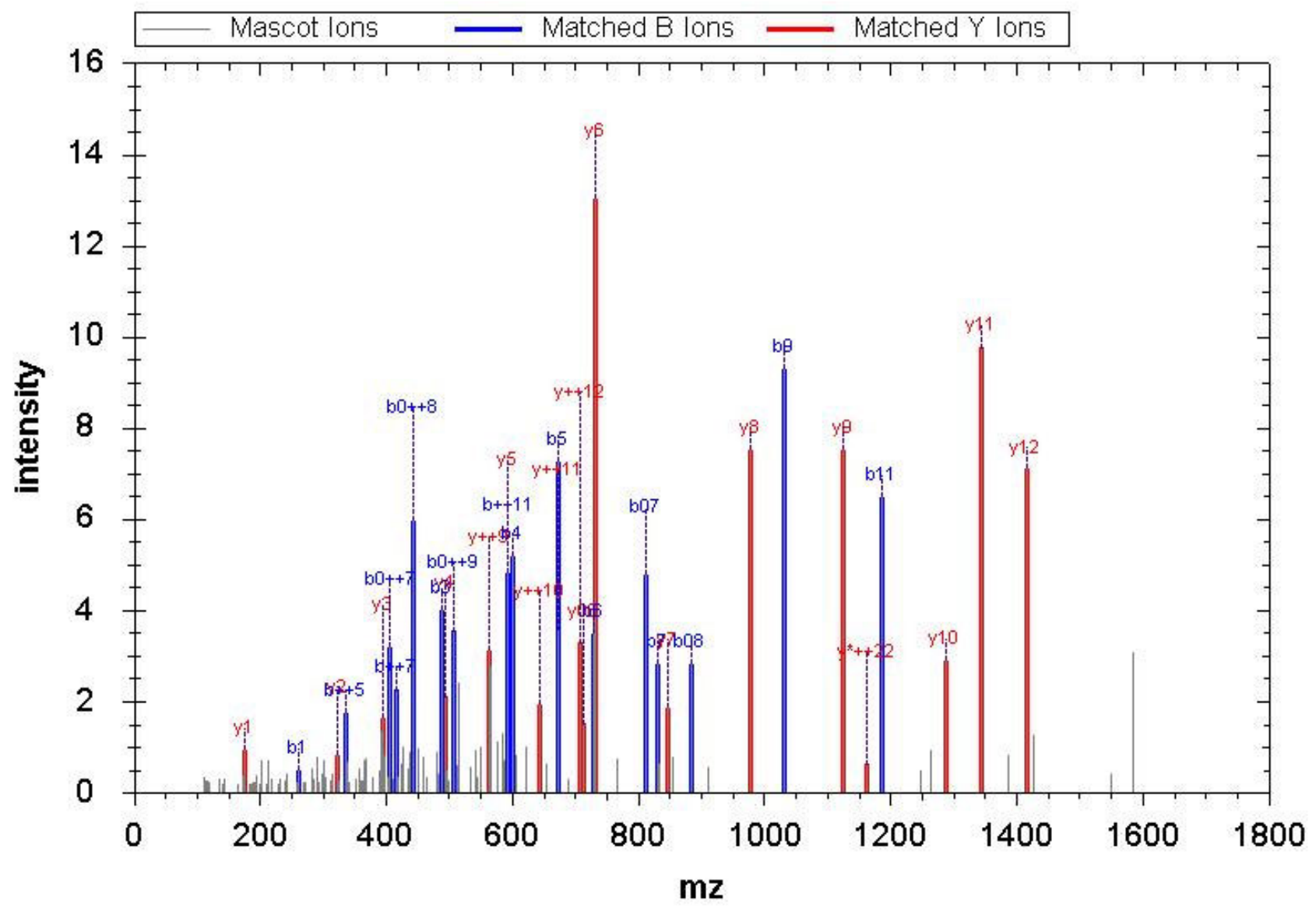

\begin{tabular}{|c|c|c|c|c|c|c|c|c|c|c|c|c|c|c|}
\hline No & $b$ & $\mathrm{~b}++$ & $b^{*}$ & $b^{*++}$ & b0 & $\mathrm{b} 0++$ & Seq & $\mathrm{y}$ & $\mathrm{y}++$ & $y^{*}$ & $y^{*++}$ & y0 & $\mathrm{y} 0++$ & RevNo \\
\hline 1 & 260.14 & 130.57 & & & 242.13 & 121.57 & $\mathrm{D}$ & & & & & & & 24 \\
\hline 2 & 373.22 & 187.11 & & & 355.21 & 178.11 & $\mathrm{~L}$ & $2,453.23$ & $1,227.12$ & $2,436.20$ & 60 & $2,435.22$ & $1,218.11$ & 23 \\
\hline 3 & 487.26 & 244.14 & 470.24 & 235.62 & 469.25 & 235.13 & $\mathrm{~N}$ & $2,340.14$ & $1,170.58$ & $2,323.12$ & $1,162.06$ & $2,322.13$ & $1,161.57$ & 22 \\
\hline 4 & 600.35 & 300.68 & 583.32 & 292.16 & 582.34 & 291.67 & $\mathrm{~L}$ & $2,226.10$ & $1,113.55$ & $2,209.07$ & $1,105.04$ & $2,208.09$ & $1,104.55$ & 21 \\
\hline 5 & 671.38 & 336.20 & 654.36 & 327.68 & 653.37 & 327.19 & A & $2,113.02$ & $1,057.01$ & $2,095.99$ & .50 & $2,095.01$ & $1,048.01$ & 20 \\
\hline 6 & 728.41 & 364.71 & 711.38 & 356.19 & 710.40 & 355.70 & G & $2,041.98$ & $1,021.49$ & $2,024.95$ & $1,012.98$ & $2,023.97$ & $1,012.49$ & 19 \\
\hline 7 & 829.45 & 415.23 & 812.43 & 406.72 & 811.44 & 406.23 & $\mathrm{~T}$ & $1,984.96$ & 992.98 & $1,967.93$ & 984.47 & $1,966.95$ & 983.98 & 18 \\
\hline 8 & 900.49 & 450.75 & 883.46 & 442.24 & 882.48 & 441.74 & A & $1,883.91$ & 942.46 & $1,866.88$ & 933.95 & $1,865.90$ & 933.45 & 17 \\
\hline 9 & $1,029.53$ & 515.27 & $1,012.51$ & 506.76 & $1,011.52$ & 506.27 & $\mathrm{E}$ & $1,812.87$ & 906.94 & 5.85 & .43 & $1,794.86$ & 897.94 & 16 \\
\hline 10 & $1,128.60$ & 564.80 & $1,111.58$ & 556.29 & $1,110.59$ & 555.80 & V & $1,683.83$ & 842.42 & $1,666.80$ & 833.91 & $1,665.82$ & 833.41 & 15 \\
\hline 11 & $1,185.62$ & 593.32 & $1,168.60$ & 584.80 & $1,167.61$ & 584.31 & G & $1,584.76$ & 792.89 & $1,567.74$ & 784.37 & $1,566.75$ & 783.88 & 14 \\
\hline 12 & $1,298.71$ & 649.86 & $1,281.68$ & 641.34 & $1,280.70$ & 640.85 & $\mathrm{~L}$ & $1,527.74$ & 764.37 & $1,510.71$ & 755.86 & $1,509.73$ & 755.37 & 13 \\
\hline 13 & $1,369.74$ & 685.38 & $1,352.72$ & 676.86 & $1,351.73$ & 676.37 & A & $1,414.66$ & 707.83 & $1,397.63$ & 699.32 & $1,396.65$ & 698.83 & 12 \\
\hline 14 & $1,426.77$ & 713.89 & $1,409.74$ & 705.37 & $1,408.76$ & 704.88 & G & $1,343.62$ & 672.31 & $1,326.59$ & 663.80 & $1,325.61$ & 663.31 & 11 \\
\hline 15 & $1,589.83$ & 795.42 & $1,572.80$ & 786.91 & $1,571.82$ & 786.41 & $\mathrm{Y}$ & $1,286.60$ & 643.80 & $1,269.57$ & 635.29 & $1,268.59$ & 634.80 & 10 \\
\hline 16 & $1,736.90$ & 868.95 & $1,719.87$ & 860.44 & $1,718.89$ & 859.95 & $\mathrm{~F}$ & $1,123.54$ & 562.27 & $1,106.51$ & 553.76 & $1,105.52$ & 553.27 & 9 \\
\hline 17 & $1,867.94$ & 934.47 & $1,850.91$ & 925.96 & $1,849.93$ & 925.47 & M & 976.47 & 488.74 & 959.44 & 480.22 & 958.46 & 479.73 & 8 \\
\hline 18 & $1,982.97$ & 991.99 & $1,965.94$ & 983.47 & $1,964.95$ & 982.98 & D & 845.43 & 423.22 & 828.40 & 414.70 & 827.42 & 414.21 & 7 \\
\hline 19 & $2,120.02$ & $1,060.52$ & $2,103.00$ & $1,052.00$ & $2,102.01$ & $1,051.51$ & $\mathrm{H}$ & 40 & 70 & 37 & 19 & 712.39 & 356.70 & 6 \\
\hline 20 & $2,221.07$ & $1,111.04$ & $2,204.05$ & $1,102.53$ & $2,203.06$ & $1,102.03$ & $\mathrm{~T}$ & 593.34 & 297.17 & 576.31 & 288.66 & 575.33 & 288.17 & 5 \\
\hline 21 & $2,320.14$ & $1,160.57$ & $2,303.11$ & $1,152.06$ & $2,302.13$ & $1,151.57$ & $\mathrm{~V}$ & 492.29 & 246.65 & 475.27 & 238.14 & & & 4 \\
\hline 22 & $2,391.18$ & $1,196.09$ & $2,374.15$ & $1,187.58$ & $2,373.17$ & $1,187.09$ & A & 393.22 & 197.12 & 376.20 & 188.60 & & & 3 \\
\hline 23 & $2,538.25$ & $1,269.63$ & $2,521.22$ & $1,261.11$ & $2,520.24$ & $1,260.62$ & $\mathrm{~F}$ & 322.19 & 161.60 & 305.16 & 153.08 & & & 2 \\
\hline
\end{tabular}


Query 90123 Hit 1

MS/MS Fragmentation of EVTSHFQVTLNDIQLQMEQHNER

Found in sp|P40222|TXLNA_HUMAN, Alpha-taxilin OS=Homo sapiens GN=TXLNA PE=1 SV=3

Match to Query 90123: 2939.437from(735.8665,4+)

Title: 743: Scan 1669 ( $\mathrm{rt}=47.1845, \mathrm{f}=2, \mathrm{i}=270$ ) [D:llab212 $\backslash$ membranelGracelJoyceliTRAQ_44_1.raw]

Data File:Submitted from 20120508-1(merge) by Mascot Daemon on JOYCE-VAIO

Monoisotopic mass of neutral peptide $\mathrm{Mr}$ (calc): 2939.437

Variable modifications:

Ions Score: 85.16 Expect: 0.000

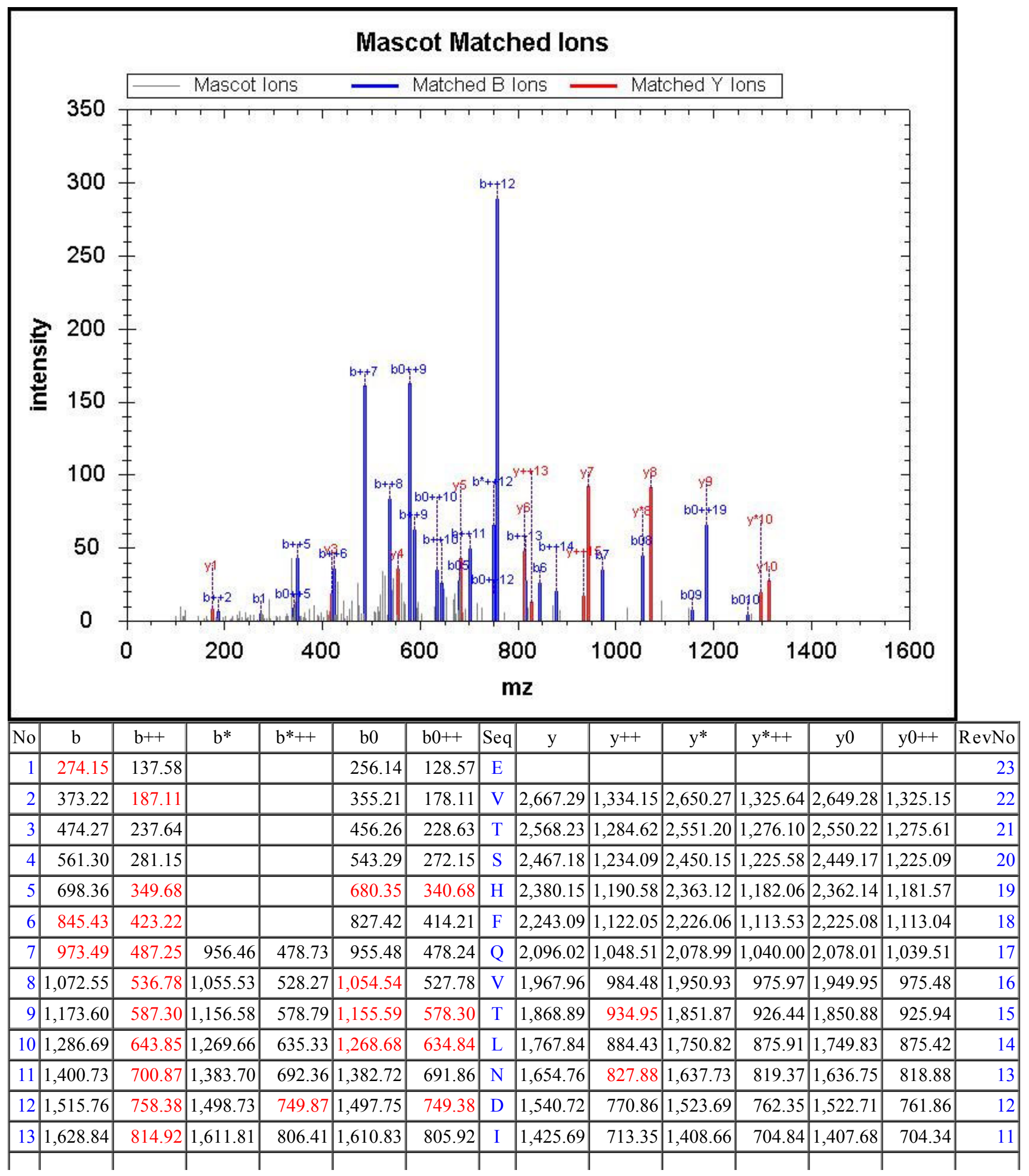




\begin{tabular}{|r|r|r|r|r|r|r|r|r|r|r|r|r|r|r|r|}
14 & $1,756.90$ & 878.95 & $1,739.87$ & 870.44 & $1,738.89$ & 869.95 & $\mathrm{Q}$ & $1,312.61$ & 656.81 & $1,295.58$ & 648.29 & $1,294.60$ & 647.80 & 10 \\
\hline \hline 15 & $1,869.98$ & 935.50 & $1,852.96$ & 926.98 & $1,851.97$ & 926.49 & $\mathrm{~L}$ & $1,184.55$ & 592.78 & $1,167.52$ & 584.26 & $1,166.54$ & 583.77 & 9 \\
\hline 16 & $1,998.04$ & 999.52 & $1,981.02$ & 991.01 & $1,980.03$ & 990.52 & $\mathrm{Q}$ & $1,071.46$ & 536.24 & $1,054.44$ & 527.72 & $1,053.45$ & 527.23 & 8 \\
\hline 17 & $2,129.08$ & $1,065.04$ & $2,112.06$ & $1,056.53$ & $2,111.07$ & $1,056.04$ & $\mathrm{M}$ & 943.41 & 472.21 & 926.38 & 463.69 & 925.39 & 463.20 & 7 \\
\hline 18 & $2,258.12$ & $1,129.57$ & $2,241.10$ & $1,121.05$ & $2,240.11$ & $1,120.56$ & $\mathrm{E}$ & 812.36 & 406.69 & 795.34 & 398.17 & 794.35 & 397.68 & 6 \\
\hline 19 & $2,386.18$ & $1,193.60$ & $2,369.16$ & $1,185.08$ & $2,368.17$ & $1,184.59$ & $\mathrm{Q}$ & 683.32 & 342.16 & 666.30 & 333.65 & 665.31 & 333.16 & 5 \\
\hline 20 & $2,523.24$ & $1,262.12$ & $2,506.22$ & $1,253.61$ & $2,505.23$ & $1,253.12$ & $\mathrm{H}$ & 555.26 & 278.14 & 538.24 & 269.62 & 537.25 & 269.13 & 4 \\
\hline 21 & $2,637.28$ & $1,319.15$ & $2,620.26$ & $1,310.63$ & $2,619.27$ & $1,310.14$ & $\mathrm{~N}$ & 418.20 & 209.61 & 401.18 & 201.09 & 400.19 & 200.60 & 3 \\
\hline 22 & $2,766.33$ & $1,383.67$ & $2,749.30$ & $1,375.15$ & $2,748.32$ & $1,374.66$ & $\mathrm{E}$ & 304.16 & 152.58 & 287.13 & 144.07 & 286.15 & 143.58 & 2 \\
\hline 23 & & & & & & & $\mathrm{R}$ & 175.12 & 88.06 & 158.09 & 79.55 & & & 1 \\
\hline
\end{tabular}

Query 58674 Hit 1

MS/MS Fragmentation of QGTFHSQQALEYGTK

Found in sp|P53597|SUCA_HUMAN, Succinyl-CoA ligase [ADP/GDP-forming] subunit alpha

Match to Query 58674: 1982.015from(661.6788,3+)

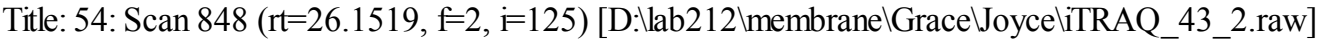

Data File:Submitted from 20120508-1(merge) by Mascot Daemon on JOYCE-VAIO

Monoisotopic mass of neutral peptide Mr(calc): 1982.015

Variable modifications:

K15 :iTRAQ4plex (K)

Ions Score: 83.82 Expect: 0.000

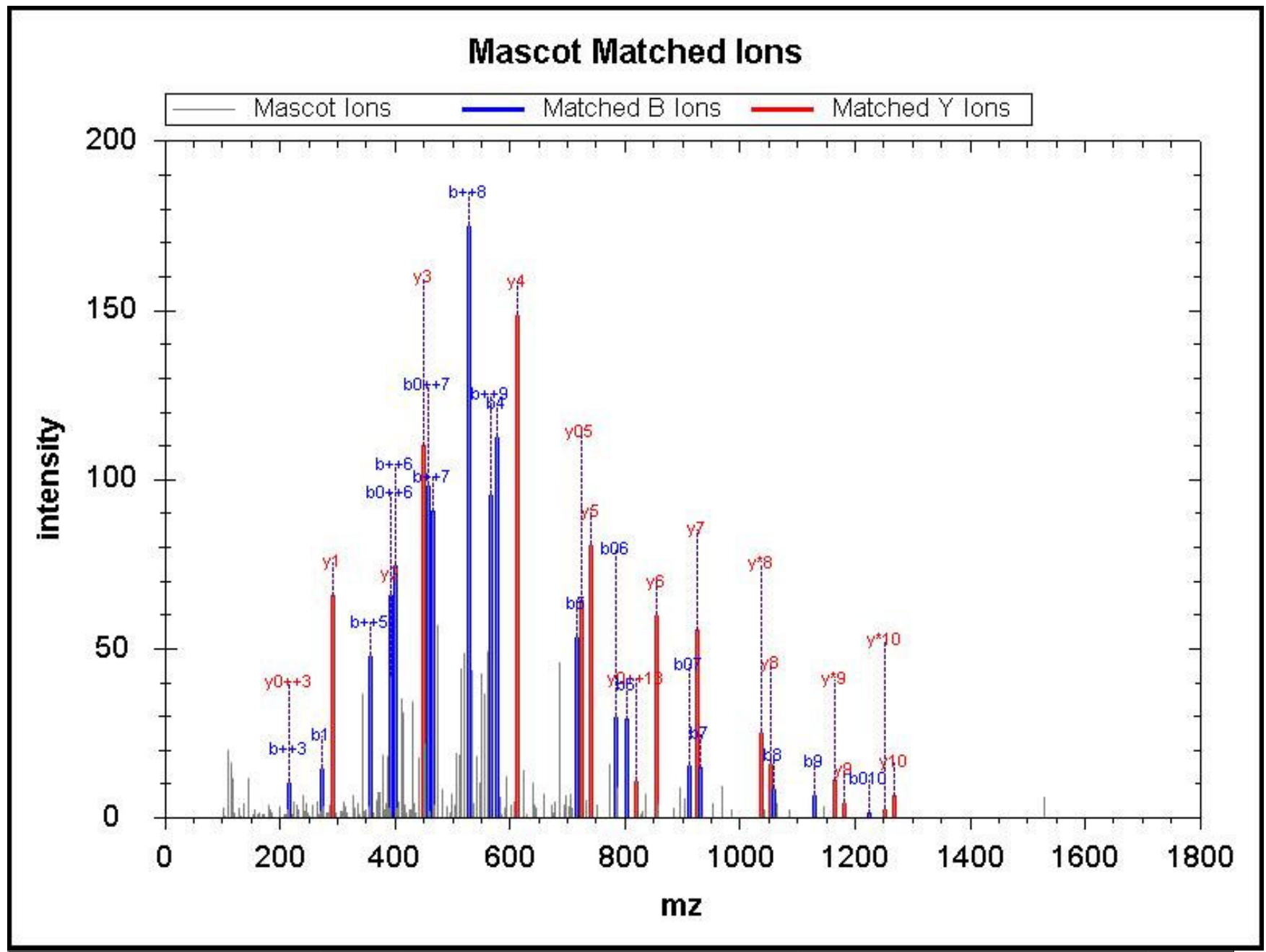

\begin{tabular}{|r|c|c|c|c|c|c|c|c|c|c|c|r|r|r|}
\hline \hline No & $\mathrm{b}$ & $\mathrm{b}++$ & $\mathrm{b}^{*}$ & $\mathrm{~b}^{*++}$ & $\mathrm{b} 0$ & $\mathrm{~b} 0++$ & Seq & $\mathrm{y}$ & $\mathrm{y}++$ & $\mathrm{y}^{*}$ & $\mathrm{y}^{*++}$ & $\mathrm{y} 0$ & $\mathrm{y} 0++$ & $\mathrm{RevNo}$ \\
\hline 1 & 273.17 & 137.09 & 256.14 & 128.57 & & & $\mathrm{Q}$ & & & & & & & 15 \\
\hline 2 & 330.19 & 165.60 & 313.16 & 157.09 & & & $\mathrm{G}$ & $1,710.86$ & 855.93 & $1,693.83$ & 847.42 & $1,692.85$ & 846.93 & 14 \\
\hline 3 & 431.24 & 216.12 & 414.21 & 207.61 & 413.23 & 207.12 & $\mathrm{~T}$ & $1,653.84$ & 827.42 & $1,636.81$ & 818.91 & $1,635.82$ & 818.42 & 13 \\
\hline 4 & 578.31 & 289.66 & 561.28 & 281.14 & 560.29 & 280.65 & $\mathrm{~F}$ & $1,552.79$ & 776.90 & $1,535.76$ & 768.38 & $1,534.78$ & 767.89 & 12 \\
\hline
\end{tabular}




\begin{tabular}{|r|r|r|r|r|r|r|r|r|r|r|r|r|r|r|r|}
\hline 5 & 715.36 & 358.19 & 698.34 & 349.67 & 697.35 & 349.18 & $\mathrm{H}$ & $1,405.72$ & 703.36 & $1,388.69$ & 694.85 & $1,387.71$ & 694.36 & 11 \\
\hline 6 & 802.40 & 401.70 & 785.37 & 393.19 & 784.39 & 392.70 & $\mathrm{~S}$ & $1,268.66$ & 634.83 & $1,251.63$ & 626.32 & $1,250.65$ & 625.83 & 10 \\
\hline 7 & 930.46 & 465.73 & 913.43 & 457.22 & 912.44 & 456.73 & $\mathrm{Q}$ & $1,181.63$ & 591.32 & $1,164.60$ & 582.80 & $1,163.62$ & 582.31 & 9 \\
\hline 8 & $1,058.51$ & 529.76 & $1,041.49$ & 521.25 & $1,040.50$ & 520.76 & $\mathrm{Q}$ & $1,053.57$ & 527.29 & $1,036.54$ & 518.78 & $1,035.56$ & 518.28 & 8 \\
\hline 9 & $1,129.55$ & 565.28 & $1,112.52$ & 556.77 & $1,111.54$ & 556.27 & $\mathrm{~A}$ & 925.51 & 463.26 & 908.48 & 454.75 & 907.50 & 454.25 & 7 \\
\hline 10 & $1,242.63$ & 621.82 & $1,225.61$ & 613.31 & $1,224.62$ & 612.82 & $\mathrm{~L}$ & 854.47 & 427.74 & 837.45 & 419.23 & 836.46 & 418.74 & 6 \\
\hline 11 & $1,371.68$ & 686.34 & $1,354.65$ & 677.83 & $1,353.67$ & 677.34 & $\mathrm{E}$ & 741.39 & 371.20 & 724.36 & 362.69 & 723.38 & 362.19 & 5 \\
\hline 12 & $1,534.74$ & 767.87 & $1,517.71$ & 759.36 & $1,516.73$ & 758.87 & $\mathrm{Y}$ & 612.35 & 306.68 & 595.32 & 298.16 & 594.34 & 297.67 & 4 \\
\hline 13 & $1,591.76$ & 796.38 & $1,574.74$ & 787.87 & $1,573.75$ & 787.38 & $\mathrm{G}$ & 449.28 & 225.15 & 432.26 & 216.63 & 431.27 & 216.14 & 3 \\
\hline 14 & $1,692.81$ & 846.91 & $1,675.78$ & 838.40 & $1,674.80$ & 837.90 & $\mathrm{~T}$ & 392.26 & 196.63 & 375.24 & 188.12 & 374.25 & 187.63 & 2 \\
\hline 15 & & & & & & & $\mathrm{~K}$ & 291.21 & 146.11 & 274.19 & 137.60 & & & 1 \\
\hline
\end{tabular}

Query 87723 Hit 1

\section{MS/MS Fragmentation of IFHDLDMLTTVQEENEPVIYNR}

Found in sp|P83111|LACTB_HUM AN, Serine beta-lactamase-like protein LACTB

Match to Query 87723: 2819.386from(940.8027,3+)

Title: 886: Sum of 2 scans in range $1927(\mathrm{rt}=53.0853, \mathrm{f}=4, \mathrm{i}=595)$ to $1928(\mathrm{rt}=53.1107, \mathrm{f}=4, \mathrm{i}=596)$

[D:llab212 \membranelGrace JoyceliTRAQ_33_2.raw]

Data File:Submitted from 20120508-1(merge) by Mascot Daemon on JOYCE-VAIO

Monoisotopic mass of neutral peptide $\operatorname{Mr}($ calc): 2819.386

Variable modifications:

Ions Score: 83.6 Expect: 0.000

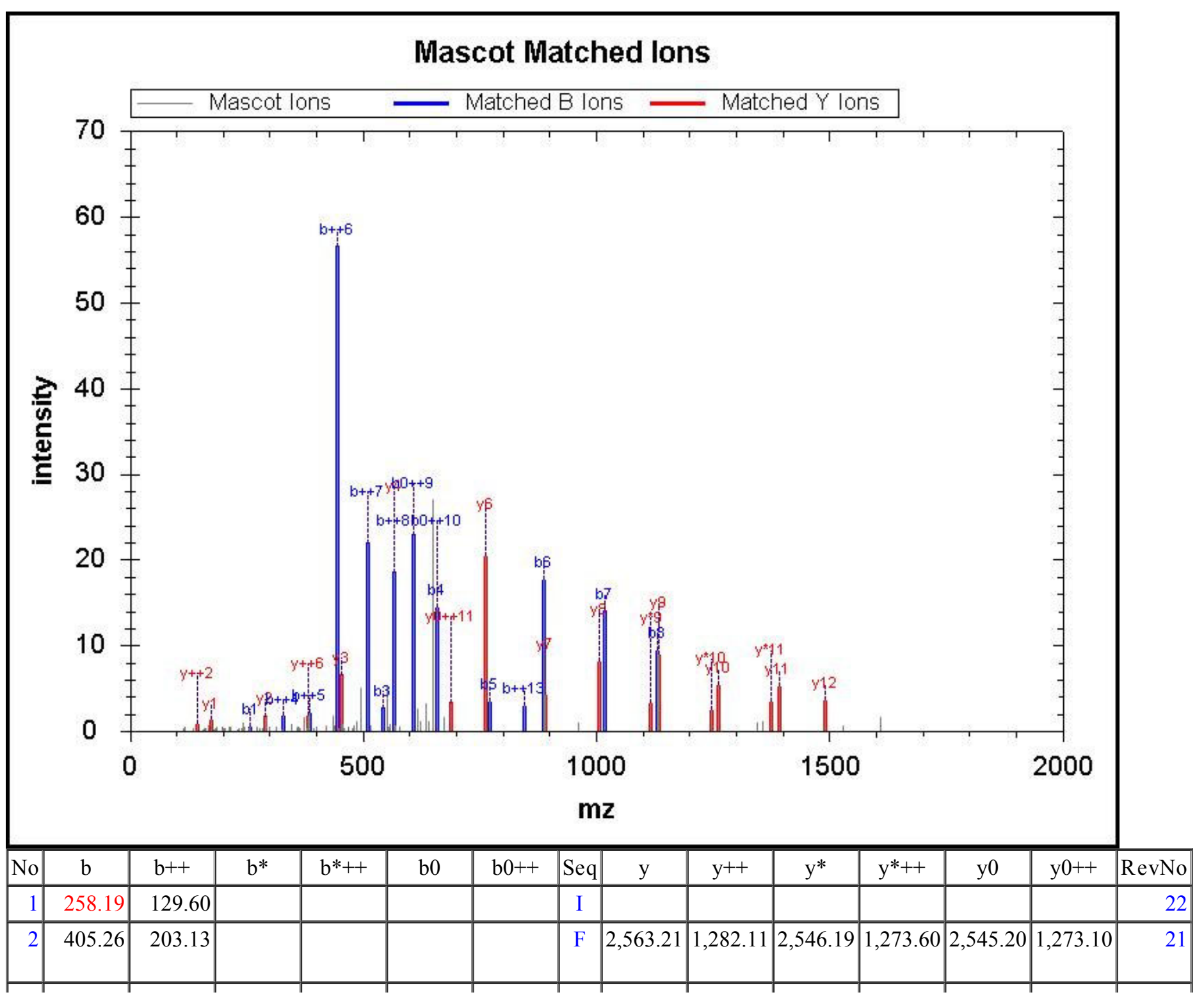




\begin{tabular}{|c|c|c|c|c|c|c|c|c|c|c|c|c|c|c|}
\hline 3 & 542.32 & 271.66 & & & & & $\mathrm{H}$ & $2,416.14$ & $|1,208.58|$ & $|2,399.12|$ & $1,200.06$ & $2,398.13$ & $1,199.57$ & 20 \\
\hline 4 & 657.35 & 329.18 & & & 639.34 & 320.17 & $\mathrm{D}$ & $2,279.09$ & $1,140.05$ & $2,262.06$ & $1,131.53$ & $2,261.08$ & $1,131.04$ & 19 \\
\hline 5 & 770.43 & 385.72 & & & 752.42 & 376.71 & $\mathrm{~L}$ & $2,164.06$ & $1,082.53$ & $2,147.03$ & $1,074.02$ & $2,146.05$ & $1,073.53$ & 18 \\
\hline 6 & 885.46 & 443.23 & & & 867.45 & 434.23 & $\mathrm{D}$ & $2,050.98$ & $1,025.99$ & $2,033.95$ & $1,017.48$ & $2,032.96$ & $1,016.99$ & 17 \\
\hline 7 & $1,016.50$ & 508.75 & & & 998.49 & 499.75 & $\mathrm{M}$ & $1,935.95$ & 968.48 & $1,918.92$ & 959.96 & $1,917.94$ & 959.47 & 16 \\
\hline 8 & $1,129.58$ & 565.30 & & & $1,111.57$ & 556.29 & $\mathrm{~L}$ & $1,804.91$ & 902.96 & $1,787.88$ & 894.44 & $1,786.90$ & 893.95 & 15 \\
\hline 9 & $1,230.63$ & 615.82 & & & $1,212.62$ & 606.81 & $\mathrm{~T}$ & $1,691.82$ & 846.42 & $1,674.80$ & 837.90 & $1,673.81$ & 837.41 & 14 \\
\hline 10 & $1,331.68$ & 666.34 & & & $1,313.67$ & 657.34 & $\mathrm{~T}$ & $1,590.78$ & 795.89 & $1,573.75$ & 787.38 & $1,572.77$ & 786.89 & 13 \\
\hline 11 & $1,430.75$ & 715.88 & & & $1,412.74$ & 706.87 & $\mathrm{~V}$ & $1,489.73$ & 745.37 & $1,472.70$ & 736.85 & $1,471.72$ & 736.36 & 12 \\
\hline 12 & $1,558.81$ & 779.91 & $1,541.78$ & 771.39 & $1,540.80$ & 770.90 & Q & $1,390.66$ & 695.83 & $1,373.63$ & 687.32 & $1,372.65$ & 686.83 & 11 \\
\hline 13 & $1,687.85$ & 844.43 & $1,670.82$ & 835.91 & $1,669.84$ & 835.42 & $\mathrm{E}$ & $1,262.60$ & 631.80 & $1,245.57$ & 623.29 & $1,244.59$ & 622.80 & 10 \\
\hline 14 & $1,816.89$ & 908.95 & $1,799.86$ & 900.44 & $1,798.88$ & 899.94 & $\mathrm{E}$ & $1,133.56$ & 567.28 & $1,116.53$ & 558.77 & $1,115.55$ & 558.28 & 9 \\
\hline 15 & $1,930.93$ & 965.97 & $1,913.91$ & 957.46 & $1,912.92$ & 956.97 & $\mathrm{~N}$ & $1,004.52$ & 502.76 & 987.49 & 494.25 & 986.51 & 493.76 & 5 \\
\hline 16 & $2,059.98$ & $1,030.49$ & $2,042.95$ & $1,021.98$ & $2,041.97$ & $1,021.49$ & $\mathrm{E}$ & 890.47 & 445.74 & 873.45 & 437.23 & 872.46 & 436.73 & 1 \\
\hline 17 & $2,157.03$ & $1,079.02$ & $2,140.00$ & $1,070.50$ & $2,139.02$ & $1,070.01$ & $\mathrm{P}$ & 761.43 & 381.22 & 744.40 & 372.71 & & & b \\
\hline 18 & $2,256.10$ & $1,128.55$ & $2,239.07$ & $1,120.04$ & $2,238.09$ & $1,119.55$ & $\mathrm{~V}$ & 664.38 & 332.69 & 647.35 & 324.18 & & & D \\
\hline 19 & $2,369.18$ & $1,185.09$ & $2,352.15$ & $1,176.58$ & $2,351.17$ & $1,176.09$ & $\mathrm{I}$ & 565.31 & 283.16 & 548.28 & 274.64 & & & + \\
\hline 20 & $2,532.24$ & $1,266.63$ & $2,515.22$ & $1,258.11$ & $2,514.23$ & $1,257.62$ & $\mathrm{Y}$ & 452.23 & 226.62 & 435.20 & 218.10 & & & 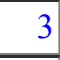 \\
\hline 21 & $2,646.29$ & $1,323.65$ & $2,629.26$ & $1,315.13$ & $2,628.28$ & $1,314.64$ & $\mathrm{~N}$ & 289.16 & 145.08 & 272.14 & 136.57 & & & 2 \\
\hline 22 & & & & & & & $\mathrm{R}$ & 175.12 & 88.06 & 158.09 & 79.55 & & & 1 \\
\hline
\end{tabular}

\section{Query 47910 Hit 1}

\section{MS/MS Fragmentation of HTLADNFNPVSEER}

Found in sp|P20645|MPRD_HUM AN, Cation-dependent mannose-6-phosphate receptor OS=Homo sapiens GN=M6PR PE=1 SV=1 Match to Query 47910: 1771.863from(591.6283,3+)

Title: 423: Scan 1002 (rt=32.0729, f=3, i=141) [D:lab212 \membranelGrace JoyceliTRAQ_39_1.raw]

Data File:Submitted from 20120508-1(merge) by Mascot Daemon on JOYCE-VAIO

Monoisotopic mass of neutral peptide $\mathrm{Mr}$ (calc): 1771.863

Variable modifications:

Ions Score: 83.43 Expect: 0.000 


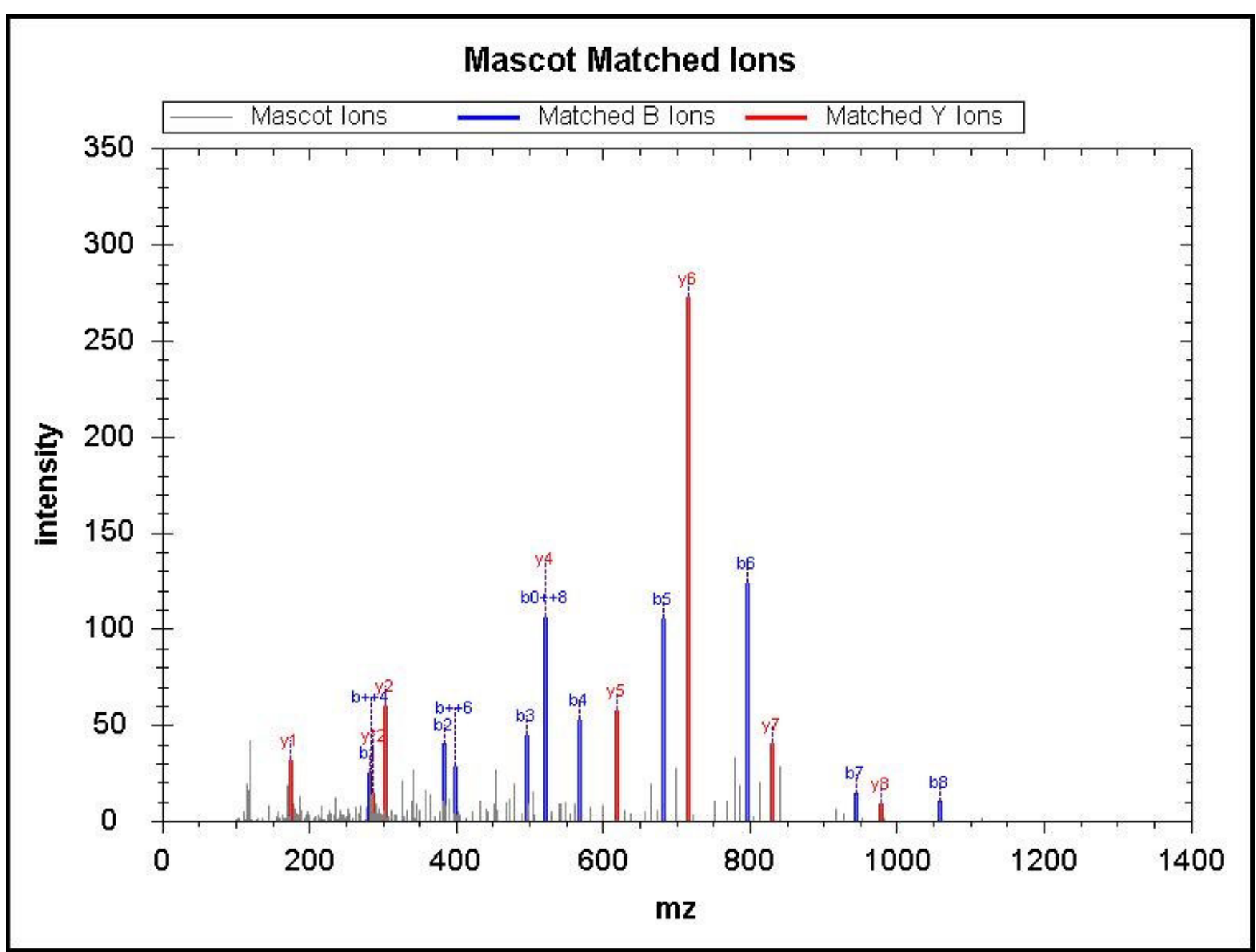

\begin{tabular}{|r|r|r|r|r|r|r|r|r|r|r|r|r|r|r|r|}
\hline \hline No & $\mathrm{b}$ & $\mathrm{b}++$ & $\mathrm{b}^{*}$ & $\mathrm{~b}^{*++}$ & $\mathrm{b} 0$ & $\mathrm{~b} 0++$ & $\mathrm{Seq}$ & $\mathrm{y}$ & $\mathrm{y}++$ & $\mathrm{y}^{*}$ & $\mathrm{y}^{*++}$ & $\mathrm{y} 0$ & $\mathrm{y} 0++$ & $\mathrm{RevNo}$ \\
\hline 1 & 282.17 & 141.59 & & & & & $\mathrm{H}$ & & & & & & & 14 \\
\hline 2 & 383.22 & 192.11 & & & 365.21 & 183.11 & $\mathrm{~T}$ & $1,491.71$ & 746.36 & $1,474.68$ & 737.84 & $1,473.70$ & 737.35 & 13 \\
\hline 3 & 496.30 & 248.65 & & & 478.29 & 239.65 & $\mathrm{~L}$ & $1,390.66$ & 695.83 & $1,373.63$ & 687.32 & $1,372.65$ & 686.83 & 12 \\
\hline 4 & 567.34 & 284.17 & & & 549.33 & 275.17 & $\mathrm{~A}$ & $1,277.58$ & 639.29 & $1,260.55$ & 630.78 & $1,259.57$ & 630.29 & 11 \\
\hline 5 & 682.36 & 341.69 & & & 664.35 & 332.68 & $\mathrm{D}$ & $1,206.54$ & 603.77 & $1,189.51$ & 595.26 & $1,188.53$ & 594.77 & 10 \\
\hline 6 & 796.41 & 398.71 & 779.38 & 390.19 & 778.40 & 389.70 & $\mathrm{~N}$ & $1,091.51$ & 546.26 & $1,074.49$ & 537.75 & $1,073.50$ & 537.25 & 9 \\
\hline 7 & 943.48 & 472.24 & 926.45 & 463.73 & 925.46 & 463.24 & $\mathrm{~F}$ & 977.47 & 489.24 & 960.44 & 480.72 & 959.46 & 480.23 & 8 \\
\hline 8 & $1,057.52$ & 529.26 & $1,040.49$ & 520.75 & $1,039.51$ & 520.26 & $\mathrm{~N}$ & 830.40 & 415.70 & 813.37 & 407.19 & 812.39 & 406.70 & 7 \\
\hline 9 & $1,154.57$ & 577.79 & $1,137.54$ & 569.28 & $1,136.56$ & 568.78 & $\mathrm{P}$ & 716.36 & 358.68 & 699.33 & 350.17 & 698.35 & 349.68 & 6 \\
\hline 10 & $1,253.64$ & 627.32 & $1,236.61$ & 618.81 & $1,235.63$ & 618.32 & $\mathrm{~V}$ & 619.30 & 310.16 & 602.28 & 301.64 & 601.29 & 301.15 & 5 \\
\hline 11 & $1,340.67$ & 670.84 & $1,323.64$ & 662.33 & $1,322.66$ & 661.83 & S & 520.24 & 260.62 & 503.21 & 252.11 & 502.23 & 251.62 & 4 \\
\hline 12 & $1,469.71$ & 735.36 & $1,452.69$ & 726.85 & $1,451.70$ & 726.36 & E & 433.20 & 217.11 & 416.18 & 208.59 & 415.19 & 208.10 & 3 \\
\hline 13 & $1,598.76$ & 799.88 & $1,581.73$ & 791.37 & $1,580.75$ & 790.88 & E & 304.16 & 152.58 & 287.13 & 144.07 & 286.15 & 143.58 & 2 \\
\hline 14 & & & & & & & R & 175.12 & 88.06 & 158.09 & 79.55 & & & 1 \\
\hline
\end{tabular}

Query 36661 Hit 1

MS/MS Fragmentation of VAELVHFLLLK

Found in sp|P43357|MAGA3_HUMAN, Melanoma-associated antigen 3 OS=Homo sapiens GN=MAGEA3 PE=1 SV=1

Match to Query 36661: 1568.99from(785.5024,2+)

Title: 1111: Sum of 2 scans in range 2384 ( $\mathrm{rt}=63.4657, \mathrm{f}=4, \mathrm{i}=749)$ to 2385 ( $\mathrm{rt}=63.4911, \mathrm{f}=4, \mathrm{i}=750$ )

[D:llab212 \membranelGrace JoyceliTRAQ40.raw]

Data File:Submitted from 20120508-1(merge) by Mascot Daemon on JOYCE-VAIO

Monoisotopic mass of neutral peptide $\mathrm{Mr}$ (calc): 1568.99

Variable modifications:

K11 :iTRAQ4plex (K)

Ions Score: 83.27 Expect: 0.000 


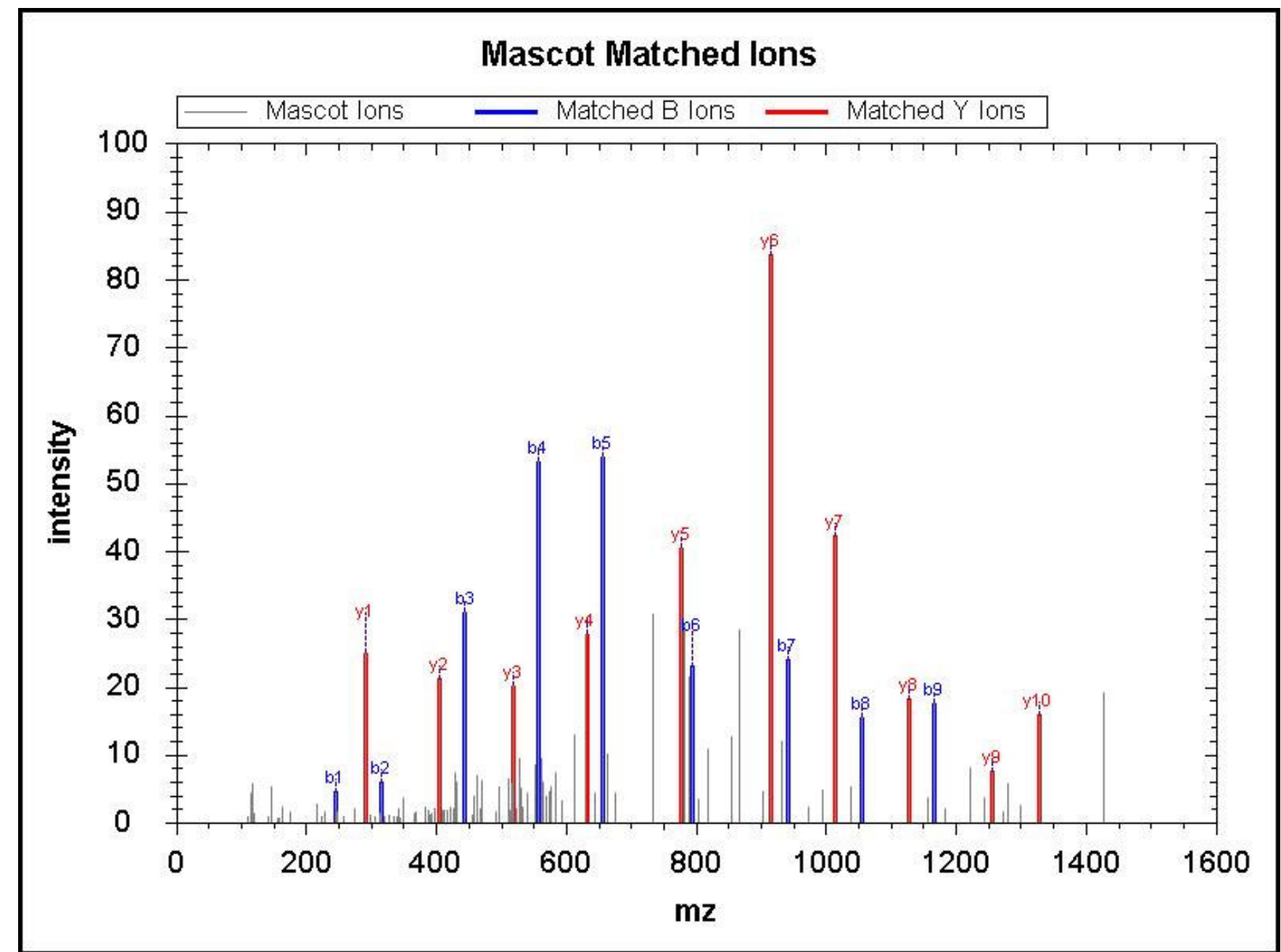

\begin{tabular}{|r|c|c|c|c|c|c|c|c|c|c|r|r|}
\hline No & $\mathrm{b}$ & $\mathrm{b}++$ & $\mathrm{b} 0$ & $\mathrm{~b} 0++$ & $\mathrm{Seq}$ & $\mathrm{y}$ & $\mathrm{y}++$ & $\mathrm{y}^{*}$ & $\mathrm{y} *++$ & $\mathrm{y} 0$ & $\mathrm{y} 0++$ & RevNo \\
\hline 1 & 244.18 & 122.59 & & & $\mathrm{~V}$ & & & & & & & 11 \\
\hline 2 & 315.21 & 158.11 & & & $\mathrm{~A}$ & $1,326.83$ & 663.92 & $1,309.80$ & 655.40 & $1,308.82$ & 654.91 & 10 \\
\hline 3 & 444.26 & 222.63 & 426.25 & 213.63 & $\mathrm{E}$ & $1,255.79$ & 628.40 & $1,238.76$ & 619.89 & $1,237.78$ & 619.39 & 9 \\
\hline 4 & 557.34 & 279.17 & 539.33 & 270.17 & $\mathrm{~L}$ & $1,126.75$ & 563.88 & $1,109.72$ & 555.36 & & & 8 \\
\hline 5 & 656.41 & 328.71 & 638.40 & 319.70 & $\mathrm{~V}$ & $1,013.66$ & 507.33 & 996.64 & 498.82 & & & 7 \\
\hline 6 & 793.47 & 397.24 & 775.46 & 388.23 & $\mathrm{H}$ & 914.59 & 457.80 & 897.57 & 449.29 & & & 6 \\
\hline 7 & 940.54 & 470.77 & 922.53 & 461.77 & $\mathrm{~F}$ & 777.54 & 389.27 & 760.51 & 380.76 & & & 5 \\
\hline 8 & $1,053.62$ & 527.31 & $1,035.61$ & 518.31 & $\mathrm{~L}$ & 630.47 & 315.74 & 613.44 & 307.22 & & & 4 \\
\hline 9 & $1,166.71$ & 583.86 & $1,148.69$ & 574.85 & $\mathrm{~L}$ & 517.38 & 259.20 & 500.36 & 250.68 & & & 3 \\
\hline 10 & $1,279.79$ & 640.40 & $1,261.78$ & 631.39 & $\mathrm{~L}$ & 404.30 & 202.65 & 387.27 & 194.14 & & & 2 \\
\hline 11 & & & & & $\mathrm{~K}$ & 291.21 & 146.11 & 274.19 & 137.60 & & & 1 \\
\hline
\end{tabular}

Query 61915 Hit 1

MS/MS Fragmentation of DKPVVLQFMDWILR

Found in sp|Q8VHL0|UT1_MOUSE, Urea transporter $1 \mathrm{OS}=$ Mus musculus $\mathrm{GN}=\mathrm{Slc} 14 \mathrm{a} 1 \mathrm{PE}=1 \mathrm{SV}=2$

Match to Query 61915: 2047.158from(683.3932,3+)

Title: 1310: Sum of 2 scans in range $3070(\mathrm{rt}=77.8649, \mathrm{f}=2, \mathrm{i}=528)$ to $3071(\mathrm{rt}=77.8903, \mathrm{f}=2, \mathrm{i}=529)$

[D:llab212\membranelGrace JoyceliTRAQ_39_1.raw]

Data File:Submitted from 20120508-1(merge) by Mascot Daemon on JOYCE-VAIO

Monoisotopic mass of neutral peptide Mr(calc): 2047.158

Variable modifications:

K2 :iTRAQ4plex (K)

Ions Score: 82.55 Expect: 0.000 


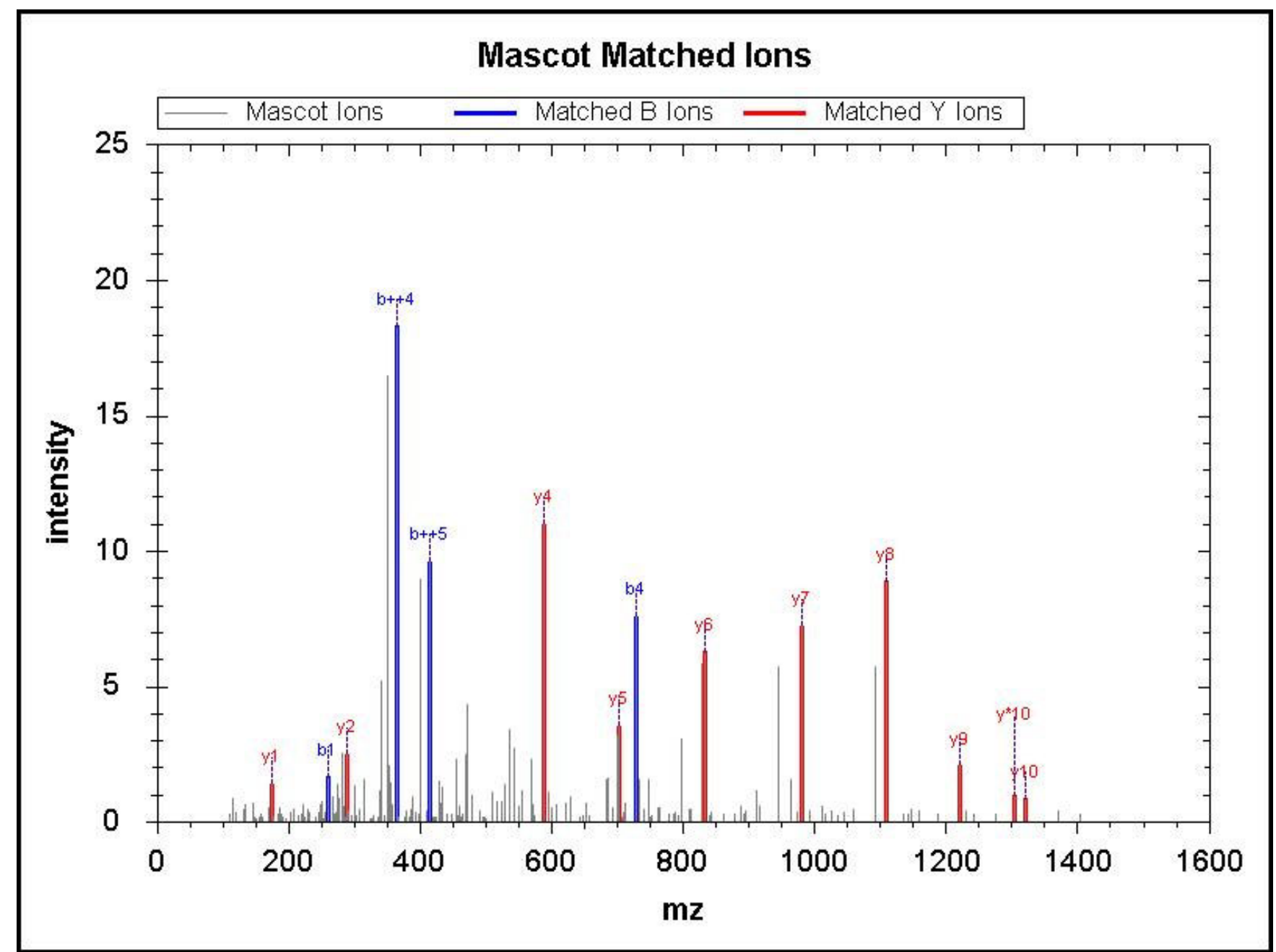

\begin{tabular}{|r|c|c|c|c|c|c|c|c|c|c|c|r|r|r|}
\hline \hline No & $\mathrm{b}$ & $\mathrm{b}++$ & $\mathrm{b}^{*}$ & $\mathrm{~b}{ }^{*++}$ & $\mathrm{b} 0$ & $\mathrm{~b} 0++$ & $\mathrm{Seq}$ & $\mathrm{y}$ & $\mathrm{y}++$ & $\mathrm{y}^{*}$ & $\mathrm{y}^{*++}$ & $\mathrm{y} 0$ & $\mathrm{y} 0++$ & $\mathrm{RevNo}$ \\
\hline 1 & 260.14 & 130.57 & & & 242.13 & 121.57 & $\mathrm{D}$ & & & & & & & 14 \\
\hline 2 & 532.33 & 266.67 & 515.31 & 258.16 & 514.32 & 257.67 & $\mathrm{~K}$ & $1,789.03$ & 895.02 & $1,772.00$ & 886.51 & $1,771.02$ & 886.01 & 13 \\
\hline 3 & 629.39 & 315.20 & 612.36 & 306.68 & 611.38 & 306.19 & $\mathrm{P}$ & $1,516.83$ & 758.92 & $1,499.81$ & 750.41 & $1,498.82$ & 749.92 & 12 \\
\hline 4 & 728.45 & 364.73 & 711.43 & 356.22 & 710.44 & 355.73 & $\mathrm{~V}$ & $1,419.78$ & 710.39 & $1,402.76$ & 701.88 & $1,401.77$ & 701.39 & 11 \\
\hline 5 & 827.52 & 414.27 & 810.50 & 405.75 & 809.51 & 405.26 & $\mathrm{~V}$ & $1,320.71$ & 660.86 & $1,303.69$ & 652.35 & $1,302.70$ & 651.85 & 10 \\
\hline 6 & 940.61 & 470.81 & 923.58 & 462.29 & 922.60 & 461.80 & $\mathrm{~L}$ & $1,221.64$ & 611.33 & $1,204.62$ & 602.81 & $1,203.63$ & 602.32 & 9 \\
\hline 7 & $1,068.67$ & 534.84 & $1,051.64$ & 526.32 & $1,050.65$ & 525.83 & $\mathrm{Q}$ & $1,108.56$ & 554.78 & $1,091.53$ & 546.27 & $1,090.55$ & 545.78 & 8 \\
\hline 8 & $1,215.73$ & 608.37 & $1,198.71$ & 599.86 & $1,197.72$ & 599.37 & $\mathrm{~F}$ & 980.50 & 490.75 & 963.48 & 482.24 & 962.49 & 481.75 & 7 \\
\hline 9 & $1,346.77$ & 673.89 & $1,329.75$ & 665.38 & $1,328.76$ & 664.89 & $\mathrm{M}$ & 833.43 & 417.22 & 816.41 & 408.71 & 815.42 & 408.22 & 6 \\
\hline 10 & $1,461.80$ & 731.40 & $1,444.77$ & 722.89 & $1,443.79$ & 722.40 & $\mathrm{D}$ & 702.39 & 351.70 & 685.37 & 343.19 & 684.38 & 342.69 & 5 \\
\hline 11 & $1,647.88$ & 824.44 & $1,630.85$ & 815.93 & $1,629.87$ & 815.44 & $\mathrm{~W}$ & 587.37 & 294.19 & 570.34 & 285.67 & & & 4 \\
\hline 12 & $1,760.96$ & 880.99 & $1,743.94$ & 872.47 & $1,742.95$ & 871.98 & $\mathrm{I}$ & 401.29 & 201.15 & 384.26 & 192.63 & & & 3 \\
\hline 13 & $1,874.05$ & 937.53 & $1,857.02$ & 929.01 & $1,856.04$ & 928.52 & $\mathrm{~L}$ & 288.20 & 144.61 & 271.18 & 136.09 & & & 2 \\
\hline 14 & & & & & & & R & 175.12 & 88.06 & 158.09 & 79.55 & & & 1 \\
\hline
\end{tabular}

Query 80933 Hit 1

MS/MS Fragmentation of LDAITDEENDMLDLAYGLTDR

Found in sp|P10109|ADX_HUM AN, Adrenodoxin

Match to Query 80933: 2526.199from(843.0737,3+)

Title: 860: Sum of 2 scans in range 2756 ( $\mathrm{rt}=68.3293, \mathrm{f}=4, \mathrm{i}=608)$ to 2757 ( $\mathrm{rt}=68.3547, \mathrm{f}=4, \mathrm{i}=609$ )

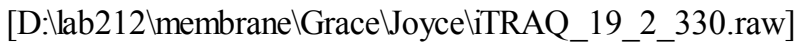

Data File:Submitted from 20120508-1(merge) by Mascot Daemon on JOYCE-VAIO

Monoisotopic mass of neutral peptide $\mathrm{Mr}$ (calc): 2526.199

Variable modifications:

Ions Score: 82.45 Expect: 0.000 
Mascot Matched lons

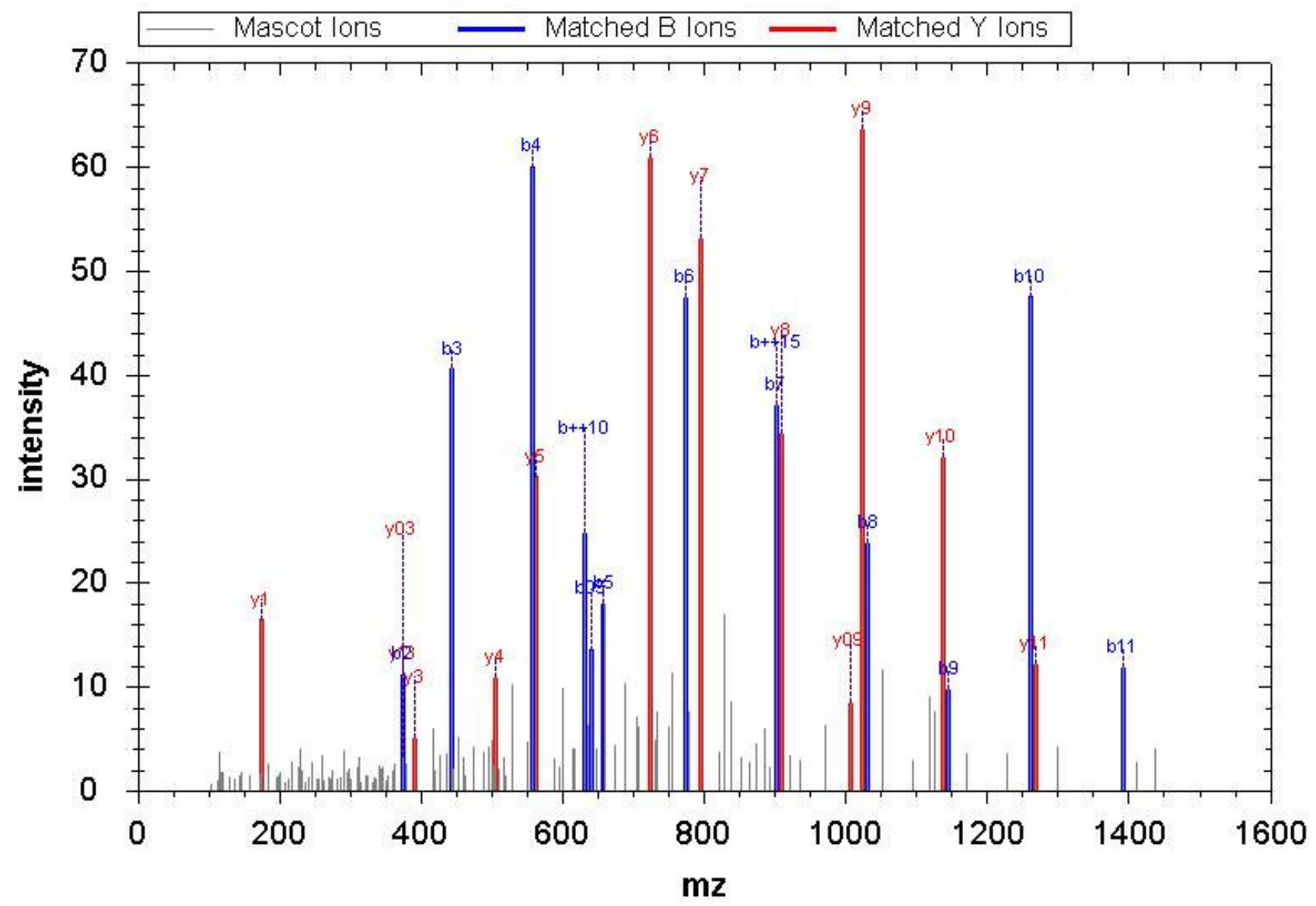

\begin{tabular}{|c|c|c|c|c|c|c|c|c|c|c|c|c|c|c|}
\hline No & $b$ & $\mathrm{~b}++$ & $b^{*}$ & $b^{*++}$ & b0 & $\mathrm{b} 0++$ & Seq & $\mathrm{y}$ & $\mathrm{y}++$ & $y^{*}$ & $y^{*++}$ & y0 & $\mathrm{y} 0++$ & RevNo \\
\hline 1 & 258.19 & 129.60 & & & & & $\mathrm{~L}$ & & & & & & & 21 \\
\hline 2 & 373.22 & 187.11 & & & 355.21 & 178.11 & D & $2,270.01$ & $1,135.51$ & $2,252.99$ & $1,127.00$ & $2,252.00$ & $1,126.50$ & 20 \\
\hline 3 & 444.26 & 222.63 & & & 426.25 & 213.63 & A & $2,154.99$ & $1,078.00$ & $2,137.96$ & $1,069.48$ & $2,136.98$ & $1,068.99$ & 19 \\
\hline 4 & 557.34 & 279.17 & & & 539.33 & 270.17 & I & $2,083.95$ & $1,042.48$ & $2,066.92$ & $1,033.96$ & $2,065.94$ & $1,033.47$ & 18 \\
\hline 5 & 658.39 & 329.70 & & & 640.38 & 320.69 & $\mathrm{~T}$ & $1,970.86$ & 985.94 & $1,953.84$ & 977.42 & $1,952.85$ & 976.93 & 17 \\
\hline 6 & 773.42 & 387.21 & & & 755.41 & 378.21 & $\mathrm{D}$ & $1,869.82$ & 935.41 & $1,852.79$ & 926.90 & $1,851.81$ & 926.41 & 16 \\
\hline 7 & 902.46 & 451.73 & & & 884.45 & 442.73 & $\mathrm{E}$ & $1,754.79$ & 877.90 & $1,737.76$ & 869.39 & $1,736.78$ & 868.89 & 15 \\
\hline 8 & $1,031.50$ & 516.25 & & & $1,013.49$ & 507.25 & $\mathrm{E}$ & $1,625.75$ & 813.38 & $1,608.72$ & 804.86 & $1,607.74$ & 804.37 & 14 \\
\hline 9 & $1,145.54$ & 573.28 & $1,128.52$ & 564.76 & $1,127.53$ & 564.27 & $\mathrm{~N}$ & $1,496.71$ & 748.86 & $1,479.68$ & 740.34 & $1,478.69$ & 739.85 & 13 \\
\hline 10 & $1,260.57$ & 630.79 & $1,243.54$ & 622.28 & $1,242.56$ & 621.78 & $\mathrm{D}$ & $1,382.66$ & 691.83 & $1,365.64$ & 683.32 & $1,364.65$ & 682.83 & 12 \\
\hline 11 & $1,391.61$ & 696.31 & $1,374.59$ & 687.80 & $1,373.60$ & 687.30 & M & $1,267.64$ & 634.32 & $1,250.61$ & 625.81 & $1,249.62$ & 625.32 & 11 \\
\hline 12 & $1,504.70$ & 752.85 & $1,487.67$ & 744.34 & $1,486.69$ & 743.85 & $\mathrm{~L}$ & $1,136.59$ & 568.80 & $1,119.57$ & 560.29 & $1,118.58$ & 559.80 & 10 \\
\hline 13 & $1,619.72$ & 810.37 & $1,602.70$ & 801.85 & $1,601.71$ & 801.36 & D & $1,023.51$ & 512.26 & $1,006.48$ & 503.75 & $1,005.50$ & 503.25 & 9 \\
\hline 14 & $1,732.81$ & 866.91 & $1,715.78$ & 858.39 & $1,714.80$ & 857.90 & $\mathrm{~L}$ & 908.48 & 454.75 & 891.46 & 446.23 & 890.47 & 445.74 & 8 \\
\hline 15 & $1,803.84$ & 902.43 & $1,786.82$ & 893.91 & $1,785.83$ & 893.42 & A & 795.40 & 398.20 & 778.37 & 389.69 & 777.39 & 389.20 & 7 \\
\hline 16 & $1,966.91$ & 983.96 & $1,949.88$ & 975.44 & $1,948.90$ & 974.95 & $\mathrm{Y}$ & 724.36 & 362.68 & 707.34 & 354.17 & 706.35 & 353.68 & 6 \\
\hline 17 & $2,023.93$ & $1,012.47$ & $2,006.90$ & $1,003.95$ & $2,005.92$ & $1,003.46$ & G & 561.30 & 281.15 & 544.27 & 272.64 & 543.29 & 272.15 & 5 \\
\hline 18 & $2,137.01$ & $1,069.01$ & $2,119.99$ & $1,060.50$ & $2,119.00$ & $1,060.00$ & $\mathrm{~L}$ & 504.28 & 252.64 & 487.25 & 244.13 & 486.27 & 243.64 & 4 \\
\hline 19 & $2,238.06$ & $1,119.53$ & $2,221.03$ & $1,111.02$ & $2,220.05$ & $1,110.53$ & $\mathrm{~T}$ & 391.19 & 196.10 & 374.17 & 187.59 & 373.18 & 187.10 & 3 \\
\hline 20 & $2,353.09$ & $1,177.05$ & $2,336.06$ & $1,168.53$ & $2,335.08$ & $1,168.04$ & $\mathrm{D}$ & 290.15 & 145.58 & 273.12 & 137.06 & 272.14 & 136.57 & 2 \\
\hline 21 & & & & & & & $\mathrm{R}$ & 175.12 & 88.06 & 158.09 & 79.55 & & & 1 \\
\hline
\end{tabular}

Query 81684 Hit 1 
MS/MS Fragmentation of FVADEELVHLLLDEVVASAALR

Found in sp|Q9UID3|FFR_HUMAN, Protein fat-free homolog OS=Homo sapiens GN=FFR PE=1 SV=2

Match to Query 81684: 2552.398from(851.8067,3+)

Title: 1266: Sum of 2 scans in range $3230(\mathrm{rt}=80.0027, \mathrm{f}=2, \mathrm{i}=539)$ to $3231(\mathrm{rt}=80.0281, \mathrm{f}=2, \mathrm{i}=540)$

[D:lab212 \membranelGrace JoyceliTRAQ 29_1.raw]

Data File:Submitted from 20120508-1(merge) by Mascot Daemon on JOYCE-VAIO

Monoisotopic mass of neutral peptide $\mathrm{Mr}$ (calc): 2552.398

Variable modifications:

Ions Score: 82.01 Expect: 0.000

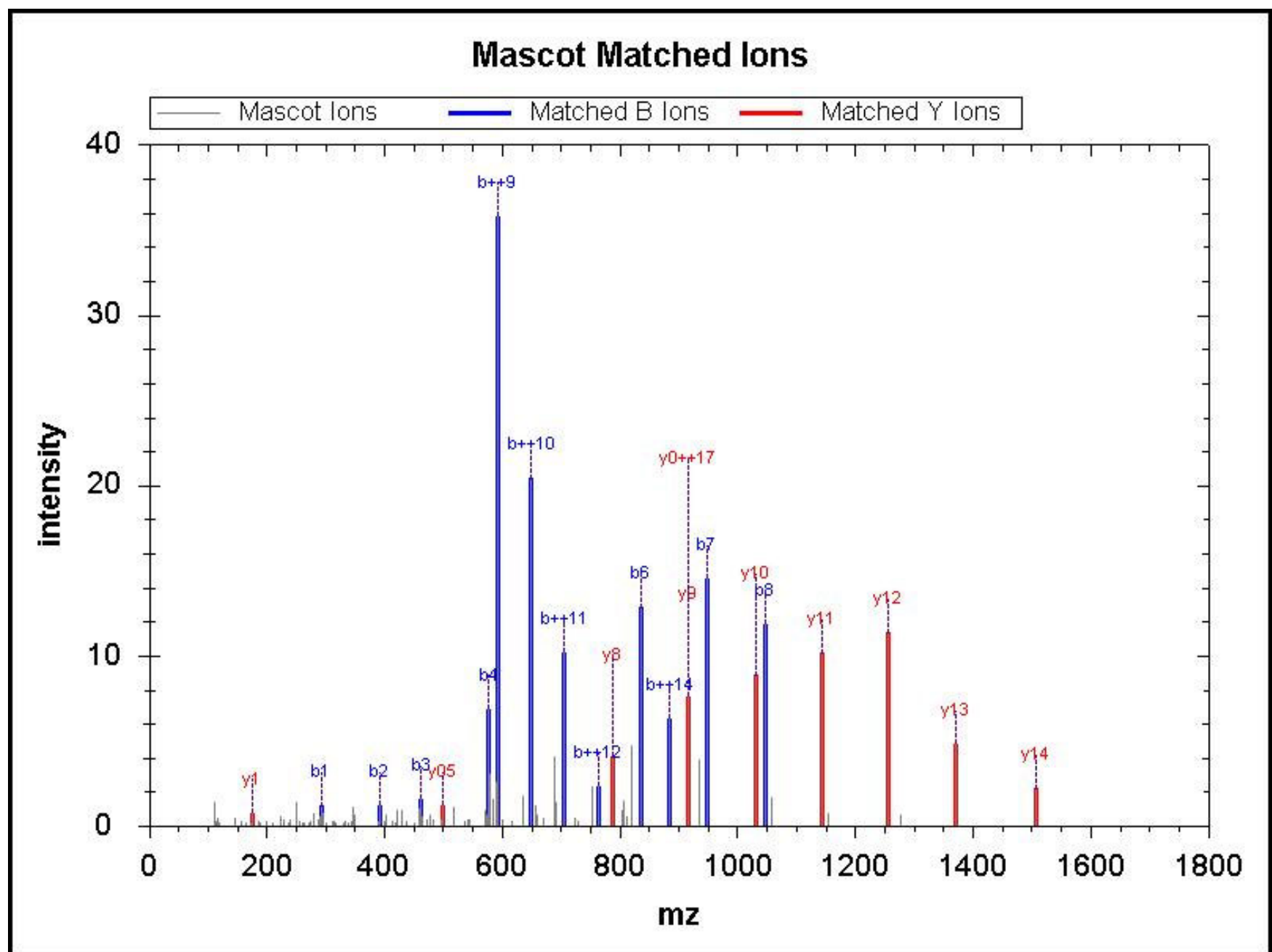

\begin{tabular}{|r|r|r|r|r|r|r|r|r|r|r|r|r|}
\hline \hline $\mathrm{No}$ & $\mathrm{b}$ & $\mathrm{b}++$ & $\mathrm{b} 0$ & $\mathrm{~b} 0++$ & $\mathrm{Seq}$ & $\mathrm{y}$ & $\mathrm{y}++$ & $\mathrm{y} *$ & $\mathrm{y}^{*++}$ & $\mathrm{y} 0$ & $\mathrm{y} 0++$ & $\mathrm{RevNo}$ \\
\hline 1 & 292.18 & 146.59 & & & $\mathrm{~F}$ & & & & & & & 22 \\
\hline 2 & 391.25 & 196.13 & & & $\mathrm{~V}$ & $2,262.23$ & $1,131.62$ & $2,245.21$ & $1,123.11$ & $2,244.22$ & $1,122.62$ & 21 \\
\hline 3 & 462.28 & 231.65 & & & $\mathrm{~A}$ & $2,163.17$ & $1,082.09$ & $2,146.14$ & $1,073.57$ & $2,145.16$ & $1,073.08$ & 20 \\
\hline 4 & 577.31 & 289.16 & 559.30 & 280.15 & $\mathrm{D}$ & $2,092.13$ & $1,046.57$ & $2,075.10$ & $1,038.05$ & $2,074.12$ & $1,037.56$ & 19 \\
\hline 5 & 706.35 & 353.68 & 688.34 & 344.67 & $\mathrm{E}$ & $1,977.10$ & 989.05 & $1,960.08$ & 980.54 & $1,959.09$ & 980.05 & 18 \\
\hline 6 & 835.40 & 418.20 & 817.38 & 409.20 & $\mathrm{E}$ & $1,848.06$ & 924.53 & $1,831.03$ & 916.02 & $1,830.05$ & 915.53 & 17 \\
\hline 7 & 948.48 & 474.74 & 930.47 & 465.74 & $\mathrm{~L}$ & $1,719.02$ & 860.01 & $1,701.99$ & 851.50 & $1,701.01$ & 851.01 & 16 \\
\hline 8 & $1,047.55$ & 524.28 & $1,029.54$ & 515.27 & $\mathrm{~V}$ & $1,605.93$ & 803.47 & $1,588.91$ & 794.96 & $1,587.92$ & 794.46 & 15 \\
\hline 9 & $1,184.61$ & 592.81 & $1,166.60$ & 583.80 & $\mathrm{H}$ & $1,506.86$ & 753.94 & $1,489.84$ & 745.42 & $1,488.85$ & 744.93 & 14 \\
\hline 10 & $1,297.69$ & 649.35 & $1,279.68$ & 640.34 & $\mathrm{~L}$ & $1,369.81$ & 685.41 & $1,352.78$ & 676.89 & $1,351.79$ & 676.40 & 13 \\
\hline 11 & $1,410.78$ & 705.89 & $1,392.76$ & 696.89 & $\mathrm{~L}$ & $1,256.72$ & 628.86 & $1,239.69$ & 620.35 & $1,238.71$ & 619.86 & 12 \\
\hline 12 & $1,523.86$ & 762.43 & $1,505.85$ & 753.43 & $\mathrm{~L}$ & $1,143.64$ & 572.32 & $1,126.61$ & 563.81 & $1,125.63$ & 563.32 & 11 \\
\hline 13 & $1,638.89$ & 819.95 & $1,620.88$ & 810.94 & $\mathrm{D}$ & $1,030.55$ & 515.78 & $1,013.53$ & 507.27 & $1,012.54$ & 506.77 & 10 \\
\hline 14 & $1,767.93$ & 884.47 & $1,749.92$ & 875.46 & $\mathrm{E}$ & 915.53 & 458.27 & 898.50 & 449.75 & 897.52 & 449.26 & 9 \\
\hline 15 & $1,867.00$ & 934.00 & $1,848.99$ & 925.00 & $\mathrm{~V}$ & 786.48 & 393.75 & 769.46 & 385.23 & 768.47 & 384.74 & 8 \\
\hline 16 & $1,966.07$ & 983.54 & $1,948.05$ & 974.53 & $\mathrm{~V}$ & 687.41 & 344.21 & 670.39 & 335.70 & 669.40 & 335.21 & 7 \\
\hline
\end{tabular}




\begin{tabular}{|r|r|r|r|r|r|r|r|r|r|r|r|r|}
17 & $2,037.10$ & $1,019.05$ & $2,019.09$ & $1,010.05$ & A & 588.35 & 294.68 & 571.32 & 286.16 & 570.34 & 285.67 & 6 \\
\hline \hline 18 & $2,124.13$ & $1,062.57$ & $2,106.12$ & $1,053.57$ & S & 517.31 & 259.16 & 500.28 & 250.64 & 499.30 & 250.15 & 5 \\
\hline 19 & $2,195.17$ & $1,098.09$ & $2,177.16$ & $1,089.08$ & A & 430.28 & 215.64 & 413.25 & 207.13 & & & 4 \\
\hline 20 & $2,266.21$ & $1,133.61$ & $2,248.20$ & $1,124.60$ & A & 359.24 & 180.12 & 342.21 & 171.61 & & & 3 \\
\hline 21 & $2,379.29$ & $1,190.15$ & $2,361.28$ & $1,181.14$ & L & 288.20 & 144.61 & 271.18 & 136.09 & & & 2 \\
\hline 22 & & & & & R & 175.12 & 88.06 & 158.09 & 79.55 & & & 1 \\
\hline
\end{tabular}

Query 63653 Hit 1

MS/MS Fragmentation of GAHLTALEMLTAFASHIR

Found in sp|Q96EL3|RM53_HUMAN, 39S ribosomal protein L53

Match to Query 63653: 2082.113from(521.5355,4+)

Title: 1116: Sum of 2 scans in range $2499(\mathrm{rt}=65.764, \mathrm{f}=4, \mathrm{i}=766)$ to 2500 ( $\mathrm{rt}=65.7894, \mathrm{f}=4, \mathrm{f}=767$ )

[D:llab212\membranelGrace \Joyce 47 _1.raw]

Data File:Submitted from 20120508-1(merge) by Mascot Daemon on JOYCE-VAIO

Monoisotopic mass of neutral peptide Mr(calc): 2082.113

Variable modifications:

Ions Score: 81.07 Expect: 0.000

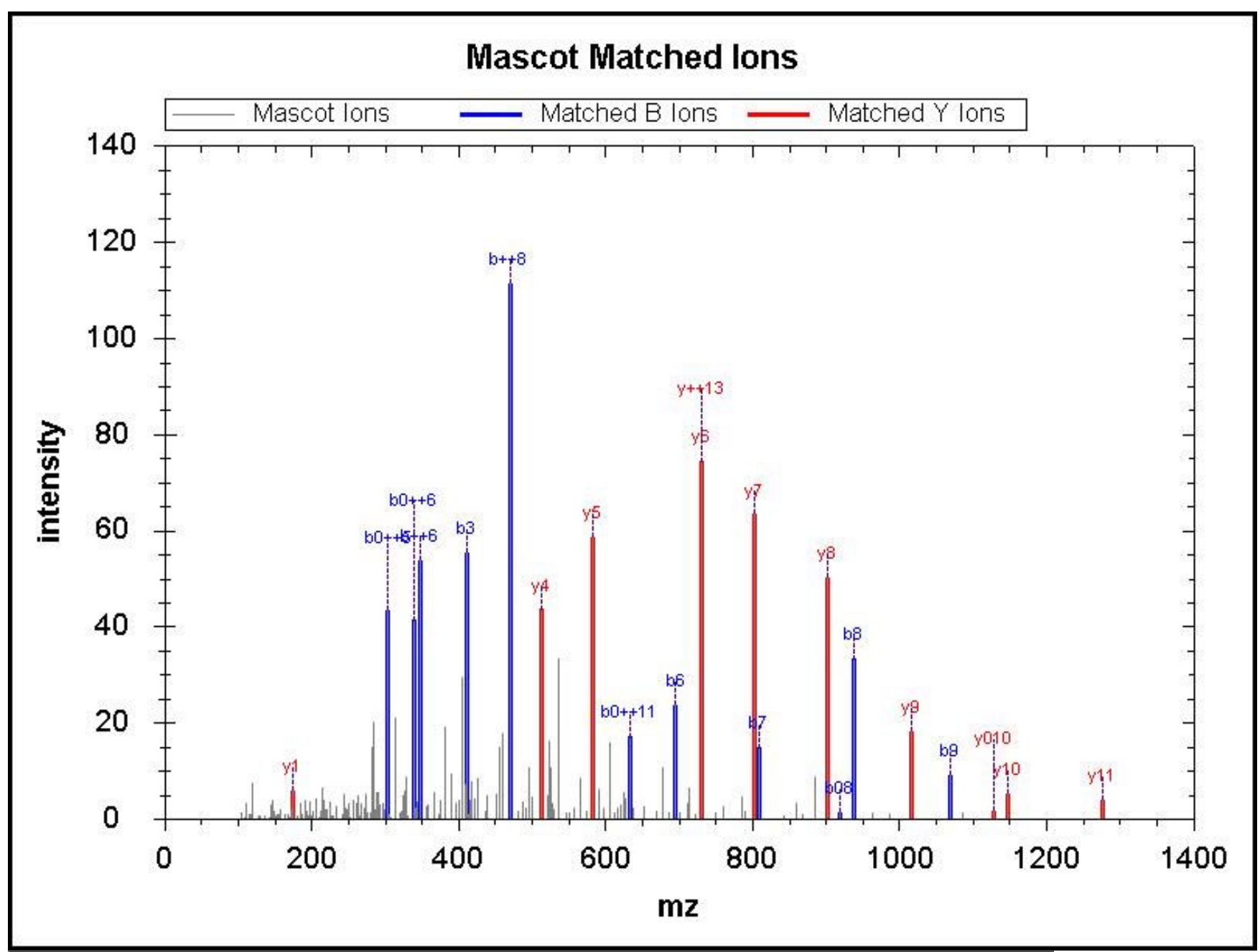

\begin{tabular}{|r|c|c|c|c|c|c|c|c|c|c|r|r|}
\hline \hline No & $\mathrm{b}$ & $\mathrm{b}++$ & $\mathrm{b} 0$ & $\mathrm{~b} 0++$ & Seq & $\mathrm{y}$ & $\mathrm{y}++$ & $\mathrm{y}^{*}$ & $\mathrm{y}^{*++}$ & $\mathrm{y} 0$ & $\mathrm{y} 0++$ & RevNo \\
\hline 1 & 202.13 & 101.57 & & & $\mathrm{G}$ & & & & & & & 18 \\
\hline 2 & 273.17 & 137.09 & & & $\mathrm{~A}$ & $1,882.00$ & 941.50 & $1,864.97$ & 932.99 & $1,863.99$ & 932.50 & 17 \\
\hline 3 & 410.23 & 205.62 & & & $\mathrm{H}$ & $1,810.96$ & 905.99 & $1,793.94$ & 897.47 & $1,792.95$ & 896.98 & 16 \\
\hline 4 & 523.31 & 262.16 & & & $\mathrm{~L}$ & $1,673.90$ & 837.46 & $1,656.88$ & 828.94 & $1,655.89$ & 828.45 & 15 \\
\hline 5 & 624.36 & 312.68 & 606.35 & 303.68 & $\mathrm{~T}$ & $1,560.82$ & 780.91 & $1,543.79$ & 772.40 & $1,542.81$ & 771.91 & 14 \\
\hline 6 & 695.40 & 348.20 & 677.39 & 339.20 & A & $1,459.77$ & 730.39 & $1,442.75$ & 721.88 & $1,441.76$ & 721.38 & 13 \\
\hline 7 & 808.48 & 404.74 & 790.47 & 395.74 & L & $1,388.74$ & 694.87 & $1,371.71$ & 686.36 & $1,370.72$ & 685.87 & 12 \\
\hline 8 & 937.52 & 469.26 & 919.51 & 460.26 & E & $1,275.65$ & 638.33 & $1,258.62$ & 629.82 & $1,257.64$ & 629.32 & 11 \\
\hline & & & & & & & & & & & &
\end{tabular}




\begin{tabular}{|r|r|r|r|r|r|r|r|r|r|r|r|r|r|}
\hline 9 & $1,068.56$ & 534.79 & $1,050.55$ & 525.78 & $\mathrm{M}$ & $1,146.61$ & 573.81 & $1,129.58$ & 565.29 & $1,128.60$ & 564.80 & 10 \\
\hline 10 & $1,181.65$ & 591.33 & $1,163.64$ & 582.32 & $\mathrm{~L}$ & $1,015.57$ & 508.29 & 998.54 & 499.77 & 997.56 & 499.28 & 9 \\
\hline 11 & $1,282.69$ & 641.85 & $1,264.68$ & 632.85 & $\mathrm{~T}$ & 902.48 & 451.75 & 885.46 & 443.23 & 884.47 & 442.74 & 8 \\
\hline 12 & $1,353.73$ & 677.37 & $1,335.72$ & 668.36 & $\mathrm{~A}$ & 801.44 & 401.22 & 784.41 & 392.71 & 783.43 & 392.22 & 7 \\
\hline 13 & $1,500.80$ & 750.90 & $1,482.79$ & 741.90 & $\mathrm{~F}$ & 730.40 & 365.70 & 713.37 & 357.19 & 712.39 & 356.70 & 6 \\
\hline 14 & $1,571.84$ & 786.42 & $1,553.83$ & 777.42 & $\mathrm{~A}$ & 583.33 & 292.17 & 566.30 & 283.66 & 565.32 & 283.16 & 5 \\
\hline 15 & $1,658.87$ & 829.94 & $1,640.86$ & 820.93 & $\mathrm{~S}$ & 512.29 & 256.65 & 495.27 & 248.14 & 494.28 & 247.65 & 4 \\
\hline 16 & $1,795.93$ & 898.47 & $1,777.92$ & 889.46 & $\mathrm{H}$ & 425.26 & 213.13 & 408.24 & 204.62 & & & 3 \\
\hline 17 & $1,909.01$ & 955.01 & $1,891.00$ & 946.00 & $\mathrm{I}$ & 288.20 & 144.61 & 271.18 & 136.09 & & & 2 \\
\hline 18 & & & & & $\mathrm{R}$ & 175.12 & 88.06 & 158.09 & 79.55 & & & 1 \\
\hline
\end{tabular}

Query 72108 Hit 1

MS/MS Fragmentation of VLYFGDHLYSDLADLMLR

Found in sp|Q9H857|NT5D2 HUMAN, 5'-nucleotidase domain-containing protein 2 OS=Homo sapiens GN=NT5DC2 PE=1 SV=1 Match to Query $72108: 2284.168$ from(762.3968,3+)

Title: 1206: Sum of 2 scans in range $2722(\mathrm{rt}=70.3926, \mathrm{f}=2, \mathrm{i}=453)$ to $2723(\mathrm{rt}=70.418, \mathrm{f}=2, \mathrm{i}=454)$

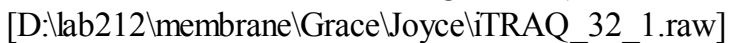

Data File:Submitted from 20120508-1(merge) by Mascot Daemon on JOYCE-VAIO

Monoisotopic mass of neutral peptide Mr(calc): 2284.168

Variable modifications:

Ions Score: 79.58 Expect: 0.000

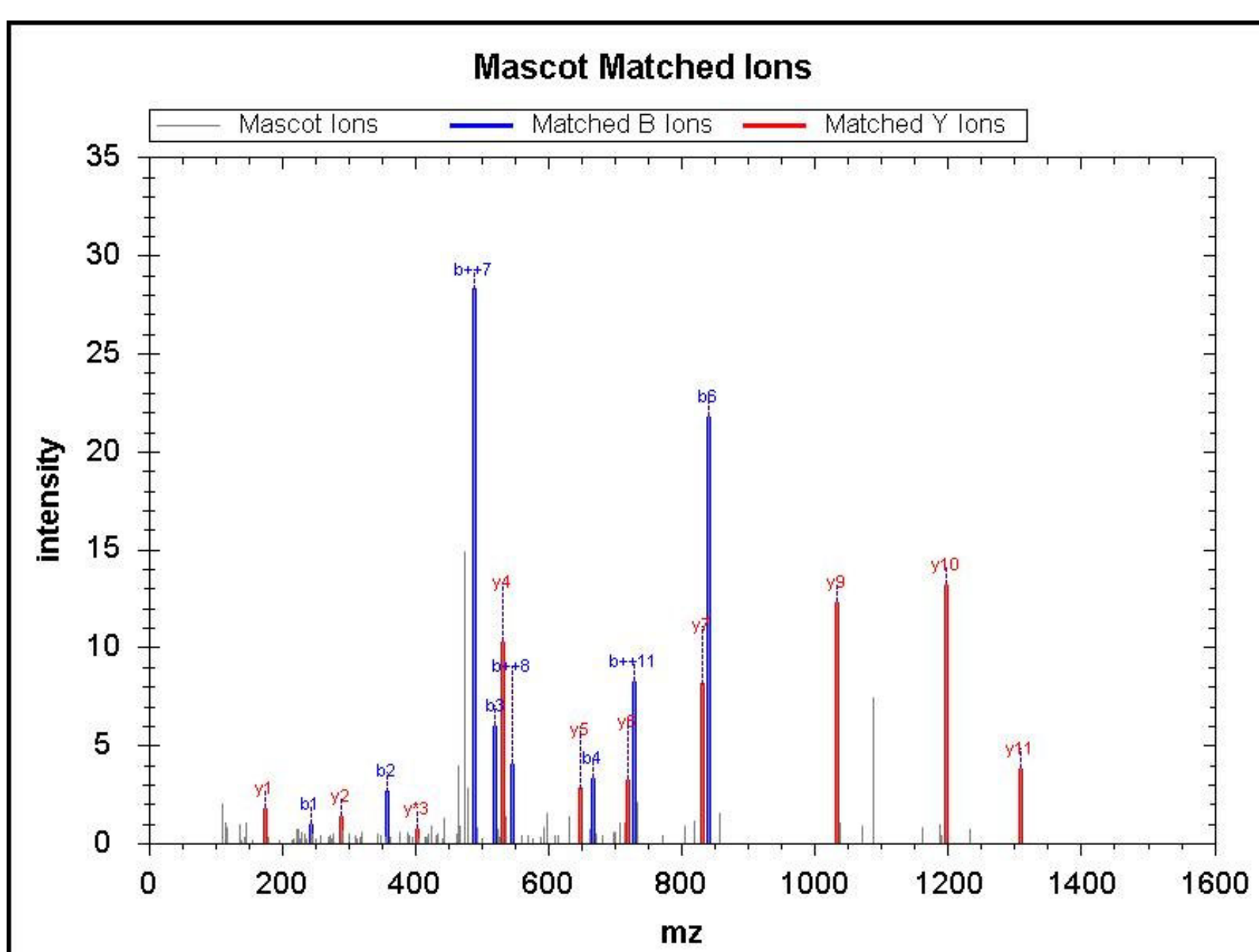

\begin{tabular}{|r|c|c|c|c|c|c|r|c|r|r|r|r|}
\hline \hline $\mathrm{No}$ & $\mathrm{b}$ & $\mathrm{b}++$ & $\mathrm{b} 0$ & $\mathrm{~b} 0++$ & $\mathrm{Seq}$ & $\mathrm{y}$ & $\mathrm{y}++$ & $\mathrm{y}^{*}$ & $\mathrm{y}^{*++}$ & $\mathrm{y} 0$ & $\mathrm{y} 0++$ & $\mathrm{RevNo}$ \\
\hline 1 & 244.18 & 122.59 & & & $\mathrm{~V}$ & & & & & & & 18 \\
\hline 2 & 357.26 & 179.13 & & & $\mathrm{~L}$ & $2,042.01$ & $1,021.51$ & $2,024.98$ & $1,012.99$ & $2,023.99$ & $1,012.50$ & 17 \\
\hline 3 & 520.33 & 260.67 & & & $\mathrm{Y}$ & $1,928.92$ & 964.96 & $1,911.89$ & 956.45 & $1,910.91$ & 955.96 & 16 \\
\hline & & & & & & & & & & & &
\end{tabular}




\begin{tabular}{|r|r|r|r|r|r|r|r|r|r|r|r|r|r|}
\hline \multicolumn{2}{|r|}{6} & 667.39 & 334.20 & & & $\mathrm{~F}$ & $1,765.86$ & 883.43 & $1,748.83$ & 874.92 & $1,747.85$ & 874.43 & 15 \\
\hline 5 & 724.42 & 362.71 & & & $\mathrm{G}$ & $1,618.79$ & 809.90 & $1,601.76$ & 801.39 & $1,600.78$ & 800.89 & 14 \\
\hline 6 & 839.44 & 420.22 & 821.43 & 411.22 & $\mathrm{D}$ & $1,561.77$ & 781.39 & $1,544.74$ & 772.87 & $1,543.76$ & 772.38 & 13 \\
\hline 7 & 976.50 & 488.75 & 958.49 & 479.75 & $\mathrm{H}$ & $1,446.74$ & 723.87 & $1,429.71$ & 715.36 & $1,428.73$ & 714.87 & 12 \\
\hline 8 & $1,089.58$ & 545.30 & $1,071.57$ & 536.29 & $\mathrm{~L}$ & $1,309.68$ & 655.34 & $1,292.66$ & 646.83 & $1,291.67$ & 646.34 & 11 \\
\hline 9 & $1,252.65$ & 626.83 & $1,234.64$ & 617.82 & $\mathrm{Y}$ & $1,196.60$ & 598.80 & $1,179.57$ & 590.29 & $1,178.59$ & 589.80 & 10 \\
\hline 10 & $1,339.68$ & 670.34 & $1,321.67$ & 661.34 & $\mathrm{~S}$ & $1,033.53$ & 517.27 & $1,016.51$ & 508.76 & $1,015.52$ & 508.27 & 9 \\
\hline 11 & $1,454.71$ & 727.86 & $1,436.70$ & 718.85 & $\mathrm{D}$ & 946.50 & 473.75 & 929.48 & 465.24 & 928.49 & 464.75 & 8 \\
\hline 12 & $1,567.79$ & 784.40 & $1,549.78$ & 775.39 & $\mathrm{~L}$ & 831.48 & 416.24 & 814.45 & 407.73 & 813.47 & 407.24 & 7 \\
\hline 13 & $1,638.83$ & 819.92 & $1,620.82$ & 810.91 & $\mathrm{~A}$ & 718.39 & 359.70 & 701.36 & 351.19 & 700.38 & 350.69 & 6 \\
\hline 14 & $1,753.86$ & 877.43 & $1,735.84$ & 868.43 & $\mathrm{D}$ & 647.35 & 324.18 & 630.33 & 315.67 & 629.34 & 315.18 & 5 \\
\hline 15 & $1,866.94$ & 933.97 & $1,848.93$ & 924.97 & $\mathrm{~L}$ & 532.33 & 266.67 & 515.30 & 258.15 & & & 4 \\
\hline 16 & $1,997.98$ & 999.49 & $1,979.97$ & 990.49 & $\mathrm{M}$ & 419.24 & 210.13 & 402.22 & 201.61 & & & 3 \\
\hline 17 & $2,111.06$ & $1,056.04$ & $2,093.05$ & $1,047.03$ & $\mathrm{~L}$ & 288.20 & 144.61 & 271.18 & 136.09 & & & 2 \\
\hline 18 & & & & & $\mathrm{R}$ & 175.12 & 88.06 & 158.09 & 79.55 & & & 1 \\
\hline
\end{tabular}

Query 63485 Hit 1

MS/MS Fragmentation of EVLEYLGNPANYPVSIR

Found in sp|Q9Y296|TPPC4_HUM AN, Trafficking protein particle complex subunit 4 OS=Homo sapiens GN=TRAPPC4 PE=1 SV=1 Match to Query 63485: 2077.102from(693.3746,3+)

Title: 593: Sum of 2 scans in range 2198 ( $\mathrm{rt}=55.7559, \mathrm{f}=4, \mathrm{i}=428)$ to $2199(\mathrm{rt}=55.7813, \mathrm{f}=4, \mathrm{i}=429)$

[D:llab212 \membranelGrace JoyceliTRAQ_19_2_330.raw]

Data File:Submitted from 20120508-1(merge) by Mascot Daemon on JOYCE-VAIO

Monoisotopic mass of neutral peptide Mr(calc): 2077.102

Variable modifications:

Ions Score: 79.46 Expect: 0.000 
Mascot Matched lons

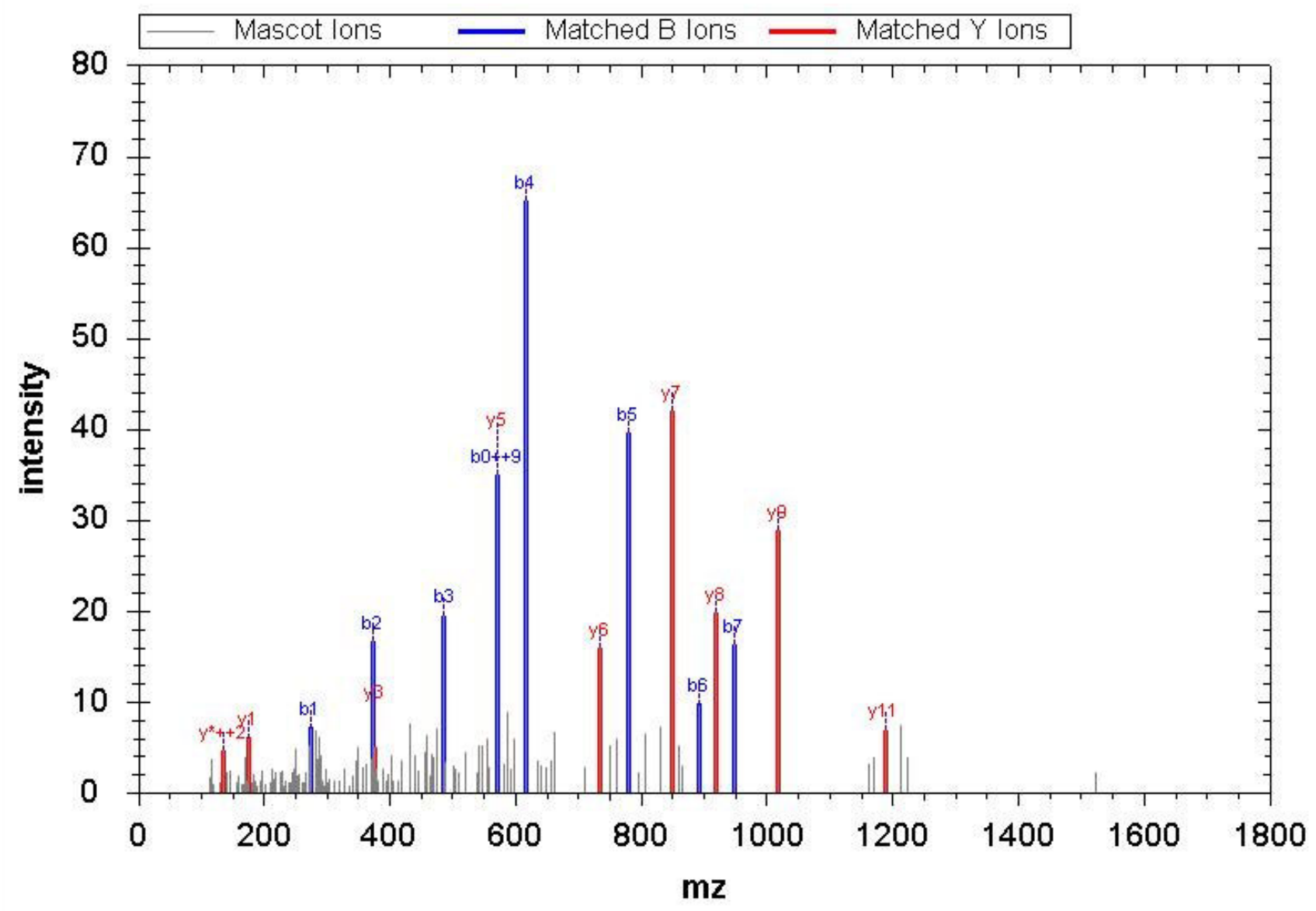

\begin{tabular}{|r|r|r|c|r|r|r|r|r|r|r|r|r|r|r|}
\hline \hline $\mathrm{No}$ & $\mathrm{b}$ & $\mathrm{b}++$ & $\mathrm{b}^{*}$ & $\mathrm{~b}++$ & $\mathrm{b} 0$ & $\mathrm{~b} 0++$ & $\mathrm{Seq}$ & $\mathrm{y}$ & $\mathrm{y}++$ & $\mathrm{y}^{*}$ & $\mathrm{y}^{*++}$ & $\mathrm{y} 0$ & $\mathrm{y} 0++$ & $\mathrm{RevNo}$ \\
\hline 1 & 274.15 & 137.58 & & & 256.14 & 128.57 & $\mathrm{E}$ & & & & & & & 17 \\
\hline 2 & 373.22 & 187.11 & & & 355.21 & 178.11 & $\mathrm{~V}$ & $1,804.96$ & 902.98 & $1,787.93$ & 894.47 & $1,786.95$ & 893.98 & 16 \\
\hline 3 & 486.30 & 243.66 & & & 468.29 & 234.65 & $\mathrm{~L}$ & $1,705.89$ & 853.45 & $1,688.86$ & 844.94 & $1,687.88$ & 844.44 & 15 \\
\hline 4 & 615.35 & 308.18 & & & 597.34 & 299.17 & $\mathrm{E}$ & $1,592.81$ & 796.91 & $1,575.78$ & 788.39 & $1,574.80$ & 787.90 & 14 \\
\hline 5 & 778.41 & 389.71 & & & 760.40 & 380.70 & $\mathrm{Y}$ & $1,463.76$ & 732.39 & $1,446.74$ & 723.87 & $1,445.75$ & 723.38 & 13 \\
\hline 6 & 891.49 & 446.25 & & & 873.48 & 437.25 & $\mathrm{~L}$ & $1,300.70$ & 650.85 & $1,283.67$ & 642.34 & $1,282.69$ & 641.85 & 12 \\
\hline 7 & 948.52 & 474.76 & & & 930.51 & 465.76 & $\mathrm{G}$ & $1,187.62$ & 594.31 & $1,170.59$ & 585.80 & $1,169.61$ & 585.31 & 11 \\
\hline 8 & $1,062.56$ & 531.78 & $1,045.53$ & 523.27 & $1,044.55$ & 522.78 & $\mathrm{~N}$ & $1,130.60$ & 565.80 & $1,113.57$ & 557.29 & $1,112.58$ & 556.80 & 10 \\
\hline 9 & $1,159.61$ & 580.31 & $1,142.59$ & 571.80 & $1,141.60$ & 571.30 & $\mathrm{P}$ & $1,016.55$ & 508.78 & 999.53 & 500.27 & 998.54 & 499.77 & 9 \\
\hline 10 & $1,230.65$ & 615.83 & $1,213.62$ & 607.31 & $1,212.64$ & 606.82 & $\mathrm{~A}$ & 919.50 & 460.25 & 902.47 & 451.74 & 901.49 & 451.25 & 8 \\
\hline 11 & $1,344.69$ & 672.85 & $1,327.67$ & 664.34 & $1,326.68$ & 663.84 & $\mathrm{~N}$ & 848.46 & 424.73 & 831.44 & 416.22 & 830.45 & 415.73 & 7 \\
\hline 12 & $1,507.76$ & 754.38 & $1,490.73$ & 745.87 & $1,489.74$ & 745.38 & $\mathrm{Y}$ & 734.42 & 367.71 & 717.39 & 359.20 & 716.41 & 358.71 & 6 \\
\hline 13 & $1,604.81$ & 802.91 & $1,587.78$ & 794.39 & $1,586.80$ & 793.90 & $\mathrm{P}$ & 571.36 & 286.18 & 554.33 & 277.67 & 553.35 & 277.18 & 5 \\
\hline 14 & $1,703.88$ & 852.44 & $1,686.85$ & 843.93 & $1,685.87$ & 843.44 & $\mathrm{~V}$ & 474.30 & 237.66 & 457.28 & 229.14 & 456.29 & 228.65 & 4 \\
\hline 15 & $1,790.91$ & 895.96 & $1,773.88$ & 887.44 & $1,772.90$ & 886.95 & $\mathrm{~S}$ & 375.24 & 188.12 & 358.21 & 179.61 & 357.22 & 179.12 & 3 \\
\hline 16 & $1,903.99$ & 952.50 & $1,886.97$ & 943.99 & $1,885.98$ & 943.49 & $\mathrm{I}$ & 288.20 & 144.61 & 271.18 & 136.09 & & & 2 \\
\hline 17 & & & & & & & $\mathrm{R}$ & 175.12 & 88.06 & 158.09 & 79.55 & & & 1 \\
\hline
\end{tabular}

Query 59379 Hit 1

MS/MS Fragmentation of AALEAIDELDLFGAHGGR

Found in sp|P46019|KPB2_HUM AN, Phosphorylase b kinase regulatory subunit alpha

Match to Query 59379: 1998.028from(667.0167,3+)

Title: 928: Sum of 2 scans in range 2039 ( $\mathrm{rt}=55.4638, \mathrm{f}=4, \mathrm{i}=621)$ to $2040(\mathrm{rt}=55.4893, \mathrm{f}=4, \mathrm{i}=622$ )

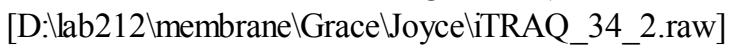

Data File:Submitted from 20120508-1(merge) by Mascot Daemon on JOYCE-VAIO 


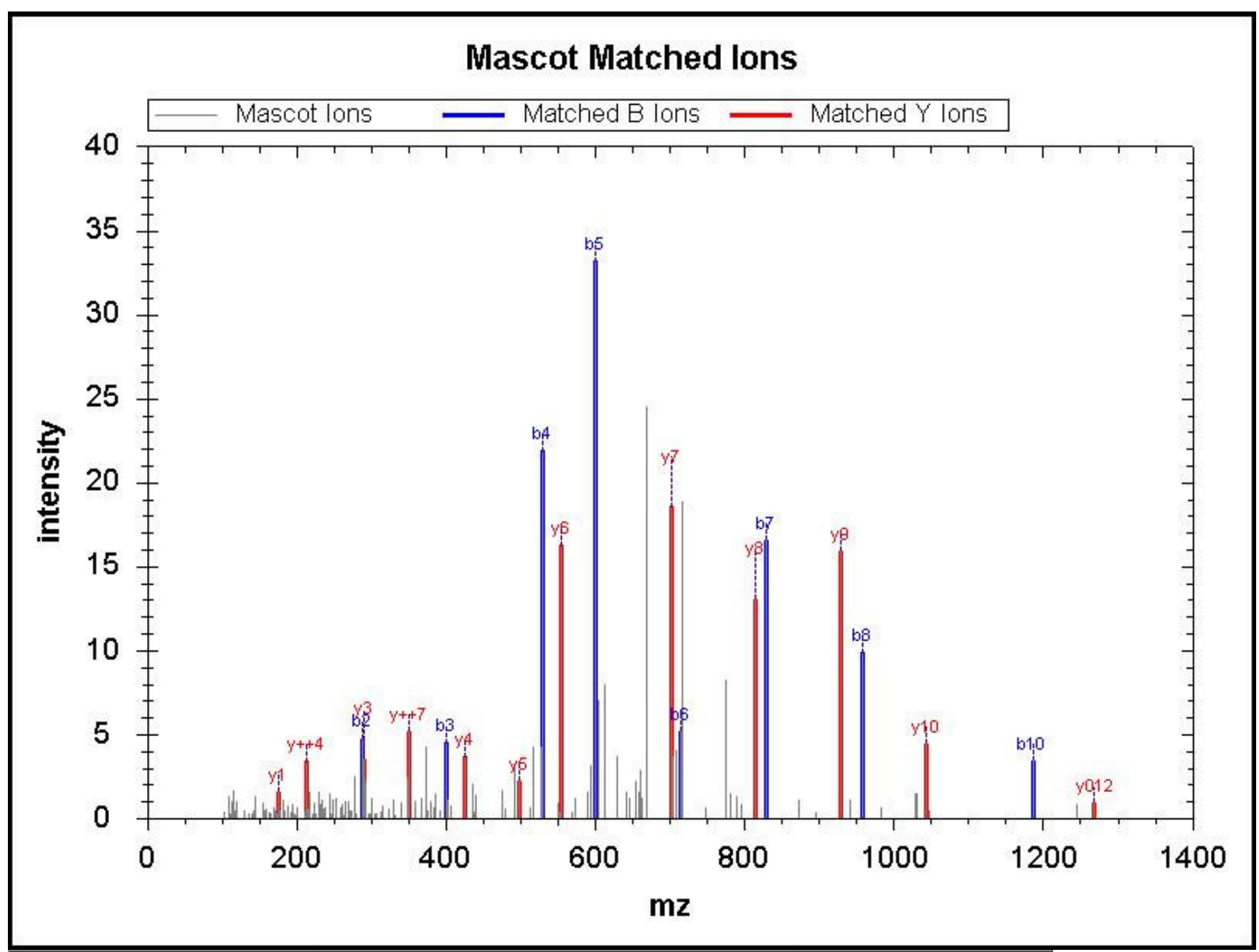

\begin{tabular}{|r|r|r|r|r|r|r|r|r|r|r|r|r|}
\hline No & $\mathrm{b}$ & $\mathrm{b}++$ & $\mathrm{b} 0$ & $\mathrm{~b} 0++$ & $\mathrm{Seq}$ & $\mathrm{y}$ & $\mathrm{y}++$ & $\mathrm{y} *$ & $\mathrm{y} *++$ & $\mathrm{y} 0$ & $\mathrm{y} 0++$ & RevNo \\
\hline 1 & 216.15 & 108.58 & & & $\mathrm{~A}$ & & & & & & & 18 \\
\hline 2 & 287.18 & 144.10 & & & $\mathrm{~A}$ & $1,783.90$ & 892.45 & $1,766.87$ & 883.94 & $1,765.89$ & 883.45 & 17 \\
\hline 3 & 400.27 & 200.64 & & & $\mathrm{~L}$ & $1,712.86$ & 856.93 & $1,695.83$ & 848.42 & $1,694.85$ & 847.93 & 16 \\
\hline 4 & 529.31 & 265.16 & 511.30 & 256.15 & $\mathrm{E}$ & $1,599.78$ & 800.39 & $1,582.75$ & 791.88 & $1,581.77$ & 791.39 & 15 \\
\hline 5 & 600.35 & 300.68 & 582.34 & 291.67 & $\mathrm{~A}$ & $1,470.73$ & 735.87 & $1,453.71$ & 727.36 & $1,452.72$ & 726.87 & 14 \\
\hline 6 & 713.43 & 357.22 & 695.42 & 348.21 & $\mathrm{I}$ & $1,399.70$ & 700.35 & $1,382.67$ & 691.84 & $1,381.69$ & 691.35 & 13 \\
\hline 7 & 828.46 & 414.73 & 810.45 & 405.73 & $\mathrm{D}$ & $1,286.61$ & 643.81 & $1,269.59$ & 635.30 & $1,268.60$ & 634.80 & 12 \\
\hline 8 & 957.50 & 479.25 & 939.49 & 470.25 & $\mathrm{E}$ & $1,171.59$ & 586.30 & $1,154.56$ & 577.78 & $1,153.57$ & 577.29 & 11 \\
\hline 9 & $1,070.59$ & 535.80 & $1,052.57$ & 526.79 & $\mathrm{~L}$ & $1,042.54$ & 521.78 & $1,025.52$ & 513.26 & $1,024.53$ & 512.77 & 10 \\
\hline 10 & $1,185.61$ & 593.31 & $1,167.60$ & 584.30 & $\mathrm{D}$ & 929.46 & 465.23 & 912.43 & 456.72 & 911.45 & 456.23 & 9 \\
\hline 11 & $1,298.70$ & 649.85 & $1,280.69$ & 640.85 & $\mathrm{~L}$ & 814.43 & 407.72 & 797.41 & 399.21 & & & 8 \\
\hline 12 & $1,445.76$ & 723.39 & $1,427.75$ & 714.38 & $\mathrm{~F}$ & 701.35 & 351.18 & 684.32 & 342.66 & & & 7 \\
\hline 13 & $1,502.79$ & 751.90 & $1,484.78$ & 742.89 & $\mathrm{G}$ & 554.28 & 277.64 & 537.25 & 269.13 & & & 6 \\
\hline 14 & $1,573.82$ & 787.42 & $1,555.81$ & 778.41 & A & 497.26 & 249.13 & 480.23 & 240.62 & & & 5 \\
\hline 15 & $1,710.88$ & 855.94 & $1,692.87$ & 846.94 & H & 426.22 & 213.61 & 409.19 & 205.10 & & & 4 \\
\hline 16 & $1,767.90$ & 884.46 & $1,749.89$ & 875.45 & G & 289.16 & 145.08 & 272.14 & 136.57 & & & 3 \\
\hline 17 & $1,824.93$ & 912.97 & $1,806.91$ & 903.96 & G & 232.14 & 116.57 & 215.11 & 108.06 & & & 2 \\
\hline 18 & & & & & R & 175.12 & 88.06 & 158.09 & 79.55 & & & 1 \\
\hline
\end{tabular}


MS/MS Fragmentation of NTFWDVDGSMVPPEWHR

Found in sp|Q9UI09|NDUAC_HUMAN, NADH dehydrogenase [ubiquinone] 1 alpha subcomplex subunit 12 OS=Homo sapiens $\mathrm{GN}=\mathrm{NDUFA} 12 \mathrm{PE}=1 \mathrm{SV}=1$

Match to Query 69319: 2216.029from(739.6837,3+)

Title: 792: Scan $1779(\mathrm{rt}=49.4788, \mathrm{f}=2, \mathrm{i}=279)$ [D:lab212 \membranelGrace JoyceliTRAQ_35_2.raw]

Data File:Submitted from 20120508-1(merge) by Mascot Daemon on JOYCE-VAIO

Monoisotopic mass of neutral peptide Mr(calc): 2216.029

Variable modifications:

Ions Score: 78.01 Expect: 0.000

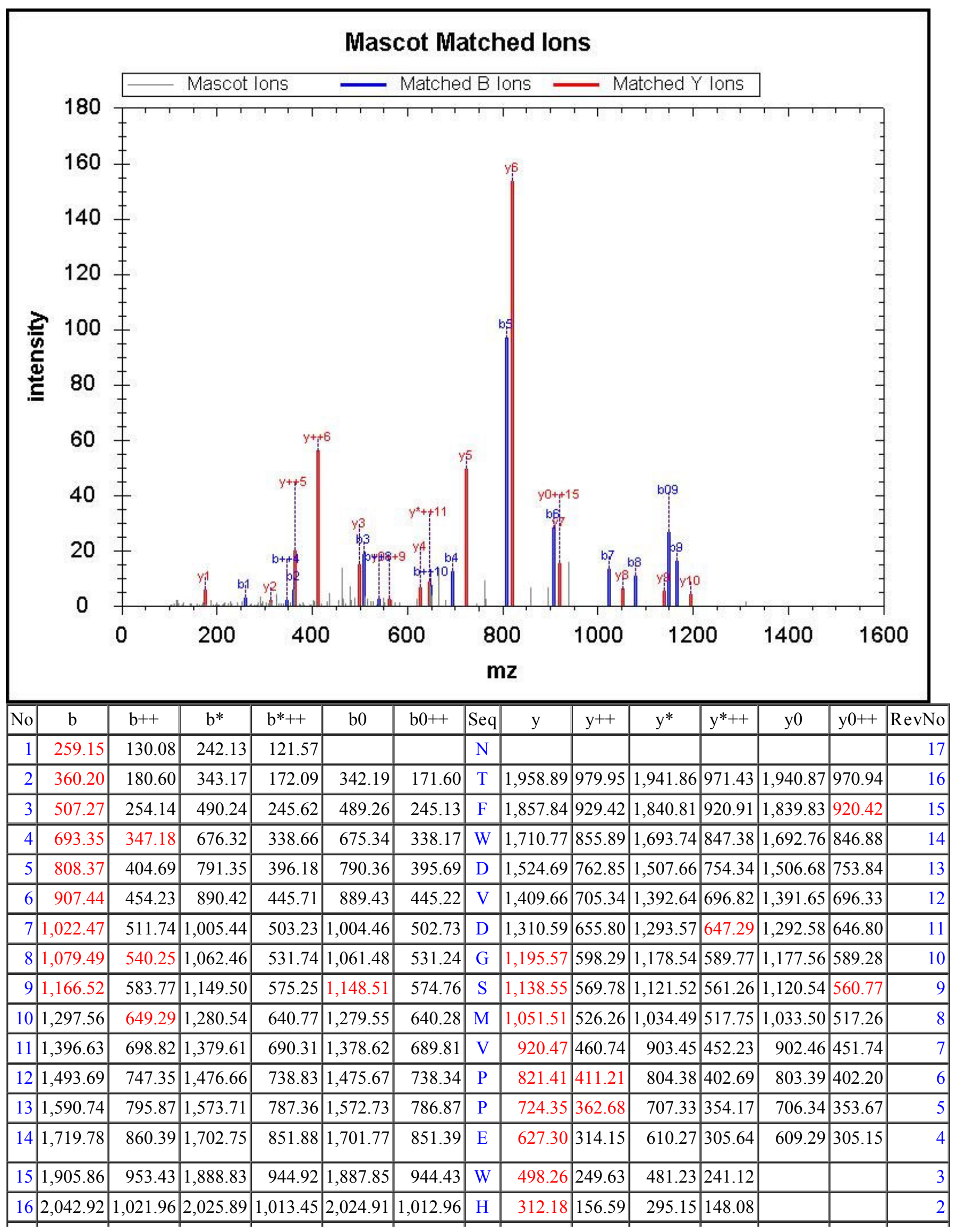


Query 76984 Hit 1

MS/MS Fragmentation of HGLTTGATLISDQWLLTTAK

Found in sp|Q61646|HPT_MOUSE, Haptoglobin OS=Mus musculus GN=Hp PE=1 SV=1

Match to Query 76984: 2414.338from(805.7866,3+)

Title: 846: Sum of 2 scans in range $1870(\mathrm{rt}=51.7318, \mathrm{f}=4, \mathrm{i}=563)$ to 1871 ( $\mathrm{rt}=51.7572, \mathrm{f}=4, \mathrm{i}=564)$

[D:llab212 \membranelGrace JoyceliTRAQ 39 2.raw]

Data File:Submitted from 20120508-1(merge) by Mascot Daemon on JOYCE-VAIO

Monoisotopic mass of neutral peptide $\mathrm{Mr}$ (calc): 2414.338

Variable modifications:

K20 :iTRAQ4plex (K)

Ions Score: 77.91 Expect: 0.000

\section{Mascot Matched lons}

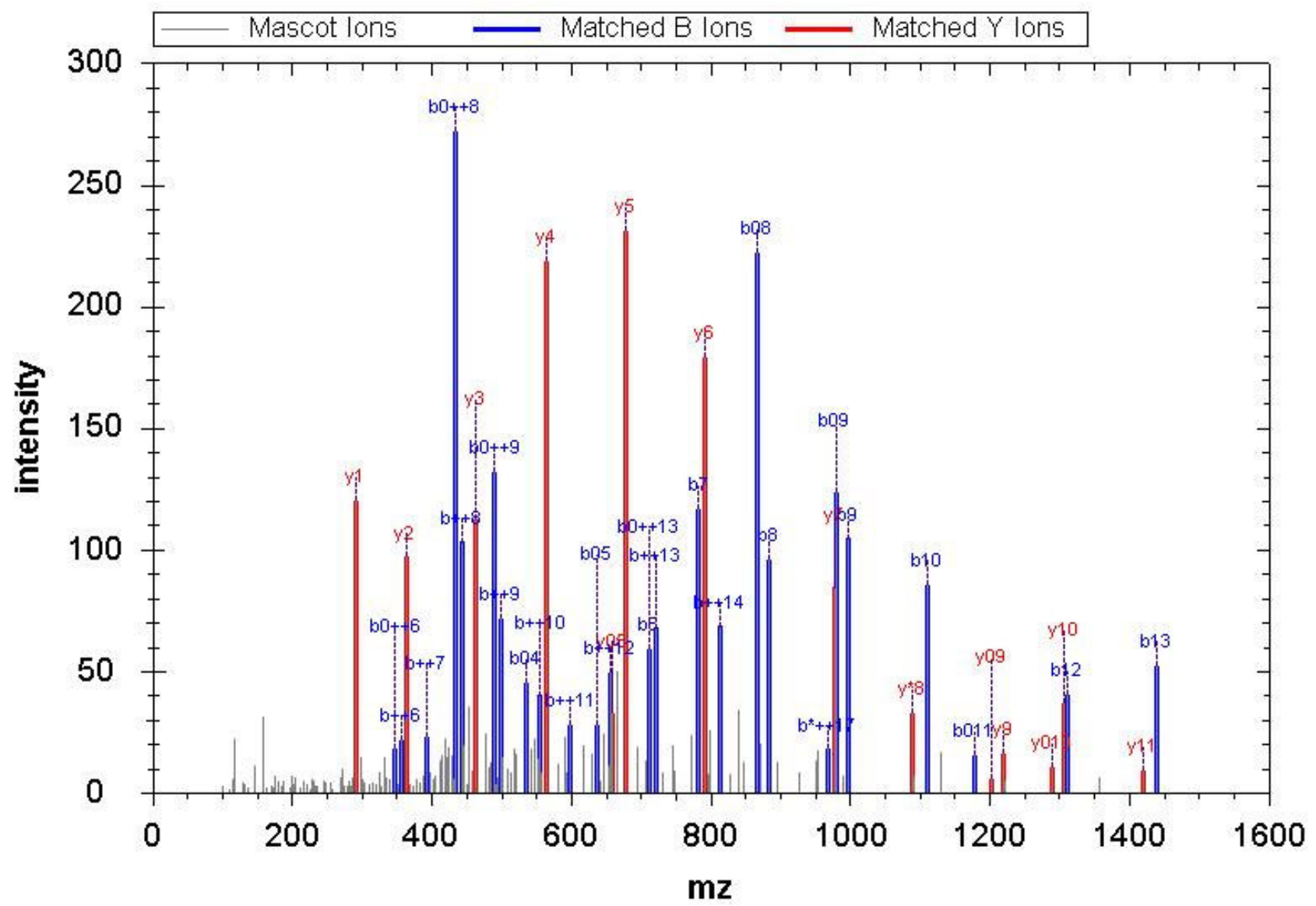

\begin{tabular}{|c|c|c|c|c|c|c|c|c|c|c|c|c|c|c|}
\hline No & $\mathrm{b}$ & $\mathrm{b}++$ & $\mathrm{b}^{*}$ & $b^{*++}$ & b0 & $\mathrm{b} 0++$ & Seq & $\mathrm{y}$ & $\mathrm{y}^{++}$ & $\mathrm{y}^{*}$ & $\mathrm{y}^{*++}$ & y0 & $\mathrm{y} 0++$ & RevNo \\
\hline 1 & 282.17 & 141.59 & & & & & $\mathrm{H}$ & & & & & & & 20 \\
\hline 2 & 339.19 & 170.10 & & & & & G & $2,134.19$ & $1,067.60$ & $2,117.16$ & $1,059.08$ & $2,116.18$ & $1,058.59$ & 19 \\
\hline 3 & 452.27 & 226.64 & & & & & $\mathrm{~L}$ & $2,077.17$ & $1,039.09$ & $2,060.14$ & $1,030.57$ & $2,059.16$ & $1,030.08$ & 18 \\
\hline 4 & 553.32 & 277.16 & & & 535.31 & 268.16 & $\mathrm{~T}$ & $1,964.08$ & 982.54 & $1,947.06$ & 974.03 & $1,946.07$ & 973.54 & 17 \\
\hline 5 & 654.37 & 327.69 & & & 636.36 & 318.68 & $\mathrm{~T}$ & $1,863.03$ & 932.02 & $1,846.01$ & 923.51 & $1,845.02$ & 923.02 & 16 \\
\hline 6 & 711.39 & 356.20 & & & 693.38 & 347.19 & $\mathrm{G}$ & $1,761.99$ & 881.50 & $1,744.96$ & 872.98 & $1,743.98$ & 872.49 & 15 \\
\hline 7 & 782.43 & 391.72 & & & 764.42 & 382.71 & A & $1,704.97$ & 852.99 & $1,687.94$ & 844.47 & $1,686.95$ & 843.98 & 14 \\
\hline 8 & 883.48 & 442.24 & & & 865.46 & 433.24 & $\mathrm{~T}$ & $1,633.93$ & 817.47 & $1,616.90$ & 808.95 & $1,615.92$ & 808.46 & 13 \\
\hline 9 & 996.56 & 498.78 & & & 978.55 & 489.78 & $\mathrm{~L}$ & $1,532.88$ & 766.94 & $1,515.85$ & 758.43 & $1,514.87$ & 757.94 & 12 \\
\hline 10 & $1,109.64$ & 555.33 & & & $1,091.63$ & 546.32 & I & $1,419.80$ & 710.40 & $1,402.77$ & 701.89 & $1,401.79$ & 701.40 & 11 \\
\hline 11 & $1,196.68$ & 598.84 & & & $1,178.66$ & 589.84 & $\mathrm{~S}$ & $1,306.71$ & 653.86 & $1,289.69$ & 645.35 & $1,288.70$ & 644.85 & 10 \\
\hline 12 & $1,311.70$ & 656.35 & & & $1,293.69$ & 647.35 & D & $1,219.68$ & 610.34 & $1,202.65$ & 601.83 & $1,201.67$ & 601.34 & 9 \\
\hline
\end{tabular}




\begin{tabular}{|r|r|r|r|r|r|r|r|r|r|r|r|r|r|r|r|r|}
\hline 13 & $1,439.76$ & 720.38 & $1,422.73$ & 711.87 & $1,421.75$ & 711.38 & $\mathrm{Q}$ & $1,104.65$ & 552.83 & $1,087.63$ & 544.32 & $1,086.64$ & 543.83 & 8 \\
\hline 14 & $1,625.84$ & 813.42 & $1,608.81$ & 804.91 & $1,607.83$ & 804.42 & $\mathrm{~W}$ & 976.59 & 488.80 & 959.57 & 480.29 & 958.58 & 479.80 & 7 \\
\hline 15 & $1,738.92$ & 869.97 & $1,721.90$ & 861.45 & $1,720.91$ & 860.96 & $\mathrm{~L}$ & 790.52 & 395.76 & 773.49 & 387.25 & 772.50 & 386.76 & 6 \\
\hline 16 & $1,852.01$ & 926.51 & $1,834.98$ & 917.99 & $1,834.00$ & 917.50 & $\mathrm{~L}$ & 677.43 & 339.22 & 660.40 & 330.71 & 659.42 & 330.21 & 5 \\
\hline 17 & $1,953.06$ & 977.03 & $1,936.03$ & 968.52 & $1,935.05$ & 968.03 & $\mathrm{~T}$ & 564.35 & 282.68 & 547.32 & 274.16 & 546.34 & 273.67 & 4 \\
\hline 18 & $2,054.10$ & $1,027.56$ & $2,037.08$ & $1,019.04$ & $2,036.09$ & $1,018.55$ & $\mathrm{~T}$ & 463.30 & 232.15 & 446.27 & 223.64 & 445.29 & 223.15 & 3 \\
\hline 19 & $2,125.14$ & $1,063.07$ & $2,108.11$ & $1,054.56$ & $2,107.13$ & $1,054.07$ & $\mathrm{~A}$ & 362.25 & 181.63 & 345.23 & 173.12 & & & 2 \\
\hline 20 & & & & & & & $\mathrm{~K}$ & 291.21 & 146.11 & 274.19 & 137.60 & & & 1 \\
\hline
\end{tabular}

Query 89707 Hit 1

MS/MS Fragmentation of LHGGTPANFLDVGGGATVHQVTEAFK

Found in sp|Q9P2R 7|SUCB1_HUMAN, Succinyl-CoA ligase [ADP-forming] subunit beta

Match to Query 89707: 2910.527 from(728.639,4+)

Title: 529: Scan 1782 ( $\mathrm{rt}=47.6093, \mathrm{f}=2, \mathrm{i}=321)$ [D:llab212 $\backslash$ membranelGracelJoyceliTRAQ_46_1.raw]

Data File:Submitted from 20120508-1(merge) by Mascot Daemon on JOYCE-VAIO

Monoisotopic mass of neutral peptide $\mathrm{Mr}$ (calc): 2910.527

Variable modifications:

K26 :TRAQ4plex (K)

Ions Score: 77.71 Expect: 0.000

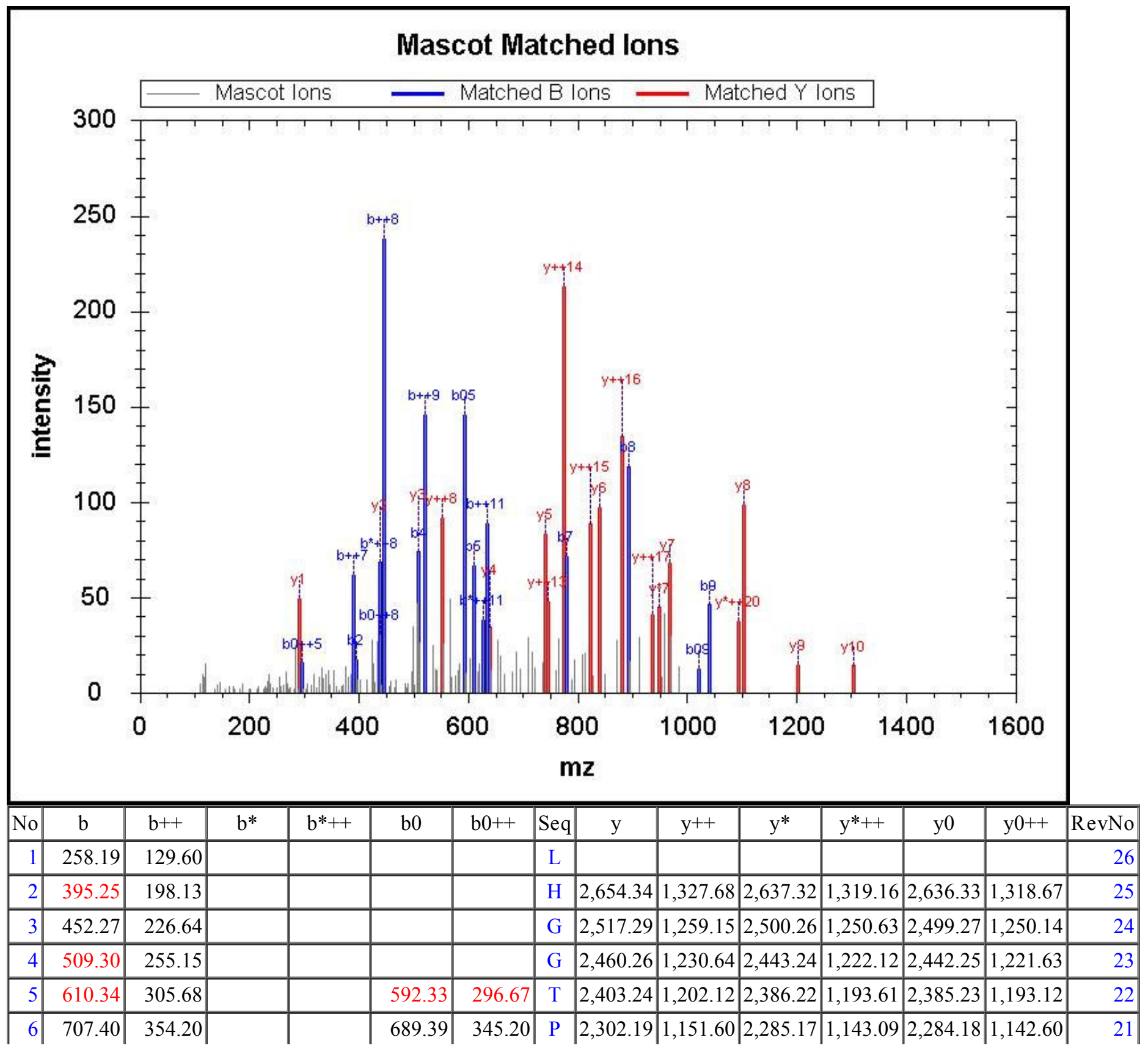




\begin{tabular}{|c|c|c|c|c|c|c|c|c|c|c|c|c|c|c|}
\hline 7 & 778.43 & 389.72 & & & 760.42 & 380.71 & A & $2,205.14$ & $1,103.07$ & $2,188.12$ & $1,094.56$ & $2,187.13$ & $1,094.07$ & 20 \\
\hline 8 & 892.48 & 446.74 & 5 & 3 & 874.47 & 437.74 & $\mathrm{~N}$ & 4.10 & 67.56 & 7.08 & $1,059.04$ & 16.09 & $1,058.55$ & 19 \\
\hline 9 & $1,039.54$ & 520.28 & 1,02 & .76 & 1,02 & .27 & $\mathrm{~F}$ & .06 & .53 & $2,003.04$ & $1,002.02$ & 2.05 & 1,0 & 18 \\
\hline 10 & $1,152.63$ & 576.82 & $1,135.60$ & 568.30 & $1,134.62$ & 567.81 & $\mathrm{~L}$ & 2.99 & 7.00 & $1,855.97$ & 928.49 & $1,854.98$ & 928.00 & 17 \\
\hline 11 & $1,267.66$ & 634.33 & $1,250.63$ & 625.82 & $1,249.64$ & 625.33 & $\mathrm{D}$ & $1,759.91$ & 880.46 & $1,742.88$ & 871.95 & $1,741.90$ & 871.45 & 16 \\
\hline 12 & $1,366.72$ & 683.87 & $1,349.70$ & 675.35 & $1,348.71$ & 674.86 & $\mathrm{~V}$ & $1,644.88$ & 822.94 & $1,627.86$ & 814.43 & $1,626.87$ & 813.94 & 15 \\
\hline 13 & $1,423.75$ & 712.38 & $1,406.72$ & 703.86 & $1,405.73$ & 703.37 & G & $1,545.81$ & 773.41 & $1,528.79$ & 764.90 & $1,527.80$ & 764.41 & 14 \\
\hline 14 & $1,480.77$ & 740.89 & $1,463.74$ & 732.37 & $1,462.76$ & 731.88 & G & $1,488.79$ & 744.90 & $1,471.77$ & 736.39 & $1,470.78$ & 735.89 & 13 \\
\hline 15 & $1,537.79$ & 769.40 & $1,520.76$ & 760.88 & $1,519.78$ & 760.39 & $\mathrm{G}$ & $1,431.77$ & 716.39 & $1,414.74$ & 707.88 & $1,413.76$ & 707.38 & 12 \\
\hline 16 & $1,608.83$ & 804.92 & $1,591.80$ & 796.40 & 1,59 & 795.91 & A & 75 & .88 & 7.72 & 9.37 & 6.74 & 8.87 & 11 \\
\hline 17 & $1,709.87$ & 855.44 & $1,692.85$ & 846.93 & $1,691.86$ & 846.43 & $\mathrm{~T}$ & $1,303.71$ & 652.36 & $1,286.69$ & 643.85 & $1,285.70$ & 643.35 & 10 \\
\hline 18 & $1,808.94$ & 904.97 & $1,791.91$ & 896.46 & $1,790.93$ & 895.97 & V & $1,202.66$ & 601.84 & $1,185.64$ & 593.32 & 4.65 & 2.83 & 9 \\
\hline 19 & $1,946.00$ & 973.50 & $1,928.97$ & 964.99 & $1,927.99$ & 964.50 & $\mathrm{H}$ & $1,103.60$ & 552.30 & $1,086.57$ & 543.79 & $1,085.59$ & 543.30 & 8 \\
\hline 20 & $2,074.06$ & $1,037.53$ & $2,057.03$ & $1,029.02$ & $2,056.05$ & $1,028.53$ & Q & 966.54 & 483.77 & 949.51 & 475.26 & 948.53 & 474.77 & 7 \\
\hline 21 & $2,173.13$ & $1,087.07$ & $2,156.10$ & $1,078.55$ & $2,155.12$ & $1,078.06$ & $\mathrm{~V}$ & 838.48 & 419.74 & 821.45 & 411.23 & 820.47 & 410.74 & 6 \\
\hline 22 & $2,274.17$ & $1,137.59$ & $2,257.15$ & $1,129.08$ & $2,256.16$ & $1,128.59$ & $\mathrm{~T}$ & 41 & 37 & 722.38 & .70 & 40 & 20 & 5 \\
\hline 23 & $2,403.22$ & $1,202.11$ & $2,386.19$ & $1,193.60$ & $2,385.21$ & $1,193.11$ & $\mathrm{E}$ & 638.36 & 319.69 & 621.34 & 311.17 & 620.35 & 310.68 & 4 \\
\hline 24 & $2,474.25$ & $1,237.63$ & $2,457.23$ & $1,229.12$ & $2,456.24$ & $1,228.63$ & A & 509.32 & 255.16 & 492.29 & 246.65 & & & 3 \\
\hline 25 & $2,621.32$ & $1,311.17$ & $2,604.30$ & $1,302.65$ & $2,603.31$ & $1,302.16$ & $\mathrm{~F}$ & 438.28 & 219.65 & 421.26 & 211.13 & & & 2 \\
\hline 26 & & & & & & & $\mathrm{~K}$ & 291.21 & 146.11 & 274.19 & 137.60 & & & 1 \\
\hline
\end{tabular}

Query 85540 Hit 1

MS/MS Fragmentation of DGNLPDIVNSGSLHEFLVNLHER

Found in sp|Q6UW02|CP20A_HUMAN, Cytochrome P450 20A1 OS=Homo sapiens GN=CYP20A1 PE=1 SV=1

Match to Query 85540: 2718.386from(680.6038,4+)

Title: 935: Sum of 2 scans in range $2061(\mathrm{rt}=56.0249, \mathrm{f}=4, \mathrm{i}=628)$ to $2062(\mathrm{rt}=56.0504, \mathrm{f}=4, \mathrm{i}=629)$

[D:llab212\membranelGrace JoyceliTRAQ_44_1.raw]

Data File:Submitted from 20120508-1(merge) by Mascot Daemon on JOYCE-VAIO

Monoisotopic mass of neutral peptide $\mathrm{Mr}$ (calc): 2718.386

Variable modifications:

Ions Score: 77.63 Expect: 0.000 


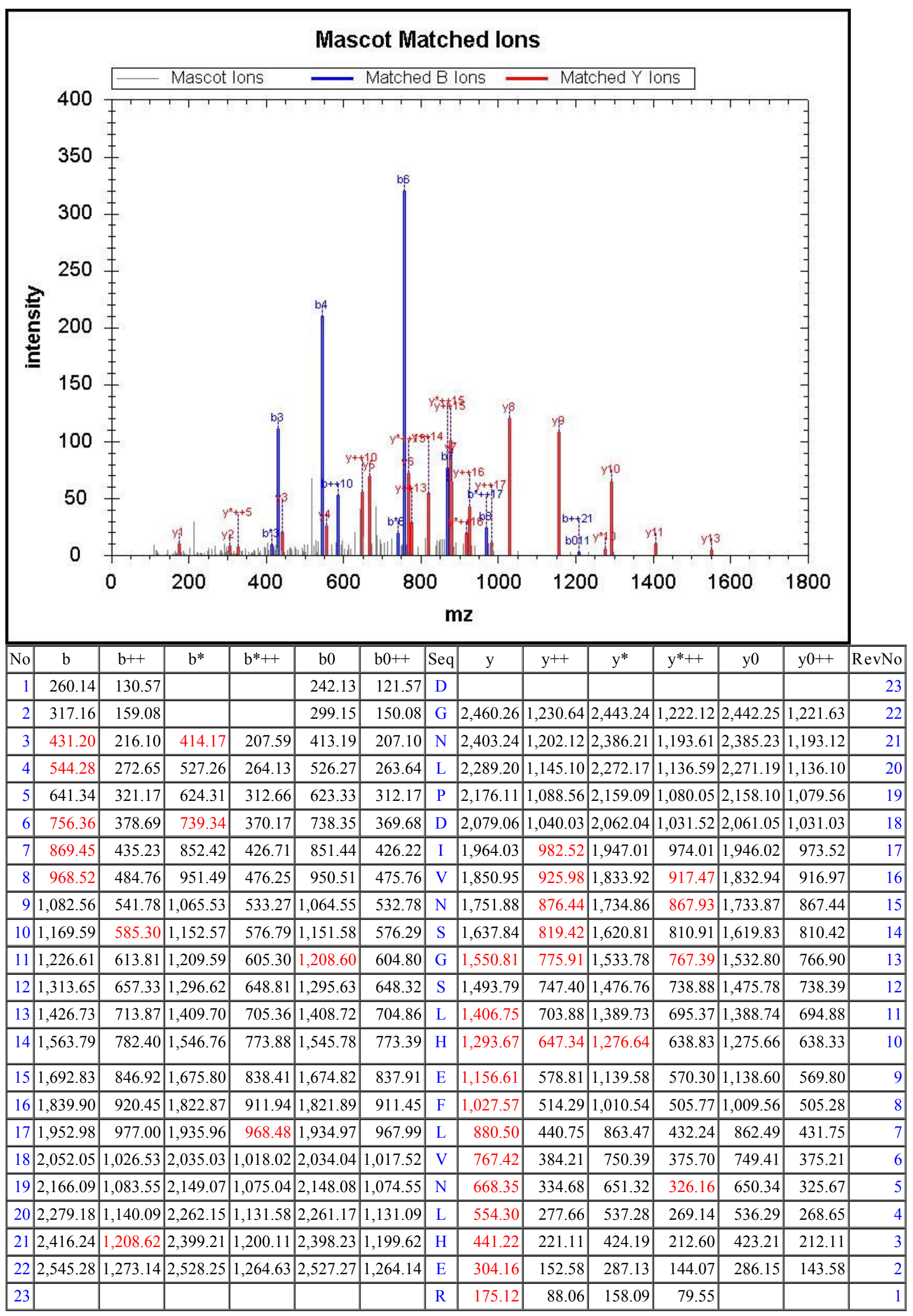


MS/MS Fragmentation of TLAYIITELDER

Found in sp|Q9Y5K8|VATD_HUMAN, V-type proton ATPase subunit D OS=Homo sapiens GN=ATP6V1D PE=1 SV=1

Match to Query 37126: 1579.856from(790.9355,2+)

Title: 735: Sum of 2 scans in range 2462 ( $\mathrm{rt}=62.8229, \mathrm{f}=3, \mathrm{i}=331$ ) to 2463 ( $\mathrm{rt}=62.8483, \mathrm{f}=3, \mathrm{i}=332$ )

[D:llab212 \membranelGrace JoyceliTRAQ19.raw]

Data File:Submitted from 20120508-1(merge) by Mascot Daemon on JOYCE-VAIO

Monoisotopic mass of neutral peptide $\mathrm{Mr}$ (calc): 1579.856

Variable modifications:

Ions Score: 77.57 Expect: 0.000

\section{Mascot Matched lons}

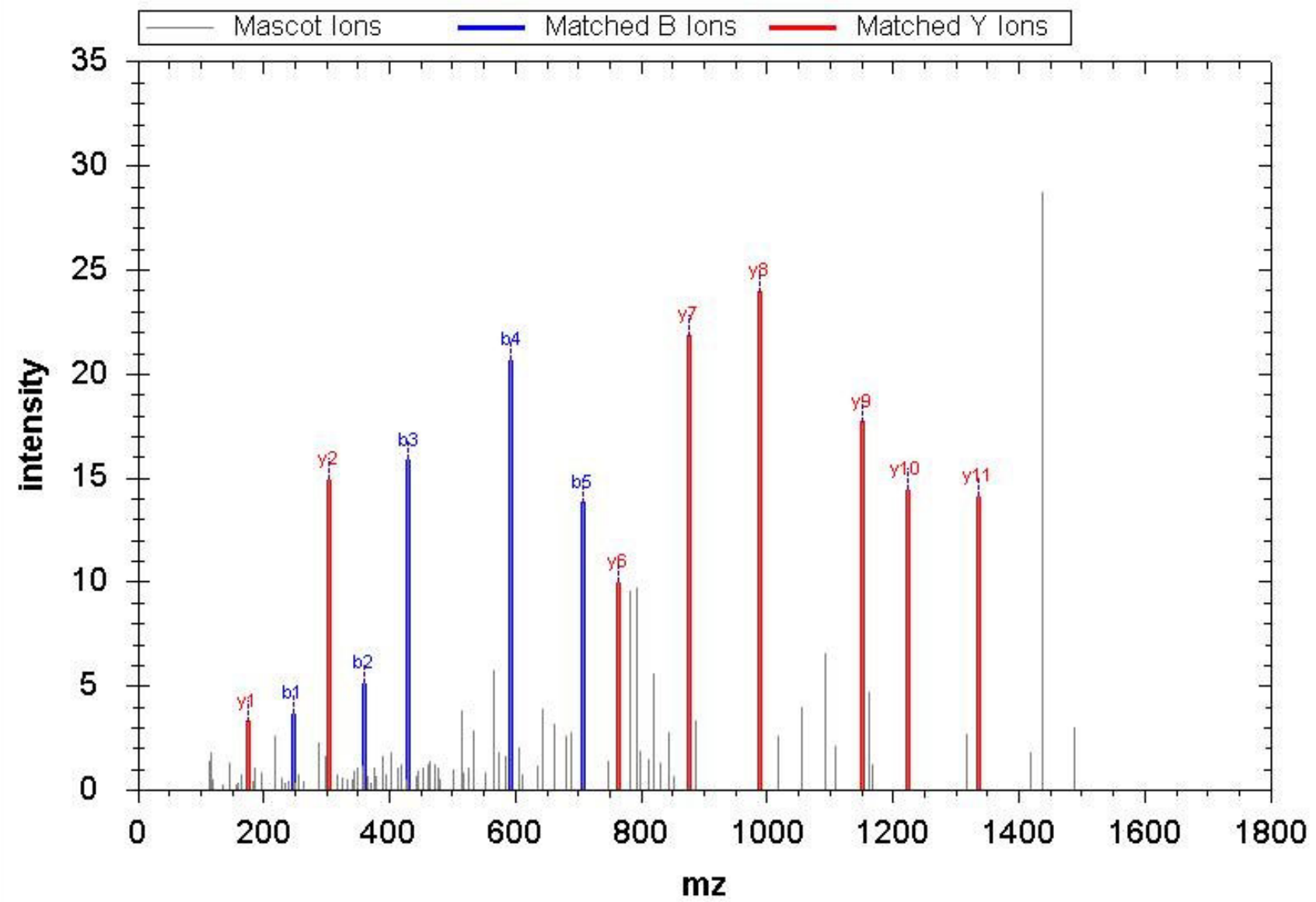

\begin{tabular}{|r|c|c|c|c|c|c|c|c|c|c|r|r|}
\hline \hline $\mathrm{No}$ & $\mathrm{b}$ & $\mathrm{b}++$ & $\mathrm{b} 0$ & $\mathrm{~b} 0++$ & $\mathrm{Seq}$ & $\mathrm{y}$ & $\mathrm{y}++$ & $\mathrm{y}^{*}$ & $\mathrm{y}^{*++}$ & $\mathrm{y} 0$ & $\mathrm{y} 0++$ & $\mathrm{RevNo}$ \\
\hline 1 & 246.16 & 123.58 & 228.15 & 114.58 & $\mathrm{~T}$ & & & & & & & 12 \\
\hline 2 & 359.24 & 180.12 & 341.23 & 171.12 & $\mathrm{~L}$ & $1,335.72$ & 668.36 & $1,318.69$ & 659.85 & $1,317.70$ & 659.36 & 11 \\
\hline 3 & 430.28 & 215.64 & 412.27 & 206.64 & $\mathrm{~A}$ & $1,222.63$ & 611.82 & $1,205.60$ & 603.31 & $1,204.62$ & 602.81 & 10 \\
\hline 4 & 593.34 & 297.17 & 575.33 & 288.17 & $\mathrm{Y}$ & $1,151.59$ & 576.30 & $1,134.57$ & 567.79 & $1,133.58$ & 567.30 & 9 \\
\hline 5 & 706.43 & 353.72 & 688.41 & 344.71 & $\mathrm{I}$ & 988.53 & 494.77 & 971.50 & 486.26 & 970.52 & 485.76 & 8 \\
\hline 6 & 819.51 & 410.26 & 801.50 & 401.25 & $\mathrm{I}$ & 875.45 & 438.23 & 858.42 & 429.71 & 857.44 & 429.22 & 7 \\
\hline 7 & 920.56 & 460.78 & 902.55 & 451.78 & $\mathrm{~T}$ & 762.36 & 381.69 & 745.34 & 373.17 & 744.35 & 372.68 & 6 \\
\hline 8 & $1,049.60$ & 525.30 & $1,031.59$ & 516.30 & $\mathrm{E}$ & 661.32 & 331.16 & 644.29 & 322.65 & 643.30 & 322.16 & 5 \\
\hline 9 & $1,162.68$ & 581.85 & $1,144.67$ & 572.84 & $\mathrm{~L}$ & 532.27 & 266.64 & 515.25 & 258.13 & 514.26 & 257.63 & 4 \\
\hline 10 & $1,277.71$ & 639.36 & $1,259.70$ & 630.35 & $\mathrm{D}$ & 419.19 & 210.10 & 402.16 & 201.58 & 401.18 & 201.09 & 3 \\
\hline 11 & $1,406.75$ & 703.88 & $1,388.74$ & 694.88 & $\mathrm{E}$ & 304.16 & 152.58 & 287.13 & 144.07 & 286.15 & 143.58 & 2 \\
\hline 12 & & & & & $\mathrm{R}$ & 175.12 & 88.06 & 158.09 & 79.55 & & & 1 \\
\hline
\end{tabular}

Query 88288 Hit 1 
Found in sp $|\mathrm{Q} 15067|$ ACOX1_HUM AN, Peroxisomal acyl-coenzyme A oxidase 1 OS=Homo sapiens GN=ACOX1 PE=1 SV=3 Match to Query 88288: 2846.373from(949.7982,3+)

Title: 927: Scan 2115 ( $\mathrm{r}=56.8103, \mathrm{f}=3, \mathrm{i}=314$ ) [D:lab212 $\backslash$ membranelGracelJoyceliTRAQ_30_1.raw]

Data File:Submitted from 20120508-1(merge) by Mascot Daemon on JOYCE-VAIO

Monoisotopic mass of neutral peptide Mr(calc): 2846.373

Variable modifications:

Ions Score: 76.94 Expect: 0.000

\section{Mascot Matched lons}

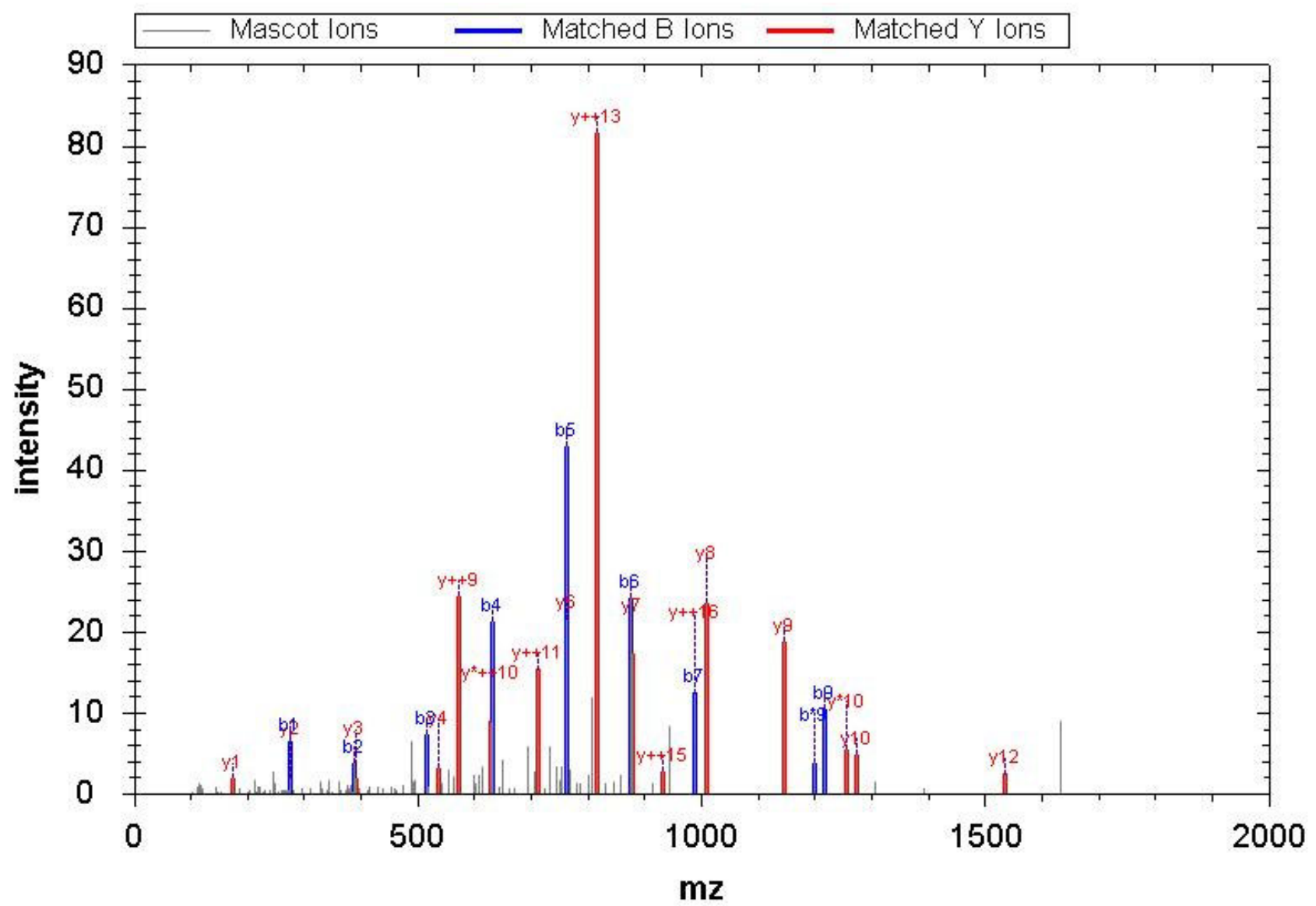

\begin{tabular}{|c|c|c|c|c|c|c|c|c|c|c|c|c|c|c|}
\hline No & $\mathrm{b}$ & $\mathrm{b}++$ & $b^{*}$ & $b^{*++}$ & b0 & $\mathrm{b} 0++$ & Seq & $\mathrm{y}$ & $\mathrm{y}^{++}$ & $\mathrm{y}^{*}$ & $y^{*++}$ & y0 & $\mathrm{y} 0++$ & RevNo \\
\hline 1 & 274.15 & 137.58 & & & 256.14 & 128.57 & E & & & & & & & 22 \\
\hline 2 & 387.24 & 194.12 & & & 369.23 & 185.12 & I & $2,574.23$ & $1,287.62$ & $2,557.20$ & $1,279.10$ & $2,556.22$ & $1,278.61$ & 21 \\
\hline 3 & 516.28 & 258.64 & & & 498.27 & 249.64 & $\mathrm{E}$ & $2,461.15$ & $1,231.08$ & $2,444.12$ & $1,222.56$ & $2,443.13$ & $1,222.07$ & 20 \\
\hline 4 & 630.32 & 315.66 & 613.29 & 307.15 & 612.31 & 306.66 & $\mathrm{~N}$ & $2,332.10$ & $1,166.56$ & $2,315.08$ & $1,158.04$ & $2,314.09$ & $1,157.55$ & 19 \\
\hline 5 & 761.36 & 381.18 & 744.34 & 372.67 & 743.35 & 372.18 & M & $2,218.06$ & $1,109.53$ & $2,201.03$ & 1.02 & $2,200.05$ & $1,100.53$ & 18 \\
\hline 6 & 874.45 & 437.73 & 857.42 & 429.21 & 856.44 & 428.72 & I & $2,087.02$ & $1,044.01$ & $2,069.99$ & $1,035.50$ & $2,069.01$ & 5.01 & 17 \\
\hline 7 & 987.53 & 494.27 & 970.50 & 485.76 & 969.52 & 485.26 & $\mathrm{~L}$ & $1,973.94$ & 987.47 & $1,956.91$ & 978.96 & $1,955.92$ & 978.47 & 16 \\
\hline 8 & $1,101.57$ & 551.29 & $1,084.55$ & 542.78 & $1,083.56$ & 542.28 & $\mathrm{~N}$ & $1,860.85$ & 930.93 & $1,843.82$ & 922.42 & $1,842.84$ & 921.92 & 15 \\
\hline 9 & $1,216.60$ & 608.80 & $1,199.57$ & 600.29 & $1,198.59$ & 599.80 & $\mathrm{D}$ & $1,746.81$ & 873.91 & $1,729.78$ & 865.39 & $1,728.80$ & 864.90 & 14 \\
\hline 10 & $1,313.65$ & 657.33 & $1,296.63$ & 648.82 & $1,295.64$ & 648.32 & $\mathrm{P}$ & $1,631.78$ & 816.39 & $1,614.75$ & 807.88 & $1,613.77$ & 807.39 & 13 \\
\hline 11 & $1,428.68$ & 714.84 & $1,411.65$ & 706.33 & $1,410.67$ & 705.84 & D & $1,534.73$ & 767.87 & $1,517.70$ & 759.35 & $1,516.72$ & 758.86 & 12 \\
\hline 12 & $1,575.75$ & 788.38 & $1,558.72$ & 779.86 & $1,557.74$ & 779.37 & $\mathrm{~F}$ & $1,419.70$ & 710.35 & $1,402.67$ & 701.84 & $1,401.69$ & 701.35 & 11 \\
\hline 13 & $1,703.81$ & 852.41 & $1,686.78$ & 843.89 & $1,685.80$ & 843.40 & Q & $1,272.63$ & 636.82 & $1,255.61$ & 8.31 & $1,254.62$ & 627.81 & 10 \\
\hline 14 & $1,840.87$ & 920.94 & $1,823.84$ & 912.42 & $1,822.85$ & 911.93 & $\mathrm{H}$ & $1,144.57$ & 572.79 & $1,127.55$ & 564.28 & $1,126.56$ & 563.79 & 9 \\
\hline 15 & $1,969.91$ & 985.46 & $1,952.88$ & 976.94 & $1,951.90$ & 976.45 & E & $1,007.52$ & 504.26 & 990.49 & 495.75 & 989.51 & 495.26 & 8 \\
\hline 16 & $2,084.94$ & $1,042.97$ & $2,067.91$ & $1,034.46$ & $2,066.92$ & $1,033.97$ & D & 878.47 & 439.74 & 861.45 & 431.23 & 860.46 & 430.73 & 7 \\
\hline 17 & $2,198.02$ & $1,099.51$ & $2,180.99$ & $1,091.00$ & $2,180.01$ & $1,090.51$ & $\mathrm{~L}$ & 763.45 & 382.23 & 746.42 & 373.71 & 745.44 & 373.22 & 6 \\
\hline 18 & $2,312.06$ & $1,156.53$ & $2,295.04$ & $1,148.02$ & $2,294.05$ & $1,147.53$ & $\mathrm{~N}$ & 650.36 & 325.68 & 633.34 & 317.17 & 632.35 & 316.68 & 5 \\
\hline
\end{tabular}




\begin{tabular}{|r|r|r|r|r|r|r|r|r|r|r|r|r|r|r|}
\hline 19 & $2,459.13$ & $1,230.07$ & $2,442.10$ & $1,221.56$ & $2,441.12$ & $1,221.06$ & $\mathrm{~F}$ & 536.32 & 268.66 & 519.29 & 260.15 & 518.31 & 259.66 & 4 \\
\hline 20 & $2,572.21$ & $1,286.61$ & $2,555.19$ & $1,278.10$ & $2,554.20$ & $1,277.61$ & $\mathrm{~L}$ & 389.25 & 195.13 & 372.22 & 186.62 & 371.24 & 186.12 & 3 \\
\hline 21 & $2,673.26$ & $1,337.13$ & $2,656.24$ & $1,328.62$ & $2,655.25$ & $1,328.13$ & $\mathrm{~T}$ & 276.17 & 138.59 & 259.14 & 130.07 & 258.16 & 129.58 & 2 \\
\hline 22 & & & & & & & $\mathrm{R}$ & 175.12 & 88.06 & 158.09 & 79.55 & & & 1 \\
\hline
\end{tabular}

Query 41527 Hit 1

MS/MS Fragmentation of LEDILESINSIK

Found in sp|Q9Y3A6|TMED5_HUM AN, Transmembrane emp24 domain-containing protein 5 OS=Homo sapiens GN=TMED5 PE=1 $\mathrm{SV}=1$

Match to Query 41527: 1660.946 from(831.4803,2+)

Title: 1020: Sum of 2 scans in range 2232 ( $\mathrm{rt}=59.7608, \mathrm{f}=4, \mathrm{i}=683)$ to 2233 ( $\mathrm{rt}=59.7862, \mathrm{f}=4, \mathrm{i}=684$ )

[D:lab212 \membranelGracelJoyceliTRAQ_28_2_327.raw]

Data File:Submitted from 20120508-1(merge) by Mascot Daemon on JOYCE-VAIO

Monoisotopic mass of neutral peptide $\mathrm{Mr}$ (calc): 1660.946

Variable modifications:

K12 :TRAQ4plex (K)

Ions Score: 76.82 Expect: 0.000

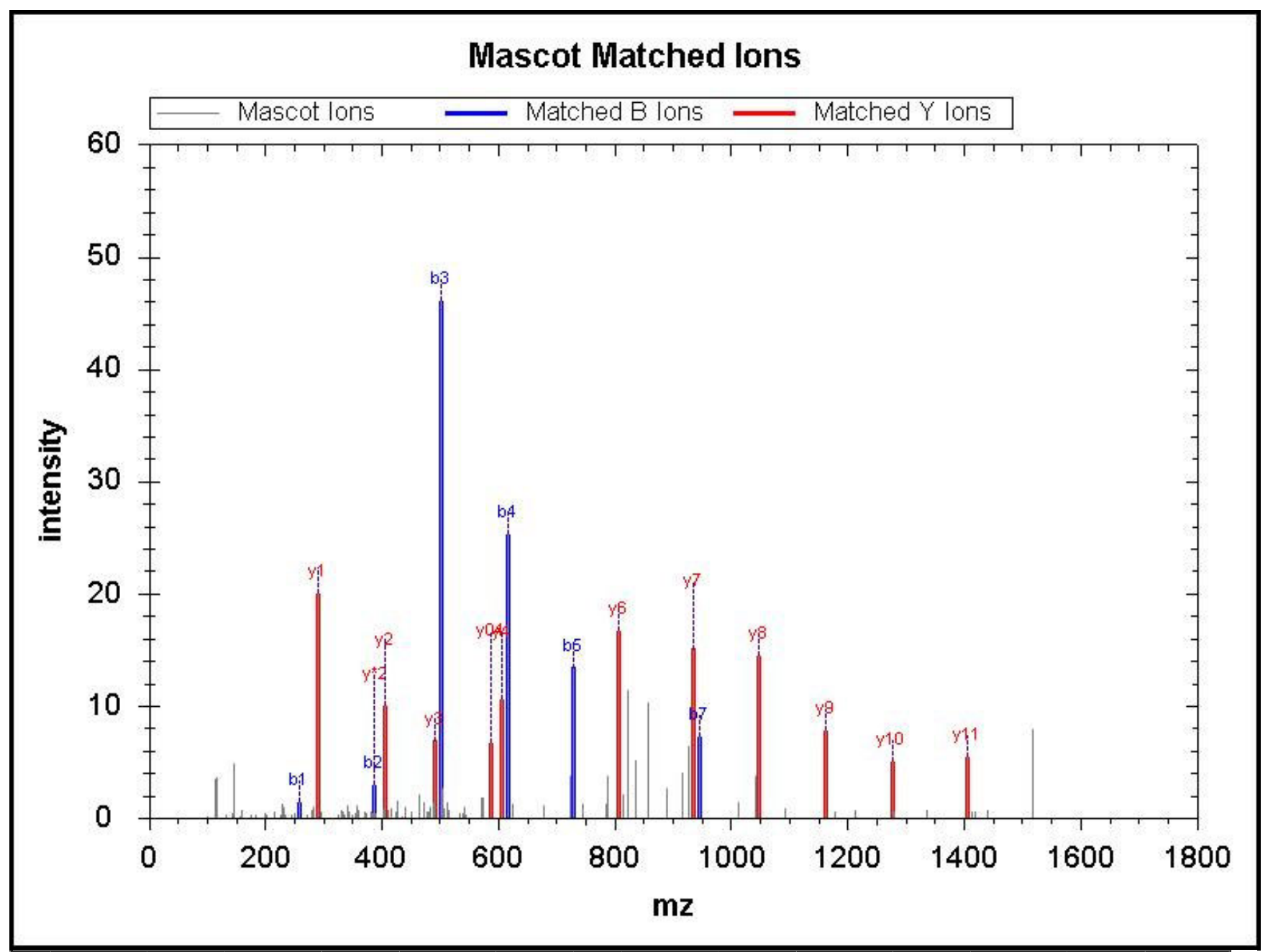

\begin{tabular}{|r|c|c|c|c|c|r|r|r|r|r|r|r|r|r|}
\hline \hline No & $\mathrm{b}$ & $\mathrm{b}++$ & $\mathrm{b} *$ & $\mathrm{~b} *++$ & $\mathrm{b} 0$ & $\mathrm{~b} 0++$ & Seq & $\mathrm{y}$ & $\mathrm{y}++$ & $\mathrm{y}^{*}$ & $\mathrm{y}^{*++}$ & $\mathrm{y} 0$ & $\mathrm{y} 0++$ & $\mathrm{RevNo}$ \\
\hline 1 & 258.19 & 129.60 & & & & & $\mathrm{~L}$ & & & & & & & 12 \\
\hline 2 & 387.24 & 194.12 & & & 369.23 & 185.12 & $\mathrm{E}$ & $1,404.77$ & 702.89 & $1,387.74$ & 694.38 & $1,386.76$ & 693.88 & 11 \\
\hline 3 & 502.26 & 251.64 & & & 484.25 & 242.63 & $\mathrm{D}$ & $1,275.73$ & 638.37 & $1,258.70$ & 629.85 & $1,257.72$ & 629.36 & 10 \\
\hline 4 & 615.35 & 308.18 & & & 597.34 & 299.17 & $\mathrm{I}$ & $1,160.70$ & 580.85 & $1,143.67$ & 572.34 & $1,142.69$ & 571.85 & 9 \\
\hline 5 & 728.43 & 364.72 & & & 710.42 & 355.71 & $\mathrm{~L}$ & $1,047.62$ & 524.31 & $1,030.59$ & 515.80 & $1,029.61$ & 515.31 & 8 \\
\hline 6 & 857.47 & 429.24 & & & 839.46 & 420.24 & $\mathrm{E}$ & 934.53 & 467.77 & 917.51 & 459.26 & 916.52 & 458.76 & 7 \\
\hline 7 & 944.51 & 472.76 & & & 926.50 & 463.75 & S & 805.49 & 403.25 & 788.46 & 394.74 & 787.48 & 394.24 & 6 \\
\hline 8 & $1,057.59$ & 529.30 & & & $1,039.58$ & 520.29 & I & 718.46 & 359.73 & 701.43 & 351.22 & 700.45 & 350.73 & 5 \\
\hline 9 & $1,171.63$ & 586.32 & $1,154.61$ & 577.81 & $1,153.62$ & 577.31 & N & 605.37 & 303.19 & 588.35 & 294.68 & 587.36 & 294.19 & 4
\end{tabular}




\begin{tabular}{|l|l|l|l|l|l|l|l|l|l|l|l|l|l|l|}
\hline 10 & $1,258.66$ & 629.84 & $1,241.64$ & 621.32 & $1,240.65$ & 620.83 & $\mathrm{~S}$ & 491.33 & 246.17 & 474.30 & 237.66 & 473.32 & 237.16 & 3 \\
\hline 11 & $1,371.75$ & 686.38 & $1,354.72$ & 677.86 & $1,353.74$ & 677.37 & $\mathrm{I}$ & 404.30 & 202.65 & 387.27 & 194.14 & & & 2 \\
\hline 12 & & & & & & & $\mathrm{~K}$ & 291.21 & 146.11 & 274.19 & 137.60 & & & 1 \\
\hline
\end{tabular}

Query 66321 Hit 1

MS/MS Fragmentation of ILDSFAAAPVPTTTLVLK

Found in sp $|\mathrm{P} 18583| \mathrm{SON}$ _HUMAN, Protein SON OS=Homo sapiens GN=SON PE=1 SV=4

Match to Query 66321:2144.268from(715.7632,3+)

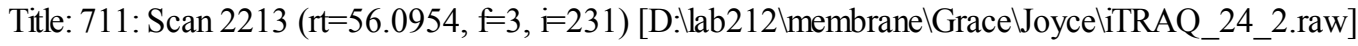

Data File:Submitted from 20120508-1(merge) by Mascot Daemon on JOYCE-VAIO

Monoisotopic mass of neutral peptide Mr(calc): 2144.268

Variable modifications:

K18 :iTRAQ4plex (K)

Ions Score: 76.43 Expect: 0.000

\section{Mascot Matched lons}

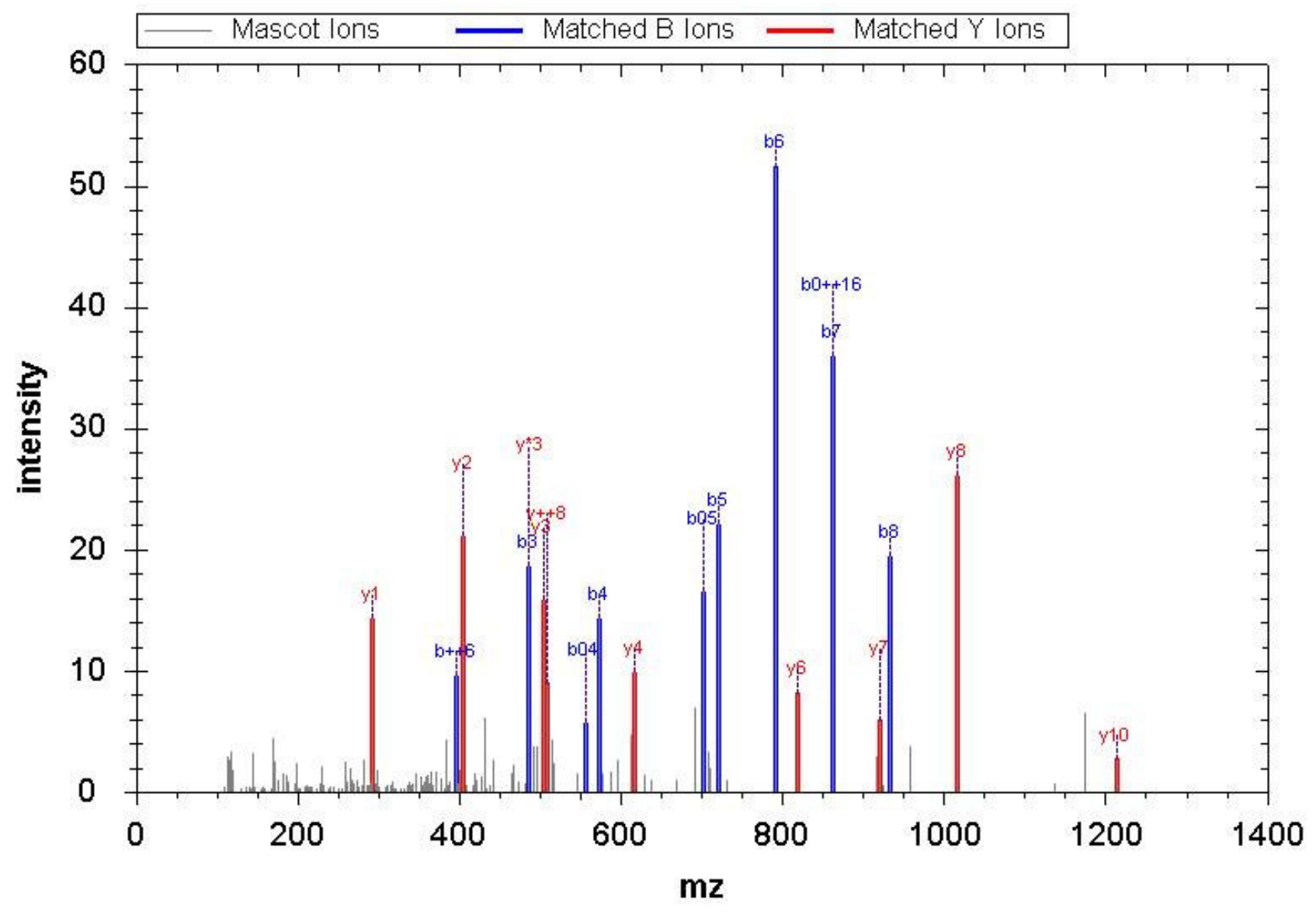

\begin{tabular}{|r|c|c|c|c|c|c|c|c|c|c|r|r|}
\hline $\mathrm{No}$ & $\mathrm{b}$ & $\mathrm{b}++$ & $\mathrm{b} 0$ & $\mathrm{~b} 0++$ & $\mathrm{Seq}$ & $\mathrm{y}$ & $\mathrm{y}++$ & $\mathrm{y}^{*}$ & $\mathrm{y} *++$ & $\mathrm{y} 0$ & $\mathrm{y} 0++$ & RevNo \\
\hline 1 & 258.19 & 129.60 & & & $\mathrm{I}$ & & & & & & & 18 \\
\hline 2 & 371.28 & 186.14 & & & $\mathrm{~L}$ & $1,888.09$ & 944.55 & $1,871.06$ & 936.04 & $1,870.08$ & 935.54 & 17 \\
\hline 3 & 486.30 & 243.66 & 468.29 & 234.65 & $\mathrm{D}$ & $1,775.01$ & 888.01 & $1,757.98$ & 879.49 & $1,757.00$ & 879.00 & 16 \\
\hline 4 & 573.34 & 287.17 & 555.33 & 278.17 & $\mathrm{~S}$ & $1,659.98$ & 830.49 & $1,642.95$ & 821.98 & $1,641.97$ & 821.49 & 15 \\
\hline 5 & 720.40 & 360.71 & 702.39 & 351.70 & $\mathrm{~F}$ & $1,572.95$ & 786.98 & $1,555.92$ & 778.46 & $1,554.94$ & 777.97 & 14 \\
\hline 6 & 791.44 & 396.22 & 773.43 & 387.22 & $\mathrm{~A}$ & $1,425.88$ & 713.44 & $1,408.85$ & 704.93 & $1,407.87$ & 704.44 & 13 \\
\hline 7 & 862.48 & 431.74 & 844.47 & 422.74 & $\mathrm{~A}$ & $1,354.84$ & 677.92 & $1,337.82$ & 669.41 & $1,336.83$ & 668.92 & 12 \\
\hline 8 & 933.52 & 467.26 & 915.51 & 458.26 & $\mathrm{~A}$ & $1,283.81$ & 642.41 & $1,266.78$ & 633.89 & $1,265.79$ & 633.40 & 11 \\
\hline 9 & $1,030.57$ & 515.79 & $1,012.56$ & 506.78 & $\mathrm{P}$ & $1,212.77$ & 606.89 & $1,195.74$ & 598.37 & $1,194.76$ & 597.88 & 10 \\
\hline 10 & $1,129.64$ & 565.32 & $1,111.63$ & 556.32 & $\mathrm{~V}$ & $1,115.72$ & 558.36 & $1,098.69$ & 549.85 & $1,097.70$ & 549.36 & 9 \\
\hline 11 & $1,226.69$ & 613.85 & $1,208.68$ & 604.84 & $\mathrm{P}$ & $1,016.65$ & 508.83 & 999.62 & 500.31 & 998.64 & 499.82 & 8 \\
\hline
\end{tabular}




\begin{tabular}{|r|r|r|r|r|r|r|r|r|r|r|r|r|r|}
12 & $1,327.74$ & 664.37 & $1,309.73$ & 655.37 & $\mathrm{~T}$ & 919.59 & 460.30 & 902.57 & 451.79 & 901.58 & 451.30 & 7 \\
\hline 13 & $1,428.79$ & 714.90 & $1,410.77$ & 705.89 & $\mathrm{~T}$ & 818.55 & 409.78 & 801.52 & 401.26 & 800.54 & 400.77 & 6 \\
\hline 14 & $1,529.83$ & 765.42 & $1,511.82$ & 756.41 & $\mathrm{~T}$ & 717.50 & 359.25 & 700.47 & 350.74 & 699.49 & 350.25 & 5 \\
\hline 15 & $1,642.92$ & 821.96 & $1,624.91$ & 812.96 & $\mathrm{~L}$ & 616.45 & 308.73 & 599.42 & 300.22 & & & 4 \\
\hline 16 & $1,741.99$ & 871.50 & $1,723.98$ & 862.49 & $\mathrm{~V}$ & 503.37 & 252.19 & 486.34 & 243.67 & & & 3 \\
\hline 17 & $1,855.07$ & 928.04 & $1,837.06$ & 919.03 & $\mathrm{~L}$ & 404.30 & 202.65 & 387.27 & 194.14 & & & 2 \\
\hline 18 & & & & & $\mathrm{~K}$ & 291.21 & 146.11 & 274.19 & 137.60 & & & 1 \\
\hline
\end{tabular}

Query 73795 Hit 1

MS/MS Fragmentation of AQVLVEDISDILEEHAEK

Found in sp|Q5VV41|ARHGG_HUMAN, Rho guanine nucleotide exchange factor 16 OS=Homo sapiens GN=ARHGEF16 PE=1 SV=1 Match to Query 73795: 2325.229from(776.0835,3+)

Title: 1057: Sum of 2 scans in range 2325 ( $\mathrm{rt}=61.764, \mathrm{f}=4, \mathrm{i}=707)$ to 2326 ( $\mathrm{rt}=61.7894, \mathrm{f}=4, \mathrm{i}=708$ )

[D:lab212 \membranelGrace JoyceliTRAQ_35_1.raw]

Data File:Submitted from 20120508-1(merge) by Mascot Daemon on JOYCE-VAIO

Monoisotopic mass of neutral peptide Mr(calc): 2325.229

Variable modifications:

K18 :iTRAQ4plex (K)

Ions Score: 76.08 Expect: 0.000

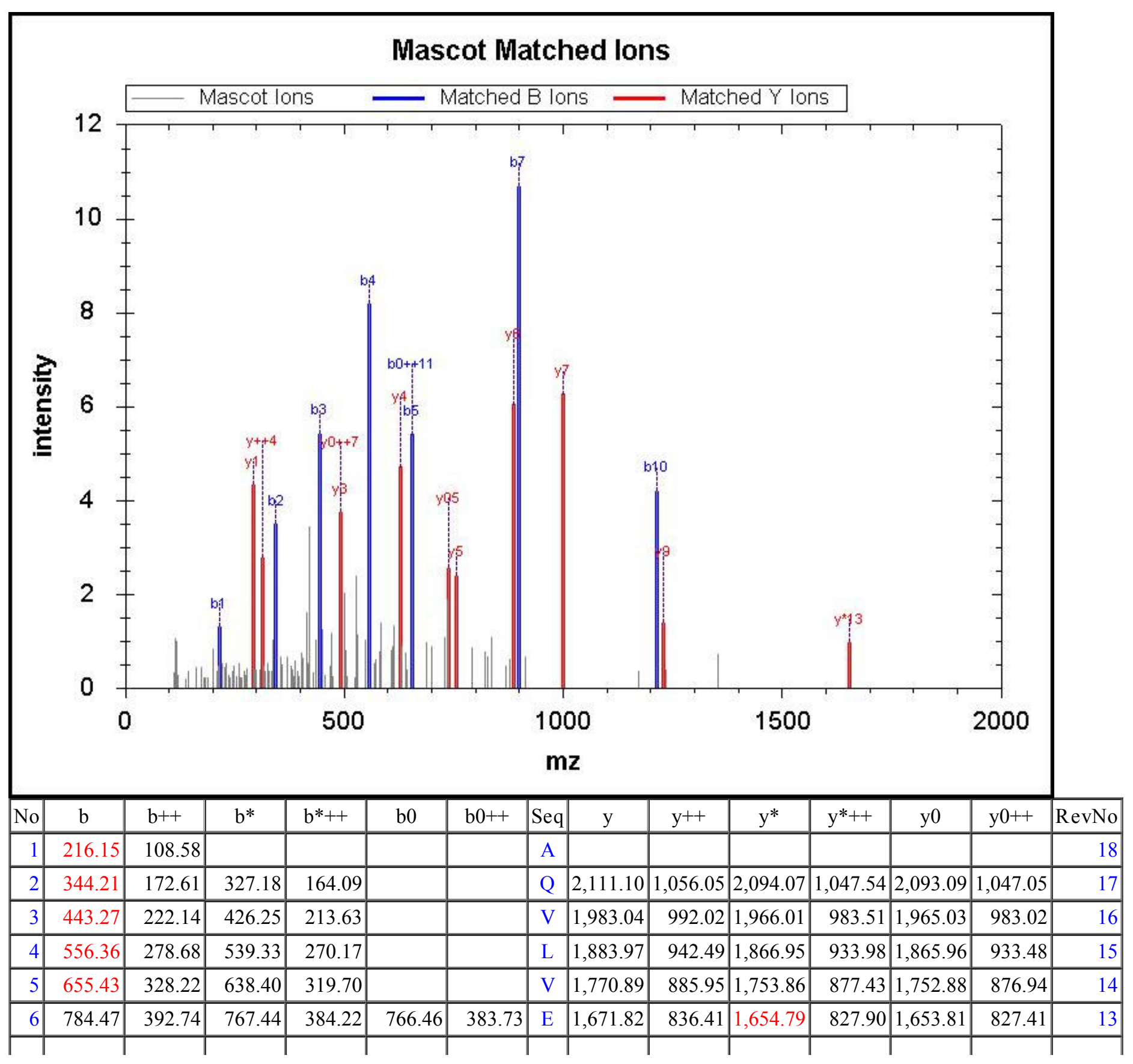




\begin{tabular}{|r|r|r|r|r|r|r|r|r|r|r|r|r|r|r|r|r|r|}
\hline 7 & 899.50 & 450.25 & 882.47 & 441.74 & 881.48 & 441.25 & $\mathrm{D}$ & $1,542.78$ & 771.89 & $1,525.75$ & 763.38 & $1,524.77$ & 762.89 & 12 \\
\hline 8 & $1,012.58$ & 506.79 & 995.55 & 498.28 & 994.57 & 497.79 & $\mathrm{I}$ & $1,427.75$ & 714.38 & $1,410.72$ & 705.87 & $1,409.74$ & 705.37 & 11 \\
\hline 9 & $1,099.61$ & 550.31 & $1,082.59$ & 541.80 & $1,081.60$ & 541.30 & $\mathrm{~S}$ & $1,314.67$ & 657.84 & $1,297.64$ & 649.32 & $1,296.66$ & 648.83 & 10 \\
\hline 10 & $1,214.64$ & 607.82 & $1,197.61$ & 599.31 & $1,196.63$ & 598.82 & $\mathrm{D}$ & $1,227.63$ & 614.32 & $1,210.61$ & 605.81 & $1,209.62$ & 605.32 & 9 \\
\hline 11 & $1,327.72$ & 664.36 & $1,310.70$ & 655.85 & $1,309.71$ & 655.36 & $\mathrm{I}$ & $1,112.61$ & 556.81 & $1,095.58$ & 548.29 & $1,094.60$ & 547.80 & 8 \\
\hline 12 & $1,440.81$ & 720.91 & $1,423.78$ & 712.39 & $1,422.80$ & 711.90 & $\mathrm{~L}$ & 999.52 & 500.26 & 982.50 & 491.75 & 981.51 & 491.26 & 7 \\
\hline 13 & $1,569.85$ & 785.43 & $1,552.82$ & 776.92 & $1,551.84$ & 776.42 & $\mathrm{E}$ & 886.44 & 443.72 & 869.41 & 435.21 & 868.43 & 434.72 & 6 \\
\hline 14 & $1,698.89$ & 849.95 & $1,681.87$ & 841.44 & $1,680.88$ & 840.94 & $\mathrm{E}$ & 757.40 & 379.20 & 740.37 & 370.69 & 739.39 & 370.20 & 5 \\
\hline 15 & $1,835.95$ & 918.48 & $1,818.92$ & 909.97 & $1,817.94$ & 909.47 & $\mathrm{H}$ & 628.35 & 314.68 & 611.33 & 306.17 & 610.34 & 305.68 & 4 \\
\hline 16 & $1,906.99$ & 954.00 & $1,889.96$ & 945.48 & $1,888.98$ & 944.99 & $\mathrm{~A}$ & 491.29 & 246.15 & 474.27 & 237.64 & 473.28 & 237.15 & 3 \\
\hline 17 & $2,036.03$ & $1,018.52$ & $2,019.00$ & $1,010.01$ & $2,018.02$ & $1,009.51$ & $\mathrm{E}$ & 420.26 & 210.63 & 403.23 & 202.12 & 402.25 & 201.63 & 2 \\
\hline 18 & & & & & & & $\mathrm{~K}$ & 291.21 & 146.11 & 274.19 & 137.60 & & & 1 \\
\hline
\end{tabular}

Query 58806 Hit 1

MS/MS Fragmentation of LGVPFQVLVATHAGLYR

Found in sp|Q96T60|PNKP_HUM AN, Bifunctional polynucleotide phosphatase/kinase OS=Homo sapiens GN=PNKP PE=1 SV=1

Match to Query 58806: 1984.135 from(662.3854,3+)

Title: 910: Sum of 2 scans in range 2002 ( $\mathrm{rt}=54.6296, \mathrm{f}=4, \mathrm{i}=609)$ to 2003 ( $\mathrm{rt}=54.655, \mathrm{f}=4, \mathrm{i}=610)$

[D:lab212 \membranelGrace JoyceliTRAQ_34_2.raw]

Data File:Submitted from 20120508-1(merge) by Mascot Daemon on JOYCE-VAIO

Monoisotopic mass of neutral peptide $\operatorname{Mr}($ calc): 1984.135

Variable modifications:

Ions Score: 74.84 Expect: 0.000

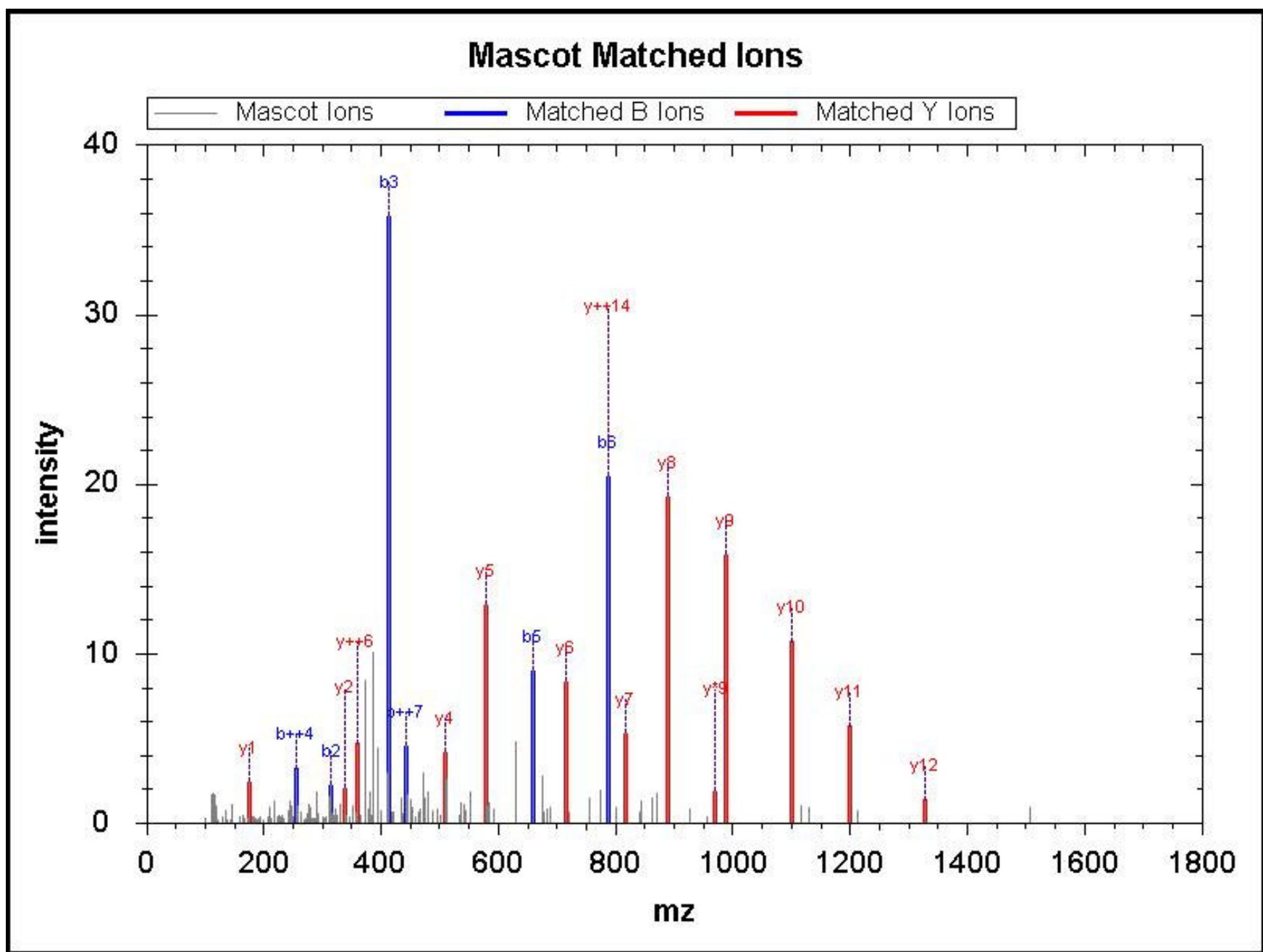

\begin{tabular}{|r|c|c|c|c|c|c|c|c|c|c|c|c|c|c|c|}
\hline \hline No & $\mathrm{b}$ & $\mathrm{b}++$ & $\mathrm{b}^{*}$ & $\mathrm{~b}^{*++}$ & $\mathrm{b} 0$ & $\mathrm{~b} 0++$ & Seq & $\mathrm{y}$ & $\mathrm{y}++$ & $\mathrm{y}^{*}$ & $\mathrm{y}^{*++}$ & $\mathrm{y} 0$ & $\mathrm{y} 0++$ & $\mathrm{RevNo}$ \\
\hline 1 & 258.19 & 129.60 & & & & & L & & & & & & & 17 \\
\hline & & & & & & & & & & & & & &
\end{tabular}




\begin{tabular}{|r|r|r|r|r|r|r|r|r|r|r|r|r|r|r|}
\hline 2 & 315.21 & 158.11 & & & & & G & $1,727.96$ & 864.48 & $1,710.93$ & 855.97 & $1,709.95$ & 855.48 & 16 \\
\hline 3 & 414.28 & 207.65 & & & & & V & $1,670.94$ & 835.97 & $1,653.91$ & 827.46 & $1,652.93$ & 826.97 & 15 \\
\hline 4 & 511.34 & 256.17 & & & & & P & $1,571.87$ & 786.44 & $1,554.84$ & 777.92 & $1,553.86$ & 777.43 & 14 \\
\hline 5 & 658.40 & 329.71 & & & & & F & $1,474.82$ & 737.91 & $1,457.79$ & 729.40 & $1,456.81$ & 728.91 & 13 \\
\hline 6 & 786.46 & 393.74 & 769.44 & 385.22 & & & Q & $1,327.75$ & 664.38 & $1,310.72$ & 655.86 & $1,309.74$ & 655.37 & 12 \\
\hline 7 & 885.53 & 443.27 & 868.50 & 434.76 & & & V & $1,199.69$ & 600.35 & $1,182.66$ & 591.84 & $1,181.68$ & 591.34 & 11 \\
\hline 8 & 998.62 & 499.81 & 981.59 & 491.30 & & & L & $1,100.62$ & 550.81 & $1,083.59$ & 542.30 & $1,082.61$ & 541.81 & 10 \\
\hline 9 & $1,097.68$ & 549.35 & $1,080.66$ & 540.83 & & & V & 987.54 & 494.27 & 970.51 & 485.76 & 969.53 & 485.27 & 9 \\
\hline 10 & $1,168.72$ & 584.86 & $1,151.69$ & 576.35 & & & A & 888.47 & 444.74 & 871.44 & 436.22 & 870.46 & 435.73 & 8 \\
\hline 11 & $1,269.77$ & 635.39 & $1,252.74$ & 626.87 & $1,251.76$ & 626.38 & T & 817.43 & 409.22 & 800.40 & 400.71 & 799.42 & 400.21 & 7 \\
\hline 12 & $1,406.83$ & 703.92 & $1,389.80$ & 695.40 & $1,388.82$ & 694.91 & H & 716.38 & 358.70 & 699.36 & 350.18 & & & 6 \\
\hline 13 & $1,477.86$ & 739.44 & $1,460.84$ & 730.92 & $1,459.85$ & 730.43 & A & 579.32 & 290.17 & 562.30 & 281.65 & & & 5 \\
\hline 14 & $1,534.89$ & 767.95 & $1,517.86$ & 759.43 & $1,516.88$ & 758.94 & G & 508.29 & 254.65 & 491.26 & 246.13 & & & 4 \\
\hline 15 & $1,647.97$ & 824.49 & $1,630.94$ & 815.98 & $1,629.96$ & 815.48 & L & 451.27 & 226.14 & 434.24 & 217.62 & & & 3 \\
\hline 16 & $1,811.03$ & 906.02 & $1,794.01$ & 897.51 & $1,793.02$ & 897.02 & Y & 338.18 & 169.59 & 321.16 & 161.08 & & & 2 \\
\hline 17 & & & & & & & R & 175.12 & 88.06 & 158.09 & 79.55 & & & 1 \\
\hline
\end{tabular}

Query 66682 Hit 1

MS/MS Fragmentation of VGLIHMLTHLAEALHQAR

Found in sp|Q9BWS9|CHID1_HUMAN, Chitinase domain-containing protein $1 \mathrm{OS}=$ Homo sapiens $\mathrm{GN}=\mathrm{CHID} 1 \mathrm{PE}=1 \mathrm{SV}=1$ Match to Query 66682: 2153.201 from(718.7408,3+)

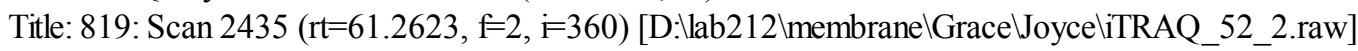

Data File:Submitted from 20120508-1(merge) by Mascot Daemon on JOYCE-VAIO

Monoisotopic mass of neutral peptide Mr(calc): 2153.201

Variable modifications:

Ions Score: 74.17 Expect: 0.000 
Mascot Matched lons

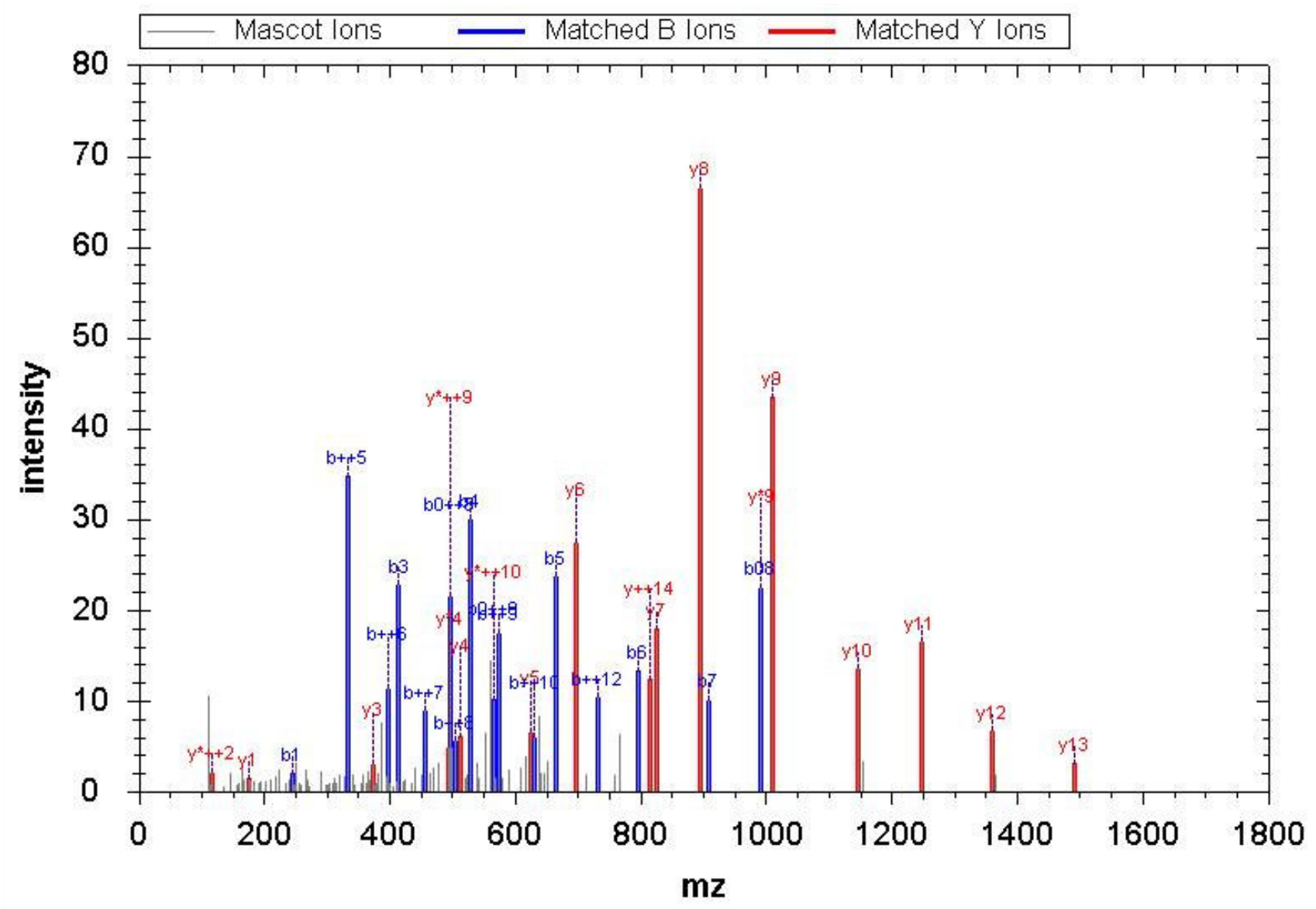

\begin{tabular}{|c|c|c|c|c|c|c|c|c|c|c|c|c|c|c|}
\hline No & $\mathrm{b}$ & $\mathrm{b}++$ & $b^{*}$ & $\mathrm{~b}^{*++}$ & b0 & $\mathrm{b} 0++$ & Seq & $\mathrm{y}$ & $\mathrm{y}++$ & $\mathrm{y}^{*}$ & $y^{*++}$ & y0 & $\mathrm{y} 0++$ & RevNo \\
\hline 1 & 244.18 & 122.59 & & & & & $\mathrm{~V}$ & & & & & & & 18 \\
\hline 2 & 301.20 & 151.10 & & & & & G & $1,911.04$ & 956.02 & $1,894.01$ & 947.51 & $1,893.03$ & 947.02 & 17 \\
\hline 3 & 414.28 & 207.65 & & & & & $\mathrm{~L}$ & $1,854.02$ & 927.51 & $1,836.99$ & 919.00 & $1,836.01$ & 918.51 & 16 \\
\hline 4 & 527.37 & 264.19 & & & & & I & $1,740.93$ & 870.97 & $1,723.91$ & 862.46 & $1,722.92$ & 861.96 & 15 \\
\hline 5 & 664.43 & 332.72 & & & & & $\mathrm{H}$ & $1,627.85$ & 814.43 & $1,610.82$ & 805.91 & $1,609.84$ & 805.42 & 14 \\
\hline 6 & 795.47 & 398.24 & & & & & M & $1,490.79$ & 745.90 & $1,473.76$ & 737.39 & $1,472.78$ & 736.89 & 13 \\
\hline 7 & 908.55 & 454.78 & & & & & $\mathrm{~L}$ & $1,359.75$ & 680.38 & $1,342.72$ & 671.86 & $1,341.74$ & 671.37 & 12 \\
\hline 8 & $1,009.60$ & 505.30 & & & 991.59 & 496.30 & $\mathrm{~T}$ & $1,246.67$ & 623.84 & $1,229.64$ & 615.32 & $1,228.65$ & 614.83 & 11 \\
\hline 9 & $1,146.66$ & 573.83 & & & $1,128.65$ & 564.83 & $\mathrm{H}$ & $1,145.62$ & 573.31 & $1,128.59$ & 564.80 & $1,127.61$ & 564.31 & 10 \\
\hline 10 & $1,259.74$ & 630.37 & & & $1,241.73$ & 621.37 & $\mathrm{~L}$ & $1,008.56$ & 504.78 & 991.53 & 496.27 & 990.55 & 495.78 & 9 \\
\hline 11 & $1,330.78$ & 665.89 & & & $1,312.77$ & 656.89 & A & 895.47 & 448.24 & 878.45 & 439.73 & 877.46 & 439.24 & 8 \\
\hline 12 & $1,459.82$ & 730.41 & & & $1,441.81$ & 721.41 & $\mathrm{E}$ & 824.44 & 412.72 & 807.41 & 404.21 & 806.43 & 403.72 & 7 \\
\hline 13 & $1,530.86$ & 765.93 & & & $1,512.85$ & 756.93 & A & 695.39 & 348.20 & 678.37 & 339.69 & & & 6 \\
\hline 14 & $1,643.94$ & 822.47 & & & $1,625.93$ & 813.47 & $\mathrm{~L}$ & 624.36 & 312.68 & 607.33 & 304.17 & & & 5 \\
\hline 15 & $1,781.00$ & 891.00 & & & $1,762.99$ & 882.00 & $\mathrm{H}$ & 511.27 & 256.14 & 494.25 & 247.63 & & & 4 \\
\hline 16 & $1,909.06$ & 955.03 & $1,892.03$ & 946.52 & $1,891.05$ & 946.03 & $\mathrm{Q}$ & 374.21 & 187.61 & 357.19 & 179.10 & & & 3 \\
\hline 17 & $1,980.10$ & 990.55 & $1,963.07$ & 982.04 & $1,962.09$ & 981.55 & A & 246.16 & 123.58 & 229.13 & 115.07 & & & 2 \\
\hline 18 & & & & & & & $\mathrm{R}$ & 175.12 & 88.06 & 158.09 & 79.55 & & & 1 \\
\hline
\end{tabular}

Query 40396 Hit 1

MS/MS Fragmentation of DPAAPEPEEQEER

Found in sp|Q969T4|UB2E3_HUMAN, Ubiquitin-conjugating enzyme E2 E3 OS=Homo sapiens GN=UBE2E3 PE=1 SV=1 Match to Query 40396: 1639.742 from(820.8785,2+)

Title: 87: Sum of 2 scans in range $395(\mathrm{rt}=18.0766, \mathrm{f}=4, \mathrm{i}=43)$ to $396(\mathrm{rt}=18.102, \mathrm{f}=4, \mathrm{i}=44)$ 
[D:llab212 \membranelGrace JoyceliTRAQ_21_2.raw]

Data File:Submitted from 20120508-1(merge) by Mascot Daemon on JOYCE-VAIO

Monoisotopic mass of neutral peptide $\operatorname{Mr}($ calc): 1639.742

Variable modifications:

Ions Score: 74.13 Expect: 0.000

\section{Mascot Matched lons}

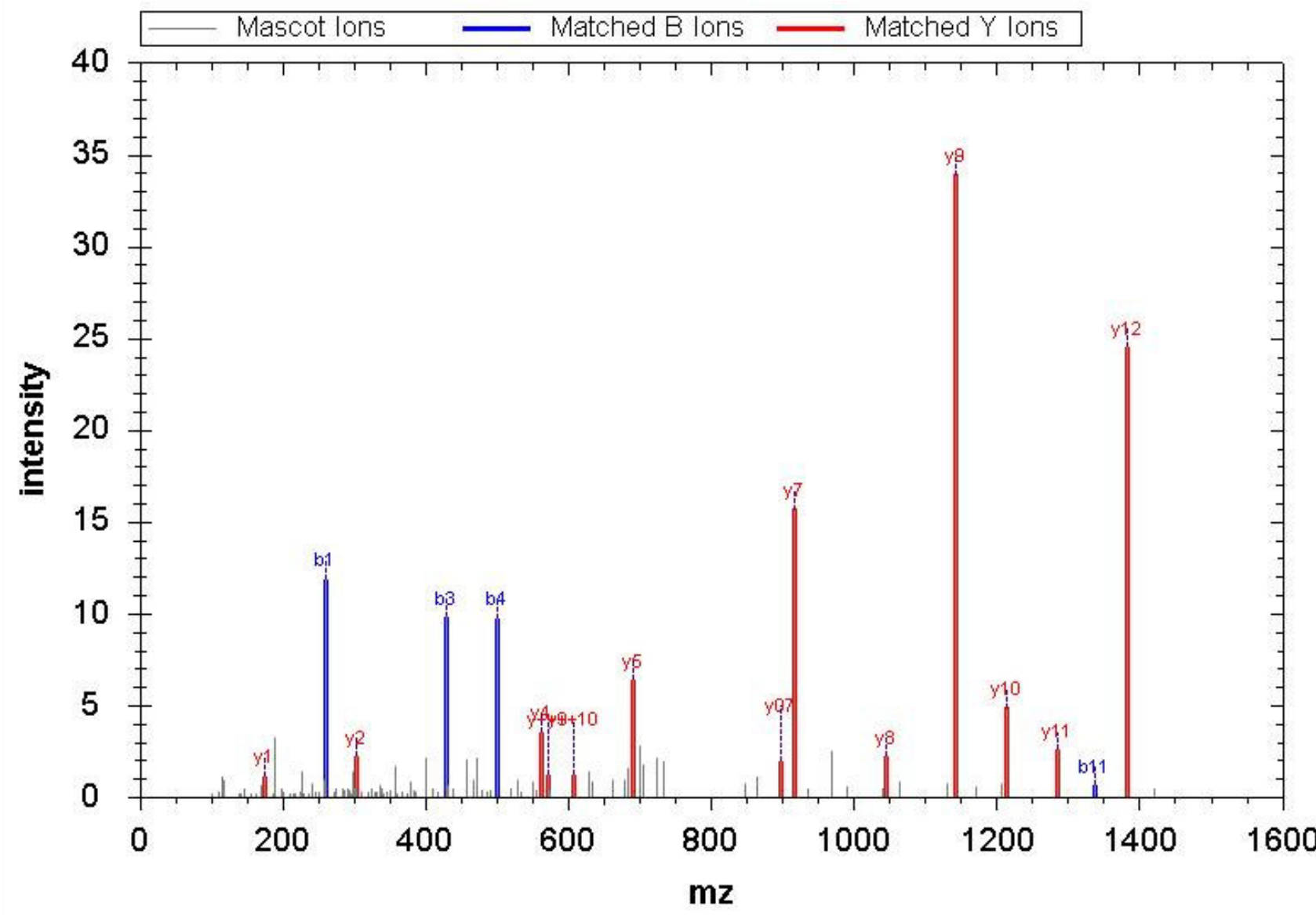

\begin{tabular}{|r|c|c|c|c|r|r|r|r|r|r|r|r|r|r|}
\hline \hline No & $\mathrm{b}$ & $\mathrm{b}++$ & $\mathrm{b}^{*}$ & $\mathrm{~b} *++$ & $\mathrm{b} 0$ & $\mathrm{~b} 0++$ & Seq & $\mathrm{y}$ & $\mathrm{y}++$ & $\mathrm{y}^{*}$ & $\mathrm{y}^{*++}$ & $\mathrm{y} 0$ & $\mathrm{y} 0++$ & $\mathrm{RevNo}$ \\
\hline 1 & 260.14 & 130.57 & & & 242.13 & 121.57 & $\mathrm{D}$ & & & & & & & 13 \\
\hline 2 & 357.19 & 179.10 & & & 339.18 & 170.09 & $\mathrm{P}$ & $1,381.62$ & 691.32 & $1,364.60$ & 682.80 & $1,363.61$ & 682.31 & 12 \\
\hline 3 & 428.23 & 214.62 & & & 410.22 & 205.61 & A & $1,284.57$ & 642.79 & $1,267.54$ & 634.28 & $1,266.56$ & 633.78 & 11 \\
\hline 4 & 499.26 & 250.14 & & & 481.25 & 241.13 & A & $1,213.53$ & 607.27 & $1,196.51$ & 598.76 & $1,195.52$ & 598.26 & 10 \\
\hline 5 & 596.32 & 298.66 & & & 578.31 & 289.66 & P & $1,142.50$ & 571.75 & $1,125.47$ & 563.24 & $1,124.49$ & 562.75 & 9 \\
\hline 6 & 725.36 & 363.18 & & & 707.35 & 354.18 & E & $1,045.44$ & 523.23 & $1,028.42$ & 514.71 & $1,027.43$ & 514.22 & 8 \\
\hline 7 & 822.41 & 411.71 & & & 804.40 & 402.70 & P & 916.40 & 458.70 & 899.37 & 450.19 & 898.39 & 449.70 & 7 \\
\hline 8 & 951.45 & 476.23 & & & 933.44 & 467.23 & E & 819.35 & 410.18 & 802.32 & 401.66 & 801.34 & 401.17 & 6 \\
\hline 9 & $1,080.50$ & 540.75 & & & $1,062.49$ & 531.75 & E & 690.31 & 345.66 & 673.28 & 337.14 & 672.29 & 336.65 & 5 \\
\hline 10 & $1,208.56$ & 604.78 & $1,191.53$ & 596.27 & $1,190.54$ & 595.78 & Q & 561.26 & 281.13 & 544.24 & 272.62 & 543.25 & 272.13 & 4 \\
\hline 11 & $1,337.60$ & 669.30 & $1,320.57$ & 660.79 & $1,319.59$ & 660.30 & E & 433.20 & 217.11 & 416.18 & 208.59 & 415.19 & 208.10 & 3 \\
\hline 12 & $1,466.64$ & 733.82 & $1,449.61$ & 725.31 & $1,448.63$ & 724.82 & E & 304.16 & 152.58 & 287.13 & 144.07 & 286.15 & 143.58 & 2 \\
\hline 13 & & & & & & & R & 175.12 & 88.06 & 158.09 & 79.55 & & & 1 \\
\hline
\end{tabular}

Query 56384 Hit 1

MS/MS Fragmentation of AIGIEPSLATYHHIIR

Found in sp|Q96EY7|PTCD3_HUMAN, Pentatricopeptide repeat-containing protein 3

Match to Query 56384: 1934.087 from $(484.529,4+)$

Title: 575: Scan $1370(\mathrm{rt}=40.3204, \mathrm{f}=3, \mathrm{i}=210)$ [D:lab212 \membranelGrace Joyce 47 _.raw]

Data File:Submitted from 20120508-1(merge) by Mascot Daemon on JOYCE-VAIO 


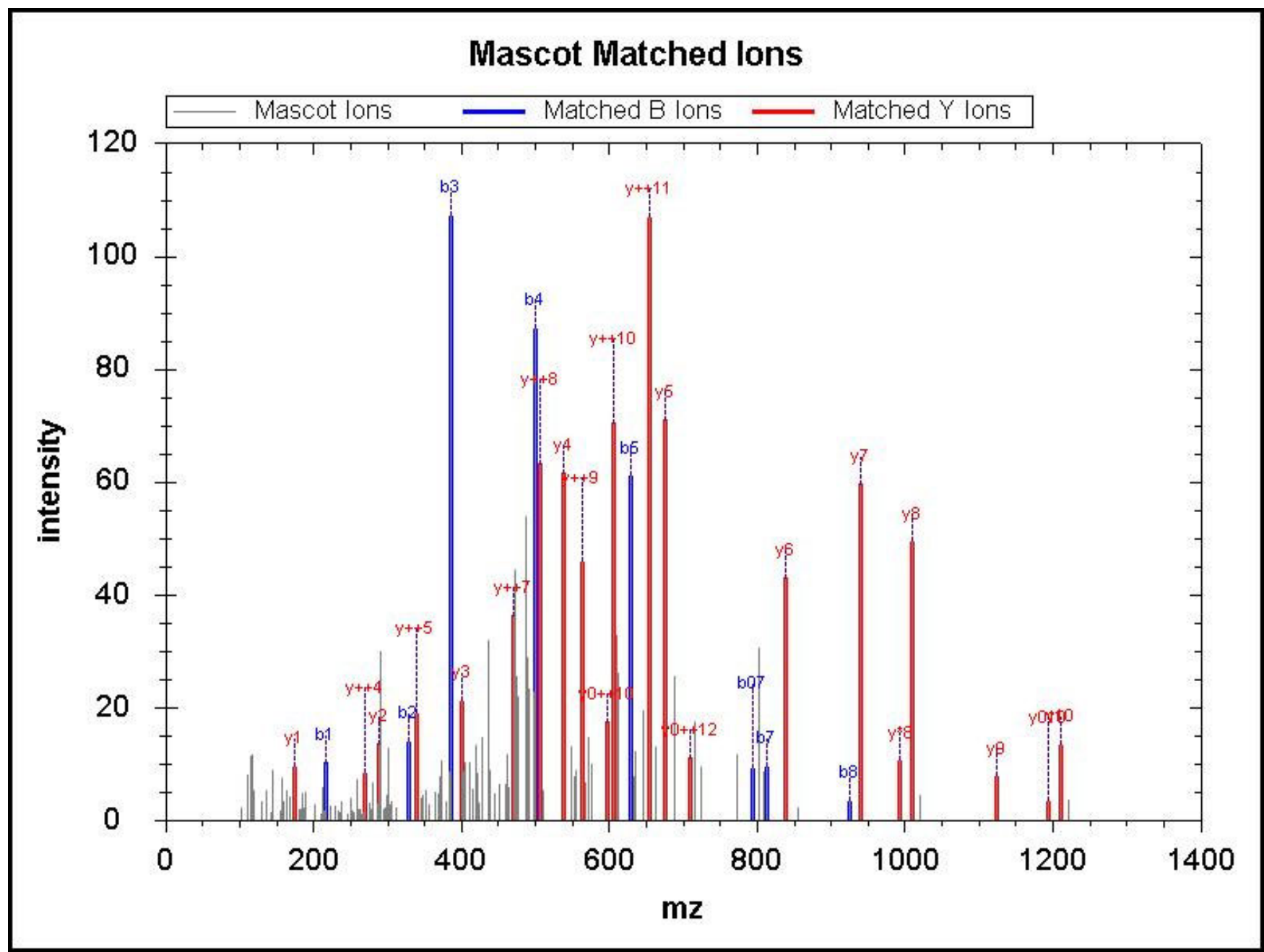

\begin{tabular}{|r|c|c|c|c|c|c|c|c|c|c|r|r|}
\hline $\mathrm{No}$ & $\mathrm{b}$ & $\mathrm{b}++$ & $\mathrm{b} 0$ & $\mathrm{~b} 0++$ & $\mathrm{Seq}$ & $\mathrm{y}$ & $\mathrm{y}++$ & $\mathrm{y}^{*}$ & $\mathrm{y}^{*++}$ & $\mathrm{y} 0$ & $\mathrm{y} 0++$ & $\mathrm{RevNo}$ \\
\hline 1 & 216.15 & 108.58 & & & $\mathrm{~A}$ & & & & & & & 16 \\
\hline 2 & 329.23 & 165.12 & & & $\mathrm{I}$ & $1,719.95$ & 860.48 & $1,702.93$ & 851.97 & $1,701.94$ & 851.48 & 15 \\
\hline 3 & 386.25 & 193.63 & & & $\mathrm{G}$ & $1,606.87$ & 803.94 & $1,589.84$ & 795.43 & $1,588.86$ & 794.93 & 14 \\
\hline 4 & 499.34 & 250.17 & & & $\mathrm{I}$ & $1,549.85$ & 775.43 & $1,532.82$ & 766.91 & $1,531.84$ & 766.42 & 13 \\
\hline 5 & 628.38 & 314.69 & 610.37 & 305.69 & $\mathrm{E}$ & $1,436.76$ & 718.89 & $1,419.74$ & 710.37 & $1,418.75$ & 709.88 & 12 \\
\hline 6 & 725.43 & 363.22 & 707.42 & 354.21 & $\mathrm{P}$ & $1,307.72$ & 654.36 & $1,290.70$ & 645.85 & $1,289.71$ & 645.36 & 11 \\
\hline 7 & 812.46 & 406.74 & 794.45 & 397.73 & $\mathrm{~S}$ & $1,210.67$ & 605.84 & $1,193.64$ & 597.32 & $1,192.66$ & 596.83 & 10 \\
\hline 8 & 925.55 & 463.28 & 907.54 & 454.27 & $\mathrm{~L}$ & $1,123.64$ & 562.32 & $1,106.61$ & 553.81 & $1,105.63$ & 553.32 & 9 \\
\hline 9 & 996.58 & 498.80 & 978.57 & 489.79 & $\mathrm{~A}$ & $1,010.55$ & 505.78 & 993.53 & 497.27 & 992.54 & 496.77 & 8 \\
\hline 10 & $1,097.63$ & 549.32 & $1,079.62$ & 540.31 & $\mathrm{~T}$ & 939.52 & 470.26 & 922.49 & 461.75 & 921.51 & 461.26 & 7 \\
\hline 11 & $1,260.70$ & 630.85 & $1,242.69$ & 621.85 & $\mathrm{Y}$ & 838.47 & 419.74 & 821.44 & 411.22 & & & 6 \\
\hline 12 & $1,397.75$ & 699.38 & $1,379.74$ & 690.38 & $\mathrm{H}$ & 675.40 & 338.21 & 658.38 & 329.69 & & & 5 \\
\hline 13 & $1,534.81$ & 767.91 & $1,516.80$ & 758.91 & $\mathrm{H}$ & 538.35 & 269.68 & 521.32 & 261.16 & & & 4 \\
\hline 14 & $1,647.90$ & 824.45 & $1,629.89$ & 815.45 & $\mathrm{I}$ & 401.29 & 201.15 & 384.26 & 192.63 & & & 3 \\
\hline 15 & $1,760.98$ & 880.99 & $1,742.97$ & 871.99 & $\mathrm{I}$ & 288.20 & 144.61 & 271.18 & 136.09 & & & 2 \\
\hline 16 & & & & & $\mathrm{R}$ & 175.12 & 88.06 & 158.09 & 79.55 & & & 1 \\
\hline
\end{tabular}

Query 70132 Hit 1

MS/MS Fragmentation of FNSVVTNPQGHYNPSTGK

Found in sp|Q02105|C1QC_MOUSE, Complement C1q subcomponent subunit C OS=Mus musculus GN=C1qc $\mathrm{PE}=2 \mathrm{SV}=1$

Match to Query 70132: 2234.125from(745.7156,3+) 
Title: 319: Scan 793 ( $\mathrm{rt}=27.1951, \mathrm{f}=3, \mathrm{i}=111)$ [D:llab212 \membranelGrace JoyceliTRAQ_38_1.raw]

Data File:Submitted from 20120508-1(merge) by Mascot Daemon on JOYCE-VAIO

Monoisotopic mass of neutral peptide $\mathrm{Mr}$ (calc): 2234.125

Variable modifications:

K18 :iTRAQ4plex (K)

Ions Score: 73.48 Expect: 0.000

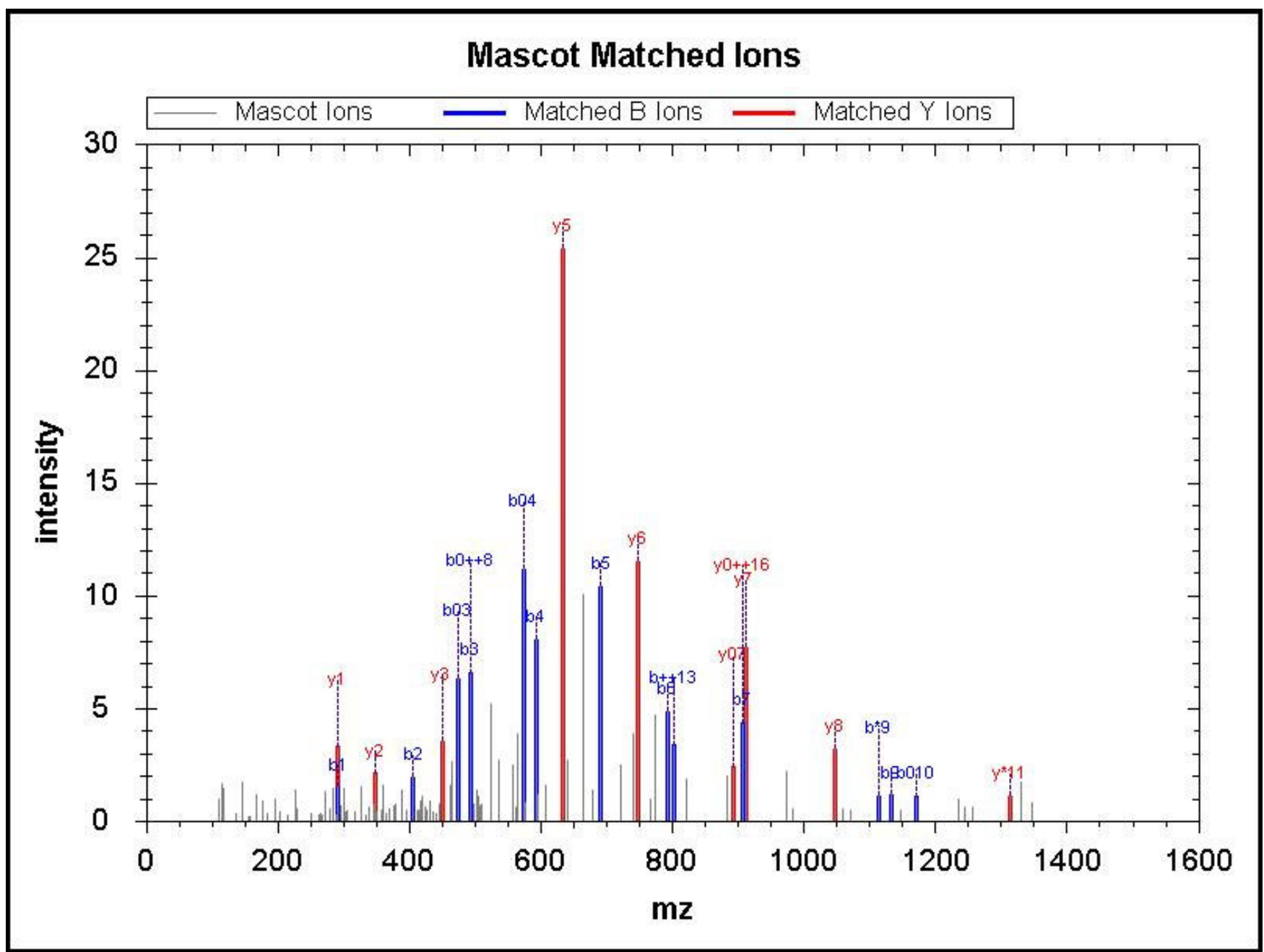

\begin{tabular}{|c|c|c|c|c|c|c|c|c|c|c|c|c|c|c|}
\hline No & $\mathrm{b}$ & $\mathrm{b}++$ & $b^{*}$ & $b^{*++}$ & b0 & $\mathrm{b} 0++$ & Seq & $\mathrm{y}$ & $\mathrm{y}^{++}$ & $y^{*}$ & $y^{*++}$ & y0 & $\mathrm{y} 0++$ & RevNo \\
\hline 1 & 292.18 & 146.59 & & & & & $\mathrm{~F}$ & & & & & & & 18 \\
\hline 2 & 406.22 & 203.61 & 389.19 & 195.10 & & & $\mathrm{~N}$ & $1,943.97$ & 972.49 & $1,926.94$ & 963.97 & $1,925.96$ & 963.48 & 17 \\
\hline 3 & 493.25 & 247.13 & 476.23 & 238.62 & 475.24 & 238.12 & $\mathrm{~S}$ & $1,829.93$ & 915.47 & $1,812.90$ & 906.95 & $1,811.92$ & 906.46 & 16 \\
\hline 4 & 592.32 & 296.66 & 575.29 & 288.15 & 574.31 & 287.66 & V & $1,742.89$ & 871.95 & $1,725.87$ & 863.44 & $1,724.88$ & 862.95 & 15 \\
\hline 5 & 691.39 & 346.20 & 674.36 & 337.69 & 673.38 & 337.19 & V & $1,643.83$ & 822.42 & $1,626.80$ & 813.90 & $1,625.82$ & 813.41 & 14 \\
\hline 6 & 792.44 & 396.72 & 775.41 & 388.21 & 774.43 & 387.72 & $\mathrm{~T}$ & $1,544.76$ & 772.88 & $1,527.73$ & 764.37 & $1,526.75$ & 763.88 & 13 \\
\hline 7 & 906.48 & 453.74 & 889.45 & 445.23 & 888.47 & 444.74 & $\mathrm{~N}$ & $1,443.71$ & 722.36 & $1,426.68$ & 713.85 & $1,425.70$ & 713.35 & 12 \\
\hline 8 & $1,003.53$ & 502.27 & 986.51 & 493.76 & 985.52 & 493.26 & $\mathrm{P}$ & $1,329.67$ & 665.34 & $1,312.64$ & 656.82 & $1,311.66$ & 656.33 & 11 \\
\hline 9 & $1,131.59$ & 566.30 & $1,114.57$ & 557.79 & $1,113.58$ & 557.29 & Q & $1,232.61$ & 616.81 & $1,215.59$ & 608.30 & $1,214.60$ & 607.81 & 10 \\
\hline 10 & $1,188.61$ & 594.81 & $1,171.59$ & 586.30 & $1,170.60$ & 585.80 & G & $1,104.56$ & 552.78 & $1,087.53$ & 544.27 & $1,086.54$ & 543.78 & 9 \\
\hline 11 & $1,325.67$ & 663.34 & $1,308.65$ & 654.83 & $1,307.66$ & 654.33 & $\mathrm{H}$ & $1,047.53$ & 524.27 & $1,030.51$ & 515.76 & $1,029.52$ & 515.27 & 8 \\
\hline 12 & $1,488.74$ & 744.87 & $1,471.71$ & 736.36 & $1,470.72$ & 735.87 & $\mathrm{Y}$ & 910.47 & 455.74 & 893.45 & 447.23 & 892.46 & 446.74 & 7 \\
\hline 13 & $1,602.78$ & 801.89 & $1,585.75$ & 793.38 & $1,584.77$ & 792.89 & $\mathrm{~N}$ & 747.41 & 374.21 & 730.39 & 365.70 & 729.40 & 365.20 & 6 \\
\hline 14 & $1,699.83$ & 850.42 & $1,682.80$ & 841.91 & $1,681.82$ & 841.41 & $\mathrm{P}$ & 633.37 & 317.19 & 616.34 & 308.67 & 615.36 & 308.18 & 5 \\
\hline 15 & $1,786.86$ & 893.94 & $1,769.84$ & 885.42 & $1,768.85$ & 884.93 & $\mathrm{~S}$ & 536.32 & 268.66 & 519.29 & 260.15 & 518.31 & 259.66 & 4 \\
\hline 16 & $1,887.91$ & 944.46 & $1,870.88$ & 935.95 & $1,869.90$ & 935.45 & $\mathrm{~T}$ & 449.28 & 225.15 & 432.26 & 216.63 & 431.27 & 216.14 & 3 \\
\hline 17 & $1,944.93$ & 972.97 & $1,927.91$ & 964.46 & $1,926.92$ & 963.96 & G & 348.24 & 174.62 & 331.21 & 166.11 & & & 2 \\
\hline 18 & & & & & & & $\mathrm{~K}$ & 291.21 & 146.11 & 274.19 & 137.60 & & & 1 \\
\hline
\end{tabular}


MS/MS Fragmentation of EILVEESNVQR

Found in sp|P60510|PP4C_HUMAN, Serine/threonine-protein phosphatase 4 catalytic subunit OS=Homo sapiens GN=PPP4C PE=1 $\mathrm{SV}=1$

Match to Query 30729: 1458.781 from(730.3979,2+)

Title: 192: Scan 991 ( $\mathrm{rt}=29.3788, \mathrm{f}=2, \mathrm{i}=105)$ [D:lab212 $\backslash$ membranelGrace $\backslash$ JoyceliTRAQ_22_2.raw]

Data File:Submitted from 20120508-1(merge) by Mascot Daemon on JOYCE-VAIO

Monoisotopic mass of neutral peptide $\mathrm{Mr}$ (calc): 1458.781

Variable modifications:

Ions Score: 73.28 Expect: 0.000

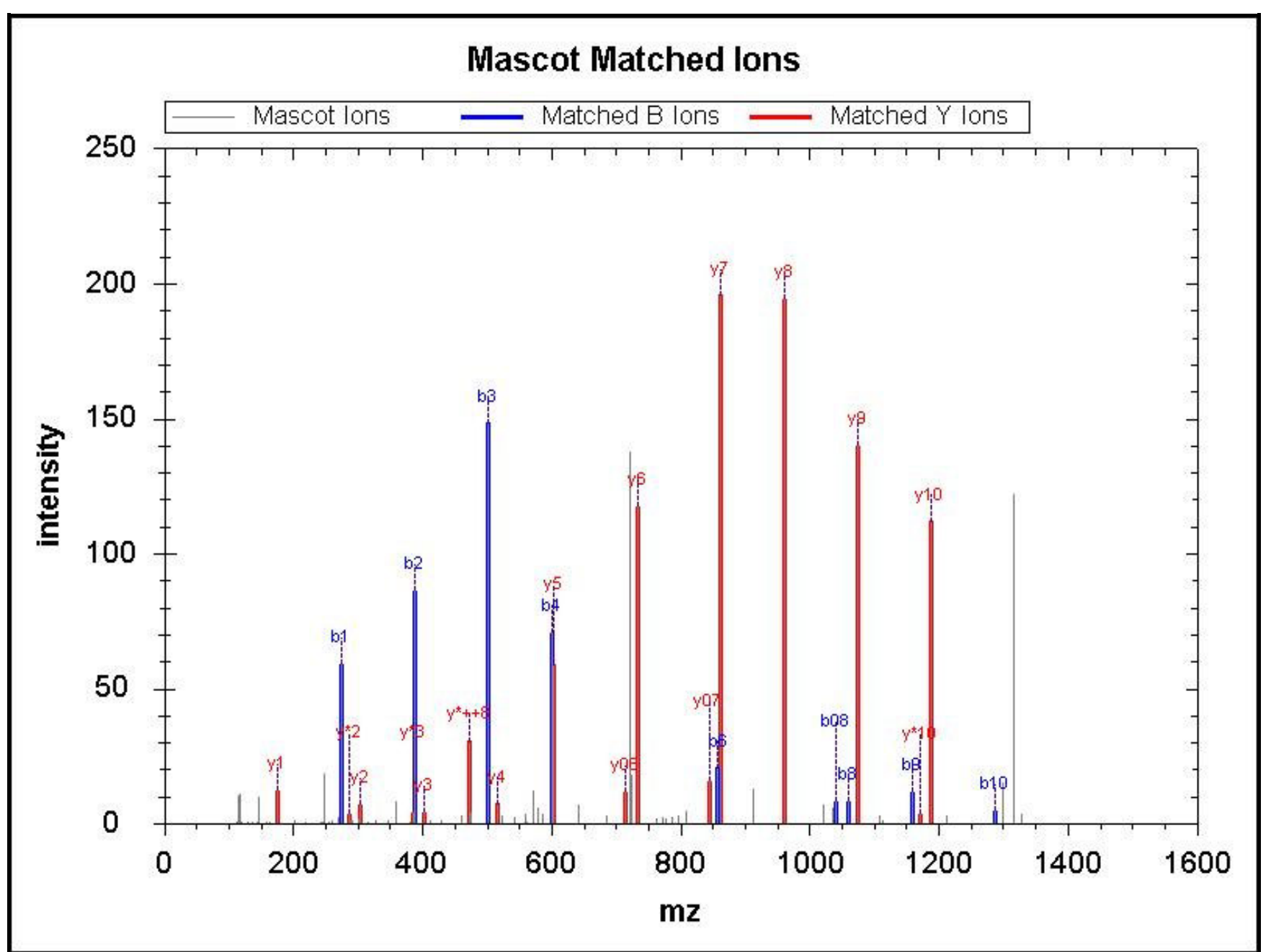

\begin{tabular}{|r|r|r|r|r|r|r|r|r|r|r|r|r|r|r|r|}
\hline $\mathrm{No}$ & $\mathrm{b}$ & $\mathrm{b}++$ & $\mathrm{b}^{*}$ & $\mathrm{~b}+++$ & $\mathrm{b} 0$ & $\mathrm{~b} 0++$ & Seq & $\mathrm{y}$ & $\mathrm{y}++$ & $\mathrm{y}^{*}$ & $\mathrm{y}^{*++}$ & $\mathrm{y} 0$ & $\mathrm{y} 0++$ & $\mathrm{RevNo}$ \\
\hline 1 & 274.15 & 137.58 & & & 256.14 & 128.57 & $\mathrm{E}$ & & & & & & & 11 \\
\hline 2 & 387.24 & 194.12 & & & 369.23 & 185.12 & $\mathrm{I}$ & $1,186.64$ & 593.83 & $1,169.62$ & 585.31 & $1,168.63$ & 584.82 & 10 \\
\hline 3 & 500.32 & 250.66 & & & 482.31 & 241.66 & $\mathrm{~L}$ & $1,073.56$ & 537.28 & $1,056.53$ & 528.77 & $1,055.55$ & 528.28 & 9 \\
\hline 4 & 599.39 & 300.20 & & & 581.38 & 291.19 & $\mathrm{~V}$ & 960.47 & 480.74 & 943.45 & 472.23 & 942.46 & 471.74 & 8 \\
\hline 5 & 728.43 & 364.72 & & & 710.42 & 355.71 & $\mathrm{E}$ & 861.41 & 431.21 & 844.38 & 422.69 & 843.40 & 422.20 & 7 \\
\hline 6 & 857.47 & 429.24 & & & 839.46 & 420.24 & $\mathrm{E}$ & 732.36 & 366.69 & 715.34 & 358.17 & 714.35 & 357.68 & 6 \\
\hline 7 & 944.51 & 472.76 & & & 926.50 & 463.75 & $\mathrm{~S}$ & 603.32 & 302.16 & 586.29 & 293.65 & 585.31 & 293.16 & 5 \\
\hline 8 & $1,058.55$ & 529.78 & $1,041.52$ & 521.26 & $1,040.54$ & 520.77 & $\mathrm{~N}$ & 516.29 & 258.65 & 499.26 & 250.13 & & & 4 \\
\hline 9 & $1,157.62$ & 579.31 & $1,140.59$ & 570.80 & $1,139.61$ & 570.31 & $\mathrm{~V}$ & 402.25 & 201.63 & 385.22 & 193.11 & & & 3 \\
\hline 10 & $1,285.68$ & 643.34 & $1,268.65$ & 634.83 & $1,267.67$ & 634.34 & $\mathrm{Q}$ & 303.18 & 152.09 & 286.15 & 143.58 & & & 2 \\
\hline 11 & & & & & & & $\mathrm{R}$ & 175.12 & 88.06 & 158.09 & 79.55 & & & \\
\hline
\end{tabular}

Query 74205 Hit 1 
Match to Query 74205: 2337.247from(780.0897,3+)

Title: 1159: Sum of 2 scans in range $2590(\mathrm{rt}=67.6027, \mathrm{f}=2, \mathrm{i}=430)$ to $2591(\mathrm{rt}=67.6281, \mathrm{f}=2, \mathrm{i}=431)$

[D:llab212 \membranelGrace JoyceliTRAQ_36_2.raw]

Data File:Submitted from 20120508-1(merge) by Mascot Daemon on JOYCE-VAIO

Monoisotopic mass of neutral peptide $\mathrm{Mr}$ (calc): 2337.247

Variable modifications:

$\mathrm{K} 18$ :iTRAQ4plex (K)

Ions Score: 73.28 Expect: 0.000

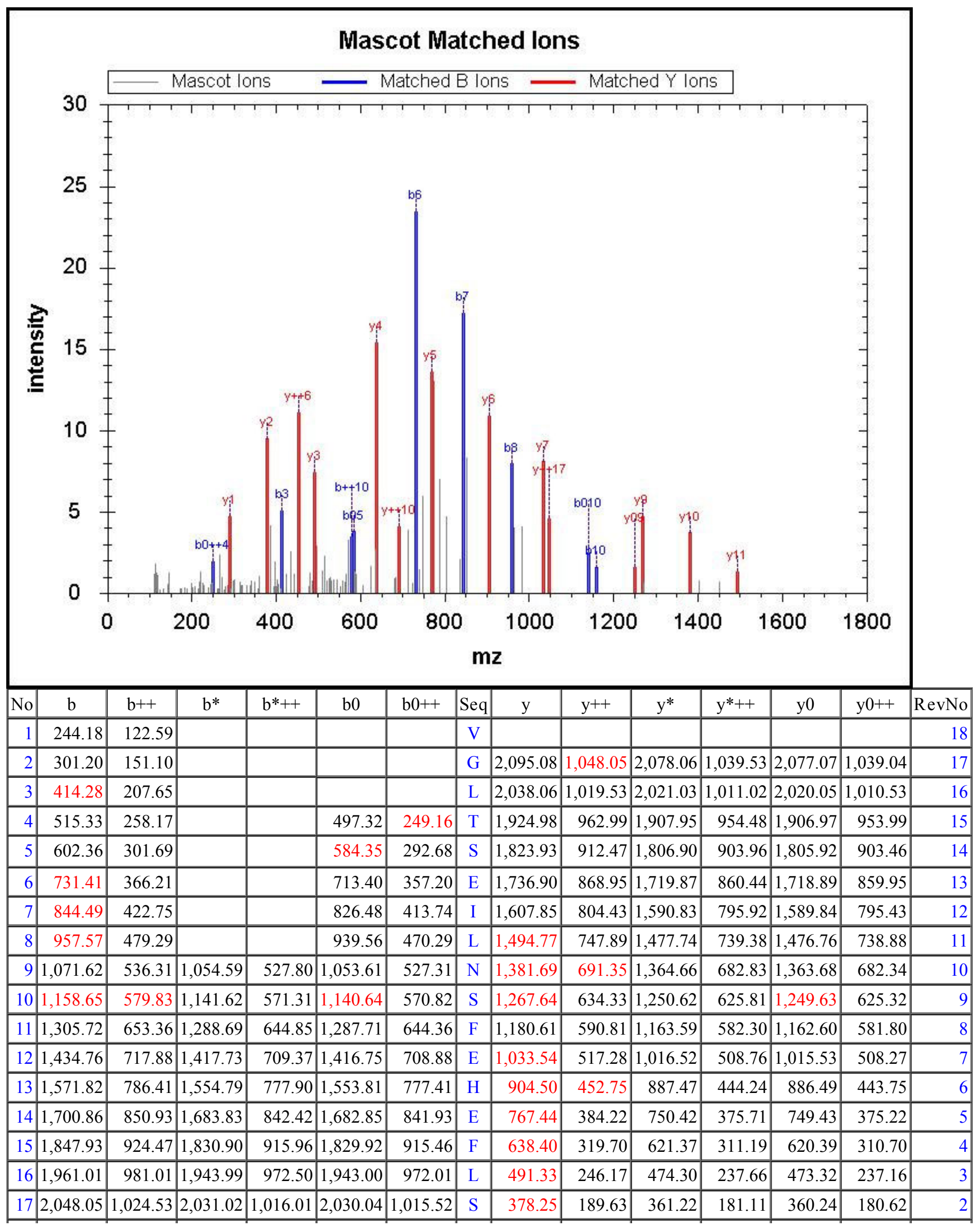


Query 76433 Hit 1

MS/MS Fragmentation of LLEEFVNHIQELQIMDER

Found in sp $|\mathrm{O} 00471| \mathrm{EXOC5}$ HUMAN, Exocyst complex component 5 OS=Homo sapiens GN=EXOC5 PE=1 SV=1

Match to Query 76433: 2399.232from(800.7513,3+)

Title: 1163: Scan $2572(\mathrm{rt}=67.2852, \mathrm{f}=2, \mathrm{i}=409)$ [D:llab212 \membranelGrace JoyceliTRAQ_33_1.raw]

Data File:Submitted from 20120508-1(merge) by Mascot Daemon on JOYCE-VAIO

Monoisotopic mass of neutral peptide $\mathrm{Mr}($ calc): 2399.232

Variable modifications:

Ions Score: 72.75 Expect: 0.000

\section{Mascot Matched lons}

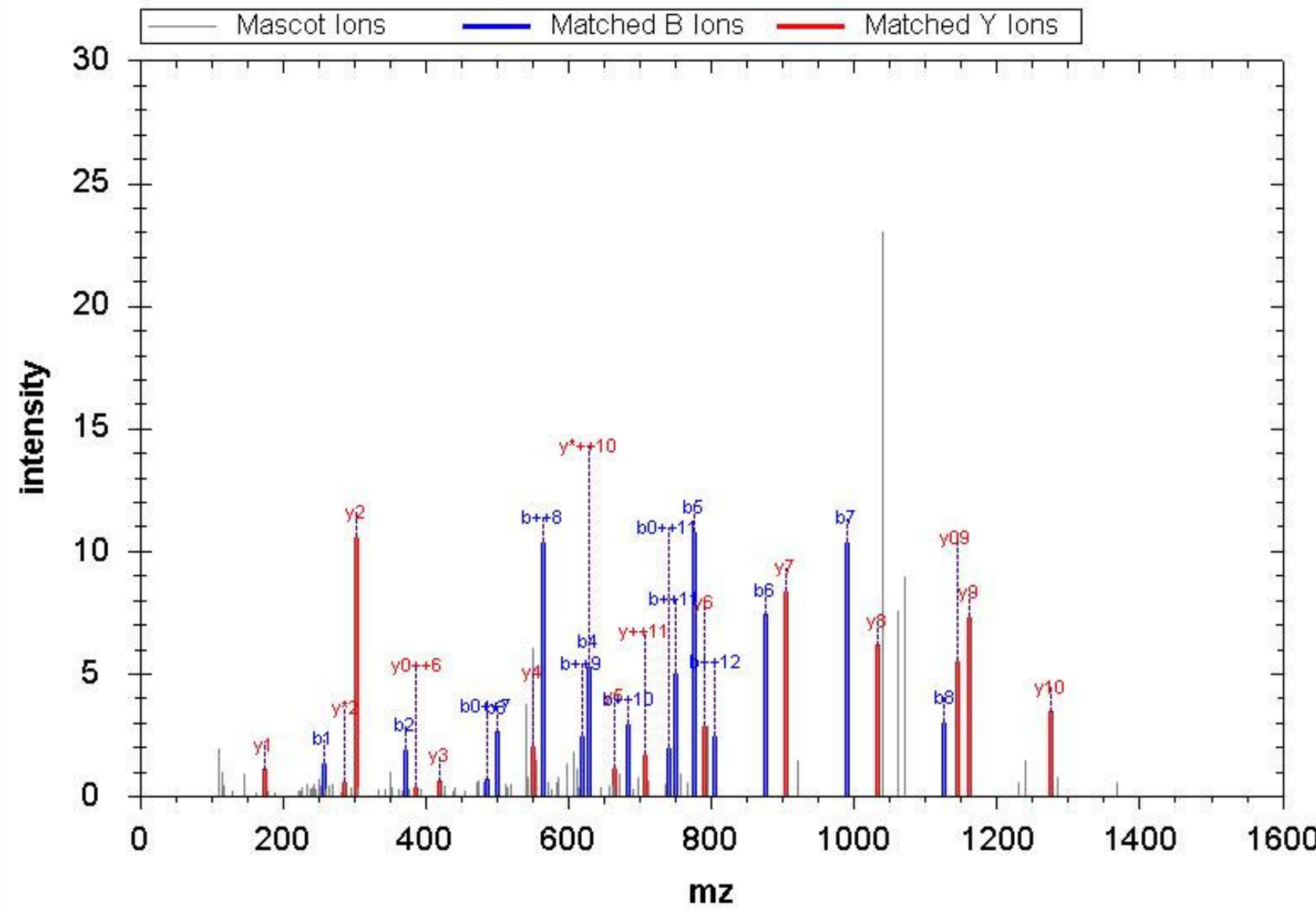

\begin{tabular}{|c|c|c|c|c|c|c|c|c|c|c|c|c|c|c|}
\hline No & b & $\mathrm{b}++$ & $b^{*}$ & $b^{*++}$ & b0 & $\mathrm{b} 0++$ & Seq & $\mathrm{y}$ & $\mathrm{y}^{++}$ & $y^{*}$ & $\mathrm{y}^{*++}$ & y0 & $\mathrm{y} 0++$ & RevNo \\
\hline 1 & 258.19 & 129.60 & & & & & $\mathrm{~L}$ & & & & & & & 18 \\
\hline 2 & 371.28 & 186.14 & & & & & $\mathrm{~L}$ & $2,143.05$ & $1,072.03$ & $2,126.02$ & $1,063.51$ & $2,125.04$ & $1,063.02$ & 17 \\
\hline 3 & 500.32 & 250.66 & & & 482.31 & 241.66 & $\mathrm{E}$ & $2,029.96$ & $1,015.49$ & $2,012.94$ & $1,006.97$ & $2,011.95$ & $1,006.48$ & 16 \\
\hline 4 & 629.36 & 315.18 & & & 611.35 & 306.18 & $\mathrm{E}$ & $1,900.92$ & 950.96 & $1,883.90$ & 942.45 & $1,882.91$ & 941.96 & 15 \\
\hline 5 & 776.43 & 388.72 & & & 758.42 & 379.71 & $\mathrm{~F}$ & $1,771.88$ & 886.44 & $1,754.85$ & 877.93 & $1,753.87$ & 877.44 & 14 \\
\hline 6 & 875.50 & 438.25 & & & 857.49 & 429.25 & V & $1,624.81$ & 812.91 & $1,607.78$ & 804.40 & $1,606.80$ & 803.90 & 13 \\
\hline 7 & 989.54 & 495.27 & 972.52 & 486.76 & 971.53 & 486.27 & $\mathrm{~N}$ & $1,525.74$ & 763.38 & $1,508.72$ & 754.86 & $1,507.73$ & 754.37 & 12 \\
\hline 8 & $1,126.60$ & 563.80 & $1,109.57$ & 555.29 & $1,108.59$ & 554.80 & $\mathrm{H}$ & $1,411.70$ & 706.35 & $1,394.67$ & 697.84 & $1,393.69$ & 697.35 & 11 \\
\hline 9 & $1,239.69$ & 620.35 & $1,222.66$ & 611.83 & $1,221.67$ & 611.34 & I & $1,274.64$ & 637.82 & $1,257.61$ & 629.31 & $1,256.63$ & 628.82 & 10 \\
\hline 10 & $1,367.74$ & 684.38 & $1,350.72$ & 675.86 & $1,349.73$ & 675.37 & Q & $1,161.56$ & 581.28 & $1,144.53$ & 572.77 & $1,143.55$ & 572.28 & 9 \\
\hline 11 & $1,496.79$ & 748.90 & $1,479.76$ & 740.38 & $1,478.78$ & 739.89 & $\mathrm{E}$ & $1,033.50$ & 517.25 & $1,016.47$ & 508.74 & $1,015.49$ & 508.25 & 8 \\
\hline 12 & $1,609.87$ & 805.44 & $1,592.84$ & 796.93 & $1,591.86$ & 796.43 & $\mathrm{~L}$ & 904.46 & 452.73 & 887.43 & 444.22 & 886.45 & 443.73 & 7 \\
\hline 13 & $1,737.93$ & 869.47 & $1,720.90$ & 860.96 & $1,719.92$ & 860.46 & Q & 791.37 & 396.19 & 774.35 & 387.68 & 773.36 & 387.18 & 6 \\
\hline 14 & $1,851.01$ & 926.01 & $1,833.99$ & 917.50 & $1,833.00$ & 917.01 & I & 663.31 & 332.16 & 646.29 & 323.65 & 645.30 & 323.15 & 5 \\
\hline
\end{tabular}




\begin{tabular}{|r|r|r|r|r|r|r|r|r|r|r|r|r|r|r|}
\hline 15 & $1,982.05$ & 991.53 & $1,965.03$ & 983.02 & $1,964.04$ & 982.53 & $\mathrm{M}$ & 550.23 & 275.62 & 533.20 & 267.10 & 532.22 & 266.61 & 4 \\
\hline 16 & $2,097.08$ & $1,049.04$ & $2,080.05$ & $1,040.53$ & $2,079.07$ & $1,040.04$ & $\mathrm{D}$ & 419.19 & 210.10 & 402.16 & 201.58 & 401.18 & 201.09 & 3 \\
\hline 17 & $2,226.12$ & $1,113.57$ & $2,209.10$ & $1,105.05$ & $2,208.11$ & $1,104.56$ & $\mathrm{E}$ & 304.16 & 152.58 & 287.13 & 144.07 & 286.15 & 143.58 & 2 \\
\hline 18 & & & & & & & $\mathrm{R}$ & 175.12 & 88.06 & 158.09 & 79.55 & & & 1 \\
\hline
\end{tabular}

Query 88597 Hit 1

MS/MS Fragmentation of DFVEAPSQMLENWVWEQEPLLR

Found in sp|P52888|THOP1_HUM AN, Thimet oligopeptidase OS=Homo sapiens GN=THOP1 PE=1 SV=2

Match to Query 88597: 2859.406from(954.1427,3+)

Title: 1204: Sum of 2 scans in range $2827(\mathrm{r}=72.6365, \mathrm{f}=4, \mathrm{i}=784)$ to $2828(\mathrm{r}=72.6619, \mathrm{f}=4, \mathrm{i}=785)$

[D:llab212 \membranelGrace Joycel20120320_TTRAQ_20-1.raw]

Data File:Submitted from 20120508-1(merge) by Mascot Daemon on JOYCE-VAIO

Monoisotopic mass of neutral peptide Mr(calc): 2859.406

Variable modifications:

Ions Score: 72.65 Expect: 0.000

\section{Mascot Matched lons}

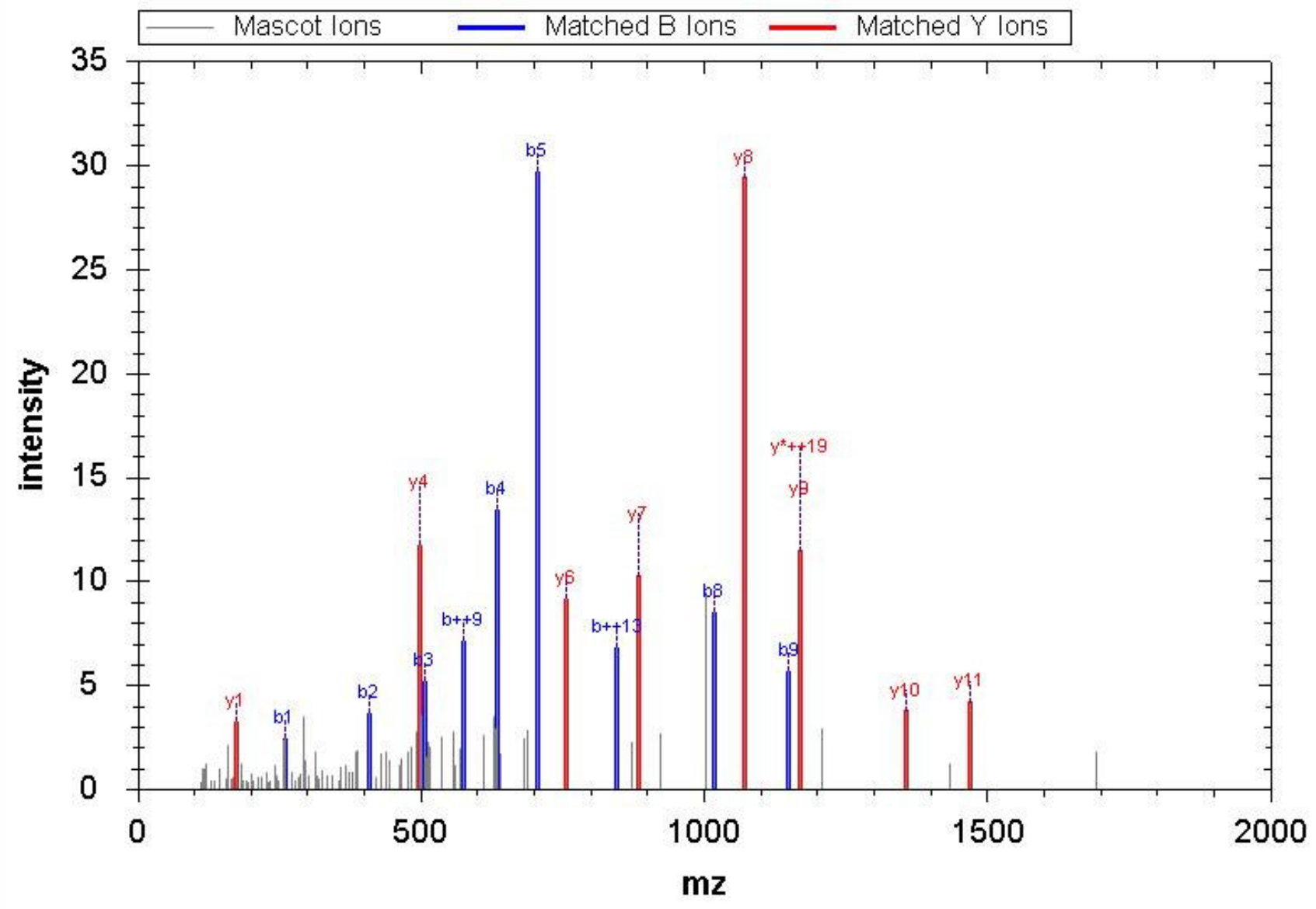

\begin{tabular}{|c|c|c|c|c|c|c|c|c|c|c|c|c|c|c|}
\hline No & $b$ & $\mathrm{~b}++$ & $b^{*}$ & $b^{*++}$ & b0 & $\mathrm{b} 0++$ & Seq & $\mathrm{y}$ & $\mathrm{y}^{++}$ & $y^{*}$ & $y^{*++}$ & y0 & $\mathrm{y} 0++$ & RevNo \\
\hline 1 & 260.14 & 130.57 & & & 242.13 & 121.57 & $\mathrm{D}$ & & & & & & & 22 \\
\hline 2 & 407.20 & 204.11 & & & 389.19 & 195.10 & $\mathrm{~F}$ & $2,601.28$ & $1,301.14$ & $2,584.25$ & $1,292.63$ & $2,583.27$ & $1,292.14$ & 21 \\
\hline 3 & 506.27 & 253.64 & & & 488.26 & 244.63 & V & $2,454.21$ & $1,227.61$ & $2,437.19$ & $1,219.10$ & $2,436.20$ & $1,218.60$ & 20 \\
\hline 4 & 635.32 & 318.16 & & & 617.31 & 309.16 & $\mathrm{E}$ & $2,355.14$ & $1,178.08$ & $2,338.12$ & $1,169.56$ & $2,337.13$ & $1,169.07$ & 19 \\
\hline 5 & 706.35 & 353.68 & & & 688.34 & 344.67 & A & $2,226.10$ & $1,113.55$ & $2,209.07$ & $1,105.04$ & $2,208.09$ & $1,104.55$ & 18 \\
\hline 6 & 803.41 & 402.21 & & & 785.40 & 393.20 & $\mathrm{P}$ & $2,155.06$ & $1,078.04$ & $2,138.04$ & $1,069.52$ & $2,137.05$ & $1,069.03$ & 17 \\
\hline 7 & 890.44 & 445.72 & & & 872.43 & 436.72 & $\mathrm{~S}$ & $2,058.01$ & $1,029.51$ & $2,040.98$ & $1,021.00$ & $2,040.00$ & $1,020.50$ & 16 \\
\hline 8 & $1,018.50$ & 509.75 & $1,001.47$ & 501.24 & $1,000.49$ & 500.75 & Q & $1,970.98$ & 985.99 & $1,953.95$ & 977.48 & $1,952.97$ & 976.99 & 15 \\
\hline 9 & $1,149.54$ & 575.27 & $1,132.51$ & 566.76 & $1,131.53$ & 566.27 & M & $1,842.92$ & 921.96 & $1,825.89$ & 913.45 & $1,824.91$ & 912.96 & 14 \\
\hline 10 & $1,262.62$ & 631.81 & $1,245.59$ & 623.30 & $1,244.61$ & 622.81 & $\mathrm{~L}$ & $1,711.88$ & 856.44 & $1,694.85$ & 847.93 & $1,693.87$ & 847.44 & 13 \\
\hline
\end{tabular}




\begin{tabular}{|r|r|r|r|r|r|r|r|r|r|r|r|r|r|r|r|r|}
\hline 11 & $1,391.66$ & 696.34 & $1,374.64$ & 687.82 & $1,373.65$ & 687.33 & $\mathrm{E}$ & $1,598.80$ & 799.90 & $1,581.77$ & 791.39 & $1,580.79$ & 790.90 & 12 \\
\hline 12 & $1,505.71$ & 753.36 & $1,488.68$ & 744.84 & $1,487.70$ & 744.35 & $\mathrm{~N}$ & $1,469.75$ & 735.38 & $1,452.73$ & 726.87 & $1,451.74$ & 726.38 & 11 \\
\hline 13 & $1,691.79$ & 846.40 & $1,674.76$ & 837.88 & $1,673.78$ & 837.39 & $\mathrm{~W}$ & $1,355.71$ & 678.36 & $1,338.68$ & 669.85 & $1,337.70$ & 669.35 & 10 \\
\hline 14 & $1,790.85$ & 895.93 & $1,773.83$ & 887.42 & $1,772.84$ & 886.93 & $\mathrm{~V}$ & $1,169.63$ & 585.32 & $1,152.60$ & 576.81 & $1,151.62$ & 576.31 & 9 \\
\hline 15 & $1,976.93$ & 988.97 & $1,959.91$ & 980.46 & $1,958.92$ & 979.97 & $\mathrm{~W}$ & $1,070.56$ & 535.79 & $1,053.54$ & 527.27 & $1,052.55$ & 526.78 & 8 \\
\hline 16 & $2,105.98$ & $1,053.49$ & $2,088.95$ & $1,044.98$ & $2,087.97$ & $1,044.49$ & $\mathrm{E}$ & 884.48 & 442.75 & 867.46 & 434.23 & 866.47 & 433.74 & 7 \\
\hline 17 & $2,234.03$ & $1,117.52$ & $2,217.01$ & $1,109.01$ & $2,216.02$ & $1,108.52$ & $\mathrm{Q}$ & 755.44 & 378.22 & 738.41 & 369.71 & 737.43 & 369.22 & 6 \\
\hline 18 & $2,363.08$ & $1,182.04$ & $2,346.05$ & $1,173.53$ & $2,345.07$ & $1,173.04$ & $\mathrm{E}$ & 627.38 & 314.19 & 610.36 & 305.68 & 609.37 & 305.19 & 5 \\
\hline 19 & $2,460.13$ & $1,230.57$ & $2,443.10$ & $1,222.06$ & $2,442.12$ & $1,221.56$ & $\mathrm{P}$ & 498.34 & 249.67 & 481.31 & 241.16 & & & 4 \\
\hline 20 & $2,573.21$ & $1,287.11$ & $2,556.19$ & $1,278.60$ & $2,555.20$ & $1,278.11$ & $\mathrm{~L}$ & 401.29 & 201.15 & 384.26 & 192.63 & & & 3 \\
\hline 21 & $2,686.30$ & $1,343.65$ & $2,669.27$ & $1,335.14$ & $2,668.29$ & $1,334.65$ & $\mathrm{~L}$ & 288.20 & 144.61 & 271.18 & 136.09 & & & 2 \\
\hline 22 & & & & & & & $\mathrm{R}$ & 175.12 & 88.06 & 158.09 & 79.55 & & & 1 \\
\hline
\end{tabular}

Query 72806 Hit 1

MS/MS Fragmentation of DVQFAVQQVLQEEHFDAR

Found in sp|Q8R 146|APEH_MOUSE, Acylamino-acid-releasing enzyme OS=Mus musculus GN=Apeh PE=2 SV=2

Match to Query 72806: 2302.143from(768.3881,3+)

Title: 872: Sum of 2 scans in range $1927(\mathrm{rt}=52.8858, \mathrm{f}=4, \mathrm{i}=583)$ to $1928(\mathrm{rt}=52.9112, \mathrm{f}=4, \mathrm{i}=584)$

[D:llab212\membranelGrace JoyceliTRAQ_32_1.raw]

Data File:Submitted from 20120508-1(merge) by Mascot Daemon on JOYCE-VAIO

Monoisotopic mass of neutral peptide $\mathrm{Mr}$ (calc): 2302.143

Variable modifications:

Ions Score: 72.6 Expect: 0.000

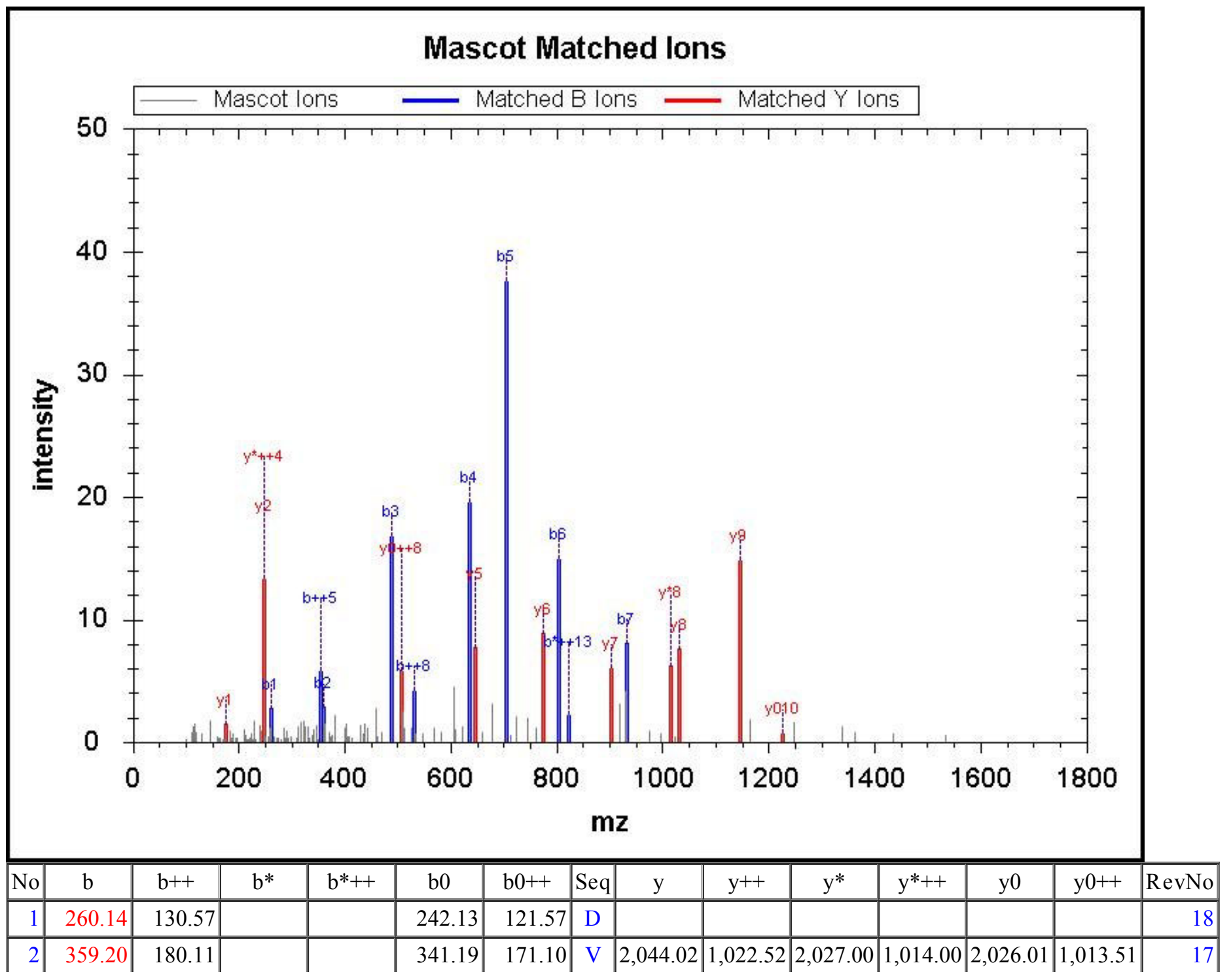




\begin{tabular}{|r|r|r|r|r|r|r|r|r|r|r|r|r|r|r|}
\hline 3 & 487.26 & 244.14 & 470.24 & 235.62 & 469.25 & 235.13 & $\mathrm{Q}$ & $1,944.96$ & 972.98 & $1,927.93$ & 964.47 & $1,926.95$ & 963.98 & 16 \\
\hline 4 & 634.33 & 317.67 & 617.31 & 309.16 & 616.32 & 308.66 & $\mathrm{~F}$ & $1,816.90$ & 908.95 & $1,799.87$ & 900.44 & $1,798.89$ & 899.95 & 15 \\
\hline 5 & 705.37 & 353.19 & 688.34 & 344.67 & 687.36 & 344.18 & $\mathrm{~A}$ & $1,669.83$ & 835.42 & $1,652.80$ & 826.91 & $1,651.82$ & 826.41 & 14 \\
\hline 6 & 804.44 & 402.72 & 787.41 & 394.21 & 786.43 & 393.72 & $\mathrm{~V}$ & $1,598.79$ & 799.90 & $1,581.77$ & 791.39 & $1,580.78$ & 790.89 & 13 \\
\hline 7 & 932.50 & 466.75 & 915.47 & 458.24 & 914.49 & 457.75 & $\mathrm{Q}$ & $1,499.72$ & 750.37 & $1,482.70$ & 741.85 & $1,481.71$ & 741.36 & 12 \\
\hline 8 & $1,060.55$ & 530.78 & $1,043.53$ & 522.27 & $1,042.54$ & 521.78 & $\mathrm{Q}$ & $1,371.67$ & 686.34 & $1,354.64$ & 677.82 & $1,353.65$ & 677.33 & 11 \\
\hline 9 & $1,159.62$ & 580.32 & $1,142.60$ & 571.80 & $1,141.61$ & 571.31 & $\mathrm{~V}$ & $1,243.61$ & 622.31 & $1,226.58$ & 613.79 & $1,225.60$ & 613.30 & 10 \\
\hline 10 & $1,272.71$ & 636.86 & $1,255.68$ & 628.34 & $1,254.70$ & 627.85 & $\mathrm{~L}$ & $1,144.54$ & 572.77 & $1,127.51$ & 564.26 & $1,126.53$ & 563.77 & 9 \\
\hline 11 & $1,400.77$ & 700.89 & $1,383.74$ & 692.37 & $1,382.75$ & 691.88 & $\mathrm{Q}$ & $1,031.45$ & 516.23 & $1,014.43$ & 507.72 & $1,013.44$ & 507.23 & 8 \\
\hline 12 & $1,529.81$ & 765.41 & $1,512.78$ & 756.89 & $1,511.80$ & 756.40 & $\mathrm{E}$ & 903.40 & 452.20 & 886.37 & 443.69 & 885.38 & 443.20 & 7 \\
\hline 13 & $1,658.85$ & 829.93 & $1,641.82$ & 821.42 & $1,640.84$ & 820.92 & $\mathrm{E}$ & 774.35 & 387.68 & 757.33 & 379.17 & 756.34 & 378.67 & 6 \\
\hline 14 & $1,795.91$ & 898.46 & $1,778.88$ & 889.95 & $1,777.90$ & 889.45 & $\mathrm{H}$ & 645.31 & 323.16 & 628.28 & 314.65 & 627.30 & 314.15 & 5 \\
\hline 15 & $1,942.98$ & 971.99 & $1,925.95$ & 963.48 & $1,924.97$ & 962.99 & $\mathrm{~F}$ & 508.25 & 254.63 & 491.22 & 246.12 & 490.24 & 245.62 & 4 \\
\hline 16 & $2,058.00$ & $1,029.51$ & $2,040.98$ & $1,020.99$ & $2,039.99$ & $1,020.50$ & $\mathrm{D}$ & 361.18 & 181.10 & 344.16 & 172.58 & 343.17 & 172.09 & 3 \\
\hline 17 & $2,129.04$ & $1,065.02$ & $2,112.02$ & $1,056.51$ & $2,111.03$ & $1,056.02$ & $\mathrm{~A}$ & 246.16 & 123.58 & 229.13 & 115.07 & & & 2 \\
\hline 18 & & & & & & & $\mathrm{R}$ & 175.12 & 88.06 & 158.09 & 79.55 & & & 1 \\
\hline
\end{tabular}

Query 64045 Hit 1

MS/MS Fragmentation of SAFLLQNLLVGHPEHK

Found in sp|Q9NZL4|HPBP1_HUM AN, Hsp70-binding protein 1 OS=Homo sapiens GN=HSPBP1 PE=1 SV=1

Match to Query 64045: 2090.196from(697.7391,3+)

Title: 652: Scan 1803 ( $\mathrm{rt}=48.8846, \mathrm{f}=2, \mathrm{i}=316$ ) [D:lab212 \membranelGracelJoyceliTRAQ_48_1.raw]

Data File:Submitted from 20120508-1 (merge) by Mascot Daemon on JOYCE-VAIO

Monoisotopic mass of neutral peptide $\mathrm{Mr}$ (calc): 2090.196

Variable modifications:

K16 :TRAQ4plex (K)

Ions Score: 72.32 Expect: 0.000 


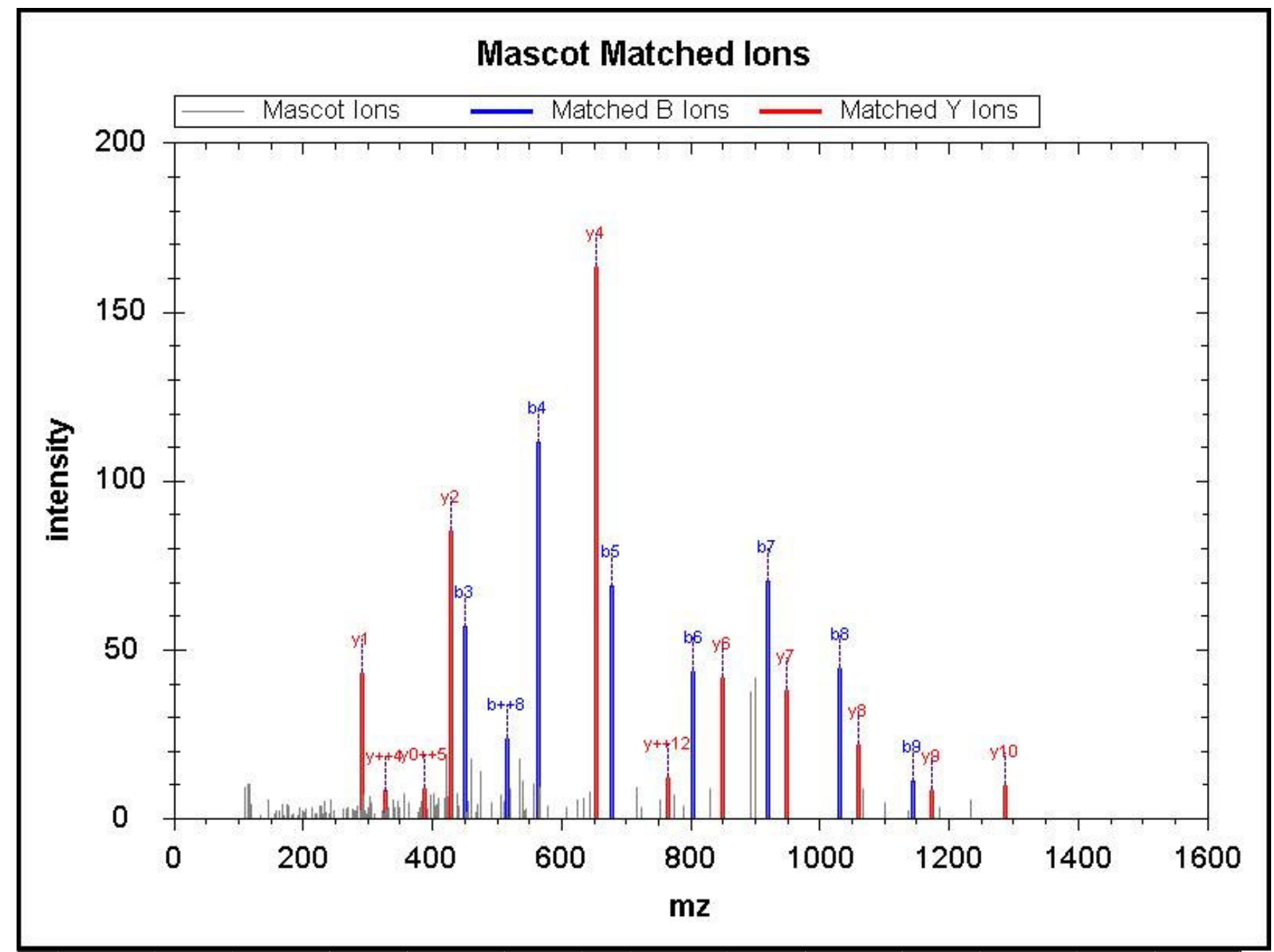

\begin{tabular}{|r|r|r|r|r|r|r|r|r|r|r|r|r|r|r|}
\hline \hline No & $\mathrm{b}$ & $\mathrm{b}++$ & $\mathrm{b}^{*}$ & $\mathrm{~b}{ }^{*++}$ & $\mathrm{b} 0$ & $\mathrm{~b} 0++$ & $\mathrm{Seq}$ & $\mathrm{y}$ & $\mathrm{y}++$ & $\mathrm{y}^{*}$ & $\mathrm{y}^{*++}$ & $\mathrm{y} 0$ & $\mathrm{y} 0++$ & $\mathrm{RevNo}$ \\
\hline 1 & 232.14 & 116.57 & & & 214.13 & 107.57 & $\mathrm{~S}$ & & & & & & & 16 \\
\hline 2 & 303.18 & 152.09 & & & 285.17 & 143.09 & $\mathrm{~A}$ & $1,860.06$ & 930.53 & $1,843.04$ & 922.02 & $1,842.05$ & 921.53 & 15 \\
\hline 3 & 450.25 & 225.63 & & & 432.24 & 216.62 & $\mathrm{~F}$ & $1,789.02$ & 895.02 & $1,772.00$ & 886.50 & $1,771.01$ & 886.01 & 14 \\
\hline 4 & 563.33 & 282.17 & & & 545.32 & 273.16 & $\mathrm{~L}$ & $1,641.96$ & 821.48 & $1,624.93$ & 812.97 & $1,623.95$ & 812.48 & 13 \\
\hline 5 & 676.41 & 338.71 & & & 658.40 & 329.71 & $\mathrm{~L}$ & $1,528.87$ & 764.94 & $1,511.85$ & 756.43 & $1,510.86$ & 755.93 & 12 \\
\hline 6 & 804.47 & 402.74 & 787.45 & 394.23 & 786.46 & 393.74 & $\mathrm{Q}$ & $1,415.79$ & 708.40 & $1,398.76$ & 699.88 & $1,397.78$ & 699.39 & 11 \\
\hline 7 & 918.52 & 459.76 & 901.49 & 451.25 & 900.51 & 450.76 & $\mathrm{~N}$ & $1,287.73$ & 644.37 & $1,270.70$ & 635.85 & $1,269.72$ & 635.36 & 10 \\
\hline 8 & $1,031.60$ & 516.30 & $1,014.57$ & 507.79 & $1,013.59$ & 507.30 & $\mathrm{~L}$ & $1,173.69$ & 587.35 & $1,156.66$ & 578.83 & $1,155.68$ & 578.34 & 9 \\
\hline 9 & $1,144.68$ & 572.85 & $1,127.66$ & 564.33 & $1,126.67$ & 563.84 & $\mathrm{~L}$ & $1,060.60$ & 530.80 & $1,043.58$ & 522.29 & $1,042.59$ & 521.80 & 8 \\
\hline 10 & $1,243.75$ & 622.38 & $1,226.73$ & 613.87 & $1,225.74$ & 613.37 & $\mathrm{~V}$ & 947.52 & 474.26 & 930.49 & 465.75 & 929.51 & 465.26 & 7 \\
\hline 11 & $1,300.77$ & 650.89 & $1,283.75$ & 642.38 & $1,282.76$ & 641.89 & $\mathrm{G}$ & 848.45 & 424.73 & 831.42 & 416.22 & 830.44 & 415.72 & 6 \\
\hline 12 & $1,437.83$ & 719.42 & $1,420.81$ & 710.91 & $1,419.82$ & 710.42 & H & 791.43 & 396.22 & 774.40 & 387.70 & 773.42 & 387.21 & 5 \\
\hline 13 & $1,534.89$ & 767.95 & $1,517.86$ & 759.43 & $1,516.88$ & 758.94 & P & 654.37 & 327.69 & 637.34 & 319.17 & 636.36 & 318.68 & 4 \\
\hline 14 & $1,663.93$ & 832.47 & $1,646.90$ & 823.95 & $1,645.92$ & 823.46 & E & 557.32 & 279.16 & 540.29 & 270.65 & 539.31 & 270.16 & 3 \\
\hline 15 & $1,800.99$ & 901.00 & $1,783.96$ & 892.48 & $1,782.98$ & 891.99 & H & 428.27 & 214.64 & 411.25 & 206.13 & & & 2 \\
\hline 16 & & & & & & & K & 291.21 & 146.11 & 274.19 & 137.60 & & & 1 \\
\hline
\end{tabular}

Query 72306 Hit 1

MS/MS Fragmentation of VLVHPPQDGEDEPTLVQK

Found in sp|Q16740|CLPP_HUMAN, Putative ATP-dependent Clp protease proteolytic subunit

Match to Query 72306: 2288.217from(763.7464,3+)

Title: 436: Sum of 2 scans in range 1038 ( $\mathrm{rt}=32.7903, \mathrm{f}=4, \mathrm{i}=291)$ to $1039(\mathrm{rt}=32.8157, \mathrm{f}=4, \mathrm{i}=292$ )

[D:llab212 \membranelGrace JoyceliTRAQ_36_1.raw]

Data File:Submitted from 20120508-1(merge) by Mascot Daemon on JOYCE-VAIO

Monoisotopic mass of neutral peptide $\mathrm{Mr}$ (calc): 2288.217 


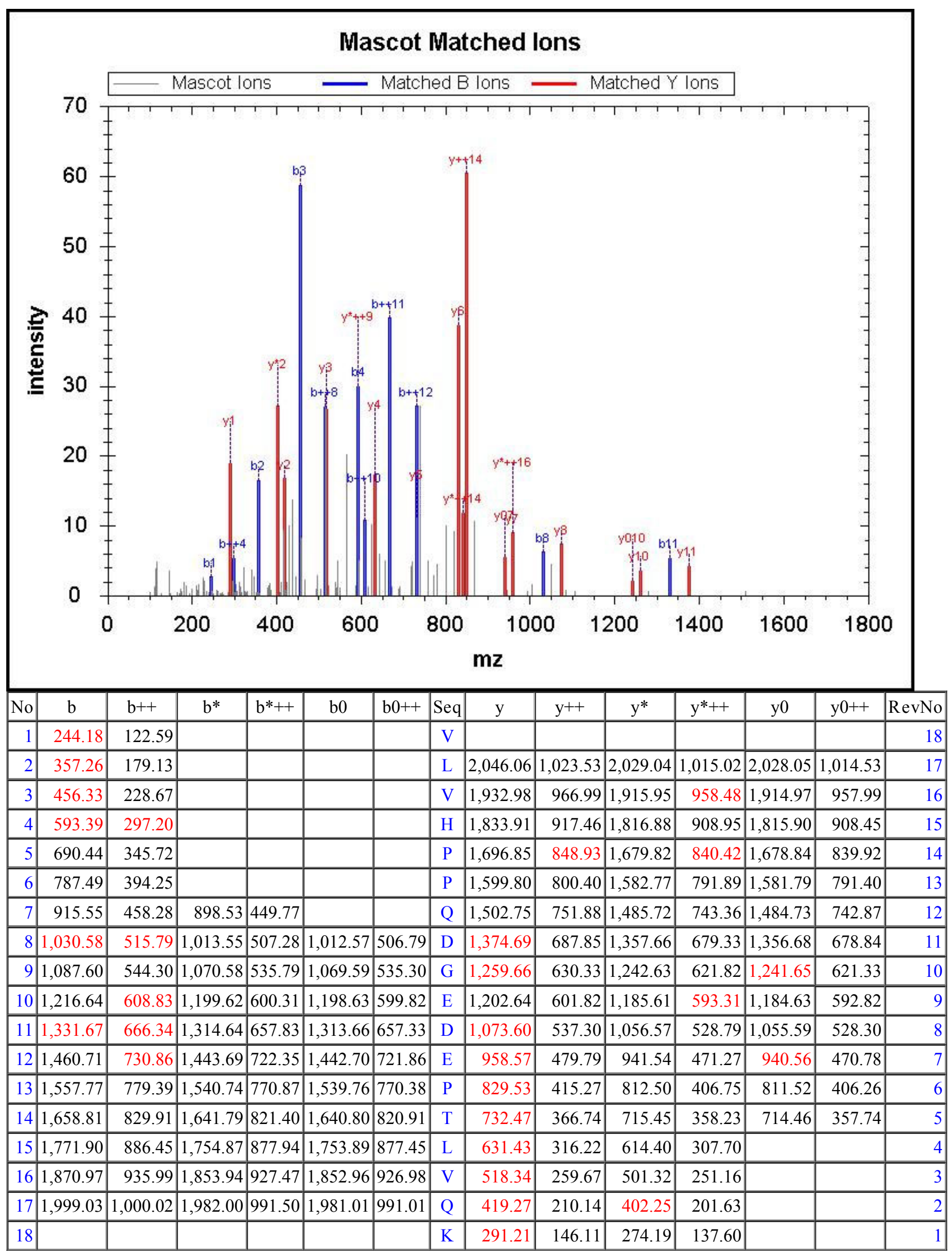

Query 12562 Hit 1 
Found in sp|Q9BTE7|DCNL5_HUMAN, DCN1-like protein 5 OS=Homo sapiens GN=DCUN1D5 PE=1 SV=1

Match to Query 12562: 1116.685 from(559.3495,2+)

Title: 856: Sum of 2 scans in range $2517(\mathrm{rt}=62.9506, \mathrm{f}=4, \mathrm{i}=555)$ to $2518(\mathrm{rt}=62.976, \mathrm{f}=4, \mathrm{i}=556)$

[D:llab212\membranelGrace JoyceliTRAQ_24_2.raw]

Data File:Submitted from 20120508-1(merge) by Mascot Daemon on JOYCE-VAIO

Monoisotopic mass of neutral peptide $\mathrm{Mr}$ (calc): 1116.685

Variable modifications:

Ions Score: 71.9 Expect: 0.000

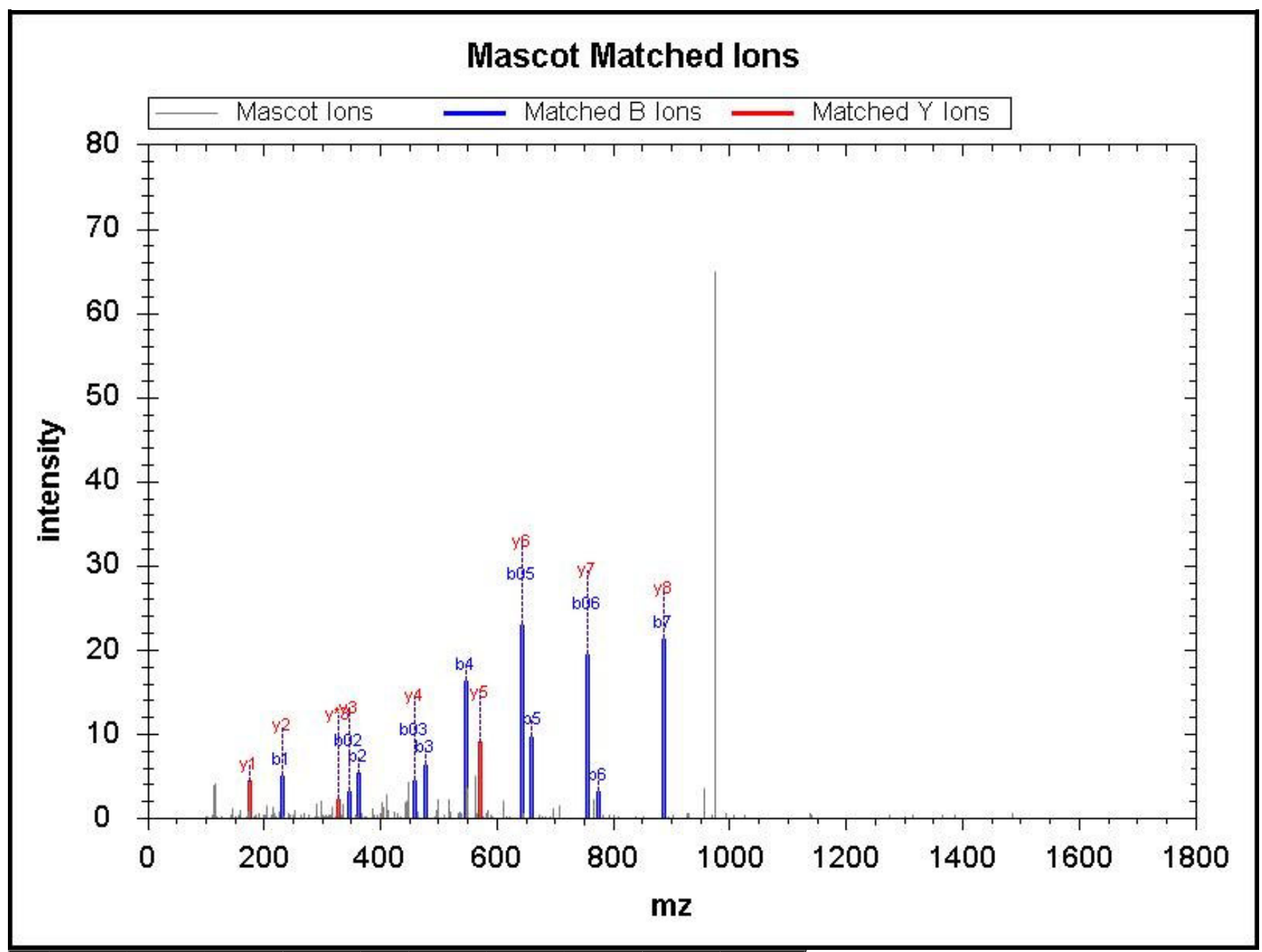

\begin{tabular}{|r|c|c|c|c|c|c|c|c|r|r|}
\hline No & $\mathrm{b}$ & $\mathrm{b}++$ & $\mathrm{b} 0$ & $\mathrm{~b} 0++$ & Seq & $\mathrm{y}$ & $\mathrm{y}++$ & $\mathrm{y}^{*}$ & $\mathrm{y}^{*++}$ & RevNo \\
\hline 1 & 232.14 & 116.57 & 214.13 & 107.57 & $\mathrm{~S}$ & & & & & 9 \\
\hline 2 & 363.18 & 182.09 & 345.17 & 173.09 & $\mathrm{M}$ & 886.55 & 443.78 & 869.53 & 435.27 & 8 \\
\hline 3 & 476.27 & 238.64 & 458.26 & 229.63 & $\mathrm{~L}$ & 755.51 & 378.26 & 738.49 & 369.75 & 7 \\
\hline 4 & 547.30 & 274.16 & 529.29 & 265.15 & $\mathrm{~A}$ & 642.43 & 321.72 & 625.40 & 313.21 & 6 \\
\hline 5 & 660.39 & 330.70 & 642.38 & 321.69 & $\mathrm{~L}$ & 571.39 & 286.20 & 554.37 & 277.69 & 5 \\
\hline 6 & 773.47 & 387.24 & 755.46 & 378.23 & $\mathrm{~L}$ & 458.31 & 229.66 & 441.28 & 221.14 & 4 \\
\hline 7 & 886.56 & 443.78 & 868.54 & 434.78 & $\mathrm{~L}$ & 345.22 & 173.12 & 328.20 & 164.60 & 3 \\
\hline 8 & 943.58 & 472.29 & 925.57 & 463.29 & $\mathrm{G}$ & 232.14 & 116.57 & 215.11 & 108.06 & 2 \\
\hline 9 & & & & & $\mathrm{R}$ & 175.12 & 88.06 & 158.09 & 79.55 & 1 \\
\hline
\end{tabular}

Query 57782 Hit 1

MS/MS Fragmentation of NDSLAGVVIADNEYPSR

Found in sp $\mid$ O15498|YKT6_HUM AN, Synaptobrevin homolog YKT6 OS=Homo sapiens GN=YKT6 PE=1 SV=1

Match to Query 57782: 1962.98from(655.3339,3+)

Title: 280: Sum of 2 scans in range $1540(\mathrm{rt}=40.9315, \mathrm{f}=4, \mathrm{i}=216)$ to $1541(\mathrm{rt}=40.957, \mathrm{f}=4, \mathrm{f}=217)$

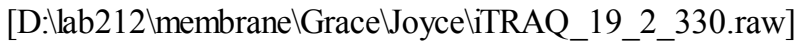

Data File:Submitted from 20120508-1(merge) by Mascot Daemon on JOYCE-VAIO

Monoisotopic mass of neutral peptide $\operatorname{Mr}($ calc): 1962.98

Variable modifications: 


\section{Mascot Matched Ions}

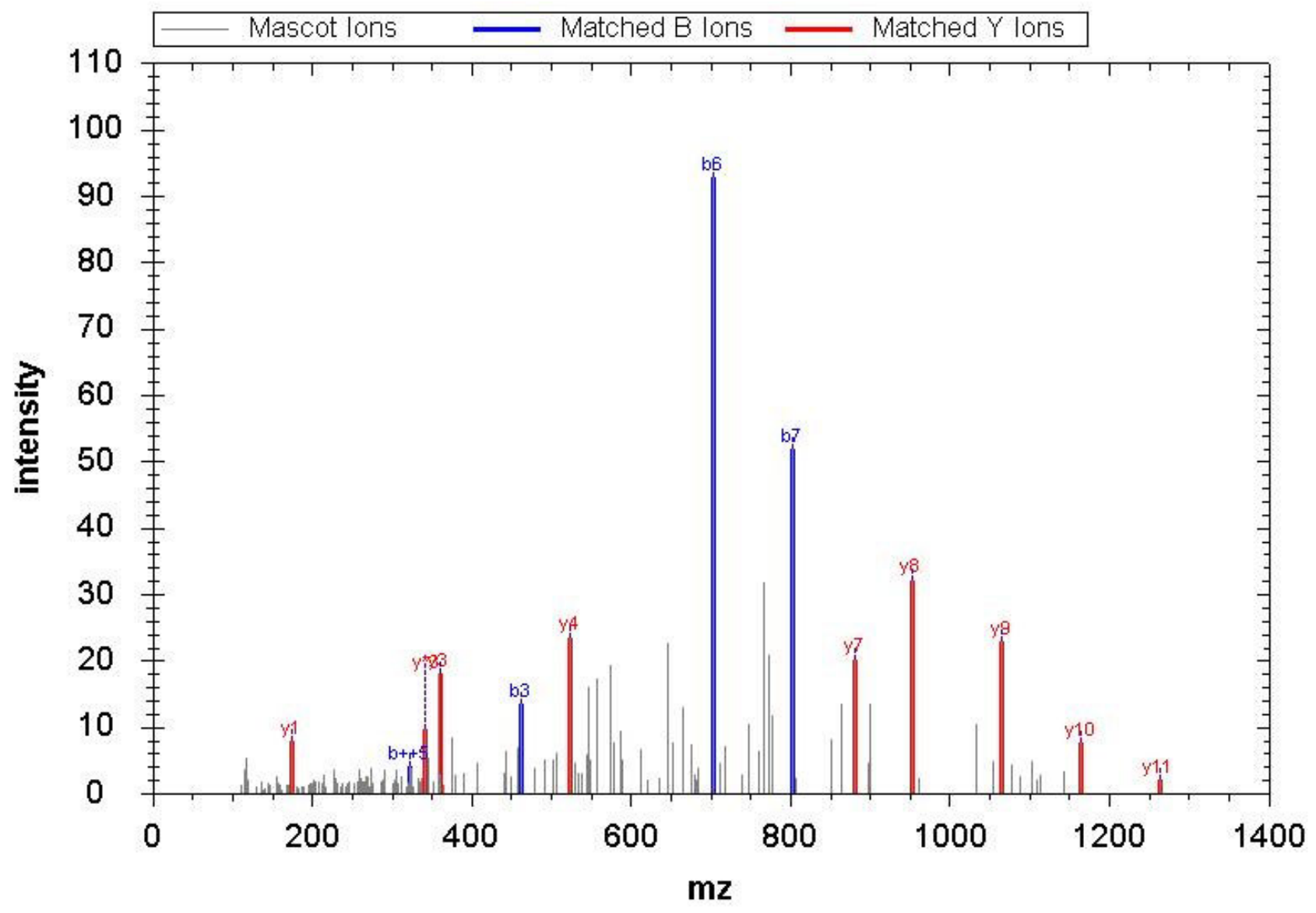

\begin{tabular}{|r|r|r|r|r|r|r|r|r|r|r|r|r|r|r|}
\hline \hline No & $\mathrm{b}$ & $\mathrm{b}++$ & $\mathrm{b} *$ & $\mathrm{~b}++$ & $\mathrm{b} 0$ & $\mathrm{~b} 0++$ & $\mathrm{Seq}$ & $\mathrm{y}$ & $\mathrm{y}++$ & $\mathrm{y}^{*}$ & $\mathrm{y}^{*++}$ & $\mathrm{y} 0$ & $\mathrm{y} 0++$ & $\mathrm{RevNo}$ \\
\hline 1 & 259.15 & 130.08 & 242.13 & 121.57 & & & $\mathrm{~N}$ & & & & & & & 17 \\
\hline 2 & 374.18 & 187.59 & 357.15 & 179.08 & 356.17 & 178.59 & $\mathrm{D}$ & $1,705.84$ & 853.42 & $1,688.81$ & 844.91 & $1,687.83$ & 844.42 & 16 \\
\hline 3 & 461.21 & 231.11 & 444.18 & 222.60 & 443.20 & 222.10 & $\mathrm{~S}$ & $1,590.81$ & 795.91 & $1,573.79$ & 787.40 & $1,572.80$ & 786.90 & 15 \\
\hline 4 & 574.30 & 287.65 & 557.27 & 279.14 & 556.28 & 278.65 & $\mathrm{~L}$ & $1,503.78$ & 752.39 & $1,486.75$ & 743.88 & $1,485.77$ & 743.39 & 14 \\
\hline 5 & 645.33 & 323.17 & 628.31 & 314.66 & 627.32 & 314.16 & $\mathrm{~A}$ & $1,390.70$ & 695.85 & $1,373.67$ & 687.34 & $1,372.69$ & 686.85 & 13 \\
\hline 6 & 702.35 & 351.68 & 685.33 & 343.17 & 684.34 & 342.68 & $\mathrm{G}$ & $1,319.66$ & 660.33 & $1,302.63$ & 651.82 & $1,301.65$ & 651.33 & 12 \\
\hline 7 & 801.42 & 401.21 & 784.40 & 392.70 & 783.41 & 392.21 & $\mathrm{~V}$ & $1,262.64$ & 631.82 & $1,245.61$ & 623.31 & $1,244.63$ & 622.82 & 11 \\
\hline 8 & 900.49 & 450.75 & 883.46 & 442.24 & 882.48 & 441.74 & $\mathrm{~V}$ & $1,163.57$ & 582.29 & $1,146.54$ & 573.77 & $1,145.56$ & 573.28 & 10 \\
\hline 9 & $1,013.57$ & 507.29 & 996.55 & 498.78 & 995.56 & 498.29 & $\mathrm{I}$ & $1,064.50$ & 532.75 & $1,047.47$ & 524.24 & $1,046.49$ & 523.75 & 9 \\
\hline 10 & $1,084.61$ & 542.81 & $1,067.59$ & 534.30 & $1,066.60$ & 533.80 & A & 951.42 & 476.21 & 934.39 & 467.70 & 933.41 & 467.21 & 8 \\
\hline 11 & $1,199.64$ & 600.32 & $1,182.61$ & 591.81 & $1,181.63$ & 591.32 & D & 880.38 & 440.69 & 863.35 & 432.18 & 862.37 & 431.69 & 7 \\
\hline 12 & $1,313.68$ & 657.34 & $1,296.66$ & 648.83 & $1,295.67$ & 648.34 & N & 765.35 & 383.18 & 748.33 & 374.67 & 747.34 & 374.17 & 6 \\
\hline 13 & $1,442.72$ & 721.87 & $1,425.70$ & 713.35 & $1,424.71$ & 712.86 & E & 651.31 & 326.16 & 634.28 & 317.65 & 633.30 & 317.15 & 5 \\
\hline 14 & $1,605.79$ & 803.40 & $1,588.76$ & 794.88 & $1,587.78$ & 794.39 & Y & 522.27 & 261.64 & 505.24 & 253.12 & 504.26 & 252.63 & 4 \\
\hline 15 & $1,702.84$ & 851.92 & $1,685.81$ & 843.41 & $1,684.83$ & 842.92 & P & 359.20 & 180.11 & 342.18 & 171.59 & 341.19 & 171.10 & 3 \\
\hline 16 & $1,789.87$ & 895.44 & $1,772.85$ & 886.93 & $1,771.86$ & 886.43 & S & 262.15 & 131.58 & 245.12 & 123.07 & 244.14 & 122.57 & 2 \\
\hline 17 & & & & & & & R & 175.12 & 88.06 & 158.09 & 79.55 & & 1 \\
\hline
\end{tabular}

Query 74286 Hit 1

MS/MS Fragmentation of QLLGHMEDLEGDAQSVLDAR

Found in sp|Q9NX46|ARHL2_HUM AN, Poly(ADP-ribose) glycohydrolase ARH3 OS=Homo sapiens GN=ADPRHL2 PE=1 SV=1 Match to Query 74286: 2340.152from(781.058,3+)

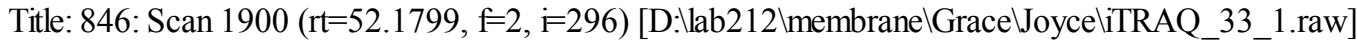




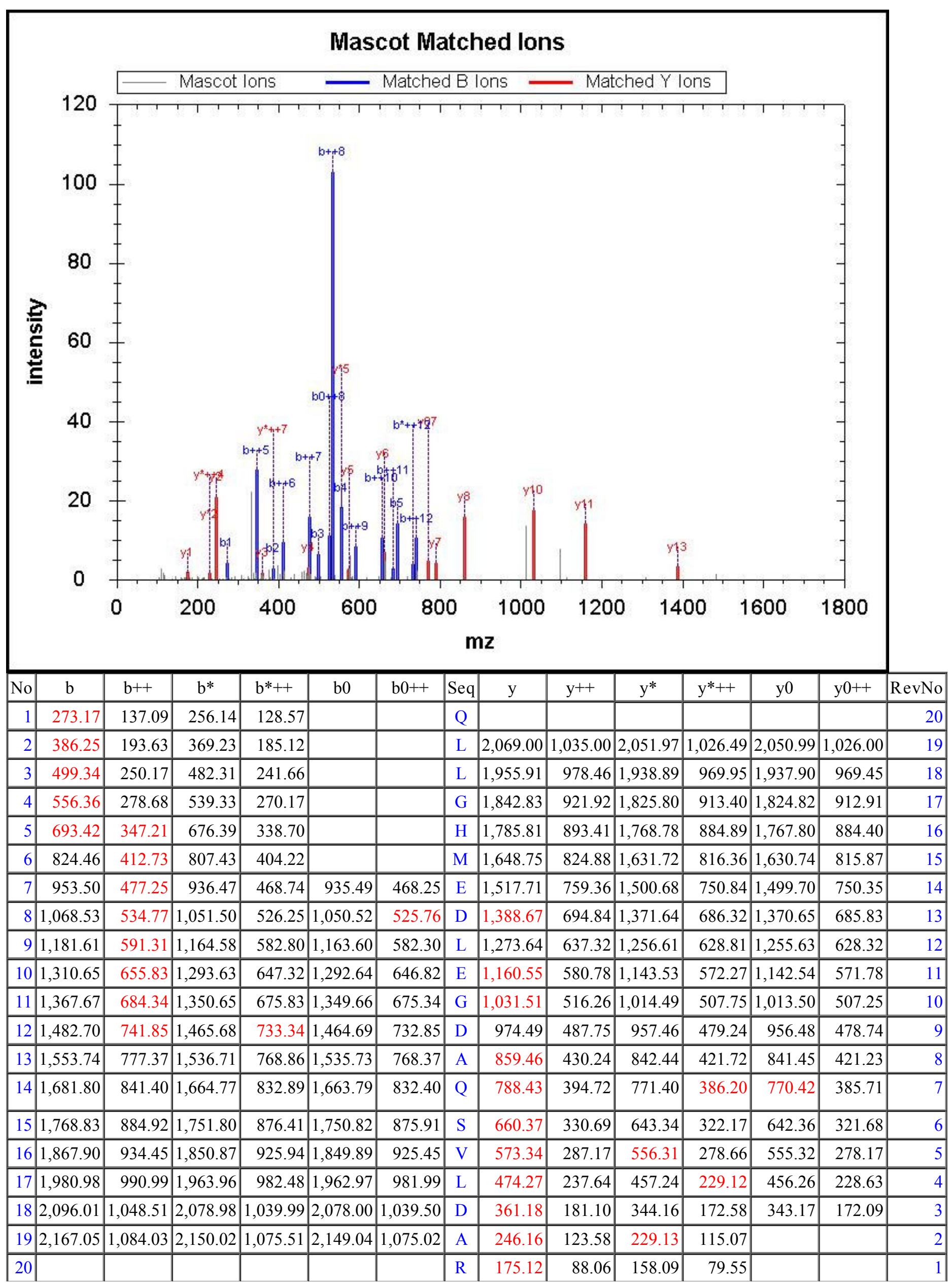

